\title{
Sixth International Workshop on Specialty Optical Fibers and Their Applications (WSOF 2019): Conference Digest
}

, "Sixth International Workshop on Specialty Optical Fibers and Their Applications (WSOF 2019): Conference Digest," Proc. SPIE 11206, Sixth International Workshop on Specialty Optical Fibers and Their Applications (WSOF 2019), 1120601 (5 November 2019); doi: 10.1117/12.2557110

SDIE Event: Sixth Workshop on Specialty Optical Fibers, 2019, Charleston, South Carolina, United States 


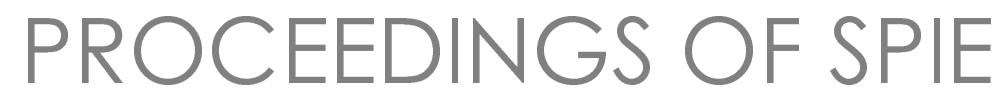

\title{
Sixth International Workshop on Specialty Optical Fibers and Their Applications (WSOF 2019)
}

\author{
John Ballato \\ Liang Dong \\ Editors
}

6-8 November 2019

Charleston, South Carolina, United States

Sponsored by

Air Force Research Laboratory (United States) • OFS Fitel, LLC (United States) • iXblue (France) Fibercore (United Kingdom) • Coherent | Nufern (United States) • Rosendahl Nextrom GmbH (Finland) • Thorlabs, Inc. (United States) - Heraeus GmbH (Germany) • 3SAE Technologies, Inc. (United States) • Fiber Optic Center, Inc. (United States) • NKT Photonics A/S (Denmark) • Fiberguide Industries, Inc. (United States) - Lightel Technologies (United States) • Lumoscribe Ltd. (Cyprus) • Optogear (Finland) • RISE A.S. (Sweden) • COMSET (United States) • FiberBridge Photonics GmbH (Germany) • SPIE

Organized by

Clemson University (United States)

Published by

SPIE

Volume 11206 
The papers in this volume were part of the technical conference cited on the cover and title page. Papers were selected and subject to review by the editors and conference program committee. Some conference presentations may not be available for publication. Additional papers and presentation recordings may be available online in the SPIE Digital Library at SPIEDigitalLibrary.org.

The papers reflect the work and thoughts of the authors and are published herein as submitted. The publisher is not responsible for the validity of the information or for any outcomes resulting from reliance thereon.

Please use the following format to cite material from these proceedings:

Author(s), "Title of Paper," in Sixth International Workshop on Specialty Optical Fibers and Their Applications (WSOF 2019), edited by Liang Dong, John M. Ballato, Proceedings of SPIE Vol. 11206 (SPIE, Bellingham, WA, 2019) Seven-digit Article CID Number.

ISSN: 0277-786X

ISSN: 1996-756X (electronic)

ISBN: 9781510631618

ISBN: 9781510631625 (electronic)

Published by

SPIE

P.O. Box 10, Bellingham, Washington 98227-0010 USA

Telephone +1 3606763290 (Pacific Time) · Fax + 13606471445

SPIE.org

Copyright @ 2019, Society of Photo-Optical Instrumentation Engineers.

Copying of material in this book for internal or personal use, or for the internal or personal use of specific clients, beyond the fair use provisions granted by the U.S. Copyright Law is authorized by SPIE subject to payment of copying fees. The Transactional Reporting Service base fee for this volume is $\$ 21.00$ per article (or portion thereof), which should be paid directly to the Copyright Clearance Center (CCC), 222 Rosewood Drive, Danvers, MA 01923. Payment may also be made electronically through CCC Online at copyright.com. Other copying for republication, resale, advertising or promotion, or any form of systematic or multiple reproduction of any material in this book is prohibited except with permission in writing from the publisher. The CCC fee code is 0277$786 \times / 19 / \$ 21.00$.

Printed in the United States of America by Curran Associates, Inc., under license from SPIE.

Publication of record for individual papers is online in the SPIE Digital Library.

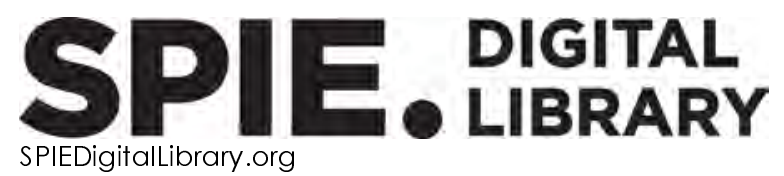

Paper Numbering: Proceedings of SPIE follow an e-First publication model. A unique citation identifier (CID) number is assigned to each article at the time of publication. Utilization of CIDs allows articles to be fully citable as soon as they are published online, and connects the same identifier to all online and print versions of the publication. SPIE uses a seven-digit CID article numbering system structured as follows:

- The first five digits correspond to the SPIE volume number.

- The last two digits indicate publication order within the volume using a Base 36 numbering system employing both numerals and letters. These two-number sets start with $00,01,02,03,04$, 05, 06, 07, 08, 09, OA, OB ... 0Z, followed by 10-1Z, 20-2Z, etc. The CID Number appears on each page of the manuscript. 


\title{
Contents
}

\author{
ix Authors \\ xiii Conference Committees \\ xvii Introduction
}

WEDNESDAY PLENARY SESSION

$1120602 \quad$ Optical fiber and glasses (101) (Plenary Paper) [11206-11]

THURSDAY PLENARY SESSION

1120603 Defense applications of high power fiber lasers (Plenary Paper) [11206-77]

RIDAY PLENARY SESSON

1120604 Bright perspectives for fluoride glass mid-infrared fiber lasers (Plenary Paper) [11206-9]

\section{SESSION 1 RBER LASERS I}

$1120605 \quad$ Next generation of DC fibers enabling high performance and reliability of industrial fiber lasers beyond 1.5kW levels (Invited Paper) [11206-72]

1120606 Fiber lasers and amplifiers for space lidarapplications (Invited Paper) [11206-76]

$1120607 \quad 2$ MW peak power ultrashort pulse amplification using tapered powder-sintered all-solid fibers: Fiber designs, technology, and results [11206-55]

1120608 Fabrication of vapor phase large core Yb doped preform with precise index control for the development of VLMA active fibers [11206-36]

1120609 Highly $\mathbf{T m}^{3+-d o p e d}$ gemanate glass and associated double clad fiber for 2 um lasers and amplifiers [11206-61] 
11206 OA Soft glass mic rostructured optical fibers and their applications (Invited Paper) [11206-73]

11206 OB Progress in mid-IR supercontinuum sources and their applications: an ovenview (Invited Paper) [11206-67]

11206 OC Optical devices based on chalcogenide fibers (Invited Paper) [11206-15]

11206 OD Design of single-mode nanohole suspended-core fibers for all-nomal dispersion supercontinuum generation [11206-32]

\section{SESSION 3 HBER SENSORS I}

11206 OE Novel optical fiber for cochlear implants (Invited Paper) [11206-27]

11206 OF Enhanced fibers for distributed sensing (Invited Paper) [11206-35]

11206 OG $\quad \mathbf{5 0 0 ^ { \circ } \mathbf { C } - r a t e d ~ o p t i c a l ~ f i b e r s ~ f o r ~ h i g h ~ t e m p e r a t u r e ~ a p p l i c a t i o n s ~ [ 1 1 2 0 6 - 1 9 ] ~}$

$11206 \mathrm{OH} \quad$ Carbon-coated optical fiber performance in hydrogen [11206-20]

\section{SESSION 4 RBER DEVICES}

11206 Ol Photonic lantems: beyond telecoms (Invited Paper) [11206-28]

112060 J History and Advancements of FG manufacturing in specialty optical fibers and their use in sensing (Invited Paper) [11206-78]

11206 OK Wedge-shaped fiber lenses with CO2 laser glass ablation [11206-14]

11206 OL Femtosec ond laser inscribed gratings for monolithic MIR fiber lasers [11206-31]

11206 OM Fibre cladding filters through femtosecond laser insc ription [11206-42]

\section{SESSION 5 FBER LASERS II}

11206 ON Reach extension of O-band transmission using BDFA (Invited Paper) [11206-69]

1120600 Recent efforts in power scaling of holmium doped fibre sources (Invited Paper) [11206-71] 
11206 OP Loss reduction in hollow-core optical fiber (Invited Paper) [11206-12]

11206 OQ Negative c unature fibers for gas-filled fiberlasers [11206-29]

11206 OR Tubular anti-resonant hollow core fiber for visible Raman spectroscopy [11206-58]

11206 OS Extruded chalcogenide antiresonant hollow core fiber for mid-IR laser delivery [11206-51]

11206 OT Non-invasive characterization of hollow-core single-ring fibers using whispering gallery mode spectrosc opy [11206-53]

\section{SESSION 7 RBER FABRICATION I}

11206 OU Microgravity fiber processing for future optical networks (Invited Paper) [11206-70]

11206 OV Cladding shaping of optical fiber preforms via CO $\mathbf{O}_{2}$ laser machining (Invited Paper) [11206-40]

11206 OW Recent progress in graded-index plastic optical fiber (Invited Paper) [11206-80]

11206 OX 3D-printing of chalcogenide preforms: a novel process for the elaboration of chalc ogenide mic rostructured optical fibers [11206-16]

11206 OY The effect of pressure on structured optical fibre drawing [11206-30]

11206 OZ Inverse modelling of mic rostructured optical fibre drawing [11206-25]

\section{SESSION 8 FBER FABRICATION II}

$1120610 \quad$ Israeli activities in specialty fibers and fiber lasers (Invited Paper) [11206-54]

1120611 3D silica lithography for doped and structured optical fibers (Invited Paper) [11206-75]

1120612 Single crystal fiber growth by laser heated pedestal growth technique (Invited Paper) [11206-13]

1120613 Titania clad fiber fatigue perfomance (Invited Paper) [11206-81]

1120614 Radiation-resistant nanoparticle erbium doped fibers for high power space laser communic ations [11206-83]

1120615 Single crystal semic onductor-core optical fiber [11206-39] 
1120616 Chalcogenide fiber for long-wave infrared supercontinuum source (Invited Paper) [11206-18]

1120617 Broadband mid-infrared supercontinuum generation in chalcogenide tapered fiber with allnomal dispersion (Invited Paper) [11206-74]

1120618 Mid-infrared supercontinuum generation in chalcogenide or heavy oxide fibers for sensing applications [11206-4]

\section{SESSION 10 FBERLASERS III}

1120619 Specialty optical fibers for generation of light with extreme properties (Invited Paper) [11206-60]

11206 1A Random lasing from optical fibers with phase-separated glass cores [11206-56]

11206 1B Challenges in preform material preparation for laser-active fibers [11206-22]

11206 1C Multic ore fiberamplifier based on the Talbot effect [11206-3]

11206 1D Cleaning protocols for fabrication of fibers for kilowatt-class lasers [11206-65]

$112061 \mathrm{E} \quad$ Improved wavelength selective filtering design in a Nd doped silica fiber for E-band amplification [11206-66]

\section{SESSION 11 RBER SENSORS II}

11206 1F Optical fibers for downhole oil and gas applications (Invited Paper) [11206-44]

$112061 G \quad$ Making optical fibers sensitive and selective to the environment via functionalization and nanostructuring (Invited Paper) [11206-82]

$11206 \mathrm{lH} \quad$ Femtosecond laser inscribed bridging cladding waveguides combining single- and multi-core optical fibres for shape sensing [11206-26]

$1120611 \quad$ Polymer end-capped fibersensors for biomedical applications [11206-79]

\section{POSTER SESSION}

11206 1] Data acquisition from a bundle of 1,000 optical fibers [11206-5]

11206 1K Fabrication of a tellunite hollow core optical fiber for mid-infrared transmission [11206-6] 
11206 1L 3D printed optical fibre preforms from silica contained resin [11206-21]

$112061 \mathrm{M}$ Wavelength dependence of transverse Anderson localization in disordered glass-air fiber [11206-23]

11206 IN Noise-like pulse pumped all-fiber supercontinuum laser source [11206-24]

1120610 Designing silic on fibertapers for effic ient mid-IR superc ontinuum [11206-33]

11206 IP Heat mitigation via anti-Stokes fluorescence cooling in core/cladding Yb-doped fiber amplifiers [11206-34]

11206 1Q Measuring the anti-Stokes cooling parameters of a Yb-doped ZBLAN fiber for radiation balancing [11206-37]

11206 IR Investigation of low-bending-loss single-mode anti-resonant hollow-core TH fiber [11206-38]

11206 1S Low-loss terahertz pulse transmission through commercially available porous tubes with PIFE [11206-41]

$112061 T \quad$ Low quantum defect fiber lasers via Yb-doped fluorosilicate optical fiber [11206-43]

$112061 U$ Long-tem behaviour of water vapour absorption in hollow core fibres [11206-45]

11206 IV All optically driven and all optical fiber modulator via luminescence-quenched Yb-doped fiber[11206-46]

11206 1W Tunable wavelength Q-switc hed all-fiber laser based on two-dimensional perovskite solution [11206-47]

11206 1X O-band bismuth-doped fiber amplifier and its temperature dependent performance [11206-50]

11206 1Y Direct extrusion of hollow-core Tht fiber using a 3D printer [11206-52]

$1120612 \quad$ Non-destructive tomography for the characterization of extruded optical fiber triplet 3D shape sensors [11206-59]

1120620 Spectroscopic properties of highly erbium doped fluorosilicate fiber [11206-64]

1120621 Applications of novel metal-derived optical fibers [11206-68] 
Downloaded From: https://www.spiedigitallibrary.org/conference-proceedings-of-spie on 26 Apr 2023

Terms of Use: https://www.spiedigitallibrary.org/terms-of-use 


\section{Authors}

Numbers in the index correspond to the last two digits of the seven-digit citation identifier (CID) article numbering system used in Proceedings of SPIE. The first five digits reflect the volume number. Base 36 numbering is employed for the last two digits and indicates the order of articles within the volume. Numbers start with 00, 01, 02, 03, 04, 05, 06, 07, 08, 09, 0A, OB...0Z, followed by 10-12, 20-2Z, etc.

Ackerman, Liam, 1A Adam, Jean-Luc, OX Ahmed, Goran, OT

Aichele, Claudia, 07

Alvarez, Oseas, 16

Amezcua-Correa, Rodrigo, $1 \mathrm{M}$

Antonio-Lopez, Jose Enrique, $1 \mathrm{M}$

Aquilina, Christophe, 18

Aubrecht, Jan, OL

Bachhuka, Akash, $1 \mathrm{G}$

Baker, Colin C., 14

Balci, Mustafa H., 15

Ballato, John, 15, 1A, 1T, 1V, 20, 21

Barnini, A., 08

Barua, P., $1 \mathrm{X}$

Bawn, Simon, $1 \mathrm{U}$

Béjot, Pierre, 18

Ben Slimen, Fedia, 09, OS

Bennett, Kevin W., 13

Benoît, Aurélien, 01

Bera, Subhabrata, 12

Beresna, Martynas, $1 Y$

Bierlich, Jörg, 07, OD

Billard, Franck, 18

Birks, Tim A., Ol

Boley, Charles, 1E

Borrelli, Nicholas F., 02

Bowen, Patrick, $\mathrm{OB}$

Boyd, Keiron, 00

Bradford, Joshua, 05

Bradley, Thomas D., OR, IU

Brambilla, Gilberto, $1 Y$

Brilland, Laurent, $\mathrm{OX}$

Buff, Andrew, 16

Burdett, Ashley A., 14

Buric, Michael, 12

Campling, Joseph, 10

Canning, John, OY, $1 \mathrm{~L}$

Cano-Velázquez, Mildred, 11

Cantwell, Patrick R., 15

Carcreff, Julie, OX

Carter, Adrian, 05, 00

Carthy, Joanna, OB

Cavillon, Maxime, 1A, 1T, 20

Chen, Bernard, OE

Chen, Michael, $\mathrm{OZ}$

Chen, Wynter, 1A

Chen, Yong, OR, $1 \mathrm{U}$

Chenard, Francois, $0 Q, 16$
Cheviré, François, $\mathrm{OX}$

Cho, Yuhtat, 07

Chorpening, Benjamin, 12

Chu, Yushi, $1 \mathrm{~L}$

Cimek, Jaroslaw, 09, 0S

Clarkson, W. Andrew, 0O, OV

Conroy, Mike, 05

Cook, Kevin, OY, $1 \mathrm{~L}$

Cook, Matthew J., 1D, $1 \mathrm{E}$

Crist, Robert, 1D, 1E

Dai, Shixun, 17

Davalos, Gabriel, 1D

Davidson, lan A., OR

Davis, Steve, 1D

Dawson, Jay, 1D, 1E

Defas-Brucil, Ricardo, 11

Désévédavy, Frédéric, 18

DiGiovanni, David J., ON

Ding, Wei, OP

Drachenberg, Derrek R., 1D, 1E

Dragic, Peter D., 1A, 1T, 1V, 20, 21

Drouin, Antoine, $1 \mathrm{Z}$

Dulashko, Yuriy, ON

Durkin, Mike, 07

D'Urso, John, OG

Ebendorff-Heidepriem, Heike, $1 G$

Enders, Michael T., OT

Eschrich, Tina, 07

Fallah Tafti, Ghazal, oY

Faucher, Olivier, 18

Feder, Ken, OF

Feurer, Thomas, 19

Friebele, E. Joseph, 14

Frosz, Michael H., OT, 1M

Fu, Xinghu, 1L

Gadret, Grégory, 18

Galdo, Elodie, OX

Gao, Shou-Fei, OP

Gautier, Antoine, OX

Ghiringhelli, Fabio, 07

Gibson, Ursula J., 15

Glaesemann, G. Scott, 13

Gouveia, Marcelo A., 1 U

Gragg, Jane, 21

Grigoleto Hayashi, Juliano, 09, OS

Guiraud, G., 08

Guitton, P., 08

Gunawardena, Dinusha, OE

Haboucha, A., 08 
Harker, Andrew T., $1 \mathrm{U}$

Harrington, Kerriane, 0 I

Hartung, Alexander, OD

Haub, John, 00

Hawkins, Thomas W., 1A, 1T, 1V, 20, 21

Hayes, John R., OR

Heidt, Alexander M., 19

Hemming, Alexander, 00

Hernández-Cordero, Juan, 11

Hochstrasser, Martin, 19

Hong, Seongjin, 1W

Honzatko, Pavel, OL

Horak, Peter, 10

Horley, Raymond J., $1 \mathrm{U}$

$\mathrm{Hu}$, Jonathan, $\mathrm{OQ}$

Huot, Laurent, OB

Inniss, Daryl, ON

Inoue, Azusa, OW

loannou, Andreas, $\mathrm{OM}, 1 \mathrm{H}$

Ishaaya, Amiel A., 10

Ismaeel, Rand, $1 Y$

Jacobsen, William, $\mathrm{OG}, \mathrm{OH}$

Jagannathan, Srinath, 1A

Jäger, Matthias, 07, OD

Jasion, Gregory T., OR, OS

Jauregui-Misas, Cesar, 1C

Jollivet, Clémence, 05

Jules, Jean-Charles, 18

Jung, Yongmin, 09

Kadoury, Samuel, 12

Kalide, Andre, 07

Kalli, Kyriacos, OL, OM, $1 \mathrm{H}$

Kanagaraj, Nithyanandan, OL

Kang, Chul, 1S

Kashyap, Raman, 12

Khitrov, Victor V., 1E

Kiani, Leily S., $1 \mathrm{E}$

Kibler, Bertrand, 18

Kim, Byungjoo, 1W

Kim, Soeun, 1S

Ko, Wing, OF

Kobelke, Jens, 07, OD

Koike, Yasuhiro, oW

Kostecki, Roman, $1 \mathrm{G}$

Kremp, Tristan, OF

Kucera, Courtney, 1T, 1V, 21

Landais, D., 08

Langner, Andreas, 07

Laurell, Fredrik, 15

Le Goffic, O., 08

Lebullenger, Ronan, OX

Lee, Yong Soo, 15

Leich, Martin, 07

Lemière, Arnaud, 18

Li, Zhao, $1 \mathrm{~F}$

Limpert, Jens, 1C

Lindner, Eric, OJ

Liu, Bo, 12

Liu, Zheng yong, $\mathrm{OE}$

Logothetis, Stephanos, 14
Lorenz, Adrian, OD

Lorenz, Martin, 07

Lorre, Pierre, $1 \mathrm{Z}$

Luo, Jiawei, ON

Luo, Xing, $1 \mathrm{~N}$

Luo, Yanhua, OY, $1 \mathrm{~L}$

LuValle, Michael J., 14

Maeng, Inhee, 1S

Mafi, Arash, 1P, 1Q

Malinowski, Andy, 07

Marshall, Andrew, 07

Mart, Cody, $1 \mathrm{E}$

Mathey, Pierre, 18

Menyuk, Curtis R., OQ

Messerly, Michael J., 1D, 1E

Mies, Eric, 00

Mikhailov, Vitaly, ON

Mobini Souchelmaei, Esmaeil, 1P, 1Q

Monberg, Eric, OF

Monteville, A., 08

Monticelli, Marcus, 1D

Moselund, Peter M., OB

Müller, Robert, 1B

N. Velazquez, M., OV

Neice, Mark, 03

Nishiharaguchi, Nobuhiko, $1 \mathrm{~K}$

Numkam Fokoua, Eric, OR

O'Leary, Stephen, OE

Oetomo, Denny, OE

Oh, Kyunghwan, 1S, 1W

Ohishi, Yasutake, OA, $1 \mathrm{~K}$

Ohodnicki, Paul, 12

Ortiz, Roy, OF

Pan, Guanyi, 20, 21

Partridge, Matthew C., OR

Pax, Paul, 1E

Peacock, Anna C., 10

Peng, Gang-Ding, OY, 11, $1 \mathrm{~L}$

Pennetta, Riccardo, OT

Peterka, Pavel, OL

Petrovich, Marco N., OR, $1 \mathrm{U}$

Peysokhan, Mostafa, IP, 1Q

Pilz, Sönke, 19

Plass, Jaqueline, 07

Poiffaut, Arthur, 12

Polleti, Francesco, 09, OR, OS, $1 \mathrm{U}$

Provino, L., 08

Puc, Gabriel S., ON

Reichel, Volker, 1B

Ren, Zhengqi, 09

Renversez, Gilles, OX

Richardson, David J., 09, OR, 1U, 1X

Rikimi, Shuichiro, OR, $1 \mathrm{U}$

Rochette, Martin, OC

Romano, Valerio, 19

Roth, Paul, 1M

Runkel, Michael, 1D, 1E

Russell, Philip St. J., OT, 1M

Ryser, Manuel, 19

Sahu, J. K., OV, $1 X$ 
Sakr, Hesham, OR, OS

Sanghera, Jasbinder S., 14

Scannell, Garth W., 13

Schartner, Erik P., $1 \mathrm{G}$

Schenkel, Nick, 1D, 1E

Schmitt, Clemens, 07

Schönfeld, Dörte, 07

Schöłz, Gerhard, 07

Schülzgen, Axel, $1 M$

Schwuchow, Anka, 07

Shardlow, P. C., OV

Sheng, Quan, 1R

Shenk, Scott D., ON

Shi, Wei, 1R

Simakov, Nikita, 00

Simoff, Deb, OF

Sintov, Yoav, 10

Smektala, Frédéric, 18

Song, Seunghan, 15

Soufiane, Abdel, OG, $\mathrm{OH}$

Spangenberg, Dirk, 19

Standish, R., OV

Starodubov, Dmitry S., OU

Stassis, Andreas, OL

Steinkopff, Albrecht, 1C

Stojanovic, Stefan, $1 \mathrm{H}$

Stokes, Yvonne, $0 Z$

Sun, Shuai, $1 \mathrm{R}$

Sun, Yingzhi, ON

Suratwala, Tayyab, 1D

Suzuki, Takenobu, 1K

Swain, Robert, 00

Talataisong, Wanvisa, $1 Y$

Tam, Hwa-Yaw, OE

Tankala, Kanishka, 05

Taunay, T., 08

Theodosiou, Antreas, $\mathrm{OL}, \mathrm{OM}, 1 \mathrm{H}$

Thipparapu, N. K., $1 \mathrm{X}$

Thomson, Robert R., Ol

Tong, Tuan Hoang, $1 \mathrm{~K}$

Trautvetter, Tom, 1B

Traynor, N., 08

Troles, Johann, OX

Tuggle, Matthew, 1A, 1V, 21

Vadivelu, Arvind N., OE

Vallée, Réal, 04

Van Putten, Lieke D., $1 Y$

VanBlarcom, Diana, 1D

Ventura, Andrea, 09, OS

Wang, $\mathrm{Pu}, \mathrm{OP}$

Wang, S., $1 \mathrm{X}$

Wang, Wenyu, OY

Wang, $Y ., 1 X$

Wang, Ying-Ying, OP

Wang, Yingying, 17

Ward, David, 1

Warren-Smith, Stephen C., $1 G$

Wei, Chengli, $O Q$

Westbrook, Paul S., OF, ON

Wheeler, Natalie V., OR, OS, $1 \mathrm{U}$
White, Nicholas, 09, 0S

Windeler, Robert S., ON

Wondraczek, Katrin, 07, 1B

Wondraczek, Lothar, 1B

Wong, Gordon K. L., IM

Wu, Hongchao, OF

Wu, Wei, 15

Wysocki, Paul F., $1 \mathrm{~F}$

Yan, Man F., ON

Yao, Jianquan, $1 R$

Yerolatsitis, Stephanos, Ol

Yu, Anthony W., 06

YU, Charles X., $1 \mathrm{E}$

Yu, Nanjie, 1T, 1V, 21

Zhang, GUo, 1R

Zhang, Jianzhong, 1L.

Zhang, Nan, 17

Zhang, Yao, 1R

Zhao, Jian, $1 \mathrm{M}$

Zheng, Wenxin, OK

Zhu, Gongwen, OK 
Downloaded From: https://www.spiedigitallibrary.org/conference-proceedings-of-spie on 26 Apr 2023

Terms of Use: https://www.spiedigitallibrary.org/terms-of-use 


\title{
Conference Committees
}

\author{
Conference Chairs
}

John Ballato, Clemson University (United States)

Liang Dong, Clemson University (United Sta tes)

Local Orga nizing Committee

John Ballato, Clemson University (United States)

Liang Dong, Clemson University (United States)

Courtney Kucera, Clemson University (United States)

Wade Hawkins, Clemson University (United States)

Technic al Program Committee

Jacques Albert, Ca rleton University (Canada)

Rodrigo Amezcua Comea, University of Central Florida

(United States)

John Ballato, Clemson University (United States)

Igor Bufetov, Fiber O ptics Resea rch Center of the RAS

(Russian Federation)

John Canning, University of Technology Sydney (Australia)

Adrian Carter, Coherent | Nufem (Australia)

David DiGiovanni, OFS Fitel, $\amalg C$ (United States)

Lang Dong, Clemson University (United States)

Peter Dragic, University of Illino is (United States)

Mark Dubinskiy, U.S. Amy Research Laboratory (United States)

Ben Eshel, Air Force Institute of Technology (United States)

Sebastien Fevrier, Université de Limoge (France)

Cliff Headley, OFS Fitel, ШC (United States)

StuartJ ackson, Macqua rie University (Australia)

Ming-J un L, Coming Incorporated (United States)

Matthias Jäger, Leibniz-Institute of Photonic Techonlogy, J ena

(Gemany)

Kyriac os Kalli, Cyprus University of Technology (Cyprus)

Jens Kristian Lyngsø, NKTPhotonic S A/S (Denma rk)

Jon Maikisch, Ha mis Corporation (United Sta tes)

Walter Margulis, RISE Acreo (Sweden)

Alexis Mendez, MCH Engineering, ШC (United States)

Mic hael J . Messenly, La wrence Livermore National Laboratory

(United States)

Daniel Milanese, Politec nic o di Torino (Italy)

Kyunghwan Oh, Yonsei University (Korea)

Yasutake Ohishi, Toyota Technical University (J apan) 
Bishnu Pal, Bennett University (India)

Francesco Poletti, University of So uthampton (United Kingdom)

Siddharth Ramachandran, Boston University (United States)

Mohammed Saad, Thorla bs (United States)

Jayanta Sahu, University of Southampton (United Kingdom)

Kunimasa Saitoh, Hokka id o University (J apan)

Axel Schulzgen, CREOL, College of Optic sand Photonics, University of Central Florida (United States)

Brandon Shaw, U.S. Naval Resea rch Laboratory (United States)

Hwa-Yaw Tam, Hong Kong Polytec hnic University (Hong Kong, China)

Ji Wang, Coming Incorporated (United States)

Michalis Zervas, Southampton University (United Kingdom)

Intemational Steering Committee

Christiano de Matos, Mackenzie Presbyterian University (Brazil)

Cristiano M. B. Cordeiro, Universid a de Esta dual de Campinas (Bra zil)

J uan Hemandez-Cordero, Universidad Nacional Autonoma de

Mexico (Mexico)

Walter Margulis, RISE Acreo (Sweden)

Hwa-Yaw Tam, Hong Kong Polytechnic University (Hong Kong, China)

Bai-Ou Guan, J inan University (C hina)

Kyriacos Kalli, Cyprus University of Tec hnology (Cyprus)

J ohn Ballato, Clemson University (United States)

Lang Dong, Clemson University (United States)

Session Chairs

Wed nesday Plenary Session

John Ballato, Clemson University (United States)

1 Fiber Lasers I

Michael J. Messerly, Lawrence Livermore National Laboratory

(United States)

2 IR Fibersand Sources I

Francois Chenard, IRflex Corporation (United States)

3 Fiber Sensors I

Paul S. Westbrook, OFS Fitel, ШC (United Sta tes)

4 Fiber Devices

Kyriacos Kalli, Cyprus University of Technology (Cyprus)

6 Thursday Plenary Session

John M. Ballato, Clemson University (United States) 
5 Fiber Lasers II

Cesar J auregui-Misas, Friedric h-Schiller-Universität J ena (Gemany)

6 Hollow-core Fibers

Yasutake Ohishi, Toyota Technolog ic al Institute (J a pan)

7 Fiber Fabric ation I

Heike Ebendorff-Heidepriem, The University of Adelaide (Australia)

Friday Plenary Session

John M. Ballato, Clemson University (United States)

$8 \quad$ IR Fibersand Sources

Peter Morten Moselund, NKTPhotonic s A/S (Denmark)

9 Fiber Lasers III

Clémence J ollivet, Coherent | Nufem (United States)

10 Fiber Sensors II

Hwa-Yaw Tam, The Hong Kong Polytechnic University (Hong Kong, China) 
Downloaded From: https://www.spiedigitallibrary.org/conference-proceedings-of-spie on 26 Apr 2023

Terms of Use: https://www.spiedigitallibrary.org/terms-of-use 


\section{Introduction}

It is with great pleasure that we introduce this Proceedingsvolume filled with papers from the 6th Intemational Workshop on Specialty Optical Fibers and their Applications (WSOF 2019). WSOF is a biennial technical workshop and exhibit focused on advances and innovations in the field of specialty optical fibers and their applications, following in the footsteps of the five previous workshops: Sao Pedro, Brazil, 2008; Oaxaca, Mexico, 2010; Sigtuna, Sweden, 2013; Hong Kong, China, 2015; and Limassol, Cyprus, 2017.

The papers contained herein represent the state-of-the-art in specialty optical fibers including novel materia ls from which they a re ma de and coated, theirdesign and modeling, theirfabrication, and theirperformance in a variety of applic ations. The committee members, sponsors, and authors hail from all over the world further validating, as if there were questions, the global nature of this field. Academia, govemmental laboratories and industry - large and small - are presented as are the most well-known and acclaimed names in our field as well as young students just beginning their careers. These facts not only capture the diversity of workshops like WSOF 2019, but also their value in creating a shared experience for all interested in specialty fiber optics in a small and familiar setting that affords oneon-one opportunities for technical disc ussions and fellowship.

We would be remiss in not gratefully acknowledging those who made this Proceedings, and the Conference, possible. Financially, the sponsors noted above were central to the success of WSOF 2019. Second, and no less central, were Thomas (Wade) Hawkins, Courtney Kucera, and Lindsay Grieshop (Clemson University), who provided inva lua ble logistic al support. 
Downloaded From: https://www.spiedigitallibrary.org/conference-proceedings-of-spie on 26 Apr 2023

Terms of Use: https://www.spiedigitallibrary.org/terms-of-use 


\title{
Optical Fiber and Glasses (101)
}

\author{
Nicholas F. Borrelli* \\ CRDC, Sullivan Park, SP-FR Corning NY 14801 USA
}

\begin{abstract}
This presentation is essentially a retrospective of my 50 years of experience in the field of optical materials while working at the Corning Incorporated Research Laboratory. This time period happened happily coincided with the emergence of what could be termed the "optical revolution." It began with the laser then the optical fiber and subsequently to all the materials and devices that are related to the present ubiquitous optical network. A number of the topics in addition to fibers will be briefly covered such as, photonic crystal fibers, nonlinear optical glass, quantum dots and polarizing glasses.
\end{abstract}

\section{Background}

My intitial research project at Corning dealt with glass lasers and not optical fiber which was then just in its beginning stage. We were the $2^{\text {nd }}$ Lab (American Optical ${ }^{1}$ was $1^{\text {st }}$ ) that demonstrated laser action with a Nd-doped glass rod laser. My project was to interpret spectroscopic properties and in particular to uncover whether one could control the radiative lifetime and gain corfficient with changes in the host glass composition. In simpler terms "were there better glass hosts?" My eventual involvement with the optical wavguide project was not in the fabrication part but rather looking at some of the unsual propagation properties that would ultimately be important e.g. polarization ${ }^{2}$ and nonlinear effects. This will be mentioned in subsequent sections.

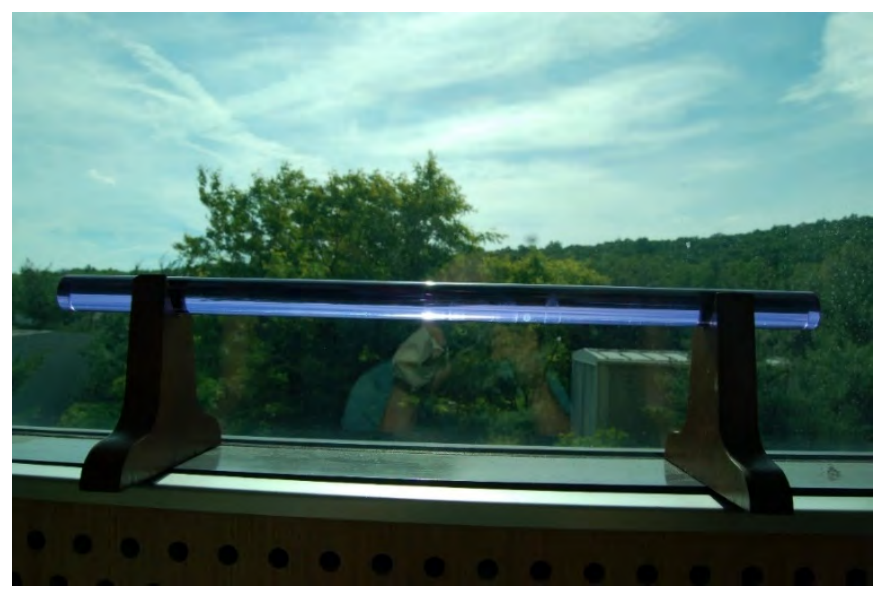

Figure 1; one of the $1^{\text {st }} \mathrm{Nd}$-doped glass lasers, circa 1964

\section{Introduction}

Many of us have lived through a period of time where arguably more technological advances have been achieved than in any other comparable period in human history. The reasons are manifold and include the fact that the field of 
quantum-based solid-state physics formulated in the 20's and 30's matured in the 50's, leading the way for people to think about such things as a solid state diode and ultimately the transistor. There was also the continuation of the technological impetus generated by WWII, things like radar and communication devices. A little later was what was called the space age culminating in the moon landing. This effort led to the need for minaturation and ultimately to integrated circuits and the personal computer. The emergence of optical waveguide fiber expanded the communication bandwith by orders of magnitude, allowing comparably more information to be transmitted. Add up all this and you have what I referred to as the optical age. Ironically we have moved back to the original AT\&T Bell Labs microwave scheme, albeit wireless rather than underground tubes; antennas rather than waveguides.

\section{Fiber}

The piecing together all these technological advances over the previous decades came the recognition that the information bandwidth of copper wire was insufficient to carry the ever growing amount of telephonic and other information. This need to increase the bandwidth, even before the invention of the Internet, was the genesis of the optical age. The Bell system and others were well aware of this limitation. If you look at the articles in the Bell System Technical Journal in the late 50's you can see their plan was to put millimeter microwave waveguide tubes underground. These would utilize reflective wall coatings, or periodic confocal dielectric lenses, or continous flowing gas gradients. How fortunate that Maxwell's equations are scalable so dielectric waveguide analysis was available ${ }^{3}$ at American Optical in 1961, even before decisions were made to jump to optical frequencies. At Standard Telephone Laboratories (ITT), Kao indicated that optical fiber transmission was possible ${ }^{4}$, and the race began to make a singlemode optical fiber with a loss of no more than $20 \mathrm{~dB} / \mathrm{km}$ (present fiber is $1 / 100^{\text {th }}$ of that number). STL later made some loss measurements on bulk fused silica rods. At that time it was difficult to pull silica into fiber strands because the required temperature to do so was much higher than that for softer compound glasses. The latter had been pulled into high-loss short fibers and bundledfor imaging applications.

The British Post Office (telephone division) talked to Corning about the possibility of producing such a fiber. I will deal with this effort in the next section. I must mention that the $\mathrm{GE}^{5}$, IBM${ }^{6}$, and $\mathrm{MIT}^{7}$ simultaneously created the first early and equally indispensable parts of the optical network in their pioneering work on solid state semiconductor diode lasers. Initially these doped GaAs diodes had high threshold currents, needed to be cooled, and had very short lifetimes. These problems were solved after $\mathrm{RCA}^{8}$ used liquid phase epitaxy in fabricating more complex structures, along with work at the loffe-Physico-Technical Institute ${ }^{9}$ and Bell Labs ${ }^{10}$, to produce $\mathrm{CW}$ room temperature lasers that could be current-modulated for sending signals. Semiconductor lasers and detectors were equally as challenging and important as the low-loss fiber to emerging optical network. All these pieces had to come together for the success of the effort.

Not too many years later a new field "photonics" came into being. This included devices to augment and expand the fiber network. Elements now like semiconductor amplifiers, fiber lasers and amplifiers ${ }^{11}$, fiber Bragg gratings ${ }^{12}$, optical switches, couplers, multiplexers and demultiplexers were all being conceived and developed. At one point in time before the 2002 bubble there was talk of an "all optical network" that is, all the switching was to be done optically. This engendered the use of nonlinear optics ${ }^{13}$ and the application to use intensity to direct and couple light. These will be discussed briefly as separate topics as well.

\section{Optical Fiber}

The challenge in the early $60^{\prime}$ s was to develop a process to deliver a core/clad glass low-loss single mode fiber for telecommunications. After a few failed attempts (that I will not go through) the Corning breakthrough was announced ${ }^{14}$. (The results were confirmed by measurements taken at STL and the BPO.) The fiber fabrication utilized a process now called chemical vapor deposition (CVD) which was invented at Corning ${ }^{15}$ a number of years earlier to 
make high purity fused silica (see Figure 2a). The idea was to use pure gases like $\mathrm{SiCl}_{4}$ and burn them in a gas/oxygen flame to produce a pure silica "soot." Initially this was by inside vapor deposition (IVD) within a silica tube that was turning and locally heated on a special lathe. Later, to scale up production, outside vapor deposition (OVD) on a bait rod was invented. In both cases the soot was subsequently consolidated at $>1200 \mathrm{C}$ to form ultrapure silica. I should add that STL and Bell Labs ${ }^{16}$ ultimately used a similar IVD process, while Nippon Telegraph and Telephone ${ }^{17}$ used OVD.

\section{Flame Hydrolysis}

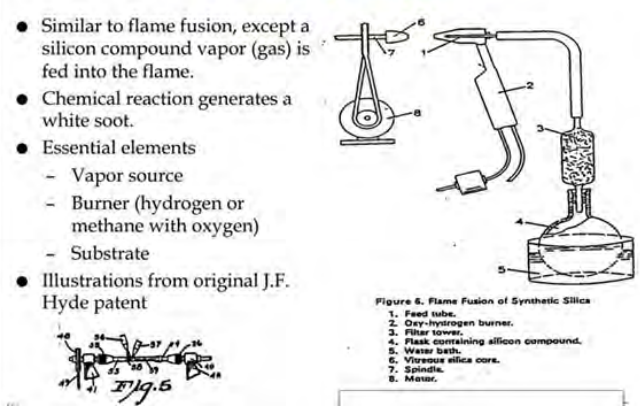

Figure 2a; copy from the original Hyde patent U.S. 741709 A $(1942)^{15}$

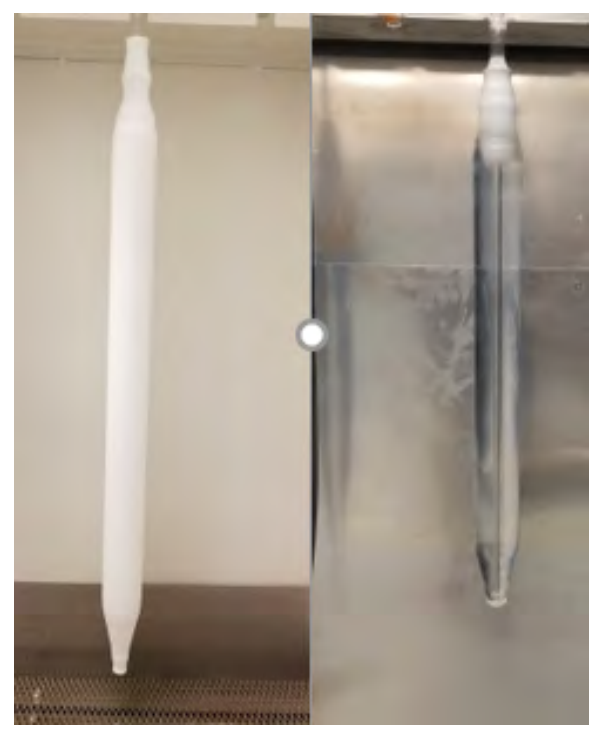

Figure 2b; a CVD-produced soot blank before (left) and after its consolidation into a preform (right)

As mentioned above the Corning Lab was interested in making glass lasers using the CVD silica method by doping with rare-earth ions. As luck would have it there was a project already underway using this process which was then converted to making what would be the waveguide core rod. This effort was then converted to depositing in a cylindrical manner and the low loss material/process was developed. The next challenge was what was to be the dopant to increase the refractive index of the core. Initially titania was used, an oxide based on Corning's experience fabricating ultra-low-expansion bulk glass. But lower loss and higher strength fiber resulted from doping with germania ${ }^{18}$, a true glass former at all doping levels. The gas $\mathrm{GeCl}_{4}$ was mixed with $\mathrm{SiCl}_{4}$ to produce $\mathrm{GeO}_{2}$ from a few percent to $20 \%$. In the OVD process the cladding was provided by the subsequent deposition of pure silica. The doping level allowed the index difference to be controlled and hence the fiber NA, the mode diameter, the cutoff wavelength, the group velocity dispersion (CVD, often specified as chromatic dispersion), nonlinear effects, etc. Higher doping also increased the zero-dispersion wavelength ${ }^{19}$ towards the lower attenuation wavelengths that we will discuss later. The 
addition of $\mathrm{F}$ to the cladding decreased its refractive index, providing an additional degree of freedom in designing index profiles, including use of a pure silica core. $P$ in the core and $B$ in the cladding were $\operatorname{tried}^{20}$ at the University of Southampton (along with liquid-core fibers) but were not pursued industrially.

There are other very important aspects that were involved, for example, the actual turning of the consolidated blank into fiber. Here Corning had at one time made thermometer tubing by a method called "redraw."

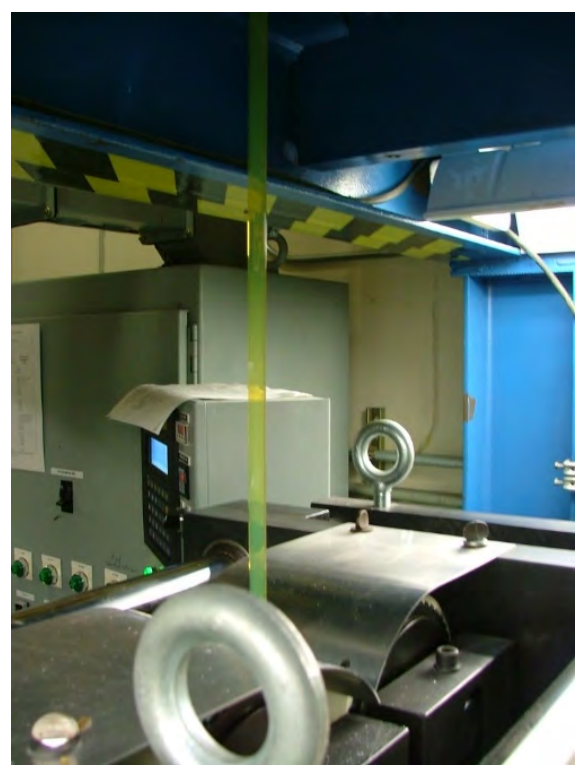

Figure 3; a glass redraw apparatus

These were towers where the glass blank would be suspended and heated. Compared to earlier fibers produced with compound glass for other applications, silica softened at much higher temperatures. Corning developed a new furnace capable of melting silica to be pulled at a rate to produce the required fiber diameter. So, two of Corning's capabilities were already available to be utilized for this new application. There was one very important part of the process that is hardly ever mentioned and yet without this process optical fiber would have never been realized. Glass fiber is very fragile and would easily break from surface flaws. Corning invented a method to polymer coat the fiber on the draw, thus allowing the fiber to be directly spun onto drums. The coated and cushioned fiber could later be put into cable with minimal microbending loss due to rough surfaces.

\section{Other Fibers, Properties/Devices}

\section{III-1 Fiber}

Initially it was all about loss, as light absorption due to fiber impurities was continually reduced due to improved manufacturing processes. The initial fibers operated in a low-loss $850 \mathrm{~nm}$ region, where light sources and detectors were readily available. Some multimode fibers for short distance, low bandwidth applications still do. But with the desire to go longer distances between repeaters and at higher modulation speeds, lower attenuation and dispersion were needed for single-mode fibers. Corning and other labs successfully reduced the $\mathrm{OH}^{\prime}$ 'water' absorption peaks around $1150 \mathrm{~nm}$ and $1400 \mathrm{~nm}$, thus opening the possibility of longer wavelengths. The initial commercial single-mode fibers had a zero-dispersion wavelength around $1310 \mathrm{~nm}$, which conveniently was at an attenuation minimum. But besides absorption, a component of total loss is Rayleigh scattering that is intrinsic to optical glass, including $\mathrm{SiO}_{2}$ and $\mathrm{GeO}_{2}$. The loss due to scattering decreases with increasing wavelength as $\lambda^{-4}$, prompting a move to operate in the 
1550nm region. (Beyond $1600 \mathrm{~nm}$, IR absorption and fiber bending loss became significant.) Semiconductor lasers and photodetectors for the $1310 \mathrm{~nm}$ and $1550 \mathrm{~nm}$ regions were becoming available.

The shift of the zero-dispersion wavelength discussed earlier occurs because designing the refractive index profile, step-index or otherwise, allows more or less of the light to travel in the cladding. ${ }^{19,21}$ This can move the zero-

dispersion wavelength to $1550 \mathrm{~nm}$, resulting in so-called dispersion-shifted fibers and dispersion-flattened fibers.

Operating near this wavelength was extremely important to allow for greater modulation speed and for nonlinear optical phenomena such as degenerate four wave mixing and other parametric processes to occur. ${ }^{13}$ An entire array of fiber profile designs have been developed over the years to enhance and/or optimize propagation performance. There was one report of a glass-ceramic fiber laser/amplifier. ${ }^{22}$

\section{III-2 Gratings}

It was found that one could induce a photorefractive effect in the Ge-based fiber by exposing a portion of the fiber to intense deep UV light through a mask. ${ }^{12}$ The mechanism involved the development of a strong UV absorption the result of a Ge-based color center in the 200-250nm wavelength region. From dispersion an index change occurred in the near IR (Kramers-Kronig). The magnitude of the induced refractive index change was sufficient to produce diffraction gratings that could act as wavelength filters and couplers.

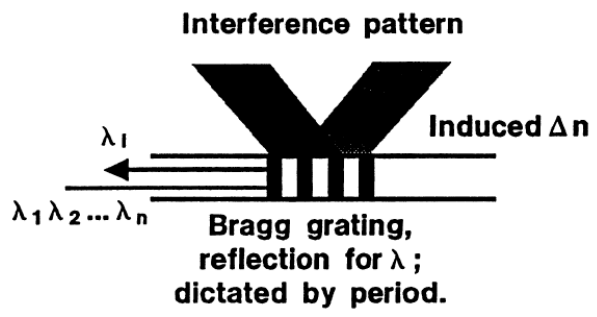

Figure 4; schematic of fiber Bragg grating, how it is fabricated, and how it functions in the fiber

\section{III-3 Photonic Crystal Fibers}

A more recent variation of waveguiding structures is the photonic crystal fiber ${ }^{23,24}$. These are fibers where the confinement of light is conventionally described in terms of diffraction as opposed to refraction. Actually, this is an artificial distinction because Maxwell's equations make no such distinction. One can always talk about effective index differences. The following set of diagrams ${ }^{25}$ makes it clear how the waveguiding concept can be viewed as a localized mode produced by a defect in an otherwise forbidden frequency gap. The representation in terms of a frequency vs. wavevector $(\omega v s . k)$ diagram is the same as one uses in electronic band structures since they are both dealing with waves in nominally periodic lattices characterized by reciprocal space parameter " $k$ " or " $\beta$ ". This is a spatial term in the formulation represented by $\exp (i k . r)$. The almost complete analogy between electrons and photons should come at no surprise, after all a wave is wave.

Referring to FIGURE 5; (a) refers to a diagram of a block of uniform glass. The shaded area represents where no propagation can occur; the dividing line in the simplest case between air and glass is just a line with a slope of $1 / \mathrm{n}_{\mathrm{g}}$. Now in (b) one adds a region of a new glass with a higher index; the line with a slope of $1 / n_{d}$. The region between the two lines can now support a mode. This is your standard waveguide. If now you make a periodic "holey" region shown in (c), one sees more structure. One is a forbidden gap above the light line due to interference phenomena and a bending of what was the $1 / \mathrm{n}_{\mathrm{g}}$ line because the light can redistribute between the air holes and the glass as a function of wavelength. If you add a defect to this structure you can again have a mode propagate in the forbidden energy gap, 
but it too has curvature which can produce interesting propagation properties. The last diagram (d) is the most interesting where the defect is now a larger air hole. This leads to propagation above the light line; phase velocity is larger than the speed of light (but the group velocity is not).
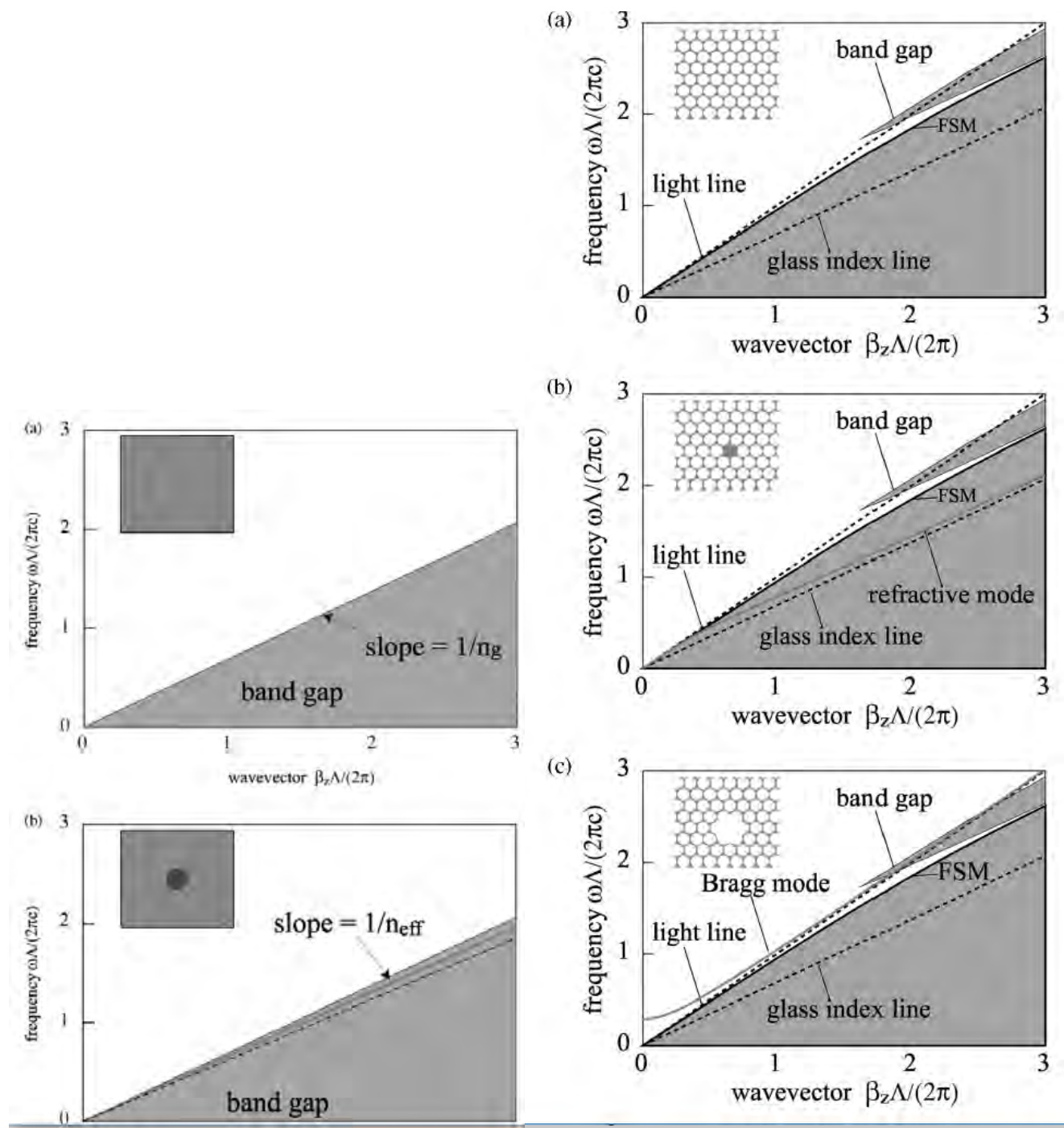

Figure 5; (a) and (b) depiction of conventional waveguide; (c), (d), photonic crystal depictions; upper holey structure, middle holey structure with a defect, and lower where the defect is a "hole" and the guiding mode is above the light line

\section{iv Optically active glasses}

Perhaps this is not the best choice of a heading title for the topic, but what really is meant in this context are glasses that interact with light to produce a variety of useful optical phenomena. A limited representative list is the following; optical nonlinearity, magneto-optics, polarizing behavior, photorefractive effect, and quantum dots in glass. These diverse phenomena derive from a variety of different glass composition systems. 


\section{iv-1 Nonlinear}

This essentially reduces to the refractive being a function of the incident light intensity; $n=n_{0}+\gamma I, \gamma$ being the nonlinear parameter in units of $\mathrm{m}^{2} / \mathrm{W}$. The glasses exhibiting high nonlinearity invariably have a large linear index because of the incorporation of highly polarizable ions such as $\mathrm{Pb}$ or $\mathrm{S} .{ }^{26,27}$ The utilization of glasses and fibers made therefrom are to produce parametric effects ranging from amplification to down conversion and in general generating new frequencies from others. ${ }^{13}$

\section{Four-Wave Interaction}

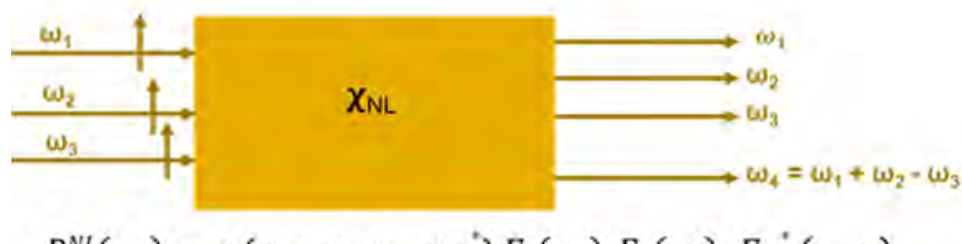

$$
P^{N L}\left(\omega_{4}\right)=\chi\left(\omega_{4} ; \omega_{1} \omega_{2}-\omega_{3}{ }^{*}\right) E_{\chi}\left(\omega_{1}\right), E_{\chi}\left(\omega_{2}\right), E_{\chi}{ }^{*}\left(-\omega_{3}\right)
$$

Figure 6: schematic of a nonlinear induced four wave mixing

The wave equation now has a nonlinear source term added to take in account interaction of the frequencies as indicated in the wave equation written below.

$\nabla x \nabla x E+\frac{\varepsilon}{c^{2}} \frac{\partial^{2} E}{\partial t^{2}}=\frac{-4 \pi}{c^{2}} \frac{\partial^{2} P^{N L}}{\partial t^{2}}$

This term accounts for the mixing of the waves, such as the example $\omega_{4}=\omega_{1}+\omega_{2}-\omega_{4}$, and when the momentum is conserved as $k_{4}=k_{1}+k_{2}-k_{3}$

\section{iv-2 Quantum dots}

Another nonlinear optical effect can be realized by "saturable absorption" which is optimally observed in glasses containing quantum dots. ${ }^{28-32}$ The splitting of the nominal band states into discrete atomic states by the ability to produce particles in nanometer size. The required size is gauged by its relation to the Bohr radius. There are many other systems reported in the literature including II-Vi, III-V, and I-VII where quantum confinement has been exhibited.
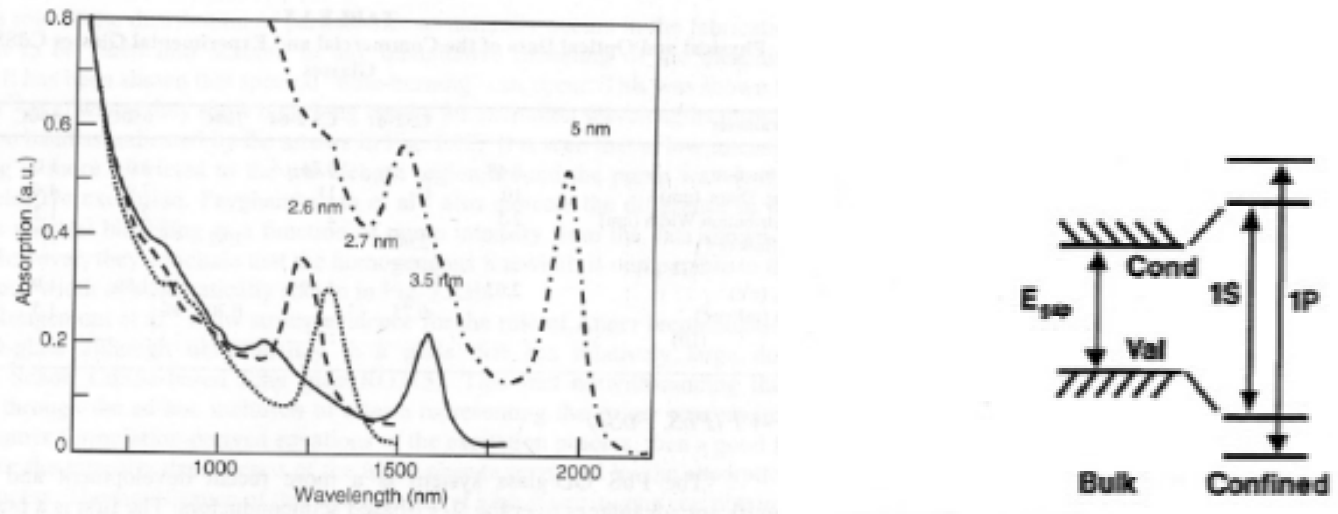

Figure 7; position of the energy level splitting as a function of the dot size $e^{32}$ 
The relation to optical applications is that the energy splitting is a good approximation to the ideal two-level system where with sufficient intensity one can produce a large ground state to excited state population difference. This is also referred to as induced transparency. Here the effective refractive index change is proportional to the difference in the population in the ground and excited states, which is directly dependent on the intensity of light.

$$
\alpha=\alpha_{0} /\left(1+\frac{I}{I_{s a t}}\right)
$$

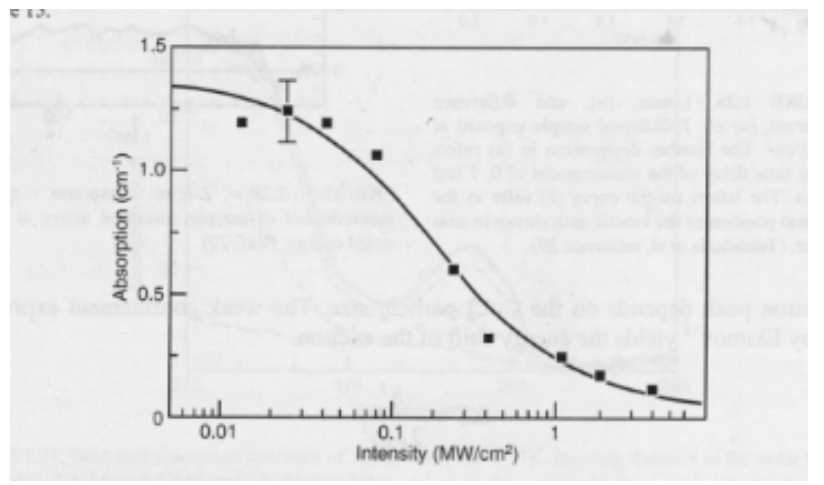

Figure 8; example of saturable absorption, transmission vs. intensity for CdSe QD glass

Although attempts to make a quantum dot fiber were not successful in the PbSe system, a quantum dot strip (not quite a fiber) has been made as shown in Figure 8.

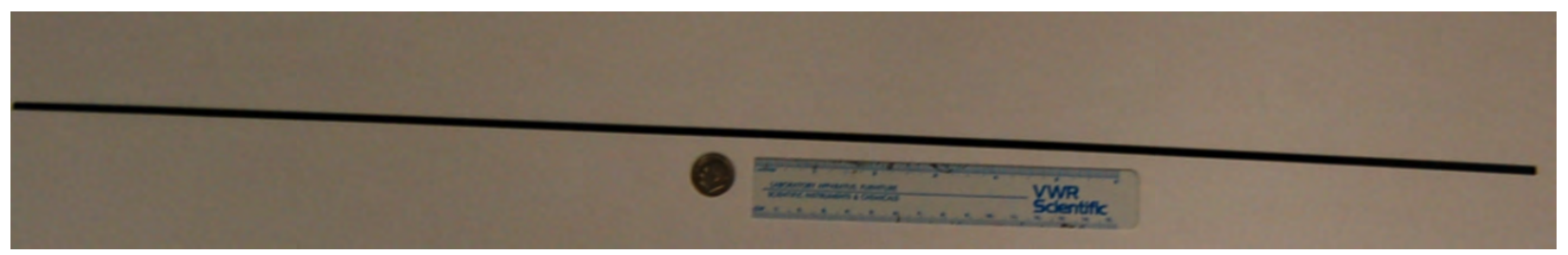

Figure 9; $1 \mathrm{~mm} \times 3 \mathrm{~mm}$ strip of PbSe quantum dot glass

\section{iv-4 Polarizer}

The last example of a novel glass with useful optical application is light polarizing glass. This glass starts out as an Aghalide containing photochromic glass ${ }^{30}$ (glass that reversibly darkens in sunlight). Although the particle size of the silver halide is not quantum dot size, the particles are small enough to render the glass transparent. This glass is stretched at a temperature where the silver halide particle is a liquid droplet. The droplet elongates and is now highly anisotropic in shape and highly birefringent. At this stage it is heated in a $\mathrm{H}_{2}$ atmosphere that reduces the $\mathrm{Ag}$-halide to elongated Ag needles, yielding the dichroic property to a point of a $50 \mathrm{~dB}$ contrast in the $\mathrm{IR}^{30}$ 


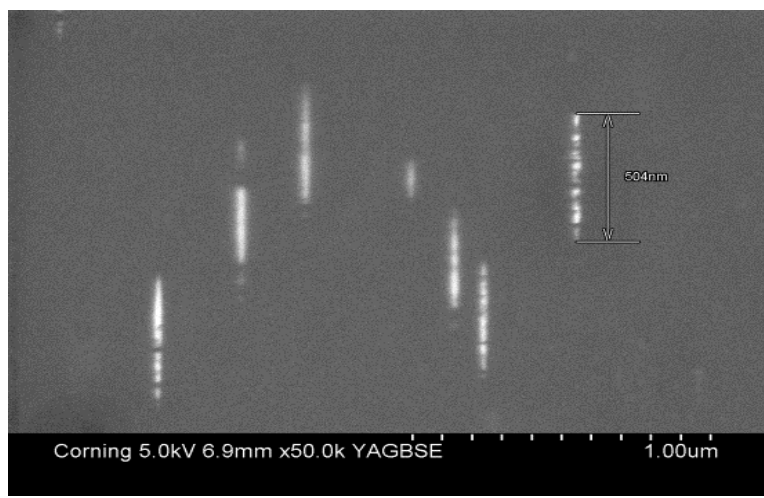

Figure 10; SEM image of the resulting elongated particles

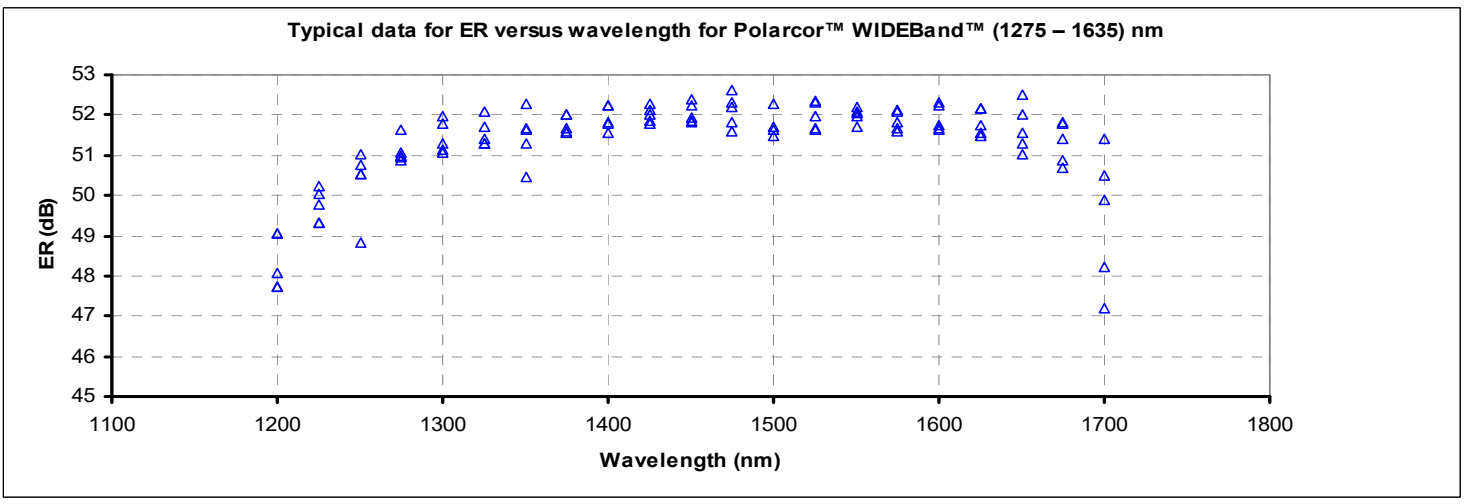

Figure 11; extinction ratio in db vs. wavelength

\section{Acknowledgement}

Felix Kapron for suggestions, comments and critical reading of the paper.

\section{References}

1. E. Snitzer (1961), Phys. Rev. Lett. 7(12), 444

2. N.F. Borrelli, F.P. Kapron, D.B. Keck (1972), IEEE J. Quantum Electronics QE-8, 222

3. E. Snitzer (1961), J. Opt. Soc. Am. 51(5), 491

4. K.C. Kao and G.A. Hockham (1966), Proc. IEE 113, 1152

5. R.N. Hall et al (1962), Phys Rev. Lett. 9, 366

6. M.I. Nathan et al (1962), Appl. Phys. Lett. 1, 62

7. T.M. Quist et al (1962), Appl. Phys. Lett. 1, 91

8. K. Kressel and H. Nelson (1969), RCA Review 30, 106

9. Z.I. Alferov et al (1970), Soviet Physics - Semiconductors (3) 9, 1107 
10. I. Hayashi et al (1970), Appl. Phys. Lett. 178(3), 109

11. R.J. Mears et al (1985), Electron. Lett. 21(17), 738

12. K.O. Hill et al (1978), Appl. Phys. Lett. 32, 647

13. P.N, Butcher and D, Cotter, "Elements of Nonlinear Optics" Cambridge Press 1990

14. F.P. Kapron. D.B. Keck and R.D. Maurer (1970), IEE Conf. 71, 48 and Appl. Phys. Lett. 17, 423

15. J.F. Hyde U.S. patents 741709 A and 2,272,342

16. W.G. French et al (1974), Bell Sys. Tech. J. 53, 951

17. M. Horiguchi and H. Osanai (1976), Electronics Lett. 12, 310

18. D.B. Keck, R.D. Maurer, P.C. Schultz (1973), Appl. Phys. Lett. 22(7), 307

19. F.P. Kapron and D.B. Keck (1971), App. Opt. 10, 1519

20. D.N. Payne and W.A. Gambling (1975), Optics Comm. 13, 422

21. V.A. Bhagavatula (1986), Tech. Digest Optical Fiber Com. Conf. OSA Washington DC

22. B. N. Samson et al (2001), Opt. Lett. 26(3) 145

23. J.D. Joanopoulus et al (1995), "Photonic Crystals" Princeton U press

24. A Yariv (1985), “Optical Elements” Holt Reinhart

25. N.F. Borrelli, (2005) “Microoptics Technology" $2^{\text {nd }} e d$

26. D. R. Uhlmann and N.J. Kreidl, (1991) “Optical Prop. of Glass, Nonlinear Optical Prop of Glass, 87

27. S. Smolorz et al (1999), J. Non-Cryst. Sol. 256,257, 31

28. D.W. Hall and N.F. Borrelli (1988), J. Appl. Phys. 5, 1650

29. P.T. Guerrio et al (1997), Appl Phys Lett. 71(12) 1595

30. N.F. Borrelli et al (1987), J. Appl Phys. 70(5) 2774

31. K. Wundke et all (2000), Appl. Phys. Lett.76(1), 10

32. N.F Borrelli and D.W. Smith (1994), J. Non Cryst. Sol. 180, 25

33. N.F. Borrelli (2015), "Photosensitive Glass and Glass-Ceramics” CRC press 
Defense applications of high power fiber lasers

Mr Mark Neice, Executive Director of the Directed Energy Professional Society (DEPS), former Director, High Energy Laser Joint Technology Office

The US Department of Defense has embraced the emerging capabilities of fiber amplifiers into their planning for technology demonstrations and experimentation campaigns. The Robust Electric Laser Initiative (RELI) funded laser source technology development, involving both spectrally and coherently combined fibers. The key to this was the development of single-mode, narrow linewidth amplifiers operating at the kW-class level, leveraged off the growth of these amplifiers from the commercial market.

Directed energy (DE) weapons have long since been a staple across Federal Government Research \& Development institutions over the past decades, and due to technological maturation a number of military $D E$ programs are showing great promise. The current posture of DE weapons across the DoD has grown in popularity over the recent years, and many strides have been made to integrate DE as a warfighting tool in support of National security needs and the Warfighter.

DoD programs to be explored are the Army High Energy Laser Mobile Demonstrator (HEL MD), the Navy Laser Weapon System demonstration (LaWS), the Marine Corp Compact Laser System experimentation campaign, and the Air Force Counter UAS experimentation campaign. Each of these early technology demonstrations has led to plans for extended experimentation campaigns to get user feedback on integration of these new capabilities into today's warfighting doctrine.

The High Energy Laser Joint Technology Office (HEL JTO) was established for the purpose of developing and executing a comprehensive investment strategy for HEL science and technology by advocating HEL development and transition, addressing joint technology requirements, stimulating inter-service research, orchestrating a portfolio of R\&D projects across government/industry/academia, establishing a community of standards \& tools, and educating the next generation of HEL technical, policy, and program leaders. The HEL JTO was chartered to advocate, address, stimulate, orchestrate, establish and educate the developmental and operational communities of the Department of Defense in High Energy Laser weapons. This is accomplished by translating military capability gaps and requirements into technology programs across industry, academia, and government agencies. These programs are chosen to advance the current state of the art in HEL technology and fill technology gaps, thus providing broad capabilities that can be transitioned into acquisition programs by the military services. Congressional language in the FY18 National Defense Authorization Act re-designated the HEL JTO to the Directed Energy Joint Transition Office (DE JTO), as multiple service programs moved into the technology demonstration phase.

The Directed Energy Professional Society (DEPS) was founded in 1999 to foster research, development and transition of Directed Energy (DE) technology for national defense and civil applications through professional communication and education. We are recognized as the premier organization for exchanging information about and advocating research, development and application of Directed Energy, which includes both high energy lasers (HEL) and high power microwaves (HPM). The Directed Energy Professional 
Society is incorporated as a nonprofit corporation in New Mexico, organized and operated exclusively for charitable, scientific, and educational purposes.

DEPS offers graduate scholarships of up to $\$ 10,000$ each, per student per academic school year. Over the past five years, DEPS has awarded more than $\$ 500 \mathrm{~K}$ to 48 students focusing their research in HEL, HPM, or USPL technologies. The number of annual scholarship awards depends on available funding, which is provided by grants from the Directed Energy Joint Transition Office (DE JTO) and the Office of Naval Research (ONR.) The traditional academic disciplines involved in DE research include, but are not limited to, physics, electrical engineering, chemistry, chemical engineering, materials sciences, optical sciences, optical engineering, and aerospace engineering.

In 2013, DEPS published the Second Edition of the Introduction to High Power Fiber Lasers, authored by Andrew Motes, Sami Shakir and Richard Berdine. This book provides a comprehensive review of current high-power fiber laser (HPFL) technology, including the history of HPFLs to an introduction of the science and engineering required for full HPFL systems, as well as the authors' predictions of future applications and research directions. This second edition also contains information on recent research that achieved increased output powers by increasing the power of individual beams and by combining multiple beams. This book describes a rapidly developing field of laser technology, and fills the very important role of providing students, researchers, and technology managers with valuable, timely, and unbiased information on the subject.

Optical fibers are an important focus of the Defense HEL applications, leveraged by the commercial development of high power ( $>1 \mathrm{~kW}$ ) fiber laser amplifiers with narrow linewidths suitable for beam combination. Research into improved fiber amplifiers continues, for both commercial manufacturing and defense applications. 


\title{
Bright Perspectives for Fluoride Glass Mid-Infrared Fiber Lasers
}

\author{
Réal Vallée \\ Center for Optics, Photonics and Lasers, Laval University, Quebec, G1V0A6, Canada
}

\begin{abstract}
We present the most recent achievements in terms of spectral coverage and output power from both cw and pulsed midIR fiber lasers with special emphasis on their basic components as well as their applications in various fields.
\end{abstract}

Keywords: fiber lasers, mid-IR coherent radiation, fiber components

*rvallee@,copl.ulaval.ca; phone 1418 656-2698

\section{Introduction}

Fiber lasers are featuring outstanding optical and mechanical properties, namely excellent beam quality, high optical conversion efficiency, ruggedness/compactness and low maintenance. They are also well known for overcoming laser diodes in terms of their brightness, which is key for several applications requiring high-intensity light-matter interactions. Accordingly, silica based $\mathrm{Yb}^{3+}$ fiber lasers emitting near 1 micron which are now producing several tens of $\mathrm{kW}$ with diffraction-limited output have revolutionized the manufacturing sectors in relation to material welding, cutting, marking and cleaning, namely in the automotive industry. More recently, lanthanide doped silica fiber lasers have also shown remarkable performance in the so-called eye-safe region, namely at 1.5 microns (with $\mathrm{Er}^{3+}$ ) or near 2.0 microns (with $\mathrm{Ho}^{3+} \mathrm{or}^{3+}$ ). However, despite their outstanding performance, silica fiber lasers are restricted, due to their limited transmission properties, to the electromagnetic spectral window spanning between $800 \mathrm{~nm}$ and $2200 \mathrm{~nm}$.

Fluoride glass optical fibers have emerged over the last two decades as the most serious contender to address the challenges related to the development of longer wavelength fiber lasers, more specifically between 2.7 and $4.0 \mu \mathrm{m}$. As lowphonon energy glasses alleviating multi-phonon decay, both zirconium fluoride $\left(\mathrm{ZrF}_{4}\right)$ and indium fluoride $\left(\mathrm{InF}_{3}\right)$ singlemode optical fibers are now available commercially with minimum losses of $10 \mathrm{~dB} / \mathrm{km}$ or less, with double-clad geometries and with lanthanide concentrations approaching $10 \mathrm{~mol} \%$. Also, fluoride fiber optical components including Fiber Bragg gratings or pump combiners are now also available thus allowing for the development of rugged monolithic Mid-IR fiber lasers.

\section{Presentation overview}

\section{High power cw emission}

Until recently, the general trend for the maximum output power from fiber lasers as a function of wavelength was following a steady exponential decrease as shown on Figure 1 (dashed line). According to this trend, watt-level operation was only achieved near $3 \mu \mathrm{m}$ from $\mathrm{Er}^{3+}$ doped $\mathrm{ZrF}_{4}$ fibers. In fact, $\mathrm{Er}^{3+} / \mathrm{ZrF}_{4}$ fiber laser used to be the most mature one of the mid-IR region. Over the last two years, developments involving both $\mathrm{Ho}^{3+}$ and $\mathrm{Dy}^{3+}$ ions (along with $\mathrm{Er}^{3+}$ ) have led to significant improvements of mid-IR fiber lasers in terms of both their maximum $\mathrm{cw}$ output power and overall spectral coverage ${ }^{1-4}$ (stars). 


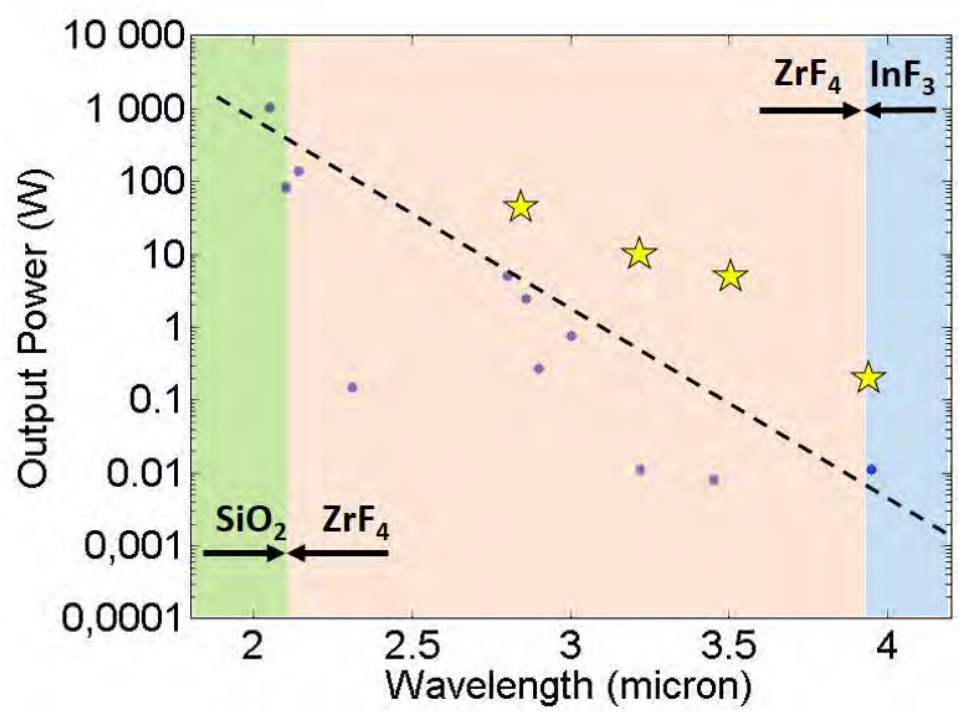

Figure 1. Maximum cw output power from mid-IR fiber lasers as a function of wavelength.

Nowadays, watt-level operation is possible over almost the entire spectral region between 2.7 and $4.0 \mu \mathrm{m}$ and the recent availability of low loss $\mathrm{InF}_{3}$ fibers is holding promises of further improvements at longer wavelength. Fluoride glass optical fibers have thus dissipated the general apprehension regarding their high power handling ability although a fiber tip photodegradation issue, especially in the neighborhood of the $\mathrm{O}-\mathrm{H}$ resonance peak, was reported as a possible hurdle to their power scaling.

\section{Pulsed laser operation}

Several mid-IR applications can not be addressed with cw FLs and are calling for their pulsed counterparts. Accordingly, several pulsed regimes of operation of mid-IR FLs were recently investigated. Both Q-switched and gain-switched FLs were demonstrated, leading to pulsed operation in the ns regime near both 3.0 and $3.5 \mu \mathrm{m}$. Pulse energies approaching the $100 \mu \mathrm{J}$ level with an average power exceeding $10 \mathrm{~W}$ were namely produced from a rugged monolithic cavity operating at $2825 \mathrm{~nm}^{5}$. Pulse energies in excess of $100 \mu \mathrm{J}$ were also produced in the sub-nanosecond regime based on an $\mathrm{Ho}^{+3}$-doped fiber based amplifier scheme. The femtosecond regime was also studied via a fiber based ring laser cavity relying on the nonlinear polarization evolution mode-locked scheme and $207 \mathrm{fs}$ pulses with $3.5 \mathrm{~kW}$ peak power near $2.8 \mu \mathrm{m}$ were produced ${ }^{6}$. Such

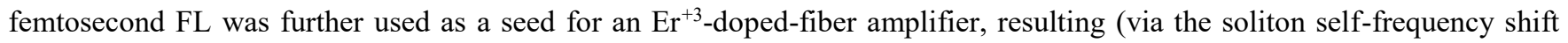
process) in a watt level femtosecond fiber source tunable from 2.8 to $3.6 \mu \mathrm{m}^{7}$.

\section{Conclusion}

Significant progresses were recently reported for both $\mathrm{cw}$ and pulsed mid-IR fiber lasers so that these sources can now address a growing number of applications that are specific to this spectral region. Further progress is also expected to occur in the near future in terms of both average power scaling (up to $100 \mathrm{~W}$ ) and wavelength coverage (up to $4 \mu \mathrm{m}$ ). 


\section{References}

[1] Fortin, V., Jobin, F., Larose, M., Bernier, M., Vallée, R., "10-W-level monolithic dysprosium-doped fiber laser at 3.24 $\mu \mathrm{m}$," Optics letters 44 (3), 491-494 (2019).

[2] Aydin, Y.O., Fortin, V., Vallée, R., Bernier, M., “Towards power scaling of $2.8 \mu \mathrm{m}$ fiber lasers,” Optics Letters 43(18), 4542-4545 (2018).

[3] Maes, F., Fortin, V., Poulain, S., Poulain, M., Carrée, J.Y., Bernier, M., Vallée, R., "Room temperature fiber laser at 3.92 $\mu \mathrm{m}$," Optica 5(7), 761-764 (2018).

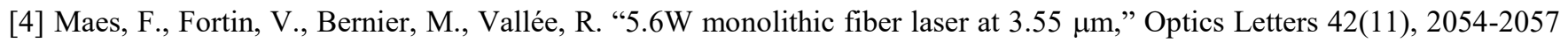
(2017).

[5] Paradis, P., Fortin, V., Aydin, Y.O., Vallée, R., Bernier, M. “10W-level gain-switched all-fiber laser at 2.8 mm,” Optics Letters 43(13), 3196-3199 (2018).

[6] Duval, S., Bernier, M., Fortin, V., Genest, J., Piché, M., Vallée, R., "Femtosecond fiber lasers reach the mid-infrared," Optica 2(7), 623-626 (2015).

[7] Duval, S., Gauthier, J.C., Robichaud, L.R., Paradis, P., Olivier, M., Fortin, V., Bernier, M., Piché, M., Vallée, R., "Wattlevel fiber-based femtosecond laser source tunable from 2.8 to $3.6 \mu \mathrm{m}$," Optics Letters 41(22), 5294-5297 (2016). 


\title{
Next Generation of DC fibers enabling high performance and reliability of industrial fiber lasers beyond $1.5 \mathrm{~kW}$ levels \\ Clemence Jollivet ${ }^{\mathrm{a}}$, Joshua Bradford ${ }^{\mathrm{a}}$, Mike Conroy ${ }^{\mathrm{a}}$, Adrian Carter ${ }^{\mathrm{a}}$, Kanishka Tankala ${ }^{\mathrm{a}}$
}

\author{
${ }^{a}$ Coherent|Nufern, 7 Airport Park Rd., East Granby, CT 06026
}

\begin{abstract}
Stimulated Raman scattering and transverse mode instability thresholds are currently limiting the maximum output power of industrial fiber lasers around $1.5 \mathrm{~kW}$. To further power scale, the choice of Large-Mode-Area Yb-doped Double-clad Fiber combined with pumping wavelength is critical. Design guidelines are discussed to maximize the achievable output power of single-cavity fiber oscillators and predict that up to $3 \mathrm{~kW}$ is achievable.

Keywords: Double-clad Fiber, Fiber Design, High Power Fiber Laser, Stimulated Raman Scattering, Transverse Mode Instability, Photodarkening

*clemence.jollivet@cohrerent.com
\end{abstract}

\section{Introduction}

The industrial fiber laser market is imposing stringent requirements on the fiber cavity and fiber components to be extremely cost-effective, manufacturable in high volume quantities and to operate reliably for extended lifetime. While the cavity design is driven by cost effectiveness, the design of the fiber components is driving the maximum achievable output power. To be cost-effective, single cavity fiber oscillator design are widely employed even though they are known to be more sensitive to thermal load and limited in terms of stability and reliability at high power levels compared to MOPA designs [1]. The highest volume of industrial fiber lasers routinely operates at $1 \mathrm{~kW}$ output power and $1.5 \mathrm{~kW}$ lasers can be found. However, scaling the output power beyond $1.5 \mathrm{~kW}$ while meeting the requirements imposed by the industrial fiber laser market is currently a challenge. Around $1.5 \mathrm{~kW}$ output power, either Stimulated Raman Scattering (SRS) or Transverse Mode Instabilities (TMI) currently limit further power scaling.

The design of the laser cavity including the choice and design of the fiber components drive the SRS and TMI thresholds. The choice and design of the Large-Mode-Area Yb-doped Double-clad Fiber (LMA-YDF) used in the cavity is the main driver for SRS or TMI effects. After summarizing the current state-of-the art of industrial fiber lasers, fiber components design available in high volumes to supply this market are reviewed. Then, we focus on the LMA-YDF and discuss how both glass/composition and waveguide designs affect SRS and TMI thresholds by introducing power scaling coefficients. In the last section, different examples of LMA-YDF designs available in high volume quantities and at cost-effective price and their impact on maximum achievable output power are provided.

\section{II. $1.5 \mathrm{~kW}$ industrial fiber laser landscape}

Industrial fiber lasers emitting in the $1 \mu \mathrm{m}$ wavelength range routinely achieve output powers up to $1.5 \mathrm{~kW}$. Various designs of fiber laser engines can be found including amplifier and oscillators supported with different pumping architectures. One of the most commonly manufactured design is comprised of a $915 \mathrm{~nm}$ bi-directionally pumped fiber oscillator. A schematic is depicted in Figure 1 where the gain fiber is spliced between high and low reflector fiber Bragg gratings which are designed in matched passive fiber to ensure efficient coupling of the laser emission and diffractionlimited beam quality. Two pump fiber combiners deliver pump radiation and the laser radiation propagates through the LR side.

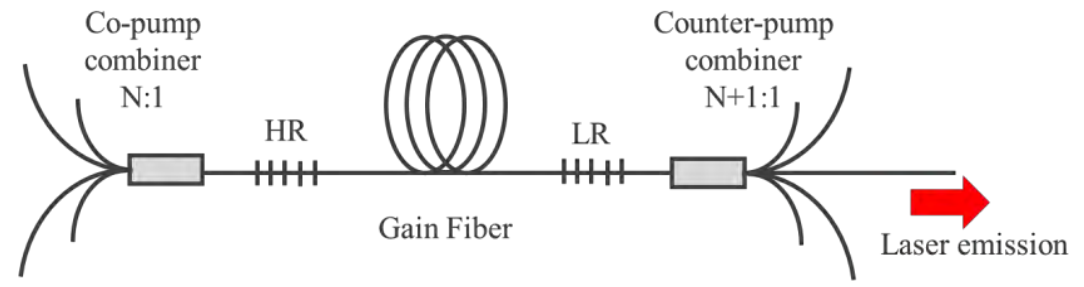

Figure 1. Typical industrial fiber oscillator architecture including two pump fiber combiners, High Reflector (HR), a length of gain fiber and Low Reflector (LR) 
The main design constraint for industrial fiber lasers being cost, single-end pumped fiber oscillators are also very popular as they require only one pump fiber combiner. The availability of pump modules at either $915 \mathrm{~nm}$ and $976 \mathrm{~nm}$ wavelengths, their brightness performances and cost play a significant role in designing the most cost-efficient systems. In addition, the availability of LMA-YDF in high volume quantities drive the cavity design while the consistency of the fiber performances drives the achievable laser output power. The LMA-YDF design currently used to achieve $1 \mathrm{~kW}$ output power comprises a $14 \mu \mathrm{m}$ diameter core, $0.08 \mathrm{NA}$ and $250 \mu \mathrm{m}$ diameter cladding and is designed to provide $0.8 \mathrm{~dB} / \mathrm{m}$ cladding absorption at $915 \mathrm{~nm}$ pump wavelength. The fiber is a so-called double-clad (DC) design since the glass cladding is surrounded by a low index fluo-acrylate polymer layer with $>0.48 \mathrm{NA}$ ensuring efficient confinement of the pump radiation. Single-end pumped fiber oscillators at $915 \mathrm{~nm}$ wavelength using LMA-YDF-14/250 offer a very price and performance competitive platform which contributes to drive the $\$ / \mathrm{W}$ target down.

On the other end, industrial fiber lasers delivering $1.5 \mathrm{~kW}$ are also available and typically use LMA-YDF a $20 \mu \mathrm{m}$ diameter core, $0.06 \mathrm{NA}$ and $400 \mu \mathrm{m}$ diameter cladding designed to provide $0.4 \mathrm{~dB} / \mathrm{m}$ cladding absorption at $915 \mathrm{~nm}$ pump wavelength (LMA-YDF-20/400). As the output power target requirements increase beyond $1.5 \mathrm{~kW}$, the selection of LMAYDF design becomes critical to overcome either SRS or TMI thresholds.

\section{Design considerations for power scaling $>1.5 \mathrm{~kW}$}

Several hero results have been demonstrated to date reporting multi-kW output power from single fiber oscillator cavity designs [2]. However, availability of high performance and high volume fiber components and cost-effective manufacturing of fiber oscillators able to consistently and reliably achieve $>1.5 \mathrm{~kW}$ output power remains a challenge.

\section{Component availability}

Availability and performances of both pump diode and pump fiber combiners are critical to support power scaling of the growing industrial fiber laser market. In terms of pump diodes, competitive pricing (around or less than $1 \$ / \mathrm{W}$ ) and performances are available for both 915 and $976 \mathrm{~nm}$ pumping wavelengths. Two standard geometries of pump delivery fibers can be found using either a $135 \mu \mathrm{m}$ pure silica core diameter, 0.22 NA and $155 \mu \mathrm{m}$ cladding diameter (MM$\mathrm{S} 135 / 155)$ or a $200 \mu \mathrm{m}$ pure silica core diameter, $0.22 \mathrm{NA}$ and $220 \mu \mathrm{m}$ cladding diameter (MM-S200/220). Table 1 summarizes diode pump modules availability for industrial applications. The output power delivered by a single diode module has recently scaled up in favor of single-end pumped fiber oscillators which are more cost-effective and easier to assemble.

Table1. Fiber-coupled pump diode modules available

\begin{tabular}{|c|c|c|}
\hline Fiber Pigtail & $\boldsymbol{\lambda}=\mathbf{9 1 5} \mathbf{~ n m}$ & $\boldsymbol{\lambda}=\mathbf{9 7 6} \mathbf{~ n m}$ \\
\hline MM-S135/155 & $270 \mathrm{~W}$ & $250 \mathrm{~W}$ \\
\hline MM-S200/220 & $370 \mathrm{~W}$ & $320 \mathrm{~W}$ \\
\hline
\end{tabular}

Pump fiber combiners manufactured with matched fibers to both the pump deliver fiber and the matched DC passive fiber are available. Different designs can be found to support co, counter or bi-directional pumping of industrial fiber lasers. Table 2 provides example of available pump fiber combiners.

Table2. Total available pump power for industrial applications using pump combiner matched to LMA-YDF-20/400

\begin{tabular}{|c|c|c|c|}
\hline Input Fiber & $\begin{array}{c}\text { Combiner designs } \\
\text { available }\end{array}$ & $\begin{array}{c}\mathbf{9 1 5} \mathbf{~ n m} \text { pump power } \\
\text { available }\end{array}$ & $\begin{array}{c}\mathbf{9 7 6} \text { nm pump power } \\
\text { available }\end{array}$ \\
\hline \multirow{2}{*}{ MM-S135/155 } & $7 \times 1$ & $3.7 \mathrm{~kW}$ bi-directional & $3.5 \mathrm{~kW}$ bi-directional \\
& $19 \times 1$ & $5.1 \mathrm{~kW}$ end-pumped & $4.7 \mathrm{~kW}$ end-pumped \\
\hline MM-S200/220 & $7 \times 1$ & $5.1 \mathrm{~kW}$ bi-directional & $4.4 \mathrm{~kW}$ bi-directional \\
\hline
\end{tabular}

Various combinations of pump architectures can be achieved with the current offering of pump diodes and pump fiber combiners. It is up to the fiber laser manufacturer to come up with co, counter or bi-directionally pumped fiber oscillators designs with the lowest cost, highest output power, best beam quality and best reliability. 


\section{Guidelines to mitigate TMI and SRS}

To ensure power scaling beyond $1.5 \mathrm{~kW}$ levels in single cavity fiber oscillators, it is critical to consider SRS and TMI thresholds which both limit the maximum achievable output power $[3,4]$. While literature has been published to analytically calculate SRS and TMI thresholds based on given system inputs, it remains challenging to generalize the use of those models in industrial applications as some of the exact systems parameters are not known. Furthermore, fiber component process capabilities and variations, which play a critical role in high volume manufacturing environments, are not often well identified and difficult to account for in complex modeling exercises.

SRS and TMI thresholds are a strongly influenced by the laser cavity design and the individual fiber component designs. The rest of this study is limited to LMA-YDF as it is well-known to be one of the major driver on SRS and TMI thresholds, along with the laser cavity design. From a fiber component standpoint, it is critical to identify which parameter drives either SRS and/or TMI thresholds in order to define a strategy towards power scaling beyond $1.5 \mathrm{~kW}$. To do so, we introduce coefficients which are function of the LMA-YDF design. The SRS coefficient, which depends on laser intensity and fiber length is noted $\boldsymbol{C}_{\boldsymbol{S R S}}$ and is defined as a function of the output power of the industrial oscillator $\boldsymbol{P}$, the cavity effective length $\boldsymbol{L}_{\text {eff }}$ and the LMA-YDF Mode-Field Diameter MFD.

$$
C_{S R S} \propto \frac{P \times L_{e f f}}{M F D^{2}}
$$

On the other hand, the TMI coefficient which involves balancing between thermal load and cavity loss is labeled $\boldsymbol{C}_{T M I}$ and is defined as a function of the output power $\boldsymbol{P}$, the laser optical efficiency $\boldsymbol{\eta}$, the cavity effective length $\boldsymbol{L}_{\text {eff }}$ and the cavity waveguide-driven loss $\boldsymbol{\alpha}_{w}$.

$$
C_{T M I} \propto \frac{P \times\left(\frac{1}{\eta}-1\right)}{L_{e f f} \times \alpha_{w}}
$$

Using the gain fiber design, it is particularity challenging to simultaneously mitigate SRS and TMI thresholds as both are counter-acting. For a given fiber oscillator architecture operating at a given output power, it is possible to calculate $\boldsymbol{C}_{S R S}$ and $\boldsymbol{C}_{T M I}$ corresponding to the power at which SRS and TMI threshold are reached respectively. These values of $\boldsymbol{C}_{\boldsymbol{S R S}}$ and $\boldsymbol{C}_{\boldsymbol{T} M I}$ can then be used as a guidelines to tune the cavity parameters in order to achieve higher power levels while simultaneously mitigating SRS or TMI thresholds.

\section{Next Generation of DC Fibers}

To mitigating SRS and TMI threshold beyond $1.5 \mathrm{~kW}$ the design of the gain fiber can be modified to tune $\boldsymbol{M F D}, \boldsymbol{L}_{\boldsymbol{e} f f}$ and $\boldsymbol{\alpha}_{w}$ accordingly. However, to be efficient, the fiber design optimization exercise must take place while considering pumping schemes which will also drive $\boldsymbol{L}$ eff and $\boldsymbol{\eta}$. All calculations and results discussed in this section assume a bidirectionally-pumped single-cavity fiber oscillator.

\section{Fiber design considerations for $915 \& 976 \mathrm{~nm}$ pumping}

This study starts from the widely used LMA-YDF-20/400 gain fiber with is designed with $17.5 \mu \mathrm{m}$ MFD at the signal wavelength $1060 \mathrm{~nm}, 0.4 \mathrm{~dB} / \mathrm{m}$ cladding absorption at $915 \mathrm{~nm}$ pumping wavelength and a 976:915 absorption ratio around 4.3. The measured cladding absorption spectrum of LMA-YDF-20/400 is depicted in green in Figure 2(a). This fiber is routinely used in bi-directionally pumped oscillators to emit $1.5 \mathrm{~kW}$ output power SRS and TMI-free. Based on the SRS and TMI coefficients defined above, various approach can be taken to further scale the gain fiber to increase the achievable output power beyond $1.5 \mathrm{~kW}$.

One possible optimization of the fiber design is to scale the MFD of this fiber while maintaining its glass composition characteristics. In practice one could use the same glass and simply change the core diameter target. Modifying the MFD primarily affecst cladding absorption, $\boldsymbol{L}$ eff and $\boldsymbol{\alpha}_{w}$. Using the SRS and TMI coefficient, it is possible to predict would be the maximum achievable output power as a function of the fiber MFD as depicted in Figure 2(b). The calculations are performed at both pumping wavelength $915 \mathrm{~nm}$ and $976 \mathrm{~nm}$ which correspond to $0.4 \mathrm{~dB} / \mathrm{m}$ and $1.7 \mathrm{~dB} / \mathrm{m}$ cladding absorption respectively (according to Figure 2(a)). Therefore, selecting different pumping wavelength has an impact on $\boldsymbol{L}_{\text {eff }}$ and $\boldsymbol{\eta}$. In Figure a(b), the green lines with circle and diamond markers represent the maximum achievable output power with $915 \mathrm{~nm}$ and $976 \mathrm{~nm}$ pumping respectively. Note the filled markers indicate SRS-driven power limitation while empty markers highlight TMI-driven power limitation. Increasing the fiber MFD from $17.5 \mu \mathrm{m}$ to $\sim 19 \mu \mathrm{m}$ favors $915 \mathrm{~nm}$ 
wavelength pumping schemes allowing to power scale from 1.5 up to $1.8 \mathrm{~kW}$ until reaching the SRS threshold. In contrast, increasing the MFD have a negative impact for $976 \mathrm{~nm}$ pumping schemes as the output power becomes TMI-limited above 1 to $1.2 \mathrm{~kW}$ due to unbalance between thermal load and cavity losses. On the other hand, scaling the MFD from $17.5 \mathrm{um}$ to $\sim 15 \mu \mathrm{m}$ favors $976 \mathrm{~nm}$ pumping schemes as $2.5 \mathrm{~kW}$ output power can be achieved before reaching the SRS threshold.
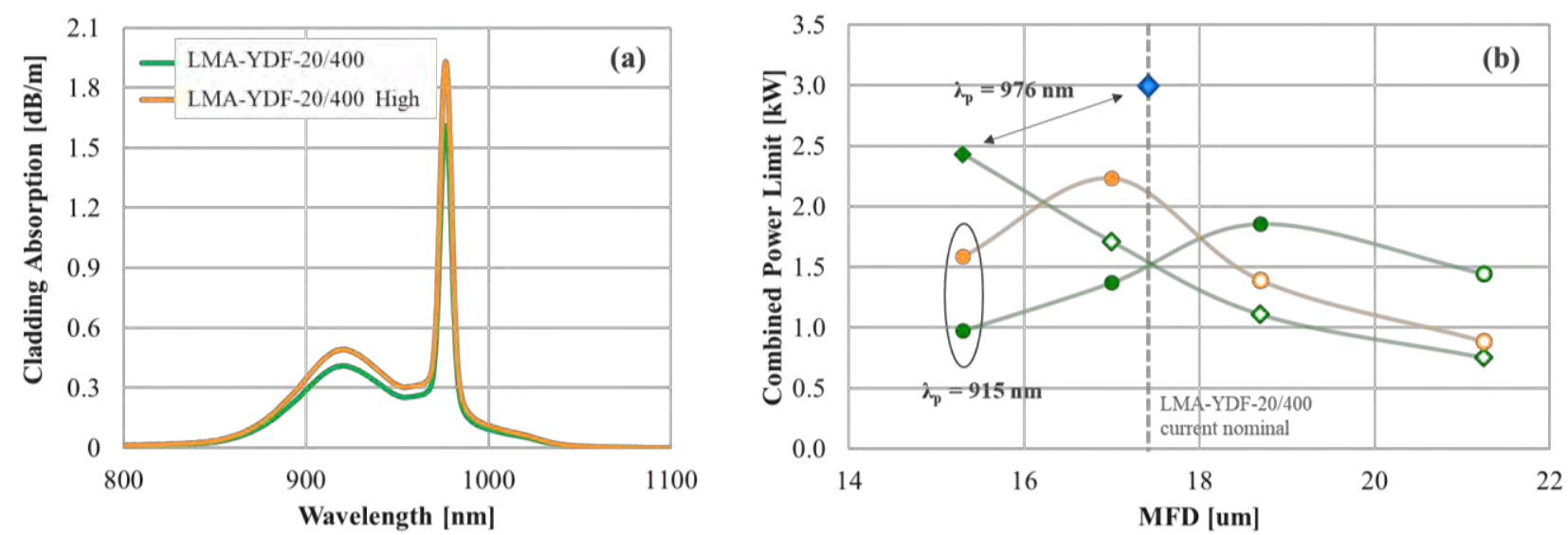

Figure 2. (a) Measured cladding absorption spectrum in LMA-YDF-20/400 targeting $0.4 \mathrm{~dB} / \mathrm{m}$ (green) and $0.48 \mathrm{~dB} / \mathrm{m}$ (orange) cladding absorption at $915 \mathrm{~nm}$ wavelength. (b) Calculated output power achievable using either $915 \mathrm{~nm}$ or $976 \mathrm{~nm}$ pumping wavelengths in a bi-directionally pumped fiber oscillator as a function of the MFD of the active fiber. The green and orange traces correspond to 0.4 and $0.48 \mathrm{~dB} / \mathrm{m} 915 \mathrm{~nm}$ cladding absorption respectively. Full and empty markers indicate SRS and TMI thresholds respectively. The bleu marker illustrates a fine-tuned LMA-YDF design with $17.5 \mu \mathrm{m}$ MFD.

Another option to further optimize the fiber design is to increase the $\mathrm{Yb}$ doping concentration in the core order to increase the cladding absorption to reduce $\boldsymbol{L}$ eff at the expense of the thermal load. The orange trace in Figure 2(a) illustrates the measure cladding absorption in LMA-YDF-20/400 with 20\% higher doping concentration. The orange line in Figure 2(b) captures the calculation results at $915 \mathrm{~nm}$ pumping wavelength. Up to $2.3 \mathrm{~kW}$ output power can be achieved before the SRS threshold is reached at the nominal $17 \mu \mathrm{m}$ MFD. While the current LMA-YDF-20/400 gain fiber $\left(\alpha_{\text {clad }}=0.4 \mathrm{~dB} / \mathrm{m}\right)$ has enabled high volume manufacturing of industrial fiber lasers up to $1.5 \mathrm{~kW}$ output power, further scaling up to $2.3 \mathrm{~kW}$ is achievable, with $915 \mathrm{~nm}$ pumping schemes, when using a fiber with higher doping concentration (e.g. $\alpha_{\text {clad }}=0.48 \mathrm{~dB} / \mathrm{m}$ ).

As a result, power scaling beyond $1.5 \mathrm{~kW}$ is achievable with LMA-YDF's but it requires fine tailoring between the pumping wavelength and the fiber design. In fact, $>2.5 \mathrm{~kW}$ can be achieved with $915 \mathrm{~nm}$ pumping by using either larger MFD LMA-YDF or higher absorption fibers. Furthermore, our calculations predict that up to $3 \mathrm{~kW}$ can be achieved with $976 \mathrm{~nm}$ pumping in a single-cavity fiber oscillator design by further tuning the LMA-YDF (blue marker in Figure 2(b)).

\section{High performance glass for improved reliability}

In addition to support the power scaling initiatives the LMA-YDF along with all other fiber components in the laser cavity must ensure performance reliability over time. For the gain fiber, it is critical to target a design with minimized photodarkening which is well known to affect power scaling due to thermal load build-up over time leading to TMI threshold [4]. PD is a common phenomenon observed in Yb-doped glass which scales with the dopant concentration and results in color-center formation during laser operation. One approach to quantify PD in LMA-YDF is to measure the redlight attenuation in the core of LMA-YDF while inverted at 50\% as a function of pump exposure time [5]. This approach enables fiber manufacturers to track PD as a function of glass composition and process improvements to minimize its effects on high power operation. The correlation between measured PD values and the laser lifetime remains challenging to establish as the design of the laser cavity and the components plays a major role in the laser lifetime performances. Therefore, for a fixed architecture, LMA-YDF with lower PD will ensure longer lifetime even though the exact duration cannot be estimated.

An example of PD performance comparison between LMA-YDF-20/400 designs is provided in Figure 3. All fibers (except for the competitor) are developed at Coherent|Nufern. Efficient techniques to mitigate PD involve glass composition optimization as well as glass manufacturing process improvements. Shown in Figure 3, the LMA-YDF20/400 GenVIII offers 4 times lower PD compared to one of the comparable competitor. Lower PD will result in extended laser lifetime when operating at high powers. The effect of glass composition improvement can be seen in Figure 3 when 
comparing GenVIII to GenX traces in blue and orange respectively. The labels "GenVIII" and "GenX" refer to different glass compositions. One can see that by optimizing the glass composition, the PD can be reduced from $-5 \mathrm{~dB} / \mathrm{m}$ to -1.5 $\mathrm{dB} / \mathrm{m}$ which. In addition, the PD measured in GenX shows a stabilization after $\sim 20$ min of accelerating testing which indicates that color center formation has reached a steady-state. Not only a LMA-YDF-20/400 GenX fiber is expected to suffer significantly less power degradation over time than GenVIII, it is also expected to extend the laser lifetime significantly from the stabilization measured. The effect of higher Yb-doping concentration is illustrated in Figure 3 between the "GenX" and the "GenX-Hi" traces where PD increases from $-1.5 \mathrm{~dB} / \mathrm{m}$ to $-3 \mathrm{~dB} / \mathrm{m}$ for $20 \%$ higher concentration.

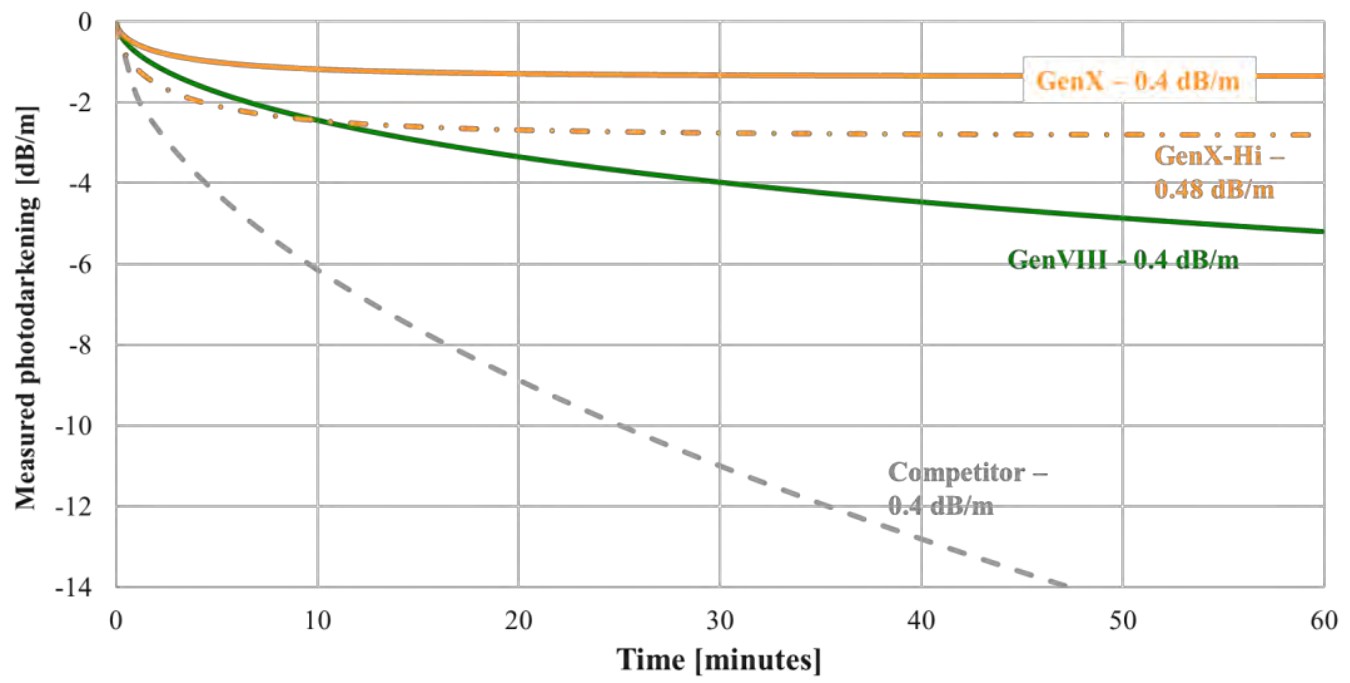

Figure 3. Accelerated PD measured in various $20 \mu \mathrm{m}$ core YDF with nominal cladding absorption of $0.4 \mathrm{~dB} / \mathrm{m}$ at $915 \mathrm{~nm}$ GenVIII and GenX correspond to two different $\mathrm{Yb}$-doped glass compositions. GenX+ has $20 \%$ higher cladding absorption that GenX.

\section{Summary \& Outlook}

Design guidelines used to tune LMA-YDF designs to power scale single-cavity fiber oscillators beyond $1.5 \mathrm{~kW}$ are discussed. Efficient mitigation of both SRS and TMI thresholds is critical and can be done by carefully selecting the design of the LMA-YDF. Furthermore, recent progress in reduction of PD supports power scaling initiatives by extending the laser lifetime. While the LMA-YDF-20/400 design currently available enables fiber laser manufacturers to achieve $1.5 \mathrm{~kW}$ output power with a wide range of pump architectures, further scaling of the output power requires carefully tuned LMA-YDF designs to the pumping wavelength.

\section{References}

[1] Friedrich Möller, Ria G. Krämer, Christian Matzdorf, Maximilian Strecker, Marco Plötner, Fabian Stutzki, Till Walbaum, Thomas Schreiber, Ramona Eberhardt, Stefan Nolte, and Andreas Tünnermann "Multi-kW performance analysis of Yb-doped monolithic single-mode amplifier and oscillator setup", Proc. SPIE 10897, Fiber Lasers XVI: Technology and Systems, 108970D (7 March 2019)

[2] Kensuke Shima, Shinya Ikoma, Keisuke Uchiyama, Yuya Takubo, Masahiro Kashiwagi, and Daiichiro Tanaka "5kW single stage all-fiber Yb-doped single-mode fiber laser for materials processing “, Proc. SPIE 10512, Fiber Lasers XV: Technology and Systems, 105120C (26 February 2018)

[3] Victor Bock, Andreas Liem, Thomas Schreiber, Ramona Eberhardt, and Andreas Tünnermann "Explanation of stimulated Raman scattering in high power fiber systems", Proc. SPIE 10512, Fiber Lasers XV: Technology and Systems, 105121F (26 February 2018) 
[4] Cesar Jauregui, Hans-Jürgen Otto, F. Stutzki, J. Limpert, and A. Tünnermann, "Simplified modelling the mode instability threshold of high power fiber amplifiers in the presence of photodarkening," Opt. Express 23, 20203-20218 (2015)

[5] Stefano Taccheo, Hrvoje Gebavi, Denis Tregoat, Thierry Robin, Beno^ıt Cadier, Daniel Milanese, and Lasse Leick "Photodarkening: measure, characterization, and figure of merit", SPIE Showroom 10.1117/2.1201209.004387 (2012) 


\title{
Fiber lasers and amplifiers for space Lidar applications
}

\author{
Anthony W. Yu* \\ NASA Goddard Space Flight Center, Greenbelt, MD 20771, USA
}

\begin{abstract}
We present current and near-term applications of high-power fiber lasers and amplifiers for NASA science and spacecraft applications. All previously flown missions are based on matured diode pumped solid-state laser transmitters. In recent years we have been exploring the fiber-based transmitter technologies for remote sensing instruments with goals toward space deployment. For instance, due to the spectroscopic nature of trace-gas lidar, and the wavelength region of interest, a fiberbased laser transmitter with a tunable front end is a more appropriate choice. Fiber-based lasers offer a number of attractive advantages for space but since they are relatively new, challenges exist in developing instruments based on them. Our current fiber-based lasers and amplifiers portfolio include laser sounders and lidars for trace gas sensing, laser communications, astrophysics, altimetry and other applications. We discuss recent experimental progress on these systems and instrument prototypes for ongoing development efforts.
\end{abstract}

Keywords: fiber laser, fiber amplifier, remote sensing, lidar, space instrument, space laser

*anthony.w.yu@nasa.gov; phone 1301 614-6248

\section{Introduction}

In the past 20+ years, NASA Goddard Space Flight Center (GSFC) has successfully developed and flown lidars for mapping of Mars, Earth, Mercury and the Moon based on diode-pump solid state laser (DPSSL) technology.[1, 2] As laser and electro-optics technologies continue to expand and mature, more sophisticated instruments that once were thought to be too complicated for space are being considered and developed. Demands for wavelengths spanning from UV to mid-infrared (MIR) to meet a broad range of science goals as well as continual development of instruments for smaller satellite platforms are high and often cannot be easily met using traditional DPSSL. The flexibility, compactness and maturity of fiber based laser architectures in meeting our diverse wavelength requirements and limited resources of small satellites are becoming more important. In this paper, we present progress on several new, space-based laser instruments under development at NASA GSFC. These include lidars for remote sensing of carbon dioxide and methane on Earth for carbon cycle and global climate change; laser communications; gravitational waves detection based on laser ranging interferometers; new generation of multiwavelength altimetry and in situ laser instruments include potential use of ultra-short pulse generation for time-of-flight mass spectrometer to study the diversity and structure of nonvolatile organics in solid samples on missions to outer planetary satellites and small bodies.

We have successfully flown missions based on DPSSL for scientific measurements. These types of laser system designs have space heritage, can be easily scaled for specific use and can be ruggedized for space deployment. At the same time as we

Sixth International Workshop on Specialty Optical Fibers and Their Applications (WSOF 2019),

edited by Liang Dong, John M. Ballato, Proc. of SPIE Vol. 11206, 1120606 · (C) 2019 SPIE

CCC code: $0277-786 X / 19 / \$ 21 \cdot$ doi: $10.1117 / 12.2550991$ 
develop DPSSL for space, we are also investing in fiber laser and amplifier technologies either through in-house development efforts or through programs such as the Small Business Innovative Research (SBIR) and Small Business Technology Transfer (STTR).[3] Fiber laser and amplifier systems have captured a large market share in recent years due to the ever increasing demands on materials processing applications, which include automobile, shipbuilding, pipeline laying, construction, electronics and aerospace. Fiber lasers and amplifiers have the potential for superior beam quality (TEM 00 ), high electricalto-optical efficiency ( $>30 \%$ wall-plug), lower maintenance, higher reliability, smaller footprint, ruggedness and easier transportability when compared to traditional DPSSL systems. All these attributes are most relevant to NASA's future missions. Critical attributes that make fiber lasers and amplifiers attractive for future space-based applications include:

- low susceptibility to optical misalignment and contamination (fusion splices);

- $\quad$ strong leverage from the laser and telecommunications industries;

- high-reliability pump laser diodes with leverages from telecommunications industry;

- pump diodes are physically separated from active laser region allowing better thermal management and distributed thermal load on gain fiber;

- availability of radiation-tolerant devices;

- tunable, diverse-wavelength reliable, low-cost, space-qualified single-frequency laser diode seed sources available for Master Oscillator Power Amplifier architecture;

- scalable to very high powers with both single-device and multi-device architectures;

- eye-safe (wavelengths longer than retinal thermal damage) versions available;

- $\quad$ high wall-plug efficiency.

Although mJ level pulse energies have been reported in fiber lasers,[4] in general the pulsed fiber-laser/amplifier opticalpeak-power is much lower than what is available from bulk solid-state lasers and new and optimized system architectures and measurement approaches are required to exploit and optimize the device capabilities. Rather than low-repetition-rate (1-100 $\mathrm{Hz}$ ) high-peak-power systems, we are investigating high-repetition-rate modest peak-power instruments for new space instrumentation. For some of the applications, especially for satellites orbiting planets that have atmosphere, backscattering from the atmosphere may restrict the repetition rate of the laser transmitter due to range ambiguity. A new measuring scheme is necessary to accommodate the use of fiber based transmitters that preferred high repetition rate operations.

Size, weight and power (SWaP) requirements are strong factors in determining the viability of a space-based instrument. Although each application has numerous factors that influence technology decisions, when considering only the electrical efficiency, the present fiber and pump diode technology favors the Yb-fiber-based laser transmitters. In addition, high electrical-efficiency also means that fewer pump diodes are required for a given optical output power, allowing for higher derating and improve reliability.

\section{Trace Gas Sensing}

\section{Fiber Laser Systems for Space Applications}

We are maturing the technology of a laser transmitter designed for use in atmospheric carbon dioxide remote-sensing. The ultimate goal is to make space-based satellite measurements with global coverage. In this program we are working on a fiberbased master oscillator power amplifier (MOPA) laser transmitter architecture. The seed laser is a wavelength-locked, single frequency, externally modulated distributed Bragg reflector (DBR) operating at $1572 \mathrm{~nm}$ followed by Er-doped amplifiers. The last amplifier stage is a polarization-maintaining, very-large-mode-area fiber with $\sim 1,000$ square microns effective area pumped 
by a Raman fiber laser. The optical output is single-frequency, one microsecond pulses with $>450 \mu \mathrm{J}$ pulse energy, $7.5 \mathrm{kHz}$ repetition rate, single spatial mode, and $>20 \mathrm{~dB}$ polarization extinction. [5]

The global and regional quantification of methane fluxes and identification of its sources and sinks has been highlighted as one of the goals of the 2017 Earth Science Decadal Survey. Detecting methane from space with an active (laser) remote sensing instrument presents several unique technology and measurement challenges. The instrument must have a single frequency, narrow-linewidth light source, and photon-sensitive detector at the right spectral region to make continuous measurements from orbit, day and night, all seasons and at all latitudes. It must have a high signal to noise ratio and must be relatively immune to biases from aerosol/cloud scattering, spectroscopic and meteorological data uncertainties, and instrument systematic errors. The technology needed for a spaceborne mission is currently being developed by NASA and industry. At GSFC, we have developed an airborne instrument to measure methane. Our instrument is a nadir-viewing lidar that uses Integrated Path Differential Absorption (IPDA), to measure a methane vibration-rotational line near $1.65 \mu \mathrm{m}$ that is relatively free of interferences from other trace gases. We sample the absorption line using multiple wavelengths from a narrow linewidth laser source and a sensitive photodetector. This measurement approach provides maximum information content about the vertical distribution of $\mathrm{CH}_{4}$, minimum bias, and sensitivity to atmospheric temperature uncertainty in $\mathrm{XCH}_{4}$ retrieval. However, technical and measurement challenges remain. We will review our progress to date and discuss the technology challenges, options and tradeoffs to measure methane from space. [6]

\section{Laser Communications}

We have recently delivered the Laser Communication Relay Demonstration (LCRD) flight modem to the project for launch in late 2020. The LCRD uses a telecommunication laser diode master oscillator seeding an Er-doped fiber amplifier. The wavelength of the LCRD system is in the $1.55 \mu \mathrm{m}$ band. LCRD will fly two optical communications terminals on a geosynchronous commercial communications satellite. Each LCRD space terminal consists of an optical module beam director, controller electronics, and two separate modems 1) an LLCD modem for Pulse Position Modulation and a multi-rate Differential Phase Shift Keying (DPSK) modem. There is also a controller / data processor between the two space terminals. Each space terminal will be communicating bi-directionally with optical communications ground stations. [7, 8]

\section{Astrophysics}

We are developing a master oscillator power amplifier (MOPA) laser transmitter for the ESA-led Laser Interferometer Space Antenna (LISA) mission.[9] Taking advantage of our space laser experience and the emerging telecom laser technology, we are developing a full laser system for the LISA mission. Our research effort has included both master oscillator (MO) and power amplifier (PA) developments, and their environmental testing and reliability for space flight. Our current baseline for the MO is a low-mass, compact micro non-planar ring oscillator (m-NPRO) laser. The amplifier uses a robust mechanical design based on optical fiber components. We have performed laser system noise tests by amplitude- and frequency-stabilizing the PA output. We will describe our progress and plans to demonstrate a TRL 6 laser system, which is an essential step toward qualifying lasers for space applications, by 2021. [10, 11]

\section{Altimetry}

We are developing a next generation space lidar system with low SWaP. The lidar transmitter is a single fiber laser with high pulse rate, high peak and average fiber laser. The time interleaved fast wavelength tuning technology is deployed in this novel design, which enables dynamic multiple ground track scanning for covering wide swath of target. A high efficiency fiber optical amplifier with high peak and average power is a key component in lidar transmitter of this development. [12] 


\section{Mass Spectroscopy}

Planetary and small body lander missions continue to seek instrumentation that comprehensively characterize the composition of the planetary surface and/or near-subsurface materials. Laser Mass Spectrometry (LMS), advanced at GSFC over the years, is typically utilized to identify and characterize trace amounts of astrobiologically relevant organic content in the acquired samples, however its ability to provide geological context by identifying the composition of the inorganic fraction is generally limited. We are currently developing a femtosecond laser to advance the capabilities of laser mass spectrometers, particularly for enhanced specificity to mineralogical rock composition and explore the potential of LMS for age dating rock samples on future planetary science missions. Femtosecond (fs) lasers are rapidly becoming an alternative to nanosecond or picosecond lasers for applications ranging from laser machining and surgeries to communications and material characterization. They owe their rise to the limited thermal and mechanical energy deposition into the probed material, which results in more precise material removal and reduced radiation damage. These characteristics make fs lasers particularly suitable for laser desorption/ionization time-of-flight mass spectrometry (LDMS) [13] and laser ablation MS (LAMS), [14] since reduced heating leads to reduced elemental fractionation and thus improved compositional accuracy, potentially enabling more accurate age dating of samples than available to date. We are current developing a fs fiber laser transmitter that would meet the systems requirements and with a path toward space deployment.

\section{Satellite Servicing}

NASA GSFC is supporting the development of an advanced 3-D imaging lidar system now baselined for NASA's RestoreL [15] project that will demonstrate an autonomous satellite-servicing capability. The 3-D imaging lidar, called the Kodiak system - formerly known as the Goddard Reconfigurable Solid-state Scanning Lidar, or GRSSLi, will provide real-time images and distance-ranging information during Restore-L. This project will demonstrate how a specially equipped robotic servicer spacecraft can extend a satellite's lifespan. [16]

\section{Conclusions}

Fiber-based laser technologies are becoming more mature and appear to have a strong future for numerous NASA space-based instrument applications. In spite of the numerous advantages of fiber-based laser transmitters there are still issues to be resolved for some NASA applications. The principle issue for many of the high-peak-power transmitters, (in particular those that require narrow bandwidth and in some cases single-frequency operation), are nonlinear effects. Continuing development are necessary to take full advantage of fiber based laser and amplifier technologies.

\section{References}

1. Yu, A.W., et al., "Fiber lasers and amplifiers for space-based science and exploration," Proc. SPIE 8237, Fiber Lasers IX: Technology, Systems, and Applications, 823713 (15 February 2012); doi: 10.1117/12.916069, and references therein.

2. Yu, A.W., "Space-based Lasers for Remote Sensing Applications," Applied Industrial Optics: Spectroscopy, Imaging and Metrology 2011, Toronto Canada, 10-14 July 201, and references therein.

3. https://sbir.nasa.gov/.

4. Lee, W., et al., "1.8 mJ, $3.5 \mathrm{~kW}$ single-frequency optical pulses at $1572 \mathrm{~nm}$ generated from an all-fiber MOPA system," Optics Letters, 43, 10, pp. 2264-2267 (2018).

5. Stephen, M., et al., "Fiber-based, Laser Transmitter Technology Maturation Program for Global Spectroscopic Trace-gas Measurements,” 2018 IEEE International Geoscience and Remote Sensing Symposium, July 201, Valencia, Spain. 
6. Riris, H, et al., "The Challenges of Measuring Methane from Space with a Lidar," Proceedings Volume 11180, International Conference on Space Optics - International Conference on Space Optics (ICSO) 2018, 09-12, October 2018, Chania; Greece.

7. Krainak, M.A., et al., "A dual format communications modem development for the Laser Communications Relay Demonstration (LCRD) program,” Proc. SPIE 8610, (2013).

8. Lafon, R., et al., "High-peak power fiber amplifier for deep-space laser communications," Proceedings Volume 10524, Free-Space Laser Communication and Atmospheric Propagation XXX; 105241C (2018) https://doi.org/10.1117/12.2290864.

9. https://lisa.nasa.gov/.

10. Numata, K., et al. ," Progress and plans for a U.S. laser system for the LISA mission,” Proceedings Volume 11180, International Conference on Space Optics - International Conference on Space Optics (ICSO) 2018, 09-12, October 2018, Chania; Greece.

11. Yu, A, et al., "Spaceborne Laser Transmitter for the Laser Interferometer Space Antenna (LISA) Mission," 2018 Conference on Lasers and Electro-Optics Pacific Rim (CLEO-PR), Hong Kong, Hong Kong, 2018.

12. NASA GSFC Internal Research and Development (IRAD) funded program.

13. Getty, S., et al., "Molecular Analyzer for Complex Refractory Organic-Rich Surfaces (MACROS)," 2017 IEEE Aerospace Conference, Big Sky, MT, United States, March 04 - March 11, 2017.

14. Li, X., et al., "Laser ablation mass spectrometer (LAMS) as a standoff analyzer in space missions for airless bodies," International Journal of Mass Spectrometry, Volumes 323-324, Pages 63-67, 1 June 2012.

15. https://sspd.gsfc.nasa.gov/restore-1.html

16. https://www.nasa.gov/feature/goddard/2018/self-driving-servicer-now-baselined-for-nasa-s-restore-1-satellite-servicingdemonstration. 


\title{
2 MW peak power ultrashort pulse amplification using tapered powder- sintered all-solid fibers: Fiber designs, technology, and results
}

\author{
Martin Leich a , André Kalide a , Tina Eschrich a, Martin Lorenz a , Anka Schwuchow a , Jens Kobelke a , \\ Jörg Bierlich ${ }^{a}$, Claudia Aichele ${ }^{a}$, Katrin Wondraczek ${ }^{a}$, Dörte Schönfeld ${ }^{b}$, Andreas Langner ${ }^{b}$, \\ Clemens Schmitt ${ }^{\mathrm{b}}$, Jaqueline Plass ${ }^{\mathrm{b}}$, Gerhard Schötz ${ }^{\mathrm{b}}$, Yuhtat Cho ${ }^{\mathrm{c}}$, Andy Malinowski ${ }^{\mathrm{c}}$, Fabio \\ Ghiringhelli ${ }^{\mathrm{c}}$, Andrew Marshall ${ }^{\mathrm{c}}$, Mike Durkin ${ }^{\mathrm{c}}$, Matthias Jäger ${ }^{\mathrm{a}}$ * \\ ${ }^{a}$ Leibniz Institute of Photonic Technology (IPHT) Jena, Albert-Einstein-Str. 9, 07745 Jena, Germany \\ ${ }^{\mathrm{b}}$ Heraeus Quarzglas GmbH \& Co. KG, Quarzstraße 8, 63450 Hanau, Germany \\ ${ }^{\mathrm{c}}$ SPI Lasers UK Ltd, 6, Wellington Park, Tollbar Way, Hedge End, SO30 2QU, Southampton, UK
}

\begin{abstract}
We investigate various all-solid fiber designs featuring very large core-to-clad ratios and powder-sintering technology for ultrashort pulse amplification up to $2 \mathrm{MW}$ peak power. Using short tapered amplifiers beam qualities of $\mathrm{M}^{2} \approx 1.5$ and better have been achieved.
\end{abstract}

Keywords: Fiber optics, specialty optical fibers, fiber amplifiers, fiber lasers, ultrashort pulses

*Matthias.jaeger@leibniz-ipht.de; phone +49 3641 206-203

\section{Introduction}

Fiber laser sources have revolutionized laser materials processing with combination of high power and excellent beam quality and have recently become the dominant laser type in this market. Peak power scaling up the MW power level has been first achieved using photonic crystal fibers about a decade ago ${ }^{1}$. However, practical aspects like bend sensitivity, spliceability etc. have prevented a general acceptance of this technology. Tapered fiber amplifiers have also been demonstrated ${ }^{2,3,4}$, relying on the adiabatic evolution of the fundamental mode in a step-index fiber for good beam quality. Alternative to these long draw-tower tapers, short adiabatic tapers allow separating the fiber drawing from tapering process, offering more flexibility and showing great potential for high peak powers ${ }^{5}$.

On the other hand, the development of new fiber technology processes such as the powder-sintering (REPUSIL) ${ }^{6}$ have enabled the production of large volumes of very homogeneously doped core material, required for very large core stepindex fibers with undisturbed mode fields. Furthermore, the inclusion of Fluorine (F) doping in the REPUSIL process ${ }^{7}$ and its negative refractive index contribution offers great flexibility in the design of tailored refractive index profiles.

We report on the investigation of various such fiber designs for ultrashort pulse amplification with a special focus on large core-to-clad ratios and refractive index control. To the best of our knowledge the peak power of $2 \mathrm{MW}$ is the highest achieved in tapered step-index fibers so far.

\section{Fiber designs}

For the amplification of ps pulses below the self-focusing limit, the threshold for stimulated Raman scattering (SRS) is the most relevant nonlinear limit. Theoretically, the SRS threshold can be extended by scaling to larger mode fields and larger pump absorption (and thus shorter effective fiber lengths). The latter can be achieved by a larger $\mathrm{Yb}_{2} \mathrm{O}_{3}$ concentration or higher core-to-clad diameter ratios (CCDR), both increasing the cladding absorption. Practically, both strategies lead to an increase of the number of guided core modes and therefore make it more difficult to maintain single transverse mode operation through adiabatic tapering.

Sixth International Workshop on Specialty Optical Fibers and Their Applications (WSOF 2019), edited by Liang Dong, John M. Ballato, Proc. of SPIE Vol. 11206, 1120607 · C 2019 SPIE

CCC code: $0277-786 X / 19 / \$ 21 \cdot$ doi: $10.1117 / 12.2548616$ 
We have investigated several approaches to reduce the number of core modes by reducing the refractive index contrast of the core vs. the inner cladding and thus the core numerical aperture (NA): by a classical pedestal design (Fig. 1a), a fully doped inner cladding (Fig 1b), and most notably also a introduction of Fluorine (F) into the REPUSIL process to reduce the core refractive index (Fig 1c) and contrasted the performance to a fiber with low Yb doping but similar core NA (Fig. 1d).

As a second strategy, we have explored very large CCDR values to significantly increase the cladding absorption at the pump wavelength. Typical double clad fibers have values of 1:20 to 1:10, while our fibers have values up to 1:3, which have been rarely investigated so far.

a)

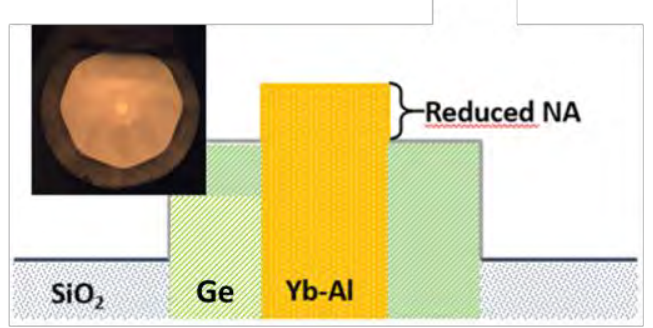

c)
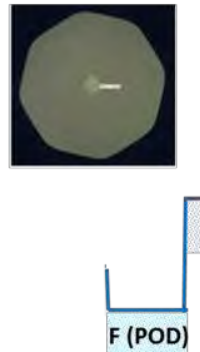

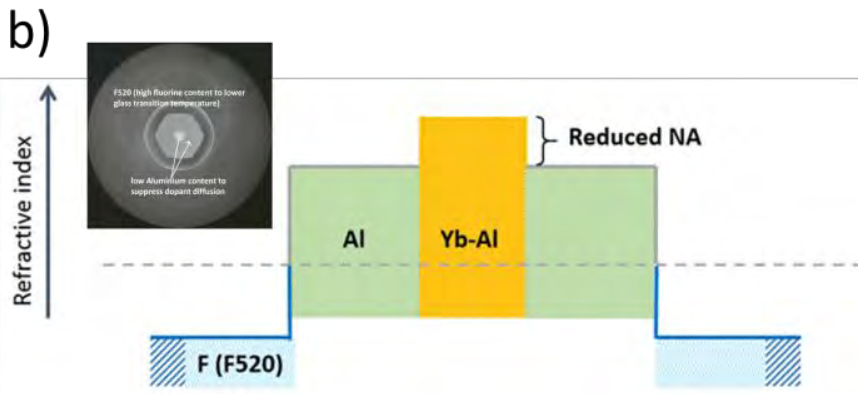

b)

d)
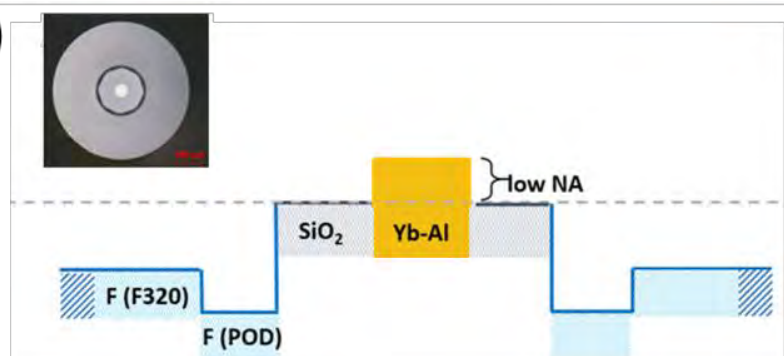

Figure 1. Investigated fiber designs: a) classical pedestal design using Germanium, b) fully doped inner cladding using Al-doped REPUSIL material, c) Fluorine-codoped core for refractive index reduction, d) low $\mathrm{Yb}_{2} \mathrm{O}_{3}$ concentration $(0.08$ $\%$ molar).

\section{Results and Discussion}

The various fibers discussed above have been tapered on the input side using a Vytran GPX-3200 and spliced directly to the seed fiber. A typical taper length is about $5 \mathrm{~cm}$, while the active fiber lengths vary between $0.5 \mathrm{~m}$ and $2 \mathrm{~m}$ depending mainly on cladding absorption. The amplification experiments were performed using a seed MOPA providing 30 to 60 ps pulses.

Figure 2 shows exemplary the amplifier results of a fiber with F-doped core/clad diameter of $41 \mu \mathrm{m} / 179 \mu \mathrm{m}$ (Fig. 1c). The corresponding thicker fiber of this preform with a $52 \mu \mathrm{m}$ core diameter exhibited up to $2 \mathrm{MW}$ peak power without SRS. This is to the best of our knowledge the highest value directly obtained from a tapered step-index fiber. The beam quality was slightly worse around $\mathrm{M}_{2}=1.5$, indicating that this new F-doped REPUSIL materials needs further refinement of the doping process to access larger core diameters.

Overall, all of the fiber types of Fig. 1 were exhibiting beam qualities of better than 2, but only the fiber type $1 \mathrm{~b}$ and $1 \mathrm{~d}$ with large CCDR were scaled in peak power above $1 \mathrm{MW}$. The various fiber designs will be compared in terms of their technology and potential. Time permitting, a discussion of mode matching, diffusion effects and the adiabatic criteria will be included. 

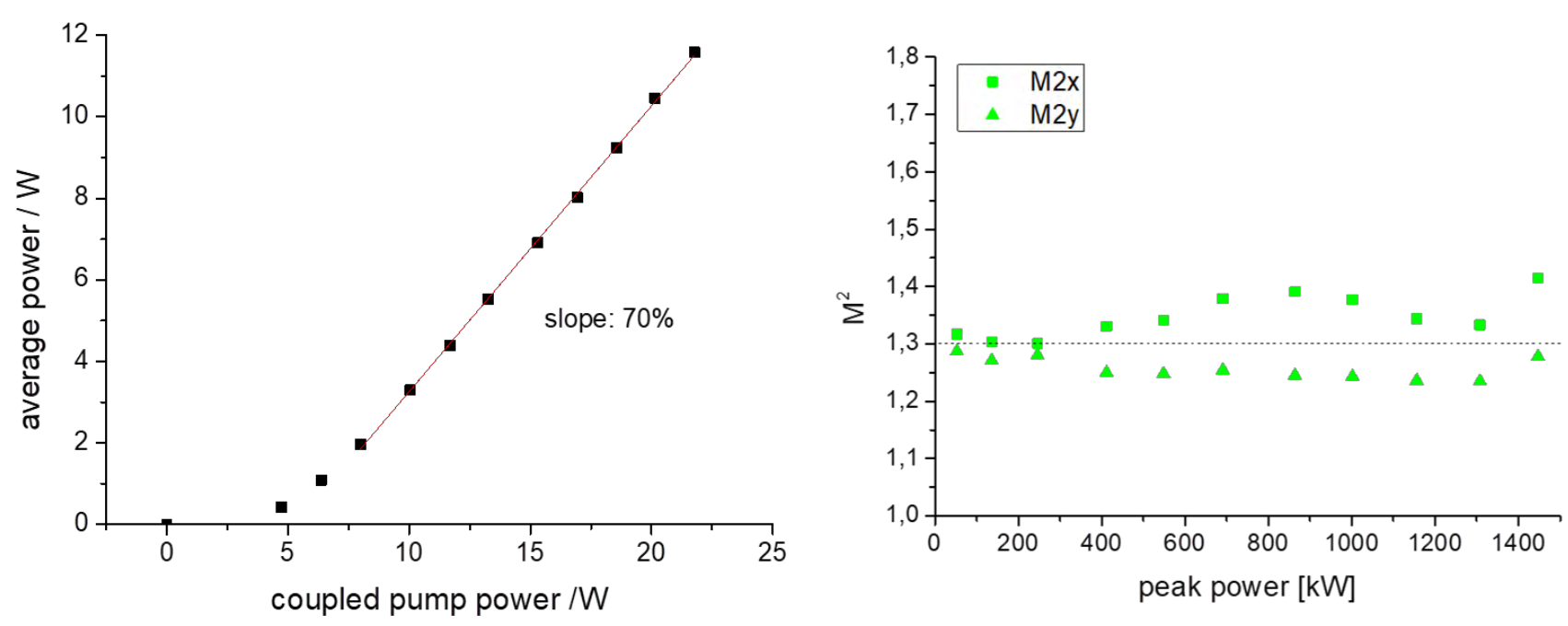

Figure 2. Slope efficiency and beam quality vs. peak power for a F-doped core fiber with $75 \mathrm{~cm}$ length.

\section{References}

[1] T. Eidam, J. Rothhardt, F. Stutzki, F. Jansen, S. Hädrich, H. Carstens, C. Jauregui, J. Limpert, and A. Tünnermann, "Fiber chirped-pulse amplification system emitting $3.8 \mathrm{GW}$ peak power," Opt. Express 19(1), 255-260 (2011).

[2] V. Filippov, Y. Chamorovskii, J. Kerttula, K. Golant, M. Pessa, and O. G. Okhotnikov, "Double clad tapered fiber for high power applications," Opt. Express 16(3), 1929-1944 (2008).

[3] K. Bobkov, A. Andrianov, M. Koptev, S. Muravyev, A. Levchenko, V. Velmiskin, S. Aleshkina, S. Semjonov, D. Lipatov, A. Guryanov, A. Kim, M. Likhachev, A. V. Andrianov, A. V. Kim, and M. E. Likhachev, "Sub-MW peak power diffraction-limited chirped-pulse monolithic Yb-doped tapered fiber amplifier," Opt. Express 25(22), 2695826972 (2017).

[4] A. Fedotov, T. Noronen, R. Gumenyuk, V. Ustimchik, Y. Chamorovskii, K. Golant, M. Odnoblyudov, J. Rissanen, T. Niemi, and V. Filippov, "Ultra-large core birefringent Yb-doped tapered double clad fiber for high power amplifiers," Opt. Express 26(6), 6581-6592 (2018).

[5] Y. Zhu, M. Leich, M. Lorenz, T. Eschrich, C. Aichele, J. Kobelke, H. Bartelt, M. Jäger, "Yb-doped large mode area fiber for beam quality improvement using local adiabatic tapers with reduced dopant diffusion," Opt. Express 26(13), 17034-17043 (2018).

[6] M. Leich, F. Just, A. Langner, M. Such, G. Schötz, T. Eschrich, and S. Grimm, "Highly efficient Yb-doped silica fibers prepared by powder sinter technology," Opt. Lett. 36(9), 1557-1559 (2011).

[7] K. Schuster, S. Grimm, A. Kalide, J. Dellith, M. Leich, A. Schwuchow, A. Langner, G. Schötz, and H. Bartelt, "Evolution of fluorine doping following the REPUSIL process for the adjustment of optical properties of silica materials," Opt. Mater. Express 5(4), 887-897 (2015). 


\title{
Fabrication of vapor phase large core Yb doped preform with precise index control for the development of VLMA active fibers
}

\author{
O. Le Goffic ${ }^{1}$, D. Landais ${ }^{1}$, A. Monteville ${ }^{1}$, L. Provino ${ }^{1}$, A. Haboucha ${ }^{1}$, A. Barnini ${ }^{2}$, P. Guitton ${ }^{2}$, G. Guiraud ${ }^{3}$, \\ N. Traynor ${ }^{3}$, T. Taunay ${ }^{1 *}$ \\ 1: Photonics Bretagne, 4 rue Louis de Broglie, 22300 Lannion, France, \\ 2: IXBLUE Photonics, Rue Paul Sabatier, 22300 Lannion, France, 3 : Azur Light Systems, 11 Avenue de Canteranne, \\ 33600 Pessac, France
}

\begin{abstract}
We report on the successful fabrication of very low NA Yb doped preforms using a commercial vapor phase delivery system using chelates precursors as rare earth sources. Two preforms with low NA 0.035 were drawn to $44 \mu \mathrm{m}$ core step index double clad PM fibers capable of operating with truly single mode regime when coiled to the proper diameter. In addition careful optimization of the glass composition with an $\mathrm{Al}_{3} \mathrm{PO}_{4}$ glass matrix enables essentially photodarkening free operation. Kilometer long uniform Yb doped PM PERFOS ${ }^{\circledR}$ fibers with mode field diameter and effective of $33 \mu \mathrm{m}$ and $880 \mu \mathrm{m}^{2}$ were successfully fabricated.
\end{abstract}

Keywords: Vapor phase, Chelates precursors, Low NA, Ytterbium, Fiber laser

* ttaunay@photonics-bretagne, +33 (0)6 99678003

\section{Introduction}

The last decade has seen the rapid development of high peak power and high pulse fiber amplifier which has been enabled by the development of large mode area (LMA) fiber amplifier architectures. This ever increasing quest for large effective area is essentially driven by the necessity to mitigate the non-linear effects such as Four-Wave mixing (FWM), Self-Phase modulation (SPM), and Stimulated Raman scattering, which all can lead to deleterious pulse amplification distortion. On the other end, state-of-the art rare-earth (RE) doped conventional manufacturing process limitations, has prevented the development of truly single mode RE doped fibers with core diameters greater than $15 \mu \mathrm{m}$ at $1 \mu \mathrm{m}^{[1]}$. Equally important is the need for offering high beam quality and excellent pointing stability. As the core diameter of LMA fibers is increased to beyond approx. $15 \mu \mathrm{m}$, manufacturing single-mode fibers becomes difficult with sufficient yield due to the required index precision obtainable even with state-of-the-art rare-earth-doped core manufacturing processes $\left(+/-10^{-4}\right)$. Consequently for the fabrication of larger SM cores numerous strategies have been applied to circumvent this technological barrier, and to date LMA photonic crystal fibers are still the only commercial viable path to reach large mode field diameters ${ }^{[2]}$. But the complex designs of most of the fibers demonstrated come at the expense of complex splicing and packaging solutions which often limit or preclude monolithic integration. Large coil diameter or incompatibilities with fused-fiber components to name a few have forced laser developers to make trade-offs between performance and practibility. Here we report on the successful fabrication of uniform long length of large core Ytterbium doped low NA step index silica preforms with the use of a commercial RE based vapor phase delivery system. The Ytterbium doped core glass matrix has been optimized to be at the same time low loss and photodarkening free while maintaining the required low NA necessary to achieve single mode behavior with good beam quality. PM step index double clad Ytterbium fibers were successfully drawn from the fabricated preforms showing minimal variation along the fiber length $(1.5 \mathrm{~km})$, and more importantly comparison with a perfect step index fiber with an equivalent core diameter show a minimal $(\sim 10 \%)$ reduction in effective area of the fabricated preform. Numerical simulations based on a commercial full-vector finite-element based modal solver demonstrates optimal coiling diameter single mode operation is

Sixth International Workshop on Specialty Optical Fibers and Their Applications (WSOF 2019), edited by Liang Dong, John M. Ballato, Proc. of SPIE Vol. 11206, 1120608 · (C) 2019 SPIE CCC code: $0277-786 X / 19 / \$ 21 \cdot$ doi: $10.1117 / 12.2548355$ 
possible for bend diameter as low as $20 \mathrm{~cm}$ while maintaining minimal loss for the fundamental loss for $44 \mu \mathrm{m}$ core PM fibers with close to $900 \mu \mathrm{m}^{2}$ effective are ( $\left.\mathrm{A}_{\text {eff }}\right)$.

\section{Results}

The major challenge in fabricating low NA RE doped preforms for VLMA fibers reside in the ability to obtain a "Defect free" radial refractive index as any imperfection will result in significant degradation of beam quality and effective area reduction ${ }^{[3]}$. Additionally in the case of Ytterbium doping to mitigate detrimental photodarkening effects and improve fiber efficiency, large concentrations of $\mathrm{Al}_{2} \mathrm{O}_{3}$ and/or $\mathrm{P}_{2} \mathrm{O}_{5}$ are required ${ }^{[4]}$, which alongside the ytterbium doping raise the core index difference well above the required NA. Fortunately, as demonstrated by DiGiovanni \& al ${ }^{[5]}, \mathrm{Al}_{2} \mathrm{O}_{3} / \mathrm{P}_{2} \mathrm{O}_{5}$ equimolar codoping leads to a core where the $\mathrm{Yb}_{2} \mathrm{O}_{3}$ is essentially responsible for the index difference. To fabricate the doped preforms we used two commercially available vapor delivery systems, a SG Controls System for the high pressure vapor halide precursors and a Lumentum, Slovenia (formerly Optacore Inc.) CDS3 system which uses chelates precursors for rare earth doping. In order to validate the $\mathrm{Al}_{2} \mathrm{O}_{3}-\mathrm{P}_{2} \mathrm{O}_{5}$ based glass modifier matrix composition for efficient Ytterbium doping, we manufactured a basic LMA Yb 10-130 type preform/double clad fiber (DCF) with a $1.1 \mathrm{~dB} / \mathrm{m}$ cladding absorption at $915 \mathrm{~nm}$. To this effect $\mathrm{GeO}_{2}$ was adding in the gas mixture during deposition to obtain the typical 0.08 NA of a LMA YB 10-130. Measured background losses at $1150 \mathrm{~nm}$ were on the order of 5 to $10 \mathrm{~dB} / \mathrm{km}$ with a surprisingly below $1 \mathrm{ppm} \mathrm{OH}$ content given the fact that no extra step was used to dehydrate the individual passes during core deposition. Photodarkening test was performed by building a core pumped $976 \mathrm{~nm}$ fiber amplifier seed by a DFB laser. After more than 120 hours the signal decrease had saturated to less than $3 \%$ which is sensibly less than similar commercially available fibers with a signal dropping generally by more than $20 \%$ after only 60 hours.

Typical refractive index profile and radial $976 \mathrm{~nm}$ core absorption along the length of fabricated preforms are shown on figure 1.a \& b. For this preform, a nearly 1:1 molar $\mathrm{Al}_{2} \mathrm{O}_{3} / \mathrm{P}_{2} \mathrm{O}_{5}$ ratio was used as core glass modifier, as can be seen on fig1.a, $250 \mathrm{~mm}$ long preform with core/clad index difference $\sim 4.5 \times 10^{-4}$ showing negligible longitudinal index variation (below 5x10-5) were successfully fabricated. For this preform type, careful adjustments of the MCVD flows were made during the 15 layers core deposition, and in particular at the end to prevent index depression due to $\mathrm{P}_{2} \mathrm{O}_{5}$ burn-off. Although not intentional, the profile presents some index gradient which could provide some $\mathrm{A}_{\text {eff }}$ resistance against bend induced degradation ${ }^{[6]}$.

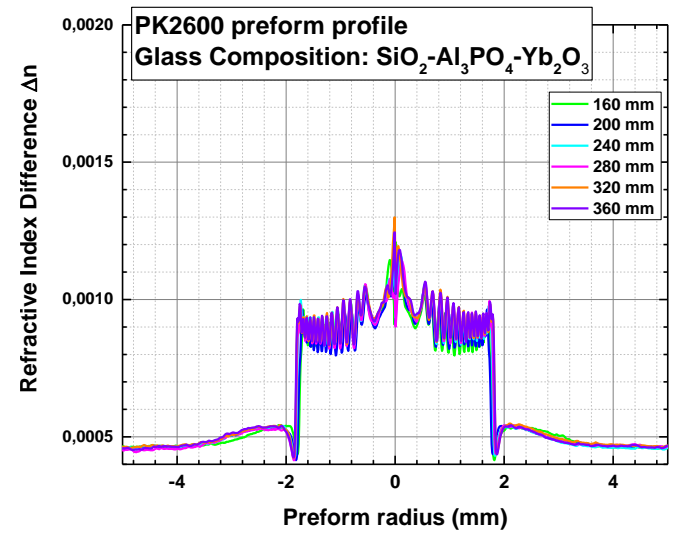

Fig.1.a: Refractive index $\mathrm{Yb}$ doped preform profiles

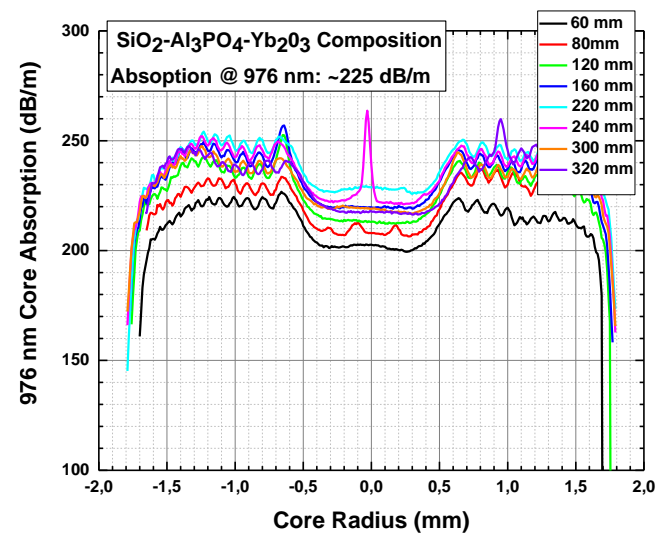

Fig.1.b: Preform $976 \mathrm{~nm}$ radial absorption profiles 
Similarly, Fig1.b represents the radial profile of the $976 \mathrm{~nm}$ core absorption of the nearly $4 \mathrm{~mm}$ core diameter preform along its length, the same overall uniformity is observed.

To optimize the potential and determine the best trade-off for the preforms fabricated we simulated the fiber properties ( $\mathrm{A}_{\text {eff, }}$ modal losses as well as cladding absorption) using an in-house full-vector finite-element based on a commercial modal solver (Comsol). Our simulations showed that for a $44 \mu \mathrm{m}$ core diameter, the effective area of the unperturbed fiber could reach $925 \mu \mathrm{m}^{2}$, for sake of comparison simulation performed on a perfect step index of equivalent diameter the effective area is equal to $1020 \mu \mathrm{m}^{2}$ or only less than $10 \%$ greater. Note that although 15 passes were used for these preforms, larger preforms could be made without any degradation which would have further improve the effective area.

Figure 2.a represents the bend losses as a function of bend diameters for the different modes for a preform whose core has been scaled to a $44 \mu \mathrm{m}$ diameter. The blue curves represents the fiber properties with the projected index induced changes associated with fiber draw. From these results one can see that truly single mode behavior can be achieved for a $\sim 20 \mathrm{~cm}$ bend diameter while maintaining low losses for the fundamental mode.

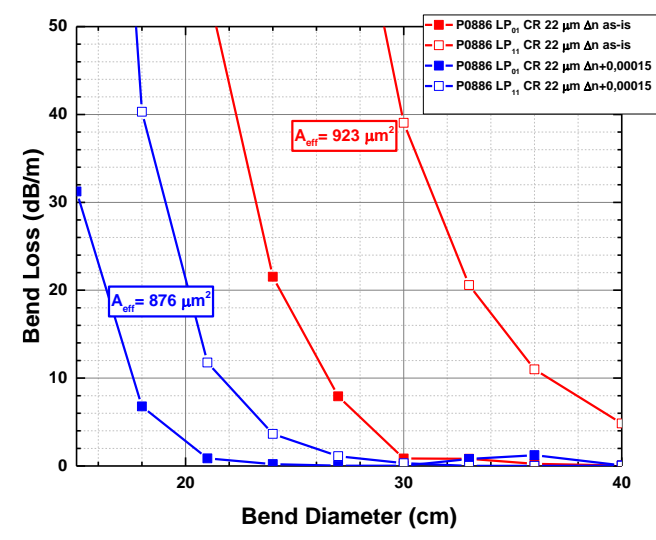

Fig. 2.a: LP01 \& LP11 bend loss as a function of bend diameter

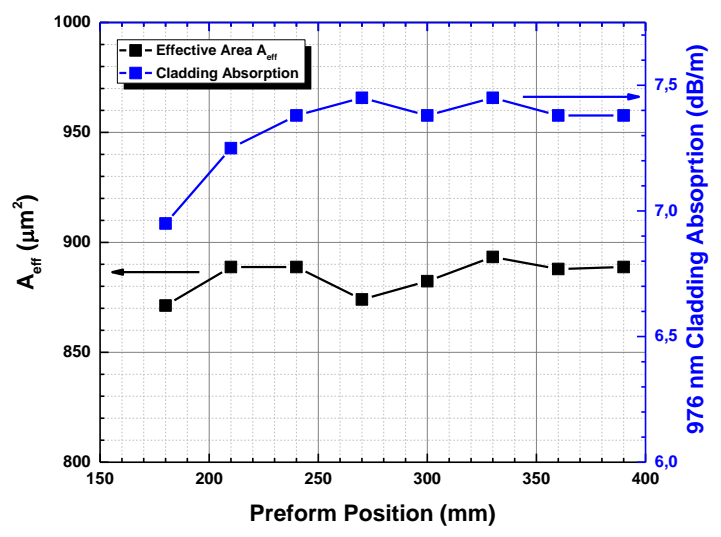

Fig. 2.b: Aeff and cladding absorption evolutions along the preform length

It is important to note that at this diameter a fiber effective area close to $700 \mu \mathrm{m}^{2}$ against $520 \mu \mathrm{m}^{2}$ for the best fiber commercially available, which in addition requires a microstructured design to achieve these performances.

Fig. 2.b represents the projected $A_{\text {eff }}$ and $976 \mathrm{~nm}$ cladding absorption for a $230 \mu \mathrm{m}$ double clad fiber diameter, one can see that minimal variations are projected for almost the entire fiber draw which is a critical parameter for fiber laser integrators.

Two preforms were consequently assembled with a standard "Stack \& Draw" method with stress rods disposed symmetrically around the core rod preform and processed to $44 \mu \mathrm{m}$ core, $230 \mu \mathrm{m}$ fiber OD nearly circular double clad fiber coated with a 0.46 low NA fluorinated coating. The measured cladding absorption, about $8 \mathrm{~dB} / \mathrm{m}$ at $976 \mathrm{~nm}$ was inline to our predictions and the cladding attenuation measured at $1300 \mathrm{~nm}$ with a multimode OTDR was $20 \mathrm{~dB} / \mathrm{km}$. Additional results, fiber efficiency and actual mode field diameter will be presented at the conference.

\section{Conclusions}

In conclusion, large and uniform $\mathrm{Yb}_{2} \mathrm{O}_{3}-\mathrm{AlPO}_{4}$ co-doped silica preform with very low NA were prepared by vapor phase deposition using $\mathrm{Yb}$-chelates precursors, and successfully drawn into VLMA double clad fibers presenting near diffraction limited $\mathrm{M}^{2}$. These PERFOS ${ }^{\circledR}$ fibers can operate with low loss truly single mode regime with small package diameter. Combined with the all-silica, microstructure free fiber design, this photodarkening free core preforms and 
double clad fibers open up the possibility to increase the output power of high energy pulse fiber amplifier with increase modal instability threshold.

\section{Acknowledgments}

This work was done in the framework of the 4F project ("French laser Fibers for Factories of the Future") funded by BPI France (PIA program).

\section{References}

[1] M.J. Li \& al, "Limit of effective area for single mode operation in Step Index LMA laser fiber", J.L.T. 27 (15), 3010$3016(2009)$

[2] T. Tanggaard \& al., "Single Mode Yb-doped LMA Photonic Bandgap rod fiber amplifier", Opt. Exp. 19 (8), 7398 7409 (2011)

[3] J. Boullet "Regime-dependent photo-darkening-induced modal degradation in high power fiber amplifier", ASSL, paper ATu4A.3, (2016)

[4] M. Likhachev \& al., "Large-Mode-Area Highly Yb-doped Photodarkening-Free $\mathrm{Al}_{2} \mathrm{O}_{3}-\mathrm{P}_{2} \mathrm{O}_{5}-\mathrm{SiO}_{2}-\mathrm{Based}$ Fiber," CLEO/Europe-EQEC 2011, paper CJ.P.24 (2011

[5] D.J. DiGiovanni \& al., "Structure and properties of silica containing aluminum and phosphorus near the $\mathrm{AlPO}_{4}$ join", J. Non-Cryst Solids, 113 (1), 58-64, (1989)

[6] J.M. Fini "Bend resistant design of conventional and microstructure fibers with very large mode area", Opt. Exp., 14 (1), 69-81 (2006) 


\title{
Highly $\mathrm{Tm}^{3+}$ doped germanate glass and associated double clad fiber for 2 um lasers and amplifiers
}

\author{
Fedia B. Slimen *, Zhengqi Ren, Andrea Ventura, Juliano Grigoleto Hayashi, Jaroslaw Cimek, \\ Nicholas White, Yongmin Jung, David Richardson, Francesco Poletti \\ Optoelectronics Research Centre, University of Southampton, SO17 1BJ, UK
}

\begin{abstract}
We report on the optimization of $\mathrm{Tm}^{3+}$ concentration in $\mathrm{Tm}^{3+}$ doped germanate single mode (SM) fibers. A record value of $8.5 \times 10^{20}$ ions $/ \mathrm{cm}^{3}(5.1 \mathrm{wt} \%)$ has been achieved. The fiber produced is promising for high power ultrashort fiber device applications.
\end{abstract}

Keywords: Germanate glasses, thulium, single mode fiber, $2 \mu \mathrm{m}$ lasers and amplifiers. *F.Ben-Slimen@soton.ac.uk

\section{I - Introduction}

Fiber lasers operating at the eyes-safe wavelength of $2 \mu \mathrm{m}$ have attracted a great deal of attention due to their potential use in applications such as LIDAR [1], laser surgery [2] and remote sensing [3]. Therefore, in recent years, considerable efforts have been devoted to the development and optimization of thulium and holmium doped glasses which emit at wavelengths around $1.95 \mu \mathrm{m}$ and $2.1 \mu \mathrm{m}$ respectively. In particular, substantial work has been dedicated to the development of effectively single mode, low numerical aperture (NA), large mode area (LMA) thulium doped fibers (TDFs) as a means to shorten active device lengths and hence to reduce the impact of optical nonlinearities that can limit the performance of single-frequency lasers and pulsed fiber laser systems operating at wavelengths around $2 \mu \mathrm{m}$ [4-6].

Only relatively low Tm-ion doping concentrations of $10^{18}-10^{19}$ ions $/ \mathrm{cm}^{3}$ can be achieved in pure silica glass [7]. Introducing co-dopants such as aluminum in the silica matrix can allow this concentration to be increased by one or two orders of magnitude. However, this generally increases the refractive index of the glass relative to pure silica and leads to the need to include a raised index pedestal around the doped core to achieve single mode guidance in a large mode area core [8]. A simpler alternative to pure or doped silica, is that of using alternative multicomponent glass system which can in principle be doped with even higher rare-earth ion concentrations, typically up to $10^{21}$ ions $/ \mathrm{cm}^{3}$ [9]. These can be more readily and directly index-matched to suitable glass cladding materials to obtain simple LMA structures, thus raising the possibility of ultrashort fiber devices ( $\sim$ few to a few 10 's of $\mathrm{cm}$ scale) to help mitigate nonlinearity. In particular, oxide glasses in the silicate [10] or germanate [6] families offer much higher levels of rare-earth ion solubility than silica glass and have thus attracted much attention in recent years. However the high phonon energy of silicate glasses $\left(\sim 1100 \mathrm{~cm}^{-1}\right)$ [11] results in fast multiphonon relaxation, which can lead to an undesirable reduction in quantum efficiency and in turn to problems with thermal management (and ultimately damage) within a fiber laser cavity. By contrast, a germanate glass host offers a comparatively low phonon energy $\left(\sim 845 \mathrm{~cm}^{-1}\right)$ [9] and hence a higher quantum efficiency, whilst providing excellent $\mathrm{Tm}^{3+}$ ion solubility, and higher IR transparency.

In this work we present our recent results on the optimization of $\mathrm{Tm}^{3+}$ concentration in a novel germanate glass composition. Note that this composition was one of the best produced germanate glasses in my recent work [12] but without optimization of $\mathrm{Tm}^{3+}$ concentration. It showed the ability to withstand the double thermal cycle necessary for the fibre fabrication without any sign of crystallisation. Now we perform the optimization and we report on the development of a high $\mathrm{Tm}^{3+}$ doping concentration $\left(8.5 \times 10^{20}\right.$ ions $\left./ \mathrm{cm}^{3}\right)$ germanate fiber which is nearly 3 times higher than that cited in

Sixth International Workshop on Specialty Optical Fibers and Their Applications (WSOF 2019), edited by Liang Dong, John M. Ballato, Proc. of SPIE Vol. 11206, 1120609 · @ 2019 SPIE

CCC code: $0277-786 X / 19 / \$ 21 \cdot$ doi: $10.1117 / 12.2548643$ 
Ref. [12], at the same time achieving a low-NA (0.07), large mode area (core diameter of $17 \mu \mathrm{m}$ ) and incorporating a double cladding to facilitate application in cladding pumped fiber lasers and amplifiers operating in the $2 \mu \mathrm{m}$ wavelength region.

\section{Glass development and characterization}

The glass samples used in this work were synthesized by the conventional melting-quenching technique using high purity chemicals (more than 99.9\%). A series of four samples with different $\mathrm{Tm}^{3+}$ concentration was preparedfrom the chemical composition of $58 \mathrm{GeO}_{2}-15 \mathrm{PbO}-13 \mathrm{ZnO}-4 \mathrm{Nb}_{2} \mathrm{O}_{5}-7 \mathrm{Na}_{2} \mathrm{O}-1.5 \mathrm{SiO}_{2}-1.5 \mathrm{Al}_{2} \mathrm{O}_{3}$. Figure 1.a depicts the absorption spectra of these glass samples in the wavelength range from 200 to $2000 \mathrm{~nm}$ using a UV-VIS-NIR double beam Varian Cary 500 spectrometer. As can be seen, there is no obvious change in the position of the $\mathrm{Tm}^{3+}$ absorption peaks, but the absorption peaks increase in proportion to the amount of $\mathrm{Tm}_{2} \mathrm{O}_{3}$ used in the glass preparation. The good linearity between the $\mathrm{Tm}^{3+}$ concentration and the peak intensities reveals the excellent solubility of the $\mathrm{Tm}$ ions in the developed germanate glass composition. Thanks to this high solubility we have measured pump absorptions at $1700 \mathrm{~nm}$ of over $1500 \mathrm{~dB} / \mathrm{m}$, which should enable amplifiers of lengths of $\sim 10 \mathrm{~cm}$ or less - ideal for many short pulse applications. Figure 1.b shows the emission spectra from 1500 to $2050 \mathrm{~nm}$ of the four germanates glasses doped with different $\mathrm{Tm}^{3+}$ concentrations. The emission was measured at room temperature under $793 \mathrm{~nm}$ pump laser excitation through a multimode silica glass fiber ( $200 \mu \mathrm{m}$ core diameter and $0.22 \mathrm{NA}$ ). As can be seen, by increasing the $\mathrm{Tm}_{2} \mathrm{O}_{3}$ content the $1.8 \mu \mathrm{m}$ emission from the ${ }^{3} \mathrm{~F}_{4}$ $\rightarrow{ }^{3} \mathrm{H}_{6}$ transition increases gradually. This phenomenon can be explained by the reduced distance between $\mathrm{Tm}^{3+}$ ions, which increases the cross-relaxation energy transfer probability $\left({ }^{3} \mathrm{~F}_{4} \rightarrow{ }^{3} \mathrm{H}_{6} \rightarrow{ }^{3} \mathrm{~F}_{4} \rightarrow{ }^{3} \mathrm{~F}_{4}\right)$ before attaining a maximum value at a $\mathrm{Tm}^{3+}$ concentration of $8.5 \times 10^{20}$ ions $/ \mathrm{cm}^{3}$. The $1.8 \mu \mathrm{m}$ emission intensity decreases with further addition of $\mathrm{Tm}_{2} \mathrm{O}_{3}$ content which may be explained by the enhanced energy transfer rate from ${ }^{3} \mathrm{~F}_{4}$ to $\mathrm{OH}-$ or impurities. The inset of Figure.1.b shows the lifetime of the $\mathrm{Tm}^{3+}:{ }^{3} \mathrm{~F}_{4}$ level in the core glass measured at room temperature using a $793 \mathrm{~nm}$ laser diode modulated with a 10-ms-duration square pulse. The fluorescence decay from the glass was collected using a multimode fiber connected to an oscilloscope equipped with an InGaAs photodetector (PDA10D-EC 1200nm-2600nm). It was measured to be $1.5 \mathrm{~ms}$ with the $\mathrm{Tm}^{3+}$ doping concentration of $8.5 \times 10^{20}$ ions $/ \mathrm{cm}^{3}$ showing a good fit with a single exponential decay function.
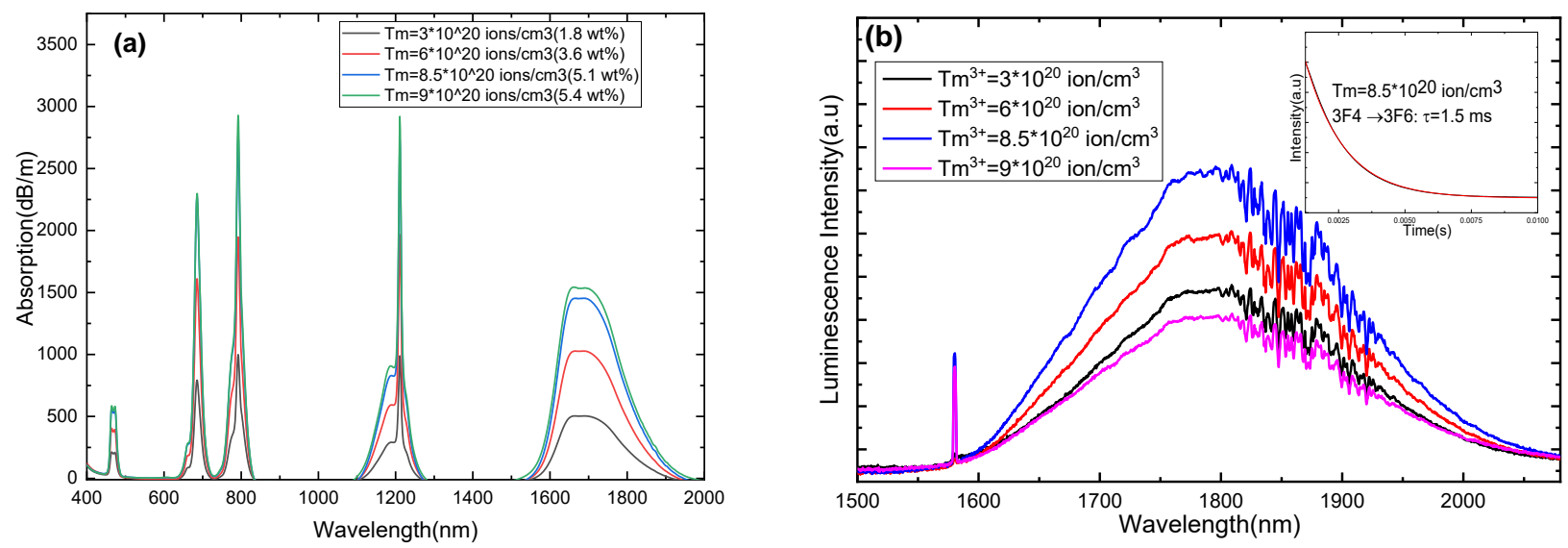

Figure 1.a) Absorption spectra of germanates glasses doped with different $\mathrm{Tm}^{3+}$ concentration. b). Emission spectra of germanate

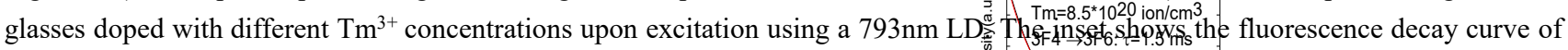
$1.8 \mu \mathrm{m}$ emission from the core glass.

The core and cladding glasses (labelled as Ge-01 and Ge-02, respectively) were then produced and the core glass was doped with $\mathrm{Tm}^{3+}$ ions at the optimal concentration of $8.5 \times 10^{20}$ ions $/ \mathrm{cm}^{3}$. The detailed Ge- 01 and Ge-02 glass compositions were designed to provide both a low refractive index contrast for LMA single mode fiber operation and 
compatible thermo-mechanical properties for fiber drawing. For each glass composition, well-mixed precursor materials were melted at $1230^{\circ} \mathrm{C}$ for about 3 hours in a platinum crucible under a dry $\mathrm{O}_{2} / \mathrm{N}_{2}$ atmosphere to reduce the $\mathrm{OH}^{-}$content. Thereafter, the molten liquid was cast into a stainless steel mold preheated to a temperature around the glass transition temperature, $\mathrm{T}_{\mathrm{g}}$. The cast glass was then annealed at $\mathrm{T}_{\mathrm{g}}-10{ }^{\circ} \mathrm{C}$ for 4 hours to remove internal stresses before it was cooled to room temperature. After the annealing process, glass samples (12 $\mathrm{mm}$ in diameter and $4 \mathrm{~mm}$ thick) were cut and polished for optical and spectroscopic characterization. For each glass composition, the glass transition (Tg) and crystallization temperature (Tx) were measured by the differential thermal analysis technique using a PerkinElmer Diamond TG/DTA instrument. Their refractive index was measured by ellipsometry using a MC05-WoolhamEllipsometer. As we can see in Table 1, the glass transition temperatures were measured to be $485^{\circ} \mathrm{C}$ and $489^{\circ} \mathrm{C}$ for glasses Ge_01 and Ge_02, respectively. Such high transition temperatures (for these glass compositions and as compared to silicate glasses) make the fabricated glasses suitable for manufacturing glass lasing structures able of handling high average powers. The NA we achieved was 0.07 , which is a good starting point to make a large core SM fibre.

Table 1. Glass transition temperature (Tg), and refractive index (n) of the developed glasses

\begin{tabular}{|c|c|c|c|c|c|}
\hline & $\begin{array}{c}\text { Density } \\
\left(\mathbf{g} / \mathbf{c m}^{\mathbf{3}}\right)\end{array}$ & $\mathbf{T}_{\mathbf{g}}\left({ }^{\mathbf{0}} \mathbf{C}\right)$ & $\left.\mathbf{T}_{\mathbf{x}}{ }^{\mathbf{0}} \mathbf{C}\right)$ & Refractive index $@ \mathbf{~ 1 . 7} \boldsymbol{\mu m}$ & NA in fibre \\
\hline Core (Ge-01) & 5.38 & 485 & 745 & 1.8025 & 0.07 \\
\cline { 1 - 4 } $\begin{array}{c}\text { Cladding (Ge- } \\
\text { 02) }\end{array}$ & 5.11 & 489 & 734 & 1.8012 & \\
\hline
\end{tabular}

\section{Preform and fiber development}

For the fabrication of $\mathrm{Tm}^{3+}$ doped germanate LMA fiber, the Ge-01 glass rod $(120 \mathrm{~mm}$ long and $11.5 \mathrm{~mm}$ in diameter) was first drawn into a cane of $1.3 \mathrm{~mm}$ diameter. The Ge-02 glass billet was then extruded into an hexagonal tube of $9.5 \mathrm{~mm}$ outer diameter (vs 2 opposites angles) and $1.8 \mathrm{~mm}$ inner diameter as shown in Figure 2.a. Note that we have chosen a hexagonal shape for our double clad fiber to facilitate pump mode mixing as needed to achieve a high pump absorption, and to provide for lower loss splicing to other centrally symmetric (e.g. circular cross section) fibers. To extrude the cladding glass, a speed of $0.1 \mathrm{~mm} / \mathrm{min}$ was used and the temperature was around $575-615^{\circ} \mathrm{C}$ in order to have a force of 1.1-2.5 kN. Both speed and temperatures were chosen to avoid bending. The Ge-01 glass rod and Ge-02 glass tube were then co-drawn into an optical fiber using a rod-in-tube technique. A cross-sectional view of the fiber is shown in Figure.2.b. The fiber core and cladding diameters were $17 \pm 0.5 \mu \mathrm{m}$ and $120 \pm 1 \mu \mathrm{m}$, respectively. An outer cladding made by a UV curable low index acrylate polymer was also added to provide mechanical protection and to allow cladding pumping. The fiber loss was measured to be $\sim 1.2 \mathrm{~dB} / \mathrm{m}$ at $980 \mathrm{~nm}$ by a cut-back method over a length of $5.3 \mathrm{~m}$. This is substantially lower than that of previously reported $\mathrm{Tm}^{3+}$ doped lead silicate glass $(7 \mathrm{~dB} / \mathrm{m})[13]$ and germanate glass $(3 \mathrm{~dB} / \mathrm{m})$ [14] fibers, and it confirms the absence of crystallization during the fiber fabrication. With this new as-drawn fiber further improvements in the 2 um laser performance are expected comparing to our previous efficient single mode fiber laser operating at $1952 \mathrm{~nm}(1.5 \mathrm{~W}$ output power with a slope efficiency of $55.9 \%$ vs absorbed pump power and using in-band pump absorption[12]). 


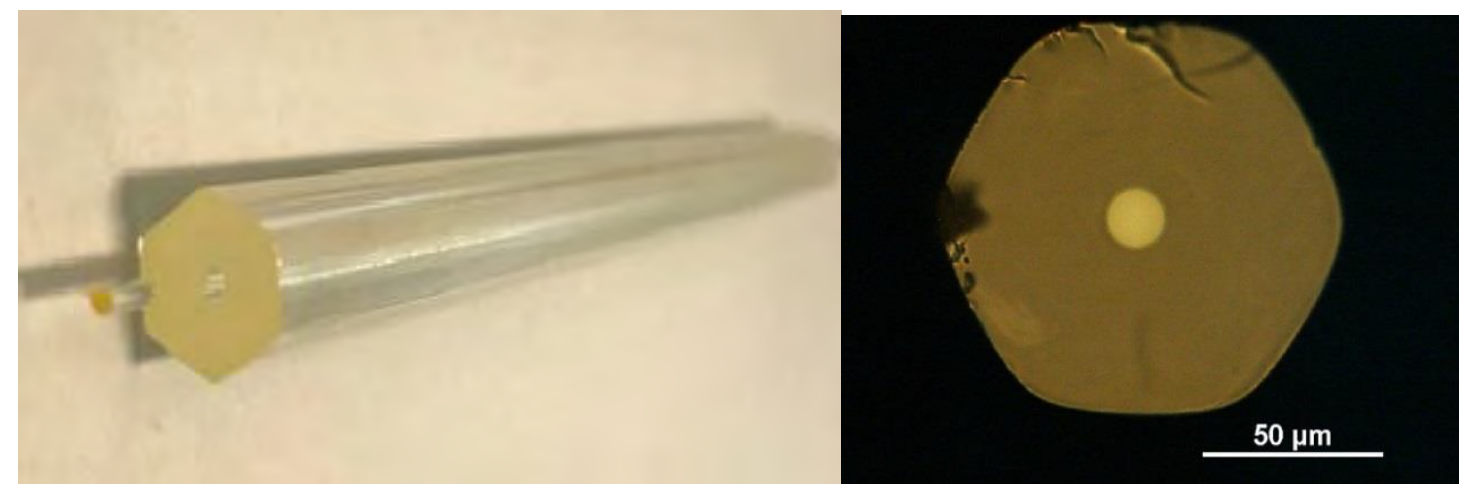

Figure 2.a) Cross sectional view of the extruded hexagonal inner cladding with the core rode inside. b). Optical microscope image of the fabricated Tm doped Germanate LMA single mode fiber.

\section{Conclusion}

Highly $\mathrm{Tm}^{3+}$ doped germanates glass SM fibers with core diameter of $17 \mu \mathrm{m}$ and a hexagonal inner cladding (diameter of $120 \mu \mathrm{m}$ ) were designed and fabricated by the rod-in-tube technique. A $\mathrm{Tm}^{3+}$ doping concentration of $8.5 \times 10^{20}$ ions $/ \mathrm{cm}^{3}$ was achieved which is the highest $\mathrm{Tm}^{3+}$ doping concentration reported to date in germanate glass fibers to the best of our knowledge. As a result of paying careful attention to optimization of the glass material quality and to the control of the fiber fabrication process a background fiber loss of $\sim 1.2 \mathrm{~dB} / \mathrm{m}$, was achieved - the lowest value reported to date for a germanate fiber. The results confirm that the as-drawn highly $\mathrm{Tm}^{3+}$ doped germanate glass SM fiber possesses high pump absorption $(\sim 1500 \mathrm{~dB} / \mathrm{m}$ at $1700 \mathrm{~nm})$, which make it a promising active medium for compact and efficient $2.0 \mu \mathrm{m}$ fiber laser.

\section{Acknowledgments}

We gratefully acknowledge funding from the U.K. Engineering and Physical Sciences Research Council under Grant EP/P030181/1 (Future Photonics Hub) and the ERC LightPipe project (grant agreement n 682724).

\section{References}

[1] Scholle, K., Heumann, E. and Huber, G., "Single mode Tm and Tm,Ho: LuAG lasers for LIDAR applications," Laser.Phy.Letters 1(6), 285-290 (2004).

[2] Jackson, S. D. and Lauto,A,” Diode pumped fiber lasers: a new clinical tool?’Lasers.Surg.Med.30(3), 184-190 (2002).

[3] Kadwani, P., Chia, J., Altal, F., Sims, R. A., Willis, C., Shah, L., Killinger, D. and Richardson, M.C., "Atmospheric absorption spectroscopy using Tm:fiber sources around 2 microns," Proc. SPIE 7924, 79240L-1-79240L-6 (2001).

[4] Wen, X., Tang, G., Wang, J., Chen, X., Qian, Q. and Yang, Z., “ Tm3+ doped barium gallo-germanate glass singlemode fibers for $2.0 \mu \mathrm{m}$ laser," Optics. Express 23(6), 7722 (2015).

[5] Wu, J., Jiang, S., Luo, T., Geng, J., Peyghambarian, N. and Barnes, N.P., "Efficient Thulium- Doped 2 um Germanate Fiber Laser," IEEE Photon. Tech. Letters 18(2), 335(2006).

[6] He, X., Xu, S., Li, C., Yang, C, Yang, Q., Mo, S., Chen, D. and Yang, Z., "1.95 $\mu \mathrm{m} \mathrm{kHz-linewidth} \mathrm{single-frequency}$ fiber laser using self-developed heavily $\mathrm{Tm}^{3+}$-doped germanate glass fiber," Opt. Express 21(18), 20800-20805 (2013).

[7] Auzel, F. and Goldner, P., "Towards rare-earth clustering control in doped glasses," Opt. Mater. 16(1-2),93-103 (2001).

[8] Jollivet, C., Farley, K., Conroy, M., Dabhi, H., Edgecumbe, J., Carter, A. and Tankala, K., "Deseign optimization of Tm-doped large-mode area fibers for power scaling of $2 \mu \mathrm{m}$ lasers and Amplifiers," Proc.SPIE 10083, 100830I-1 -100830I7 (2017). 
[9] Wen, X., Tang, G., Yang, Q., Chen, X., Qian, Q., Zhang, Q. and Yang, Z., "Highly Tm ${ }^{3+}$ doped germanate glass and its single mode fiber for $2.0 \mu \mathrm{m}$ laser," Sci. Rep 6(20344), 1-10 (2016).

[10] Lee, Y. W., Ling, H. Y., Lin, Y. H. and Jiang, S., "Heavily $\mathrm{Tm}^{3+}$-doped silicate fiber with high gain per unit length," Opt. Mater.Express 5(3), 549-557 (2015).

[11] Li, M., Bai, G., Guo, Y., Hu, L. and Zhang, J., "Investigation on $\mathrm{Tm}^{3+}$-doped silicate glass for $1.8 \mu \mathrm{m}$ emission, "J. Lumin 132(7), 1830-1835 (2012).

[12] Ben Slimen, F., Chen, S., Lousteau, J., Jung, Y., White, N., Alam, S., Richardson, D. J. and Poletti, F., "Highly efficient Tm3+ doped germanate large mode area single mode fiber laser," Opt.Mat.Express 9(8), 367705-367715(2019).

[13] Liu, X., Wang, X., Wang, L., Kuan, P. W., Li, M., Li, W., Fan, X., Li, K., Hu, L. and Chen, D., "Realization of 2 $\mu \mathrm{m}$ laser output in Tm3+ doped lead silicate double cladding fiber, " Mater.Lett 125(3), 12-14, (2014).

[14] Fan, X., Kuan, P., Li, K., Zhang, L., Li, W. and Hu, L., "A 2 um continuous wave and passively Q-switched fiber laser in thulium-doped germinate glass fibers," Laser. Phys 24(8), 085107 (2014). 


\title{
Soft glass microstructured optical fibers and their applications
}

\author{
Yasutake Ohishi* \\ Research Center for Advanced Photon Technology, Graduate School of Engineering, \\ Toyota Technological Institute, 2-12-1 Hisakata, Tempaku-ku, Nagoya, 468-8511, Japan
}

\begin{abstract}
We demonstrate the mid-infrared supercontinuum generation in the normal dispersion regime by using a chalcogenide double clad fiber pumped with femtosecond pulse, and mid-infrared wavelength conversion using a chalcogenide suspended-core fiber pumped with pico-second pulse.
\end{abstract}

Keywords: Nonlinear optics, Highly nonlinear fiber, Supercontinuum generation, Four-wave mixing *ohishi@toyota-ti.ac.jp; phone: +81 52 809-1860

\section{Introduction}

Recently, interest in mid-infrared (MIR) light source for many applications, such asoptical coherence tomography, metrology [1], and bio-photonic diagnostics [2] has been promoted rapidly. For these applications, a spatially coherent light source is suitable to realize high spatial resolution imaging because it can be focused to a diffraction limited spot size. To realize the high spatial coherent light source, supercontinuum (SC) generation in an optical fiber has been receiving an attention. In this decade, researchers have developed various type of fiber such as photonic crystal fiber, microstructured optical fiber, and rib-waveguide to obtain the MIR SC spectrum. However, intrinsic transmission window of fused silica restrains the SC expansion beyond $3 \mu \mathrm{m}$. To extend the SC spectrum beyond $3 \mu \mathrm{m}$, alternative materials have been proposed, such as tellurite and fluoride glasses. These glasses have large advantages because of their wide transmittance window, and high nonlinearity. In 2008, P. Domachuk et al. demonstrated SC generation beyond 4 $\mu \mathrm{m}$ by using a femtosecond pump source [3]. For obtaining the SC further into the MIR region, chalcogenide glasses are attractive with a wide transmission window beyond $10 \mu \mathrm{m}$. Many researchers have demonstrated SC generation in chalcogenide fibers [4,5]. In 2016, Cheng et al. demonstrated MIR SC extending from 2.0 to $15.1 \mu \mathrm{m}$ in a chalcogenide step index fiber [6]. SC generation in the normal dispersion regime in soft glass optical fiber has been studied recently [7].

There are other MIR light sources, such as quantum cascade lasers, cascaded Raman lasers, and fiber optical parametric oscillators (FOPOs). FOPOs usually employ the degenerate four-wave mixing (DFWM) in fibers. In DFWM process, two photons at the pump frequency $\left(\boldsymbol{\omega}_{p}\right)$ are annihilated to generate signal and idler photons at the signal frequency $\left(\omega_{s}\right)$ and the idler frequency $\left(\omega_{i}\right)$, respectively. Wavelength conversion based on DFWM uses high-intensity pump, which is much higher than the intensity of input signal and generates the idler at the frequency of $\boldsymbol{\omega}_{i}=2 \boldsymbol{\omega}_{p}-\boldsymbol{\omega}_{s}$. Recently, FOPOs in the MIR range have received attention. Generation of MIR light by DFWM have been demonstrated in silica-based fibers [8,9]. However, the intrinsic transmission window and low optical nonlinearity of silica limit the MIR light generation above $3 \mu \mathrm{m}$. To generate the MIR light, chalcogenide glass is an attractive candidate because of their wide transmission window up to around $18 \mu \mathrm{m}$ and their high optical nonlinearity.

In the present study, we use chalcogenide $\mathrm{As}_{2} \mathrm{~S}_{5}$ suspended-core fiber (Ch-SCF) to generate a MIR light via DFWM using a $1.958 \mu \mathrm{m}$ pico-second pump source. We fabricate a Ch-SCF whose zero-dispersion wavelength (ZDW) is 2.12 $\mu \mathrm{m}$. We also demonstrate far-detuned parametric wavelength conversion from 1.27 to $4.3 \mu \mathrm{m}$. To the best of our knowledge, it is the first observation of MIR wavelength conversion whose detuning frequency is over $80 \mathrm{THz}$ in an optical fiber.

Sixth International Workshop on Specialty Optical Fibers and Their Applications (WSOF 2019), edited by Liang Dong, John M. Ballato, Proc. of SPIE Vol. 11206, 112060A · (C) 2019 SPIE CCC code: $0277-786 \mathrm{X} / 19 / \$ 21 \cdot$ doi: $10.1117 / 12.2550227$ 
Here, we demonstrate the highly coherent MIR SC generation using chalcogenide double clad fiber (Ch-DCF) with the normal dispersion [10] and also demonstrate far-detuned parametric wavelength conversion from 1.27 to $4.3 \mu \mathrm{m}$ using a Ch-SCF [11]. We show the potential of chalcogenide optical fibers for the MIR coherent light generation in this presentation.

\section{Mid-infrared Supercontinuum Generation in Chalcogenide Fiber}

Figures 1(a) shows refractive index profiles of $\mathrm{As}_{2} \mathrm{Se}_{3}$, $\mathrm{AsSe}_{2}$, and $\mathrm{As}_{2} \mathrm{~S}_{5}$ glasses. Figure 1(b) shows a cross-section photograph of the Ch-DCF. The core, first cladding, and second cladding are made of $\mathrm{As}_{2} \mathrm{Se}_{3}, \mathrm{AsSe}_{2}$, and $\mathrm{As} 2 \mathrm{~S}$, respectively. The diameters of core and first cladding were 7.8 and $30.7 \mu \mathrm{m}$. Figure 1(c) shows a calculated chromatic dispersion profile of the Ch-DCF. The Ch-DCF has a near-zero flattened chromatic dispersion in the normal dispersion regime, from -20 to $0 \mathrm{ps} / \mathrm{nm} / \mathrm{km}$ in the wavelength range from $3.8 \mu \mathrm{m}$ to the ZDW of $12.5 \mu \mathrm{m}$. At the wavelength of 5.6 $\mu \mathrm{m}$, the chromatic dispersion closes to zero dispersion.

In the measurement, a difference frequency generation (DFG) unit which can deliver $<200$-fs pulse with a tunable wavelength range from 2.5 to $11 \mu \mathrm{m}$ was used as a pump source. The beam was free-space coupled into a $2.8 \mathrm{~cm}-\mathrm{long}$ Ch-DCF with a black diamond lens, anti-refractive coating in the MIR range, a focal length of $4.0 \mathrm{~mm}$, and NA of 0.56. The output from the Ch-DCF was collimated by a ZnSe lens that has a focal length of $12 \mathrm{~mm}$, and was launched into a monochromator to measure the SC signal with a liquid nitrogen cooled HgCdTe (MCT) detector. The signal was amplified by a lock-in amplifier.

We measured MIR SC in Ch-DCF with a different pump wavelength of 8,10 , and $12 \mu \mathrm{m}$. Averaged pump power for each wavelength was $2.0,1.5$, and $1.2 \mathrm{~mW}$ that correspond to the peak power of 10, 7.5, and $6 \mathrm{MW}$, respectively. Considering the Fresnel reflection and the geometrical losses, estimated maximum-coupled peak power was 1.8, 1.3, and 1.1 MW, respectively. Figure 2 shows the measured SC spectrum. When the pump wavelength was $8 \mu \mathrm{m}$, the SC was extending from 4 to $10 \mu \mathrm{m}$ at $-50 \mathrm{~dB}$ and the small peak was obtained around $3 \mu \mathrm{m}$. The broadening of SC spectrum is dominated by self-phase modulation and optical wave breaking. The whole spectrum existed in the normal dispersion regime. When the pump wavelength was 10 and $12 \mu \mathrm{m}$, the $\mathrm{SC}$ was extending from 2 to $14 \mu \mathrm{m}$ and from 4 to $14 \mu \mathrm{m}$ at $40 \mathrm{~dB}$, respectively. The long wavelength side of the SC spectrum existed in the anomalous dispersion range.
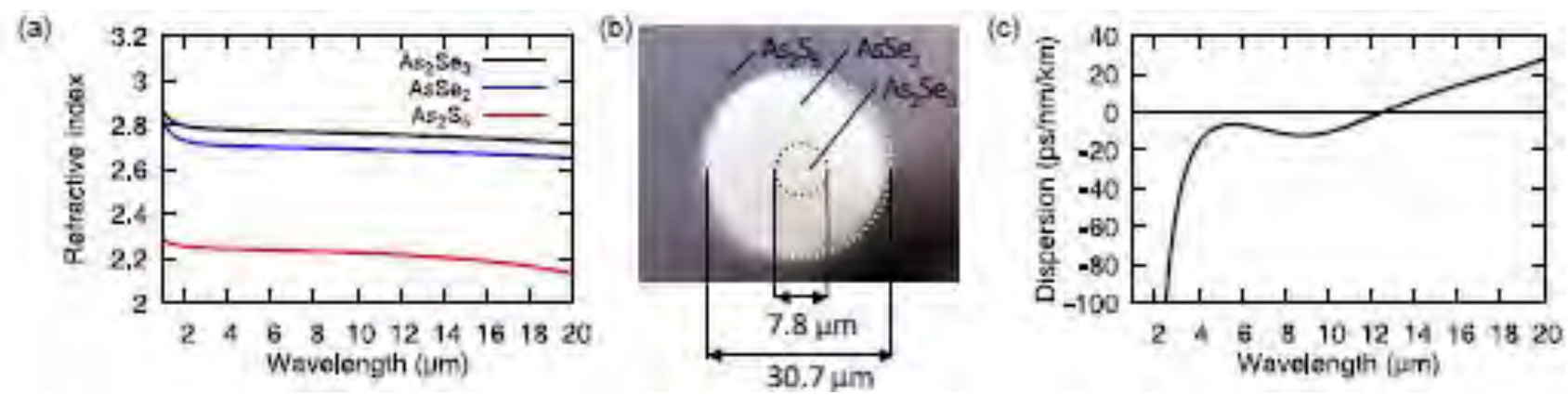

Figures 1(a) shows refractive index profiles of $\mathrm{As}_{2} \mathrm{Se}_{3}, \mathrm{AsSe}_{2}$, and $\mathrm{As}_{2} \mathrm{~S}_{5}$ glasses. Figure 1(b) shows a cross-section photograph of the Ch-DCF. The core, first cladding, and second cladding are made of $\mathrm{As}_{2} \mathrm{Se}_{3}, \mathrm{AsSe}_{2}$, and As2 $\mathrm{S}_{5}$, respectively. The diameters of core and first cladding were 7.8 and $30.7 \mu \mathrm{m}$. Figure 1(c) shows a calculated chromatic dispersion profile of the Ch-DCF. The Ch-DCF has a near-zero flattened chromatic dispersion in the normal dispersion regime, from -20 to $0 \mathrm{ps} / \mathrm{nm} / \mathrm{km}$ at the wavelength range from $3.8 \mu \mathrm{m}$ to the ZDW of $12.5 \mu \mathrm{m}$. At the wavelength of 5.6 $\mu \mathrm{m}$, the chromatic dispersion closes to zero dispersion. 
Figure 3 shows the simulated first-order coherence with the pump wavelength of $10 \mu \mathrm{m}$ and coupled pump peak power of 1.3 MW. We can see the obtained SC is coherent in the whole spectral range.
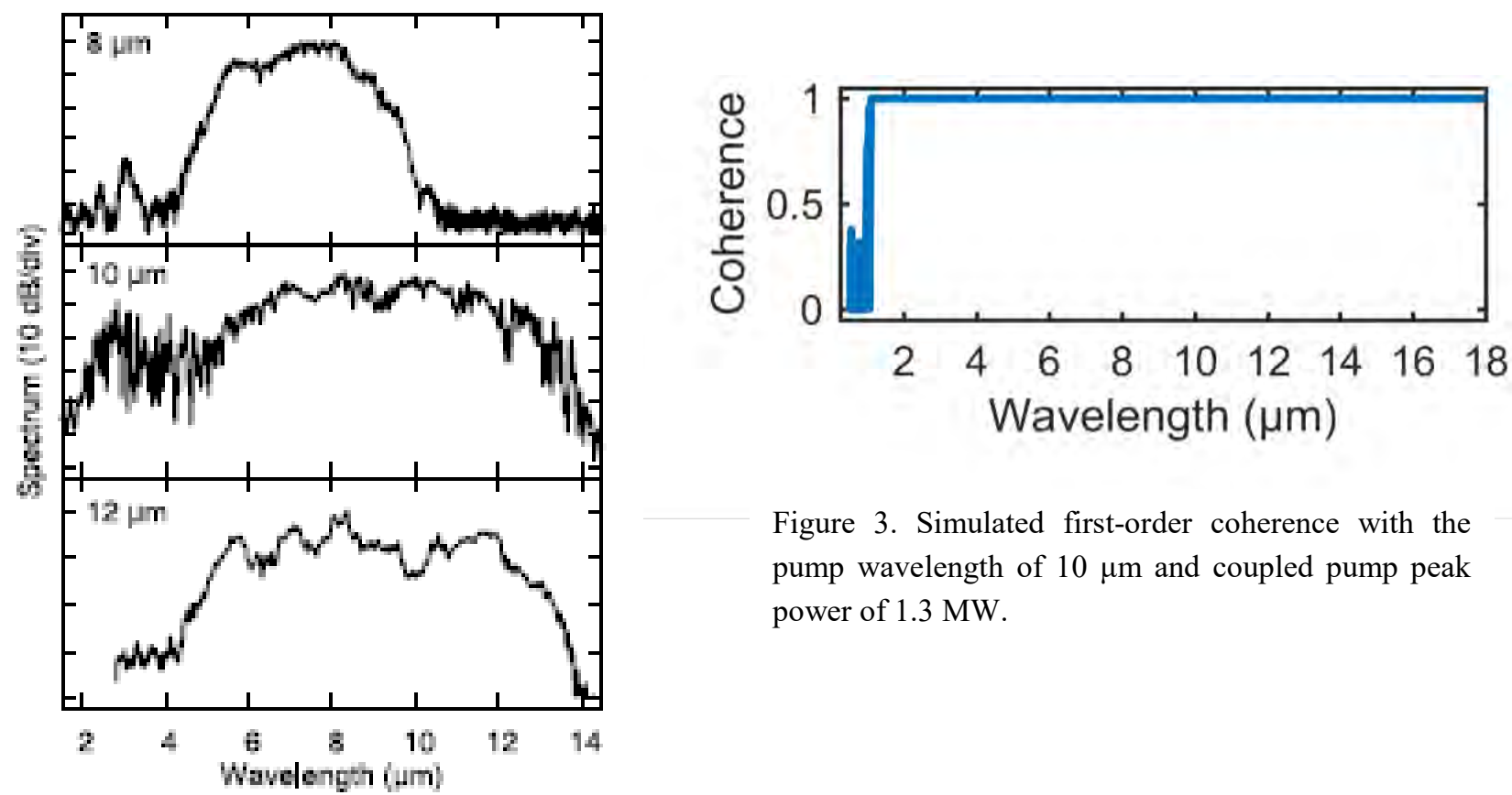

Figure 2. Measured SC spectra by pumping at the wavelength of 8,10 , and $12 \mu \mathrm{m}$.

\section{Near-infrared to Mid-infrared Wavelength Conversion in Chalcogenide Fiber}

Figures 4(a) shows the refractive index and material dispersion curves of $\mathrm{As}_{2} \mathrm{~S}_{5}$ glass. The ZDW of $\mathrm{As}_{2} \mathrm{~S}_{5}$ glass is 5.2 $\mu \mathrm{m}$. Figure 4(b) shows a cross-section photograph of the Ch-SCF which was observed by an optical microscope. The core diameter " $\mathrm{d}$ " was $3.25 \mu \mathrm{m}$, and the width of the bridge "w" was $0.4 \mu \mathrm{m}$. Figure 4(c) shows a calculated chromatic dispersion curve of the fundamental mode in the Ch-SCF. The zero-dispersion wavelength (ZDW) was about $2.12 \mu \mathrm{m}$. In the experiment, a mode-locked laser was used as a pump source. The pump pulse had a full width of at half maximum of $\sim 2.7 \mathrm{ps}$, a repetition rate of $32 \mathrm{MHz}$, and an average power of $250 \mathrm{~mW}$. The signal light was provided with the tunable $\mathrm{CW}$ laser whose wavelength was fixed at $1.27 \mu \mathrm{m}$. The beams of the pump and the signal were combined by a beam splitter. The combined beam was coupled into the Ch-SCF with an aspheric lens whose focal length and numerical aperture were $5.95 \mathrm{~mm}$ and 0.56 , respectively. The coupled average power of the pump beam was estimated to be $31 \mathrm{~mW}$ with considering the Fresnel reflection on the face of $\mathrm{Ch}-\mathrm{SCF}$ and the coupling efficiency of the lens. Fiber length used in the experiment was $21 \mathrm{~cm}$. The output beam from the Ch-SCF was coupled into the $30 \mathrm{~cm}$-long large core ZBLAN fiber and was measured with FTIR spectrometer and optical spectrum analyzer (OSA). Figure 5(a) shows a measured spectrum. The long-wavelength side idler appeared at the wavelength of $4.27 \mu \mathrm{m}$. A detuning frequency of the long-wavelength side idler was about $83 \mathrm{THz}$ which was almost same as the detuning frequency between the signal and pump wave. Figure 5(b) shows simulated spectra in the experimental condition, which were calculated using a generalized nonlinear Schrödinger equation (GNLSE). The GNLSE considered frequency dependent nonlinear coefficient. Blue curve indicated the averaged output spectrum from the Ch-SCF. Individual simulated 20 spectra employed in the average spectrum were also shown in gray curves. The long-wavelength side idler was obtained at the wavelength of $4.3 \mu \mathrm{m}$. In 
the experiment, when the $\mathrm{CW}$ laser was not launched into the Ch-SCF, the idler wave was not obtained. This was also shown in the numerical simulation. These results indicate that the idler wave at $4.27 \mu \mathrm{m}$ in the experiment was generated via wavelength conversion in the $\mathrm{Ch}-\mathrm{SCF}$.

(a)

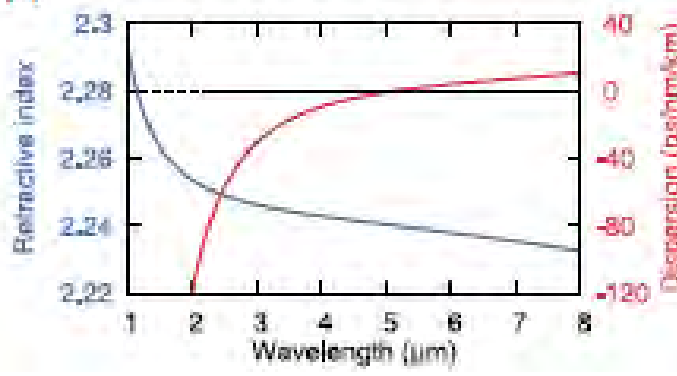

(b)

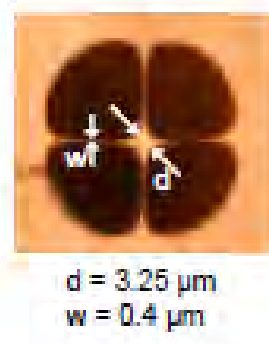

(c)

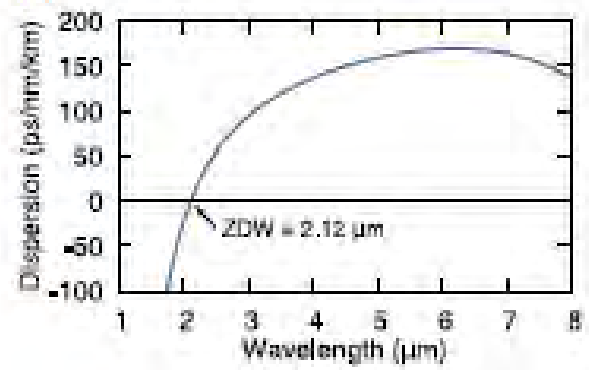

Figure 4. (a) Refractive index and material dispersion spectra of $\mathrm{As}_{2} \mathrm{~S}_{5}$ glass. (b) Cross-section photograph of the Ch-SCF. (c) Chromatic dispersion spectrum of the fundamental mode in the Ch-SCF. The ZDW was located at $2.12 \mu \mathrm{m}$.

(a)

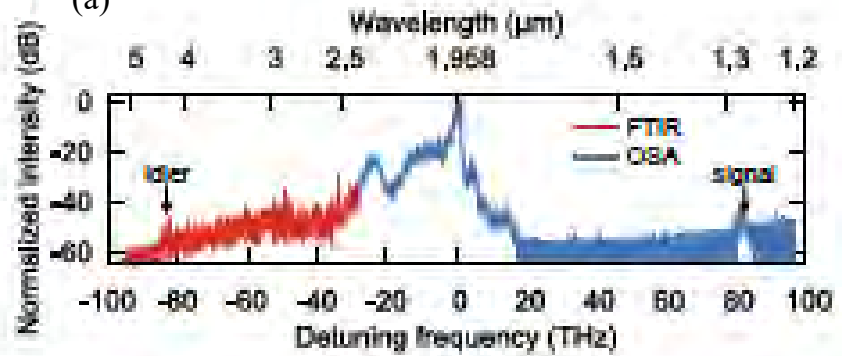

(b)

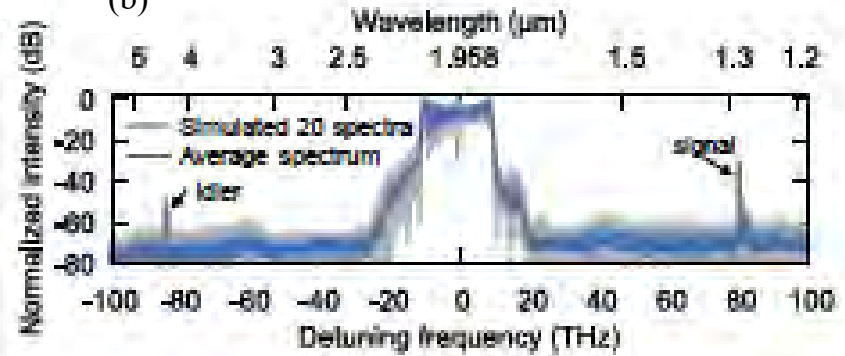

Figure 5. (a) Experimental observation of the wavelength conversion when the pump wavelength is at $1.958 \mu \mathrm{m}$ in the normal dispersion regime. Red and blue curve indicate measured spectrum with FTIR and OSA, respectively. (a) Numerical observation of the wavelength conversion in the Ch-SCF. Blue curve indicates an average spectrum. Individual realizations employed in the average spectrum are shown in gray curves.

\section{Conclusion}

We experimentally demonstrated the MIR SC extending from 2 to $14 \mu \mathrm{m}$ in $2.8 \mathrm{~cm}$-long Ch-DCF pumped with femtosecond pulse pumping at $10 \mu \mathrm{m}$. Most SC spectrum existed in the normal dispersion regime. The results indicate that the Ch-DCF is a promising medium to generate the highly coherent MIR SC generation. We also demonstrated a parametric wavelength conversion from 1.27 to $4.27 \mu \mathrm{m}$ using a Ch- SCF with pico-second pulse pumping. The detuning frequency from the pump frequency was about $83 \mathrm{THz}$. The experimental observation was confirmed by solving the GNLSE which considered frequency dependent nonlinear coefficient. The result reported here is the first observation of MIR wavelength conversion whose detuning frequency is over $80 \mathrm{THz}$ in an optical fiber and indicates that the potential of Ch-SCF for wavelength conversion from near-infrared to MIR. 


\section{Acknowledgment}

This work was partly supported by Japan Society for the Promotion of Science (JSPS) (15H02250, 17K18891, 18H01504). The author would like to acknowledge Dr. Takenobu Suzuki, Dr. Kenshiro Nagsaka, Dr. Hoang Tuan Tong, Dr. Than Singh Saini and Dr. Xing Luo at Toyota Technological Institute for their collaboration.

\section{Reference}

[1] R. Su, M. Kirillin, E. W. Chang, E. Sergeeva, S. H. Yun, and L. Mattsson, "Perspectives of mid-infrared optical coherence tomography for inspection and micrometrology of industrial ceramics," Opt. Express 22, 15804-15819 (2014). [2] M. Verdonck, A. Denayer, B. Delvaux, S. Garaud, R. De Wind, C. Desmedt, C. Sotiriou, K.Willard-Gallob and E. Goormaghtigh, "Characterization of human breast cancer tissues by infrared imaging," The Analyst 141, 606-619 (2016). [3] P. Domachuk, N. A.Wolchover, M. Cronin-Golomb, A. Wang, A. K. George, C.M.B. Cordeiro, J.C. Knight, and F. G. Omenetto, "Over $4000 \mathrm{~nm}$ bandwidth of mid-IR supercontinuum generation in sub-centimeter segments of highly nonlinear tellurite PCFs," Opt. Express 16, 7161-7168 (2008).

[4] C. R. Petersen, U. Møller, I. Kubat, B. Zhou, S. Dupont, J. Ramsay, T. Benson, S. Sujecki, N. Abdel-Moneim, Z. Tang, D. Furniss, A. Seddon, and O. Bang, "Mid-infrared supercontinuum covering the 1.4-13.3 $\mu \mathrm{m}$ molecular fingerprint region using ultra-high NA chalcogenide step-index fibre," Nat. Photon. 8, 830-834 (2014).

[5] B. Zhang, Y. Yu, C. Zhai, S. Qi, Y. Wang, A. Yang, X. Gai, R. Wang, Z. Yang, and B. Luther-Davies, "High Brightness 2.2-12 $\mu \mathrm{m}$ Mid- Infrared Supercontinuum Generation in a Nontoxic Chalcogenide Step-Index Fiber," J. Am. Ceram. Soc. 99, 2565-2568 (2016).

[6] T. Cheng, K. Nagasaka, T. H. Tuan, X. Xue, M. Matsumoto, H. Tezuka, T. Suzuki, Y. Ohishi, "Mid-infrared supercontinuum generation spanning 2.0 to $15.1 \mu \mathrm{m}$ in a chalcogenide step-index fiber," Opt. Lett. 41, 2117-2120 (2016). [7] M. Klimczak, B. Siwicki, P. Skibiński, D. Pysz, R. Stępień, A. Heidt, C. Radzewicz and R. Buczyński, "Coherent supercontinuum generation up to $2.3 \mu \mathrm{m}$ in all-solid soft-glass photonic crystal fibers with flat all-normal dispersion ," Opt. Express. 22, 18824-18832 (2014).

[10] K. Nagasaka, L. Liu, T. H. Tuan, T. Cheng, M. Matsumoto, H. Tezuka, T. Suzuki, and Y. Ohishi, "Supercontinuum generation in chalcogenide double-clad fiber with near zero-flattened normal dispersion profile", Journal of Optics, 19, 19 (2017).

[11] K. Nagasaka, T. H. Tuan, N. P. T. Hoa, M. Matsumoto, S. Cho. T. Suzuki, and Y. Ohishi, "Near-infrared to Midinfrared wavelength conversion by chalcogenide suspended-core fiber”, CLEO2018, JTu2A.126 (2018). 


\title{
Progress in Mid-IR Supercontinuum sources and their applications
}

\section{- an overview.}

\author{
Peter M. Moseund*a , Joanna Carthy ${ }^{\mathrm{a}}$, Laurent Huot ${ }^{\mathrm{a}}$, Patrick Bowen ${ }^{\mathrm{a}}$ \\ ${ }^{a}$ NKT Photonics A/S, Blokken 84 - DK-3460 Birkerød - Denmark
}

\begin{abstract}
In this talk we will summarize the areas where mid-IR supercontinuum has been applied and demonstrated its strength since its commercial breakthrough two years ago and where we expect its application to grow in the coming years.
\end{abstract}

Keywords: Mid-IR, Supercontinuum, ZBLAN, Spectroscopy, Microscopy

*Pmm@nktphotonics.com; phone (+45) 43483900

\section{Introduction}

We are now approaching the $50^{\text {th }}$ anniversary for the first demonstration of supercontinuum generation ${ }^{1}$ and the commercial introduction of visible supercontinuum generation one and a half decade ago has demonstrated how these "white laser" sources can benefit a wide array of applications, especially within bio-photonics and metrology in the visible spectrum. More recently mid-IR supercontinuum sources also made their commercial breakthrough about two years ago after a decade of gradually increasing research activity. In this talk we will summarize the results achieved with these new sources in the mid-IR and where we expect the main new applications to arise. We will mainly focus on the areas of spectroscopy and imaging.

\section{Spectroscopy}

One of the key advantages with operating in the mid-IR spectrum is the high number of chemically specific absorption lines present in this range. This has lead the mid-IR to become the preferred region for a wide range of spectroscopy applications but particularly for laser based gas spectroscopy which is able to achieve the highest sensitivities only in this range. One of the advantages that laser based spectroscopy has, is that a collimated beam can have a long interaction length with a gas and thus allow the detection of very small variations in absorbance. However, the monochromatic nature of a laser also means that when one desires to perform spectroscopy on multiple species simultaneously one typically require a new laser for every additional specie which has to be detected. As mid-IR lasers are generally expensive this leads to a very high system cost for multispecies detection systems. Mid-IR supercontinuum sources combine the beam quality of a laser with a ultra-wide spectrum and can therefore allow the probing of a wide range of absorption lines with a single light source and through this approach broadband multi-species trace gas detection spectroscopy reaching sub-ppm level sensitivity have recently been demonstrated and applied to the control of fruit storage atmosphere ${ }^{2}$.

Beside the poor choice of high brightness light sources in the mid-IR another challenge for sensitive mid-IR spectroscopy has been the low sensitivity and poor signal to noise ratio (SNR) of mid-IR detectors. A new method to overcome this limitation has been the adaptation of sum-frequency generation based up-conversion detectors. Under some circumstances these have demonstrated significantly better performance than traditional detectors and as they work best with a highly directional, high peak power light source these are particularly suitable to be combined with SC sources as has been demonstrated both in spectroscopy ${ }^{3,4}$ or imaging setups ${ }^{5,6}$.

Sixth International Workshop on Specialty Optical Fibers and Their Applications (WSOF 2019),

edited by Liang Dong, John M. Ballato, Proc. of SPIE Vol. 11206, 112060B · C 2019 SPIE

CCC code: $0277-786 \times / 19 / \$ 21 \cdot$ doi: $10.1117 / 12.2548736$ 


\section{Imaging}

The most important application area for visible supercontinuum sources has been imaging technologies, in particular confocal fluorescence microscopy. It is therefore not surprising that imaging modes such as microscopy and OCT are also some of the first application for mid-IR SC in particular as these imaging modalities greatly benefit from high brightness light sources such as supercontinuum and there was previously very few practical high brightness broadband light sources covering the mid-IR.

In the lack of more practical light sources mid-IR synchrotron beamlines have traditionally been applied to microscopy for a number of proof of principle diagnostic and analytical applications. However recently, mid-IR SC sources have been demonstrated to considerably surpass synchrotrons in brightness while also matching or surpassing them in signal to noise ratio ${ }^{7}$. This has lead to mid-IR SC driven microscopy being proposed as a path to an in situ optical biopsy ${ }^{8}$ where absorption spectroscopy based high resolution imaging should provide equivalent or better diagnostic information than is currently provided by the analysis of stained tissue biopsies.

Mid-IR SC is also being applied to imaging outside bioimaging particularly for nondestructive testing where for instance mid-IR SC based OCT have been presented as a new method to gain 3D imaging inside samples which are nontransparent or too scattering in other parts of the optical spectrum ${ }^{9,10}$.

\section{Conclusion}

Mid-IR Supercontinuum sources are still a very new technology and it is still unclear where they will eventually make the biggest impact. However as presented they are currently being adopted in a wide range of applications and we are confident that their unique characteristics of wide continuous spectrum and high brightness will lead to their mainstream acceptance in the optical labs.

\section{References}

[1] Alfano R. and Shapiro, S. "Emission in the region 4000 to $7000^{\circ} \mathrm{A}$ via four-photon coupling in glass," Physical Review Letters 24(11), 584-587 (1970).

[2] Eslami Jahromi, K., Pan, Q., Khodabakhsh, A., Sikkens, C., Assman, P., Cristescu, S.M., Moselund, P.M., Janssens, M., Verlinden, B.E., et al., "A Broadband Mid-Infrared Trace Gas Sensor Using Supercontinuum Light Source: Applications for Real-Time Quality Control for Fruit Storage," Sensors 19(10), 2334 (2019).

[3] Moselund, P. M. , Petersen, C., Leick, L., Dam, J. S. , Tidemand-Lichtenberg, P. , and Pedersen, C., "Highly Stable, All-fiber, High Power ZBLAN Supercontinuum Source Reaching $4.75 \mu \mathrm{m}$ used for Nanosecond mid-IR Spectroscopy," in Advanced Solid-State Lasers Congress, Optical Society of America, paper JTh5A.9.

[4] Pan, Q., Jahromi, K. E., Abbas, M. A., Khodabakhsh, A., Cristescu, S. M., and F. J. M. Harren, "Towards Broadband Multi-species Trace Gas Detection Using a Mid-infrared Supercontinuum Source,"in Conference on Lasers and ElectroOptics,OSA Technical Digest (online)(Optical Society of America, 2018), paper ATh3O.2. (2018)

[5] Huot, L., Moselund, P. M., Tidemand-Lichtenberg, P., Leick, L.and Pedersen, "Upconversion imaging using an allfiber supercontinuum source," Opt. Lett. 41, 2466-2469 (2016) 
[6] Huot, L., Moselund, P. M., Tidemand-Lichtenberg, P. and Pedersen, C. "Pulsed upconversion imaging of midinfrared supercontinuum light using an electronically synchronized pump laser," Appl. Opt. 58, 244-249 (2019)

[7] Borondics, F., Jossent, M., Sandt,C., Lavoute, L.., Gaponov, ., Hideur, A. ., Dumas, P., and Février, S., "Supercontinuum-based Fourier transform infrared spectromicroscopy," Optica 5, 378-381 (2018)

[8] Seddon, A.B., Benson, T. M., Sujecki, S., Abdel-Moneim, N., Tang, Z., Furniss, D., Sojka, L., Stone, N., Jayakrupakar, N., Lloyd, G.R., Lindsay, I., Ward, J., Farries, M., Moselund, P.M., Napier, B, Lamrini,,S. Møller, U., Kubat, I., Christian, R. P., and Bang, O., "Towards the mid-infrared optical biopsy", Proc. SPIE 9703, Optical Biopsy XIV: Toward Real-Time Spectroscopic Imaging and Diagnosis, 970302 (2016)

[9] Zorin, I., Su,R., Prylepa, A., Kilgus,J., Brandstetter, M., and Heise, B., "Mid-infrared Fourier-domain optical coherence tomography with a pyroelectric linear array," Opt. Express 26, p. 33428 (2018)

[10] Israelsen, N. M., Petersen, C. R., Barh, A., Jain, D., Jensen, M., Hannesschläger, G., Tidemand-Lichtenberg, P., Pedersen, C., Podoleanu, A., Bang, O., " Real-time high-resolution mid-infrared optical coherence tomography " Light: Science \& Applications 8, Article number: 11, (2019) 


\title{
Optical devices based on chalcogenide fibers
}

\author{
Martin Rochette \\ Department of Electrical and Computer Engineering, McGill University, Montréal (QC), Canada, H3A $2 A 7$ \\ Authore-mail address: martin.rochette@mcgill.ca
}

\begin{abstract}
This talk features recent achievements of the Nonlinear Photonics Group at McGill University towards the fabrication of chalcogenide-based mid-infrared optical fiber components such as nonlinear gain fibers, optical fiber couplers and optical fiber filters.

OCIS codes: (060.2320) Fiber optics amplifiers and oscillators; Nonlinear optics, fibers; (140.3070) Infrared and far-infrared lasers.
\end{abstract}

\section{Introduction}

In a quest for the fabrication of all-fiber optical fiber sources that operate in the mid-infrared, optical fiber components such as gain media, optical fiber couplers, and optical fiber filters must be developed. Fiber components made from chalcogenide glass are of high importance in this context, owing to their transparency in the 1-13 $\mu \mathrm{m}$ wavelength range and their high-nonlinear gain. In this talk, I present recent achievements of the Nonlinear Photonics Group at McGill University towards the fabrication of all-fiber nonlinear gain media, optical fiber couplers, and optical filter compatible with the mid-infrared.

\section{References}

1. Zhang, K., et al., "Chalcogenide Fabry-Perot Fiber Tunable Filter," IEEE Photon. Technol. Lett. 30(23), 2013-2017 (2018).

2. Rochette, M., "Design and fabrication of nonlinear optical waveguides for far-detuned wavelength conversion to the mid-infrared," Advanced Optical Technologies 7(5), 321-326 (2018).

3. Tavakoli, F., et al.,"Broadband and Wavelength-Dependent Chalcogenide Optical Fiber Couplers," IEEE Photonics Technology Letters 29(9), 735-738 (2017).

4. Li, L., et al., "Mid-infrared wavelength conversion from AsSe microwires," Optics Letters 42(3), 639-642 (2017).

5. Li, L., et al., "Chalcogenide optical microwires cladded with fluorine-based CYTOP," Optics Express 24(17), 18931-18937 (2016).

6. Godin, T., et al., "Far detuned mid-infrared frequency conversion via normal dispersion modulation instability in chalcogenide microwire," Optics Letters 39(07), 1885-1888 (2014).

7. Al Kadry, A., et al., "Mid-infrared sources based on the soliton self-frequency shift," Journal of the Optical Society of America B 29(6), 1347-1355 (2012).

\footnotetext{
Sixth International Workshop on Specialty Optical Fibers and Their Applications (WSOF 2019), edited by Liang Dong, John M. Ballato, Proc. of SPIE Vol. 11206, 112060C · C 2019 SPIE

CCC code: $0277-786 X / 19 / \$ 21 \cdot$ doi: $10.1117 / 12.2543832$
} 


\title{
Design of single-mode nanohole suspended-core fibers for all-normal dispersion supercontinuum generation
}

\author{
Alexander Hartung*, Jörg Bierlich, Adrian Lorenz, Jens Kobelke, Matthias Jäger \\ Leibniz-Institute of Photonic Technology, Albert-Einstein-Straße 9, 07745 Jena, Germany
}

\begin{abstract}
Suspended-core optical fibers are designed to be all-normal dispersive and single-mode by a proper sizing of a central hole and the suspending struts. Realization strategies are discussed particularly regarding material composition and fabrication sequence and finally tested.
\end{abstract}

Keywords: fiber optics, specialty fibers, suspended-core fiber, all-normal dispersion, fabrication

\section{Introduction}

A current trend in supercontinuum (SC) generation is the realization of finite width, high power spectral density SC for e.g. nonlinear spectroscopic applications. In this case, fibers based on fused silica with its low nonlinearity and high damage threshold typically are most reasonable choice.

Based on fused silica photonic crystal fibers (PCFs) are so far the only established option to provide flexible allnormal dispersion ${ }^{1}$. But despite their in theory flexible all-normal dispersion scientific work about supercontinuum generation in pure fused silica PCFs is mainly concentrated around a pump wavelength of $1 \mu \mathrm{m}{ }^{2-5}$. The reason is a vanishing light confinement for geometries that are dispersion-optimized for pump wavelengths above $1.2 \mu \mathrm{m}$.

We discuss an alternative silica-based fiber design, namely nanohole suspended core optical fibers, as a promising option to provide both all-normal dispersion and continued light confinement up to a wavelength of $2 \mu \mathrm{m}$ and above.

\section{Results}

Suspended-core fibers are micro-structured fibers having very few large air holes surrounding a central core as shown in Fig. 1. Such a fiber is typically used for evanescent field sensing purposes. To provide all-normal dispersion a small hole needs to be located in the center of the fiber core. The fiber geometry is described by the number of suspending strands $\mathrm{N}$ and their thickness $\mathrm{t}$, the incircle core diameter $\mathrm{D}$ and the incircle hole diameter $\mathrm{d}$.
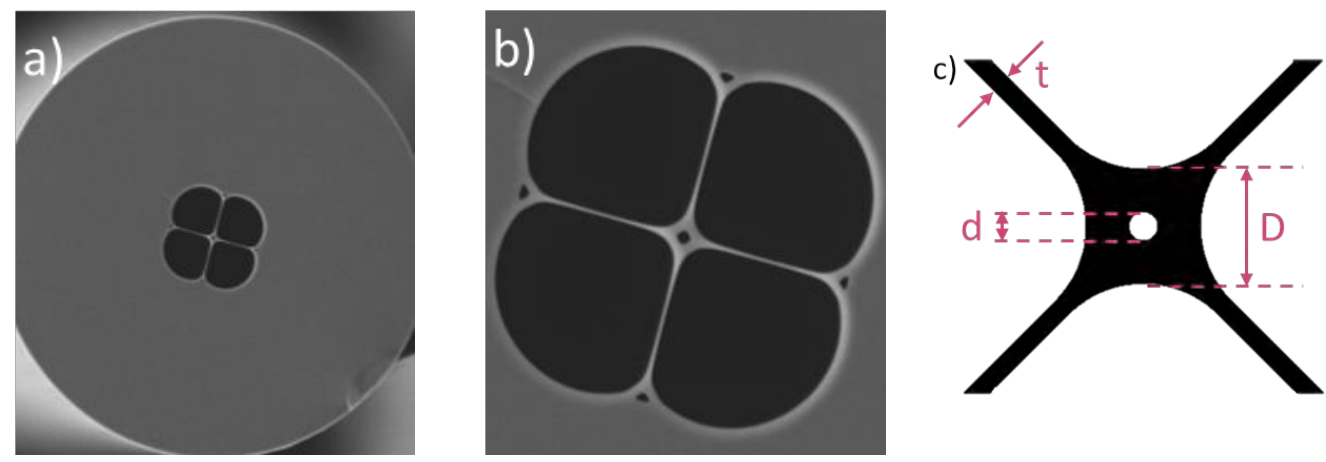

Figure 1: Scanning electron microscopy images of a) the full suspended-core fiber and b) the central section containing air holes. Design parameters are depicted in c).

Sixth International Workshop on Specialty Optical Fibers and Their Applications (WSOF 2019), edited by Liang Dong, John M. Ballato, Proc. of SPIE Vol. 11206, 112060D · @ 2019 SPIE

CCC code: $0277-786 X / 19 / \$ 21 \cdot$ doi: $10.1117 / 12.2548296$ 
Based on these design parameters all-normal dispersion can be created basically at any region within the transparency range of fused silica (Fig. 2a). The main dispersion defining parameters are the core diameter D and the hole diameter $\mathrm{d}$. There are two main differences regarding the dispersion compared to PCFs. Firstly, there is a steep slope maintained at the long wavelength edge and hence a dispersion maximum point is maintained up to $2 \mu \mathrm{m}$ and above. Secondly, there is a shoulder formation at the short wavelength side of the dispersion curve for geometries optimized around $2 \mu \mathrm{m}$ pump wavelength extended the expected SC to shorter wavelengths. The light confinement capability is demonstrated in Fig. $2 \mathrm{~b}$. While for PCFs the mode area explodes for dispersion curves shifted above $1.2 \mu \mathrm{m}$ demonstrating the vanishing guiding capability, the mode area is kept small and hence light guidance is maintained in case of SCFs.
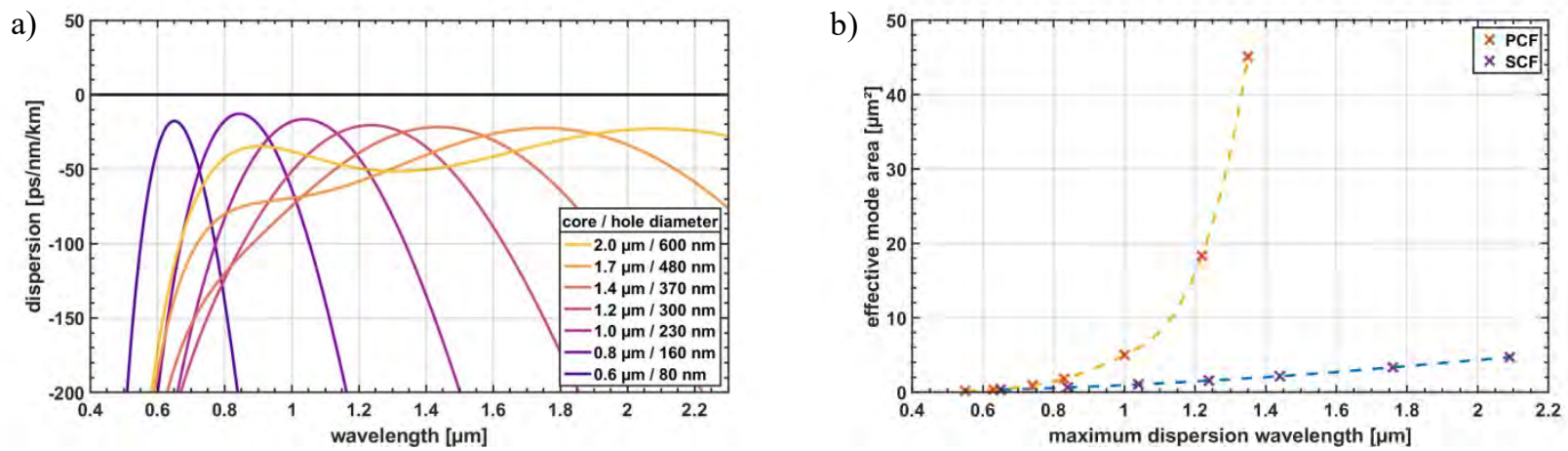

Figure 2: A) Dispersion curves for various combinations of core and hole diameters for four suspending struts with a thickness of $1 / 6^{\text {th }}$ of the core diameter. B) Effective mode area as a function of dispersion high point location also known as maximum dispersion wavelengths.

The thickness $t$ of the suspending struts only has a minor impact on the overall dispersion behavior. For this reason this parameter can effectively be used to define the mode content of the SCFs (Fig. 3). While the SCFs are multi-modal with thin struts and guide typically around ten polarization modes, all polarization modes except the two fundamental ones can be delocalized using a strut thickness around $1 / 4^{\text {th }}$ of the core diameter.

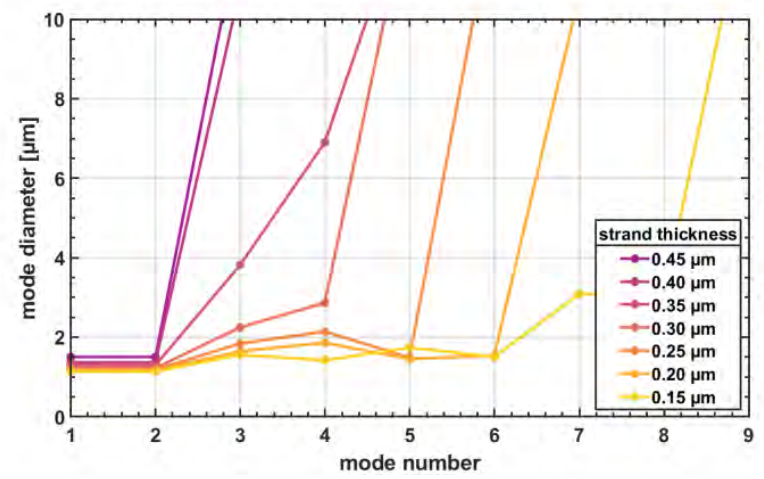

Figure 3: Modal diameter simulation for the first nine modes (including polarization discrimination) for various strut thicknesses. The fiber has a core diameter of $2 \mu \mathrm{m}$. Modes with significant larger diameter are not located in and hence guided by the core.

For the realization of such fibers we combine pure fused silica capillaries with a highly Fluorine doped fused silica cladding tube. The allows an overall viscosity increase of the central strut and core structure during processing and a good control over the central hole size by the processing temperature. In addition an intermediate cane drawing step assists the realization of comparable large struts thickness in combination with small core diameters. 


\section{References}

[1] Hartung, A., Heidt, A. M. and Bartelt, H., "Design of all-normal dispersion microstructured optical fibers for pulse-preserving supercontinuum generation.," Opt. Express 19(8), 7742-7749 (2011).

[2] Heidt, A. M., "Pulse preserving flat-top supercontinuum generation in all-normal dispersion photonic crystal fibers," J. Opt. Soc. Am. B 27(3), 550 (2010).

[3] Tu, H. and Boppart, S. A., "Coherent fiber supercontinuum for biophotonics," Laser Photon. Rev. 7(5), 628-645 (2013).

[4] Hooper, L. E., Mosley, P. J., Muir, A. C., Wadsworth, W. J. and Knight, J. C., "Coherent supercontinuum generation in photonic crystal fiber with all-normal group velocity dispersion.," Opt. Express 19(6), 4902-4907 (2011).

[5] Wang, H., Fleming, C. P. and Rollins, A. M., "Ultrahigh-resolution optical coherence tomography at 1.15 mum using photonic crystal fiber with no zero-dispersion wavelengths.," Opt. Express 15(6), 3085-3092 (2007). 


\title{
Novel optical fibre for cochlear implants
}

\author{
Hwa-Yaw Tam*a, Zhengyong Liu ${ }^{\mathrm{a}}$, Dinusha Serandi Gunawardena ${ }^{\mathrm{a}}$, Arvind N Vadivelu ${ }^{\mathrm{b}, \mathrm{c}}$, Bernard \\ Chen', Denny Oetomo ${ }^{\mathrm{b}}$, Stephen O’Leary ${ }^{\mathrm{c}}$ \\ aPhotonics Research Centre, Department of Electrical Engineering, The Hong Kong Polytechnic \\ University, Hung Hom, Kowloon, Hong Kong, China \\ bepartment of Mechanical Engineering, University of Melbourne, Parkville, VIC 3010, Australia \\ ${ }^{c}$ Department of Otolaryngology, University of Melbourne and the Royal Victorian Eye and Ear \\ Hospital, VIC 3002, Australia
}

\begin{abstract}
We report the application of low-bending loss fibres inscribed with fibre Bragg grating sensor arrays integrated inside a cochlear implant's electrode array for contact force measurement to reduce insertion trauma during cochlear surgery.
\end{abstract}

Keywords: Fibre-optic sensor, fibre Bragg grating, bending loss, cochlear implant, contact force measurement *hwa-yaw.tam@polyu.edu.hk; phone +852 27666175

\section{Introduction}

Hearing Loss is a rising global health issue which affects both young and old equally. The World Health Organization has predicted that by 2050, 1 in 10 people will have disabling hearing defects. Cochlear implantation surgery is the existing standard of reinstating hearing in deaf patients. A cochlear implant (CI) consists of an electrode array (EA) which is implanted into the inner ear (cochlea) bypassing the missing or defective sensory receptors by directly stimulating the auditory nerve and thereby, restoring hearing in patients suffering from hearing deficiencies. This sensitive surgical process is solely dependent on the human tactile perception and the experience of the surgeons.

The accurate placement of the EA is not only crucial in reducing trauma, but also in future explantation and reimplantation of the CIs. The lifetime of CIs is generally about 20 years; hence, replacement is necessary for several times in a young person's life. Rupture or any other damages to the delicate cochlear structures are usually irreversible and may lead to losses in residual hearing. To reduce the trauma experienced by a patient during CI surgeries, a number of different techniques have been reported. For instance, automated CI insertion tools with embedded force sensors ${ }^{1}$ or usage of optical fibre sensors in a $\mathrm{CI}^{2}$. The former fails to detect the contact force between the $\mathrm{CI}$ and the cochlea and the latter uses standard $125 \mu \mathrm{m}$ fibre which do not have adequate sensitivity nor sufficient flexibility, stiffening the CI and making it unsuitable for the application.

In this work, we report the integration of a specially designed optical fibre with fibre Bragg grating array in the CI to measure the contact force between the CI and the inner wall of cochlea. The intra-cochlear sensor scheme is preferable to provide real-time force feedback, for in-vivo implantation of CI.

\section{Integration of FBG sensors in the cochlear implant}

Figure 1(a) schematically illustrates the anatomic structure of a cochlea and the scala tympani (ST) inside the cochlea ${ }^{1}$. Figure 1(b) shows the image of an actual cochlear implant currently used in surgical implantation. The total effective length with 22 electrodes is about $20 \mathrm{~mm}$. Basically, the bending radii of the spiral ST varies from $\sim 3 \mathrm{~mm}$ to $\sim 1.5 \mathrm{~mm}$. Therefore, such extreme scenario requires special optical fibre with ultralow bending loss. In this work, an optical fibre with $50 \mu \mathrm{m}$ cladding dimeter and $\sim 9 \mu \mathrm{m}$ core is designed and fabricated. Smaller outer dimeter makes the fibre more

Sixth International Workshop on Specialty Optical Fibers and Their Applications (WSOF 2019), edited by Liang Dong, John M. Ballato, Proc. of SPIE Vol. 11206, 112060E · C 2019 SPIE CCC code: $0277-786 X / 19 / \$ 21 \cdot$ doi: $10.1117 / 12.2547877$ 
flexible. The fibre was fabricated using "rod-in-tube" method by inserting a Germanium doped core rod in jacket tube. The core is surrounded by a low index trench to reduce the bending loss. The measured bending loss is $\sim 0.3 \mathrm{~dB} /$ turn for $2.5 \mathrm{~mm}$ bending radius. The schematic design of the CI with FBG sensors is shown in Fig. 1(c), where the FBG close to the apex is used to measure the tip contact force when it touches the inner wall.
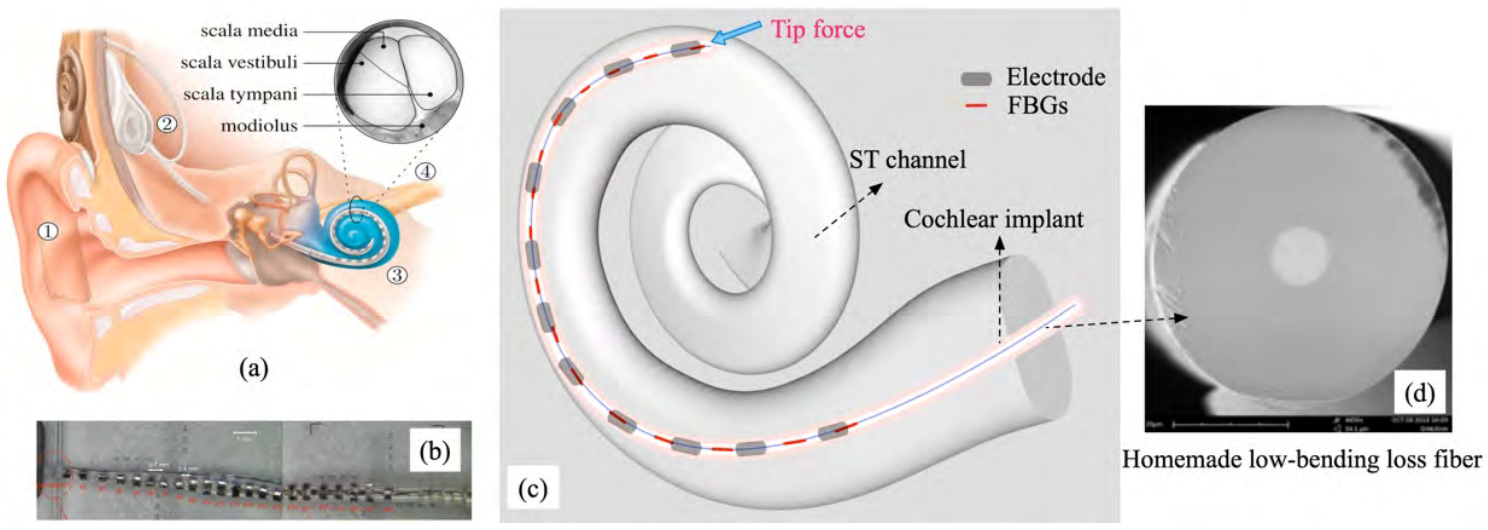

Figure 1. (a) Schematic figure of a cochlea with an implant system ${ }^{1}: 1$ ) a speech processor, 2) internal receiver, 3) the electrode array, 4) the auditory nerve. (b) The microscopic photo of an actual cochlear implant used for surgery. (c) The schematic figure of the cochlear implant integrated with fibre Bragg grating (FBG) sensors. (d) SEM photo of the fabricated fibre.

Figure 2(a) shows the experimental setup of the in-vitro tests in the laboratory using ST models printed by 3D printers. The shape and size of the ST models are identical to that of the human cochlea shown in Fig. 1(a). The CI integrated with FBG sensors and electrodes fixed on a translation stage is precisely inserted into the ST phantom and an interrogator is used to measure the Bragg wavelength change during the insertion. Figure 2(e) and (f) show the measured tip contact force using an external force gauge and the internal FBG sensor. It is observed that the intra-cochlear FBG sensors can measure the force precisely and give more details of the force change during the entire insertion process.
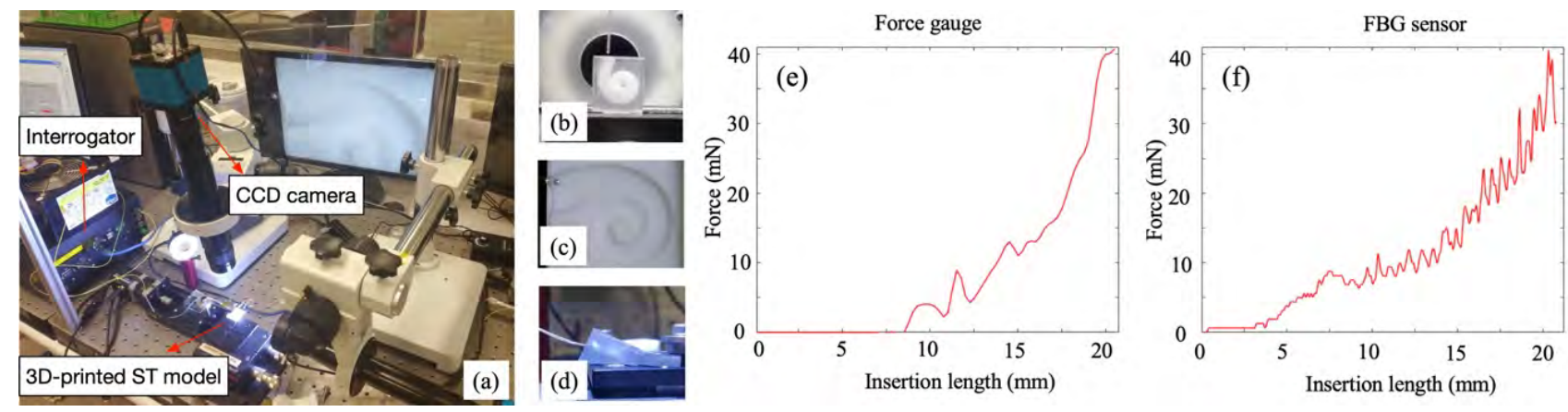

Figure 2. (a) Experimental setup used for the in-vitro tests, (b) a 3D scala tympani (ST) phantom printed by a 3D printer, (c) 2D ST phantom, and (d) 3D ST phantom considering otologic positioning during surgery. (e) and (f) plot the measured tip contact force using external force gauge and internal FBG sensor as a function of insertion length, respectively.

\section{Conclusion}

In this work, we demonstrate a smart cochlear implant integrated with FBG sensors to provide real-time contact force during CI implantation. The fibre is designed with ultralow bending loss to avoid too much signal attenuation and has reduced stiffness. Such smart CI integrated with the optical fibre sensors is promising to avoid trauma to the inner tissue of the cochlea by keeping the actual contact force below the rupture level during the implantation. 


\section{Reference}

[1] Kobler, J. P., Beckmann, D., Rau, T. S., Majdani, O. and Ortmaier, T., “An automated insertion tool for cochlear implants with integrated force sensing capability," Int. J. Comput. Assist. Radiol. Surg. 9(3), 481-494 (2014).

[2] Wade, S. A., Fallon, J. B., Wise, A. K., Shepherd, R. K., James, N. L. and Stoddart, P. R., "Measurement of forces at the tip of a cochlear implant during insertion," IEEE Trans. Biomed. Eng. 61(4), 1177-1186 (2014). 


\title{
Enhanced fibers for distributed sensing
}

\author{
Paul Westbrook*a, Ken Feder ${ }^{\mathrm{a}}$, Tristan Kremp ${ }^{\mathrm{a}}$, Hongchao $\mathrm{Wu}^{\mathrm{a}}$, Wing $\mathrm{Ko}^{\mathrm{a}}$, Roy Ortiz ${ }^{\mathrm{a}}$, Eric \\ Monberg $^{a}$, Debra Simoff \\ ${ }^{a}$ OFS Labs, Somerset NJ \\ ${ }^{\mathrm{b}} \mathrm{OFS}$, Avon, CT
}

\begin{abstract}
We discuss the use of enhanced fibers in distributed sensing applications. Processing of fibers over lengths from $1 \mathrm{~m}$ to more than $1 \mathrm{~km}$ can increase the signal to noise ratio in many sensing applications. We discuss enhancements observed with optical frequency domain reflectometry. Applications include temperature, strain, shape and position sensing, and distributed acoustic sensing.
\end{abstract}

Keywords: fiber sensor, fiber optics, sensing

westbrook@ofsoptics.com.com; phone 1732 748-7495

\section{Introduction}

It has been almost 50 years since optical fibers were first recognized as excellent candidates for sensing. As the possibility of low attenuation optical fiber became a reality for telecommunications, researchers understood that optical fiber could also provide very long and stable optical light paths with high sensitivity to external conditions. In particular, long spools of fiber were used to measure acoustic signals and these spools also allowed for a new type of sensitive gyroscope. It was also recognized that a long linear length of fiber could be used to measure temperature, strain, and other measurands, effectively turning the length of fiber into a continuous sensor. Such sensors became known as distributed sensors, and today they form a significant field of research in fiber optics and a growing commercial market for photonics. In addition to their unique distributed sensing capability, other benefits of optical fibers include small diameter, no electrical connections or susceptibility to electromagnetic interference, as well as resistance to corrosives, temperature, and high tensile strain. Applications include monitoring of oil and gas wells, pipelines, civil structures, and security perimeters. While these applications typically require sensing over hundreds of meters or more, there are also applications that require continuous sensing over shorter lengths, such as fiber shape and position sensing in medical and other industries. In most applications, access to a single end of the fiber is available and reflected signals are used to sense conditions along the fiber path. Various reflected signals may be used, including Raman, Brillouin and elastic scattering from Rayleigh scattering or index modulations added to the core after fiber draw. This paper will focus on elastic scattering in fibers which has been used in several applications, including distributed temperature [1] and strain [2] sensing, as well as sensing of fiber bend and shape $[3,4]$. It is known that elastic scattering may be increased through post processing of an optical fiber. In recent years there has been a growing interest in extending UV post processing techniques similar to fiber grating exposure to distributed sensors. While fiber gratings have been demonstrated as point sensors and in arrays since the $1980 \mathrm{~s}$, the use of continuous or quasi continuous arrays in sensing is a more recent innovation. Such quasi continuous arrays act as a new type of enhanced optical fiber suitable for sensing applications, typically by increasing the elastic scattering observed in the optical fiber. As with discrete fiber gratings, it is possible to design the optical spectrum to suit a given application. Multi-core fibers have 
also been applied to various sensing applications, most significantly in fiber shape sensing. Shape sensing uses a fiber with several cores to measure the state of bend and twist along a segment of fiber typically a few meters in length. This information is used to reconstruct the shape and position of the fiber. Shape sensing often requires continuous or nearly continuous sensing along the fiber length in order to produce accurate results.

UV post processing can greatly improve the signal to noise ratio (SNR) in many distributed sensing applications, both in multi-core and single-core fibers [5,6]. Moreover, when compared with Rayleigh scattering, enhanced scattering can offer a more easily interpreted back-scatter signal, making the optical interrogation schemes more stable. Continuous, very low increases in scattering can act in a manner similar to Rayleigh scattering, providing an elastic scattering signal that can be used to sense local strain and temperature. Typically, such sensor arrays are interrogated using swept wavelength interferometry or optical time domain reflectometry.

In this work, we review advances in distributed sensing fibers with continuous enhancements in scattering over lengths from a few meters to lengths in excess of 1 kilometer.

\section{Enhanced fibers}

We first discuss enhanced multicore fibers. In recent years, a new type of distributed sensing has been developed to measure the shape of a fiber [4]. In contrast to typical distributed sensing applications, which can require $1 \mathrm{~km}$ of fiber and have meter-scale resolution, shape sensing typically requires only a few meters of fiber, but requires resolution at the mm scale. Because these measurements are often continuous along the fiber, shape sensing is a type of short-range distributed sensing. Fig. 1 shows the typical elements of a shape sensing application. A hexagonal array of seven cores is drawn with a permanent twist of typically tens of revolutions per meter. The offset cores allow for a measurement of the fiber bend radius at all points along the fiber. The introduction of a permanent twist into the fiber allows the interrogator to determine the direction of the local twist state of the fiber. Both twist and bend sensitivity are required for a complete reconstruction of the fiber shape. Continuous gratings are imprinted into all of the cores simultaneously and their local Bragg condition is measured using optical frequency domain reflectometry (OFDR). As the scan length and range (bandwidth) determine its resolution, OFDR is also referred to as swept wavelength interferometry. With scan ranges of several nm, OFDR can reach sensor spatial resolutions of less than $1 \mathrm{~mm}$ over many meters. In Fig. $1 \mathrm{~b}$ we show an array measured via OFDR using a Luna OBR. Within the grating, the signal is a factor of more than $20 \mathrm{~dB}$ above the background Rayleigh scattering. Moreover, the phase derivative of the signal is very smooth, with very little noise. This is expected, since the spatial phase derivative is related to the local Bragg wavelength. The plot also shows a region with bare fiber that only exhibits the inherent Rayleigh scattering. This signal is much lower, and the phase derivative is much noisier. While the Rayleigh signal can also be used in continuous distributed measurements, the increased noise from these two factors can limit speed and accuracy of measurements in a given interrogation scheme.

Fig. 1c shows an example of shape reconstruction [7]. The shape sensing fiber used was similar to that in Fig. 1a. Grating arrays were inscribed over $10 \mathrm{~cm}$ of this fiber, and a set of reference and calibration measurements were performed for all of the cores of the fiber. The fiber was then wrapped around a post. Such a helical path is inherently threedimensional and requires measurements of both bend and twist along the fiber. Signals collected from four of the cores were then used to reconstruct the shape of the fiber using an algorithm similar to that in Ref. [8]. 


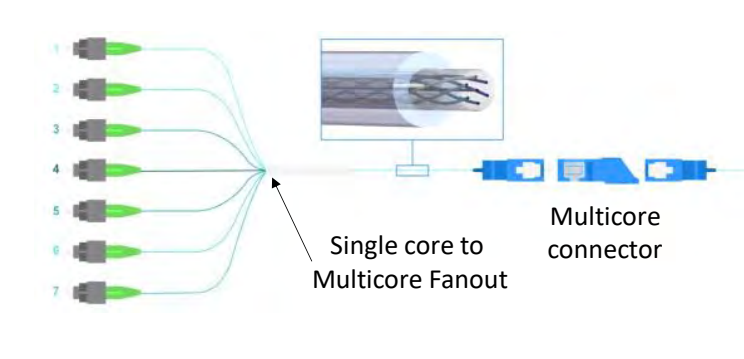

(a)

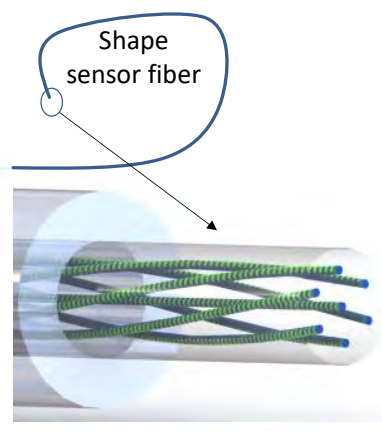

(b)

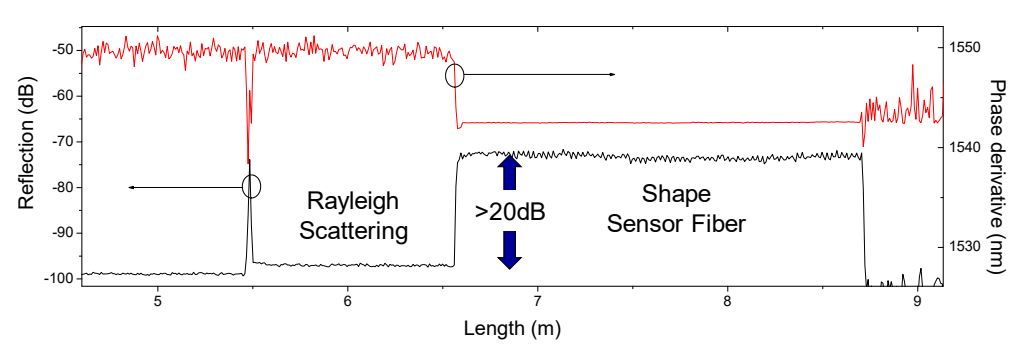

(c)

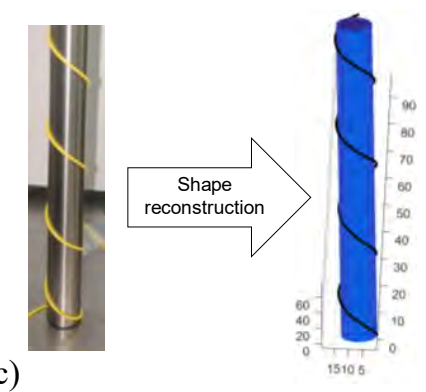

Figure 1. (a) Shape sensing components: Single- to multi-core fanout, multi-core connectors, and shape sensor fiber. (b) OFDR trace showing increased scattering (black trace) and improved signal-to-noise ratio of the spatial phase derivative signal (red curve) in the shape sensor fiber. (c) Shape reconstruction: Fiber wound around a post (left); reconstructed shape (right).

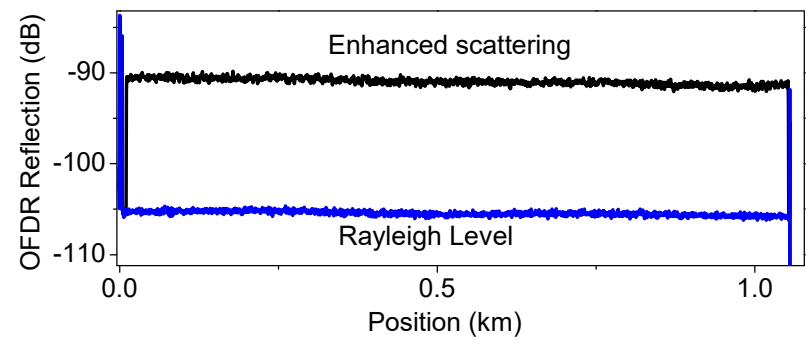

Figure 2. OFDR trace showing more than $10 \mathrm{~dB}$ increase in reflected power over $1.1 \mathrm{~km}$ of UV processed fiber (black curve). The blue curve shows the level of the native Rayleigh scattering in the bare fiber.

While meter-long sensor arrays are appropriate for many shape sensing applications, distributed acoustic sensing (DAS) applications typically require fiber lengths in excess of $100 \mathrm{~m}$. Using similar post processing methods, it is also possible to inscribe very long lengths of fiber with continuous enhanced backscattering [9]. The enhanced scattering from such gratings can improve various sensing applications, including distributed temperature, strain and acoustic sensors [5,6]. Importantly, such arrays can be inscribed while maintaining relatively low attenuation in standard single-mode fibers. Fig. 2 shows an 
OFDR trace of a 1.1-kilometer-long fiber with continuously enhanced scattering. With differing levels of UV processing intensity, it is possible to control the amount of enhancement of the scattering from a few $\mathrm{dB}$ to more than $20 \mathrm{~dB}$ above the native Rayleigh scattering. Attenuation remains at a level close to that of a standard single-mode fiber. Such gratings have been shown to improve the accuracy and speed of applications such as static and dynamic distributed temperature and strain sensing that require a strong scattering signal along the entire length of the sensor fiber [6].

\section{Conclusion}

Sensing applications that rely on optical backscattering can be greatly improved through the use of post-processing techniques similar to UV processing through phase masks and other interferometric techniques. These methods can process long lengths of fiber in excess of $1 \mathrm{~km}$, providing enhanced SNR performance over long lengths. They also allow for the creation of enhanced scattering in multi-core fibers. Such multi-core fibers are useful in applications that require sensing of shape and position of optical fibers.

\section{References}

[1] D. K. Gifford et al., "Distributed fiber-optic temperature sensing using Rayleigh backscatter." ECOC Proceedings. Vol. 3. 511-512, 2005.

[2] M. Froggatt et al., "High-spatial-resolution distributed strain measurement in optical fiber with Rayleigh scatter," Appl. Opt. 37, 1735-1740 (1998).

[3] M. J. Gander et al., "Bend measurement using Bragg gratings in multi-core fiber," Electron. Lett. 36, 120-121 (2000).

[4] R. G. Duncan et al., "High accuracy fiber-optic shape sensing," Proc. SPIE 6530, 65301S-11 (2007).

[5] P. S. Westbrook et al., "Continuous multicore optical fiber grating arrays for distributed sensing applications," J.

Lightwave Technol. 35, 1248-1252 (2017).

[6] V. A. Handerek et al., "Improved Optical Power Budget in Distributed Acoustic Sensing Using Enhanced Scattering Optical Fibre," In Optical Fiber Sensors, OFS-26, paper TuC5, (2018).

[7] P. S. Westbrook et al., "Distributed sensing over meter lengths using twisted multicore optical fiber with continuous Bragg gratings," Furukawa Electric Review 48, 26-32 (2017).

[8] J. P. Moore and M. D. Rogge. "Shape sensing using multi-core fiber optic cable and parametric curve solutions," Optics Express 20, 2967-2973 (2012).

[9] P. S. Westbrook et al., "Kilometer length low loss enhanced back scattering fiber for distributed sensing," Proc. SPIE 10323, 25th International Conference on Optical Fiber Sensors, OFS-25 PD, 103239Q (8 June 2017). 


\title{
$500^{\circ} \mathrm{C}$-Rated Optical Fibers for High Temperature Applications
}

\author{
William Jacobsen*, Abdel Soufiane, John D’Urso \\ AFL Specialty Fibers, 15 Centennial Drive, North Grafton, MA, United States
}

\begin{abstract}
Optical fibers for high temperature applications $\left(>300^{\circ} \mathrm{C}\right)$ have typically been metal-coated. These fibers suffer high attenuation $(>10 \mathrm{~dB} / \mathrm{km})$, and are limited in length. This paper describes a low-attenuation metal-coated fiber operating at temperatures up to $500^{\circ} \mathrm{C}$.
\end{abstract}

Keywords: metal coating, harsh environment, DTS, supercritical geothermal, downhole

*bill.jacobsen@aflglobal.com; phone 1508 890-7105

\section{Introduction}

Silica-based glass optical fibers without coating can withstand temperatures greater than $600^{\circ} \mathrm{C}$. However, glass fibers need to be protected from the environment. Standard telecom fibers are typically coated with acrylate that allow their use in temperatures up to $85^{\circ} \mathrm{C}$. Specialty optical fibers can be produced with a polyimide coating, which allows these fibers to be used in environments up to $300^{\circ} \mathrm{C}$. This type of fiber has been used extensively in the oil and gas industry to provide important communications and sensing functions for reservoir management.

For temperatures above $300^{\circ} \mathrm{C}$, metal coatings would be attractive. Those produced to date have been deemed unsuitable for geothermal well deployment due to high attenuation values at low temperatures ${ }^{1}$. Downstream oil processing can also benefit from high temperature measurements requiring low attenuation fibers that perform beyond $300^{\circ} \mathrm{C}$. This attenuation as well as significant attenuation changes during cycling is generally attributed to microbending and the large mismatch of the coefficients of thermal expansion between the metal coating and the glass fiber ${ }^{2}$. Among other things, thinner metal coatings could help to mitigate these issues; however, the production of long lengths of high quality metal-coated fiber with controlled thickness of the coating is non-trivial ${ }^{2}$.

In this paper, a metal-coated fiber capable of withstanding temperatures up to $500^{\circ} \mathrm{C}$ will be demonstrated. In addition, it will be shown that this fiber can be cycled between room temperature and $500^{\circ} \mathrm{C}$, while maintaining low attenuation even at low temperatures.

\section{Fiber Design}

It has been demonstrated since the early 1980s that hydrogen ingression in silica-based glass induces losses in optical fibers at specific wavelengths due to the absorption of a variety of hydrogen related species ${ }^{3}$. Common silica fibers used in communications such as standard single-mode (SM) and standard graded-index multimode (MM) suffer a dramatic optical degradation in presence of hydrogen even at room temperature. The cores of these fibers are typically

Sixth International Workshop on Specialty Optical Fibers and Their Applications (WSOF 2019),

edited by Liang Dong, John M. Ballato, Proc. of SPIE Vol. 11206, 112060G · (C) 2019 SPIE

CCC code: $0277-786 X / 19 / \$ 21 \cdot$ doi: $10.1117 / 12.2547386$ 
doped with refractive index increasing elements such as germanium and phosphorus. Depending on the temperature and $\mathrm{H}_{2}$ concentration, once hydrogen diffuses in the fiber core, it can migrate to interstitial sites of the structure and/or bond with existing defects in the glass such as $\mathrm{SiO}, \mathrm{GeO}$ and P-O. The overall fiber loss reaches hundreds of decibels per kilometer, which makes it unusable for any light transmission applications.

AFL took an innovative approach to prevent the optical degradation of optical fibers immersed in a harsh environment by modifying and optimizing the design of the glass component of the fiber itself. In particular, the approach consists of eliminating the dopants that create more defects in the glass structure such as germanium, phosphorus, and boron. The fiber is designed with only silica in the core, along with fluorine doping to achieve the graded index profile of the multimode fiber ${ }^{4}$. This fiber is produced by AFL and is branded as Verrillon ${ }^{\circledR}$ VHM5000; it is a $0.2 \mathrm{NA} 50 / 125 \mu \mathrm{m}$ GI-MMF.

VHM5000 was the base fiber used with this metal coating. It had a gold-based coating with a wall thickness of approximately 3 - $5 \mu \mathrm{m}$ which is well below the typical coating thickness of $15-25 \mu \mathrm{m}$ for commercially available metal-coated fibers. A cross-sectional SEM image demonstrating the good concentricity and integrity of the coating process is shown in Figure 1.

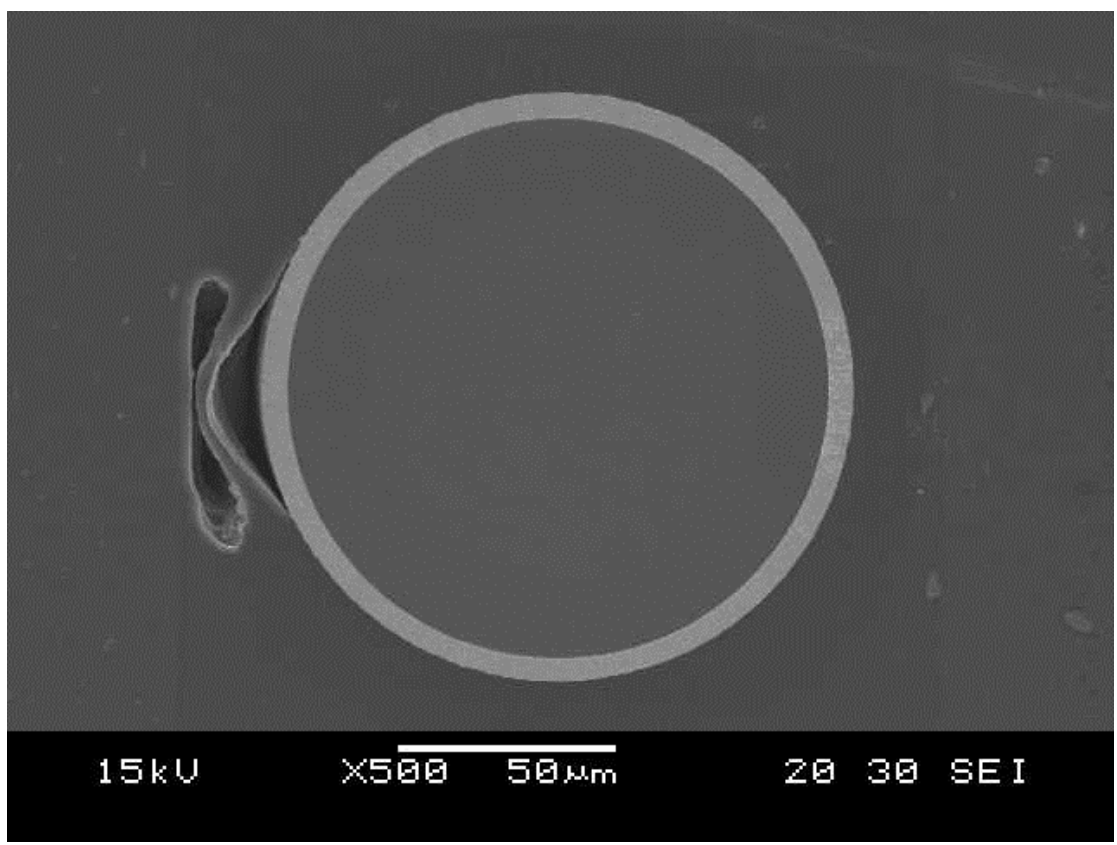

Figure 1: SEM cross-section of fiber produced using the current process

Metal-coated fibers can have optical losses in as-drawn condition as high as $20-100 \mathrm{~dB} / \mathrm{km}$ at room temperature ${ }^{2}$. Figure 2 shows the spectral attenuation of VHM5000 with a gold-based coating shown in Figure 1, at room temperature, measured on $88 \mathrm{~m}$ of fiber. Fiber was measured in a $300 \mathrm{~mm}$ diameter loose coil. 


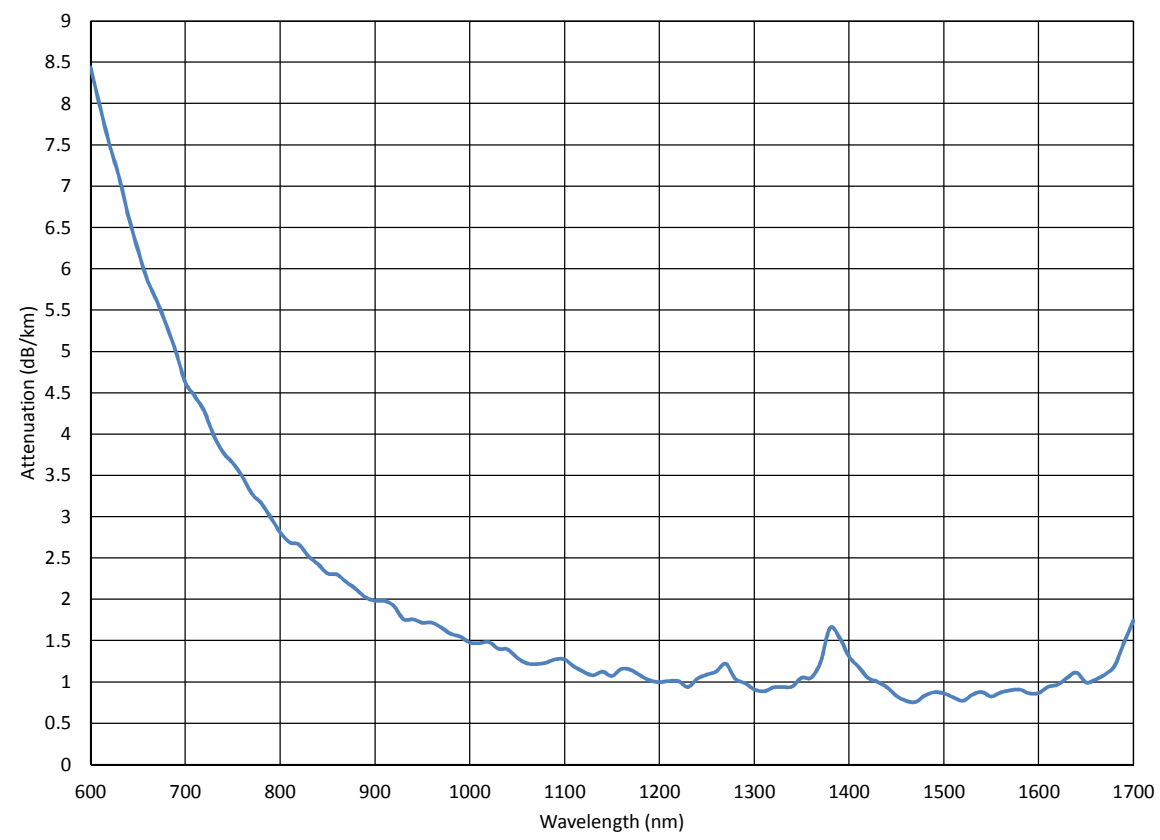

Figure 2: Spectral attenuation of VHM5000 with gold-based coating, $88 \mathrm{~m}$ long, 300mm diameter loose coil, room temperature

The spectral attenuation of this fiber with a gold-based coating shows attenuation levels similar to standard acrylate or polyimide coated multimode fibers, as opposed to the significantly higher levels shown by other commercially available metal-coated multimode fibers.

Metal-coated fibers also have the tendency to 'cold bond' to other metals, or itself, at temperatures significantly below their melting temperature. AFL has a patent-pending process which prevents these metal-coated fibers from bonding. This process was applied to all the fibers in these tests.

\section{Results and Discussion}

Figure 3 shows six temperature cycles of VHM5000 with gold based coating, between room temperature and $375^{\circ} \mathrm{C}$. Data was acquired every 5 minutes using an OTDR. The fiber was in a $114 \mathrm{~mm}$ loose coil, 40 meters in length. Each cycle consisted of a $30^{\circ} \mathrm{C}$ / hour ramp to $375^{\circ} \mathrm{C}$, the temperature was held at $375^{\circ} \mathrm{C}$ for 24 hours, and then it was ramped down $30^{\circ} \mathrm{C} /$ hour until $60^{\circ} \mathrm{C}$. At that point, the oven was allowed to return to room temperature, and then the next cycle was started. $850 \mathrm{~nm}$ was the wavelength that was monitored. 


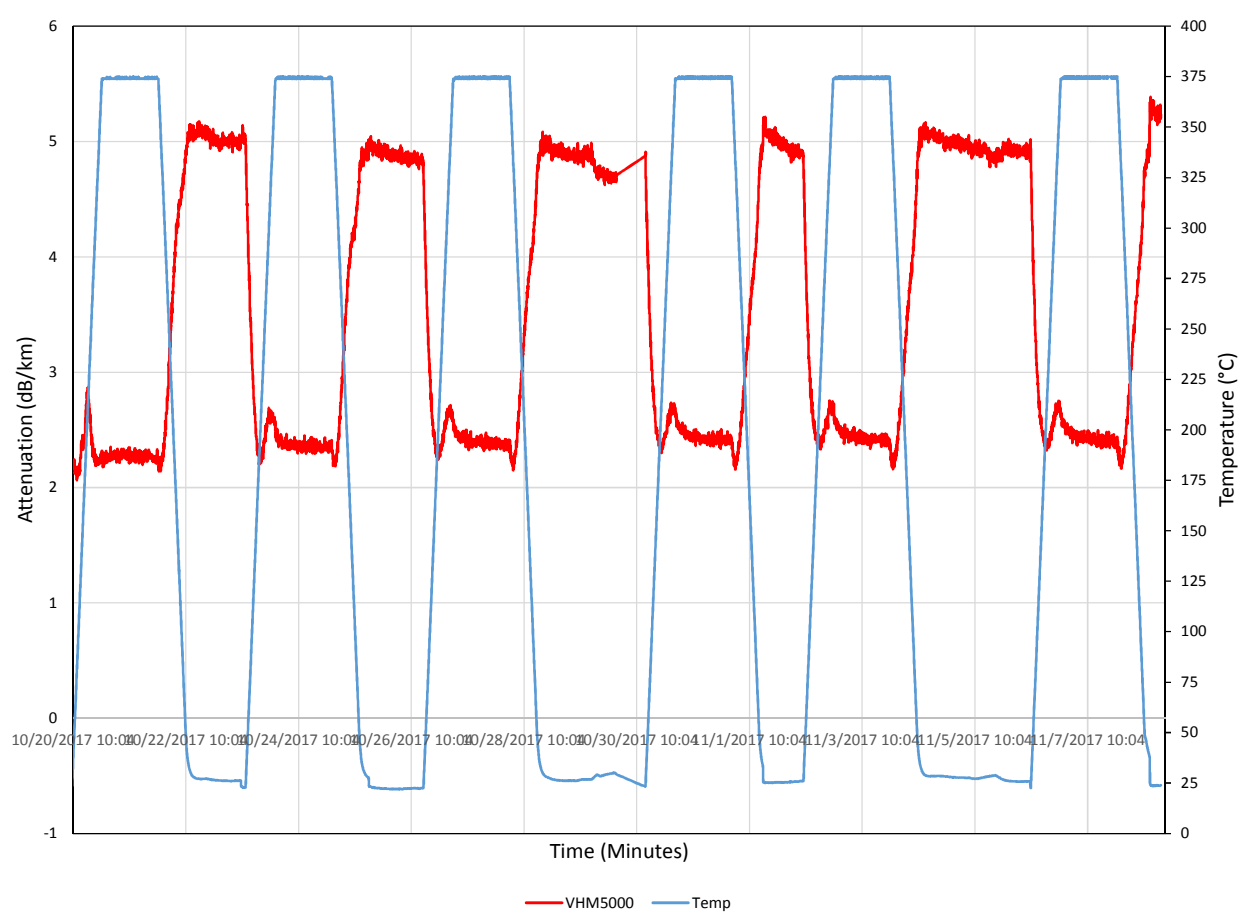

Figure 3: VHM5000 with gold-based coating, 40m long, $850 \mathrm{~nm}$ attenuation, six temperature cycles between room temperature and $375^{\circ} \mathrm{C}$

43 meters of VHM5000 gold-based coated fiber was put in a $500^{\circ} \mathrm{C}$ oven for 900 hours. An OTDR was connected to the fiber at the conclusion of the 900 hours, and a $500^{\circ} \mathrm{C}$ cycle was run. Figure 4 shows this temperature cycle,

between room temperature and $500^{\circ} \mathrm{C}$. Data was acquired every 5 minutes. The fiber was in a $114 \mathrm{~mm}$ loose coil. The cycle consisted of a $30^{\circ} \mathrm{C}$ / hour ramp to $500^{\circ} \mathrm{C}$, the temperature was held at $500^{\circ} \mathrm{C}$ for 34 hours, and the oven was stopped and allowed to return to room temperature on its own. $850 \mathrm{~nm}$ was the wavelength that was evaluated. 


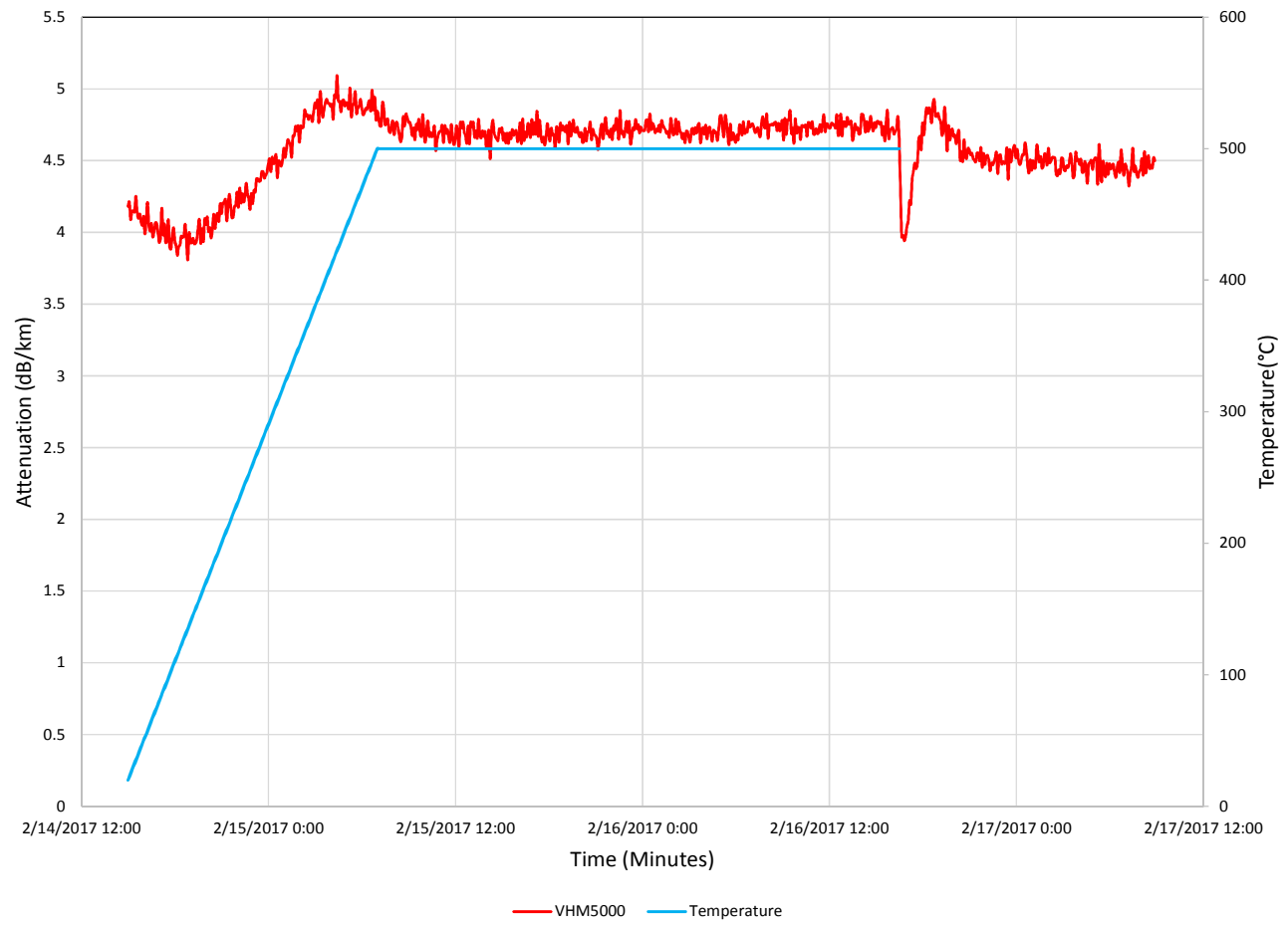

Figure 4: VHM5000 with gold-based coating, $43 \mathrm{~m}$ length, $850 \mathrm{~nm}$ attenuation, 34 hours at $500^{\circ} \mathrm{C}$ after soaking at $500^{\circ} \mathrm{C}$ for 900 hours

\section{Conclusions}

A low attenuation metal-coated optical fiber capable of withstanding temperatures up to $500^{\circ} \mathrm{C}$ was demonstrated. Performance was validated using an OTDR. Temperature cycling showed that the metal-coated fiber could withstand the expansion and contraction of the metal coating repeated multiple times. Attenuation at both room temperature and high temperature was significantly lower than any reported attenuation in metal-coated fibers. The 900 hour soak, and subsequent evaluation of the fiber, showed that the fiber still performed well after long-term exposure at $500^{\circ} \mathrm{C}$. In addition, this process is capable of producing long length of fiber, up to $3.5 \mathrm{~km}$ continuous.

\section{References}

[1] Reinsch, T., and Henninges, J. "Temperature Dependent Characterization of Optical Fibres for Distributed Temperature Sensing in Hot Geothermal Wells,” Measurement Science and Technology, 21, (2010).

[2] Bogatyrev, V.A., and Semjonov, S. "Metal-Coated Fibers," Specialty Optical Fibers Handbook, Academic Press, 491-512 (2007).

[3] Stone, J., Chraplyvy, A.R., and Burrus, C.A. "Gas-in-glass-a new Raman-gain medium: molecular hydrogen in solid-silica optical fibres," Opt. Lett., 7, 297-299 (1982).

[4] Weiss, J. "Downhole geothermal well sensors comprising a hydrogen-resistant optical fiber." U.S. Patent No. 6853798 B1, (2005). 


\title{
Carbon-Coated Optical Fiber Performance in Hydrogen
}

\author{
William Jacobsen*, Abdel Soufiane \\ AFL Specialty Fibers, 15 Centennial Drive, North Grafton, MA, United States
}

\begin{abstract}
A study of the lifetime of carbon-coated optical fibers is presented. Multimode fibers are interrogated in a chamber containing hydrogen, at three different temperatures. Lifetime results are calculated. Results show that carbon is effective as a hydrogen barrier up to $150^{\circ} \mathrm{C}$.
\end{abstract}

Keywords: optical fiber, harsh environment, carbon, hydrogen, hermetic, permeation time constant

*bill.jacobsen@aflglobal.com; phone 1508 890-7105

\section{Introduction}

In the 1980s, it was realized that hydrogen ingression could cause significant losses in optical fiber. In 1985, AT\&T reported results for hermetic fibers with carbon coatings; the coatings were deposited by thermally decomposing carbon-containing gases onto the fiber surface during fiber draw ${ }^{1}$. The time dependence of the concentration of hydrogen in the glass fiber is given by LeMaire et $\mathrm{al}^{2}$ :

$$
\Delta \alpha_{1.24}(\mathrm{t}, \mathrm{T}) /\left\{\Delta \alpha_{1.24}(\infty, \mathrm{T}) \times \mathrm{P}_{\mathrm{H} 2}\right\}=\left[1-\exp \left\{-\left(\mathrm{t}-\tau_{\mathrm{i}}\right) / \tau_{\mathrm{f}}\right\}\right]
$$

where $\Delta \alpha_{1.24}(\mathrm{t}, \mathrm{T})$ is the change in attenuation (at $1.24 \mu \mathrm{m}$ ) after the fiber has been exposed to a hydrogen environment for time $\mathrm{t}$ at temperature $\mathrm{T} ; \Delta \alpha_{1.24}(\infty, \mathrm{T})$ is the change in attenuation when equilibrium has been reached between the fiber and the environment; and $\mathrm{P}_{\mathrm{H} 2}$ is the hydrogen pressure. $\tau_{\mathrm{i}}$ is the time constant for initial lag (before any increase in attenuation is observed), while $\tau_{\mathrm{f}}$ is the rate at which the attenuation increases at any given temperature $\mathrm{T}$. Also for relatively thin coatings, $\tau_{\mathrm{i}}$ is generally much less than $\tau_{\mathrm{f}}$. For relatively short times, equation (1) becomes

$$
\Delta \alpha_{1.24}(\mathrm{t}, \mathrm{T}) /\left\{\Delta \alpha_{1.24}(\infty, \mathrm{T}) \times \mathrm{P}_{\mathrm{H} 2}\right\}=\left[\mathrm{t} / \tau_{\mathrm{f}}\right]
$$

A plot of the left hand side of equation (2) vs. time " $\mathrm{t}$ " should yield a line with slope $1 / \tau_{\mathrm{f}}$. $\tau_{\mathrm{f}}$ and $\tau_{\mathrm{i}}$ contain all the information of interest regarding the permeation characteristics of the carbon coating ${ }^{3}$. Since $\tau_{\mathrm{f}}$ is significantly larger than $\tau_{\mathrm{i}}$, this paper concentrates on finding $\tau_{\mathrm{f}}$ for carbon-coated multimode fibers at three different temperatures. Once $\tau_{\mathrm{f}}$ and the maximum saturation level of $\mathrm{H}_{2}$ in glass are determined, fiber lifetime in $\mathrm{H}_{2}$ can be predicted.

\section{Optical Fiber}

\section{Experimental Description}

The fiber used in this test was a standard $50 \mu \mathrm{m}$ core / $125 \mu \mathrm{m}$ clad graded-index multimode fiber, containing only germanium as a dopant (AFL Verrillon ${ }^{\circledR}$ VHM2000). Both carbon-coated and non carbon-coated fibers used in this test were produced from the same glass preform during the same draw run. The fiber had mid-temp dual acrylate coating

\footnotetext{
Sixth International Workshop on Specialty Optical Fibers and Their Applications (WSOF 2019),

edited by Liang Dong, John M. Ballato, Proc. of SPIE Vol. 11206, 112060H · (c) 2019 SPIE

CCC code: $0277-786 \mathrm{X} / 19 / \$ 21 \cdot$ doi: $10.1117 / 12.2547390$
} 
applied, which is rated for service up to $150^{\circ} \mathrm{C}$. Non carbon-coated fibers were required in order to determine what the hydrogen saturation level was at each temperature.

\section{Testing Apparatus}

The apparatus consists of a stainless steel chamber with high-pressure optical fiber feed-throughs. An ANDO AQ4303B White Light Source is connected to a DiCon GP700 Optical Switch. Up to eight fibers can be connected to the switch, although one fiber is used as a reference fiber. The switch is also connected to an ANDO AQ-6315E Optical Spectrum Analyzer (OSA). A computer, using LabView, is used to interrogate the fibers, real-time, in user-defined intervals. The chamber can be pressurized up to $102 \mathrm{~atm}(1500 \mathrm{psi})$ and heated to a temperature of up to $300^{\circ} \mathrm{C}$. A schematic of the system is in Figure 1.

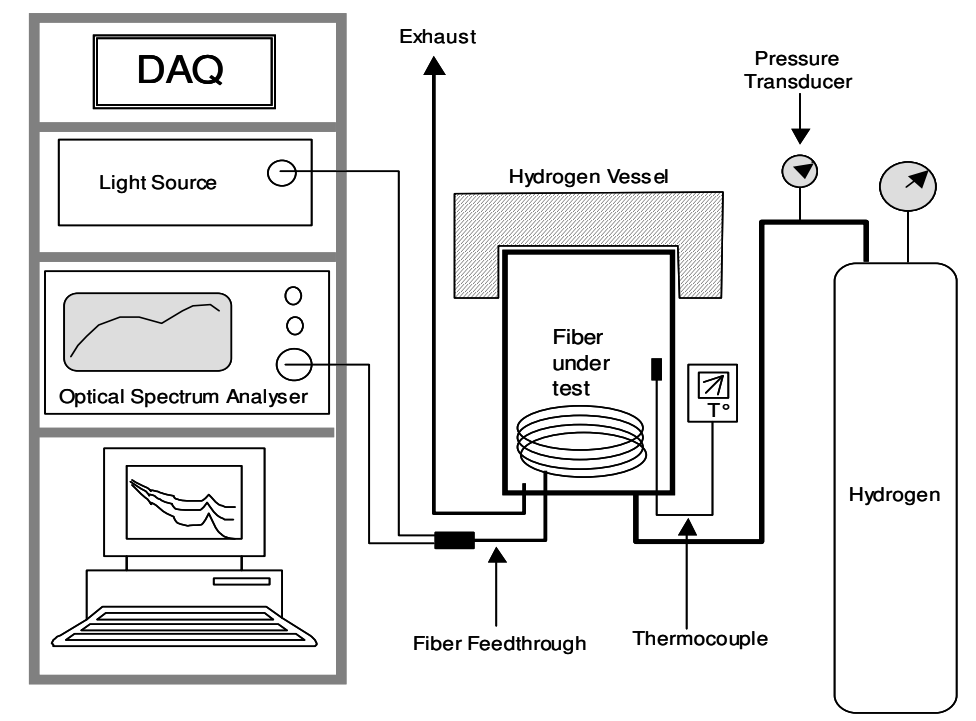

Figure 1. Real-Time Hydrogen Ingression Test Setup

\section{Test Setup and Conditions}

Three tests were performed at three different temperatures: $150^{\circ} \mathrm{C}, 135^{\circ} \mathrm{C}$, and $120^{\circ} \mathrm{C}$. For each test, there was one $50 \mathrm{~m}$ loose coil of fiber without carbon, along with one $500 \mathrm{~m}$ loose coil of fiber with carbon. Coils were $89 \mathrm{~mm}$ in diameter. The short $50 \mathrm{~m}$ length without carbon was required to determine what the maximum amount of free $\mathrm{H}_{2}$ that could be incorporated into the fiber; 50m was long enough to get good data, taking into account the limitations of the dynamic range of the OSA.

In order to make temperature the only variable, each test was initially loaded with different pressures of $\mathrm{H}_{2}$. The goal was that once the chamber reached the temperature, the pressure would be the same for all three tests. For these tests, the $\mathrm{H}_{2}$ pressure at the temperature of interest was 84.7atm (1245psi). 84.7atm of pure $\mathrm{H}_{2}$ is not a 'typical' harsh environment; it was intentionally harsh in order to accelerate the test duration.

Fibers were interrogated by LabView every hour. Tests were run for different lengths of time; they were run until a time where it was felt that $\tau_{\mathrm{f}}$ could be calculated accurately. 


\section{Results and Discussion}

\section{Determination of Maximum $\mathrm{H}_{2}$ Saturation}

Fiber lifetime in $\mathrm{H}_{2}$ is calculated based on induced loss at $1244 \mathrm{~nm}$, the molecular $\mathrm{H}_{2}$ absorption peak. For the most part, loss seen at $1244 \mathrm{~nm}$ is due to free, unreacted $\mathrm{H}_{2}$. The $50 \mathrm{~m}$ coils of non carbon-coated fiber were saturated with $\mathrm{H}_{2}$ within hours after exposure; the induced loss seen for each of these coils at each temperature is critical to calculating fiber lifetime. Figure 2 shows the induced loss at $1244 \mathrm{~nm}$ of all three non carbon-coated fibers, at the same pressure of pure $\mathrm{H}_{2}$.

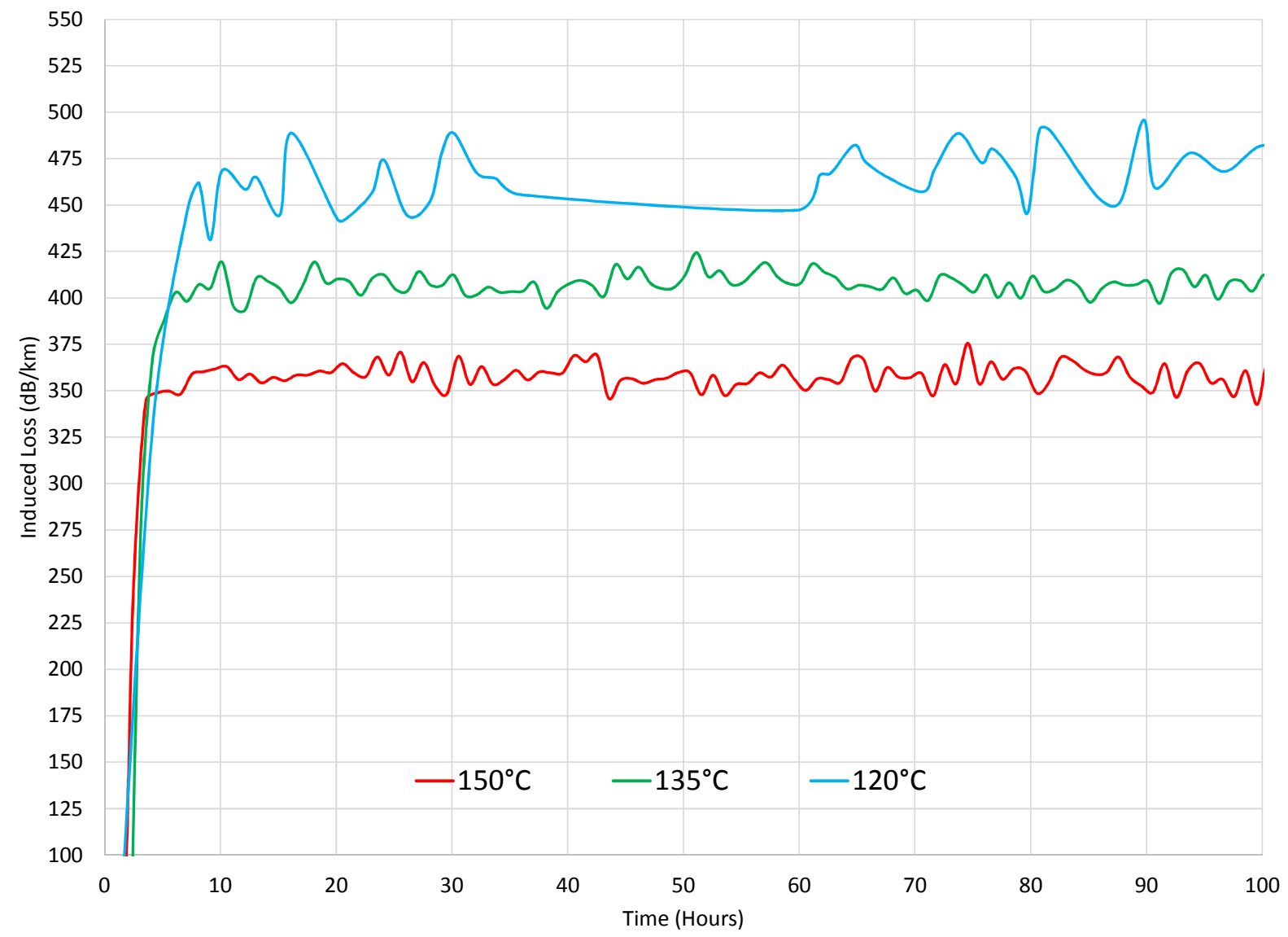

Figure 2. Induced loss @ 1244nm of three non carbon-coated VHM2000 fibers, exposed to 84.7atm pure $\mathrm{H}_{2}$ at different temperatures

The results of the non-carbon coated fiber test illustrates a very important concept; that the solubility of $\mathrm{H}_{2}$ in silica decreases with an increase in temperature. This has a profound impact on the meaning of 'fiber lifetime'. 


\section{Determination of $\mathbf{H}_{2}$ Saturation Rate}

In order to calculate fiber lifetime, $1 / \tau_{\mathrm{f}}$, the change of induced loss over time for the carbon-coated fibers is also needed. This value is required in order to determine how long it will take to completely saturate the fiber with $\mathrm{H}_{2}$. Figure 3 shows the induced loss at 1244nm of all three carbon-coated fibers, at 84.7atm (1245psi) of pure $\mathrm{H}_{2}$.

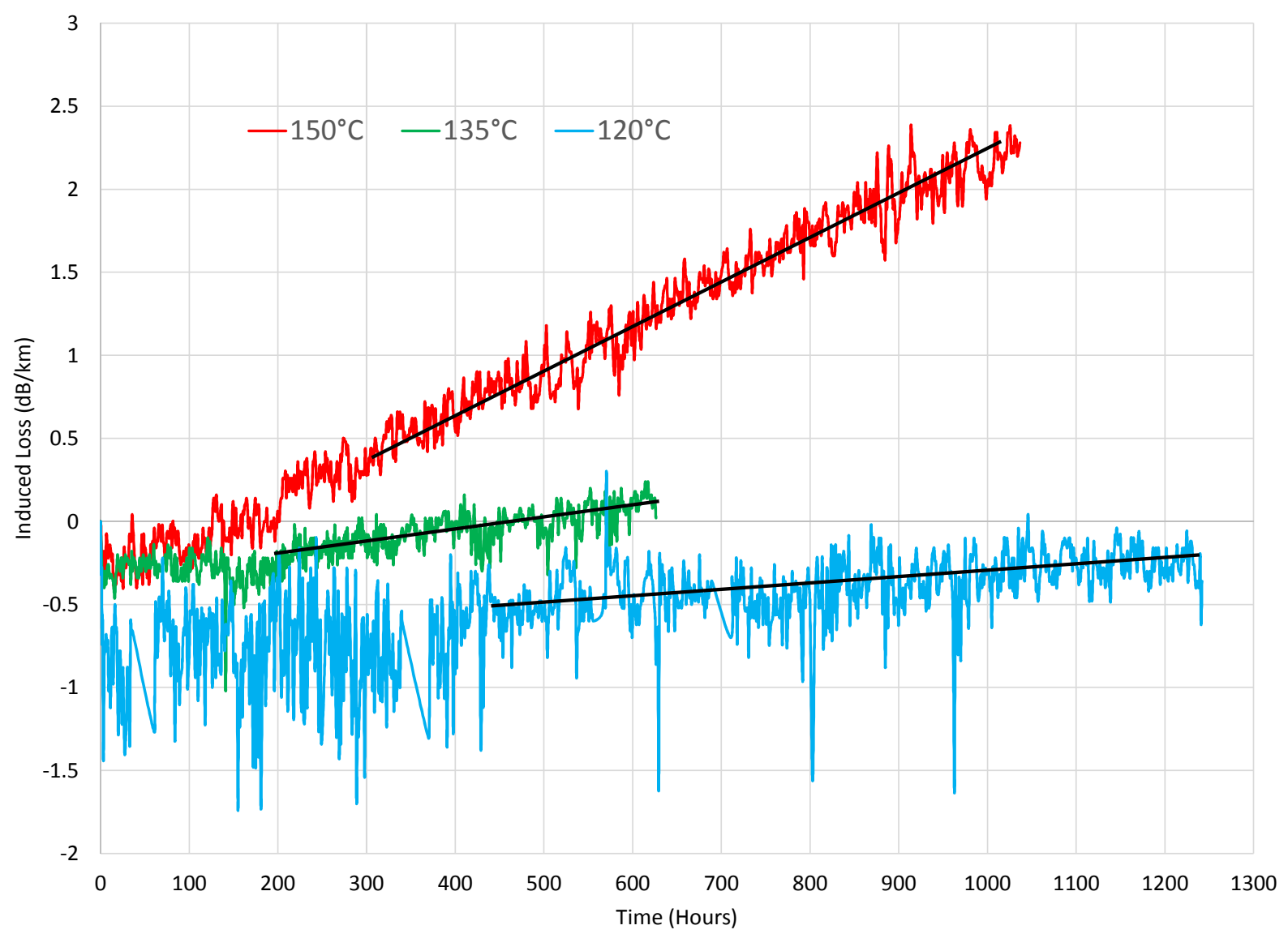

Figure 3. Induced loss @ 1244nm of 3 carbon-coated VHM2000 fibers, exposed to 84.7atm pure $\mathrm{H}_{2}$ at different temperatures

Based on the change in induced loss over time and the maximum amount of $\mathrm{H}_{2}$ that can be absorbed into the glass at that particular temperature, the following slopes $\left(1 / \tau_{\mathrm{f}}\right)$ and fiber lifetimes have been calculated in Table 1 . 


\begin{tabular}{|c|c|c|c|}
\hline Temperature & Slope - Induced Loss $/$ Time $\left(\mathbf{1} / \tau_{\mathrm{f}}\right)$ & Max $\mathbf{H}_{2}$ Induced Loss & Fiber Lifetime \\
\hline $120^{\circ} \mathrm{C}$ & $0.000359 \mathrm{~dB} / \mathrm{km} / \mathrm{hr}$ & $463 \mathrm{~dB} / \mathrm{km}$ & 147.1 years \\
\hline $135^{\circ} \mathrm{C}$ & $0.0008727 \mathrm{~dB} / \mathrm{km} / \mathrm{hr}$ & $406 \mathrm{~dB} / \mathrm{km}$ & 53.1 years \\
\hline $150^{\circ} \mathrm{C}$ & $0.002615 \mathrm{~dB} / \mathrm{km} / \mathrm{hr}$ & $352 \mathrm{~dB} / \mathrm{km}$ & 15.4 years \\
\hline
\end{tabular}

Table 1: Slope $\left(1 / \tau_{\mathrm{f}}\right)$ and fiber lifetime calculations for carbon-coated VHM2000 fibers, exposed to 84.7 atm pure $\mathrm{H}_{2}$ at different temperatures

It can been seen that at lower temperatures, it takes much longer for $\mathrm{H}_{2}$ to penetrate the carbon layer and get into the glass. However, once it gets into the glass, the glass can hold large amounts of $\mathrm{H}_{2}$. Conversely, at higher temperatures, it does not take as long for the $\mathrm{H}_{2}$ to penetrate the carbon layer, but the $\mathrm{H}_{2}$ solubility is much lower. The fact that slope and maximum $\mathrm{H}_{2}$ induced loss are inversely related means that fiber lifetime gets much longer at lower temperatures. The fiber lifetime is not a linear increase as a function of temperature.

\section{Fiber Lifetime}

The definition of 'Fiber Lifetime', as discussed in this paper, is the point where the carbon-coated fiber reaches maximum saturation level of $\mathrm{H}_{2}$. Is this a realistic measurement of fiber lifetime? The answer is no.

The primary cause of optical loss is not due to the presence of 'free $\mathrm{H}_{2}$ ' in the glass itself; it has to do with the reaction of $\mathrm{H}_{2}$ with dopants and defects contained in the optical fiber itself. Glass chemistry, along with the process used to manufacture the preform, have the greatest impact on fiber lifetime in $\mathrm{H}_{2}$. In the case presented here, VHM2000 has a core doped with germanium only. However, the presence of $\mathrm{GeOH}$, created by the reaction of $\mathrm{H}_{2}$ and the defects in $\mathrm{GeO} 2$, cause significant optical losses at the shorter wavelengths. As a result, many of the multimode fibers used in harsh environments traditionally operate at $1064 \mathrm{~nm}$; this wavelength region has been shown to be most 'immune' to the presence of $\mathrm{H}_{2}$. Figure 4 shows the effect of $\mathrm{H}_{2}$ on non carbon-coated VHM2000 fibers, at 1064nm. 


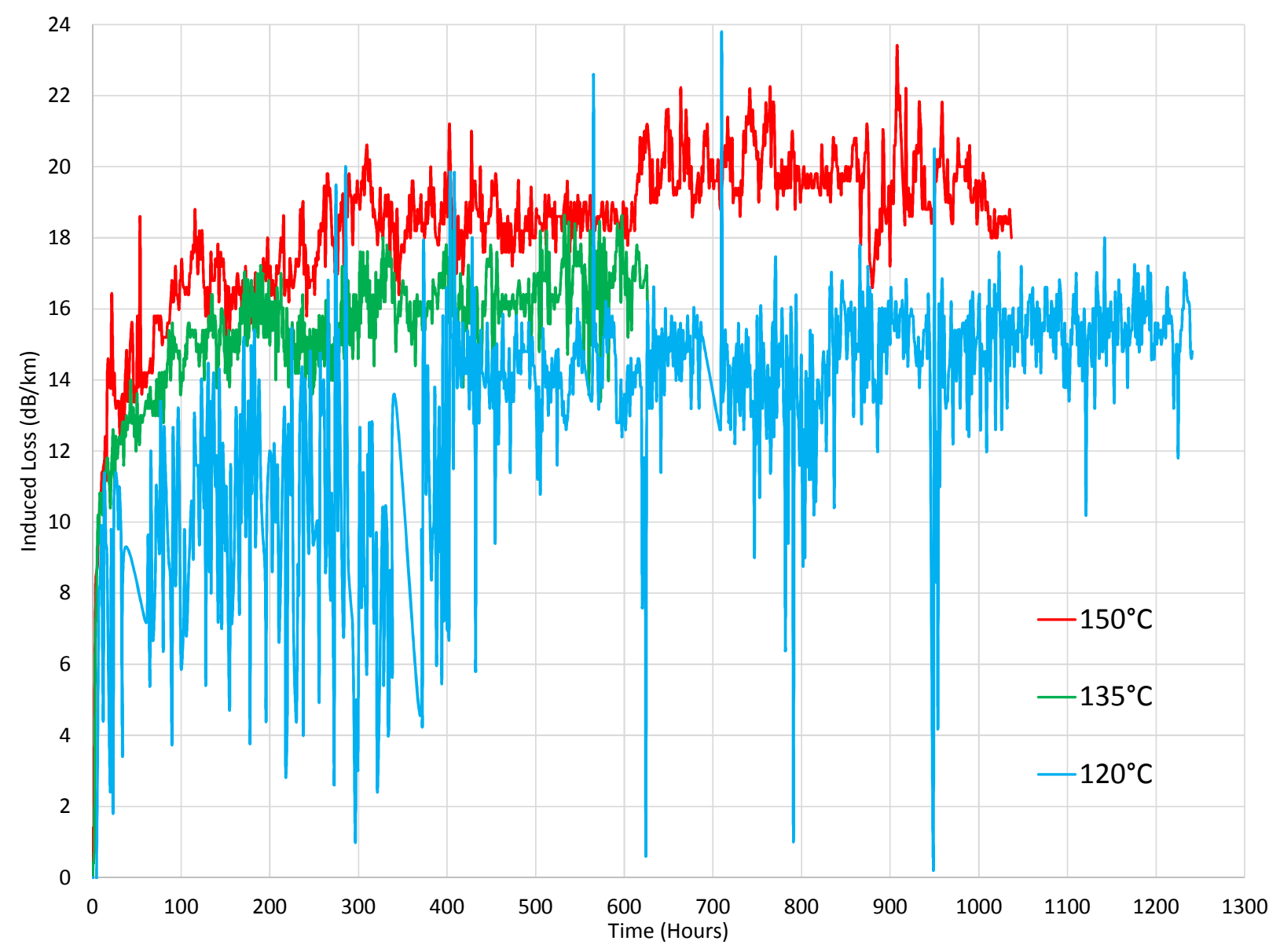

Figure 4. Induced loss @ 1064nm of 3 non carbon-coated VHM2000 fibers, exposed to 84.7atm pure $\mathrm{H}_{2}$ at different temperatures

It can be seen that the losses at $1064 \mathrm{~nm}$ without carbon are still significant, even though $1064 \mathrm{~nm}$ is a preferred wavelength of operation in $\mathrm{H}_{2}$ environments. It can also be seen that the maximum loss appears to stabilize, so a maximum loss at $1064 \mathrm{~nm}$ can be determined. Figure 5 shows the effect of $\mathrm{H}_{2}$ on carbon-coated VHM2000 fibers, at $1064 \mathrm{~nm}$. 


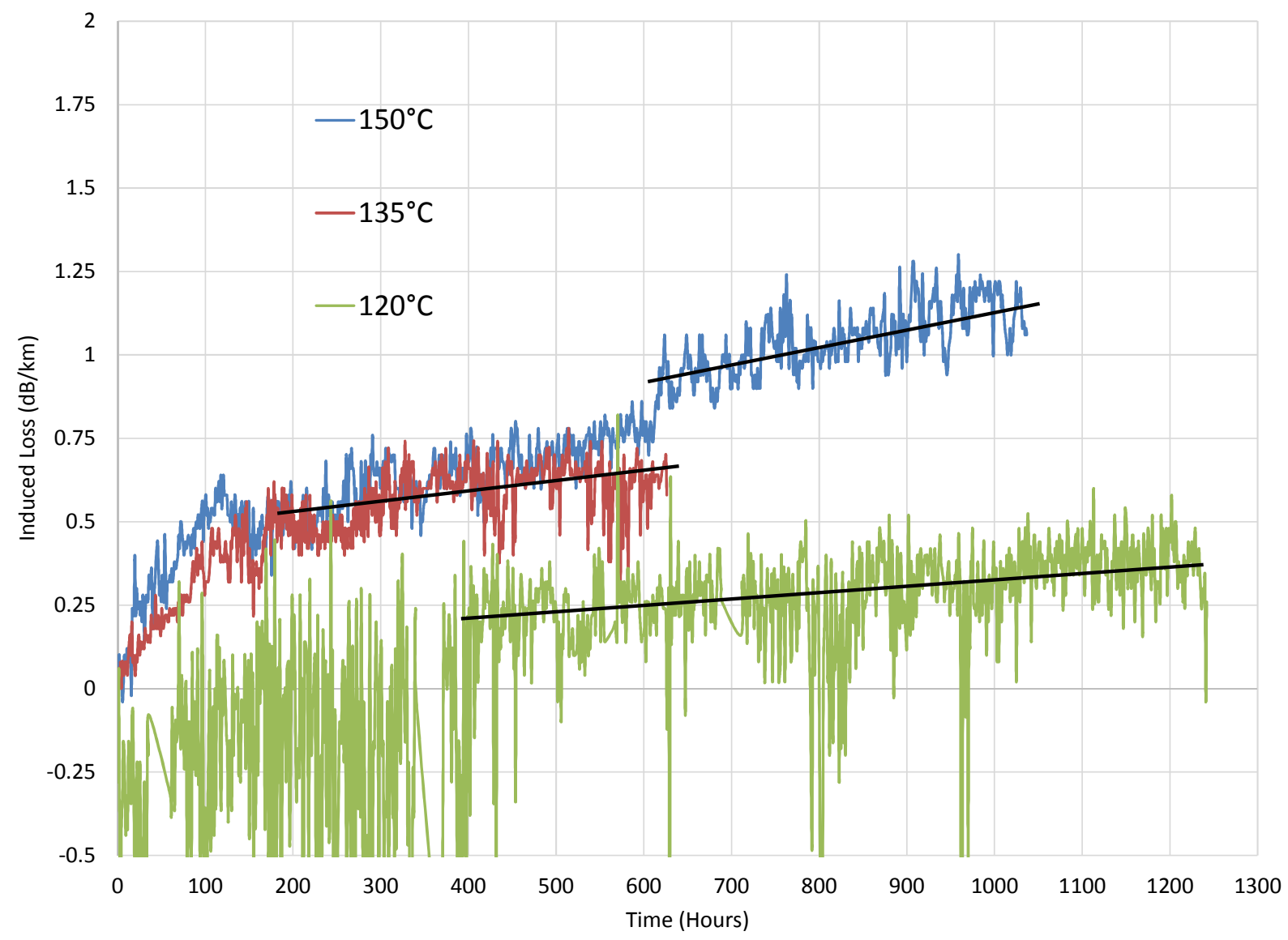

Figure 5. Induced loss @ 1064nm of 3 carbon-coated VHM2000 fibers, exposed to 84.7atm pure $\mathrm{H}_{2}$ at different temperatures

It can be seen that, in at least two of the three tests, the loss at $1064 \mathrm{~nm}$ is slowly increasing over time. Given that the maximum loss is known, the time it takes to reach maximum loss can be calculated. Table 2 shows these results.

\begin{tabular}{|c|c|c|c|}
\hline Temperature & Slope - Induced Loss / Time & Max Induced Loss & $\sim$ Time To Max Loss \\
\hline $120^{\circ} \mathrm{C}$ & $0.00025 \mathrm{~dB} / \mathrm{km} / \mathrm{hr}$ & $16 \mathrm{~dB} / \mathrm{km}$ & 7.5 years \\
\hline $135^{\circ} \mathrm{C}$ & $0.00037 \mathrm{~dB} / \mathrm{km} / \mathrm{hr}$ & $17 \mathrm{~dB} / \mathrm{km}$ & 5.1 years \\
\hline $150^{\circ} \mathrm{C}$ & $0.000584 \mathrm{~dB} / \mathrm{km} / \mathrm{hr}$ & $19.5 \mathrm{~dB} / \mathrm{km}$ & 3.7 years \\
\hline
\end{tabular}

Table 2: Slope and time to maximum loss calculations for carbon-coated VHM2000 fibers, exposed to 84.7atm pure $\mathrm{H}_{2}$ at different temperatures, $1064 \mathrm{~nm}$

The results seen at $1064 \mathrm{~nm}$ show a much different result versus performance at $1244 \mathrm{~nm}$. For example, a DTS measurement device has a dynamic range at $1064 \mathrm{~nm}$ of $14 \mathrm{~dB}$. If $2 \mathrm{~km}$ of VHM2000 is deployed in a $120^{\circ} \mathrm{C}, 84.7 \mathrm{~atm}$ pure $\mathrm{H}_{2}$ condition, it would function properly for about 3.25 years before it would be unable to take measurements. The previous 'Fiber Lifetime' calculation, using $1244 \mathrm{~nm}$ to show $\mathrm{H}_{2}$ ingression, was 147 years. 
The loss at $1244 \mathrm{~nm}$, therefore, is more of an indicator of the presence of $\mathrm{H}_{2}$ as opposed to a predictor of fiber performance.

\section{Conclusions}

Germanium-doped multimode fiber (VHM2000), with and without carbon, was exposed to 84.7atm of pure $\mathrm{H}_{2}$ at three different temperatures. Slope $\left(1 / \tau_{\mathrm{f}}\right)$ and fiber lifetime calculations were derived from the induced loss at $1244 \mathrm{~nm}$ (' $\mathrm{H}_{2}$ Peak'). These results were examined by evaluating fiber performance at $1064 \mathrm{~nm}$; induced loss at $1064 \mathrm{~nm}$ caused by the reaction of $\mathrm{H}_{2}$ with $\mathrm{GeO}$ defects was shown to have a more profound impact on fiber lifetime. Carbon is an effective method of preventing $\mathrm{H}_{2}$ ingression into glass; the conditions the fibers were exposed to were intentionally harsh in order to get readings within a reasonable time frame. This study used an accelerated aging process and is not indicative of real-life conditions due to the excessive partial pressure of $\mathrm{H}_{2}$ used.

\section{References}

[1] Lemaire, PJ, Lindholm, EA, "Hermetic Optical Fibers: Carbon-Coated Fibers," Specialty Optical Fibers Handbook, Academic Press, 453-490 (2007).

[2] LeMaire P.J., Kranz, S., Walker, K. L., Huff, G., DiMarcello, F. V., “ Hydrogen permeation in optical fibers with hermetic carbon coatings," Electron Lett., vol. 24, 1323-1324 (1988).

[3] Wang, C., Drenzek, G., Majid, I., Wei, K., Bolte, D., Soufiane, A. "High-Performance Hermetic Optical Fiber for Downhole Applications." SPE91042, ,(2004). 


\title{
Photonic Lanterns: Beyond Telecoms
}

\author{
Stephanos Yerolatsitis*a, Aurélien Benoît ${ }^{\mathrm{b}}$, Kerrianne Harrington ${ }^{\mathrm{a}}$, Robert R. Thomson ${ }^{\mathrm{b}}$, Tim A. \\ Birks $^{\mathrm{a}}$ \\ aDepartment of Physics, University of Bath, Claverton Down, Bath BA2 7AY, UK \\ ${ }^{b}$ SUPA, Institute of Photonics and Quantum Sciences, Heriot-Watt University, Edinburgh, EH14 \\ $4 \mathrm{AS}, \mathrm{UK}$
}

\begin{abstract}
Photonic lanterns, applied in areas such as telecommunications, astronomical and biomedical instrumentation, provide a way to bridge multimode and multicore channels. Here, we discuss the fabrication and potential uses of a six-mode modeselective photonic lantern.
\end{abstract}

Keywords: fibre post-processing, mode-selective photonic lanterns

*s.yerolatsitis@bath.ac.uk

\section{Introduction}

Recent interest in space division multiplexing has led to the development of mode selective photonic lanterns as a way to couple light from several separate channels into the modes of a multimode fibre, or vice versa $[1,2]$. They therefore act as a bridge between two regimes: the multicore and multimode. The simplest version of a mode selective photonic lantern can be fabricated using three dissimilar core fibres. The fibres are threaded through a fluorine-doped silica capillary, and heated and stretched together, forming a fused multimode core. The guided mode of each dissimilar core has a different propagation constant. Therefore, in an adiabatic transition, light in each input core evolves into just one of the modes of the fused output core in order of its respective propagation constant [2].

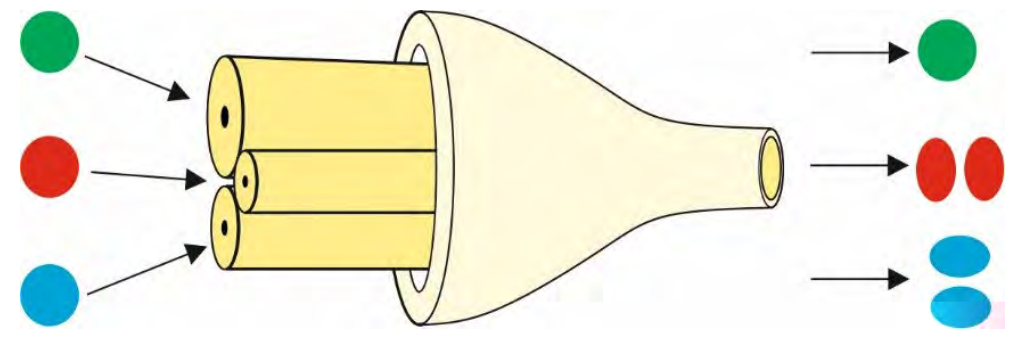

Figure 1. Schematic mode-selective photonic lantern made by fusing and tapering three dissimilar SMFs in an F-doped capillary.

\section{Higher-order mode-selective photonic lanterns}

For mode-selective photonic lanterns the adiabaticity of the transition is critical. In order to increase the number of multiplex modes, either the transition length needs to be increased or the cladding diameter of each fibre needs to be reduced [2,3]. As fibres with reduced cladding diameter are hard to handle, multicore fibres with dissimilar cores can be used instead [3]. 
(a)

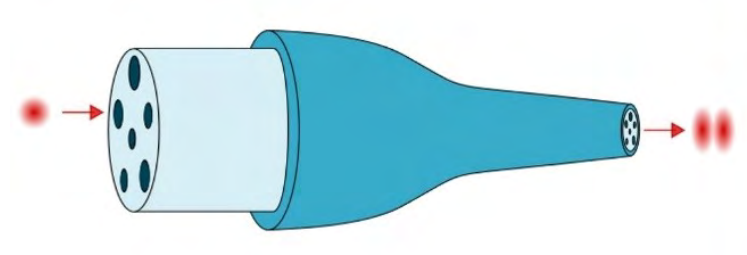

(b)

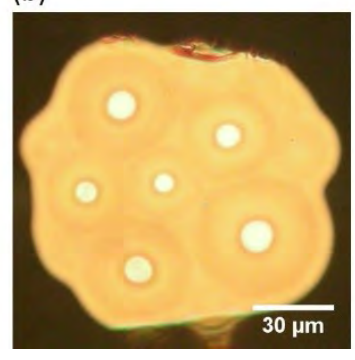

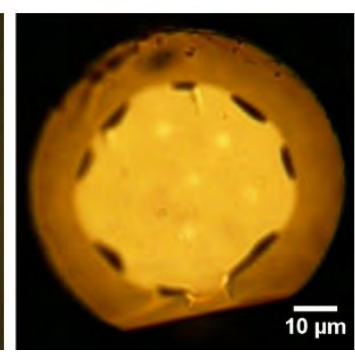

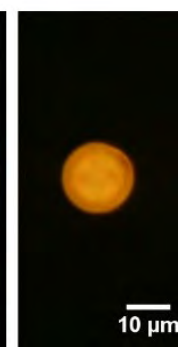

Figure 2. (a) Schematic mode-selective photonic lantern made by tapering a multicore fibre with dissimilar cores in a fluorine-doped jacket. (b) Cross-sectional micrographs of (left) the 6-core fibre, (centre) a point partway down the photonic lantern's taper transition and (right) the lantern's multimode output.

We fabricated a multicore fibre with 6 dissimilar Ge-doped step-index cores $(\mathrm{NA}=0.11)$, Figure 2(b). The core diameters $(11,10.3,9.5,8.3,7.3$ and $6.5 \mu \mathrm{m})$ were chosen to maximise their dissimilarity, with the largest core to be small enough single moded at $1550 \mathrm{~nm}$, and the smallest core large enough to have tolerable bend loss. The minimum core-tocore separation was $26 \mu \mathrm{m}$, calculated to ensure $<0.1 \%$ crosstalk at $1550 \mathrm{~nm}$. A photonic lantern was made by tapering the fibre in a fluorine-doped capillary (numerical aperture NA $=0.22$ relative to undoped silica), cleaved to yield a 6-moded multimode core at $1550 \mathrm{~nm}$. The taper profile was linear and $3 \mathrm{~cm}$ long. The taper ratio of 0.09 ensured that the $\mathrm{V}$-values of the residual single mode cores were $\leq 0.22$ : too small to guide light. Light from a supercontinuum source was buttcoupled into each single mode core in turn from a small-core fibre. The near-field intensity distribution patterns in the multimode output for $1550 \mathrm{~nm}$ light, Figure. 3, resembled the pure LP modes expected from excitation in each core, confirming the mode-selective nature of the photonic lantern.

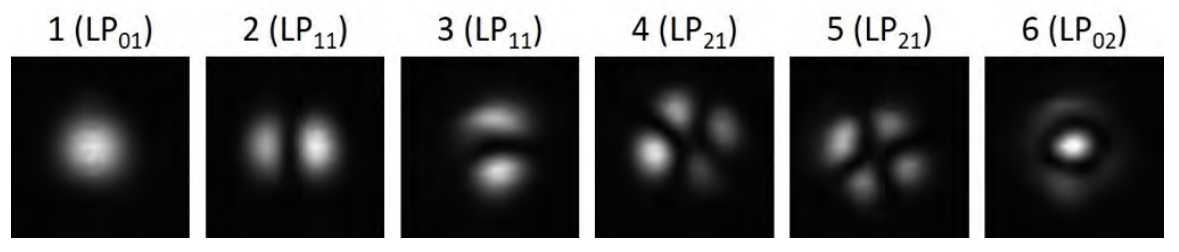

Figure 3. Near-field images of the output of a 6-mode photonic lantern for $1550 \mathrm{~nm}$ light in each input core in turn, the cores being numbered 1-6 in decreasing order of diameter with the expected mode in parentheses.

\section{From telecommunications to the stars}

Although mode-selective photonic lanterns have been developed for telecommunication applications, the first photonic lantern was actually designed and fabricated for astronomical instrumentation [1]. In similar manner, mode-selective photonic lanterns can be used in a variety of applications such as biomedical applications and astronomical instrumentation. For example, in astronomical instrumentation, multimode fibre fed spectrographs are used to collect light from hundreds of objects across the telescope focal plane [4]. A limiting factor for these spectrographs is the focal-ratio-degradation [5], phenomenon resulting of mode coupling along the fibre. Due to energy transfer by mode coupling from lower-order to higher order modes, the light that exits a multimode fibre has a lower focal-ratio (or higher numerical aperture N.A.) than the input light. As a result, the spectrograph must be larger to accommodate the faster beam with increasing the cost of the optics. In a complete mode-selective photonic lantern device (MSPL device, based on a multicore fibre with two adiabatic transitions at each end), the light is guided in the multicore region for most of its length and so exhibits minimal coupling. To obtain a fair comparison, the FRD behaviour is also investigated on a 6-mode step-index multimode fibre with the same fibre length of $7 \mathrm{~m}$. 
(a)

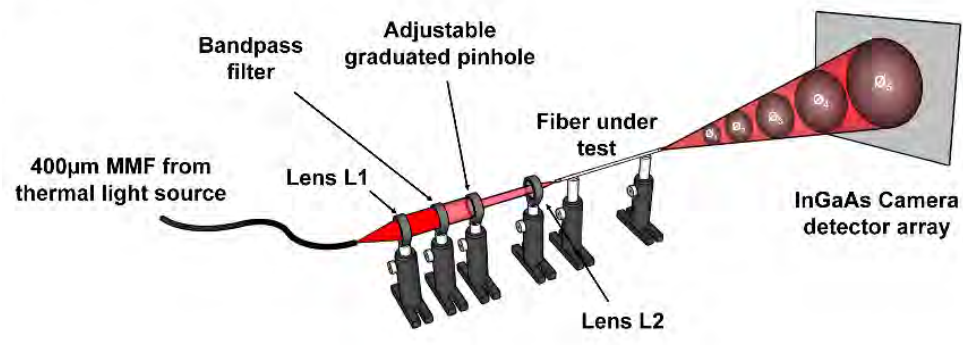

(b)

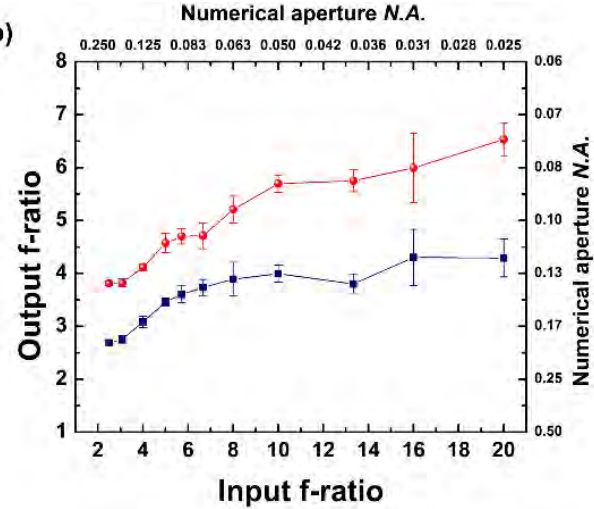

Figure 4. (a) Schematic of the FRD measurement experimental setup. (b) Output f-ratio versus input f-ratio measured for the 6-mode step-index fibre (blue squares) and the 6-mode MSPL device (red points). Points are an average of five measurements with the maximum and minimum values measured represented by the error bars.

To characterise the FRD of a multimode fibre and the MSPL device, the experimental setup as shown in Figure 4(a) was built. A thermal incoherent light source was collimated, and passed through a bandpass filter (1550 $\mathrm{nm} \pm 20 \mathrm{~nm})$. An adjustable graduated pinhole was used to control the input f-ratio. The f-ratio of the light exiting from the output of the device was measured using an InGaAs camera placed on a translation stage and a virtual knife edge technique. Figure 4(b) presents the evolution of the output f-ratio as a function of the input f-ratio for a 6-mode step-index fibre and a 6-moded MSPL device, measured at $95 \%$ of the beam size. The use of mode-selective photonic lanterns clearly demonstrates, for the first time, a way to control and mitigate FRD in fibre-fed spectrographs [6].

This is one example of the use of mode-selective photonic lanterns outside of telecommunications. We envisage many other further applications including sensing and biomedical instrumentation.

\section{References}

[1] Birks, T. A., et al. "The photonic lantern." Advances in Optics and Photonics 7(2), 107-167 (2015)

[2] Yerolatsitis, S., et al. "Adiabatically-tapered fiber mode multiplexers." Optics Express 22(1), 608-617 (2014)

[3] Yerolatsitis, S., et al. "Mode-selective Photonic Lanterns from Multicore Fibres. " in Optical Fiber Communication Conference, Los Angeles, California, paper Tu3J.6 (2017).

[4] Colless, M., et al. "The 2dFgalaxy redshift survey: spectra and redshifts." Monthly Notices of the Astronomical Society 328, 1039-1063 (2001)

[5] Carrasco, E., et al., "A method for determining the focal ratio degradation of optical fibers for astronomy." Monthly Notices of the Astronomical Society 271, 1-12 (1994)

[6] Benoit, A., et al. "A focal-ratio-degradation (FRD) resistant multimode fibre link using mode-selective photonic lanterns" in Conference on Lasers and Electro-Optics Europe (CLEO Europe), paper CJ-14.6 (2019) 
"History and Advancements of FBG manufacturing in specialty optical fibers and their use in sensing"

Eric Linder, FBGS Technologies, GmbH (Germany)

The discovery of a periodic refractive index change after laser laser exposure in optical fibers dates back more than 40 years. These later on called "Fiber Bragg Gratings" (FBGs) were finding their use in into many telecommunication and sensing applications. The talk will review historic and latest advancements of FBG manufacturing in specialty optical fibers and their use in sensing technology. Furthermore we will give an outlook on future sensing applications using specialty optical fibers. 


\title{
Wedge-shaped fiber lenses with $\mathrm{CO}_{2}$ Laser glass ablation
}

\author{
Gongwen Zhu* and Wenxin Zheng
}

\author{
AFL, 260 Parkway East, Duncan, SC, USA 29334
}

\begin{abstract}
We present a novel method of fabricating wedge-shaped fiber lenses with $\mathrm{CO}_{2}$ laser glass ablation. Compared with conventional method of fiber polishing, $\mathrm{CO}_{2}$ laser glass ablation is a chemical free, contamination-free, noncontact manufacturing process which provides scratch-free microstructured fiber tips.
\end{abstract}

Keywords: Lensed fiber, $\mathrm{CO}_{2}$ laser ablation, Microstructured fibers, optical coupling

*Gongwen.Zhu@aflglobal.com; phone 1864 486-7099

\section{Introduction}

Wedge-shaped fiber lenses are widely used in a number of applications such optical coupling [1-2], fiber sensors and illumination, etc. Conventionally wedge-shaped fiber lenses are fabricated by fiber polishing which may introduce contamination or involve using hazardous chemicals. $\mathrm{CO}_{2}$ laser glass ablation, on the other hand, is a chemical free, contamination-free, noncontact manufacturing process. In addition to the glass ablation, $\mathrm{CO}_{2}$ laser can also polish the fiber tips to efficiently reduce the scattered light. In this paper we present wedge-shaped fiber tips with $\mathrm{CO}_{2}$ laser glass ablation using LZM-120A+ glass processing system.

\section{Fabrication}

The schematic of $\mathrm{CO}_{2}$ laser glass ablation in LZM-120A+ is shown in figure 1. The $\mathrm{CO}_{2}$ laser beam is first expanded to about $10 \mathrm{~mm}$, then focused to a small spot size with a diameter of roughly $60 \mu \mathrm{m}$. The focused laser beam will provide enough power density to directly ablate the silica glass. The optical fiber can rotate continuously and move in the longitudinal direction (parallel to the fiber) as well. The $\mathrm{CO}_{2}$ laser beam can move both towards and perpendicular to the fiber, thus creating fiber tips with different shapes. The movement of the fiber and $\mathrm{CO}_{2}$ laser beam, and the lasing time can all be precisely controlled.

The fabrication of wedge-shaped fiber lenses includes the following steps. This is a contamination-free process which does not involve using any hazardous chemicals.

1) Prepare a cleaved fiber and adjust the $\mathrm{CO}_{2}$ laser beam position so it focuses on the fiber. The focus adjustment can be automated in LZM-120A+.

2) Move the fiber to a predefined position, transversally (perpendicular to the fiber) scan the fiber with the focused $\mathrm{CO}_{2}$ laser beam. The focused beam have enough power density to ablate the silica glass.

3) Move the fiber to a new position, transversally scan the fiber with the focused $\mathrm{CO}_{2}$ laser beam. Then move $\mathrm{CO}_{2}$ laser beam closer to the fiber and scan the fiber again. Repeat step 3) until desired shape is achieved.

4) Rotate the fiber $180^{\circ}$ and repeat step 2) and 3) to process the other side of the fiber.

5) Repeat 2) to 4) if necessary to get a smooth the fiber tip when necessary.

Sixth International Workshop on Specialty Optical Fibers and Their Applications (WSOF 2019),

edited by Liang Dong, John M. Ballato, Proc. of SPIE Vol. 11206, 112060K · (C) 2019 SPIE

CCC code: $0277-786 \mathrm{X} / 19 / \$ 21 \cdot$ doi: $10.1117 / 12.2543425$ 


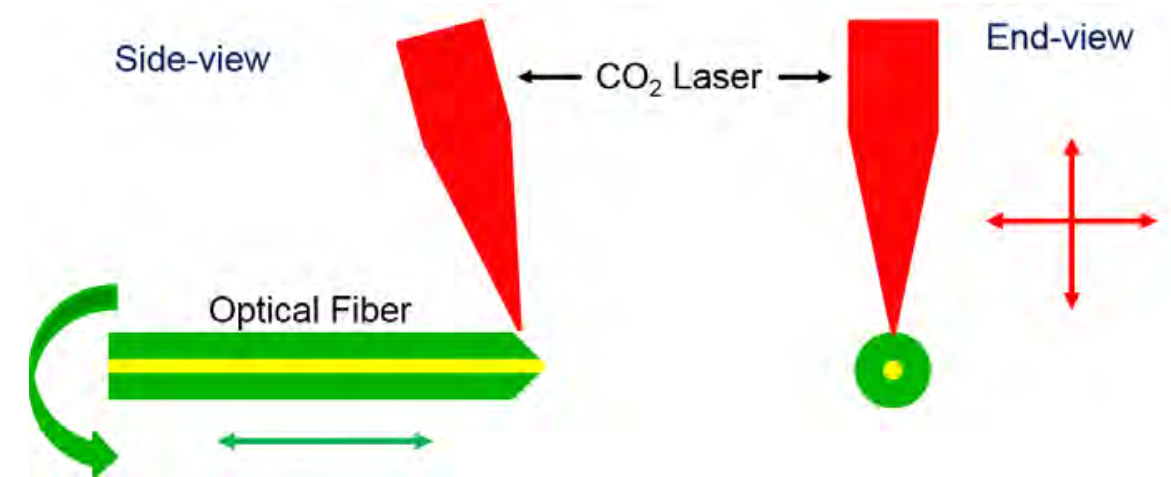

Figure 1. The schematic of wedge-shaped fiber lenses based on $\mathrm{CO}_{2}$ laser glass ablation. The green arrows show the rotating and moving directions of the optical fiber; and the red arrows represent the moving directions of the $\mathrm{CO}_{2}$ laser beam.

\section{Results}

A roughly $90^{\circ}$ wedge-shaped fiber lens is fabricated with the described method and the results are shown in Figure 2 . The images are taken in two perpendicular directions with the splicer and a microscope respectively. The output beam profile is elliptical as shown in Figure 2(e). The measured aspect ratio is about 1:4. Other aspect ratios can also be achieved by precisely controlling the movement of the fiber and $\mathrm{CO}_{2}$ laser beam, and fine tuning the process. The wedge-shaped fiber lenses are ideal for coupling light from laser diodes to single-mode fibers.
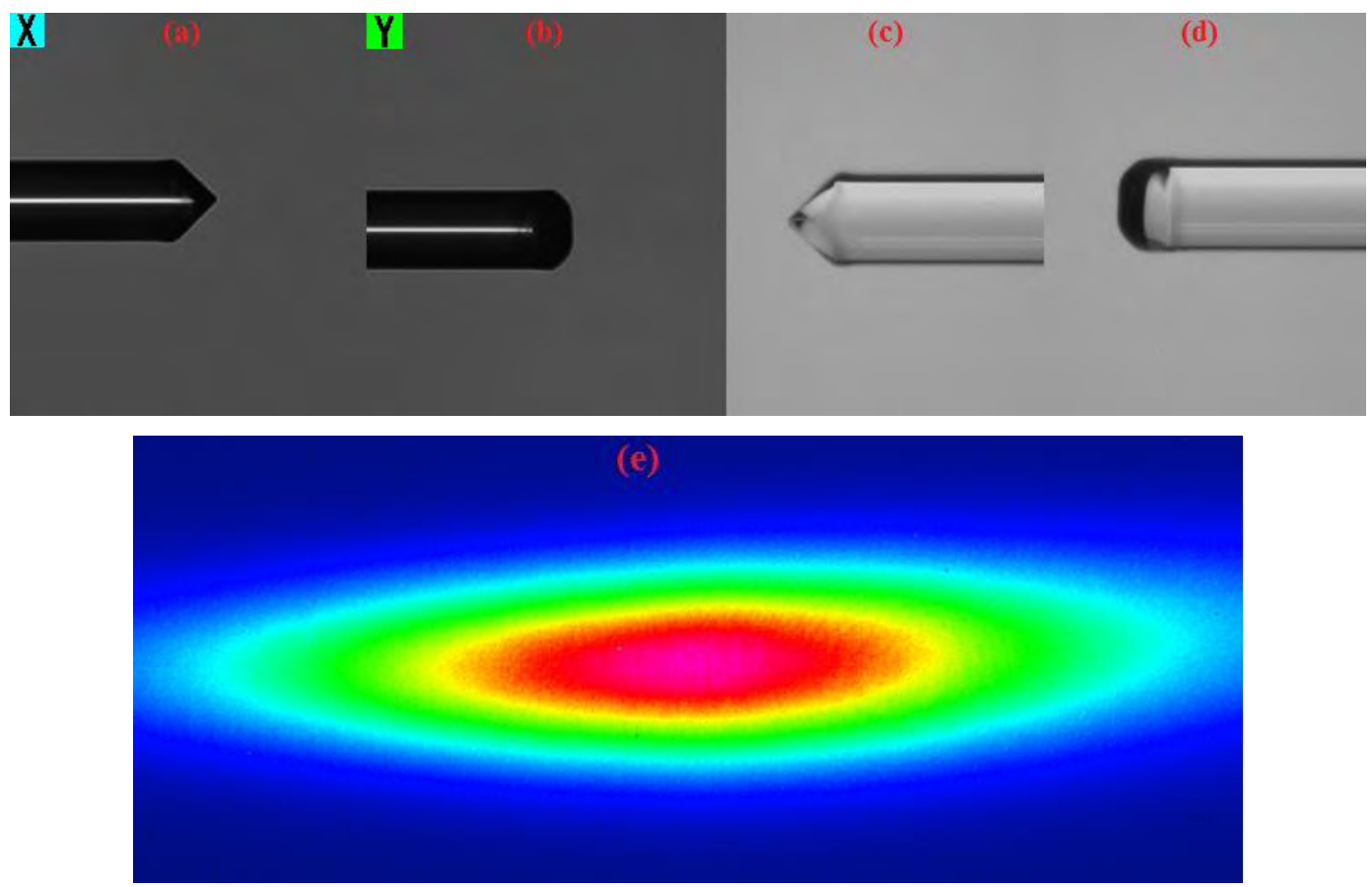

Figure 2. (a) \& (b) The images of the wedge-shaped fiber lens in the splicer; (c) \& (d) the images in the microscope; (e) the beam profile taken with a Gentec camera. 


\section{Conclusion}

In this paper we present a simple method of fabricating wedge-shaped fiber lenses based on $\mathrm{CO}_{2}$ laser glass ablation with a commercially available glass processing system LZM-120A+. This non-contaminating and chemical-free process is ideal for fabricating many microstructured fiber optic components.

\section{Reference}

[1] He, Y., Mondal, S. K., and Shi, F. G., "Design optimization of wedge-shaped lensed fibers for fiber-laser coupling: fresnel reflection and non-Gaussian mode effects,” IEEE J. Light. Technol. 21(10), 2271-2275 (2003).

[2] Yoda, H. and Shiraishi, K., "A new scheme of a lensed fiber employing a wedge-shaped graded-index fiber tip for the coupling between high-power laser diodes and single-mode fibers," IEEE J. Light. Technol. 19(12), 1910-1917 (2001). 


\title{
Femtosecond laser inscribed gratings for monolithic MIR fiber lasers
}

\author{
Antreas Theodosiou* ${ }^{* a, b}$, Jan Aubrecht ${ }^{\mathrm{c}}$, Nithyanandan Kanagaraj ${ }^{\mathrm{c}}$, Pavel Peterka ${ }^{\mathrm{c}}$, Pavel Honzátko ${ }^{\mathrm{c}}$, \\ Andreas Stassis ${ }^{\mathrm{d}}$, Kyriacos Kalli*a \\ aPhotonics \& Optical Sensors Research Laboratory, Cyprus University of Technology, Nicolaou \\ Saripolou 33, 3036, Limassol, Cyprus \\ ${ }^{\mathrm{b}}$ Lumoscribe LTD, Margatitas Liasidou 12, 8036, Paphos Cyprus \\ ${ }^{\mathrm{c}}$ Institute of Photonics and Electronics of the CAS, v.v.i., Chaberská 57, 18251 Prague, Czech \\ Republic \\ ${ }^{\mathrm{d}}$ Department of Mechanical Engineering Cyprus University of Technology Nicolaou, Kitiou \\ Kiprianou 45, 3041 Limassol, Cyprus
}

\begin{abstract}
We present our recent developments regarding monolithic fibre lasers, incorporating gratings as reflectors and polarizing elements via the plane-by-plane femtosecond laser inscription method. Designs are considered for continuous and pulsed laser operation in the mid-infrared wavelength range.

Keywords: monolithic fibre lasers, mode-locked lasers, femtosecond laser, Bragg gratings

*theodosiou.antreas@lumoscribe.com; kyriacos.kalli@cut.ac.cy
\end{abstract}

\section{Introduction}

The advent of fibre lasers is undoubtedly the most important recent laser development. There are numerous advantages on offer that make fibre lasers increasingly attractive, such as their high efficiency, excellent output beam quality, compactness, low maintenance requirements, efficient cooling and spectral adaptability [1]. A more sophisticated design for fibre lasers is the incorporation of the resonator mirrors, such as fibre Bragg gratings (FBGs) written directly into the active fibre. In this way, the laser can be potentially improved in terms of durability, as there are no splices to the FBGs that would otherwise result in device failure under high optical pump and/or laser signal power. Currently, significant interest is devoted to fibre lasers operating $\sim 2 \mu \mathrm{m}$ and further in the mid-infrared (MIR) wavelengths. In this paper, we present our recent results regarding the inscription of uniform Bragg gratings in Thulium doped fibre (TDF) for the demonstration of monolithic fibre lasers, and the inscription of a $45^{\circ}$ degree tilted FBG (TFBG) for mode locked MIR fibre lasers. All components are developed using the femtosecond laser, plane-by-plane (pl-by-pl) inscription method.

\section{Inscription and Results}

For the continuous wave laser, a high reflectivity FBG ( $>95.5 \%)$ was inscribed directly into the Tm-doped single mode fibre (fabricated in-house: $3500 \mathrm{ppm}$ of $\mathrm{Tm}^{3+}$ and $4.5 \mathrm{~mol} \%$ of $\mathrm{Al}_{2} \mathrm{O}_{3}$ ), using the plane-by-plane femtosecond laser inscription method [2,3] and we characterized the in-fibre laser configuration in terms of the power slope efficiency (PSE). The laser cavity was formed using the high reflector FBG (HR-FBG) and the perpendicularly cleaved active fibre at the output end, to produce a low reflectivity mirror through the Fresnel reflection (Fig. 1a). The initial length between the HR-FBG and the fibre end was $\sim 4$ metres and was gradually shortened; the PSE was measured for fibre lengths from 3.8 to $0.42 \mathrm{~m}$. The laser lines for individual fibre lengths are shown in Fig. 1b. The PSE varied from 53 to $58 \%$; this is an improvement over previously demonstrated fibre lasers using FBGs in passive fibres.

A $45^{\circ}$ TFBG was inscribed in order to perform sufficient selective polarisation dependent loss in a 9.3-m long all-fibre TDF laser for a mode-locking. The TFBG was inscribed with total length $\sim 10 \mathrm{~mm}$ and a grating period of $\sim 2.69 \mu \mathrm{m}$ with

Sixth International Workshop on Specialty Optical Fibers and Their Applications (WSOF 2019),

edited by Liang Dong, John M. Ballato, Proc. of SPIE Vol. 11206, 112060L · C 2019 SPIE

CCC code: $0277-786 X / 19 / \$ 21 \cdot$ doi: $10.1117 / 12.2548293$ 
PDL $\sim 5 \mathrm{~dB}$ (Fig. 2a). The TFBG was fusion spliced with a TDF as shown in Fig. $2 b$. The experimental setup for the demonstration of the mode locked laser shown in Fig. 2b. The optical spectrum of the laser was centred at $1875 \mathrm{~nm}$ with a FWHM of $5.5 \mathrm{~nm}$ (Fig. 2c), while the signal to noise ratio was $>45 \mathrm{~dB}$ (Fig. 2d) with very high stability for room temperature operation. The autocorrelation trace of the mode-locked pulse train corresponding to pulse duration of $862 \mathrm{fs}$ and the RF spectrum at the fundamental frequency was found to be $22.34 \mathrm{MHz}$.
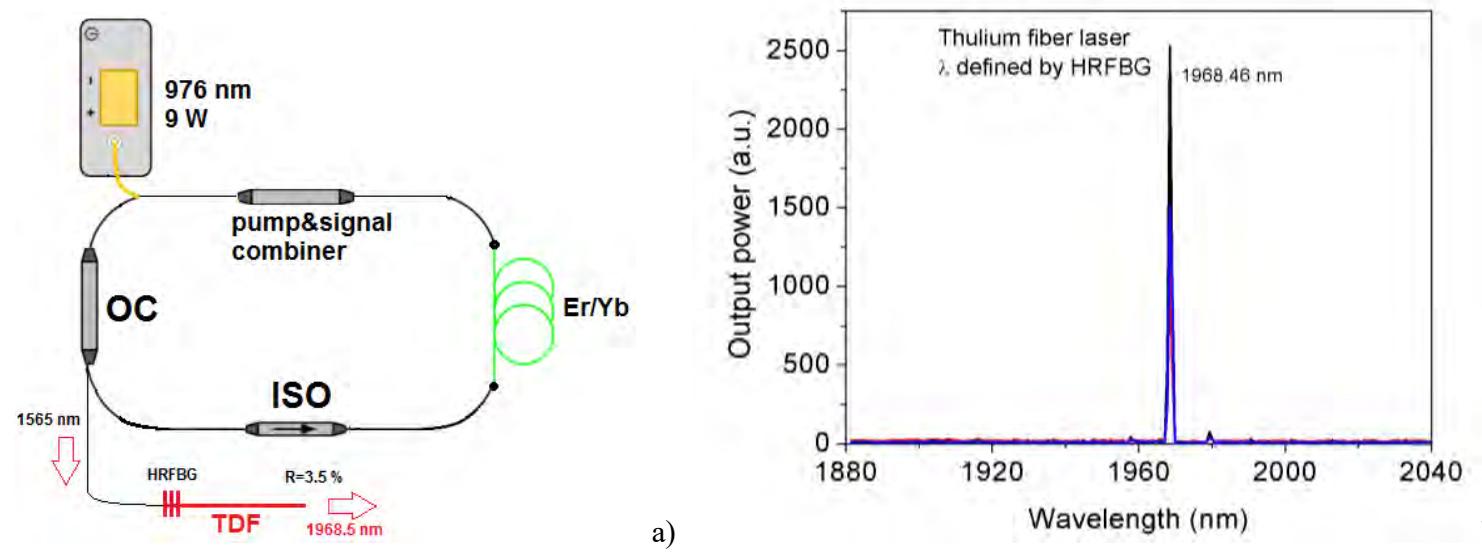

Figure 1. a) Schematic diagram of the experimental setup, and b) output of Tm fibre laser.
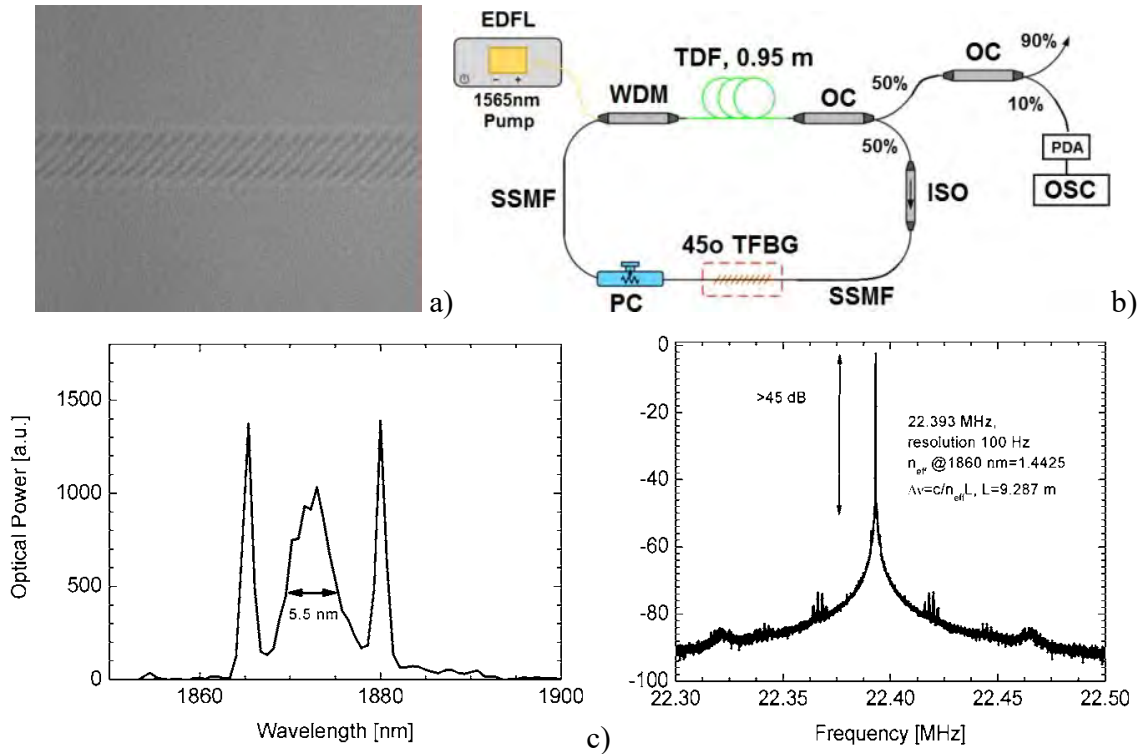

b)

Figure. 2. a) Microscope picture of the TFBG inscribed using the pl-by-pl inscription method, b) schematic diagram of mode locked fibre laser system. c) Optical spectrum of the laser output and d) RF spectrum at $100 \mathrm{~Hz}$ resolution.

\section{References}

[1] Zervas, M and Codemard, C, "High Power Fiber Lasers: A Review," IEEE J. Selec. Topics in Quant. Electr. 20(5), 219-241, (2014).

[2] Theodosiou, A., Aubrecht, J., Peterka, P., Kasik, I., Todorov, F., Moravec, O., Honzatko, P., Kalli, K., "Er/Yb Double-Clad Fiber Laser With fs-Laser Inscribed Plane-by-Plane Chirped FBG Laser Mirrors", IEEE Phot. Techn. Lett., 31(5), 409-412 (2019)

[3] Theodosiou, A., Aubrecht, J., Peterka, P., and Kalli, K., "Femtosecond laser plane-by-plane Bragg gratings for monolithic Thulium-doped fibre laser operating at $1970 \mathrm{~nm}$ ”, Proc. SPIE, 11029, 110290F (2019). 


\title{
Fibre cladding filters through femtosecond laser inscription
}

\author{
K. Kalli ${ }^{\text {* }}$, A. Theodosiou ${ }^{\mathrm{a}}$, A. Ioannou ${ }^{\mathrm{a}, \mathrm{b}}$ \\ ${ }^{\mathrm{a}}$ Photonics and Optical Sensors Research Laboratory, Cyprus University of Technology, Limassol, \\ Cyprus; ${ }^{b}$ University of Mons, 7000, Belgium
}

\begin{abstract}
We inscribe several in-cladding-fibre filters using the same key femtosecond laser parameters, via an "inscribe and step", plane-by-plane (Pl-by-Pl) approach, leading to ultra-compact waveguides and Mach Zehnders (MZs) that can support functional, integrated fibre Bragg gratings (FBGs).

Keywords: Femtosecond laser inscription, fibre Bragg gratings, optical sensing *kyriacos.kalli@cut.ac.cy; phone 35725002609
\end{abstract}

\section{Introduction}

We use a femtosecond (fs) laser and single inscription recipe to simply create sensors located in the cladding of optical fibres, such as cladding waveguides (CWGs), Mach-Zehnder interferometers (MZIs), both with integrated fibre Bragg gratings (FBGs) as embedded waveguide Bragg gratings (WBGs), via the "inscribe and step", plane-by-plane (Pl-by-Pl) inscription approach [1-3]. We overcome a significant impediment to fs-laser inscription of optical components; namely that waveguides and reflective components and combinations thereof require significantly different laser processing parameters during fabrication. For example, waveguides demand high repetition rate $(100 \mathrm{kHz}-\mathrm{MHz})$ inscription at low laser pulse energies ( 100-250nJ/pulse), whereas point-by-point ( $\mathrm{PbP})$ fibre gratings require low repetition rates $(\mathrm{kHz})$ and relatively high pulse energies ( $>500 \mathrm{~nJ} /$ pulse) for inscription. Both aforementioned cases are dependent on different laser focusing conditions, and the numerical aperture of the final lens greatly affects the energy deposition for material change. To resolve this issue we have developed a method that uses the same key laser-inscription parameters made applicable to any type of miniature optical component, for which the focusing conditions and final inscription lens are common throughout, ensuring reliability and repetition in component development. We show that the compound sensors maintain their unique sensing capabilities, and that the sensor response can be tailored, e.g. for bend sensing.

\section{Results}

All inscriptions were performed using a fs laser (HighQ laser femtoREGEN, $\lambda=517 \mathrm{~nm}$, pulse duration=220 fs, repetition rate: $1-100 \mathrm{kHz}$ ), for a pulse energy of $110 \mathrm{~nJ}$. The un-stripped fibres were mounted on an air-bearing translation system (Aerotech) for accurate two-axis motion; synchronization of the laser pulse and stage motion proved necessary for laser processing of the cladding waveguide (straight and curved) and the FBG. We increased or decreased the number of pulses/ $\mu \mathrm{m}$ according to which component was required, using the direct-write, plane-by-plane ( $P l-b y-P l)$, "inscribe and step" method was used to build all components in a piece-wise process [1-3], Fig. 1a.

Sixth International Workshop on Specialty Optical Fibers and Their Applications (WSOF 2019),

edited by Liang Dong, John M. Ballato, Proc. of SPIE Vol. 11206, 112060M · (C) 2019 SPIE

CCC code: $0277-786 X / 19 / \$ 21 \cdot$ doi: $10.1117 / 12.2548477$ 


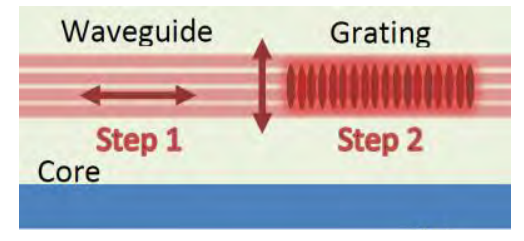

Fig. 1a. Step 1: "Inscribe and step" waveguide inscription in the fibre cladding. Step 2: Repeat for grating inscription on orthogonal axis.

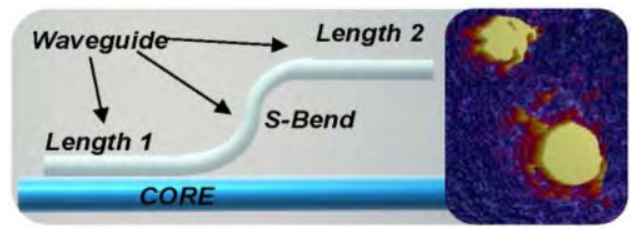

Fig. 1b. Waveguide design (left) in the fibre cladding. Output light profile of the waveguide, measured using a profilometer (right), with light connected to the distal end of the fibre sample.

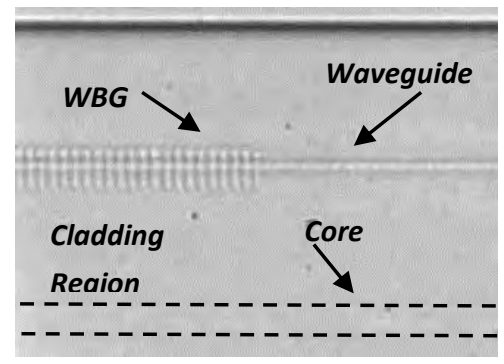

Fig. 1c. Microscope image of the waveguide integrated WBG.

The cladding waveguides run parallel to the core, exchanging power through evanescent wave coupling, thereby reducing loss and ensuring repeatability, Fig. 1b. The first and third sections are straight, the former coupling light from the core to the cladding and vice versa, and the latter section incorporates the FBG (WBG); they are joined by an S-bend that defines where the WBG will be located from the core region. The inscription procedure is repeated several times with sub-micron translation steps perpendicular to the fibre axis between each cladding waveguide portion, building the waveguide until the desired width is complete - an "inscribe and step" process (Fig. 1a). Fig. 1b shows light exiting the inscribed waveguide, obtained for a $250-\mu \mathrm{m}$ coupling length and $0.3-\mu \mathrm{m}$ translation step between each plane, respectively; for an index change of $4 \times 10^{-3}$. To inscribe the WBG, we used direct-write, $P l-b y-P l$ inscription [1-3], writing a uniform FBG transversely to the cladding waveguide as the second "inscribe and step" process (2000 planes were written with $\sim 2.18-\mu \mathrm{m}$ Bragg period for a $4^{\text {th }}$ order grating). Fig. 1c shows a microscope image of the resulting inscription. Fig. 2a shows the WBG growth in reflection during inscription for various grating lengths; the lower inset shows the final grating reflection spectrum, for a length of $4.5 \mathrm{~mm}$. The grating reflects at $1583.6 \mathrm{~nm}$ with a full width at half maximum bandwidth of $\sim 0.5 \mathrm{~nm}$. The WBG's effective refractive was $\mathrm{n}_{\mathrm{eff}}=1.4506$.

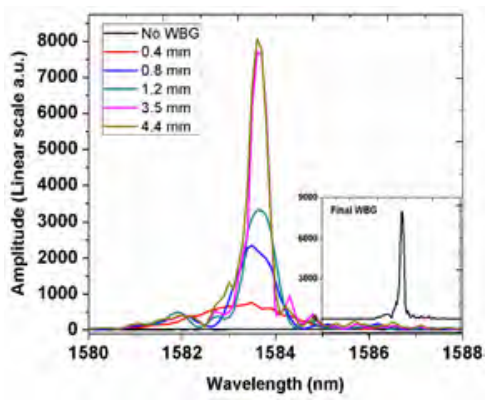

Fig. 2a. WBG growth with increasing length. Inset: Final WBG spectrum.

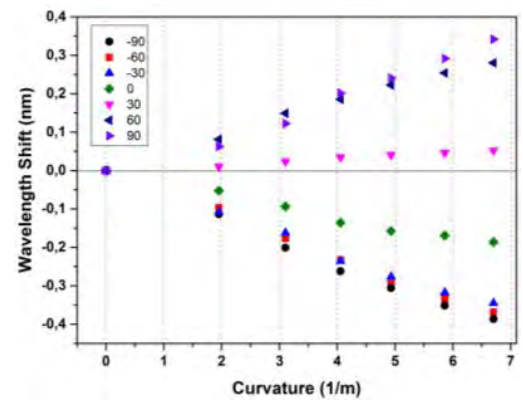

Fig $2 \mathrm{~b}$. Bend response with sensor rotation: WBG at $25 \mu \mathrm{m}$ from the core; sensitivities are $53 \mathrm{pm} / \mathrm{m}^{-1}$ and $-58.6 \mathrm{pm} / \mathrm{m}^{-1}$.

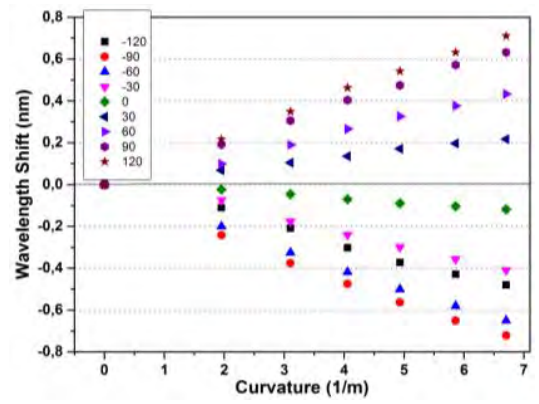

Fig. 2c. Bend response with sensor rotation: WBG at $45 \mu \mathrm{m}$ from the core; sensitivities are $106.6 \mathrm{pm} / \mathrm{m}^{-1}$ and $-107.6 \mathrm{pm} / \mathrm{m}^{-1}$.

Two similar CWG-WBG devices were inscribed and tested for their curvature response with respect to sensor rotation angle, for waveguides and WBGs located 25 and $45 \mu \mathrm{m}$ from the fibre core, respectively (Fig. 2b \& 2c). The WBG bend sensitivity is related to its angular position and location in the cladding and can be readily tailored. For the sensor located $25 \mu \mathrm{m}$ from the core, the maximum positive and negative bend sensitivities were $53 \mathrm{pm} / \mathrm{m}^{-1}$ and $-58.6 \mathrm{pm} / \mathrm{m}^{-1}$, respectively; the WBG closest to the cladding edge ( $45 \mu \mathrm{m}$ from the core) displays a greater sensitivity with maximum positive and negative values of $106.6 \mathrm{pm} / \mathrm{m}^{-1}$ and $-107.6 \mathrm{pm} / \mathrm{m}^{-1}$, respectively. This is maximised when the WBG lies along the vertical axis, breaking the fibre symmetry and thereby experiencing the largest degree of bend. 


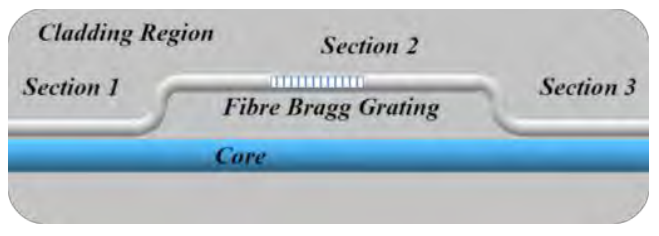

Fig. 3a. Schematic of the MZI-WBG structure; coupling from and to the fibre core is controlled in Sections 1 and 3, respectively.

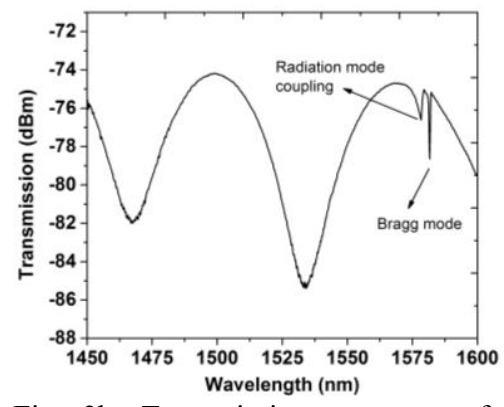

Fig. 3b. Transmission spectrum of composite MZI and WBG.

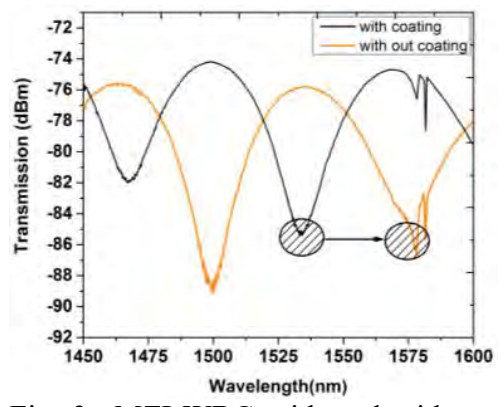

Fig. 3c MZI-WBG with and without the outer polymer jacket.

The MZI is similar to the CWG, and uses the same recipe, with the S-bend mirrored to recouple light back into the core, Fig. 3a, and Fig. 3b shows the transmission spectrum of the combined MZI with WBG. The WBG strength is tailored to match that of the MZI, so that both filters may be interrogated using the same high-resolution instrumentation. The compound sensor has the advantage that each element maintains its basic sensing characteristics, as each sensor operates in a fundamentally different way. When the outer coating of the fibre was removed the MZI transmission dips shift by $\sim 50 \mathrm{~nm}$, indicative of a refractive index sensitivity $\sim 107 \mathrm{~nm} / \mathrm{RIU}$; the wavelength shift of the WBG was only $0.2 \mathrm{~nm}$. To measure the MZI-WBG temperature and humidity response, the sensor was placed in a climate chamber. The wavelength shift of the MZI fringe located at $\sim 1530 \mathrm{~nm}$ gave a large negative wavelength shift of $-490 \mathrm{pm} /{ }^{\circ} \mathrm{C}$, whereas the $\mathrm{FBG}$ response was $\sim 10.5 \mathrm{pm} /{ }^{\circ} \mathrm{C}$, which is very similar to the response for an $\mathrm{FBG}$ inscribed in the fibre core.

We have presented fs-laser inscription applicable to critical filtering and wave-guiding components in optical fibres, e.g. cladding waveguides (CWGs) and interferometers (MZIs), incorporating embedded waveguide Bragg gratings (WBGs); all inscribed using the same key fs-laser parameters, via an "inscribe and step", $P l$-by-Pl approach. The allfibre, ultra-compact optical components proved to be robust sensing devices. The inscription process offers several advantages; ensuring a flexible, single inscription process, reliability and repetition in device manufacture. The method provides immense and control in the grating fabrication parameters and for the customization of a sensing device and its sensitivity, e.g. tailored bend sensing. This ensures that in contrast to other FBG inscription using a fs laser there is no need to change the laser energy as the device type is changed. We consider that this is an important step for compact, smart optical fibre sensors.

\section{References}

[1] Theodosiou, A., Lacraz, A., Polis, M, Kalli, K., Tsangari, M., Stassis, A., and Komodromos, M., "Modified fs-laser inscribed FBG array for rapid mode shape capture of free-free vibrating beams," IEEE Photonics Technology Letters 28(14), 1509-1512 (2016).

[2] Theodosiou, A., Lacraz, A., Stassis, A., Koutsides, C., Komodromos, M., and Kalli, K., "Plane-by-Plane femtosecond laser inscription method for single-peak Bragg gratings in multimode CYTOP polymer optical fiber," Journal of Lightwave Technology 35(24), 5404-5410 (2017).

[3] Ioannou, A., Theodosiou, A., Caucheteur, C., and Kalli, K., "Direct writing of plane-by-plane tilted fiber Bragg gratings using a femtosecond laser," Optics Letters 42(24), 5198 - 5200 (2017). 


\title{
Reach extension of O-band transmission using BDFA
}

\author{
Vitaly Mikhailov*, Jiawei Luo, Man F. Yan, Yingzhi Sun, Gabriel S. Puc, Scott D. Shenk, \\ Yuriy Dulashko, Robert S. Windeler, Paul S. Westbrook, Daryl Inniss, David J. DiGiovanni \\ OFS Laboratories, 19 Schoolhouse Rd, Somerset, New-Jersey, 08873, USA
}

\begin{abstract}
We developed a simple single-stage silica-based BDFA with 40nm gain bandwidth, $19 \mathrm{~dB}$ gain, $20 \mathrm{dBm}$ output power, $5 \mathrm{~dB}$ noise figure and $20 \%$ power conversion efficiency (PCE). We demonstrated that the amplifier can be used to extend 400GBASE-LR-8 transmission distance beyond $50 \mathrm{~km}$ of G.652 fiber.
\end{abstract}

Keywords: Bismuth doped fiber amplifier, O-band transmission, LAN-WDM, Broadband optical amplification *vmikhailov@ofsoptics.com; phone 1732 748-7498

\section{Introduction}

O-band is extensively used for low cost data transmission. The advantage of O-band is that the transmitter wavelength(s) are located near the fiber's zero dispersion wavelength $\left(\lambda_{0}\right)$, thus neither optical nor electronic chromatic dispersion compensation is required. Recently O-band transponder aggregate bit rate was increased to $425 \mathrm{~Gb} / \mathrm{s}$, for example by using 8 LAN WDM 26.6 Gbaud/s PAM-4 modulated channels [1]. The use of WDM and complex modulation format reduces both per-channel power at the receiver and receiver sensitivity, thus making optical amplification desired. Semiconductor optical amplifiers (SOA) can be used to boost O-band signals but they introduce significant distortion due to self- and cross- gain modulation [2] and although amplification of a large number dispersion broadened channels has been demonstrated [3], SOAs are not suitable for transmission of intensity modulation formats like PAM-4 operating near $\lambda_{0}$ with relatively small channel count. Praseodymium doped fiber amplifiers (PDFA) with 1280-1320 nm bandwidth were demonstrated [4], but they require non-silica host glass that makes PDFA complicated and expensive.

In this paper we present a simple single-stage Bi-doped silica fiber amplifier (BDFA) with more than $80 \mathrm{~nm}$ 6-dB gain bandwidth. The amplifier has up to $19 \mathrm{~dB}$ gain, $20 \mathrm{dBm}$ output power, $20 \% \mathrm{PCE}$ and $5 \mathrm{~dB}$ noise-figure, similar to an EDFA of the same complexity. BDFA can extend 425 Gb/s 400GBASE-LR- 8 transmission (LAN WDM $8 \times 26.6$ Gbaud/s PAM- 4 channels) to $50 \mathrm{~km}$ distance with long-term measured BER of $6 \times 10^{-5} \mathrm{BER}$.

\section{Amplifier design}

To amplify the LR8 signals we built a simple counter-pumped amplifier using 80 meters of silica fiber with core codoped with bismuth and phosphorus, fused WDM and input optical isolator. WDM central wavelength was selected at $1272 \mathrm{~nm}$ to induce up to $4 \mathrm{~dB}$ loss at long wavelengths, while $1195 \mathrm{~nm}$ pump wavelength was chosen to increase the gain of the short wavelength channels [5]. Fig's. 1(a,b) shows gain (G) and noise figure (NF) at 500 and $800 \mathrm{~mW}$ pump power respectively. Over IEEE standardized 1272-1310 nm wavelength range [1], the amplifier has maximum $19 \mathrm{~dB}$ gain, $2 \mathrm{~dB}$ gain flatness and $5 \mathrm{~dB}$ noise figure with $5.5 \mathrm{~dB}$ peak value at around $1272 \mathrm{~nm}$.

\section{Transmission performance over G.652 fiber}

To evaluate the BDFA transmission performance a Finisar 400GBASE-LR8 transceiver and a Viavi ONT-604 tester were used. The tester generates $16 \times 26.6 \mathrm{~Gb} / \mathrm{s}^{31}-1$ PRBS OOK data lanes at the transmitter side and detects BER for each of 16 receiver side lanes. The LR8 combines 16 OOK data lanes into $8 \times 26.6$ Gbaud/s PAM- 4 channels and transmit them using

Sixth International Workshop on Specialty Optical Fibers and Their Applications (WSOF 2019), edited by Liang Dong, John M. Ballato, Proc. of SPIE Vol. 11206, 112060N · C 2019 SPIE

CCC code: $0277-786 X / 19 / \$ 21 \cdot$ doi: $10.1117 / 12.2548909$ 
the set of eight directly modulated lasers. At the receiver side, eight WDM channels are demultiplexed, detected and converted into 16 digital signal lanes. The transceiver signal $(11.7 \mathrm{dBm})$ was launched into $40-55 \mathrm{~km}$ of G.652 fiber (loss $0.33 \mathrm{~dB} / \mathrm{km} @ 1310 \mathrm{~nm}, \lambda_{0}=1312 \mathrm{~nm}$ ) and post amplified by the BDFA. The optical spectra after the transmitter, $40 \mathrm{~km}$ fiber span and amplifier are shown in fig. 1(c). The average fiber loss was $14.6 \mathrm{~dB}$ (incl. connectors) while short wavelength channels suffer up to $2 \mathrm{~dB}$ higher loss compared to the long wavelength channels. To investigate BER degradation from OSNR and estimate link loss margin a VOA was inserted between the fiber and BDFA. The total receiver power was maintained at optimal $6 \mathrm{dBm}$ level with $3 \mathrm{~dB}$ difference between best and worst channels. It has been found, that for transmitter power of $11.7 \mathrm{dBm}$ and $40 \mathrm{~km}$ fiber span, up to $6.8 \mathrm{~dB}$ loss can be added to the link before KP4-FEC BER threshold is reached $\left(2.4 \times 10^{-4}\right.$ BER). Fig. 1(d) shows average BER vs total receiver power for $40-55 \mathrm{~km}$ G.652 fiber transmission. Measured long term $(>8 \mathrm{~h})$ BER for $40 \mathrm{~km}$ distance was $5 \times 10^{-6}$ and it was possible to measure BER in all lanes for up to $55 \mathrm{~km}$ 's, while the error floor increases with the distance to $1.3 \times 10^{-4}$ level. To further increase the span length, the signal was pre-amplified by another BDFA with the total output power of $20.8 \mathrm{dBm}$ (pump wavelength and power are 1215 $\mathrm{nm}$ and $800 \mathrm{~mW}$ respectively). Only 8-15 data lanes were included in BER calculation while channels 1-4 where still transmitted. The transmission distance was limited by performance of the short wavelength channels and measured $81.5 \mathrm{~km}$ long-term error floor was $3 \times 10^{-5}$.

In summary, we have presented a single-stage $80 \mathrm{~m}$ long Bi-doped fiber amplifier with $19 \mathrm{~dB}$ gain, $20 \mathrm{dBm}$ output power, and $5 \mathrm{~dB}$ noise figure. To the best of our knowledge, this is the first fiber amplifier operating over IEEE standardized part of O-band (1272-1310 nm) with parameters comparable to commercially available EDFAs of the same complexity.
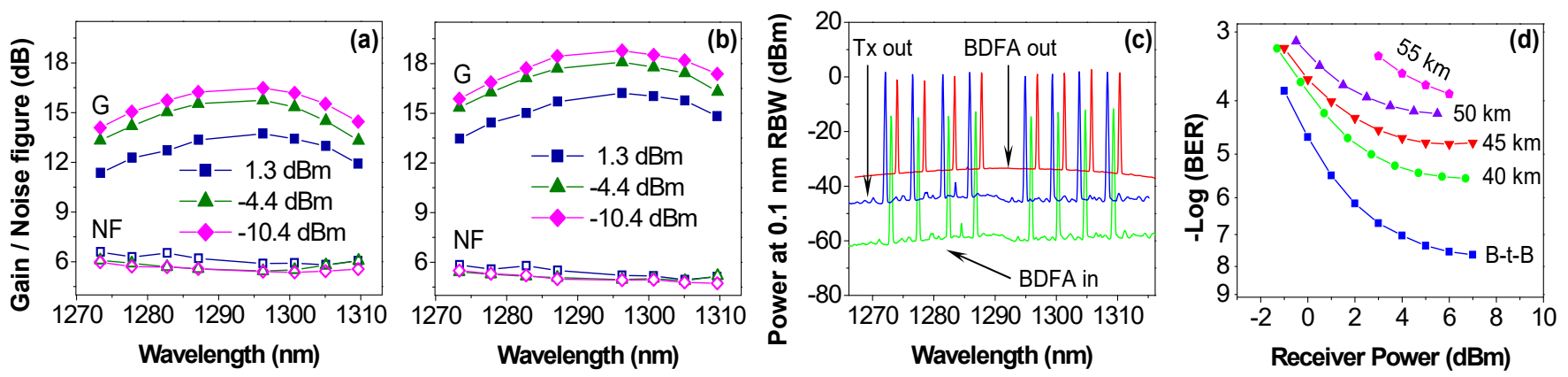

Figure 1. (a,b) BDFA gain and noise figure for 500 and $800 \mathrm{~mW}$ pump power; (c) $40 \mathrm{~km}$ transmission spectra, note wavelength shift was added to increase visibility; (d) BER vs power for 40-55 km distances.

\section{References}

[1] IEEE 802.3bs-2017 - IEEE Standard for Ethernet Amendment 10: Media Access Control Parameters, Physical Layers, and Management Parameters for $200 \mathrm{~Gb} / \mathrm{s}$ and $400 \mathrm{~Gb} / \mathrm{s}$ Operation, IEEE Standard, 2017.

[2] A.A.M Saleh et al., Effects of semiconductor optical amplifier nonlinearity on the performance of high-speed intensity modulation lightwave systems, IEEE Trans. Com., vol. 38, 1990.

[3] J. Renaudier, 100nm ultra-wideband optical fiber transmission systems using semiconductor optical amplifiers, Proc. ECOC 2018, Mo4G.5.

[4] S. Barthomeuf, et al., High Optical Budget 25Gbit/s PON with PAM4 and Optically Amplified O-Band Downstream Transmission, ECOC'18, Mo4B.2.

[5] V. Mikhailov, et al., Simple Broadband Bismuth Doped Fiber Amplifier (BDFA) to Extend O-band Transmission Reach and Capacity, OFC'19, M1J.4 


\title{
Recent efforts in power scaling of holmium doped fibre sources
}

\author{
Nikita Simakov ${ }^{\mathrm{a}}$, Alexander Hemming ${ }^{\mathrm{a}}$, Adrian Carter $^{\mathrm{b}}$, Keiron Boyd $^{\mathrm{a}}$, Robert Swain ${ }^{\mathrm{c}}$, Eric Mies ${ }^{\mathrm{c}}$, \\ W. Andrew Clarkson ${ }^{\mathrm{d}}$, and John Haub ${ }^{\mathrm{a}}$ \\ aDefence Science and Technology Group, Edinburgh, South Australia 5111, Australia \\ ${ }^{\mathrm{b}}$ Coherent|Nufern, 7 Airport Park Rd, East Granby, Ct 06026 USA; \\ 'Sub-micron Engineering, PO Box: 509, Marlboro New Jersey 07746 USA; \\ ${ }^{\mathrm{d}}$ Optoelectronics Research Centre, University of Southampton, Southampton, SO17 1BJ, UK
}

\begin{abstract}
This paper summarizes recent efforts in the development of core-pumped holmium sources. This includes the development of high power single-mode thulium-pump lasers, components optimized for core-pumping and efficiency optimization of core-pumped holmium fibres.
\end{abstract}

Keywords: Holmium, pulsed, eye-safe, fibre

\section{Introduction}

In order to scale to higher peak powers in holmium doped fibres, it becomes increasingly important to manage various limiting and deleterious nonlinear effects. Core-pumped configurations offer shorter fibre lengths in comparison to cladding-pumped systems providing benefits for pulsed and narrow linewidth systems at power levels suitable for a range of applications. However, in order to realise monolithic, high power operation of such a core-pumped amplifier it is necessary to power scale the thulium-doped fibre pump laser, the pump and signal combiner, and optimize the composition of the active holmium-doped fibre. This presentation will provide an overview of relevant nonlinear effects in the $\mathrm{CW}$ and ns-pulsed regimes and discuss the power scaling of the various components required and the subsequent core-pumped holmium sources.

\section{Limiting non-linear processes}

Table 1 summarizes the three main non-linear mechanisms that apply for a $1-10 \mathrm{~ns}$ pulse with a peak power of $10 \mathrm{~kW}$ propagating in a fibre with an effective mode-field diameter of $20 \mu \mathrm{m}$ (effective area of $\sim 300 \mu \mathrm{m}^{2}$ ) [1]. The dominant mechanisms are modulation instability and stimulated Brillouin scattering (SBS) with stimulated Raman scattering (SRS) experiencing a reduced gain coefficient. The magnitude of the gain for the modulation instability, SBS or SRS depends on the spectral bandwidth of the source.

Table 1: Summary of non-linear mechanisms for a pulse at $2.1 \mu \mathrm{m}$, with $10 \mathrm{~kW}$ peak power and propagating in a fibre with an effective mode area of $\sim 300 \mu \mathrm{m}^{2}$. ( $g_{H o}$ is the gain of the holmium fibre in the amplifier and will contribute to the gain of the various nonlinear effects that are in the gain band)

\begin{tabular}{|c|c|c|}
\hline Nonlinear mechanism & Gain (dB/m) & Comment \\
\hline Modulation Instability & $23+g_{H o}$ & $\begin{array}{c}\text { Very little can be done to directly suppress this effect. } \\
\text { Peak gain occurs within } \pm 15 \mathrm{~nm} \text { around signal }\end{array}$ \\
\hline Stimulated Raman Scattering & 6.5 & $\begin{array}{c}\text { Assuming that the fibre has an OH content of } \sim 1 \mathrm{ppm} \\
\text { Peak gain occurs at } 2.3 \mu \mathrm{m} \text { with a } 2.1 \mu \mathrm{m} \text { signal }\end{array}$ \\
\hline Stimulated Brillouin Scattering & $72(7.2)+g_{H o}$ & $\begin{array}{c}1 \mathrm{GHz},(10 \mathrm{GHz}) \text { master oscillator bandwidth } \\
\text { Inhomogeneous broadening can be used } \\
\text { Peak gain at } \sim 8 \mathrm{GHz}(0.1 \mathrm{~nm}) \text { from signal }\end{array}$ \\
\hline
\end{tabular}

Sixth International Workshop on Specialty Optical Fibers and Their Applications (WSOF 2019), edited by Liang Dong, John M. Ballato, Proc. of SPIE Vol. 11206, 1120600 · C 2019 SPIE CCC code: $0277-786 X / 19 / \$ 21 \cdot$ doi: $10.1117 / 12.2549915$ 


\section{Core-pumped architecture}

Previous holmium-doped fibre experiments using pulsed sources in a cladding-pumped configuration resulted in an output with an extremely broad spectral bandwidth - largely attributed to modulation instability [2]. Figure 1 shows a schematic of an alternate forward core-pumped holmium-doped fibre amplifier configuration. Here a $2.1 \mu \mathrm{m}$ master oscillator and a $1.95 \mu \mathrm{m}$ pump laser are launched into the 2 inputs of a wavelength division multiplexer (WDM) and the master oscillator is subsequently amplified in the holmium-doped fibre spliced to the output of the WDM.

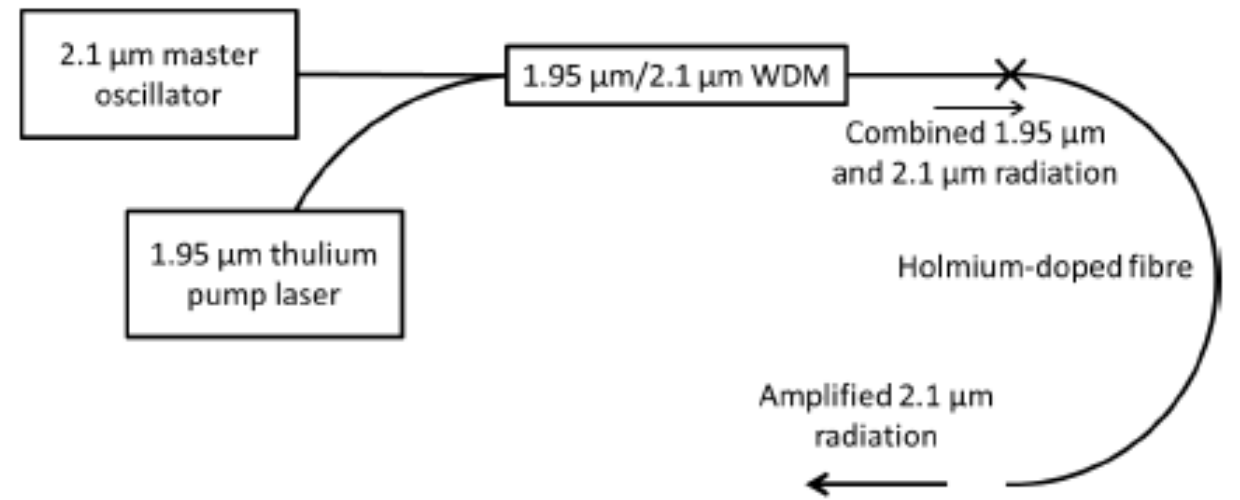

Figure 1: Forward pumped, core-pumped configuration where the master oscillator and the thulium pump laser are coupled into the fundamental mode of the core of a holmium-doped fibre via a fused biconic taper.

Such a configuration reduces the required length of the active fibre from the order of 5-10 $\mathrm{m}$ of a typical claddingpumped system, to $<3 \mathrm{~m}$. In addition, the mode-field diameters of the $1.95 \mu \mathrm{m}$ pump and the $2.1 \mu \mathrm{m}$ are very similar, resulting in excellent spatial overlap. This architecture requires improved fibre efficiency to mitigate the higher heat load associated with this reduction in fibre length.

\section{Power scaling of pump sources and couplers}
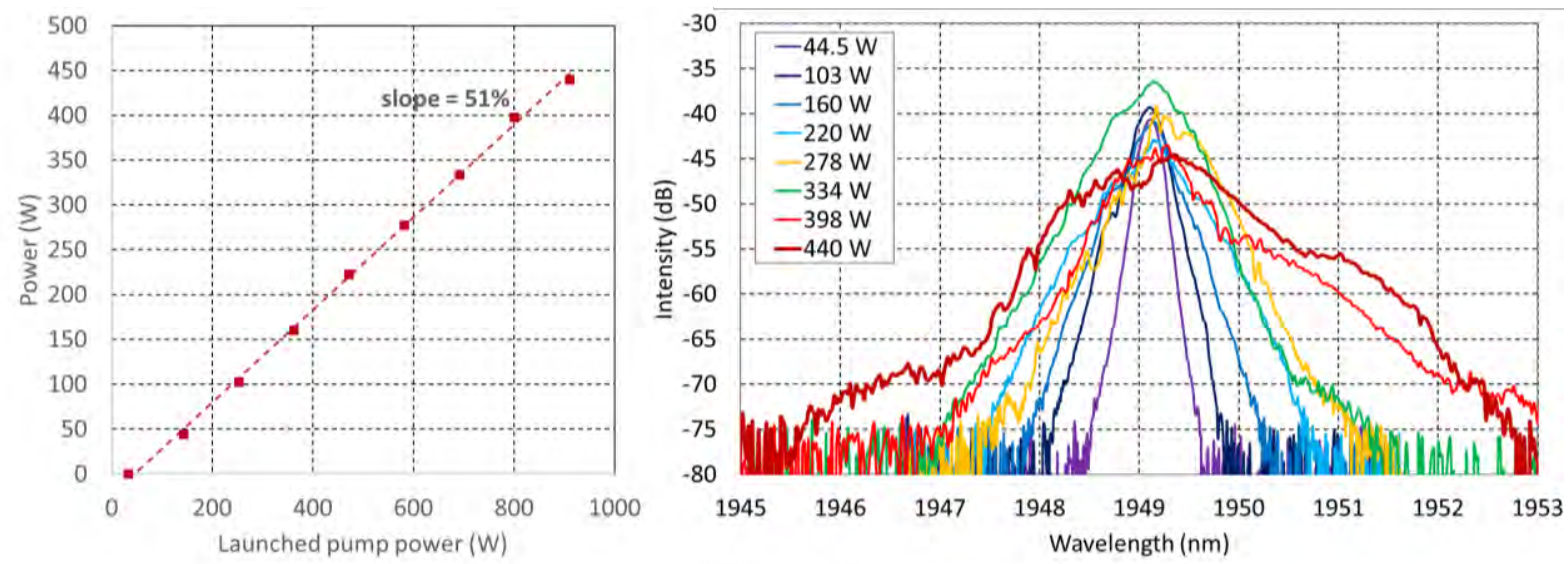

Figure 2: Slope efficiency of a $1.95 \mu \mathrm{m}$ monolithic single mode thulium laser oscillator and the corresponding output spectrum.

In order to enable the power scaling of such core-pumped architectures it is necessary to power scale both the WDM couplers and the $1.95 \mu \mathrm{m}$ thulium-doped fibre pump lasers. Figure 2 shows the output power versus launched $0.79 \mu \mathrm{m}$ diode pump power of a $1.95 \mu \mathrm{m}$ thulium-doped fibre laser and the corresponding output spectrum [3]. It is essential for 
the output bandwidth of the thulium pump laser to remain sufficiently small $(<10 \mathrm{~nm})$ in order to be able to be efficiently transmitted through the passband of the WDM coupler.

The WDM couplers were fabricated in-house based on a fused biconic taper configuration and operated at pump powers of $>150 \mathrm{~W}$ with no degradation in performance. To the best of our knowledge, this is the highest power operation of a WDM in this wavelength region and the highest power operation of a robustly single mode thulium doped fibre laser operating at $1.95 \mu \mathrm{m}$.

\section{Core pumped pulsed operation}

The core pumped architecture was demonstrated using a 5 ns master oscillator. Here the system was pumped up to 55 $\mathrm{W}$ and produced $17 \mathrm{~W}$ of output power. A slope efficiency curve is shown in Fig 3(a). The output pulse duration was $5 \mathrm{~ns}$ and the repetition rate was $100 \mathrm{kHz}$ producing a peak power of $\sim 36 \mathrm{~kW}$. Figure 3(b) shows the reduction of spectral broadening that the core-pumped approach has enabled in comparison to previous attempts with a cladding pumped system [2].
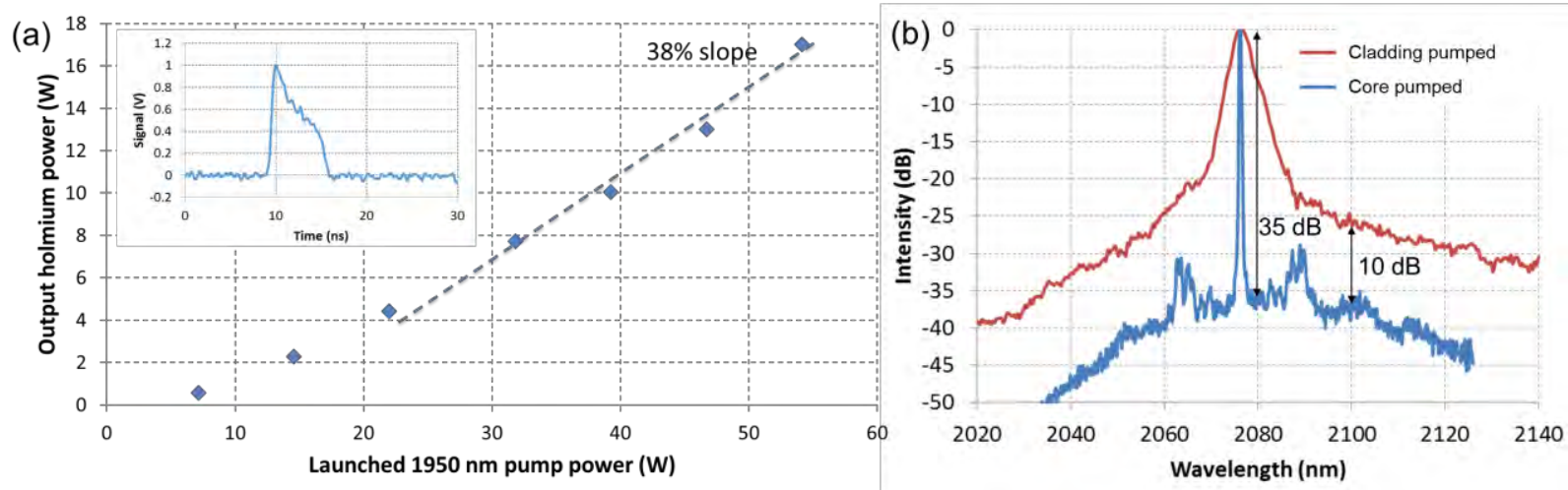

Figure 3: (a) Output power vs launched pump power from a pulsed holmium amplifier; Inset: Output pulse showing a 5 ns duration and exhibiting significant gain shaping. (b) Output spectrum at $36 \mathrm{~kW}$ peak power showing the reduction of spectral broadening comparing a previous cladding-pumped amplifier with a core-pumped amplifier approach.

\section{Conclusion}

With the development of the necessary components, we have been able to demonstrate the power scaling of corepumped holmium doped fibres in the ns-pulsed regimes. This has been enabled by the development of robustly singlemode pump lasers, high power 1.95/2.1 $\mu \mathrm{m}$ WDM couplers and AR-coated end-caps. We will discuss recent results and future opportunities for core-pumped holmium amplifiers in both the ns-pulsed and CW regimes, and their applicability to a range of applications.

\section{References}

[1] Simakov, Nikita (2017) Development of components and fibres for the power scaling of pulsed holmium-doped fibre sources. Doctoral Thesis, 190pp.

[2] Alexander Hemming, Jim Richards, Nikita Simakov, Alan Davidson, Neil Carmody, John Haub, and Adrian Carter, "Pulsed operation of a resonantly pumped, linearly polarised, large mode area holmium-doped fibre amplifier," Opt. Express 22, 7186-7193 (2014)

[3] Alexander Hemming, Nikita Simakov, Keiron Boyd, Robert Swain, Eric Mies, Alan Davidson, Neil Carmody, Kevin Farley, Adrian Carter, John Haub, "Power scaling of single-mode, high power thulium-doped fibre lasers", SPIE Photonics West, 10897-6, (2019) 


\title{
Loss Reduction in Hollow-Core Optical Fiber
}

\author{
Ying-ying Wang*a,b, Shou-fei Gao ${ }^{\mathrm{a}, \mathrm{b}}$, Wei Ding ${ }^{\mathrm{b}}$ and Pu Wang \\ ${ }^{\text {a}}$ National Center of Laser Technology, Institute of Laser Engineering, Beijing University of \\ Technology, Beijing 100124, China \\ ${ }^{b}$ Guangdong Provincial Key Laboratory of Optical Fiber Sensing and Communications, Institute of \\ Photonics Technology, Jinan University, Guangzhou 510632, China
}

\begin{abstract}
We review our recent progress in achieving ultralow loss hollow-core optical fiber in both the near-IR and the visible wavelengths. The loss of the green-guiding fiber strides across the Rayleigh scattering loss limit of silica glass fiber.

Keywords: hollow-core fiber, microstructured fiber, transmission loss, ultralow loss, Rayleigh scattering *dearyingyingwang@hotmail.com;
\end{abstract}

\section{Introduction}

Pursuing ultralow loss has been a long term goal in fiber optics. The loss record of silica glass fiber (SGF) almost approaches the intrinsic Rayleigh scattering loss (RSL) limit $(0.14 \mathrm{~dB} / \mathrm{km})[1,2]$, showing little space for further optimization. Hollow-core photonic crystal fiber (HC-PCF) [3] has been considered as a potential candidate for achieving ultralow loss below the fundamental RSL limit of SGF, since $\sim 99.9 \%$ of light is guided in the air core. However, the lowest demonstrated transmission loss of hollow-core photonic bandgap fiber (HC-PBGF) is $1.7 \mathrm{~dB} / \mathrm{km}$ [4] (with one mention of loss of $1.2 \mathrm{~dB} / \mathrm{km}$ in [5]), which is intrinsically limited by the surface scattering loss (SSL) due to the frozenin capillary wave [5]. Furthermore, the usable bandwidth of this fiber is less than $20 \mathrm{~nm}$, a main impediment in applications. Though broader bandwidth (160-200 nm) HC-PBGF has also been fabricated in recent years, the loss level is raised to $3.5-5 \mathrm{~dB} / \mathrm{km}[6]$.

Recently, the appearance of hollow core negative-curvature fiber (HC-NCF), which originates from the Kagome type broadband HCF in 2002 [7], reignites the hope of achieving ultralow loss. Since the discovery of the important negativecurvature (or hypocycloid) core wall shape [8], great progresses have been made in the design and fabrication of low loss HC-NCF, pushing the loss of HC-NCF to the same level of HC-PBGF, e.g., $2 \mathrm{~dB} / \mathrm{km}$ at $1512 \mathrm{~nm}$ in the conjoined-tube NCF [9] and $1.3 \mathrm{~dB} / \mathrm{km}$ at $1450 \mathrm{~nm}$ in the nested NCF [10]. Although these results are still one order of magnitude higher than silica's RSL, it is possible to beat the RSL limit at visible wavelengths because the loss in NCF follows the scaling law of $\lambda^{-1}$ while the RSL follows $\lambda^{-4}$. Here, we review our recent progress in conjoined-tube NCF (CTF for short) in both the near-IR and visible. Several CTFs are fabricated in our group with losses of $2 \mathrm{~dB} / \mathrm{km}$ at $1550 \mathrm{~nm}, 2.7 \mathrm{~dB} / \mathrm{km}$ at 1150 $\mathrm{nm}, 3.8 \mathrm{~dB} / \mathrm{km}$ at $680 \mathrm{~nm}$ and $4.9 \mathrm{~dB} / \mathrm{km}$ at $558 \mathrm{~nm}$ respectively. The loss in the visible stay beneath the RSL limit of SGF. 


\section{Results and Discussion}

Fig. 1 shows three fabricated CTFs. Fiber $a$ has a core diameter of $30.5 \mu \mathrm{m}$. The average glass wall thicknesses of $t_{1}, t_{2}$ and $t_{3}$ are $1.12 \mu \mathrm{m}, 1.06 \mu \mathrm{m}$ and $1.16 \mu \mathrm{m}$ respectively. The loss spectrum is obtained by a cut-back measurement from $330 \mathrm{~m}$ to $5 \mathrm{~m}$. A supercontinuum (SC) source is butt-coupled to the $330 \mathrm{~m}$ long CTF, which is looped on the drum during the measurement with bending radius of $16 \mathrm{~cm}$. The output of CTF is coupled to an optical spectral analyzer (OSA) using a magnetic clamp bare fiber adaptor. Multiple cleaves at the output end show little variation in spectra for both $330 \mathrm{~m}$ and $5 \mathrm{~m}$ long fiber respectively. Fig. 1(b) shows the measured loss spectra of $2 \mathrm{~dB} / \mathrm{km}$ at $1512 \mathrm{~nm}, 3.7 \mathrm{~dB} / \mathrm{km}$ at $1550 \mathrm{~nm}$, and $13.4 \mathrm{~dB} / \mathrm{km}$ at $1310 \mathrm{~nm}$. The usable bandwidth with the loss lower than $16 \mathrm{~dB} / \mathrm{km}$ spans from $1302 \mathrm{~nm}$ to $1637 \mathrm{~nm}$, covering half of the $\mathrm{O}$ band, and full E, S, C, L telecom bands. Spectral oscillations probably stem from slight inconsistence of the AR conditions for different glass layers which could be optimized by more sophisticated control of the glass membrane thicknesses. Other trivial oscillations distributed over the whole transmission band are a result of the Fano resonances introduced by the glass web connections.
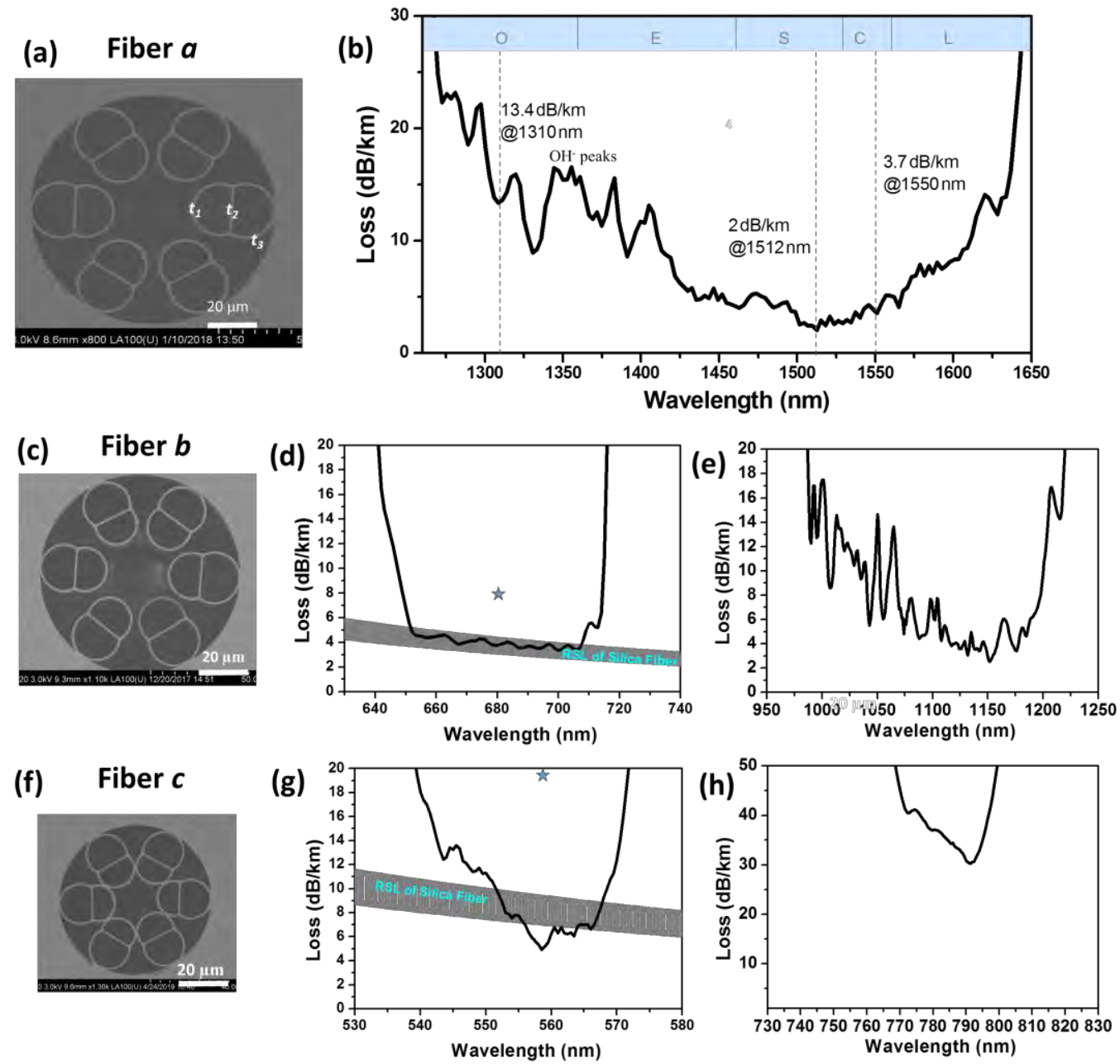

Fig.1. Ultralow loss CTFs. (a, c, f) Scanning electron microscope (SEM) image of fiber $a, b$ and $c$ respectively. (b, d, e, g, h) Measured loss spectra. The gray stripes in $(\mathrm{d})$ and $(\mathrm{g})$ outline the RSL range of SGF. The star symbols represents the loss level of commercial SGF. 
Fiber $b$ has a core diameter of $25 \mu \mathrm{m}$ and the average glass wall thicknesses of $t_{1}, t_{2}$ and $t_{3}$ are $0.84 \mu \mathrm{m}, 0.75 \mu \mathrm{m}$ and $0.82 \mu \mathrm{m}$ respectively. The loss spectrum is obtained in similar manner with fiber $a$. Two transmission bands are manifest in Fig. 1(d\&e). In the first band, from $960 \mathrm{~nm}$ to $1276 \mathrm{~nm}$, corresponding to the $2^{\text {nd }}$ order AR band of all the glass membranes [11], the minimum loss of $2.7 \mathrm{~dB} / \mathrm{km}$ appears at $1150 \mathrm{~nm}$ [Fig. 1(e)], and a number of loss peaks can be attributed to Fano resonance induced extra losses [12]. The $3^{\text {rd }}$ order AR band, from $653 \mathrm{~nm}$ to $706 \mathrm{~nm}$ [Fig. 1(d)], exhibits a much smoother loss spectrum with those Fano resonance induced loss peaks fading out. In this red-guiding band, the propagation attenuation is measured to be 3.4-4.6 dB/km, the same level of the RSL of SGF.

For fiber $c$, the core diameter has decreased remarkably to $18 \mu \mathrm{m}$, while the glass wall thicknesses are $0.98 \mu \mathrm{m}\left(t_{1}\right), 0.7$ $\mu \mathrm{m}\left(t_{2}\right)$ and $0.84 \mu \mathrm{m}\left(t_{3}\right)$, respectively. The minimum losses in the two bands are $30 \mathrm{~dB} / \mathrm{km}$ at $790 \mathrm{~nm}$ and $4.9 \mathrm{~dB} / \mathrm{km}$ at $558 \mathrm{~nm}$, respectively. This fiber far surpasses the RSL limit in the $555-564 \mathrm{~nm}$ wavelength region. At $558 \mathrm{~nm}$, the loss $(4.9 \mathrm{~dB} / \mathrm{km})$ is $2 \mathrm{~dB}$ lower than the RSL limit of SGF, indicating that fiber $c$ undoubtedly breaks a long-sustained optical fiber loss limit set by silica glass material. It should be also noted that commercial SGFs typically have higher attenuations than the above figures, e.g., $8-10 \mathrm{~dB} / \mathrm{km}$ at $680 \mathrm{~nm}$ and $20-30 \mathrm{~dB} / \mathrm{km}$ at $558 \mathrm{~nm}$ (labeled as star symbols in Fig.1), which are 2-5 times higher than our CTFs.

\section{Conclusion}

In conclusion, since SGF cannot exceed the fundamental RSL limit, HCF undertakes the task of extending the range of possibilities in optical fibers. In this work, by leveraging the higher-order-band light guidance and the $\lambda^{-1}$ wavelength dependence of the CL, along with employing our celebrated CTF geometry, we reviewed several ultralow loss HCF. In particular, an optical fiber loss beneath the RSL limit of SGF by $2 \mathrm{~dB}$ at green wavelength was fabricated for the first time. Apart from the fundamental interest of conquering the RSL limit of SGF, an ultralow loss fiber also gains great momentums from the application aspect.

\section{References}

1. K. Saito et al, "Limit of the Rayleigh scattering loss in silica fiber," Appl. Phys. Lett. 83, 5175-5177 (2003).

2. Y. Tamura et al "The first $0.14-\mathrm{dB} / \mathrm{km}$ loss optical fiber and its impact on submarine transmission," J. Lightwave Technol. 36, 44-49 (2018).

3. J. C. Knight, "Photonic Crystal Fibers", Nature 424, 847-851 (2003).

4. B. Mangan et al, in Optical Fiber Communication Conference, PD24 (Los Angeles, CA, USA, 2004).

5. P. J. Roberts et al, Ultimate low loss of hollow-core photonic crystal fibers. Opt. Express 13, 236-244 (2005).

6. F. Poletti et al, Towards high-capacity fiber-optic communications at the speed of light in vacuum. Nature Photon. 7(4), 279-284 (2013).

7. F. Benabid et al, Stimulated Raman scattering in hydrogen-filled hollow-core photonic crystal fiber. Science 298, 399-402 (2002).

8. Y. Y. Wang et al, Low loss broadband transmission in hypocycloid-core Kagome hollow-core photonic crystal fiber. Opt. Lett. 36, 669-671 (2011). 
9. S. F. Gao, Y. Y. Wang, W. Ding, D. L. Jiang, S. Gu, X. Zhang, and P. Wang, "Hollow-core conjoined-tube negativecurvature fibre with ultralow loss," Nat. Commun. 9, 2828 (2018).

10. T. D. Bradley, J. R. Hayes, Y. Chen, G. T. Jasion, S. R. Sandoghchi, R. Slavik, E. N. Fokoua, S. Bawn, H. Sakr, I. A. Davidson, A. Taranta, J. P. Thomas, M. N. Petrovich, D. J. Richardson, and F. Poletti, "Record low loss $1.3 \mathrm{~dB} / \mathrm{km}$ data transmitting antiresonant hollow core fibre," in 44th European Conference and Exhibition on Optical Communications (ECOC) (2018).

11. N. M. Litchinitser, A. K. Abeeluck, C. Headley, and B. J. Eggleton, "Antiresonant reflecting photonic crystal optical waveguides," Opt. Lett. 27(18), 1592-1594 (2002).

12. L. Vincetti and V. Setti, "Extra loss due to Fano resonances in inhibited coupling fibers based on a lattice of tubes," Opt. Express 20, 14350-14361 (2012). 


\title{
Negative curvature fibers for gas-filled fiber lasers
}

\author{
Chengli Wei ${ }^{\mathrm{a}}$, Francois Chenard ${ }^{\mathrm{b}}$, Curtis R. Menyuk ${ }^{\mathrm{c}}$, Jonathan $\mathrm{Hu}^{* \mathrm{~d}}$ \\ a Department of Computer Science, Engineering and Physics, University of Mary Hardin-Baylor, \\ 900 College Street, Belton, TX 76513, USA \\ bIRflex Corporation, 300 Ringgold Industrial Parkway, Danville, VA 24540, USA \\ ${ }^{\mathrm{c}}$ Department of Computer Science and Electrical Engineering, University of Maryland, \\ 5200 Westland Blvd., Baltimore, MD 21227, USA \\ ${ }^{\mathrm{d}}$ Department of Electrical and Computer Engineering, Baylor University, \\ One Bear Place \#97356, Waco, TX 76798, USA
}

\begin{abstract}
We find optimal structure for chalcogenide negative curvature fibers with different thicknesses and gaps between cladding tubes to yield low transmission loss at wavelengths of $1.5 \mu \mathrm{m}$ and $4.5 \mu \mathrm{m}$ simultaneously for gas-filled fiber lasers.

Keywords: negative curvature fiber, fiber laser, simulation, chalcogenide glass, gas laser

*Jonathan hu@baylor.edu; phone 1254 710-1853

\section{Introduction}

Mid-infrared lasers are widely used in sensing, medical, and defense applications [1]. The invention of hollow-core fibers and their ability to host gases for long interaction lengths and micrometer-scale mode areas made possible the use of new gas-filled hollow-core fiber lasers at mid-infrared wavelengths [2]. Using a pump at $1.5 \mu \mathrm{m}$, emission at around $4.5 \mu \mathrm{m}$ has been realized in hollow-core fibers filled with $\mathrm{H}_{2}$ or $\mathrm{N}_{2} \mathrm{O}$ gases [3-5]. Recently, negative curvature fibers with a broad bandwidth and low loss have drawn much attention because it is possible to fill the fibers with gas or liquid [6-9]. It has been shown that negative curvature fibers that are made with chalcogenide glass have lower loss than negative curvature fibers made with silica glass if the wavelength is longer than $4.5 \mu \mathrm{m}$ [10]. In this paper, we computationally study the leakage loss in the negative curvature fibers made with $\mathrm{As}_{2} \mathrm{~S}_{3}$ chalcogenide glass. We change the tube thickness and gap between the cladding tubes to find the optimum structure that can simultaneously provide low transmission loss at wavelengths of $1.5 \mu \mathrm{m}$ and $4.5 \mu \mathrm{m}$. We study negative curvature fibers with both six and eight tubes.
\end{abstract}

\section{Simulation and results}

\section{Negative curvature fiber with six cladding tubes}

We first simulate the leakage loss as a function of gap and tube thickness for negative curvature fibers with six cladding tubes. In our simulation, the core diameter is $100 \mu \mathrm{m}$. The refractive indices of $\mathrm{As}_{2} \mathrm{~S}_{3}$ glass are 2.44 and 2.41 at wavelengths of $1.5 \mu \mathrm{m}$ and $4.5 \mu \mathrm{m}$, respectively [11]. The material losses of $\mathrm{As}_{2} \mathrm{~S}_{3}$ glass are $2 \mathrm{~dB} / \mathrm{m}$ and $0.15 \mathrm{~dB} / \mathrm{m}$ at wavelengths of $1.5 \mu \mathrm{m}$ and $4.5 \mu \mathrm{m}$, respectively [12]. Figure 1(a) shows the loss at wavelength of $1.5 \mu \mathrm{m}$. When the tube thickness increases from $0.5 \mu \mathrm{m}$ to $2.5 \mu \mathrm{m}$, there are several high loss regions and low loss regions, which correspond to resonant and antiresonant conditions, respectively [13]. In the low transmission region, when the gap increases from 2 $\mu \mathrm{m}$ to $30 \mu \mathrm{m}$, the loss decreases first, and then increases, which means that an appropriate gap should be selected to realize low loss transmission [14]. Figure 2(b) shows the loss at wavelength of $4.5 \mu \mathrm{m}$. For a larger wavelength, we see less loss peaks as thickness changes from 0.5 to $2.5 \mu \mathrm{m}$, which agrees with the resonant condition, $2 t\left(n^{2}-1\right)^{1 / 2}=m \lambda$, where $t$ is the tube thickness, $\lambda$ is the resonant wavelength, and $m$ is the order of resonance $[13,15]$.

Sixth International Workshop on Specialty Optical Fibers and Their Applications (WSOF 2019),

edited by Liang Dong, John M. Ballato, Proc. of SPIE Vol. 11206, 112060Q · C 2019 SPIE

CCC code: $0277-786 \mathrm{X} / 19 / \$ 21 \cdot$ doi: $10.1117 / 12.2548000$ 

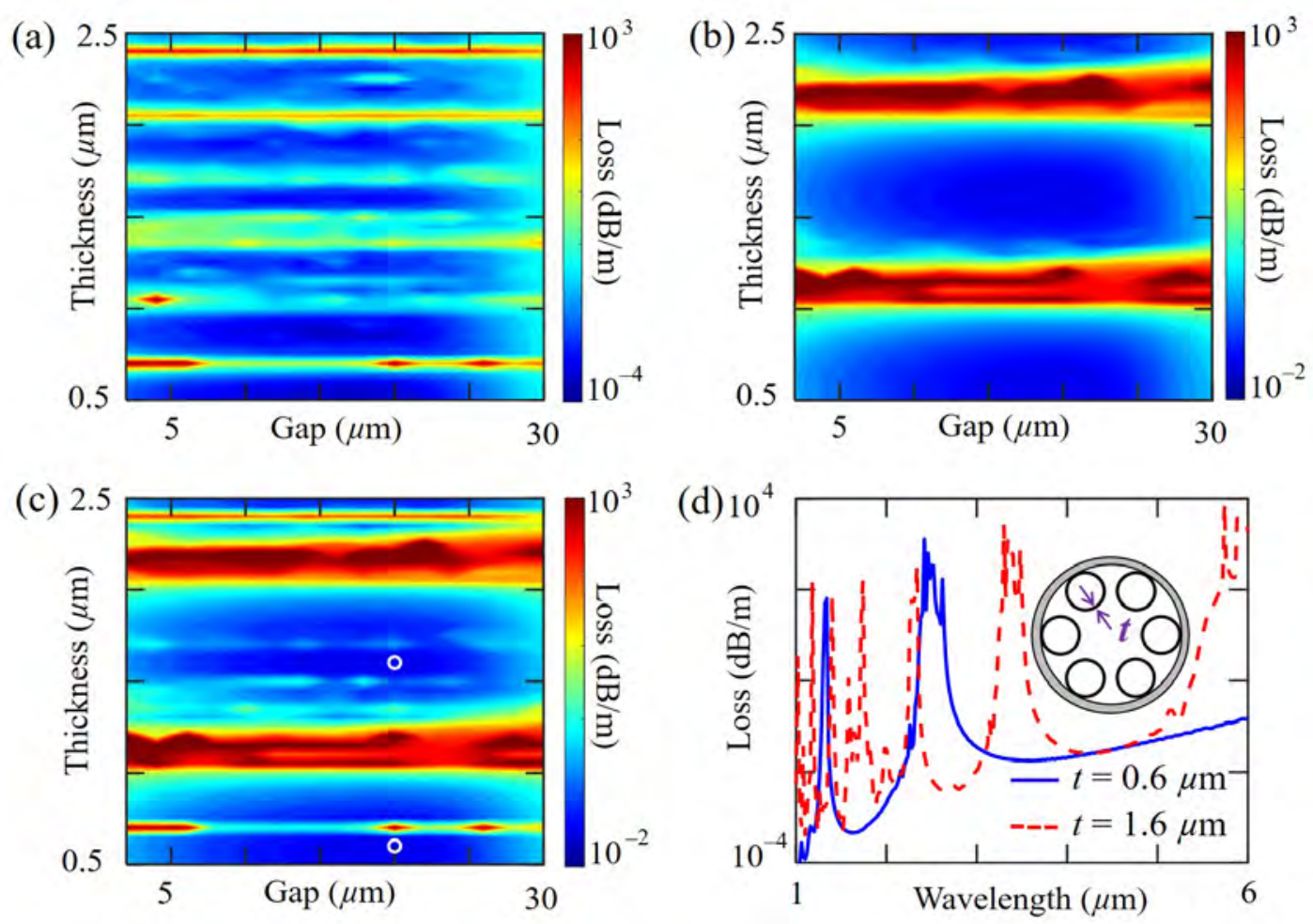

Figure 1. Contour plot of leakage loss at wavelengths of (a) $1.5 \mu \mathrm{m}$ and (b) $4.5 \mu \mathrm{m}$ using negative curvature fibers with six cladding tubes. (c) Contour plot of sum of leakage loss at wavelengths of $1.5 \mu \mathrm{m}$ and $4.5 \mu \mathrm{m}$. The white circles indicate the parameters that give low sum losses. (d) Loss as a function of wavelength for a fiber with a tube thickness of $0.6 \mu \mathrm{m}$ and a fiber with a tube thickness of $1.6 \mu \mathrm{m}$, corresponding to the white circles in Fig. 1(c). The gap is $20 \mu \mathrm{m}$. Inset shows a schematic of the cross section of the fiber with six cladding tubes.

In order to find a gap and a tube thickness that can realize low loss for both wavelengths of $1.5 \mu \mathrm{m}$ and $4.5 \mu \mathrm{m}$, we sum the losses for wavelengths at $1.5 \mu \mathrm{m}$ and $4.5 \mu \mathrm{m}$ and show the new contour plot in Fig. 1(c). In the hollow-core fiber lasers filled with $\mathrm{H}_{2}$ or $\mathrm{N}_{2} \mathrm{O}$ gases, the pump light propagates in the fiber at $1.5 \mu \mathrm{m}$ before the conversation of light at 4.5 $\mu \mathrm{m}$. Hence, the sum of the loss at the wavelengths of $1.5 \mu \mathrm{m}$ and $4.5 \mu \mathrm{m}$ gives a good indication of the overall loss of the fiber laser. Both the high-loss pattern features of Figs. 1(a) and 1(b) can be observed in Fig. 1(c). There are several lowloss regions when the tube thickness changes. We use two white circles to mark the fiber parameters that give low loss in Fig. 1(c), which can provide low loss transmission for both wavelengths of $1.5 \mu \mathrm{m}$ and $4.5 \mu \mathrm{m}$. We further ran simulations for fiber loss as a function of wavelength using the optimum fiber parameters that are marked with white circles in Fig. 1(c). In Fig. 1(d), we show the result with a gap of $20 \mu \mathrm{m}$. The blue solid curve and red dashed curve show the losses for the fibers with tube thicknesses of $0.6 \mu \mathrm{m}$ and $1.6 \mu \mathrm{m}$, respectively. For both fibers, the losses are about of $10^{-3} \mathrm{~dB} / \mathrm{m}$ and $10^{-2} \mathrm{~dB} / \mathrm{m}$ at wavelengths of $1.5 \mu \mathrm{m}$ and $4.5 \mu \mathrm{m}$, respectively. At a wavelength of $1.5 \mu \mathrm{m}$, a fiber with a thinner tube wall of $0.6 \mu \mathrm{m}$, corresponding to lower order of antiresonance, yields a significant wider bandwidth of 0.8 $\mu \mathrm{m}$ than a narrower bandwidth of $0.1 \mu \mathrm{m}$ for a fiber with a thicker tube wall of $1.6 \mu \mathrm{m}$, corresponding to higher order of antiresonance. Here, we evaluate bandwidth as the transmission window with a loss below $10^{-2} \mathrm{~dB} / \mathrm{m}$. 

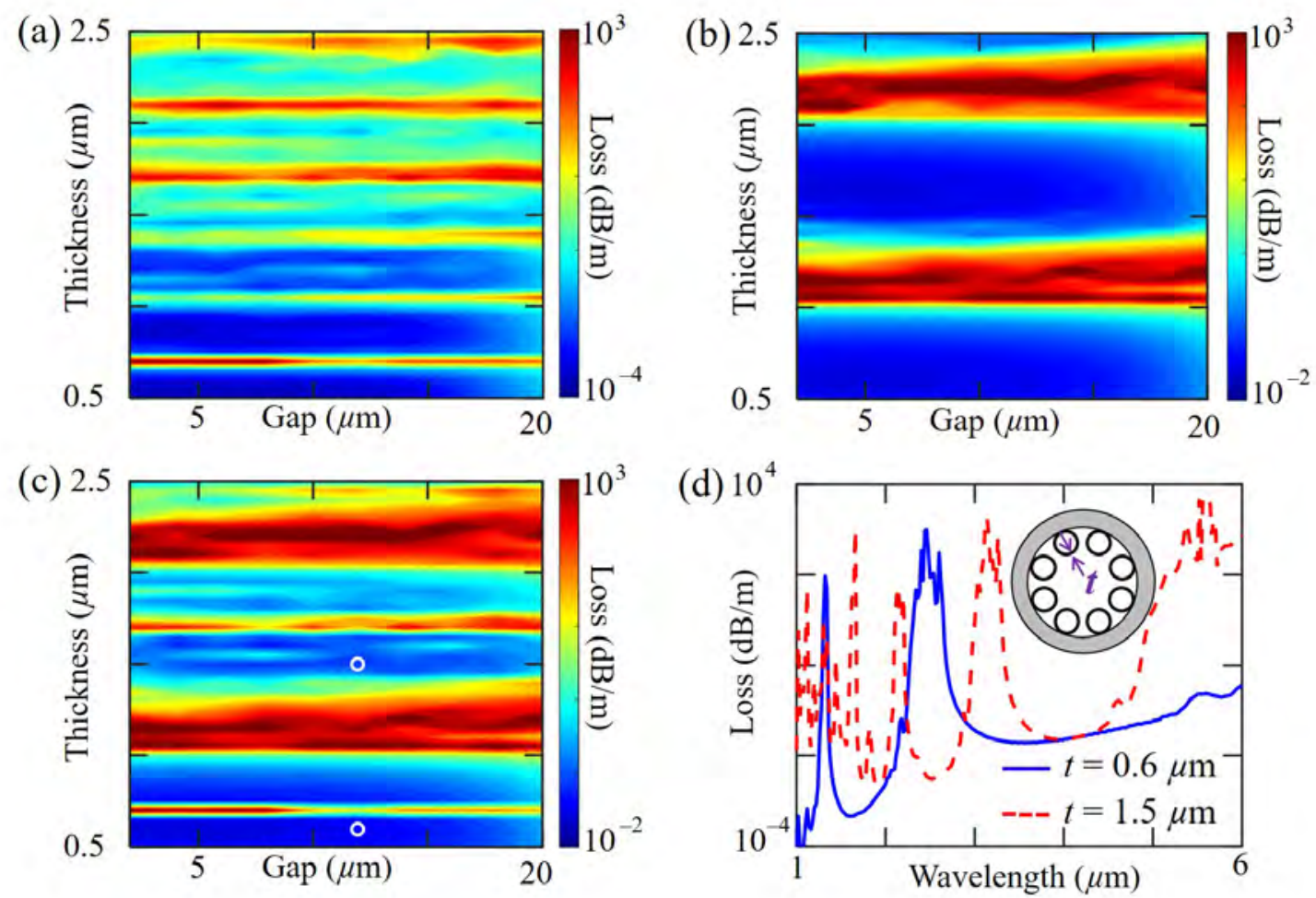

Figure 2. Contour plot of leakage loss at wavelengths of (a) $1.5 \mu \mathrm{m}$ and (b) $4.5 \mu \mathrm{m}$ using negative curvature fibers with eight cladding tubes. (c) Contour plot of sum of leakage loss at wavelengths of $1.5 \mu \mathrm{m}$ and $4.5 \mu \mathrm{m}$. The white circle indicates the parameters that gives a low sum loss. (d) Loss as a function of wavelength for a fiber with a tube thickness of $0.6 \mu \mathrm{m}$ and a fiber with a tube thickness of $1.5 \mu \mathrm{m}$, corresponding to the white circles in Fig. 2(c). The gap is $12 \mu \mathrm{m}$. Inset shows a schematic of the cross section of the fiber with eight cladding tubes.

\section{Negative curvature fiber with eight cladding tubes}

We now study the leakage loss for negative curvature fibers with eight cladding tubes. Figures 2(a) and 2(b) show the loss at wavelengths of $1.5 \mu \mathrm{m}$ and $4.5 \mu \mathrm{m}$, respectively. Figure 2(c) shows the sum of leakage loss at wavelengths of 1.5 $\mu \mathrm{m}$ and $4.5 \mu \mathrm{m}$ as a function of gap and tube thickness. Again, the fiber parameters that yield low loss are marked with white circles in Fig. 2(c). Figure 2(d) shows the loss as a function of wavelength for both two fibers with a gap of $12 \mu \mathrm{m}$. The blue solid curve and red dashed curve show the losses for the fibers with tube thicknesses of $0.6 \mu \mathrm{m}$ and $1.5 \mu \mathrm{m}$, respectively. Both fibers yield a loss that is lower than $0.1 \mathrm{~dB} / \mathrm{m}$ at wavelengths of $1.5 \mu \mathrm{m}$ and $4.5 \mu \mathrm{m}$. When the wavelength is $1.5 \mu \mathrm{m}$, the losses in a fiber with a tube thickness of $1.5 \mu \mathrm{m}$ is 40 times higher than the fiber loss in a fiber with a tube thickness of $0.6 \mu \mathrm{m}$. When the tube diameter is small, a thick tube does not serve as an antiresonant layer well and cannot confine the light well at a shorter wavelength [10].

\section{Conclusion}

In conclusion, we study negative curvature fibers with six and eight cladding tubes in order to simultaneously obtain low loss transmission at wavelengths of $1.5 \mu \mathrm{m}$ and $4.5 \mu \mathrm{m}$. Using six cladding tubes, negative curvature fibers with tube thicknesses of $0.6 \mu \mathrm{m}$ and $1.6 \mu \mathrm{m}$ yield a similar loss at both $1.5 \mu \mathrm{m}$ and $4.5 \mu \mathrm{m}$. However, the fiber with the thinner tube thickness of $0.6 \mu \mathrm{m}$ yields a broader bandwidth of $0.4 \mu \mathrm{m}$ by contrast to the fiber with the thicker tube thickness of 1.6 $\mu \mathrm{m}$ that yields a narrower bandwidth of $0.1 \mu \mathrm{m}$. Using eight cladding tubes, a negative curvature fiber with a tube 
thickness of $0.6 \mu \mathrm{m}$ yields a 40 times lower loss at wavelength of $1.5 \mu \mathrm{m}$ than does a fiber with a tube thickness of 1.5 $\mu \mathrm{m}$. Our study shows that it is possible to realize low loss transmission for wavelengths at $1.5 \mu \mathrm{m}$ and $4.5 \mu \mathrm{m}$ using one negative curvature fiber, which is crucial for gas-filled fiber lasers that require low-loss transmission of both pump and lasing wavelengths.

\section{References}

[1] Sorokina, I. T. and Vodopyanov, K. L., [Solid-state mid-infrared laser sources], Springer, Berlin, (2003).

[2] Nampoothiri, A. V. V., Jones, A. M., Fourcade-Dutin, C., Mao, C., Dadashzadeh, N., Baumgart, B., Wang, Y. Y., Alharbi, M., Bradley, T., Campbell, N., Benabid, F., Washburn, B. R., Corwin, K. L., and Rudolph, W., "Hollow-core optical fiber gas lasers (HOFGLAS): a review [Invited]," Opt. Mater. Express 2(7), 948-961 (2012).

[3] Gladyshev, A. V., Kosolapov, A. F., Khudyakov, M. M., Yatsenko, Y. P., Kolyadin, A. N., Krylov, A. A., Pryamikov, A. D., Biriukov, A. S., Likhachev, M. E., Bufetov, I. A., and Dianov, E. M., "4.4 $\mu \mathrm{m}$ Raman laser based on hollow-core silica fibre," Quantum Electron. 47(5), 491-494 (2017).

[4] Astapovich, M. S., Kolyadin, A. N., Gladyshev, A. V., Kosolapov, A. F., Pryamikov, A. D., Khudyakov, M. M., Likhachev, M. E., and Bufetov, I. A., "Efficient 1556 to $4400 \mathrm{~nm}$ hydrogen Raman laser based on hollow-core silica fiber,” Proc. 2018 International Conference Laser Optics (ICLO), 312-312 (2018).

[5] Aghbolagh, F. B. A., Nampoothiri, V., Debord, B., Gerome, F., Vincetti, L., Benabid, F., and Rudolph, W., "Mid IR hollow core fiber gas laser emitting at $4.6 \mu \mathrm{m}$," arXiv:1811.01140 (2018).

[6] Wang, Y., Couny, F., Roberts, P. J., and Benabid, F., "Low loss broadband transmission in optimized core-shaped Kagome hollow-core PCF," in Conference on Lasers and Electro-Optics (CLEO), OSATechnical Digest (CD) (Optical Society of America, 2010), paper CPDB4.

[7] Wang, Y., Wheeler, N. V., Couny, F., Roberts, P. J., and Benabid, F., "Low loss broadband transmission in hypocycloid-core Kagome hollow-core photonic crystal fiber,” Opt. Lett. 36(5), 669-671 (2011).

[8] Yu, F., and Knight, J. C., "Negative curvature hollow-core optical fiber," IEEE J. Sel. Top. Quantum Electron. 22(2), 4400610 (2016).

[9] Wei, C., Young, J. T., Menyuk, C. R., and Hu, J., "Temperature sensor based on liquid-filled negative curvature optical fibers," OSA Continuum 2(7), 2123-2130 (2019)

[10] Wei, C., Hu, J., and Menyuk, C. R., "Comparison of loss in silica and chalcogenide negative curvature fibers as the wavelength varies," Front. Phys. 4, 30 (2016).

[11] Ta'eed, V., Baker, N. J., Fu, L., Finsterbusch, K., Lamont, M. R. E., Moss, D. J., Nguyen, H. C., Eggleton, B. J., Choi, D.-Y., Madden, S., and Luther-Davies, B., "Ultrafast all-optical chalcogenide glass photonic circuits," Opt. Express 15(15), 9205-9221 (2007).

[12] Hu, J., Menyuk, C. R., Shaw, L. B., Sanghera, J. S., and Aggarwal, I. D., "Computational study of a 3-5 $\mu$ m source that is created by using supercontinuum generation in $\mathrm{As}_{2} \mathrm{~S}_{3}$ chalcogenide fibers with a pump at $2 \mu \mathrm{m}$," Opt. Lett. 35(17), 2907-2909 (2010).

[13] Wei, C., Weiblen, R. J., Menyuk, C. R., and Hu, J., "Negative curvature fibers,” Adv. Opt. Photon. 9(3), 504-561 (2017).

[14] Wei, C., Menyuk, C. R., and Hu, J., "Impact of cladding tubes in chalcogenide negative curvature fibers," IEEE Photon. J. 8(3), 2200509 (2016).

[15] Archambault, J. L., Black, R. J., Lacroix, S., and Bures, J., "Loss calculations for antiresonant waveguides," J. Light. Technol. 11(3), 416-423 (1993). 


\title{
Tubular Anti-resonant Hollow Core Fiber for Visible Raman Spectroscopy
}

\author{
Ian A. Davidson*, Matthew Partridge, John R. Hayes, Yong Chen, Thomas D. Bradley, \\ Hesham Sakr, Shuichiro Rikimi, Gregory T. Jasion, Eric Numkam Fokoua, Marco Petrovich, \\ Francesco Poletti, David J. Richardson and Natalie V. Wheeler \\ Optoelectronics Research Centre, University of Southampton, Southampton, SO17 1BJ, U.K.
}

\begin{abstract}
We report low loss, wide bandwidth, tubular anti-resonant hollow core fibers with a low macro- and micro- bend sensitivity tailored for gas sensing using visible wavelength Raman spectroscopy. The fibers show record low bend sensitivity for anti-resonant fibers operating in this spectral region. A minimum loss of $23.6 \mathrm{~dB} / \mathrm{km}$ is measured at $570 \mathrm{~nm}$ for a fiber spooled on a $30 \mathrm{~cm}$ diameter drum.

Keywords: hollow core fibers, microstructured fibers, Raman spectroscopy, gas sensing *I.A.K.Davidson@soton.ac.uk
\end{abstract}

\section{Introduction}

Hollow core fibers (HCFs) have been extensively investigated for use in gas detection via various techniques in applications ranging from environmental and industrial process monitoring to early stage disease detection through breath analysis [1,2]. As gas can be loaded into the hollow core region of HCFs and interact with the light guided there over very long path lengths (determined only by the fiber's length or attenuation) HCFs can provide enhanced sensitivity. Optical gas detection through Raman spectroscopy is a very promising gas detection technique allowing simultaneous detection of multiple gas species, as Raman scattering from the molecules in a gas sample produces a spectrum with distinct features which can be directly correlated to the gas composition. While Raman scattering in gas is very weak, this can be overcome by the long path length provided by a HCF, enabling high sensitivity gas detection, as well as multi-species identification. So far, the lowest limit of detection reported is $0.2 \mathrm{ppm}$ for methane gas [3].

In most published work on Raman sensing using HCF, the fiber used was a hollow core photonic bandgap fiber (HCPBGF) along with a visible wavelength pump laser, as the Raman scattering co-efficient scales with $\lambda^{-4}$. However, in this spectral region, HC-PBGFs have three key drawbacks for Raman gas sensing applications: firstly, the core size is typically very small $(\sim 5 \mu \mathrm{m}$ [4]) which limits how quickly the fiber can be filled with gas; secondly, HC-PBGF attenuation scales with $\lambda^{-3}$ [5] and thirdly, the fiber's low loss bandwidth is usually limited to $\sim 50 \mathrm{~nm}$ in the visible [4], limiting the range of Raman signals (and hence gas species) which can be simultaneously detected.

Recently, anti-resonant HCFs (AR-HCFs) have emerged as true competitors to HC-PBGFs for many applications. In 2018 an AR-HCF with a record low loss for any HCF design was reported (1.3 dB/km at $1450 \mathrm{~nm}[6])$. As well as reduced attenuation, AR-HCFs can provide wider operating bandwidths and larger core diameters than HC-PBGFs. Possible drawbacks include higher bend sensitivity and lower Raman capture efficiency. Currently, state-of-the-art visible-guiding AR-HCFs have demonstrated low attenuation (13.8 dB/km at $539 \mathrm{~nm}$ [7]) but these fibers are more bend sensitive than the fibers described here, which could limit their suitability for practical deployment. Gao et al. [8], have reported a "conjoined tube" fiber, where each tubular unit consists of two tubes joined together resulting in fiber with a very low loss of $3.8 \mathrm{~dB} / \mathrm{km}$ at $680 \mathrm{~nm}$, showing that the low loses demonstrated in these more structurally complex fibers can scale to the visible wavelength range.

Here, we report the fabrication and characterization of two tubular AR-HCFs, designed for Raman sensing, which rival the optical properties of state-of-the-art tubular fibers, when considering attenuation, macro- and micro- bend loss, moving these fibers closer to deployment in practical devices.

Sixth International Workshop on Specialty Optical Fibers and Their Applications (WSOF 2019), edited by Liang Dong, John M. Ballato, Proc. of SPIE Vol. 11206, 112060R · C 2019 SPIE CCC code: $0277-786 X / 19 / \$ 21 \cdot$ doi: $10.1117 / 12.2548629$ 


\section{Fiber Design, Fabrication and Characterization}

\section{Fiber Design and Fabrication}

For this work we chose to use a 7 element tubular HC-ARF as modelling indicates this is the best compromise between loss, macro-bend loss, and higher order mode suppression [9] for this fiber type. We also chose to limit the core diameter to around $20 \mu \mathrm{m}$, as this appears to be a good balance between overall attenuation (limited by confinement loss, which decreases with core size) and bend sensitivity (which increases with core size, for a given wavelength). Furthermore, this core size is substantially larger than that of a visible wavelength HC-PBGF, enabling faster gas filling times [10]. For the Raman sensing application, we use a 532nm pump laser. The largest Raman shift we aim to detect is for the vibrational hydrogen Stokes line, which, for this pump wavelength, is at $683 \mathrm{~nm}$. Hence, we chose to design our fiber to operate in the second anti-resonant transmission window in order to provide the required bandwidth, without the complexity of targeting fundamental window operation, which for visible wavelengths requires very thin glass membranes and that represents a far harder fabrication challenge.

The two fibers reported here were fabricated in a multi-stage process. Hereaus F300 tubes were initially drawn into a series of uniform capillaries. These were then stacked and fused into an initial preform with a diameter $\sim 2 \mathrm{~cm}$. This stack was then drawn into millimeter-scale canes, and these canes jacketed to make a final fiber preform. During fiber drawing a multi-zone pressurization system was used to control the fibers' final structure [11]. Both fibers were drawn from nominally identical canes, with only the dimensions of the jacket tube (and hence fiber glass outer diameter, OD) changing.

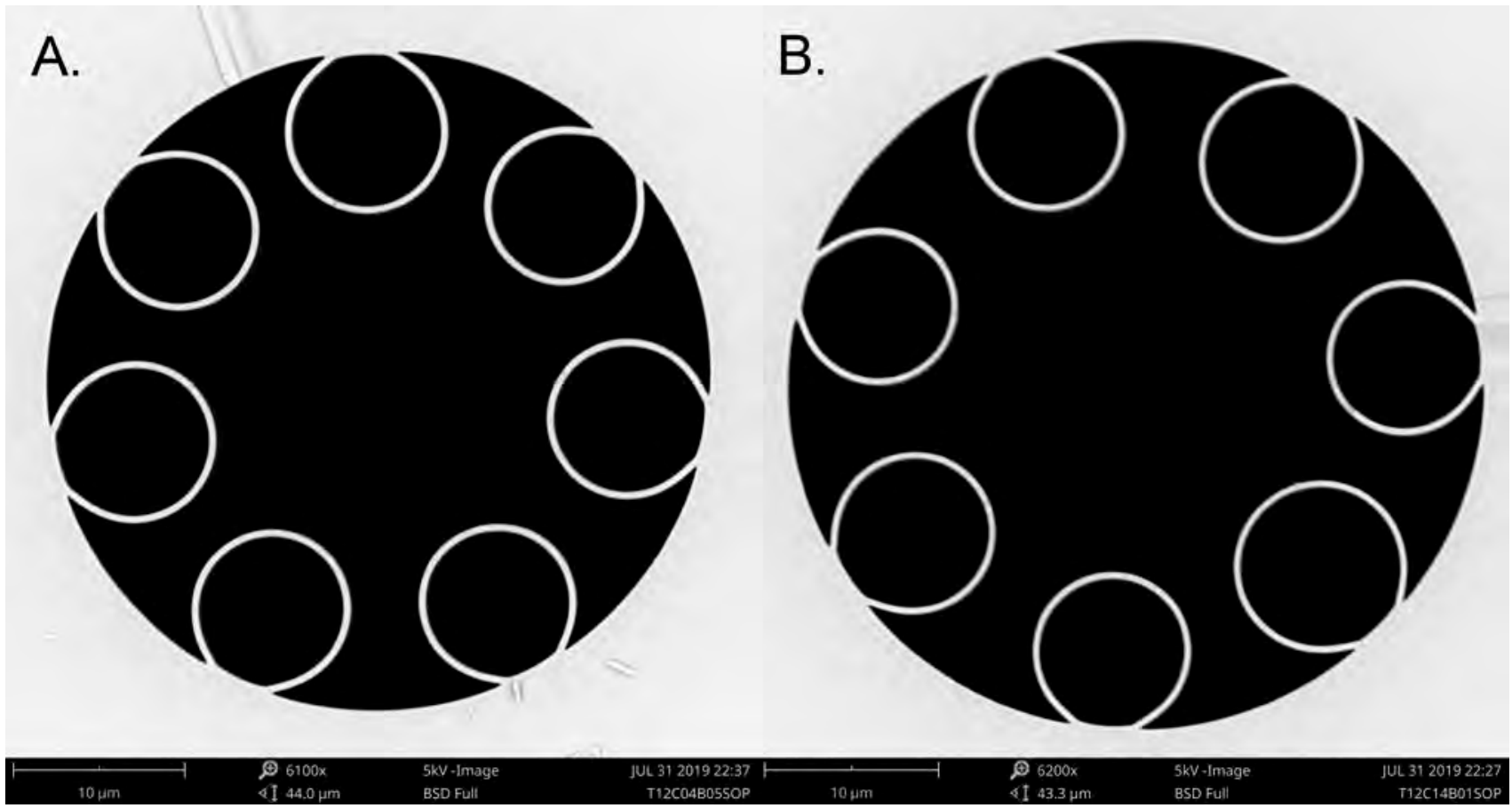

Figure. 1 SEM images of fibers A and B 
Table I: Key structural and optical parameters of fibers A and B (reported here) compared to state-of-the-art tubular fibers from the literature $[7,12]$.

\begin{tabular}{|l|l|l|l|l|}
\hline & Fiber A & Fiber B & Fiber C [7] & Fiber D [12] \\
\hline $\begin{array}{l}\text { Minimum Loss } \\
\text { (wavelength) }\end{array}$ & $\begin{array}{l}35.9 \mathrm{~dB} / \mathrm{km} \\
(619 \mathrm{~nm})\end{array}$ & $\begin{array}{l}23.6 \mathrm{~dB} / \mathrm{km} \\
(570 \mathrm{~nm})\end{array}$ & $\begin{array}{l}13.8 \mathrm{~dB} / \mathrm{km} \\
(539 \mathrm{~nm})\end{array}$ & $\begin{array}{l}80 \mathrm{~dB} / \mathrm{km} \\
(532 \mathrm{~nm})\end{array}$ \\
\hline Loss at $532 \mathrm{~nm}$ & $65.7 \mathrm{~dB} / \mathrm{km}$ & $26.0 \mathrm{~dB} / \mathrm{km}$ & $\sim 17 \mathrm{~dB} / \mathrm{km}$ & $80 \mathrm{~dB} / \mathrm{km}$ \\
\hline 3dB bandwidth & $62 \mathrm{~nm}$ & $71 \mathrm{~nm}$ & $\sim 40 \mathrm{~nm}$ & $\sim 45 \mathrm{~nm}$ \\
\hline $\begin{array}{l}\text { Macro-bend loss } \\
(16 \mathrm{~cm} \text { bend diameter, } 532 \mathrm{~nm})\end{array}$ & $0.1 \mathrm{~dB} / \mathrm{m}$ & $0.12 \mathrm{~dB} / \mathrm{m}$ & $\begin{array}{l}\sim 1.4 \mathrm{~dB} / \mathrm{m} \\
(\text { at } 15 \mathrm{~cm} \text { diameter })\end{array}$ & $0.2 \mathrm{~dB} / \mathrm{m}$ \\
\hline Core diameter $(\mu \mathrm{m})$ & $20.5 \pm 0.2$ & $20.6 \pm 0.1$ & 41 & 26 \\
\hline Cladding tube gap $(\mu \mathrm{m})$ & $3.5 \pm 0.4$ & $3.8 \pm 0.6$ & $2.2-4$ & 7 \\
\hline Membrane thickness $(\mathrm{nm})$ & $440 \pm 10$ & $428 \pm 30$ & 610 & 210 \\
\hline Fiber outer diameter $(\mu \mathrm{m})$ & 140 & 223 & $\begin{array}{l}\text { Not Available in } \\
\text { Literature }\end{array}$ & 200 \\
\hline
\end{tabular}

\section{Fiber Characterization}

\section{(1) Structural Characterization}

The physical dimensions of fibers A and B were measured from scanning electron micrograph (SEM) images (see Figure 1) taken using a Phenom ProX desktop SEM system. The key structural parameters are detailed in Table I. As expected the features of the microstructure are very similar (as the fibers are from similar canes). One small difference is that the membrane thicknesses in fiber B are slightly thinner, which results in the transmission window shifting to shorter wavelengths.
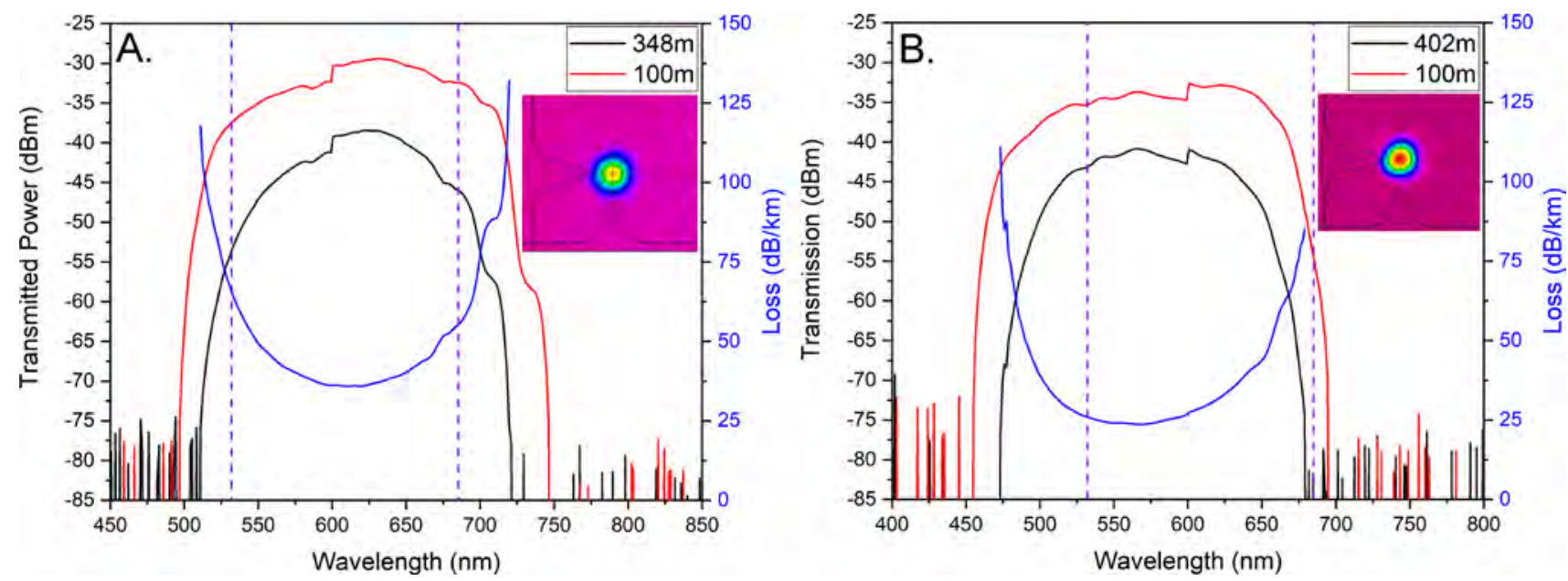

Figure 2. The transmission (red and black curves) and loss (blue curves) are shown for fiber A and B respectively, purple lines have been added to show the 532 and $683 \mathrm{~nm}$ wavelengths as a guide to the eye. The obvious step feature at $600 \mathrm{~nm}$ is due to the OSA switching gratings at that wavelength. Inset are beam profile images.

(2) Transmission and Loss Measurements

The fibers' transmission and loss (Figure 2) were characterized using a NKT SuperK COMPACT super-continuum laser system and a Yokogawa AQ-6315A optical spectrum analyzer (OSA) using an SMF28 launch fiber. As expected, the 
transmission window of fiber B is shifted $\sim 40 \mathrm{~nm}$ towards shorter wavelengths. Fiber attenuation was measured using the cutback technique; fibers $\mathrm{A}$ and B were cut from $348 \mathrm{~m}$ and $402 \mathrm{~m}$ respectively to $100 \mathrm{~m}$, to ensure the modal content was consistent between both lengths and to enable comparison between the two measurements. During the measurements the fibers were spooled on $\sim 30 \mathrm{~cm}$ diameter bobbins under $20 \mathrm{~g}$ of tension. The key loss values for both fibers are summarized in Table I.

The beam profile images inset in Figure 2 were recorded using a silicon CCD camera (Spiricon Scorpion, SCOR 20) and aspheric collimating lens (Thorlabs C260TME). The fibers were illuminated with a $632.8 \mathrm{~nm}$ HeNe laser (Melles Griot 05-LHR-151-181), which was directly launched into the core of the HCF using another aspheric lens (Thorlabs C260TME).

The low-loss bandwidth of these fibers is essential for their use in Raman spectroscopic gas measurements as it enables the simultaneous detection of multiple species that is a key feature of this technique. An example of this is shown in Figure 3, where a 10m length of fiber A was filled with 3 bar of $3 \%$ hydrogen in nitrogen gas, and then pumped with $145 \mathrm{~mW}$, and clearly shows the vibrational Raman emission ( $1^{\text {st }}$ Stokes) lines of both the nitrogen (at $\left.2330 \mathrm{~cm}^{-1}\right)$ and hydrogen $\left(\right.$ at $\left.4160 \mathrm{~cm}^{-1}\right)$, along with some trace oxygen $\left(580 \mathrm{~nm} / 1556 \mathrm{~cm}^{-1}\right)$ and water vapor $\left(\mathrm{OH}^{-}\right.$at $\left.660 \mathrm{~nm} / 3645 \mathrm{~cm}^{-1}\right)$.

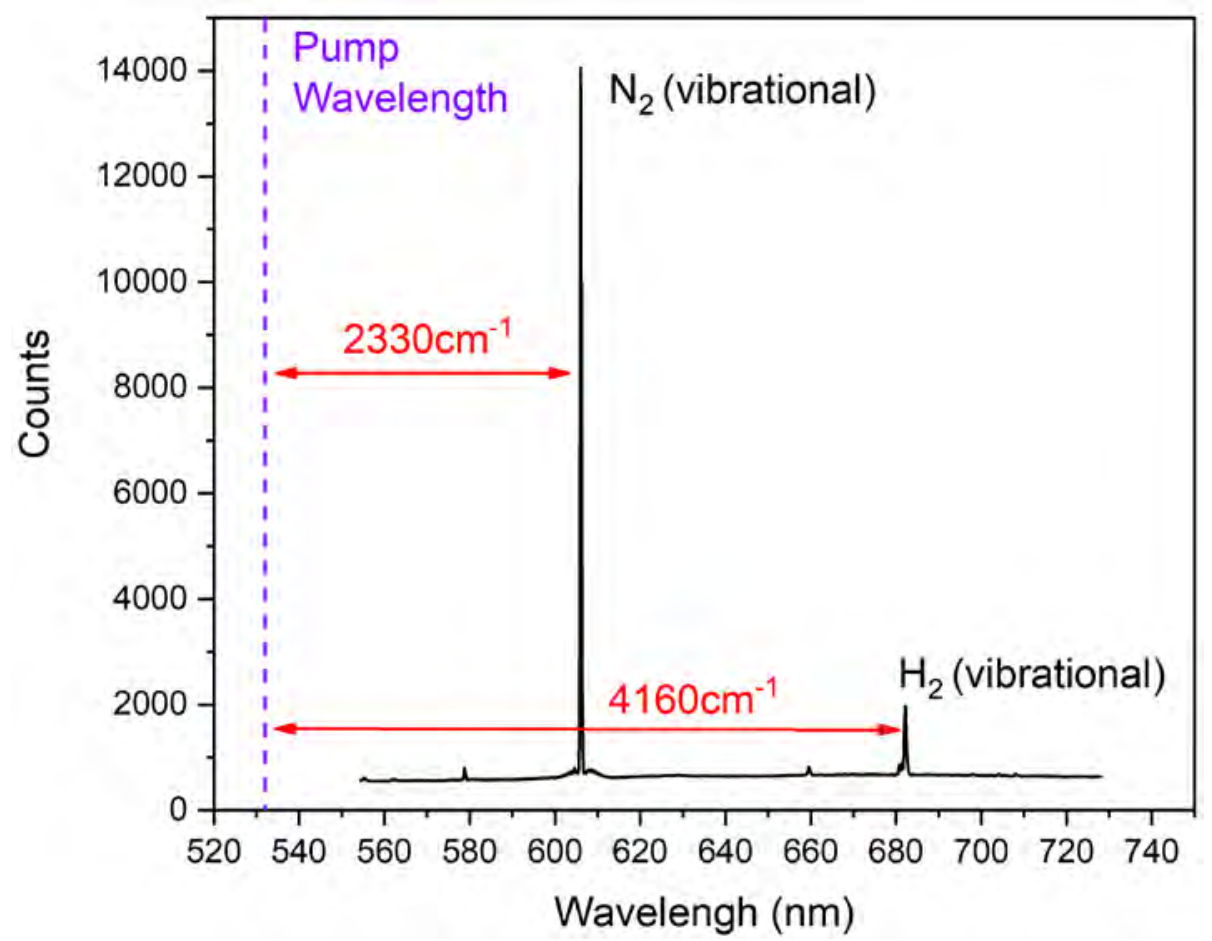

Figure 3. Results of a Raman measurements made with fiber A showing the nitrogen and hydrogen vibrational Raman emission $\left(1^{\text {st }}\right.$ Stokes) lines at $\sim 2330 \mathrm{~cm}^{-1}\left(\mathrm{~N}_{2}\right)$ and $4160 \mathrm{~cm}^{-1}\left(\mathrm{H}_{2}\right)$.

(3) Macro-bend Measurements

Macro-bend measurements were made using a series of precision inscribed grooves in an acrylic plate. Light from a halogen lamp was launched into the fiber using an SMF28 patch cable mounted on a micro-positioning stage. The transmitted power was measured using the same OSA as above. As the fiber length used was only $4 \mathrm{~m}$, and the modal content of the fiber was not directly measured, the reported bend-losses represent the worst case scenario, as higher order modes are expected to be more bend-loss sensitive than the fundamental. 
The results of the macro-bend measurements are shown in Figure 4 at $532 \mathrm{~nm}$ and $683 \mathrm{~nm}$, the extremes of our desired operating wavelength range. The results show that down to bend diameters of $13 \mathrm{~cm}$, fibers A and B show very low bend sensitivity. Furthermore, in Figure 4, the macro-bend performance of two state-of-the-art tubular ARFs from the literature, fibers $\mathrm{C}$ and $\mathrm{D}[7,12]$ are also shown. Comparing fibers A and B with these state-of-the-art fibers, shows that fibers $\mathrm{A}$ and $\mathrm{B}$ demonstrate much lower bend sensitivity.
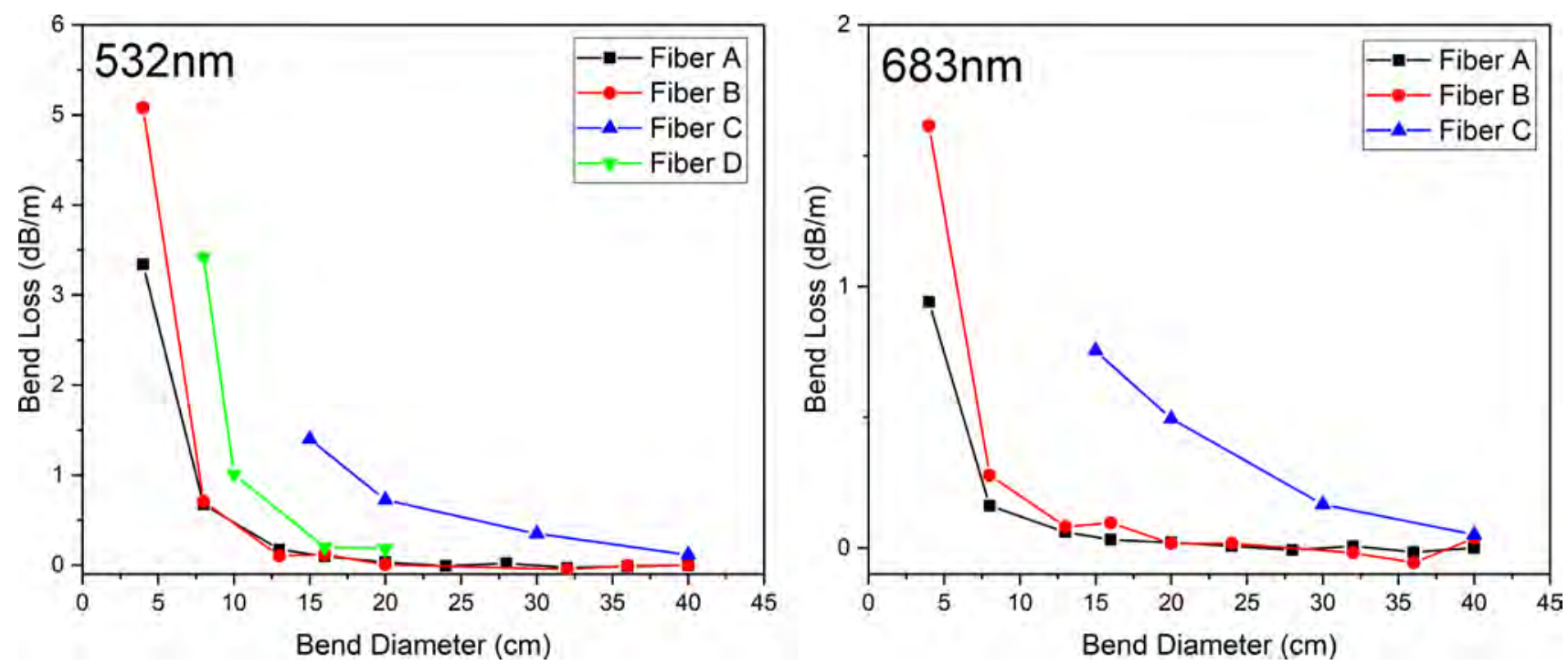

Figure 4. Macro-bend loss vs. bend diameter for fibers A-D. The data for fibers C and D were taken from ref. [7] and [12].

(4) Micro-bend Measurements

Micro-bend sensitivity is another important fiber loss mechanism, and relates to losses induced by subjecting a fiber to millimeter and sub-millimeter lengths scale bends. The dynamics of this mechanism are different to those of traditional macro-bending (induced by centimeter or larger length scale bends) and hence this needs to be evaluated separately [13]. It can become a factor when a fiber is being deployed in a non-ideal environment, outside the lab for example, where the fiber may end up pressed against a rough surface or point strains may induces localized fiber distortions.

Micro-bend measurements were made using a purpose built system with structural perturbations at a fixed spatial frequency over which fibers can be tensioned. Set amounts of stress were applied to $100 \mathrm{~m}$ of each fiber (in roughly $25 \mathrm{MPa}$ steps) and the transmission through the fiber measured at each step. The transmission set-up used for these measurements was identical to that used for the cutback measurements. The results for both fibers are shown in Figure 5.

As can clearly be seen in these measurements, fiber B is substantially less sensitive to micro-bend, as it is necessary to apply nearly $50 \mathrm{MPa}$ of fiber stress (200g of tension) before any transmission degradation is seen, whereas the transmission of fiber A starts to degrade when even minimal amounts of fiber stress/tension are applied. This effect is further magnified as the smaller glass dimension of fiber A (compared to B) mean even a minimal tension (of the order of $10-20 \mathrm{~g})$ is sufficient for performance degradation to occur. 

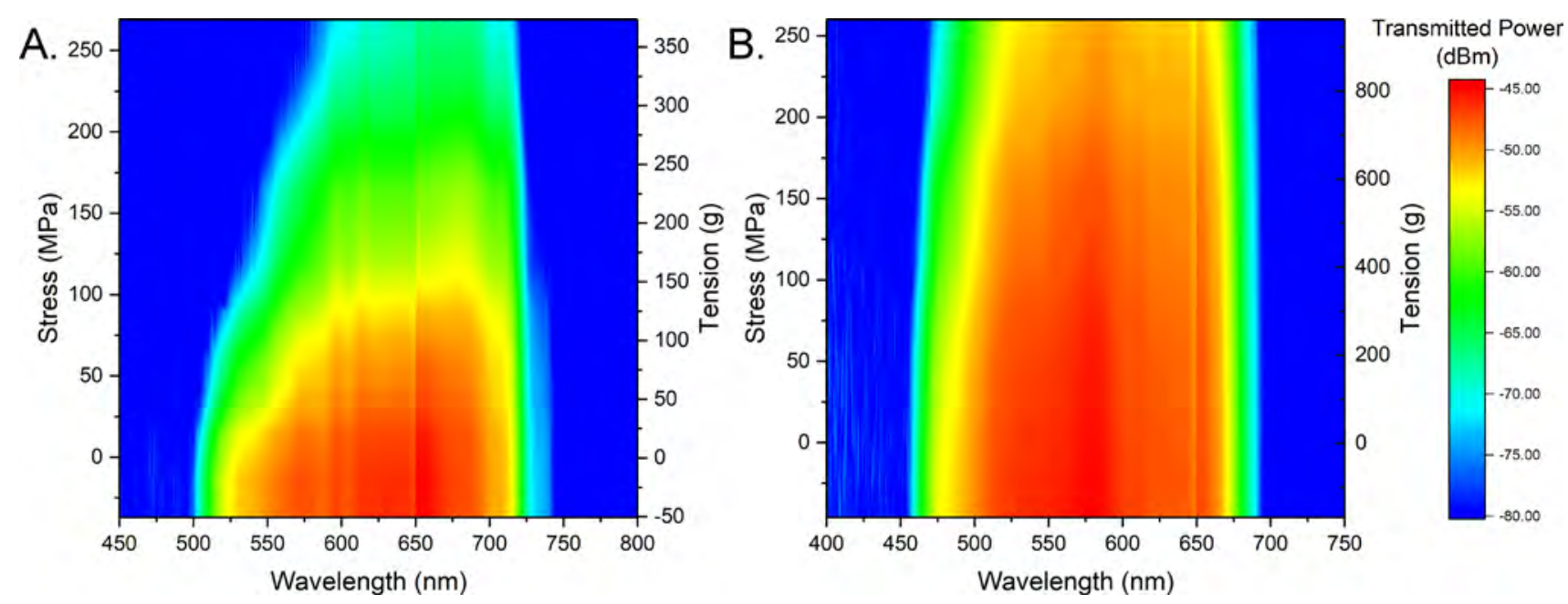

Figure 5. Micro-bend measurements of Fiber A (left) and Fiber B (right). The linear feature at 650nm is due to the OSA switching gratings at that wavelength for this measurement range.

Although the fibers reported here perform well, it is likely further improvements can be made in terms of loss without compromising their low bend sensitivity through design and fabrication optimization. This is an area that to explore in future through efforts to further optimize the combination of core diameter, tubular element gaps and size, and fiber OD, to see how far we can push this technology. Ultimately, the introduction of nested elements (an additional tube within the existing tubular elements) during fabrication seems a more complete solution to the problem of realizing low loss fibers with excellent bend-loss performance, albeit at the expense of greater fabrication complexity $[6,8,14]$.

\section{Conclusions}

We report on work towards realizing practical, low loss, wide bandwidth HC-ARFs with reduced bend sensitivity for the visible spectral window. Two fibers are reported, A and B, which have minimum losses of $35.9 \mathrm{~dB} / \mathrm{km}$ and $23.6 \mathrm{~dB} / \mathrm{km}$ respectively and exhibit record low bend sensitivity for visible-guiding tubular AR-HCF. This performance makes these fibers highly attractive for a wide range of applications, including Raman spectroscopy. As structurally the key difference between the two fibers is the glass cladding thickness, the micro-bend performance has been characterized and it was found that, due to the thicker cladding of fiber B, its micro-bend sensitivity is substantially reduced; this likely also accounts for the difference in the two fibers overall attenuation. Apart from demonstrating progress in HCF fabrication at these wavelengths, these results also demonstrate the need for thorough HCF characterization beyond simple attenuation measurements (when in a loosely spooled condition) to fully assess their suitability for deployment in practical devices, which is very timely considering recent breakthroughs in the fabrication of low loss HCFs.

\section{Acknowledgements}

We gratefully acknowledge support from Innovate UK (project 103973), EPSRC Programme grant Airguide (EP/P030181/1), the Royal Society, and the Royal Academy of Engineering. We also thank Mark Mason and Adrian Potasnik for assistance with fiber rewinding and technical support. 


\section{References}

[1] Hodgkinson, J. and Tatam, R. P., "Optical gas sensing: a review," Meas. Sci. Technol. 24, 012004, 1-59 (2013).

[2] Jin, W., Ho, H.L., Cao, Y.C., Ju, J. and Qi, L.F., "Gas detection with micro- and nano- engineered optical fibers," Optical Fiber Technology, 19(6) B, 741-759 (2013).

[3] Hanf, S., Keiner, R., Yan, D., Popp, J. and Frosch, T., "Fiber-Enhanced Raman Multigas Spectroscopy: A Versatile Tool for Environmental Gas Sensing and Breath Analysis," Analytical Chemistry 86(11), 5278-5285 (2014).

[4] Moseley, P.J., Huang, W.C., Welch, M.G., Mangan, B.J., Wadsworth, W.J. and Knight, J.C., "Ultrashort pulse compression and delivery in a hollow-core photonic crystal fiber at 540nm wavelength," Optics Letters, 35(21) 3589-3591 (2010).

[5] Chen, Y., Wheeler, N. V., Baddela, N. K., et al., "Understanding Wavelength Scaling in 19-Cell Core hollow-core photonic bandgap fibers," Proc. Optical Fiber Communication Conference, OSA Technical Digest (online) (Optical Society of America, 2014), M2F.4 (2014).

[6] Bradley, T. D., Hayes, J. R., Chen, Y., et al., "Record low-loss $1.3 \mathrm{~dB} / \mathrm{km}$ data transmitting antiresonant hollow core fibre," Proc. Eur. Conf. on Optical Communication, PDP Th3F.2, (2018).

[7] Chafer, M., Delahaye, F., Amrani, F., Debord, B., Gerome, F. and Benabid, F., "1 km long HC-PCF with losses at the fundamental Rayleigh scattering limit in the green wavelength range," Conference on Lasers and Electro-Optics (CLEO) 2018, SF1K.3, (2018).

[8] Gao, S.-F., Wang Y. Y., Ding W., Hong Y. F., and Wang P., "Hollow-Core Conjoined-Tube Negative-Curvature Fiber with Loss approaching Rayleigh Scattering Limit of Silica." In CLEO: Science and Innovations, pp. STh1L-6. Optical Society of America (2019).

[9] Hayes, J R., Sandoghchi, S.R., Bradley, T.D., Liu, Z., Slavík, R., Alonso Gouveia, M., Wheeler, N.V. et al. "Antiresonant hollow core fiber with an octave spanning bandwidth for short haul data communications." Journal of Lightwave Technology 35(3), 437-442 (2017)

[10] Dicaire, I., Beugnot, J.C. and Thevenaz, L., "Analytical modeling of the gas-filling dynamics in photonic crystal fibers," Applied Optics, 49(24) 4604- 3609, (2010).

[11] Jasion, G.T., Hayes, J.R., Wheeler, N.V., Chen, Y., Bradley, T.D., Richardson, D.J., and Poletti, F., "Fabrication of tubular antiresonant hollow core fibers: modelling, draw dynamics and process optimization," Opt. Express 27, 20567-20582 (2019)

[12] Gao, S., Wang, Y.Y., Liu, X-L., Hong, C., Gu, S. and Wang, P., "Nodeless Hollow-Core Fiber for Visible Spectral Range," Optics Letters, 42(1) 61-64 (2017).

[13] Fokoua, E. N., Chen, Y., Richardson, D. J., \& Poletti, F., "Microbending effects in hollow-core photonic bandgap fibers". In ECOC 2016; 42nd European Conference on Optical Communication (pp. 1-3). VDE (2016).

[14] Sakr, H., Bradley, T. D., Hong, Y., Jasion, G. T., et al. "Ultrawide Bandwidth Hollow Core Fiber for Interband Short Reach Data Transmission” In Optical Fiber Communication Conference (pp. Th4A-1). Optical Society of America (2019) 


\title{
Extruded Chalcogenide Antiresonant Hollow Core Fiber for Mid-IR Laser Delivery
}

\author{
Juliano G. Hayashi", Andrea Ventura, Gregory T. Jasion, Jaroslaw Cimek, Fedia B. Slimen, Nicholas \\ White, Hesham Sakr, Natalie V. Wheeler, and Francesco Poletti \\ Optoelectronics Research Centre, University of Southampton, SO17 1BJ, UK
}

\begin{abstract}
We report modeling and fabrication of chalcogenide antiresonant hollow core fibers aimed at laser delivery in the midIR $(4.5-11 \mu \mathrm{m})$. The results indicate that, with further improvements in fiber's structure, very promising performances could be achieved.
\end{abstract}

Keywords: hollow core microstructured fiber, chalcogenide glass, mid-IR, extrusion.

*igh1m18@soton.ac.uk;

\section{I - Introduction}

The delivery of mid-IR laser radiation in a flexible optical fiber and with good beam quality would be beneficial for several applications, such as laser cutting, medical laser surgery [1], non-linear imaging microscopy [2], and additive manufacturing [3]. Antiresonant hollow core microstructured fibers (AR-HCFs) [4] are a promising candidate for such tasks due to some unique properties: very simple design (amenable to fabrication using different techniques), extremely low overlap of the fundamental optical mode with the cladding material (down to $2-5 \times 10^{-5}$ ), ultra-low nonlinearities, low scattering, high damage threshold, excellent beam-quality, and potentially very low-loss and effectively single-mode operation [5]. AR-HCFs made of silica glass can exhibit low-loss when operating at wavelengths $(\lambda)$ as large as $\sim 4.5 \mu \mathrm{m}$ $[6,7]$. At longer wavelengths, the attenuation of silica ultimately limits the achievable losses to several $\mathrm{dB} / \mathrm{m}$. A different glass is required to reduce such losses and extend the fiber's operation further into the mid-IR. Unlike silica, mid-IR transmitting glasses are usually not available in tubes, making preform assembly via the stack-and-draw technique more challenging. Besides, they have inferior mechanical strength compared to silica, and considerably steeper viscosity vs temperature curves. Consequently, the fabrication of mechanically robust microstructure fibers made of these materials, with transversally regular and longitudinally uniform structure, is noticeably challenging $[4,8]$. Here, we present our initial work on the fabrication of low-loss AR-HCFs made of chalcogenide glass (IG3, $\mathrm{Ge}_{30} \mathrm{As}_{13} \mathrm{Se}_{32} \mathrm{Te}_{25}$ ) for laser delivery applications in the mid-IR $(4.5-11 \mu \mathrm{m})$. The use of a single step preform extrusion allows reproducible and regular preform fabrication, while the incorporation of a fluorinated polymer coating co-drawn with the fiber helps to improve its mechanical properties as strength and flexibility, protects the glass from the external environment, and addresses safety concerns due to the toxicity of the glass.

\section{II - Results and discussion}

The AR-HCF preforms were fabricated by extrusion from commercial billets of chalcogenide glass IG3 $\left(\mathrm{Ge}_{30} \mathrm{As}_{13} \mathrm{Se}_{32} \mathrm{Te}_{25}\right.$ - Vitron [9], $28 \mathrm{~mm}$ in diameter and $33 \mathrm{~mm}$ in height). High quality preforms with no visible

Sixth International Workshop on Specialty Optical Fibers and Their Applications (WSOF 2019),

edited by Liang Dong, John M. Ballato, Proc. of SPIE Vol. 11206, 112060S · C 2019 SPIE

CCC code: $0277-786 X / 19 / \$ 21 \cdot$ doi: $10.1117 / 12.2548580$ 
crystallization and symmetric structures, as seen in Fig. 1(a), were obtained after the optimization of the die design and the extrusion parameters (temperature and load). The preform shown in Fig. 1(a) has an outside diameter (OD) of $14 \mathrm{~mm}$, a core diameter around $6.5 \mathrm{~mm}$, a usable length of $25 \mathrm{~cm}$, and six capillary elements. The capillaries have ODs varying from 1.6 to $1.9 \mathrm{~mm}$ and thicknesses varying from 470 to $650 \mu \mathrm{m}$, indicating that more work is needed to improve the regularity of the die and the extrusion process. The chalcogenide preform was inserted in a FEP (Fluorinated Ethylene Propylene) polymer tube with an $\mathrm{ID}=15 \mathrm{~mm}$ and an $\mathrm{OD}=17 \mathrm{~mm}$ to enhance its mechanical strength, allowing fiber drawing under moderate/high pulling tension, which is required to avoid undesired deformation of the fiber's structure. The co-drawing of the IG3 glass and FEP polymer, which is not trivial and has been previously established by our research group in the fabrication of mid-IR fiber bundles [10], considerably improves the mechanical strength and flexibility of the drawn fibers. Before the fabrication attempt of the fibers, COMSOL Multiphysics was employed to study the optical loss (including both confinement and glass absorption) of different IG3 AR-HCF structures. Figure 1(b) shows the target AR-HCF that resulted from numerical optimization. The fiber has six non-touching capillaries with a thickness of $7.5 \mu \mathrm{m}(\mathrm{OD}=115 \mu \mathrm{m}, \mathrm{ID}=100 \mu \mathrm{m})$, a core diameter of $190 \mu \mathrm{m}$ and an overall $\mathrm{OD}=500 \mu \mathrm{m}$. According to Fig. 1(c), our proposed structure exhibits five transmission bands between 4.5-11 $\mu \mathrm{m}$ with the following overall losses of the fundamental mode: $0.04 \mathrm{~dB} / \mathrm{m}$ at $\lambda=5 \mu \mathrm{m}, 0.02 \mathrm{~dB} / \mathrm{m}$ at $\lambda=5.8 \mu \mathrm{m}, 0.05 \mathrm{~dB} / \mathrm{m}$ at $\lambda=7 \mu \mathrm{m}, 0.02 \mathrm{~dB} / \mathrm{m}$ at $\lambda=8.4 \mu \mathrm{m}$, and $0.06 \mathrm{~dB} / \mathrm{m}$ at $\lambda=10.6 \mu \mathrm{m}$ (blue curve). Such expected losses are very promising, particularly at $\lambda=10.6 \mu \mathrm{m}$, where a loss about two orders of magnitude smaller than the smallest measured loss reported in the literature for AR-HCFs made of mid-IR glasses seems possible [11].

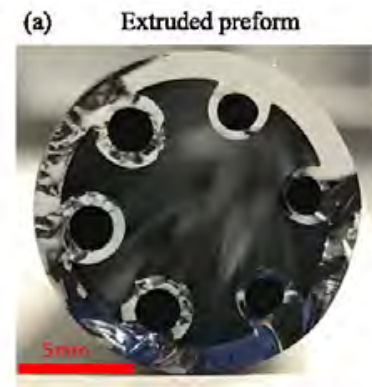

(d) Fiber 1 (2 mbar - inside cap.)

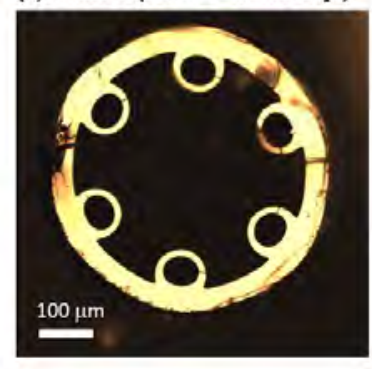

(b) Proposed fiber

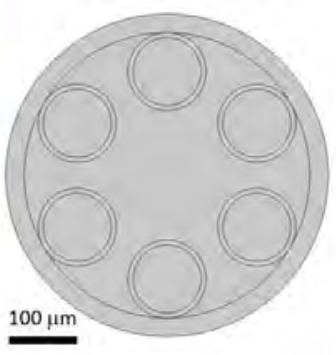

(e) Fiber 2 (6 mbar - inside cap.)

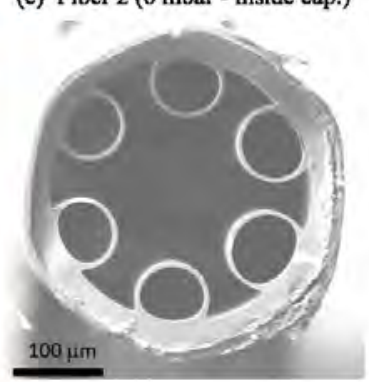

(c)

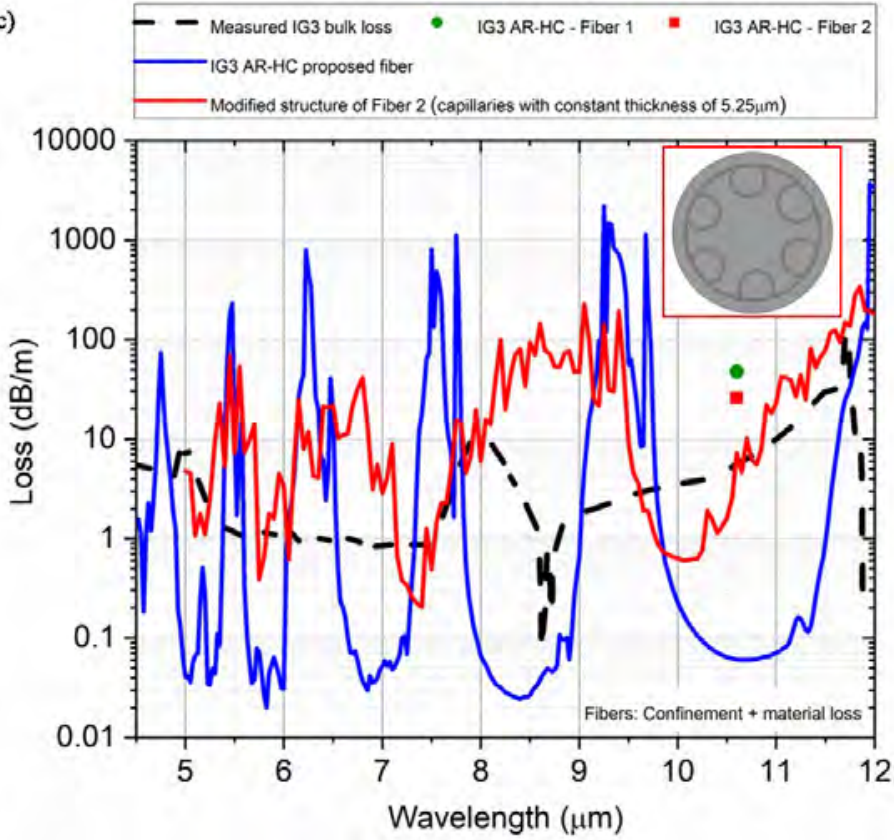

Figure 1. (a) IG3 AR-HCF preform fabricated by extrusion of a cylindrical IG3 billet. The preform has OD=14 mm and length of $25 \mathrm{~cm}$. (b) Our proposed structure targeted for low-loss performance in the mid-IR (4.5-11 $\mu \mathrm{m})$. (c) Material loss of bulk IG3 glass (black dash line), simulated overall losses (material + confinement) of our proposed structure (blue curve), fabricated fibers (green dot - Fig. 1(d), red square - Fig. 1(e)), and the modified structure of Fiber 2 (capillaries with constant thickness of $5.25 \mu \mathrm{m}$ - inset). Cross-sectional images of the IG3 AR-HC fibers fabricated pressuring the inside of the capillaries of the preform seen in (a) with 2 mbar (d) and 6 mbar (e). 
In the first fabrication attempted of our proposed structure, the six capillaries of the preform seen in Fig 1(a) were pressurized with argon during the drawing $\left(\mathrm{T} \sim 360^{\circ} \mathrm{C}\right)$, while the core of the preform was kept at atmospheric pressure. Fiber 1 (Fig. 1(d)) was achieved applying 2 mbar of pressure, resulting in an AR-HCF structure with a core diameter $\sim 285 \mu \mathrm{m}$, capillaries with IDs varying from 52 to $65 \mu \mathrm{m}$, and thicknesses varying from 14 to $17 \mu \mathrm{m}$. Fiber 2 (Fig. 1(e)) was achieved by applying 6 mbar of pressure, resulting in an AR-HCF structure with core diameter $\sim 160 \mu \mathrm{m}$, capillaries with IDs varying from 60 to $85 \mu \mathrm{m}$, and thicknesses varying from 7.5 to $4.5 \mu \mathrm{m}$. In both cases, mechanically robust and longitudinally uniform fibers of several meters to tens of meters were obtained, indicating that the draw process is stable and reproducible. Numerical modeling of both fibers from the fabricated cross-sectional images indicates a fundamental mode loss of $47 \mathrm{~dB} / \mathrm{m}$ (Fiber 1) and $26 \mathrm{~dB} / \mathrm{m}$ (Fiber 2) at $\lambda=10.6 \mu \mathrm{m}$ (green dot and red square in Fig. 1(c), respectively). As expected, Fiber 1 exhibits high loss due to the large thickness of the capillaries and the large lateral gaps between them, which results in the need to operate in very high order (hence lossy) antiresonant window and with high confinement losses, respectively. Surprisingly, Fiber 2 also exhibits a high loss in spite of its high symmetry, small thickness of the capillaries (mostly varying from 5-6 $\mu \mathrm{m}$ ), and a small gap between the capillaries $(\sim 35-40 \mu \mathrm{m})$. To

clarify this high loss, we also simulated a modified version of Fiber 2 containing capillaries with a constant thickness of $5.25 \mu \mathrm{m}$ (inset of Fig. 1(c) and red curve). Contrary to the Fiber 2 structure, this modified version exhibits noticeable transmission bands between $4.5-11 \mu \mathrm{m}$ with the following overall losses of the fundamental mode: $1.10 \mathrm{~dB} / \mathrm{m}$ at $\lambda=5.2 \mu \mathrm{m}, 0.38 \mathrm{~dB} / \mathrm{m}$ at $\lambda=5.75 \mu \mathrm{m}, 0.20 \mathrm{~dB} / \mathrm{m}$ at $\lambda=7.4 \mu \mathrm{m}, 0.63 \mathrm{~dB} / \mathrm{m}$ at $\lambda=10 \mu \mathrm{m}$ (red curve in Fig. $1(\mathrm{c})$ ). At $\lambda=10.6 \mu \mathrm{m}$, the modified Fiber 2 exhibits loss of $7.2 \mathrm{~dB} / \mathrm{m}$, which indicates that the non-uniformities in the thickness of the capillaries of Fiber 2 are responsible for part of the deviation of the loss in comparison to our proposed/targeted structure. The remaining deviation of the loss between the modified Fiber 2 and our proposed fiber is probably due to a combination of two issues: the core diameter $(160 \mu \mathrm{m}$ instead of the $190 \mu \mathrm{m}$ of the proposed structure) and the high contact area between the capillaries and the glass jacket. A further numerical study is in progress to fully clarify this.

\section{III - Conclusion}

We have demonstrated the successful fabrication of symmetric IG3 AR-HC preforms ( $25 \mathrm{~cm}$ long) obtained by direct extrusion. Initial fabrication attempts pressurizing the six capillaries in the preform resulted in two sets of reasonably symmetric AR-HC fibers. Whilst their structure is still far from the optimized target, the stability of the fiber draw and the mechanical robustness of the achieved fibers, resulting from the incorporation of a polymer coating, indicate that the proposed fabrication approach has the potential to produce high performance fibers. Numerical modeling indicates that the loss of the fabricated fibers at $\lambda=10.6 \mu \mathrm{m}$ is still high, probably due to the sub-optimum core size, to variations in the thickness of the capillaries along their circumference, and the high contact area between the capillaries and the glass jacket. Future work will focus on the improvement of the preform extrusion (more symmetric capillaries with smaller contact area with the glass jacket), on fine-tuning of the fabrication parameters and the full characterization of the fibers in the mid-IR.

\section{Acknowledgment}

We gratefully acknowledge funding from the U.K. Engineering and Physical Sciences Research Council under Grant EP/P030181/1 (Future Photonics Hub), the ERC LightPipe project (grant agreement $n^{\circ}$ 682724) and the Royal Society (Newton International Fellowship - NF170629). 


\section{References}

[1] Oh, H.S. and Kim, J.S., [Clinical Application of $\mathrm{CO}_{2}$ Laser: Optimisation and Application], 357 (2012).

[2] Petrov, G.I. and Yakovlev, V.V., "Resonantly enhanced coherent anti-Stokes Raman scattering”, Proc. SPIE 10498, 104981R (2018).

[3] Zavala-Arredondo, M., Boone, N., Willmott, J., Childs, D.T., Ivanov, P., Groom, K.M. and Mumtaz, K., "Laser diode area melting for high speed additive manufacturing of metallic components," Mater. Des. 117, 305-315 (2017).

[4] Shiryaev, V. S., Kosolapov, A. F., Pryamikov, A. D., Snopatin, G. E., Churbanov, M. F., Biriukov, A. S., Kotereva, T. V., Mishinov, S.V., Alagashev, G. K., and Kolyadin, A. N., "Development of technique for preparation of As $2 \mathrm{~S}_{3}$ glass preform for hollow core microstructured optical fibers", J. Optoelectron. Adv. M. 16 (9-10), 1020 - 1025 (2014).

[5] Poletti, F., "Nested antiresonant nodeless hollow core fiber," Opt. Express 22(20), 23807-23828 (2014).

[6] Kolyadin, A., Kosolapov, A. F., Pryamikov, A. D., Biriukov, A. S., Plotnichenko, V. G., and Dianov, E. M., "Light transmission in negative curvature hollow core fiber in extremely high material loss region," Opt. Express 21(8), 9514-9519 (2013).

[7] Yu, F., and Knight, J. C., "Spectral attenuation limits of silica hollow core negative curvature fiber," Opt. Express 21(18), 2146621471 (2013).

[8] Kosolapov, A. F., Pryamikov, A. D., Biriukov, A. S., Shiryaev, V. S., Astapovich, M. S., Snopatin, G. E., Plotnichenko, V. G., Churbanov, M. F. and Dianov, E. M., "Demonstration of CO2-laser power delivery through chalcogenide-glass fiber with negative curvature hollow core,” Opt. Express 19(25), 25273 - 25278 (2011).

[9] Vitron, https://www.vitron.de/datasheets/VITRON\%20IG-3\%20Datenblatt\%20Juni\%202014\%20.pdf (31 July 2019).

[10] Ventura, A., Slimen, F.B., Lousteau, J., White, N., Masoudi, A., Janicek, P. and Poletti, F., "Flexible Mid-IR fiber bundle for thermal imaging of inaccessible areas," Opt. Express 27(15), 20259-20272 (2019).

[11] Gattass, R.R., Rhonehouse, D., Gibson, D., McClain, C.C., Thapa, R., Nguyen, V.Q., Bayya, S.S., Weiblen, R.J., Menyuk, C.R., Shaw, L.B. and Sanghera, J.S., "Infrared glass-based negative-curvature anti-resonant fibers fabricated through extrusion," Opt. Express 24(22), 25697-25703 (2016). 


\title{
Non-invasive characterization of hollow-core single-ring fibers using whispering gallery mode spectroscopy
}

\author{
Michael H. Frosz*a, Riccardo Pennetta ${ }^{\mathrm{a}}$, Michael T. Enders ${ }^{\mathrm{a}}$, Goran Ahmed ${ }^{\mathrm{a}}$, Philip St.J. Russella,b \\ ${ }^{a}$ Max Planck Institute for the Science of Light and ${ }^{b}$ Department of Physics, Friedrich-Alexander- \\ Universität, Staudtstr. 2, 91058 Erlangen, Germany
}

\begin{abstract}
Non-invasive whispering gallery mode spectroscopy is applied to measure the internal microstructure of a hollow-core fiber with cladding capillaries. The capillaries are measured with sub-micron accuracy in sub-cm steps along the fiber.

Keywords: hollow-core microstructured fibers; photonic crystal fibers; fiber drawing

*michael.frosz@mpl.mpg.de; phone +49 091317133219

\section{Introduction}

Single-ring hollow-core photonic crystal fiber (SR-PCF), consisting of a ring of thin-walled glass capillaries surrounding a central hollow core, can offer remarkably low transmission loss [1], and is finding applications in, e.g., wavelength conversion and pulse compression in gases, and high-power beam delivery. We recently presented a novel technique for continuously measuring the capillary diameter (from which all other structural parameters can be deduced) during fiber drawing in real-time [2]. This is expected to improve the yield of useful fiber lengths, as well as offering better control of structural uniformity along the fiber. The technique relies on exciting whispering gallery modes (WGMs) in the capillary walls, measuring the spectrum, and fast data analysis to extract the capillary circumference. We here report the use of the technique for post-draw measurements in carefully studying variations between capillaries at a fixed cross-section of a SR-PCF, as well as in quantifying fluctuations in capillary size along the fiber in sub-cm steps.
\end{abstract}

\section{Whispering gallery mode spectroscopy}

Analysis shows that the frequency spacing between successive WGMs fulfils the condition:

$$
\frac{\delta v}{v}=\frac{v_{\mathrm{G} m}}{\pi d_{c} v}=\frac{c}{\pi d_{c} v}\left(n_{m}(v)+v \frac{\partial n_{m}(v)}{\partial v}\right)^{-1}=\frac{c}{\pi d_{c} v} \frac{1}{n_{\mathrm{G} m}(v)},
$$

where $n_{\mathrm{G} m}$ is the group index, $v_{\mathrm{G} m}$ the group velocity, $v$ and $\lambda$ the optical frequency and wavelength, $c$ the vacuum velocity of light, $d_{\mathrm{c}}$ the mean diameter of the capillary and $n_{\mathrm{m}}$ the frequency-dependent effective index of the $m$-th waveguide mode in the capillary walls, which can be calculated using standard planar waveguide theory [3]. Figure 1(a) shows how WGMs can be excited by simply illuminating the fiber from the side with a collimated beam. The WGM spectrum can then be measured by collecting the light exiting the fiber at an angle specific to the capillary. Using a fixed collection angle $\left(\sim 60^{\circ}\right.$ shifted from the input beam $)$ and rotating the fiber, we measured the sizes of all the capillaries in a SR-PCF while also scanning along the fiber ( $60 \mathrm{~cm}$ length), as shown in Fig. 1(b).

Sixth International Workshop on Specialty Optical Fibers and Their Applications (WSOF 2019), edited by Liang Dong, John M. Ballato, Proc. of SPIE Vol. 11206, 112060T · (C) 2019 SPIE

CCC code: $0277-786 \mathrm{X} / 19 / \$ 21 \cdot$ doi: $10.1117 / 12.2548603$ 

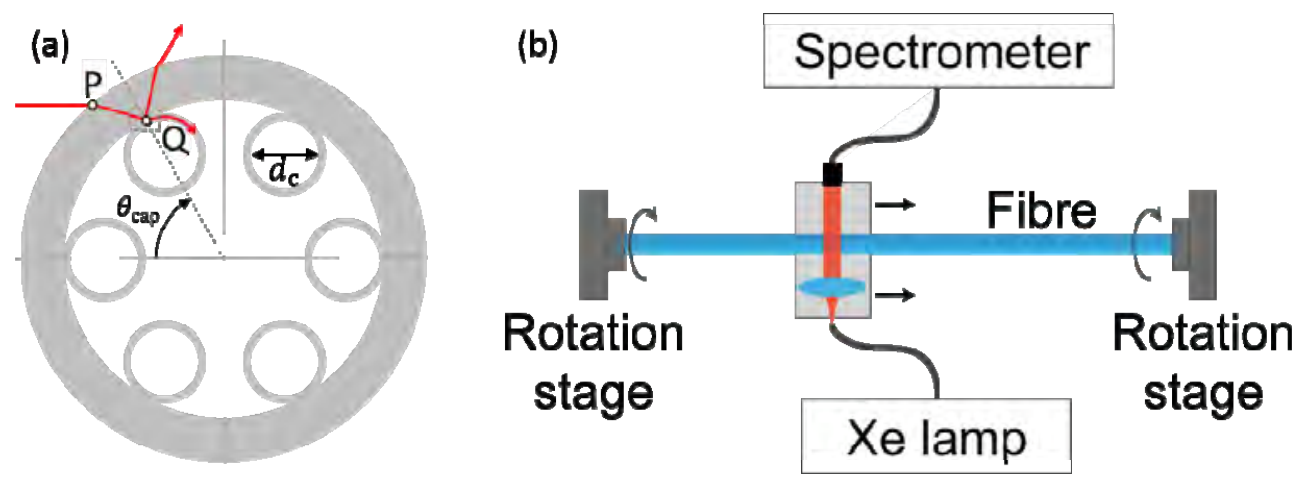

Figure 1: (a) Path of a ray refracting at point $\mathrm{P}$, which at point $\mathrm{Q}$ excites a WGM inside a capillary. The ray exits the fiber at a specific angle, allowing detection of the WGM spectrum of the selected capillary. (b) Setup used to scan along a fiber length while rotating the fiber to selectively measure individual capillaries.

Figure 2(a) shows a SR-PCF with 10 capillaries of varying size as well as two gaps, which were used to uniquely identify each capillary in the $360^{\circ}$ rotational scan WGM-measurement shown in Fig. 2(b). Figure 2(c) shows the result of tracking one particular capillary along the fiber length.
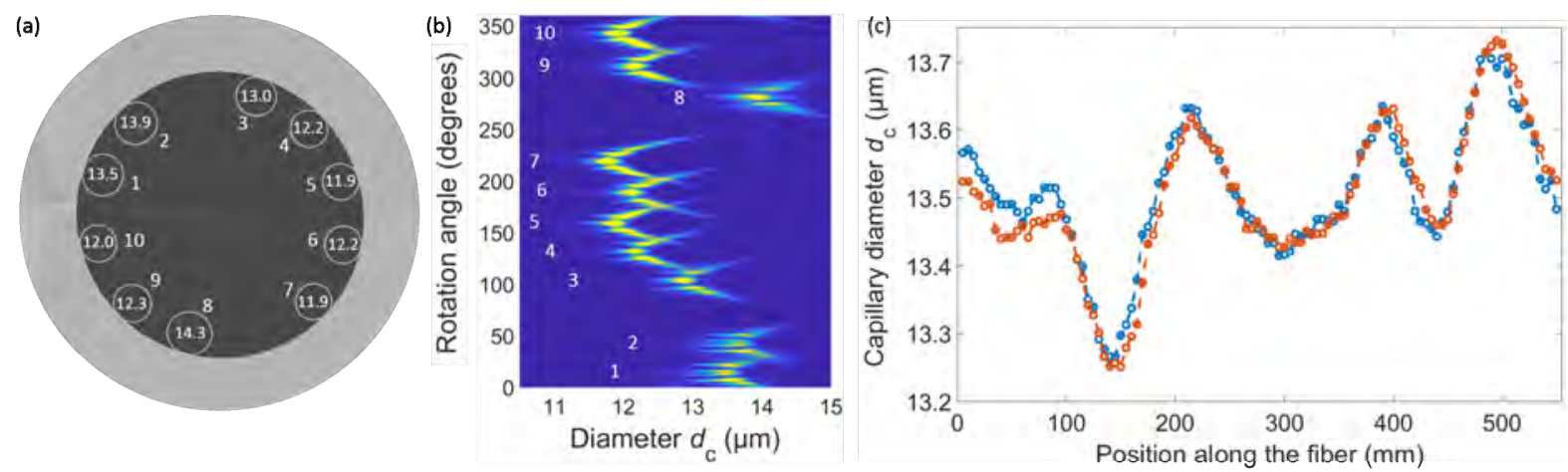

Figure 2: (a) Scanning electron micrograph (SEM) of the SR-PCF. The effective diameter of each capillary, estimated from the SEM, is notated in the diagram. Comparison with (b) shows that each capillary can be identified with good agreement. (b) An example of the scattered signals detected during a measurement in which the SR-PCF was rotated. The angle was scanned in steps of $0.1^{\circ}$. (c) Diameter of a single capillary along a $550 \mathrm{~mm}$ length of SR-PCF, obtained by finding the vertex of a parabola fitted to the V-shaped signals (Fig. 2(b)). The fiber was scanned in $5 \mathrm{~mm}$ steps. Two measurements are shown, the second being made after turning the fiber end-to-tail in the set-up.

\section{References}

[1] B. Debord, A. Amsanpally, M. Chafer, A. Baz, M. Maurel, J. M. Blondy, E. Hugonnot, F. Scol, L. Vincetti, F. Gérôme, and F. Benabid, "Ultralow transmission loss in inhibited-coupling guiding hollow fibers," Optica 4, 209-217 (2017).

[2] M. H. Frosz, R. Pennetta, M. T. Enders, G. Ahmed, and P. St.J. Russell, "Non - invasive Real - time Characterization of Hollow - core Photonic Crystal Fibres Using Whispering Gallery Mode Spectroscopy," in 2019 European Conference on Lasers and Electro-Optics and European Quantum Electronics Conference, (Optical Society of America, 2019), CJ-3.6.

[3] H. Kogelnik, "Theory of Dielectric Waveguides," in Integrated Optics, T. Tamir, ed. (Springer, Berlin, Heidelberg, 1975). 


\title{
Microgravity Fiber Processing for Future Optical Networks
}

\author{
Dmitry Starodubov*, K. McCormick, M. Dellosa, E. Erdelyi, and L. Volfson \\ FOMS Inc., 3525 Del Mar Heights Road, \#236, San Diego, CA 92130
}

\begin{abstract}
The growing demand for higher capacity data links defines the need for increasing the bandwidth of optical fiber communications. Microgravity processing of challenging glass compositions with potentially lower insertion loss at longer wavelengths is explored.
\end{abstract}

Keywords: optical fiber, low loss, microgravity, space manufacturing

*dstarodubov@gmail.com; phone: 1 (805) 501-9399

\section{Introduction}

Optical fibers carry the bulk of the modern data transmissions. The continuous increase of the bandwidth demand sets the pace for the increase of the transmission capacity for the data links. The projections of the power consumption for the multi-Tbit/s transmission line of the near future with the current generation of the technology would exceed 10kW and the bandwidth of the transmission will be limited by the nonlinear effects in the optical fibers that will set the nonlinear Shannon limit of the transmission capacity ${ }^{[1]}$. While the growing complexity of nonlinearity mitigation techniques is addressing the immediate needs of the data management the ultimate solution for the next generation of optical data links requires new optical fibers with lower insertion loss. Photonic crystal fiber structures are promising candidates for demonstrating the lower insertion loss; however the resonant structure of the design makes it challenging to achieve the desired transmission properties across the broad spectral regions that will be needed for high bandwidth communications. The presence of physical boundary with corresponding defects in the optical path makes the photonic crystal fibers susceptible to environmental conditions and challenges their use as the high bandwidth media of the future. The remaining alternative is the use of the low phonon energy glass materials for the optical fiber links. The fluoride glass compositions have the promise of insertion loss that could be an order of magnitude lower than silica fibers ${ }^{[2]}$. The proper processing of such glass materials however is challenging. In addition to the challenge of purification of the glass producing materials these glasses react with moisture, have fairly narrow processing temperature range and demonstrate phase separation with formation of crystalline phase. One of the suggested ways to improve the quality of the glass processing is to produce the optical fibers in microgravity environment ${ }^{[3]}$. The initial tests of the fiber processing in the microgravity of the parabolic airplane fight confirmed the validity of the approach ${ }^{[4]}$. The fabrication of long fiber spans would require longer durations of uninterrupted microgravity environment and therefore suggesting the fiber manufacturing tests on the orbital platform of International Space Station (ISS) ${ }^{[5]}$.

\section{Approach: Optical Fiber Fabrication on International Space Station}

In our first set of experiments we focused on evaluation of drawing the optical fiber from fiber preform in microgravity. The manufacturing hardware for the orbital platform of ISS has to comply with restrictions of size, weight and power limitations of the delivery and return from the orbit. Additionally the hardware operation on board of the station has to

Sixth International Workshop on Specialty Optical Fibers and Their Applications (WSOF 2019),

edited by Liang Dong, John M. Ballato, Proc. of SPIE Vol. 11206, 112060U · C 2019 SPIE

CCC code: $0277-786 X / 19 / \$ 21 \cdot$ doi: $10.1117 / 12.2549503$ 
meet the strict safety requirements of operation in the station environment that is the habitat of the station's crew. The challenge of processing fluoride glasses inside the high humidity environment of ISS is that the reaction of the fluoride glass with water vapor can release hydrofluoric acid. The manufacturing process requires three levels of containment to ensure the safety of the crew. The containment has to be developed as a part of the manufacturing payload design since the facilities of the station are not designed for hydrofluoric acid barrier.

The resulting fiber manufacturing payload represents the $12 \times 11 \times 32$ inches container with $25 \mathrm{~kg}$ weight and two fiber manufacturing experiments with processing capacity of up to $50 \mathrm{~km}$ of fiber. FOMS team launched two payloads to ISS on NG-11 mission and executed four remotely operated experiments in April of 2019. The results are shown in Figure 1.

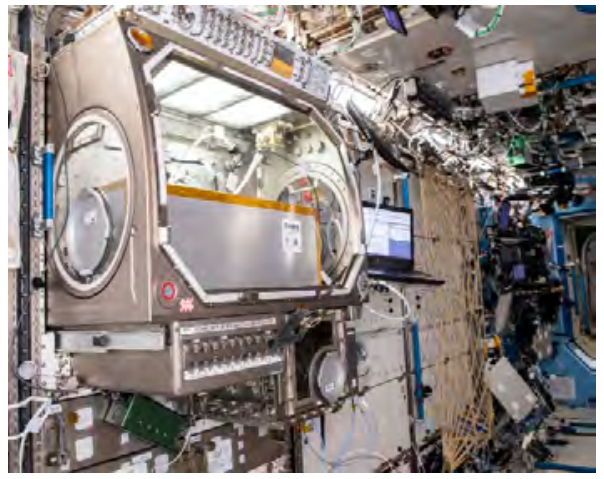

(a)

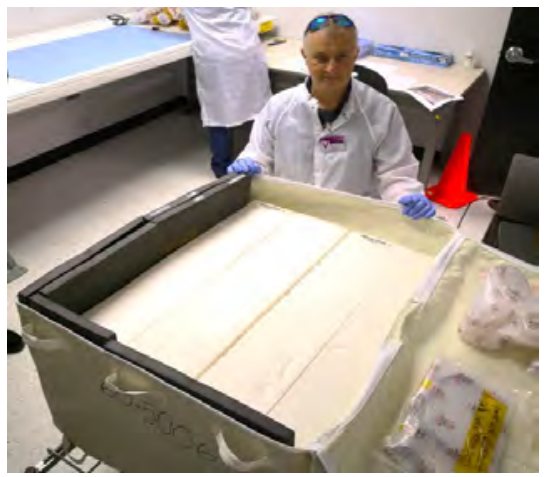

(b)

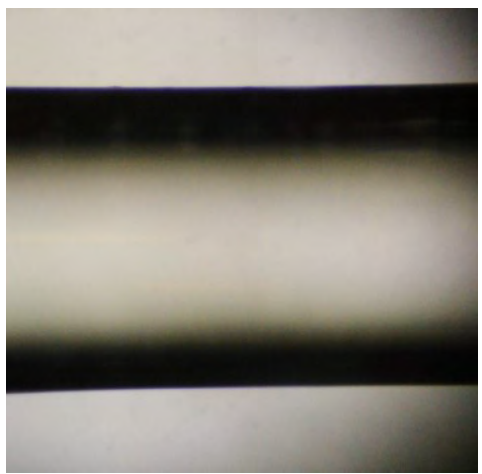

(c)

Figure 1. (a) Fiber manufacturing payload by FOMS Inc. inside Microgravity Science Glovebox on board of ISS, (b) unpacking of two manufacturing units in Houston, TX after returning back to Earth, (c) fluoride optical fiber produced in microgravity.

The fiber draw process was initiated on the ground and then re-started in space. The challenges included the mechanical failure of the fiber connection, the compensation of the crucible position for the microgravity conditions, the correction of the operating temperature settings with suppressed convection and the remote control of the experiment from the ground. The team successfully completed the calibration of the manufacturing hardware and demonstrated the first optical fiber manufacturing on the orbit. The microgravity fiber demonstrated better uniformity than the fiber produced on the ground.

The team is planning the follow up missions that will define the path towards volume manufacturing of optical fibers in space. This development is supported by ISS National Lab (CASIS) grant and NASA SBIR program.

\section{References}

[1] Ellis, A., "The nonlinear Shannon limit and the need for new fibers", Nonlinear Opt. and Appl., Proc. SPIE 8434 (2012).

[2] France, P. W., "Fluoride glass optical fibres", Blackie, 1990.

[3] Tucker, D., et al., "Effects of Gravity on ZBLAN Glass Crystallization," Ann. N.Y. Acad. Sci., vol. 1027, p. 1, 2004.

[4] Starodubov, D. S., Mechery, S., Miller, D., Ulmer, C., Willems, P., Ganley, J., and Tucker, D., "ZBLAN Fibers: From Zero Gravity Tests to Orbital Manufacturing," in Imaging and Applied Optics 2014, OSA Technical Digest, paper AM4A.2.

[5] Starodubov, D., McCormick, K., Dellosa, M., Erdelyi, E., Volfson, L., "Facility for orbital material processing," Proc. SPIE 10641, Sensors and Systems for Space Applications XI, 106410T (2 May 2018). 


\title{
Cladding Shaping of Optical Fiber Preforms via $\mathrm{CO}_{2}$ Laser Machining
}

\author{
P. C. Shardlow*, R. Standish, M. N. Velazquez, J. Sahu, and W. A. Clarkson \\ Optoelectronics Research Centre, University of Southampton, Southampton, SO17 1BJ, UK
}

\begin{abstract}
Shaping optical fiber preforms utilizing $\mathrm{CO}_{2}$ laser ablation is able to significantly reduce process times, improve surface qualities and facilitate fabrication of complicated cladding geometries including convex and concave surfaces.

Keywords: Fiber design and fabrication, Fiber Lasers, Optical fabrication, $\mathrm{CO}_{2}$ Laser, Preform shaping, Reuleaux polygon

*peter.shardlow@soton.ac.uk

\section{Introduction}

Double clad optical fiber for use in high power laser systems consist of a core material doped with a laser active ion, an outer cladding glass into which the pump is coupled and a low index outer polymer that guides the pump. Unfortunately using circular shaped outer claddings leads to some modes of the pump experiencing poor overlap with the core, and as such poor absorption. This can be readily improved by shaping the outer cladding in order to scramble the pump modes. This promotes better overlap, and subsequently absorption, of the pump light by the core. This shaping is traditionally undertaken via diamond or ultrasonic milling of the outer glass into an octagon or hexagon. This process often leaves a rough surface that is subsequently fire polished. We report on our developments in utilizing a $\mathrm{CO}_{2}$ laser to ablate the preform in order to customize the fiber cladding shape [1]. This approach is shown to allow fabrication of novel cladding structures, which can include diameter reduction, concave and convex surfaces. This allows the fabrication of novel cladding structures that are not feasible with classical fabrication techniques [2,3]. This is coupled with a significant increase in the processing speeds and removal of necessity of fire polishing prior to fiber drawing.
\end{abstract}

\section{II $\mathrm{CO}_{2}$ Laser Preform Processing System}
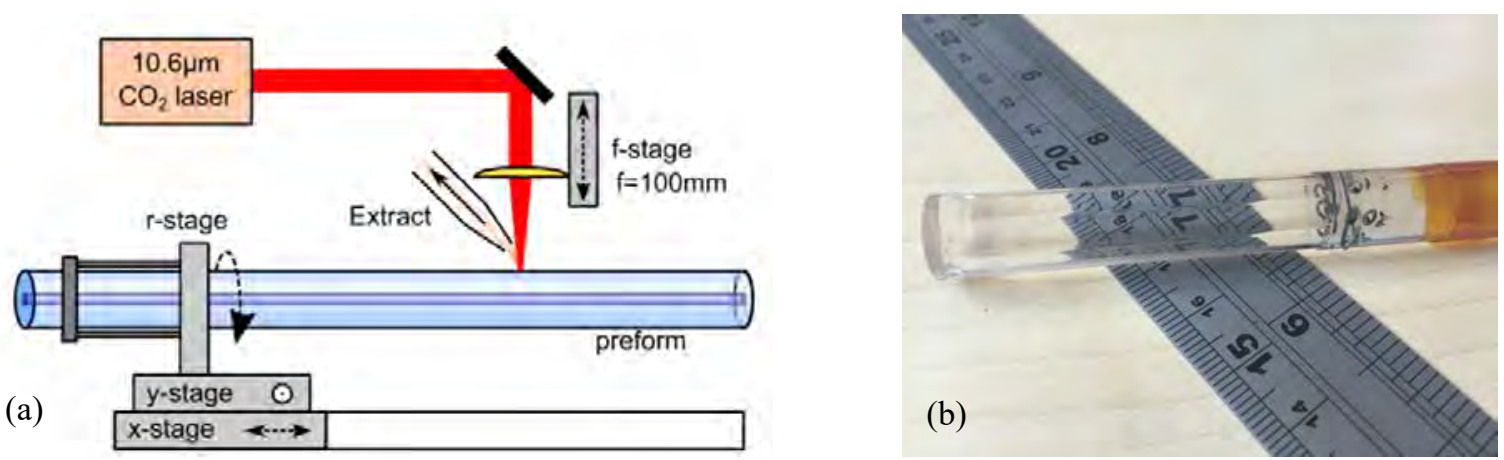

Figure 1 (a) Showing a conceptual schematic of the $\mathrm{CO}_{2}$ preform processing system. The preform itself is mounted within a rotation stage mounted onto $\mathrm{x}-\mathrm{y}$ translation stages. The $\mathrm{QCW}$ pulsed $\mathrm{CO}_{2}$ laser is directed via a focusing lens onto the top surface of the preform. (b) An example of a $12 \mathrm{~mm}$ diameter preform shaped to an octagonal outer profile.

Sixth International Workshop on Specialty Optical Fibers and Their Applications (WSOF 2019), edited by Liang Dong, John M. Ballato, Proc. of SPIE Vol. 11206, 112060V · (C) 2019 SPIE CCC code: $0277-786 X / 19 / \$ 21 \cdot$ doi: $10.1117 / 12.2548443$ 
Figure 1a shows a conceptual schematic of the $\mathrm{CO}_{2}$ preform processing system. A silica clad preform of typical diameter of $6-25 \mathrm{~mm}$ is mounted with two high temperature gaskets within a rotation stage mounted onto an $\mathrm{x}-\mathrm{y}$ translation stage. The $x$ stage is a high speed linear motor with translation speeds of up to $300 \mathrm{~mm} / \mathrm{s}$, and the y stage is a slower stepper motor driven stage for repositioning of the preform between high speed machining passes. The output from a $\mathrm{CO}_{2}$ laser (Coherent Diamond G100 10.6um) is directed to a focusing lens mounted above the preform providing a focused spot on the surface of the preform. The focusing lens is mounted onto a translation stage so that the position of the focal spot can be adjusted to control the spot intensity on the preform surface. An extract is employed to safely remove the ablated silica from the work-piece and to avoid reconsolidation of silica soot onto the preform during processing. The $\mathrm{CO}_{2}$ laser is operated in a QCW (Quasi Continuous Wave) mode providing pulses with peak power of $\sim 300 \mathrm{~W}$ with a rise and fall time below 90 us. The laser is modulated at a speed of up to $3 \mathrm{KHz}$ with the QCW pulse length utilized to control the amount of material removed per machining pass. Fully automated generation of raster scanning profiles to machine the circular preform to the desired cross section are employed based on a Bresenham based algorithm to discretize the preform [4].

\section{III $\mathrm{CO}_{2}$ Laser Preform Processing Benefits}
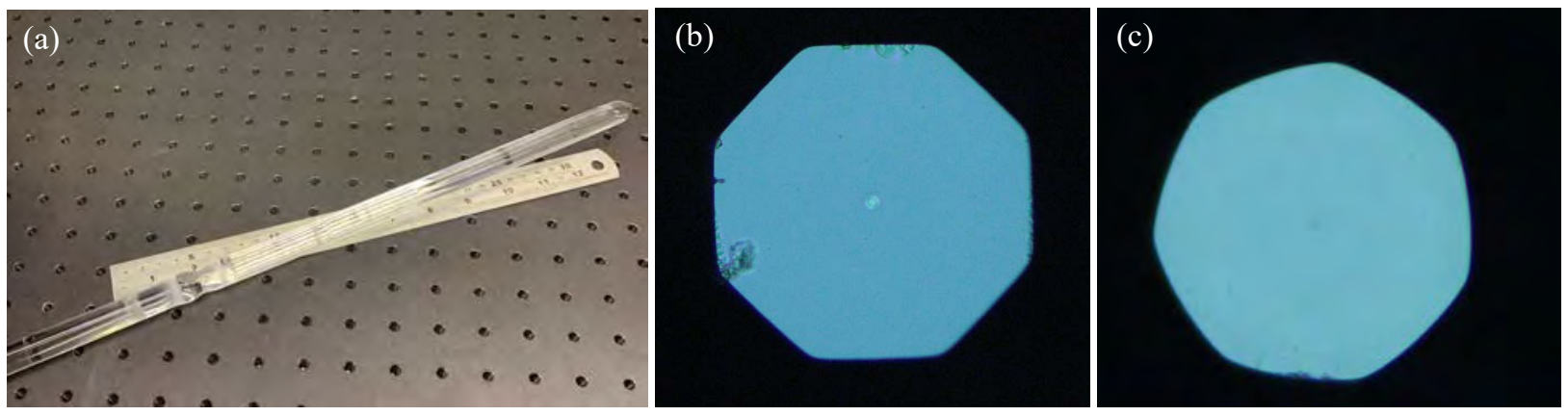

Figure 2. (a) Image of a $300 \mathrm{~mm}$ long preform shaped into three sections with varying outer cladding geometries. (b) End face image of a fiber drawn from a $\mathrm{CO}_{2}$ laser shaped preform, (c) End face image of a fiber drawn from a 7 sided Reuleaux polygon $\mathrm{CO}_{2}$ laser shaped preform.

\section{Surface Quality}

Figure $1 \mathrm{~b}$ shows an example of a $12 \mathrm{~mm}$ outer diameter preform shaped to an octagonal cladding utilizing the $\mathrm{CO}_{2}$ preform processing system. This shows the surface quality directly from the machining system and shows an optically transparent polished surface with significant improvements over traditional milling approaches and allows drawing without further fire polishing prior to drawing.

\section{Processing Speed}

Shaping of a circular preform to an N sided polygon is fully automated. Significant speed improvements over mechanical milling are reported due to primarily an increased machining speed and additionally no requirement for remounting the preform between machining of each facet due to low mechanical stress. As a result a $12 \mathrm{~mm}$ outer diameter preform of $180 \mathrm{~mm}$ in length takes less 90 minutes to octagonalize. 


\section{Novel Geometries}

As well as providing traditional polygon shaping it is possible to customize the raster scan profile in order to produce more complicated cladding shapes, such as convex and concave surfaces, as well as creating multiple shaped sections in a single preform. Figure 2c shows such a fibre drawn from a part of a preform shaped into a 7 sided Reuleaux polygon. This shape is of particular interest as Reuleaux polygons are of a mathematical class of shapes that have a constant diameter despite not being circularly symmetric. As such the fiber profilometers on the draw tower measure constant diameters irrespective of Reuleaux fiber orientation, allowing easier automation and more accurate diameter drawing of shaped fibers.

\section{Conclusion}

In this talk we review our developments in utilizing $\mathrm{CO}_{2}$ laser based ablation processing for shaping the cladding geometries of optical fiber preforms. Improved surface qualities, significant process speed improvements and novel

cladding geometry fabrication are reported. Improving draw diameter accuracy, shaping of close core to cladding area fibers and reduction of $\mathrm{OH}$ contamination in cladding glasses will also be reported.

\section{References}

[1] P. C. Shardlow, R. Standish, J. Sahu, and W. A. Clarkson, "Cladding Shaping of Optical Fibre Preforms via CO2 Laser Machining," in 2015 European Conference on Lasers and Electro-Optics - European Quantum Electronics Conference, (Optical Society of America, 2015), paper CJ_P_29.

[2] Muendel, Martin H. "Optimal inner cladding shapes for double-clad fiber lasers." in Conference on Lasers and Electro-Optics, (1996)

[3] James J. Morehead and Martin H. Muendel, "Nearly circular pump guides," Proc. SPIE 7914, Fiber Lasers VIII: Technology, Systems, and Applications, 79142Y (2011)

[4] J. Kennedy, "A Fast Bresenham Type Algorithm For Drawing Ellipses," Santa Monica College, https://dai.fmph.uniba.sk/upload/0/01/Ellipse.pdf 


\title{
Recent Progress in Graded-Index Plastic Optical Fiber
}

\author{
Yasuhiro Koike*, Azusa Inoue \\ ${ }^{a}$ Graduate School of Science and Technology, Keio Photonics Research Institute (KPRI), Keio \\ University, 7-1, Shin-Kawasaki, Saiwai-ku, Kawasaki, Kanagawa, 212-0032, Japan
}

\begin{abstract}
Recently, we proposed low-noise graded-index plastic optical fiber (GI POF) utilizing distinctive mode coupling owing to microscopic polymer properties. Here we develop a low-noise GI POF to realize indoor optical wiring for nextgeneration broadcast and communication in home and building.
\end{abstract}

Keywords: plastic optical fiber (POF), mode coupling, scattering, noise *koike@appi.keio.ac.jp; phone 81-44-580-1563

\section{Introduction}

Recently, ultrahigh-definition (UHD) broadcastings are being introduced in some countries [1]. The UHD broadcast signals are received with antennas, frequency-down-converted, and multiplexed with other broadcast signals to be transmitted through cables into homes, buildings, and broadcasting stations. Moreover, in households, the nextgeneration wireless local-area and mobile networks (e.g., IEEE 802.11ac, 5G) are being introduced [2,3]. In these wireless communications, carrier frequencies tend to be increased, decreasing cell sizes of the wireless networks. These trends are demanding indoor optical wiring to transmit various radio frequency (RF) signals based on radio over fiber (RoF). On the other hand, UHD device connections require uncompressed video transmission at bit rates exceeding 100 $\mathrm{Gb} / \mathrm{s}$.

A GI POF has been a promising transmission medium for indoor applications because of its flexibility, safety, and high bandwidth [4,5], which enables 40-Gb/s data transmission through a 100-m GI POF. Recently, we proposed a lownoise GI POF to achieve significantly stable and robust data transmission [6-8]. This stabilization is mainly attributed to strong mode coupling closely related to the core-material properties of the GI POF. Here, we demonstrate that transmission quality of baseband and subcarrier transmission (RoF) can be significantly improved by using our developed graded-index plastic optical fiber (GI POF).

\section{Low-noise GI POF}
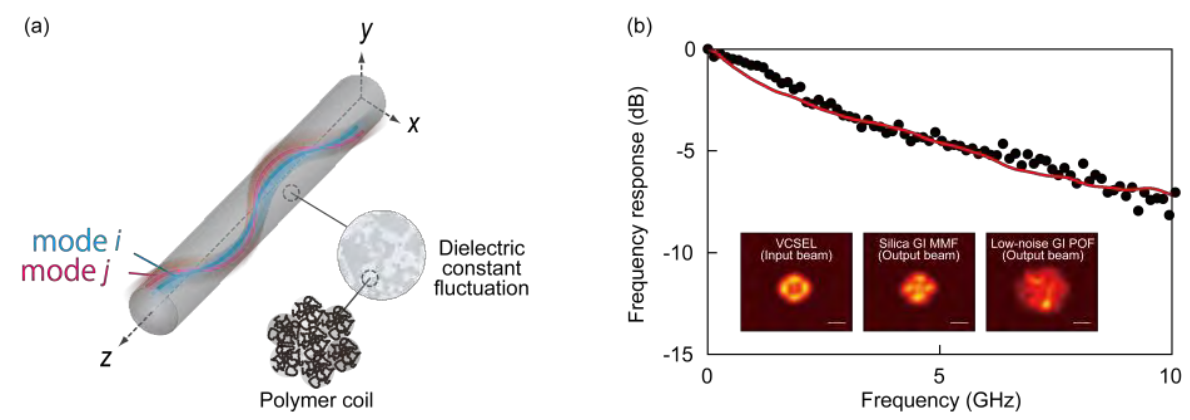

Figure 1. (a) Schematic of distinctive mode coupling in low-noise GI POF. (b) Frequency response of the GI POF. measured data (black closed circles) were fitted to calculated curve (red solid line) using our developed coupled power equation for the GI POF. Insets show near-field patterns of VCSEL and, output beam from 1-m Silica GI MMF and 1-m GI POF

Polymers are composed of significantly large polymer coils. This microscopic structure of polymer result in hierarchical density fluctuations. In low-noise GI POFs, random mode coupling is strongly induced by the microscopic inhomogeneities and is pronounced even for optical fiber lengths below several meters. We fabricated a low-noise GI

\footnotetext{
Sixth International Workshop on Specialty Optical Fibers and Their Applications (WSOF 2019), edited by Liang Dong, John M. Ballato, Proc. of SPIE Vol. 11206, 112060W · (C) 2019 SPIE CCC code: $0277-786 X / 19 / \$ 21 \cdot$ doi: $10.1117 / 12.2551502$
} 
POF with a core diameter of $\sim 50 \mu \mathrm{m}$, NA of $\sim 0.2$, and GI profile index-exponent of $\sim 2.0$, which are almost the same as those of a reference silica GI MMF, by the co-extrusion method. Using our developed coupled power equation for the distinctive mode coupling in the low-noise GI POFs, we estimated average power coupling coefficient taken over all the guided mode pairs at $7.6 \times 10^{-5} \mathrm{~m}^{-1}$. This mode coupling of the low-noise GI POF is pronounced even for a fiber length of $1 \mathrm{~m}$, as shown in the inset of Fig. 1 (b). The GI POF has an attenuation of $\sim 6.5 \times 10^{-2} \mathrm{~dB} / \mathrm{m}$ and a bandwidth of $\sim 750$ $\mathrm{GHz} \cdot \mathrm{m}$, whereas the silica GI MMF has an attenuation of $\sim 2.3 \times 10^{-3} \mathrm{~dB} / \mathrm{m}$ and a bandwidth above $2650 \mathrm{GHz} \cdot \mathrm{m}$ (device limit of the component analyzer).
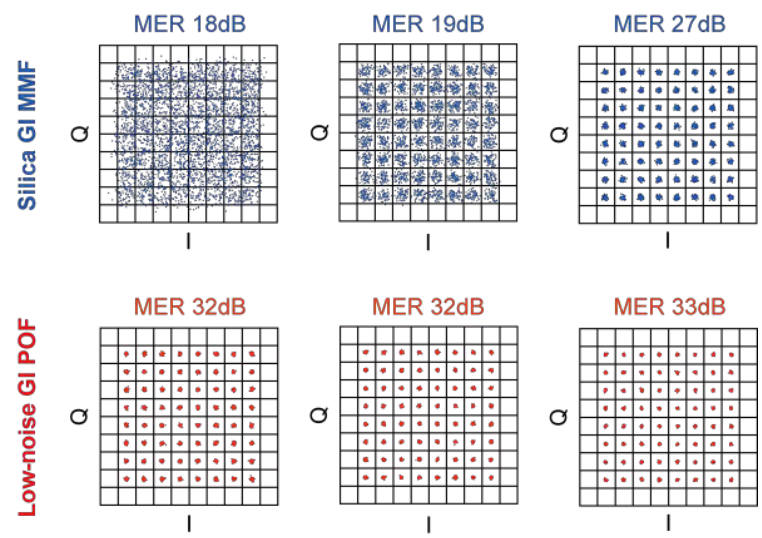

(a) $1 \mathrm{GHz}$

(b) $2 \mathrm{GHz}$

(c) $3 \mathrm{GHz}$

Figure 2. Constellation map for signal transmitted through 100-m silica GI MMF and 100-m low-noise GI POF. Carrier frequencies are (a) $1 \mathrm{GHz}$, (b) $2 \mathrm{GHz}$, and (c) $3 \mathrm{GHz}$.

We composed an optical link of a 100-m fiber, an 850-nm multimode vertical-cavity surface emitting laser (VCSEL), and a 12-GHz GaAs photodiode (PD) without amplifiers, where coupling loss and modal noise can be ignored [8]. We evaluated constellation maps and modulation error ratios (MERs) of a RF signal transmitted through the low-noise GI POF and silica GI MMF. The modulation format and Nyquist bandwidth of the transmitted signal are 64 QAM and 5.3 $\mathrm{MHz}$, respectively. Signals detected with the PD were amplified by a 20-dB built-in preamplifier of the signal analyzer. As shown in Fig. 2, the low-noise GI POF allows for higher quality RF transmission than the silica GI MMF despite its higher attenuation for all the carrier frequencies. This can be attributed to strong mode coupling of the low-noise GI POF, which can decrease interferometric noise including reflection noise [6].

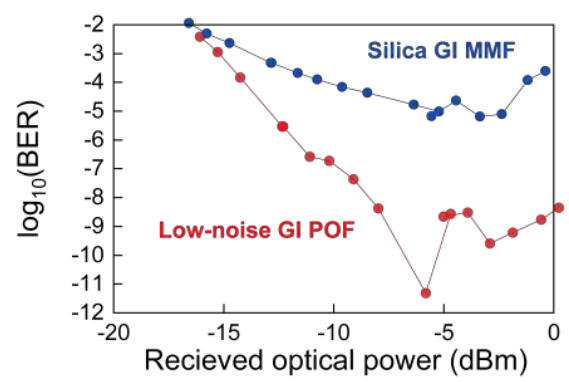

Figure 3. BER as functions of received optical power for MMF links with silica GI MMF and low-noise GI POF.

Furthermore, to evaluate baseband transmission through the low-noise GI POF, VCSEL is directly modulated at a bias current of $5 \mathrm{~mA}$ with a 10-Gb/s non-return-to-zero (NRZ) data (231-1 PRBS pattern length) to evaluate the transmission qualities of the MMF link. BERs of transmitted data through a low-noise GI POF and a silica GI MMF were measured for a low modulation voltage of $0.1 \mathrm{~V}$. Both fiber lengths are $1 \mathrm{~m}$. In these experiments, we used a PD with a 
transimpedance amplifier. Figure 3 shows BERs for silica GI MMF and low-noise GI POF as functions of received optical power. In silica GI MMF, the optical-feedback-induced instabilities degrade transmission qualities [5]. However, low-noise GI POF significantly decreases BERs for all the received optical powers because of its strong mode coupling or noise reduction effect.

\section{Conclusion}

We developed a low-noise GI POF to achieve higher-quality subcarrier and baseband transmission than conventional multimode fibers. Our developed GI POF will realize indoor optical wiring for next-generation broadcast and communication in home and building.

\section{References}

[1] K. Motohashi, "Media, culture and industry in the 4K/8K smart TV era," New Breeze 26(2), 5-7 (2014).

[2] R. Waterhouse and D. Novak, "Realizing 5G: Microwave photonics for 5G mobile wireless systems," IEEE Microw. Mag. 16(8), 84-92 (2015).

[3] Yasuhiro, K., [Fundamentals of Plastic Optical Fibers], Wiley-VCH, Weinheim, (2015).

[4] Y. Koike and A. Inoue, "High-speed graded-index plastic optical fibers and their simple interconnects for $4 \mathrm{~K} / 8 \mathrm{~K}$ video transmission," J. Lightwave Technol. 13(6), 1551-1555 (2016).

[5] A. Inoue and Y. Koike, "Low-noise graded-index plastic optical fiber for significantly stable and robust data transmission,” J. Lightwave Technol. 36(24), 5887-5892 (2018).

[6] A. Inoue and Y. Koike, "Intrinsically stabilized plastic optical fiber link subject to external optical feedback," IEEE Photonics J. 11(1), 7201207 (2019).

[7] A. Inoue and Y. Koike, "Unconventional plastic optical fiber design for very short multimode fiber link," Opt. Express 27(9), 12061-12069 (2019).

[8] K. Muramoto, A. Inoue, and Y. Koike, "Nonlinear distortion reduction effect of graded-index plastic optical fiber," IEEE Photon. Technol. Lett. 31(10), 791-794 (2019).

[9] A. Inoue, T. Sassa, K. Makino, A. Kondo, and Y. Koike, "Intrinsic transmission bandwidths of graded-index plastic optical fiber," Opt. Lett. 37(13), 2583-2585 (2012).

[10] A. Inoue, T. Sassa, R. Furukawa, K. Makino, A. Kondo, et al. "Efficient group delay averaging in graded-index plastic optical fiber with microscopic heterogeneous core,” Opt. Express 21(14), 17379-17385 (2013). 


\title{
3D-printing of chalcogenide preforms: a novel process for the elaboration of chalcogenide microstructured optical fibers
}

\author{
Julie Carcreff ${ }^{a}$, François Cheviré ${ }^{a}$, Elodie Galdo ${ }^{a}$, Ronan Lebullenger ${ }^{a}$, Antoine Gautier ${ }^{\text {a }}$, Jean-Luc \\ Adam $^{\mathrm{a}}$ Laurent Brilland $^{\mathrm{b}}$, Gilles Renverse ${ }^{\mathrm{c}}$ and Johann Troles*a \\ aUniv Rennes, CNRS, ISCR-UMR 6226, F-35000 Rennes, France \\ b Selenoptics, 263 Avenue Gal Leclerc, 35042 Rennes, France \\ 'Aix-Marseille Univ, CNRS, Centrale Marseille, Institut Fresnel, UMR 7249, 13013 Marseille, France
}

\begin{abstract}
The elaboration of chalcogenide microstructured optical fibers (MOFs) permits to combine the mid-infrared transmission of chalcogenide glasses up to $18 \mu \mathrm{m}$ to the unique optical properties of MOFs due to the high degree of freedom in the design of their geometrical structure. In this context, we have shown that chalcogenide preforms can be obtained by an original additive manufacturing process, and that such as-prepared preforms can be drawn into chalcogenide optical fibers. Those results open a new way for the elaboration of chalcogenide MOFs, especially for hollowcore chalcogenide MOFs
\end{abstract}

Keywords: chalcogenide glasses, microstructured optical fibers, 3D printing

\section{Introduction}

In recent years, a growing interest has settled for optical materials and fibers for the mid infrared (mid-IR) region. This interest originates from societal needs for health and environment for instance, and also from demand for defense applications. Indeed, the mid-IR spectral region contains the atmospheric transparent windows $(3-5 \mu \mathrm{m})$ and $(8-12 \mu \mathrm{m})$ where thermal imaging (military and civilian) can take place. Furthermore, the infrared window is well-suited for sensing (bio)-molecules, whose fingerprints are located at wavelengths between 2 and $15 \mu \mathrm{m}$. The development of mid-IR transparent materials and optical fibers is essential. Chalcogenide glasses are good candidates for the realization of new and innovative mid-IR systems. In this context, chalcogenide fibers have attracted great interest in the field of versatile mid-IR fiber transmission, supercontinuum generation, and sensing [1].

An original way to obtain fibers is to design microstructured optical fibers (MOFs). Such fibers present unique optical properties thanks to the high degree of freedom in the design of their geometrical structure. The elaboration of chalcogenide MOFs permits to combine the mid-IR transmission of chalcogenide glasses to the light propagation properties of MOFs. Different methods for the realization of chalcogenide preforms and microstructured fibers have been reported, such as the stack and draw method [2], drilling method [3], extrusion processes [4] and molding method [5].

We have investigated an alternative way for fabricating complex microstructured preform by using an original 3D printing process. It has been shown recently that bulk $\mathrm{As}_{2} \mathrm{~S}_{3}$ can be obtained by additive manufacturing process [6]. In the present study, we have 3D-printed a holey chalcogenide preform, and shown that this preform can be drawn into a chalcogenide optical fiber that can transmit light. The attenuation of the printed fiber will be compared to the optical transmission of chalcogenide fiber drawn from a classical melt-quenched preform.

Sixth International Workshop on Specialty Optical Fibers and Their Applications (WSOF 2019), edited by Liang Dong, John M. Ballato, Proc. of SPIE Vol. 11206, 112060X · (C) 2019 SPIE

CCC code: $0277-786 X / 19 / \$ 21 \cdot$ doi: $10.1117 / 12.2543952$ 


\section{Elaboration of chalcogenide preform and fiber by 3D printing}

\section{3D manufacturing of the preform}

A new method has been investigated for producing preform for mid-IR fibers: the additive manufacturing. This method can be implemented with $\mathrm{Te}_{20} \mathrm{As}_{30} \mathrm{Se}_{50}$ glass (TAS), which is very stable against crystallization. Likewise, its low glass transition temperature $\left(\mathrm{T}_{\mathrm{g}}\right)$ facilitates its shaping at low temperature and makes it a good candidate to explore 3D printing of chalcogenide glasses

The thermal properties of TAS, i.e. $\mathrm{Tg}$ around $140^{\circ} \mathrm{C}$ and an extrusion temperature. around $270-300^{\circ} \mathrm{C}$ are close to the ones of commercial printable polycarbonate $\left(\mathrm{Tg}_{\mathrm{g}}\right.$ around $140-150^{\circ} \mathrm{C}$ and extrusion temperature. around $\left.260-310^{\circ} \mathrm{C}\right) . \mathrm{In}$ order to manufacture TAS glass preforms, a customizable commercial RepRap-style 3D printer (Anet A8) has been modified accordingly with a copper hot end (nozzle diameter $=0.4 \mathrm{~mm}$ ) that can reach printing temperature up to $400^{\circ} \mathrm{C}$ and a sodalime glass bed that can be heated slightly above the TAS $\mathrm{T}_{\mathrm{g}}$ for adhesion purpose. 400-mm long TAS rods (1.75 and $3 \mathrm{~mm}$ in diameter), produced by the fiber-drawing method, were utilized to supply the feeding mechanism especially customized for brittle materials. As a proof of concept of the printability of chalcogenide glass preforms, a basic tube of glass has been elaborated, at first. The material is deposited uniformly layer by layer in a continuous circular motion at constant linear speed and extrusion rate under ambient conditions. The resulting piece has an external diameter of 8.2 $\mathrm{mm}$ and an internal diameter of $3 \mathrm{~mm}$ for a height of $15 \mathrm{~mm}$. The tube surface is bright, suggesting that neither crystallization, nor defects are discernible to the naked eye. Only the stripes due to the different layers of the additive process are clearly visible, as can be seen in Figure 1.
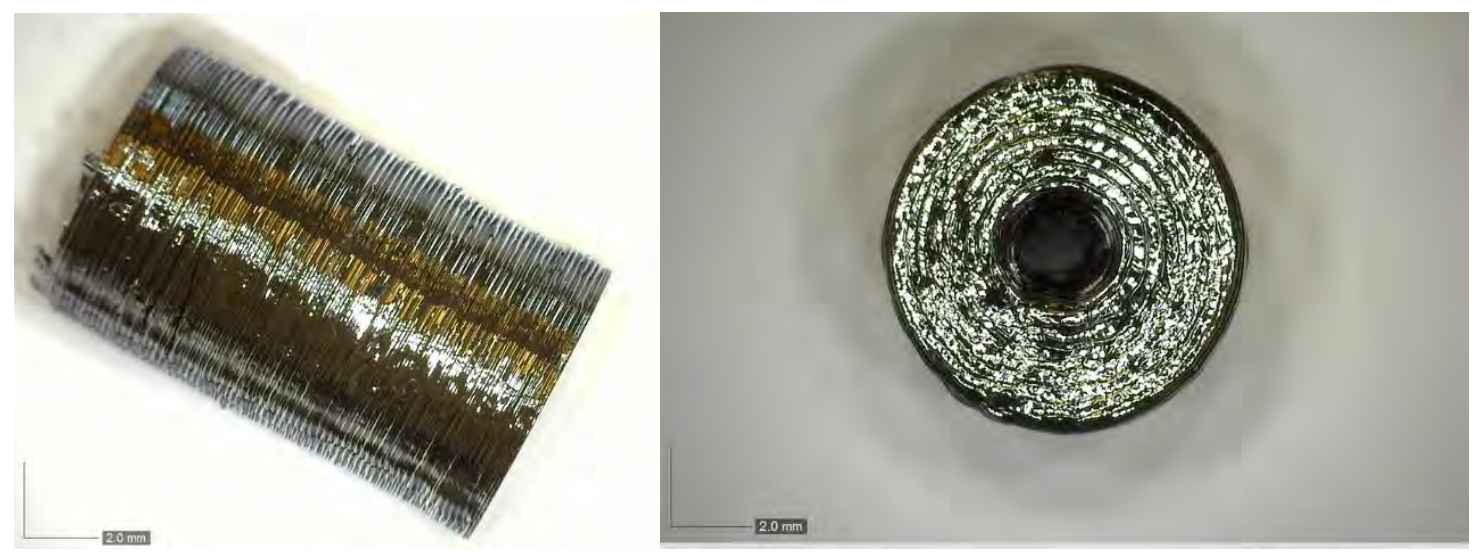

Fig 1. Photos of the chalcogenide glass hollow preform obtained by 3D printing.

\section{Drawing and optical characterizations of the "printed" fiber}

The printed preform has been drawn into a hollow-core fiber by using a drawing tower especially built for soft glasses such as chalcogenide glasses. Few meters long samples with an inner diameter around $70 \mu \mathrm{m}$ and an outer diameter around $280 \mu \mathrm{m}$ (Fig2a) have been produced. The attenuation curve of the "printed" fibers has been recorded and compared to the attenuation losses measured in the TAS glass cane used for printing the preform (fig $2 \mathrm{~b}$ ). The measured optical losses for the "printed" fiber are much higher than the initial optical losses, by nearly a factor of 4 in the 7-8 $\mu \mathrm{m}$ region. The additional losses are mainly due to scattering effects induced by the presence of bubbles 
(fig 2) and also water and oxygen pollution during the 3D printing that takes place in air. One can note that the initial glass used for this first attempt was not a high purity chalcogenide glass.
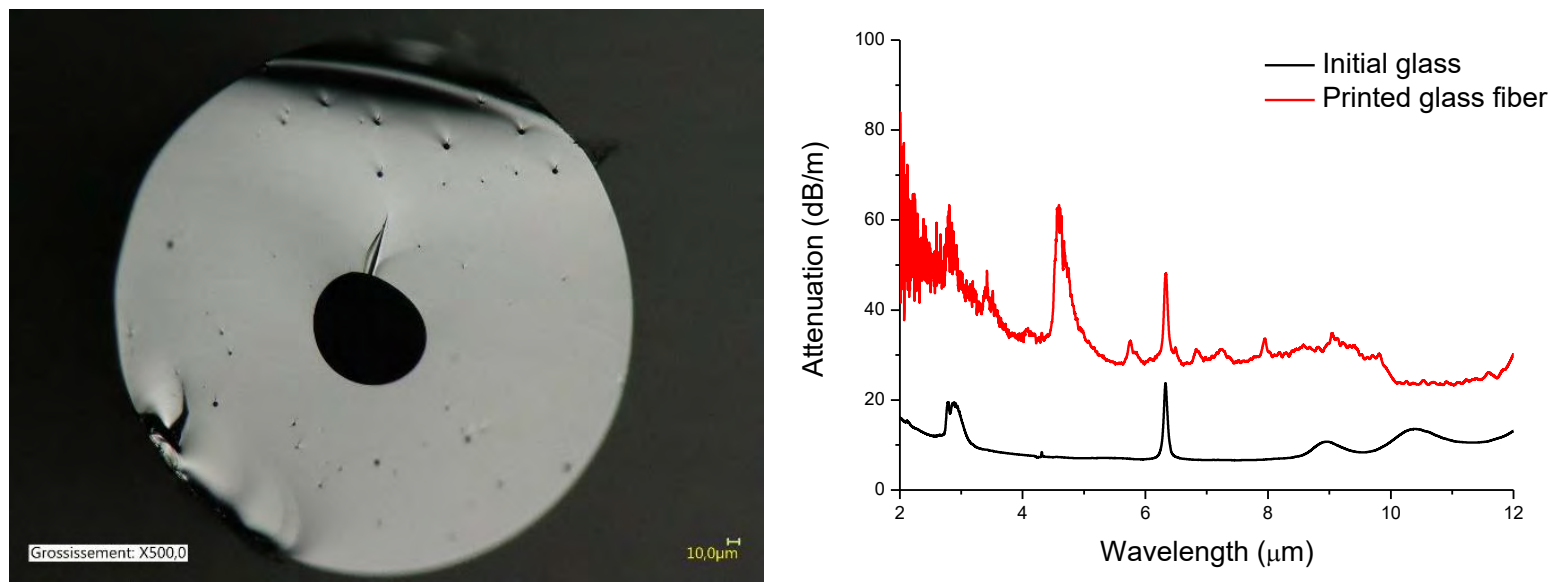

Fig 2 : 3D-printed chalcogenide hollow fiber : (a) fiber cross section, (b) : Optical loss of the chalcogenide (black) and the printed hollow fiber (red).

Those results open a new way for the elaboration of chalcogenide MOFs, especially for the preparation of hollow core chalcogenide MOFs.

\section{References}

[1] G. Tao, H. Ebendorff-Heidepriem, A. M. Stolyarov et al., "Infrared fibers," Advances in Optics and Photonics, 7(2), 379-458 (2015).

[2] L. Brilland, J. Troles, P. Houizot et al., "Interfaces impact on the transmission of chalcogenide photonic crystal fibres," Journal of the Ceramic Society of Japan, 116(10), (2008).

[3] M. El-Amraoui, G. Gadret, J. C. Jules et al., "Microstructured chalcogenide optical fibers from As2S3 glass: towards new IR broadband sources," Opt. Express, 18(25), 26655-26665 (2010).

[4] B. Wu, Z. M. Zhao, X. S. Wang et al., "Mid-infrared supercontinuum generation in a suspended-core telluriumbased chalcogenide fiber," Optical Materials Express, 8(5), 1341-1348 (2018).

[5] P. Toupin, L. Brilland, J. Trolès et al., "Small core Ge-As-Se microstructured optical fiber with single-mode propagation and low optical losses," Optical Materials Express, 2(10), 1359-1366 (2012).

[6] E. Baudet, Y. Ledemi, P. Larochelle et al., "3D-printing of arsenic sulfide chalcogenide glasses," Optical Materials Express, 9(5), 2307-2317 (2019). 


\title{
The Effect of Pressure on Structured Optical Fibre Drawing
}

\author{
Ghazal Tafti $^{\text {a* }}$, John Canning ${ }^{\mathrm{a}, \mathrm{b}}$, Wenyu Wang ${ }^{\mathrm{a}}$, Yanhua Luo ${ }^{\mathrm{a}}$, Kevin Cook ${ }^{\mathrm{a}, \mathrm{b}}$, Gang-Ding Peng ${ }^{\mathrm{a}}$ \\ ${ }^{a}$ National Fibre Facility, Photonics \& Optical Communication, School of Electrical Engineering and \\ Telecommunications, University of New South Wales, Kensington, NSW 2052, Australia \\ ${ }^{b}$ interdisciplinary Photonics Laboratories ( $\left.i \mathrm{PL}\right)$, School of Electrical \& Data Engineering \& Tech \\ Lab, UTS and School of Chemistry, The University of Sydney, NSW 2007 \& 2006 Australia
}

\begin{abstract}
The role of internal capillary on structured optical fibre drawing is explored. A single-capillary function modified to cater for multi-capillary structural constraint within a larger single capillary draw is proposed and shown to give reasonable fits with experiment.

Keywords: Structured Optical Fibre (SOF); photonic crystal fibres, Structure formation; Drawing condition; Experimental investigation; Simple modelling.

\section{Introduction}

The structure of structured optical fibres (SOF) [1-7] is usually characterised by periodic arrays of microscopic air-holes running along its entire length. Changes in the size and distribution of the air-holes can significantly alter the optical properties of SOFs. This provides flexibility in design and allows for the development of SOFs with important features, including endlessly single-mode [5], high-nonlinearity [7] and temperature-independent high-birefringence [8]. The design and fabrication of SOFs are of interest in telecommunications [9], optical fibre sensing $[10,11]$, and fibre lasers $[12,13]$. The final structure of SOF depends on the drawing process which involves a complex interplay between furnace temperature, pressurisation, drawing tension, feeding rate and drawing speed. Changes in these parameters or conditions directly affect the viscosity flow, surface tension, and shear force. They can significantly alter the air hole geometry and hence the properties of the fibre.

Although numerous papers describe the fabrication process of SOFs, there have been only a few research studies attempted to rigorously investigate the effects of drawing parameters [1417]. Several models based on the Navier-Stokes and convectiondiffusion equations have been developed to describe the fluid flow in the neck-down region at the drawing process [18-20]. Fitt et al. [19] used a simplified model to describe the drawing

process of a hollow fibre with a single axisymmetric hole, relating it to an idealized single capillary. They compared their solutions with the experimental results of drawing a single capillary to verify their model at different drawing pressures. Later, a number of studies have been conducted using this single capillary analogy to purportedly predict the fabrication process of SOFs. A fundamental problem with this approach is that the arbitrary fitting of a single capillary model to experimental data has no clear physical justification because there is no reason why the internal multi-capillary structure of the SOF can be ignored.

This paper aims to extend the simple capillary model to make it suitable for analytical SOF studies by taking into account the internal structure and the role of pressure. The SOF studied is composed of four hexagonal rings of air-holes surrounding the solid-core. A comparison between the experimental and predicted results is presented. The effect of a net counter-pressure from the surrounding holes on an air-hole within the drawn SOF is the likely explanation for the difference. A simple intuitive correction of the Fitt's single capillary approach, using a structural constraint to capture the role of the multiple-capillary structure within the SOF, is shown to be sufficient opening a pathway to quantify constraint and optimise actual SOF design.
\end{abstract}

\section{Design and Fabrication of Structured Optical Fibres}

There are several methods to fabricate SOFs including stacking of capillaries [14-17], extrusion, [21], sol-gel casting [22], drilling [23-25] and potentially the most disruptive and flexible of all, 3D printing [26, 27]. The capillary stacking technique, the most widely adopted method, is used in this study. Stacking allows relatively fast, low-cost, clean, and flexible fabrication although it has significant limitations on design flexibility beyond periodic arrays. The SOF we draw for this work is made up of capillaries (inner diameter $2 \phi_{c}=1.56 \mathrm{~mm}$ ) stacked to form a hexagonal pattern, inserted into the jacketing tube (Figure 1(a)). The interstitial spaces situated between the capillaries were removed by fusing on a modified chemical vapor deposition (MCVD) lathe. The fused preform was drawn into SOFs using an optical fibre drawing tower (furnace hot zone length $L=4 \mathrm{~cm}$ ). Feeding rate, drawing speed, furnace temperature, and drawing pressure were adjusted to control the shape and structure of SOFs. Drawing pressure controlled airholes' dimensions by adjusting collapse or expansion.

\section{Single Capillary Draw Analogy}

Fitt et al. [19] used a simplified closed-form Navier Stokes expression and convection-diffusion equations that govern the viscous fluid flow in the neck down region of a furnace drawing capillary (Figure 1(b)). 


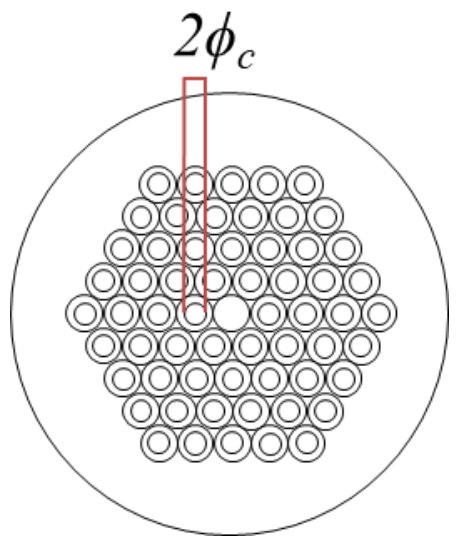

(a)

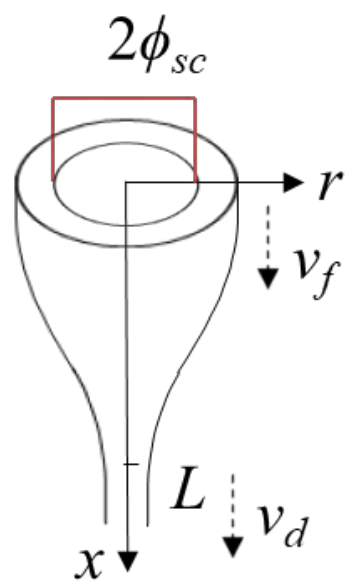

(b)

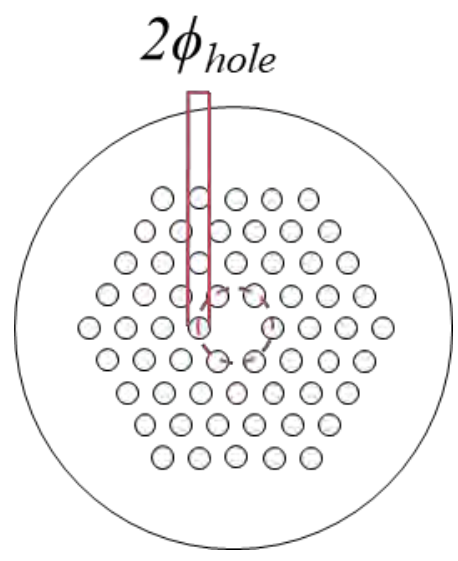

(c)

Figure 1 Illustration of (a) an SOF structure with the inner capillary diameter $2 \phi_{c}$; (b) a single capillary with inner diameter, $2 \phi_{s c}$ at the neck-down region; (c) a fabricated hexagonal structured optical fibre, SOF with inner diameter $2 \phi_{\text {hole. }}$

The model takes into account the interplay between furnace temperature, pressurisation, drawing tension, feeding rate, and drawing speed. According to this single capillary model, the inner radius of the drawn capillary $\phi_{\text {hole }}(\mathrm{m})$ is defined as a function of drawing parameters by [19]:

$$
\begin{gathered}
\phi_{\text {hole }}(x)=\exp \left(\frac{-\beta x}{2 L}-P \exp \left(\frac{-\beta x}{L}\right)\right)\left[\phi_{s c} \exp (P)\right. \\
-\int_{0}^{x} G \exp \left(\frac{-\beta u}{2 L}\right. \\
\left.+P \exp \left(\frac{-\beta u}{L}\right)\right) d u
\end{gathered}
$$

where $G=\gamma /\left(2 \mu v_{f}\right), \beta=\log \left(v_{d} / v_{f}\right), \quad P=L P_{d} /\left(2 \beta \mu v_{f}\right), \mu$ $\left(\mathrm{N} \cdot \mathrm{s} \cdot \mathrm{m}^{-2}\right)$ is viscosity, $\gamma\left(\mathrm{N} \cdot \mathrm{m}^{-1}\right)$ is surface tension and $v_{f}\left(\mathrm{~m} \cdot \mathrm{s}^{-1}\right)$ is preform feeding speed, $v_{d}\left(\mathrm{~m} \cdot \mathrm{s}^{-1}\right)$ is the drawing speed, and $P_{d}$ $\left(\mathrm{N} \cdot \mathrm{m}^{-2}\right)$ is the drawing pressure applied to the air-holes within the preform, $L(\mathrm{~m})$ is the furnace hot zone length whilst $\phi_{S C}(\mathrm{~m})$ is the inner radius of the capillary and $x(\mathrm{~m})$ is the distance along the axis of a capillary from the start point of the neck-down region. The surface tension of the silica is $\gamma=0.3 \mathrm{~N} / \mathrm{m}$. The viscosity of the silica is approximately given by $\mu=0.1 \times$ $10^{-6.24+26900 /(T+273)}$ where $T$ is the drawing temperature $\left({ }^{\circ} \mathrm{C}\right)$ [28]. In this model, the length of the hot zone is assumed to be much larger than the radius of the outer capillary, $\phi_{o c}$, so $\phi_{o c} / L$ $<<1$. Theoretically, there exist two pressure thresholds, collapse $P_{d, \text { coll }}$ and expansion $P_{d, \text { exp }}$, that determine capillary collapse
$\left(P_{d}<P_{d, \text { coll }}\right)$ and expansion $\left(P_{d}>P_{d, \exp }\right)$, respectively, satisfying [19]:

$$
\begin{gathered}
G \int_{0}^{x} \exp \left(\frac{-\beta u}{2 L}+\frac{L P_{d, \text { coll }}}{2 \beta \mu v_{f}} \exp \left(\frac{-\beta u}{L}\right)\right) d u \\
=\phi_{s c} \exp \left(\frac{L P_{d, \text { coll }}}{2 \beta \mu v_{f}}\right)
\end{gathered}
$$

$P_{d, \exp }>\frac{\gamma}{\phi_{s c}}+\frac{\beta \mu v_{f}}{L}$

An obvious difference between the SOF and the single capillary model is the role of the multi-capillary internal structure inside the main outer capillary structure of the fibre this difference exists because each capillary expands in its own right against each other creating a cylindrical web with an effective counteracting pressure that should lead to an overall pressure differing from that of the single capillary analogy. Here, we quantify the difference between the single capillary and the actual SOF without making assumptions on parameters to obtain an arbitrary fit. More importantly, this difference should be a direct measure of the degree of constraint in the structure since it is what causes the deviation from a singular capillary of similar dimensions.

\section{Experimental and Predicted Results and Discussion}

To study the relation between the drawing conditions and the final air-hole dimensions/structures of SOFs, drawing temperature, $T_{d}$, drawing pressure, $P_{d}$, preform feeding speed, $v_{f}$, and drawing speed, $v_{d}$, were adjusted. Velocities $v_{f}$ and $v_{d}$ were set at $v_{f}=0.5 \mathrm{~mm} / \mathrm{min}$ and $v_{d}=15 \mathrm{~m} / \mathrm{min}$ to produce a hexagonal pattern of air-holes in the fibre with outer diameter $\phi_{f} \approx 125 \mu \mathrm{m}$.
The air-hole size in a SOF can be tuned by adjusting $P_{d}$ and/or $T_{d} . P_{d}$ can also be changed indirectly through $v_{d}$ which changes drawing tension and therefore internal pressure via Poisson's ratio. The response of control by $T_{d}$ is slow but that by $P_{d}$ is fast. Therefore, $P_{d}$ is explored as a suitable parameter for controlling the eventual air-hole structure of SOFs. 


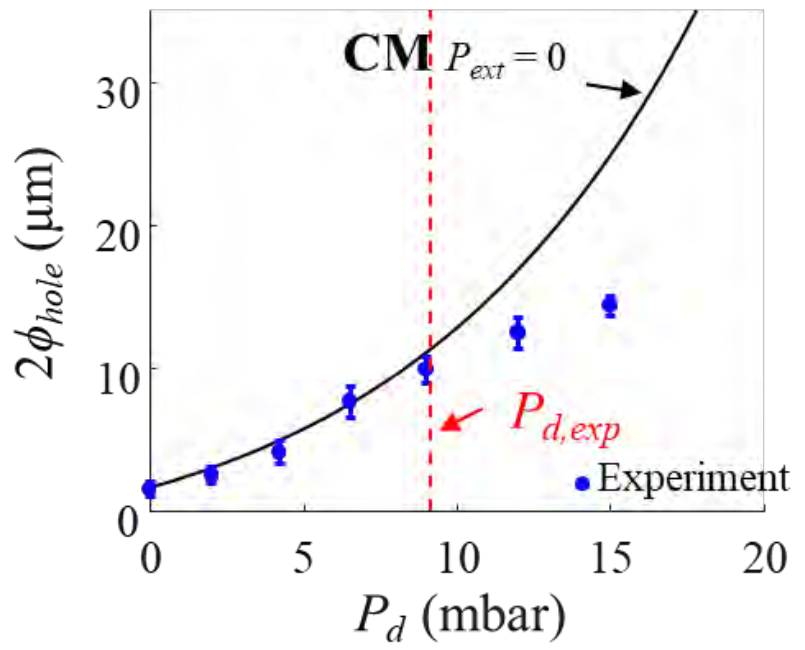

(a)

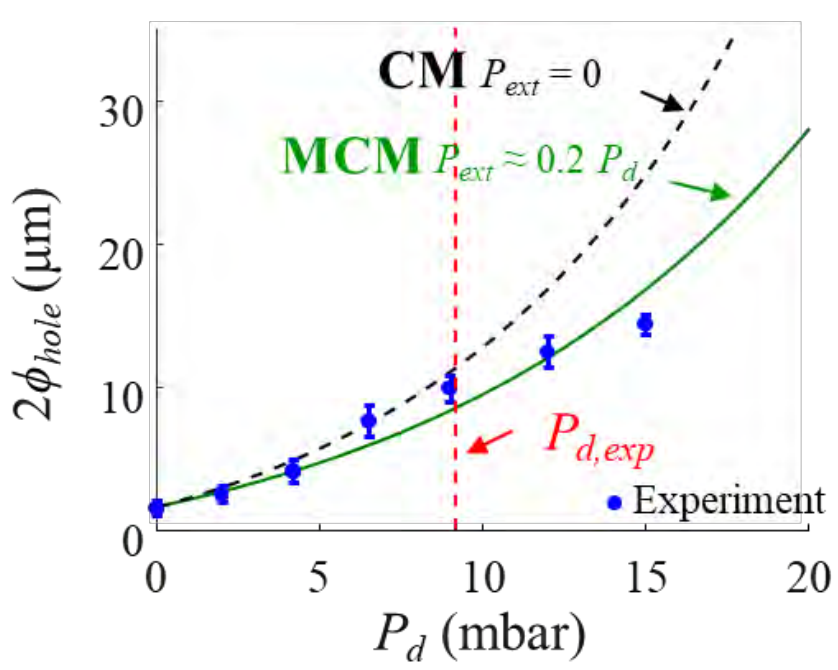

(b)

Figure 2. Experimental vs predicted results of the single capillary model at $T_{d}=1860{ }^{\circ} \mathrm{C}, P_{d}=(0.1-16)$ mbar: (a) Ignoring $P_{\text {ext }}$ and (b) with $P_{\text {ext }} \approx 0.2 P_{d}$.

To maintain a desirable level of surface tension, whilst not making the fibre brittle at too low $T_{\mathrm{d}}$, the furnace temperature had to lie between $1855<T<1880^{\circ} \mathrm{C}$. Here, $T_{d}=$ $(1860-1870){ }^{\circ} \mathrm{C}$ and $P_{d}=(0.1-16)$ mbar were considered.

Scanning electron microscope (SEM) and optical microscope were used to image the SOFs. Image analysis characterised the fibre geometry and determined the air-hole size. The average size of the air-holes in the first ring from the core is used for comparison (Figure 1(c)). One would expect the changes in dimension here to be approximated by a constrained single capillary with a diameter close to that of the ring diameter. Figure 2(a) shows the experimental results from the SOF drawn and also the results for a single capillary analysis (CM) under different drawing pressures at $T_{d}=1860{ }^{\circ} \mathrm{C}$. The expansion pressure thresholds of a single drawn capillary were calculated from Eq. (3). Within experimental error there is good agreement between experimental and predicted results prior to rapid expansion (i.e. when the effective ring of holes starts to expand rapidly). However, when $P_{d}>P_{d, \text { exp }}$, the size of the air-hole predicted by the Fitt model deviates significantly from that of the experimental results.

To model the system in its entirety using a multi-capillary model would be extremely complicated. Considering that the net effect of the structure is to oppose the expansion from the beginning, we can introduce an external pressure $P_{\text {ext }}$ to quantify the net counter-pressure to a hole's expansion from the lattice holes in the SOF structure. The value of $P_{\text {ext }}$ depends on the position of the air-holes in the SOF, the size of the holes, and the internal drawing pressure. Hence in a multiple air-hole SOF, the effective draw pressure, $P_{d, \text { eff }}\left(P_{d, \text { eff }}=P_{d}-P_{\text {ext }}\right)$ determines a hole's size, corresponding to $P_{d}$ in a single capillary structure. By applying this $P_{d, e f f}$ we introduce a modified single capillary model (MCM) for SOF, providing a simple, intuitive and direct measure of multiple capillary structure contributions, which define a so-called structural constraint parameter, SC, that can be used to optimise designs. In terms of the normalized pressure parameter $P_{S C}$ :

$P_{S C}=\frac{L\left(P_{d}-P_{e x t}\right)}{2 \beta \mu v_{f}}$

Based on this modified single capillary model, the external pressure $P_{\text {ext }}$ will increase the expansion limit by:

$P_{d, \exp }>\frac{\gamma}{\phi_{s c}}+\frac{\beta \mu v_{f}}{L}+P_{\text {ext }}$

in which $P_{d, \exp }$ is the internal drawing pressure leading to expansion of the entire structure. From the experimental results, this external pressure can be defined as a function of the internal drawing pressure and predicted to be $P_{\text {ext }} \approx 0.2 P_{d}$, as shown in Figure 2(b).

Furthermore, Figure 3 (a) and (b) show the experimental results and the predicted results from the SOF (fused inner capillaries diameter $2 \phi_{c}=1.45 \mathrm{~mm}$ ) at different drawing conditions $T_{d}=1865{ }^{\circ} \mathrm{C}$ and $1870{ }^{\circ} \mathrm{C}, v_{f}=0.5 \mathrm{~mm} / \mathrm{min}, v_{d}=16.5$ $\mathrm{m} / \mathrm{min}$, respectively. Seen from Figure 3 , good agreement is also observed between the experimental and predicted results by using $P_{e x t} \approx 0.2 P_{d}$. 


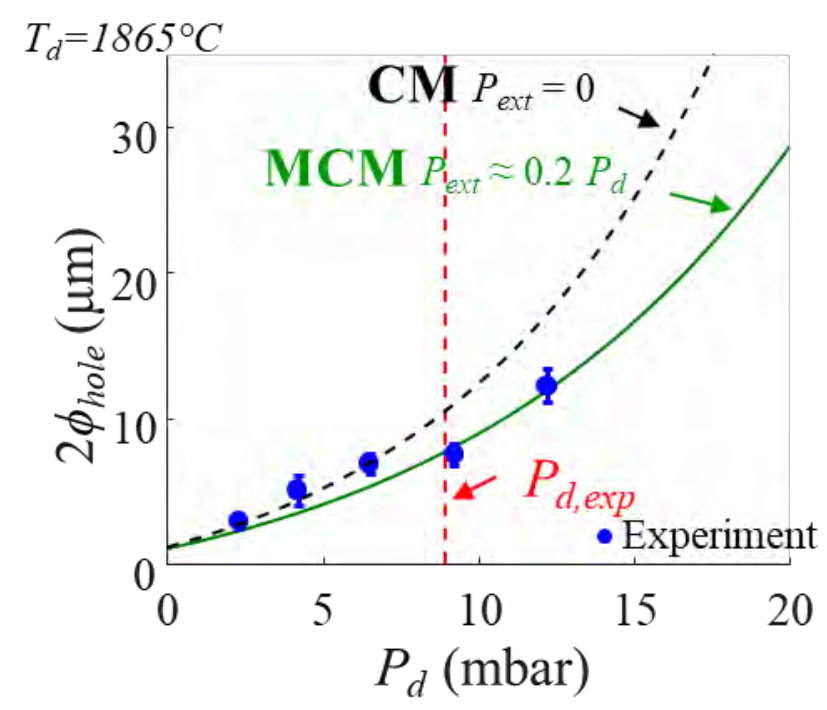

(a)

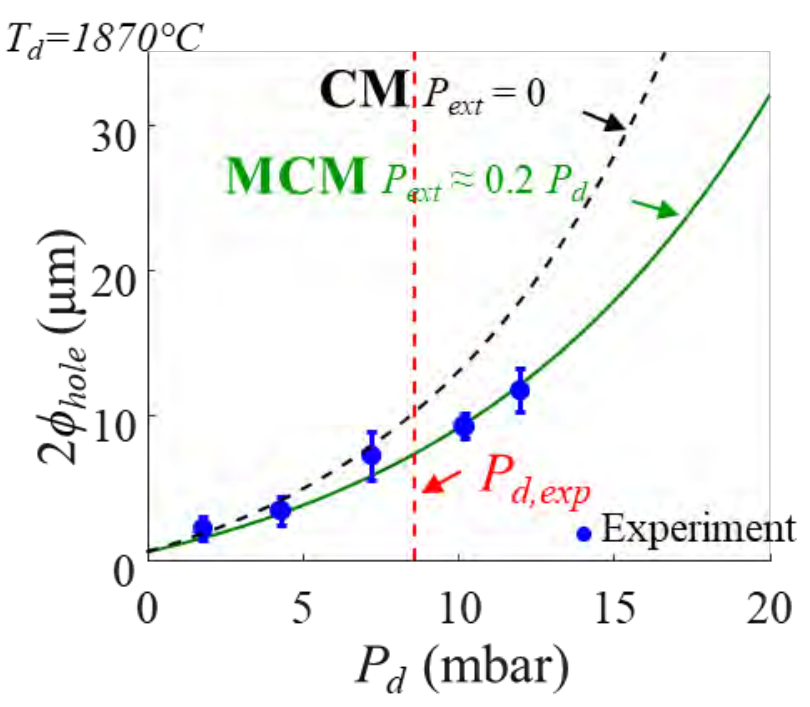

(b)

Figure 3. Experimental vs predicted results of the single capillary model (CM) and the modified single capillary model (MCM) at $P_{d}=$ (0.1-16) mbar: (a) $T_{d}=1865^{\circ} \mathrm{C}$ and (b) $T_{d}=1870{ }^{\circ} \mathrm{C}$.

\section{Conclusion}

Using an extended single-capillary function, a simple analytical approach to model SOF structure with regard to drawing parameters and conditions is reported. A single capillary analogy alone is insufficient because it ignores the expected contribution from an effective counter pressure arising from the multi-capillary interior. However, in order to retain the simplicity of the single capillary approach whilst being able to describe the actual pressures for a multi-capillary system of the type in a SOF, the single capillary model has been extended to include a structural constraint parameter arising from the internal capillary structure. This assumes the dominant force on the fibre remains the net expansion of the entire system with the structure acting as a constraint, consistent with observations. An effective external pressure was defined and added to the single capillary model leading to generate agreement between the experimental and predicted results here, when external pressure is close to $1 / 5$ of drawing pressure. The model retains simplicity but has physical meaning supporting it with structural constraint as a direct measure of interest for the study of SOFs. It explains the origin of hole flattening often observed in the drawn fibre.

The model can be used to explore and quantify the variations in more complex structures and obtain information that will relate to the ability to tune, for example, stresses and strains in the final fibre. In our experiments, SOFs were fabricated under different drawing conditions and the structurerelated results are shown to give agreement with the simple analytical fit. Further refinements are possible by closely studying the regime below rapid expansion in more detail and exploring the impact of different hole sizes and designs. More complex asymmetric profiles can be modeled using axially variant models. This work suggests the impact of hole flattening and so on can be controllably increased or reduced, if not eliminated, by optimising the temperature and drawing pressures that influence the magnitude of constraint.

Acknowledgment: The authors thank Air Force Office of Scientific Research (AFOSR), in partnership of the Asian Office of Aerospace R\&D (AOARD) and the High Energy Laser Joint Technology Office (HELJTO), for grant (FA2386-16-1-4031).

\section{References}

[1] Kaiser, P. and Astle, H. W., "Low-loss single-material fibers made from pure fused silica," Bell Syst. Tech. J. 53(6), 10211039 (1974).

[2] Canning, J., [Optical Fiber: Structured], Encyclopedia of Optical and Photonic Engineering, Taylor \& Francis, (2015)

[3] Canning, J., [Structured Optical Fibres and the Application of their Linear and Non-Linear Properties], Selected Topics in Photonic Crystals and Metamaterials, Italy, 389-452 (2011)

[4] Knight, J. C., Birks, T. A., Russell, P. S. J. and Atkin, D. M., "All-silica single-mode optical fiber with photonic crystal cladding," Opt. Lett. 21(19), 1547-1549 (1996).

[5] Birks, T. A., Knight, J. C., and Russell, P. S. J., "Endlessly single-mode photonic crystal fiber," Opt. Lett. 22(13), 961-963 (1997). 
[6] Buczynski, R., "Photonic crystal fibers," Acta. Phys. Pol. A 106(2), 141-168 (2004).

[7] Stone J., "Photonic crystal fibres and their applications in the nonlinear regime," Ph.D. dissertation (School of Physics, University of Bath, 2009).

[8] Michie, A., Canning, J., Lyytikainen, K., Åslund, M., and Digweed, J., "Temperature independent highly birefringent photonic crystal fibre," Opt. Express 12(21), 5160-5165 (2004).

[9] Matsui, T., Nakajima, K., and Fukai, C., "Applicability of photonic crystal fiber with uniform air-hole structure to highspeed and wide-band transmission over conventional telecommunication bands," J. Light. Technol. 27(23), 5410-5416 (2009).

[10] Michie, A., Canning, J., Bassett, I., Haywood, J., Digweed, K., Ashton, B., Stevenson, M., Digweed, J., Lau, A. and Scandurra, D., "Spun elliptically birefringent photonic crystal fibre for current sensing," Meas. Sci. Technol. 18(10), 3070 (2007).

[11] Chen, T., Chen, R., Jewart, C., Zhang, B., Cook, K., Canning, J. and Chen, K.P., "Regenerated gratings in air-hole microstructured fibers for high-temperature pressure sensing," Opt. Lett. 36(18), 3542-3544 (2011).

[12] Cook, K., Canning, J., Holdsworth, J., \& Dewhurst, C. "Stable CW Single-Mode Photonic Crystal Fiber DFB Ring Laser," J. Electron. Sci and Technol 6(4), 442-444 (2008).

[13] Groothoff, N., Canning, J., Ryan, T., Lyytikainen, K. and Inglis, H., "Distributed feedback photonic crystal fibre (DFB-PCF) laser," Opt. Express 13(8), 2924-2930 (2005).

[14] Tafti, G., Wang, W., Karpisz, T., Canning, J., Cook, K., Luo, Y., Wang, S. and Peng, G., "Fabrication and structure of Hi-Bi Micro-structured optical fibre," Proc. ANZCOP, 142 (2017).

[15] Lyytikainen, K., Canning, J., Digweed, J. and Zagari, J., "Geometry control of air-silica structured optical fibres," Proc. COIN/ACOFT, 137-140 (2003).

[16] Lyytikainen, K., "Control of complex structural geometry in optical fibre drawing," Ph.D. dissertation (School of Physics and Optical Fibre Technology Centre, University of Sydney, 2004).

[17] Wang, W., Karpisz, T., Tafti, G., Canning, J., Cook, K., Luo, Y. and Peng, G., "Optimal pressure for tuning the lattice structure of photoinic crystal fibre," Proc. ANZCOP, 141 (2017).

[18] Lyytikainen, K., Canning, J., Digweed, J. and Zagari, J., "Geometry control of air-silica structured optical fibres using pressurization," Proc. IMOC 2, 1001-1006 (2003).

[19] Fitt, A.D., Furusawa, K., Monro, T.M., Please, C.P. and Richardson, D.J., "The mathematical modelling of capillary drawing for holey fibre manufacture," J. Engrg. Math. 43(2-4), 201-227 (2002).

[20] Chen, Y. and Birks, T.A., "Predicting hole sizes after fibre drawing without knowing the viscosity," Opt. Mater. Express 3(3), 346-356 (2013).

[21] Ebendorff-Heidepriem, H. and Monro, T.M., "Extrusion of complex preforms for microstructured optical fibers," Opt. Express 15(23), 15086-15092 (2007).

[22] Zhu, Y., Bise, R.T., Kanka, J., Peterka, P. and Du, H., "Fabrication and characterization of solid-core photonic crystal fiber with steering-wheel air-cladding for strong evanescent field overlap," Opt. Commun. 281(1), 55-60 (2008).

[23] Kostecki, R., Ebendorff-Heidepriem, H., Warren-Smith, S.C. and Monro, T.M., "Predicting the drawing conditions for microstructured optical fiber fabrication," Opt. Mater. Express 4(1), 29-40 (2014).

[24] Denisov, A.N., Kosolapov, A.F., Senatorov, A.K., Pal'tsev, P.E.E. and Semjonov, S.L., "Fabrication of microstructured optical fibres by drawing preforms sealed at their top end," Quant. Electron. 46(11), 1031-1039 (2016).

[25] Canning, J., Buckley, E. and Lyytikainen, K., "Multiple source generation using air-structured optical waveguides for optical field shaping and transformation within and beyond the waveguide," Opt. Express 11(4), 347-358 (2003).

[26] Cook, K., Canning, J., Leon-Saval, S., Reid, Z., Hossain, M.A., Comatti, J.E., Luo, Y. and Peng, G.D., "Air-structured optical fiber drawn from a 3D-printed preform," Opt. Lett. 40(17), 3966-3969 (2015).

[27] Canning, J., Cook, K., Luo, Y., Leon-Saval, S., Peng, G., Comatti, E., Hossain, M.A. and Reid, Z., "3D printing optical fibre preforms," Proc. ACP, ASu4B.2 (2015).

[28] Bansal, N.P. and Doremus, R.H., [Handbook of glass properties], Academic Press, New York, (1986). 


\title{
Inverse modelling of microstructured optical fibre drawing
}

\author{
Yvonne Stokes*a, Michael Chen ${ }^{\mathrm{a}}$ \\ ${ }^{a}$ School of Mathematical Sciences, The University of Adelaide, Australia, 5005
}

\begin{abstract}
Drawing of a fibre with desired structure is a difficult inverse problem, requiring determination of preform geometry and draw parameters. A mathematical model is described which enables solution of this inverse problem in a very efficient way. An excellent prediction of preform and draw parameters for a desired fibre may be obtained reducing the need for expensive experimental trials.

Keywords: microstructured optical fibres, inverse problem, mathematical modelling, asymptotic methods, free-boundary problem, extensional flow.
\end{abstract}

*yvonne.stokes@adelaide.edu.au; phone 618 8313-4808

\section{Introduction}

We consider the drawing of a microstructured optical fibre from a preform, containing a pattern of air channels, as depicted in Figure 1. We take the $x$-axis to be directed vertically downwards along the axis of the preform/fibre. The preform, with cross-sectional area $S_{i n}$, enters a heater at $x=0$ at a slow feed speed $U_{i n}$. Between $x=0$ and $x=L_{h}$ heat is applied so that the preform becomes a deformable viscous fluid thread, and from $x=L_{h}$ to $x=L>L_{h}$ the thread cools to a solid. At $x=L$ the thread is pulled at a much larger draw speed $U_{\text {out }}$ by a take-up roller. Over the so-called neck-down region $0 \leq x \leq L$ the preform deforms greatly to form a fibre, due primarily to the draw ratio $D=U_{\text {out }} / U_{\text {in }} \gg 1$ which results in reduction of the cross-sectional area $S$ with $x$. By mass conservation, the cross-sectional area of the fibre at $x=L$ is $S_{\text {out }}=S_{\text {in }} / D$. However, surface tension and/or any pressure applied within the channels, also deforms the geometry. Thus, in order to manufacture a fibre with some desired geometry it is necessary to determine a preform geometry and the draw parameters that will yield the desired fibre. The crucial draw parameters are the draw ratio $D$, the pulling tension $\mathcal{T}$ and the constant pressure $p_{H}$ applied within the channels. The effect of gravity is negligible and, hence, is not considered.

This is a difficult inverse problem. Starting with an initial estimate/guess for the preform and draw parameters, an experimental trial-and-error process might be used to iteratively find a satisfactory answer, but this is costly in both time and resources. Similarly, a trial-and-error process employing numerical simulation, as in [10], might be used but, again, this is costly in terms of computational resources and is not yet practical for fibres with many air channels. However, noting that the neck-down geometry is often slender, such that the parameter $\epsilon=\sqrt{S_{i n}} / L$ is much less than unity, the authors and coworkers have exploited this property and used asymptotic methods to obtain an extensional-flow model that may be solved very efficiently for fibres with any internal geometry. The modelling approach is described in $[3,11]$ (building on earlier work including $[5,6,7,8,9])$, while numerical techniques that enable efficient handling of arbitrary internal geometry are described in $[1,2]$. Importantly, the model enables direct solution of the inverse problem. Furthermore, it gives understanding of the key parameters that may be used to control the result.

\section{Asymptotic mathematical model}

The fibre drawing process described above is, essentially, a steady-state viscous flow problem and we assume that the softened fibre material (typically a glass) behaves as an incompressible Newtonian fluid with viscosity $\mu$, that depends on temperature, and constant surface tension $\gamma$. Application of asymptotic methods to the flow and temperature equations (see $[3,11,12]$ for details), shows that the axial velocity $u$ and temperature are uniform in any cross section and so change with axial position $x$ only. Since the viscosity $\mu$ depends on temperature it is also a function of $x$ only. Furthermore the model becomes a 1D problem for the change in

Sixth International Workshop on Specialty Optical Fibers and Their Applications (WSOF 2019),

edited by Liang Dong, John M. Ballato, Proc. of SPIE Vol. 11206, 112060Z · C 2019 SPIE

CCC code: $0277-786 X / 19 / \$ 21 \cdot$ doi: $10.1117 / 12.2547746$

Proc. of SPIE Vol. 11206 112060Z-1 


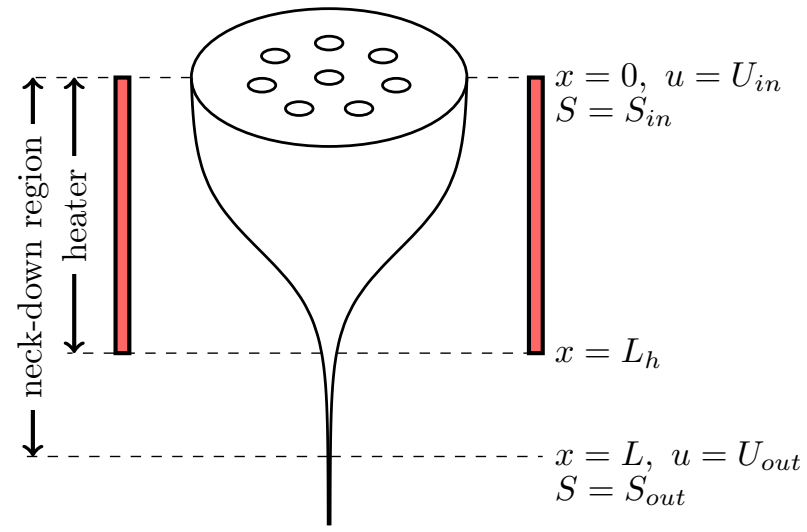

Figure 1: Schematic diagram of the neck-down region, $0 \leq x \leq L$, over which the initial cross-sectional area of the thread $\chi_{i n}^{2}$ reduces to that of the fibre $\chi_{\text {out }}^{2}$ due to the large draw speed $U_{\text {out }}$ relative to the feed speed $U_{\text {in }}$.

cross-sectional area due to axial stretching and a 2D cross-plane problem for the deformation in the cross-section due to surface tension and channel pressurisation.

For the purposes of modelling we define the scaled cross-sectional area as

$$
\chi^{2}=S / S_{\text {in }}
$$

where $\chi$ is the square root of the dimensionless cross-sectional area and $\chi=1$ at $x=0$. We also introduce scaled pulling tension, channel pressure and surface tension parameters, denoted by asterisks and given by

$$
\mathcal{T}^{*}=\frac{\mathcal{T}}{6 \gamma \sqrt{S_{\text {in }}}}, \quad \mathcal{P}^{*}=\frac{p_{H} \sqrt{S_{\text {in }}}}{\gamma} \quad \text { and } \quad \gamma^{*}=\frac{\gamma L}{\bar{\mu} U_{i n} \sqrt{S_{\text {in }}}},
$$

where

$$
\bar{\mu}=L / \int_{0}^{L} \frac{1}{\mu(x)} d x
$$

is the harmonic mean of the viscosity over $0 \leq x \leq L$ which is used to scale the viscosity, i.e. $\mu^{*}=\mu / \bar{\mu}$. Then $\chi$ is given by the ordinary differential equation

$$
\frac{d \chi}{d \tau}-\frac{1}{12} \chi \Gamma=-\mathcal{T}^{*}, \quad \chi(0)=1,
$$

where $\tau$ is a new independent variable related to the scaled axial position $x^{*}=x / L$ by

$$
\frac{d x^{*}}{d \tau}=\frac{\mu^{*}}{\gamma^{*} \chi}, \quad x^{*}(0)=0,
$$

and $\chi(\tau) \Gamma(\tau)$ is the sum of the lengths of all boundaries in the cross-section at position $x(\tau)$. In addition, from $S_{\text {out }}=S_{\text {in }} / D$, we have the boundary condition $\chi=1 / \sqrt{D}$ at $x^{*}=1$, which is used to determine the tension parameter $\mathcal{T}^{*}$. We define $\tau_{\text {out }}$ as the value of $\tau$ for which $x^{*}(\tau)=1$.

The function $\Gamma(\tau)$ is determined from a scaled cross-plane problem which removes the change in crosssectional area due to the draw ratio and models the change in the geometry of a cross-section of unit area, due to both surface tension and channel pressurisation, over $0 \leq \tau \leq \tau_{\text {out }}$. This is a classical two-dimensional free-boundary Stokes-flow problem driven by unit surface tension and channel pressure $\mathcal{P}^{*}$. The equations for conservation of mass and momentum in the fluid are

$$
v_{y}+w_{z}=0, \quad v_{y y}+v_{z z}=p_{y}, \quad w_{y y}+w_{z z}=p_{z},
$$

with boundary conditions

$$
\begin{aligned}
G_{\tau}^{(i)}+v G_{y}^{(i)}+w G_{z}^{(i)} & =0, \\
\left(-p+2 v_{y}\right) G_{y}^{(i)}+\left(v_{z}+w_{y}\right) G_{z}^{(i)} & =-\left(\kappa^{(i)}+\mathcal{P}^{(i)} \chi\right) G_{y}^{(i)}, \\
G_{y}^{(i)}\left(v_{z}+w_{y}\right)+G_{z}^{(i)}\left(-p+2 w_{z}\right) & =-\left(\kappa^{(i)}+\mathcal{P}^{(i)} \chi\right) G_{z}^{(i)},
\end{aligned}
$$




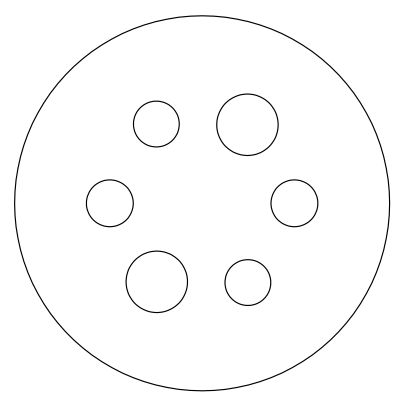

Figure 2: Schematic of the preform geometry. The preform outer diameter is $3.93 \mathrm{~mm}$. The six internal holes are each described by their diameter $d$ and their position $(x, y)$, where the centre of the outer boundary is $(x, y)=$ $(0,0)$. Clockwise from top right the six holes are $(d, x, y)=(611,484,822),(469,968,1.12),(458,477,-828)$, $(611,477,823),(468,-966,-0.986)$ and $(459,-477,827) \mu \mathrm{m}$. Reprinted, with permission, from Chen et al., J. Lightwave Tech. 34(24), 5651- 5656 [4, fig. 4].

where $v$ and $w$ are the velocities in the $y$ and $z$ directions, respectively, $p$ is the pressure in the fluid region, $G^{(0)}(\tau, y, z)=0$ is the external boundary subject to external pressure $\mathcal{P}^{(0)}=0, G^{(i)}(\tau, y, z)=0, i=1, \ldots, N$, are the $N$ interior boundaries subject to the channel pressure $\mathcal{P}^{(i)}=\mathcal{P}^{*}, i=1, \ldots, N$, and $\kappa^{(i)}$ is the curvature of the $i$ th boundary. Here subscripts denote differentiation with respect to the subscript variables. The solution of this cross-plane problem yields both $\Gamma(\tau)$, as well as the evolution of the cross-sectional geometry. We note that if the channels are not pressurised $\left(\mathcal{P}^{*}=0\right)$ the cross-plane problem completely decouples from the axial stretching problem and may be solved first but, otherwise, there is full coupling and both problems must be solved simultaneously.

A variety of methods can be used to solve the cross-plane problem depending on the geometry. There is an analytic solution for an unpressurised tube [11] and an efficient computational routine is available where the shape of the channels is restricted to be ellipses [2]. For very general channel shapes a spectral method [1] can be used to compute their evolution efficiently.

\section{Model validation}

The validity of our model is demonstrated in [4] which describes a comparison of results from our model with experimental results and 3D finite element simulations of Luzi et al. [10] for the six-hole preform shown in Figure 2, which has a cross-sectional area of $S_{i n}=1.088 \times 10^{-5} \mathrm{~m}^{2}$. The feed and draw speeds were $U_{\text {in }}=3.333 \times 10^{-4} \mathrm{~m} \mathrm{~s}^{-1}$ and $U_{\text {out }}=3.117 \times 10^{-1} \mathrm{~m} \mathrm{~s}^{-1}$, for a draw ratio of $D=935$. The silica glass used for the preform had a surface tension of $\gamma=0.3 \mathrm{~N} \mathrm{~m}^{-1}$, the neck-down length was taken to be $L=0.1 \mathrm{~m}$ and the harmonic mean of the viscosity over the neck-down was calculated to be $\bar{\mu}=1.13 \times 10^{6} \mathrm{Pas}$. We solved our model numerically using the spectral method of [1] for the cross-plane problem. Figure 3 overlays the fibre geometries yielded by our model, the experiments and the 3D finite-element simulations for the values of pulling tension $\mathcal{T}$ and channel pressurisation $p_{H}$ there shown. Our model performed extremely well and better than the finite-element simulation. This provides excellent validation of the asymptotic modelling approach. For further details see [4].

\section{Solution of the inverse problem}

Given a desired fibre geometry, what preform and draw parameters will yield it? This is an inverse problem which is of great practical importance. To solve it we specify the fibre geometry, rather than the preform geometry, and run our model backwards for some choice of the draw parameters to find the preform geometry that is required for the choice of parameters. The choice of the draw ratio immediately gives the cross-sectional area of the preform from the cross-sectional area of the fibre while the pulling tension and channel pressurisation determine the difference in shape between the preform and fibre. 

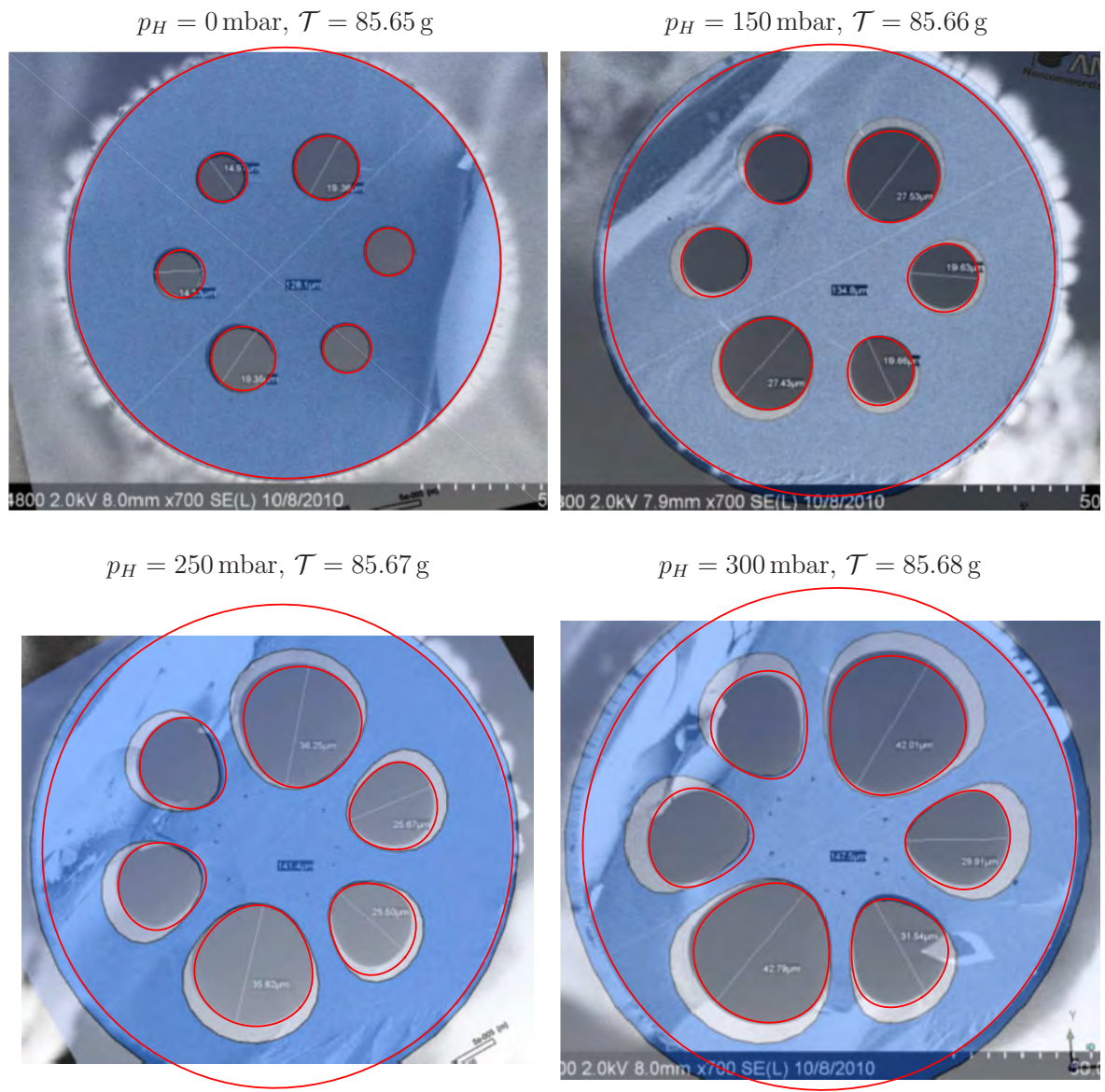

$(100 \mathrm{~Pa}=1 \mathrm{mbar})$

Figure 3: Experimental microscope images of the fibre cross-section, overlaid with the finite element simulation of [10] (pale blue transparency) and the results of the new asymptotic simulation (thin red lines). Shown are the four values of pressurisation from [10, Fig. 3]. For each example the pressurisation applied is shown in the caption above the image, along with the fibre tension as calculated by the iterative scheme. (c) 2016 IEEE. Reprinted, with permission, from Chen et al., J. Lightwave Tech. 34(24), 5651- 5656 [4, fig. 4].

(a)

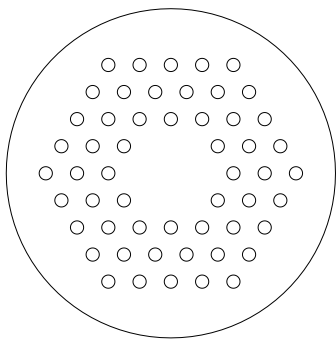

(b)

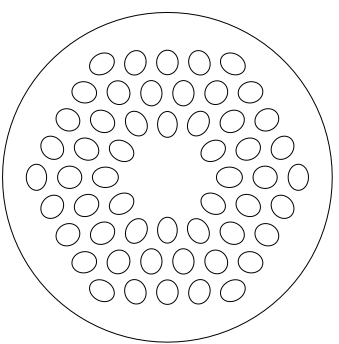

(c)

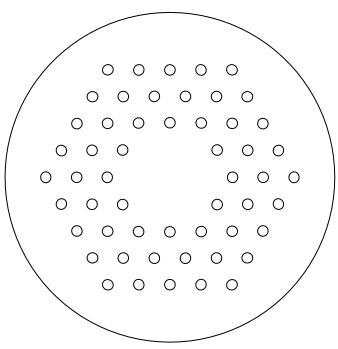

Figure 4: (a) Desired geometry of the fibre which has cross-sectional area $S_{\text {out }}=2.39 \times 10^{-8} \mathrm{~m}^{2}$. Required preform geometry when using a pulling tension $\mathcal{T}=35 \mathrm{~g}$ and channel pressurisation (b) $p_{H}=0$, (c) $p_{H}=$ $3000 \mathrm{~Pa}$. The remaining parameters for the displayed solutions are $D=3000, S_{\text {in }}=7.18 \times 10^{-5} \mathrm{~m}^{2}, U_{\text {in }}=$ $2.33 \times 10^{-2} \mathrm{~m} \mathrm{~s}^{-1}$ and $\gamma=0.23 \mathrm{~N} \mathrm{~m}^{-1}$. 
Figure 4(b,c) shows two preform geometries that may be used to obtain the fibre geometry shown in Figure 4(a) which has cross-sectional area $S_{\text {out }}=2.39 \times 10^{-8} \mathrm{~m}^{2}$. The draw ratio has been set at $D=3000$ so that the preform has cross-sectional area $S_{i n}=7.18 \times 10^{-5} \mathrm{~m}^{2}$. A tension of $\mathcal{T}=35 \mathrm{~g}$ is required and there must be no channel pressurisation for preform (b) while, for preform (c) the channel pressure must be $p_{H}=3000 \mathrm{~Pa}$. Not surprisingly, the size of the preform channels decreases as the pressurisation is increased. A benefit of pressurising the channels is that they are (nearly) circular in the preform which may render the preform more easily manufactured. These solutions were computed using the elliptic pore model [2] for the cross-plane problem. For more detail on this example see [3].

\section{Conclusions}

Asymptotic methods have been used to obtain an efficient model of drawing of microstructured optical fibres containing many air channels. The model comprises a 1D problem for the change in cross-sectional area due to stretching and a $2 \mathrm{D}$ problem for the deformation in the cross-section due to surface tension and channel pressurisation. The model may be used to determine the fibre geometry given a preform geometry and draw parameters (the forward problem) or to solve the inverse problem of determining a preform geometry and draw parameters for a desired fibre geometry. The crucial parameters that control the draw are the draw ratio $D$, which determines the change in cross-sectional area from preform to fibre, and the pulling tension $\mathcal{T}$ and channel pressurisation $p_{H}$ which determine the deformation of the cross-sectional shape. The model has been demonstrated to be accurate where the geometry through the neck-down region is sufficiently slender.

It is noteworthy that the crucial parameters required to draw a given fibre from a given preform may be determined without the need to know anything about the temperature of the fibre material or the exact neckdown length; see [11] for a detailed discussion of this. When using a draw tower fitted with a tension-measuring device, the furnace temperature can be adjusted to yield the required pulling tension. This is of great practical value since determining the temperature and, therefore, the viscosity, through the neck-down region is extremely difficult, if not impossible. We also note that there is not a unique viscosity, hence temperature, profile for a given fibre draw. Rather, the model indicates that all temperature profiles which yield viscosity profiles with the same harmonic mean through the neck-down region give the same fibre from a given preform. The temperature profile affects the evolution of the geometry along the neck-down region but not the end result. Should the geometry through the neck-down region be important, or the draw tower not provide for measurement of pulling tension, then a temperature model must be coupled to the flow model discussed herein. This is considered in $[12]$.

\section{References}

[1] Buchak, P., Crowdy, D.G., "Surface-tension-driven Stokes flow: A numerical method based on conformal geometry," J. Comput. Phys. 317, 347-361 (2016).

[2] Buchak, P., Crowdy, D.G., Stokes, Y.M., Ebendorff-Heidepriem, H., "Elliptical pore regularisation of the inverse problem for microstructured optical fibre fabrication," J. Fluid Mech. 778, 5-38 (2015).

[3] Chen, M.J., Stokes, Y.M., Buchak, P., Crowdy, D.G., Ebendorff-Heidepriem, H., "Microstructured optical fibre drawing with active channel pressurisation," J. Fluid Mech. 783, 137-165 (2015).

[4] Chen, M.J., Stokes, Y.M., Buchak, P., Crowdy, D.G., Ebendorff-Heidepriem, H., "Asymptotic modelling of a six-hole MOF," J. Lightwave Technol. 34, 5651-5656 (2016).

[5] Cummings, L.J., Howell, P.D., "On the evolution of non-axisymmetric viscous fibres with surface tension, inertia and gravity," J. Fluid Mech. 389, 361-389 (1999).

[6] Dewynne, J.N., Ockendon, J.R., Wilmott, P., "A systematic derivation of the leading-order equations for extensional flows in slender geometries," J. Fluid Mech. 244, 323-338 (1992).

[7] Dewynne, J.N., Howell, P.D., Wilmott, P., "Slender viscous fibres with inertia and gravity," Q. Jl Mech. appl. Math. 47, 541-555 (1994). 
[8] Fitt, A.D., Furusawa, K., Monro, T.M., Please, C.P., Richardson, D.A., "The mathematical modelling of capillary drawing for holey fibre manufacture," J. Engng. Maths 43, 201-227 (2002).

[9] Griffiths, I.M., Howell, P.D., "Mathematical modelling of non-axisymmetric capillary tube drawing," J. Fluid Mech. 605, 181-206 (2008).

[10] Luzi, G., Epple, P., Scharrer, M., Fujimoto, K., Rauh, C., Delgado, A., "Numerical solution and experimental validation of the drawing process of six-hole optical fibers including the effects of inner pressure and surface tension," J. Lightwave Technol. 30, 1306-1311 (2012).

[11] Stokes, Y.M., Buchak, P., Crowdy, D.G., Ebendorff-Heidepriem, H., "Drawing of microstructured optical fibres: circular and non-circular tubes," J. Fluid Mech. 755, 176-203 (2014).

[12] Stokes, Y.M., Wylie, J.J., Chen, M.J., "Coupled fluid and energy flow in fabrication of microstructured optical fibres," J. Fluid Mech. 874, 548-572 (2019). 


\title{
ISRAELI ACTIVITIES IN SPECIALTY FIBERS AND FIBER LASERS
}

\author{
Amiel A. Ishaaya ${ }^{[1]}$, Yoav Sintov ${ }^{[2]}$
}

Within the present talk, we review the main activities that are presently taking place in Israel in the field of specialty fibers and fiber lasers. We start by presenting the objectives and infrastructure of the newly built Israel Center of Advanced Photonics (ICAP). An example of a recent ICAP activity concerning fs inscription of Fiber Bragg Gratings (FBGs) will be presented. The objective of this activity was to inscribe an FBG through the polymer coating, without stripping the fiber, while maintaining the coating functionality, especially in double clad fibers. Next, we review the main activities within the ALTIA Consortium, with an emphasis on the newly developed high power UV and Green lasers for micromachining applications. Examples of laser micromachining applications of the consortium partners will be presented. Next, we present the high power Raman fiber laser development at Soreq, which aims to reach more than $5 \mathrm{~kW}$ from a single fiber source. So far $1.2 \mathrm{~kW}$ output power has been achieved with good beam quality and significant brightness enhancement compared to the pump source. Finally, we present two novel Yb-doped double clad fiber designs. The first is a large area multicore fiber with six doped cores which was designed and fabricated jointly by BGU and NTU, and the second design is a very large mode double clad fiber where the amplification takes place in the clad.

[1] School of Electrical and Computer Engineering, Ben-Gurion University in the Negev, BeerSheva 8410501, Israel. Email: ishaaya@bgu.ac.il

[2] ICAP/Soreq, Yavne 81800, Israel.Email: sintovy@soreq.gov.il 


\title{
3D silica lithography for Doped and Structured Optical Fibers
}

\author{
Gang-Ding Peng* \\ Photonics and Optical Communications, School of Electrical Engineering and Telecommunications, \\ University of New South Wales, Sydney, NSW 2052, Australia
}

\begin{abstract}
This presentation will report on recent progresses and discuss current challenges in research and development of 3D silica lithography based new fabrication technologies for future doped and structured optical fibers.

Keywords: 3D silica lithography, 3D silica printing, optical fiber fabrication, optical fiber manufacture, doped optical fiber, structured optical fiber

*G.Peng@unsw.edu.au; phone 61293854014
\end{abstract}

\section{Introduction}

Chemical vapor deposition (CVD) based silica fiber fabrication has been the prevailing traditional optical fiber fabrication way and produced most of commercial optical fibers and fiber amplifiers so far. As the Internet evolves into a so-called ubiquitous "Internet of things," or IoT, the role of optical fibers is expanding from a mainly passive telecommunications transmission medium to multifunctional host for fiber sensing, fiber devices and lasers, and beyond. This is creating a great demand for optical fibers of diversified materials and sophisticated structures. Nevertheless the traditional silica fiber fabrication, based on CVD as well as rod-and tube stacking, has limited capability in both material and structure flexibility for diverse and custom-designed functionalities. The recent research and development of 3D silica lithography using digital light processing and related technologies show a new pathway to future doped and structured optical fibers [1].

3D printing technology allows new possibilities in fiber fabrication [2]. The early work in this field was on 3D printing plastic fibers [3]-[6]. The recent development of 3D silica printing lithography [7]-[11] for glass applications shows enormous potential in fabricating silica with greater freedom in both structure and material formation, while highly possible with superior optical transparency. Although 3D polymer fiber work can be easily applied to lower-temperature soft glasses such as borosilicate or chalcogenides, silica is a very hightemperature material could mean more significant challenges. High purity silica is also extremely difficult to shape and dope, something presently inaccessible to 3D printing. As of the time of writing, there are no reports of 3D printed silica optical fiber preforms or fibers although there are recent works toward 3D printing optical glasses [12].

Here we reported our progress in demonstrating the first successful 3D printed step-index silica optical preforms and the fibers drawn from these preforms. Although the quality and design are far from desirable so far, we believe that 3D printing based technology could offer great potential to produce new silica fibers for future applications.

\section{3D Printed Silica Preform Fabrication}

Our new pathway to manufacture silica optical fibers is to use Digital Light Processing (DLP) or Stereo Lithography (SLA) based 3D printing technology, as depicted in Figure 1. This pathway will include several steps:

1. Material preparation and processing: UV curable solution doped with nanoparticles, rare earths and / or other functional dopants; 
2. 3D printing of structured and / or doped silica preform;

3. Sintering and consolidation of silica preform;

4. Drawing optical fiber from the 3D printed and thermally processed preform.
By incorporating 3D printing into traditional fiber technology, one could realise both structured and doped fiber fabrication, and may produce specialty silica fibers that require custom design structures and material compositions.

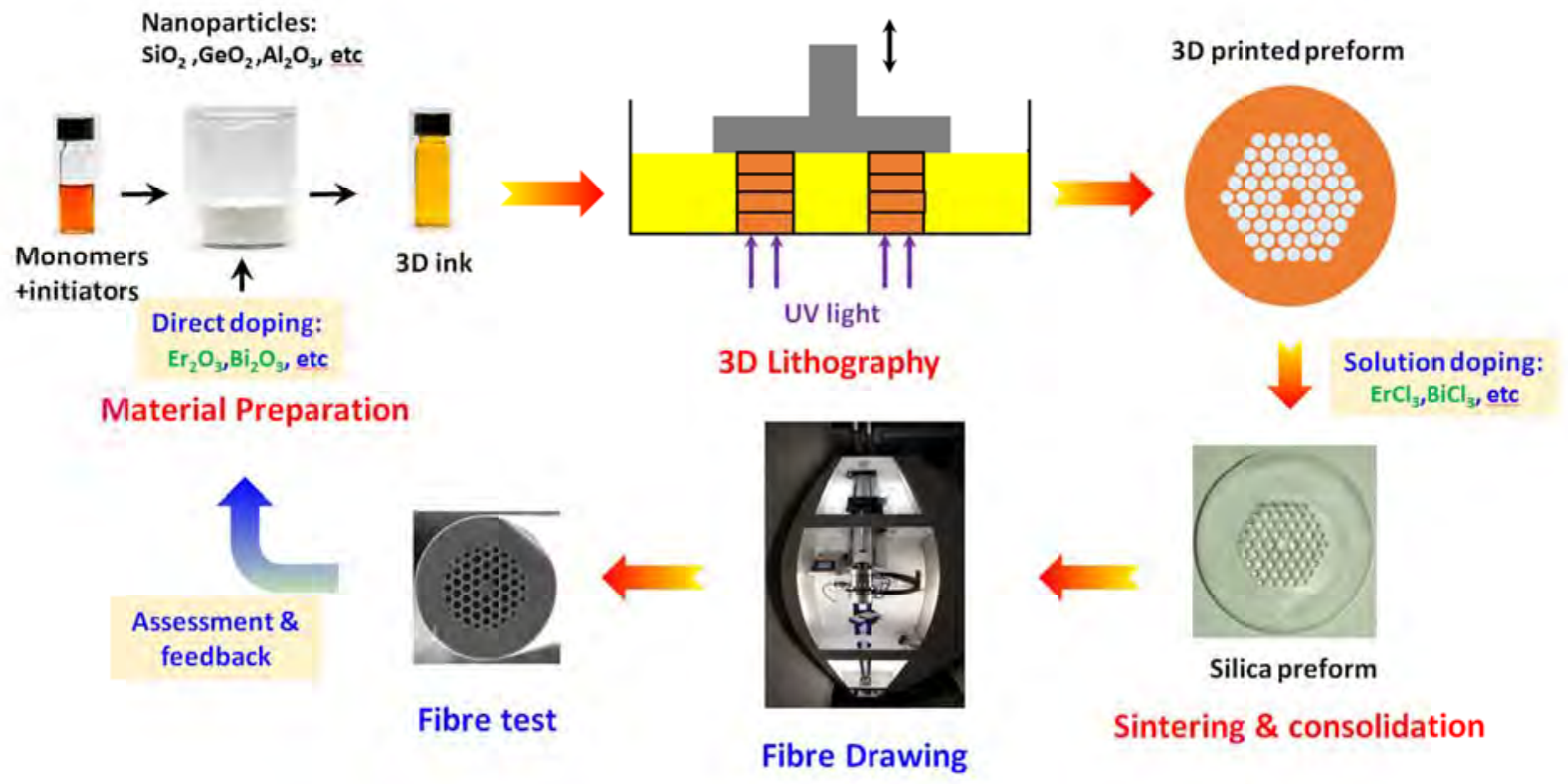

Figure 1. 3D lithography-based new pathway to silica optical fiber fabrication.

In terms of material preparation for silica stereolithograohy, proper material formulation is needed for conditioning photo- or thermal polymerization for optimization of 3D printing speed and resolution, as well as polymer debinding and silica sintering processes. Researchers in Karlsruhe Institute of Technology (KIT) initiated and demonstrated very effective techniques [7][9]. They reported the first SLA-based 3D printed, transparent fused silica glass with several millimeter scale and micrometer resolution, achieved by suspending nanoparticle silica in ultraviolet (UV) sensitized monomer.

We used material preparation processes similar to that reported by KIT, although our debinding and, especially, sintering processes are quite different. In our first 3D printed silica optical preforms and fibers [13]-[14], we used amorphous silica nanoparticles (Aerosil OX50 from Evonika) with a mean diameter of $40 \mathrm{~nm}$ and dispersed them into initiated monomers to form a UV curable resin.
The UV curable resin is a mixture of 2-hydroxyethyl methacrylate (HEMA), 2-phenoxyethanol (POE), tetra(ethylenglycol) diacrylate (TEGDA), diphenyl $(2,4,6-$ trimethylbenzoyl) phosphine oxide (DPO) and hydroquinone (Hyd). Compositions of the printing resin are tested with varying amount of $\mathrm{SiO} 2$, up to $40 \mathrm{wt} \%$ of $\mathrm{SiO} 2$ nanoparticles, which can mix well with HEMA (up to $40 \mathrm{wt} \%)$, POE ( $20 \mathrm{wt} \%)$, TEGDA (5 8 $\mathrm{wt} \%)$, DPO $(\sim 0.2 \mathrm{wt} \%)$ and Hyd $(\sim 0.1 \mathrm{wt} \%)$.

The mixed resin can work well with our Digital Light Processing (DLP) 3D printer (Asiga Freeform Pro 2) Taking advantage of 3D printing and selecting proper writing parameters, different designs and arbitrary structures of special fiber preforms can be printed, such as multicore or photonic crystal fibers. Figure 2 shows two samples of the 3D printed fiber preforms with high concentrations of silica nanoparticles. This shows that silica fiber preforms can be 3D printed to required structures of good quality. 


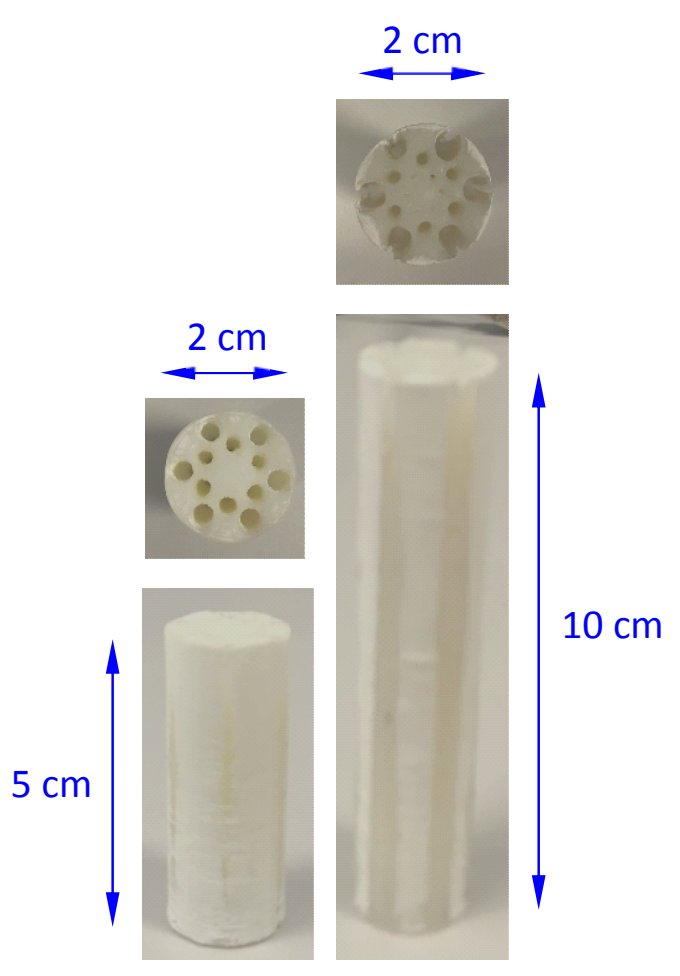

Figure 2. Raw silica fiber preforms fabricated using the UV curable resin with a DLP 3D printer.

Figure 3 and Figure 4 show two samples of the 3D printed fiber preforms before and after their sintering at different temperature in oven. Since our sintering is a process that temperature increases progressively and slowly, the sintering temperature mentioned here and hereafter stands for the final stage temperature in a full sintering process. Evidently these preforms experience significant changes in materials and sizes through the sintering process. In general, sintering at $800{ }^{\circ} \mathrm{C}$ or above the polymer contents, and monomer residual if any, will be removed. For sintering at $1200{ }^{\circ} \mathrm{C}$ or above the
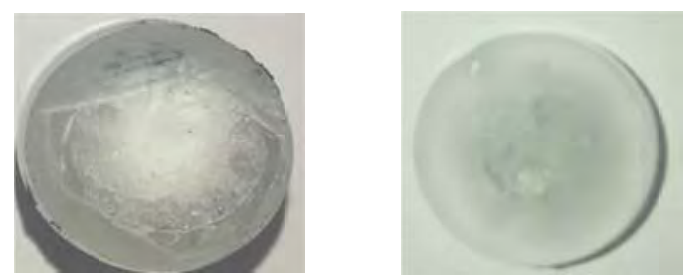

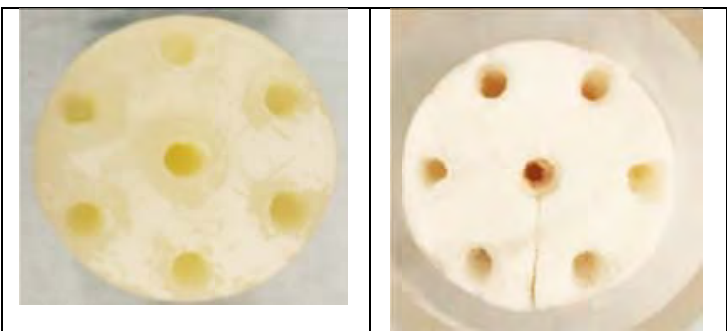

(a) before sintering

(b) after sintering

Figure 3. 3D printed silica fiber preforms before and after sintering at $600^{\circ} \mathrm{C}$ for about 4 hours.

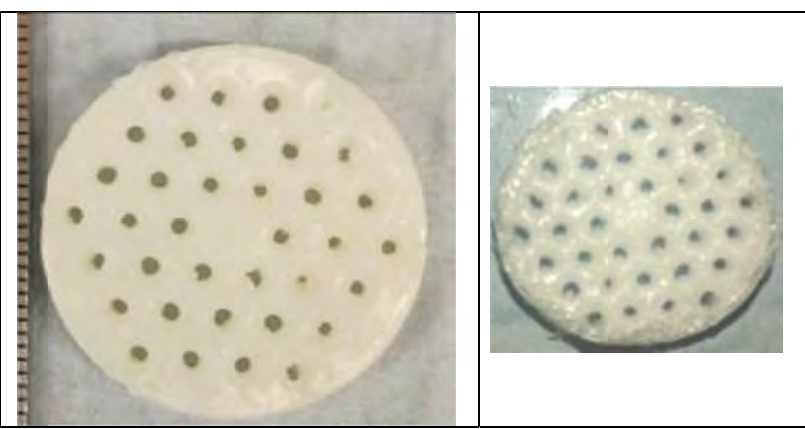

(a) before sintering

(b) after sintering

Figure 4. 3D printed silica fiber preforms before and after sintering at $1300^{\circ} \mathrm{C}$ for about 4 hours.

vitrification of silica will become significant. However, as seen in Figure 4 in the case that, after the preform is sintered at $1300^{\circ} \mathrm{C}$ for about 4 hours, the vitrification of silica remains incomplete that the preforms are opaque and could contain air bubbles. Hence we investigated further with various sintering conditions and processes. $3 \mathrm{D}$ printed preforms sintered at $1600^{\circ} \mathrm{C}$ for $20 \sim 30$ minutes, are shown as examples of incomplete or nonideal vitrification cases in Figure 5. How to minimize and remove possible non-ideal defects or air bubbles remains a great challenge in this regard.
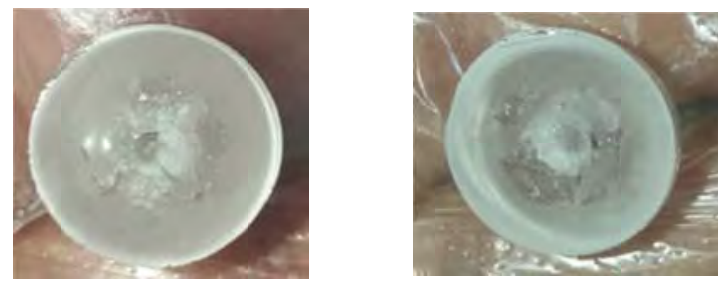

Figure 5. Sliced 3D printed silica fiber preforms showing non-ideal and incomplete cases of silica vitrification. The preforms are sintered at $1600^{\circ} \mathrm{C}$ for $20 \sim 30$ mins. 


\section{Step-Index Silica Optical Fiber from 3D Printed Preform}

We have demonstrated the feasibility of the proposed pathway by successfully drawing the first batch of stepindex silica optical fibers from 3D printed preform recently [14]. In this drawing, 3D printed silica preforms were first thermally processed to get polymer removed and silica partially sintered. The processed preforms were then inserted into a fused silica tube (Heraeus F300) which would be drawn together with the 3D printed preforms and becomes the outer cladding of fiber. To avoid possible crystallization at high sintering temperature, the final sintering process at higher temperature is carried out within the furnace at fiber draw tower and just before fiber drawing. We inspected the remaining preform left from fiber drawing and figured out how the 3D printed silica preform had progressed through the final sintering stage before fiber drawing. We established that we have achieved good vitrification of the 3D silica preform through the final 'on-tower' sintering process as expected.

Figure 6 shows the measured refractive index profile of the fiber tip at the start of fiber drawing. This tip is thicker than all other sections of the optical fiber and its index profile can be measured using our preform index analyzer (Photon Kinetics PK2600). As seen from Figure 6, there exist three distinguishable layers: the outer cladding layer formed by fused silica tube; the 3D printed inner cladding (the region within the blue dot - dash line) and the higher index core.

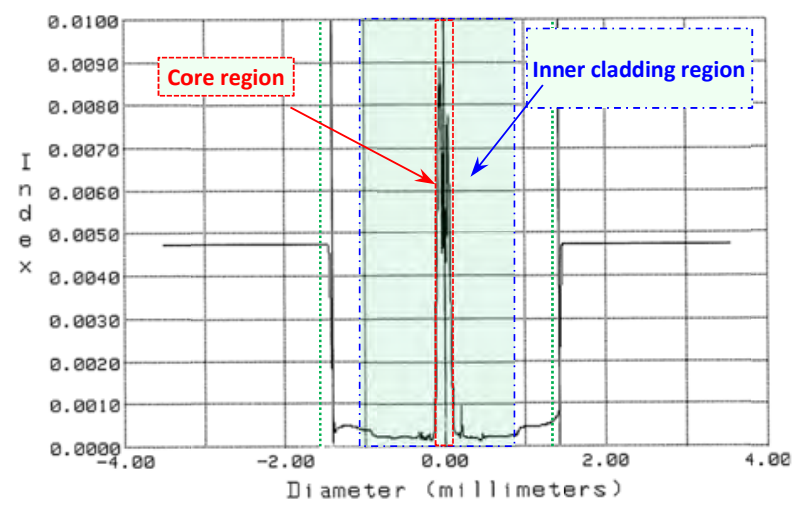

Figure 6. Index profile of the fiber tip at the start of fiber draw.
The fiber's geometry was inspected using a desktop optical microscope. The near field photo of a silica fiber sample drawn from one of the 3D printed preforms is shown in Figure 7. The cross section as seen from Figure 7 is uniform with good waveguide structure and free from air bubbles. The diameter of this fiber sample is $147 \mu \mathrm{m}$. The core is partly deformed and its diameter is about 8 $\mu \mathrm{m}$.

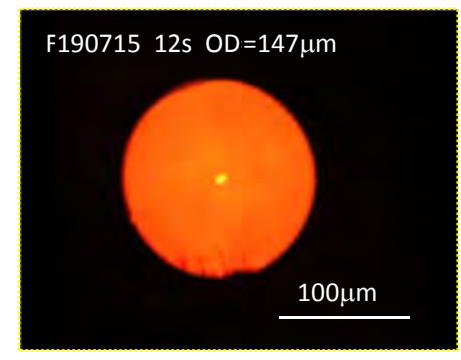

Figure 7 The near field photo of a fiber sample.

We have examined the fiber's light transmission, simply using a $532 \mathrm{~nm}$ laser as shown in Figure 8 . Although the fiber sample guides light with proper waveguide structure, as clearly seen in Figure 8, the light scattering is quite significant and bright spots are scattered along fiber length. Figure 8 indicates that the fiber is not very uniform and has high scattering loss. The far field from the fiber is projected onto a screen through a lens. The far field is seen as a green multimode beam /spot which is included in the inset of Figure 8.

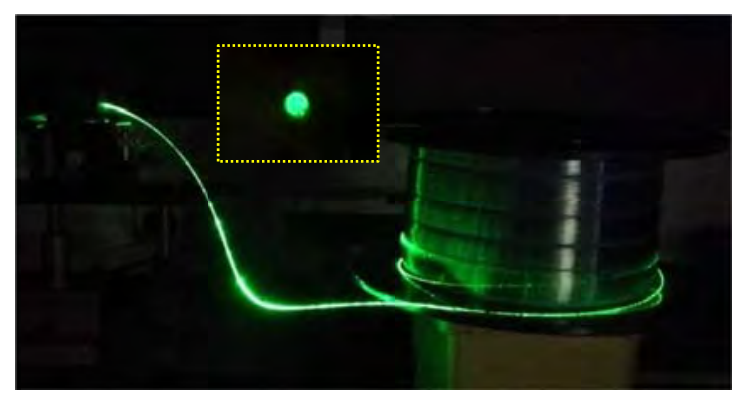

Figure 8 The light transmission of the optical fiber when launched with $532 \mathrm{~nm}$. The inset is the far field pattern of the fiber.

The spectral attenuation of a few fiber samples draw from the $3 \mathrm{D}$ printed silica preforms has been measured. 
All the fiber samples tested have shown high attenuation and high scattering loss. A typical example of the spectral attenuation is shown in Figure 9. The fiber sample tested shows a very high loss coefficient, $\sim 6 \mathrm{~dB} / \mathrm{m}$, at $1550 \mathrm{~nm}$. In addition, high loss of $\sim 20 \mathrm{~dB} / \mathrm{m}$ is observed at 1380 $\mathrm{nm}$ which is attributed to the $\mathrm{OH}$ overtone.

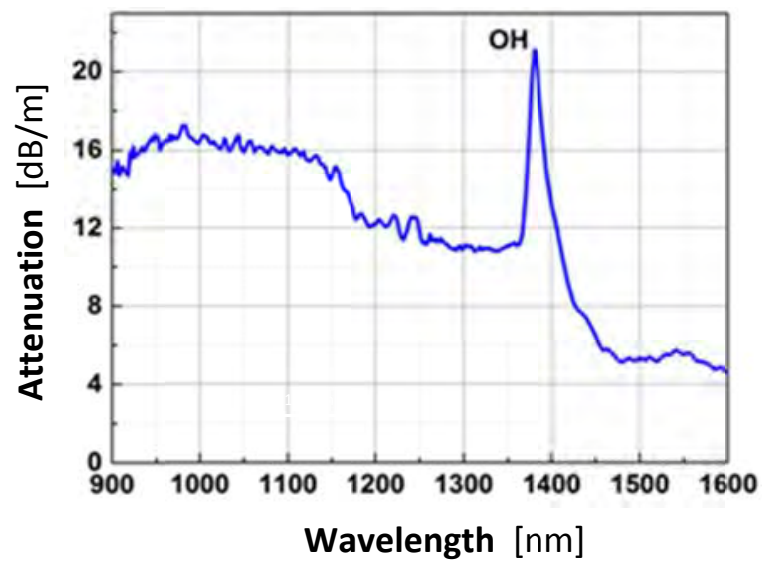

Figure 9. Measured spectral attenuation of one of the $1^{\text {st }}$ step-index fiber draw from 3D printed silica preforms.

Several short 3D printed preforms were drawn in this $1^{\text {st }}$ successful fiber drawing which is achieved after a few failed earlier attempts. In total over $2 \mathrm{~km}$ of the fibers were produced. Based on our initially test and assessment, fiber quality (structure, material and transparency), rather than quantity, is the key remaining challenge in future development.

\section{Doped Silica Optical Fiber from 3D Printed Preform}

Following a similar process to the fabrication of the undoped 3D step-index fiber, we have successfully made the first bismuth and erbium co-doped silica optical fibers from 3D printed preform.

In our preliminary experiment, both bismuth and erbium related emission features have been observed as shown in Figure 10. Both the emission around $1430 \mathrm{~nm}$ that is related to bismuth active center related to silicon (BAC-Si) and the emission around $1530 \mathrm{~nm}$ that is from $\mathrm{Er}^{3+}$ are quite typical. They are similar to that from $\mathrm{Bi} / \mathrm{Er}$ codoped fiber made from solution doping based on conventional CVD technology. The experimental observation of typical emission characteristics of $\mathrm{Bi}$ and Er indicates good possibility and feasibility for us to achieve optical amplification and lasing from the $\mathrm{Bi} / \mathrm{Er}$ codoped fiber drawn from 3D printed preform.

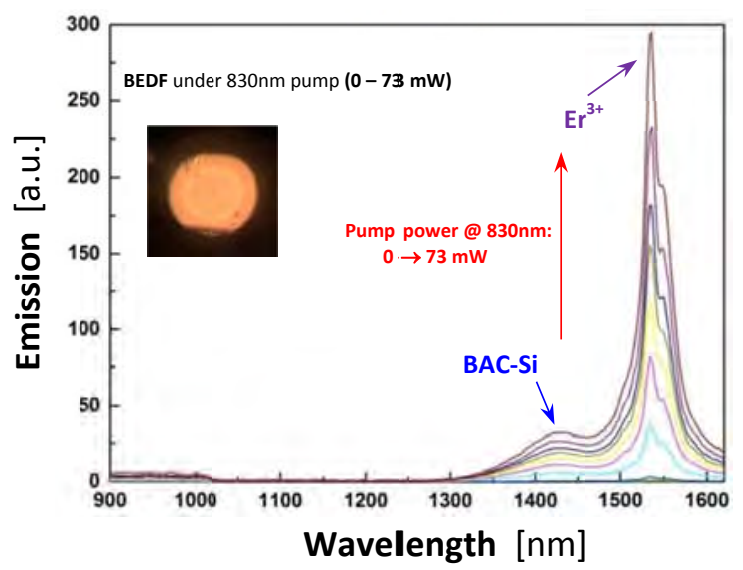

Figure 10. Spectral emission from one Bi/Er co-doped optical fiber draw from 3D printed silica preform.

In this first doped fiber draw from 3D silica preform, the dopants are directly and conveniently mixed into the UV curable resin. This is the first experimental demonstration of doping directly in UV curable resin, one of the two options proposed in the pathway depicted in Figure 1. We believe that the new pathway through 3D printed preform can make the fabrication of doped silica optical fibers substantially easier and more controllable.

\section{Future Work}

The development of 3D silica lithography or 3D silica printing brings about great opportunity by enabling both custom-design structure and material in special optical fiber fabrication. We have successfully demonstrated the first silica optical fiber preforms, step-index fibers and, $\mathrm{Bi}$ and Er co-doped fibers based on 3D silica lithography.

Though there remain many significant challenges, such as high background loss, high $\mathrm{OH}$ absorption and significant structure deformation, these preliminary progresses suggest good feasibility and flexibility and show great potential of the new pathway through 3D silica printing for future custom-design special optical fibers. 
Acknowledgement: Many of my colleagues: Yushi Cui, Xinghu $\mathrm{Fu}$, John Canning, Yanhua Luo, Jianxian Wen and Jianzhong Zhang, have made main contributions to design, fabrication and test in this research.

\section{References}

[1] Peng, G. D., Luo, Y., Zhang, J., Wen, W., Chu, Y., Cook, K. and Canning, J., "3D Silica Lithography for Future Optical Fiber Fabrication", in Peng, G. D. (ed.), Handbook of Optical Fibers, Springer Nature, Singapore, 637-653, https://doi.org/10.1007/978-981-10-7087-7 79, (2019)

[2] Bikas, H., Stavropoulos, P. and Chryssolouris, G., "Additive manufacturing methods and modelling approaches: a critical review," The International Journal of Advanced Manufacturing Technology, 83(1-4), pp.389-405 (2016)

[3] Cook, K., Canning, J., Leon-Saval, S., Reid, Z., Hossain, M. A., Comatti, J.-E., Luo, Y. and Peng, G. D., "Airstructured optical fibre drawn from a 3D-printed preform," Opt. Lett. 40, 3966-3969 (2015)

[4] Cook, K., Balle, G., Canning, J., Chartier, L., Athanaze, T., Hossain, M. A., Han, C., Comatti, J.-E., Luo, Y. and Peng, G. D., "Step-index optical fibre drawn from 3D printed preforms," Opt. Lett. 41, 4554-4557 (2016)

[5] Canning, J., Hossain, M. A., Han, C., Chartier, L., Cook, K. and Athanaze, T., "Drawing optical fibres from 3D printers", Opt. Lett., vol. 41 (23), 2016, pp. 5551-5554 (2016)

[6] Flanagan, P., Cook, K., Canning, J., "3D Printed Photonic Ribs: a new platform for devices, sensors and more", Asia Pacific Optical Sensors (APOS2018), Matsue City, Shimane, Japan (2018)

[7] Kotz, F., Arnold, K., Bauer, W., Schild, D., Keller, N., Sachsenheimer, K.,Nargang, T. M., Richter, C., Helmer, D., and Rapp, B. E., "Three-dimensional printing of transparent fused silica glass", Nature 544, 337 (2017)

[8] Kotz, F., Plewa, K., Bauer, W., Schneider, N., Keller, N., Nargang, T., Helmer, D., Sachsenheimer, K., Schäfer, M., Worgull, M. and Greiner, C., "Liquid glass: a facile soft replication method for structuring glass." Advanced Materials 28.23: 4646-4650 (2016)

[9] Kotz, F., Schneider, N., Striegel, A., Wolfschläger, A., Keller, N., Worgull, M., Bauer, W., Schild, D., Milich, M., Greiner, C. and Helmer, D., "Glassomer-Processing Fused Silica Glass Like a Polymer", Advanced Materials, 30(22), p.1707100 (2018)

[10] Nguyen, D. T., Meyers, C., Yee, T. D., Dudukovic, N. A., Destino, J. F., Zhu, C., Duoss, E. B., Baumann, T. F., Suratwala, T., Smay, J. E. and Dylla-Spears, R., "3DPrinted Transparent Glass", Advanced Materials 29, 1701181 (2017)

[11] Destino, J. F., Dudukovic, N. A., Johnson, M. A., Nguyen, D. T., Yee, T. D., Egan, G. C., Sawvel, A. M., Steele, W. A., Baumann, T. F., Duoss, E. B., Suratwala, T., and Dylla-
Spears, R. "3D Printed Optical Quality Silica and SilicaTitania Glasses from Sol-Gel Feedstocks", Advanced Materials Technologies 3, 1700323 (2018)

[12] Cooperstein, I., Shukrun, E., Press, O., Kamyshny, A., and Magdassi, S., "Additive Manufacturing of Transparent Silica Glass from Solutions," ACS Applied Materials \& Interfaces 10, 18879-18885 (2018)

[13] Chu, Y., Canning, J., Fu, X., Luo, Y., Cook, K., Zhang, J. and Peng, G. D., "3D printed optical fibre preforms from silica contained resin", $5^{\text {th }}$ WSOF, (2019)

[14] Chu, Y., Fu, X., Luo, Y., Canning, J., Tian, Y., Cook, K., Zhang, J. and Peng, G. D., "Step-index silica optical fibre drawn from 3D printed preforms", paper in preparation 


\title{
Single crystal fiber growth by Laser Heated Pedestal Growth Technique
}

\author{
Subhabrata Bera*a,b, \\ Paul Ohodnicki ${ }^{\mathrm{a}}$, Bo Liu ${ }^{\mathrm{c}, \mathrm{d}}$, Michael Buric ${ }^{\mathrm{c}}$, Benjamin Chorpening ${ }^{\mathrm{c}}$ \\ ${ }^{a}$ National Energy Technology Laboratory, 626 Cochrans Mill Road, Pittsburgh, PA 15236, USA \\ bLeidos Research Support Team, 626 Cochrans Mill Road, Pittsburgh, PA 15236, USA \\ 'National Energy Technology Laboratory, 3610 Collins Ferry Road, Morgantown, WV 26505, USA \\ ${ }^{\mathrm{d}}$ West Virginia University Research Co., 3610 Collins Ferry Road, Morgantown, WV 26505, USA
}

\begin{abstract}
Single crystal (SC) fibers combine the superior material properties of crystals with the advantages of a fiber geometry, and offer have great potential in harsh environment sensing as well as high-power laser gain media. Advances in the laser heated pedestal growth of SC fibers are discussed, along with results in various passive and active fiber applications.
\end{abstract}

Keywords: crystal fibers, solid state lasers, harsh environment sensing, sapphire, YAG, LHPG, spinel

*subhabrata.bera@netl.doe.gov

\section{Introduction}

Single crystal (SC) fibers present an interesting alternative to conventional glass fibers for both active and passive fiber applications. Glass fibers are not stable at the high temperature or energy fluences associated with applications like harsh environment sensing or high-power delivery systems respectively. In high power active fiber applications, limitations in power scaling in silica glass fibers arising from thermal lensing, onset of nonlinear phenomena, thermal shock and degradation of the fiber core, etc. hinder performance at high power densities. Since these inadequacies are related to the amorphous structure of the silica glass in the fiber medium, there has been a considerable push to move the harsh environment sensing fiber and the fiber laser architecture to a crystalline material medium. SC fibers grown from materials like sapphire or yttrium aluminum garnet (YAG) are thermally, chemically and mechanically more robust compared to conventional glass fibers, making them ideal for sensing applications in boilers, jet engines and nuclear reactors[1]. Additionally, high thermal conductivity and low nonlinear gain coefficients of the crystalline material, coupled with the high surface-area-to-volume ratio of the fiber geometry make SC fibers suitable for application involving high optical power density[2].

\section{Fiber Growth}

Among the processes available for growing crystals with high aspect ratio, the laser heated pedestal growth (LHPG) technique is the most suitable for growing small diameter crystal fibers with very long lengths[3]. LHPG, in essence, is a modified optical float-zone technique for growing single crystals where the melt is created by focusing a high-power laser on the tip of the starting feed rod. A seed crystal fiber is dipped into this melt and drawn up, forming a single crystal fiber. For a sustaining growth operation, both the fiber and the source feedstock must be simultaneously and continuously moved in the same direction to maintain a zero-mass accumulation in the molten zone. The diameter of the fiber is monitored by

Sixth International Workshop on Specialty Optical Fibers and Their Applications (WSOF 2019), edited by Liang Dong, John M. Ballato, Proc. of SPIE Vol. 11206, 1120612 · C 2019 SPIE

CCC code: $0277-786 X / 19 / \$ 21 \cdot$ doi: $10.1117 / 12.2543420$ 
a non-contact micrometer and its instantaneous value is controlled by varying the speeds of the fiber and the source rod automatically by utilizing a PID loop. The principal advantage of the LHPG technique over other crystal fiber growing techniques is the absence of a crucible, avoiding the possibility of contamination due to the refractory material of the crucible. However, due to the absence of a crucible, the molten zone is held together by surface tension and hence, is susceptible to mechanical perturbations during growth.



Figure 1: Schematic of Laser Heated Pedestal Growth Set-up

A pair of zinc-selenide transmissive axicons convert the output beam from an amplitude stabilized $\mathrm{cw} \mathrm{CO}_{2}$ laser to a collimated ring. This collimated ring is then focused on top of the source rod by a parabolic mirror to form the melt from which the SC fiber is grown. The stability and the uniformity of the molten zone is very critical to the quality of the single crystal fiber that is grown. Hence it is crucial to control and stabilize the $\mathrm{CO}_{2}$ laser of the LHPG. The output power of the laser is controlled by varying the operating current through a PID feedback loop. The position of the melt is kept constant while the seed and the source feedstock are moved out and into it respectively. Two sets of independent pulling mechanisms are required for the translation of the source pedestal and the growing SC fiber respectively. As the fiber is pulled continuously from the melt, the source pedestal must be fed simultaneously and continuously in the region of the focus of the laser beam to maintain a zero-net mass flux in the melt.

\section{Fiber Quality}

While SC fibers have theoretically shown a lot of promise, in practice the high background losses in such waveguides have been a major obstacle in fabricating highly efficient SC fiber-based devices. Previously losses in YAG SC fiber waveguides have been reported to be about $3 \mathrm{~dB} / \mathrm{m}$ to several $\mathrm{dB} / \mathrm{cm}$. Attenuation losses of such magnitude severely limit the length of fiber that can be used, and thereby constraining real-life applications. However, over the years, improvements in the growth process have helped in significantly reducing attenuation losses. Simulation aided careful alignment of the optical components of the growth apparatus have led to the development of fibers of record low losses[4, 5].

For sensor applications, along with low attenuation losses, fibers of significant lengths are desirable. While a single $10 \mathrm{~cm}$ source rod can theoretically yield about $900 \mathrm{~cm}$ of crystal fiber, in practice, the length obtained is much smaller as perturbations during the growth process can lead to significant localized scattering spots which can force the termination of fiber growth. In recent years, improvements in the growth process control algorithms have been developed to combat this issue and now automatic growth control can not only erase regions of local diameter fluctuations but also stitch fiber growths without introducing significant losses, leading to the possibility of "endless SC fibers"[6]. 


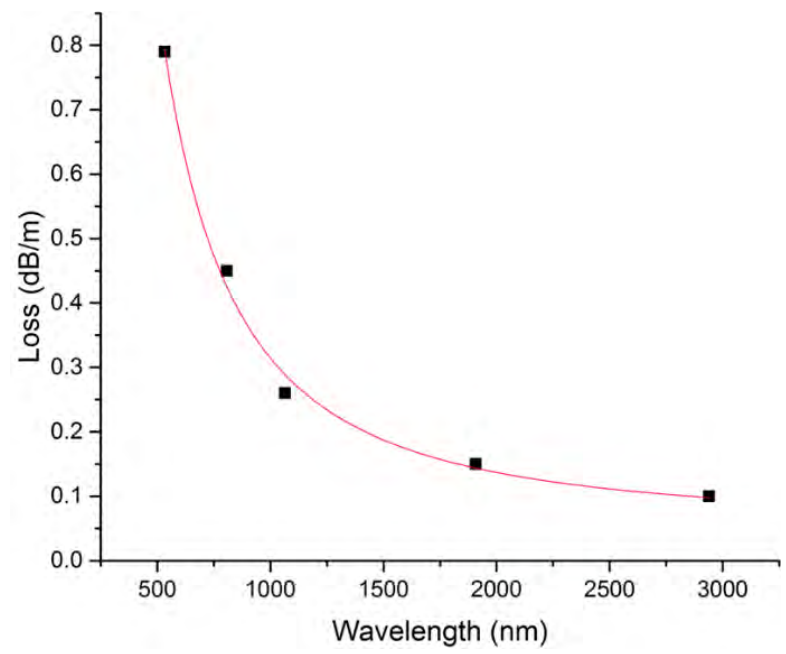

Figure 2: Total attenuation losses for a 1 meter long $330 \mu \mathrm{m}$ diameter YAG SC fiber at different wavelengths

\section{Doped Crystal Fibers}

One of the major driving forces in the resurgence of the research in crystal fibers has been due to the interest in developing high-power solid-state lasers[7, 8]. YAG SC fibers doped with various rare-earth ions are being extensively investigated. Due to similar size of the yttrium ion compared to most lanthanides, varying levels of the dopants can be introduced into the YAG matrix. Fiber lasers constructed with doped crystal fiber gain media have demonstrated excellent lasing characteristics.

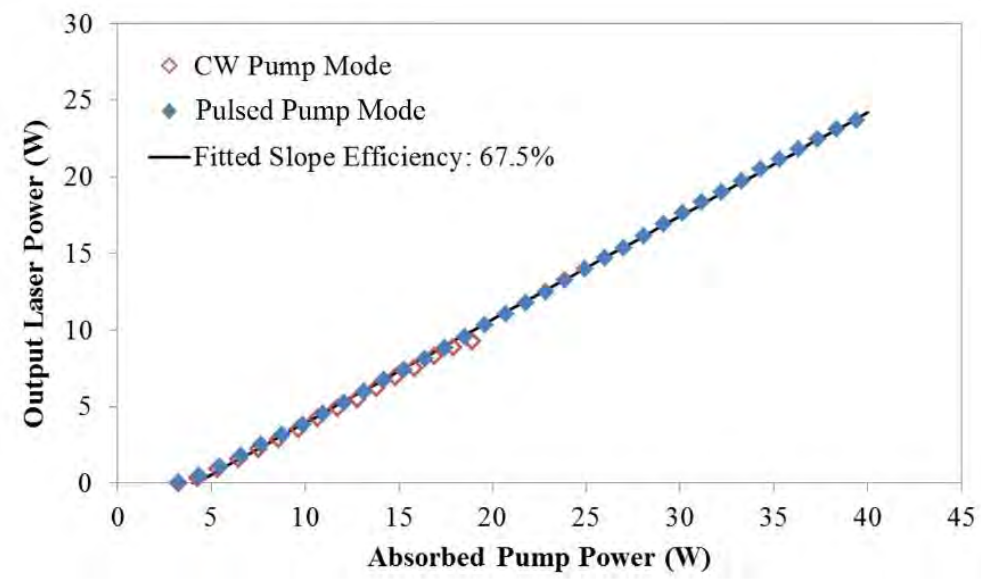

Figure 3: Slope efficiency of Ho:YAG SC fiber[9]

The output characteristics of a solid-state laser depends strongly on the concentration of dopants in the gain medium. If the dopant concentration is too low, the pump does not get sufficiently absorbed while if the concentration is too high, a part of the output is reabsorbed by the laser active ions. The background losses in YAG SC fibers is still considerably high, limiting the maximum length of the fiber gain medium. Hence varying the concentration of dopant for a given length is the more practical approach to optimizing the output gain of the SC fiber gain medium. While ceramic rods of tailored dopant concentration can be fabricated in-house, the binders required to make these materials contribute to significant increase in attenuation losses. A new hybrid method to grow high purity SC YAG fibers with varied RE dopant concentrations using a low cost, low temperature sol-gel based method has been developed. A sol-gel derived layer of a suitable RE dopant was deposited on a $330 \mu \mathrm{m}$ diameter previously grown SC fiber by dip-coating, which was then 
regrown to SC fibers with diameters of about $120 \mu \mathrm{m}$, to introduce the RE ions into the YAG matrix. Not only can the dopant concentration in these fibers was changed by varying the thickness of the sol-gel derived layer, the several dopants can be simultaneously introduced by dipping into different sol-gel materials.

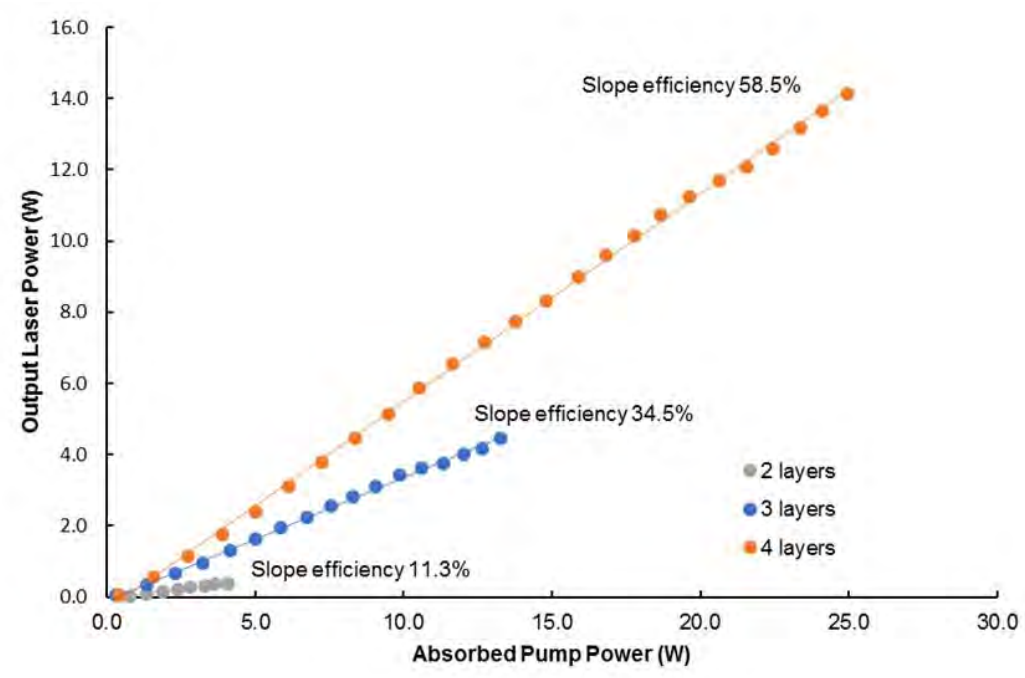

Figure 4: Slope efficiency of Ho:YAG fibers grown from a preform with different number of HoAG layers[10]

Control of diffusion is difficult to achieve during the growth of the SC fibers, hence complicating efforts to obtain a gradedindex fiber through a rod-in-tube approach[11]. However, it has been observed that certain dopants have the tendency to move towards the central axis of the fiber after growth. This effect is likely due to the thermo-capillary and convection currents in the melt zone during growth. In YAG, larger dopant ions like $\mathrm{Nd}^{3+}$ ions move towards the center whereas smaller ions like $\mathrm{Cr}^{4+}$ ions move towards the periphery[12, 13].
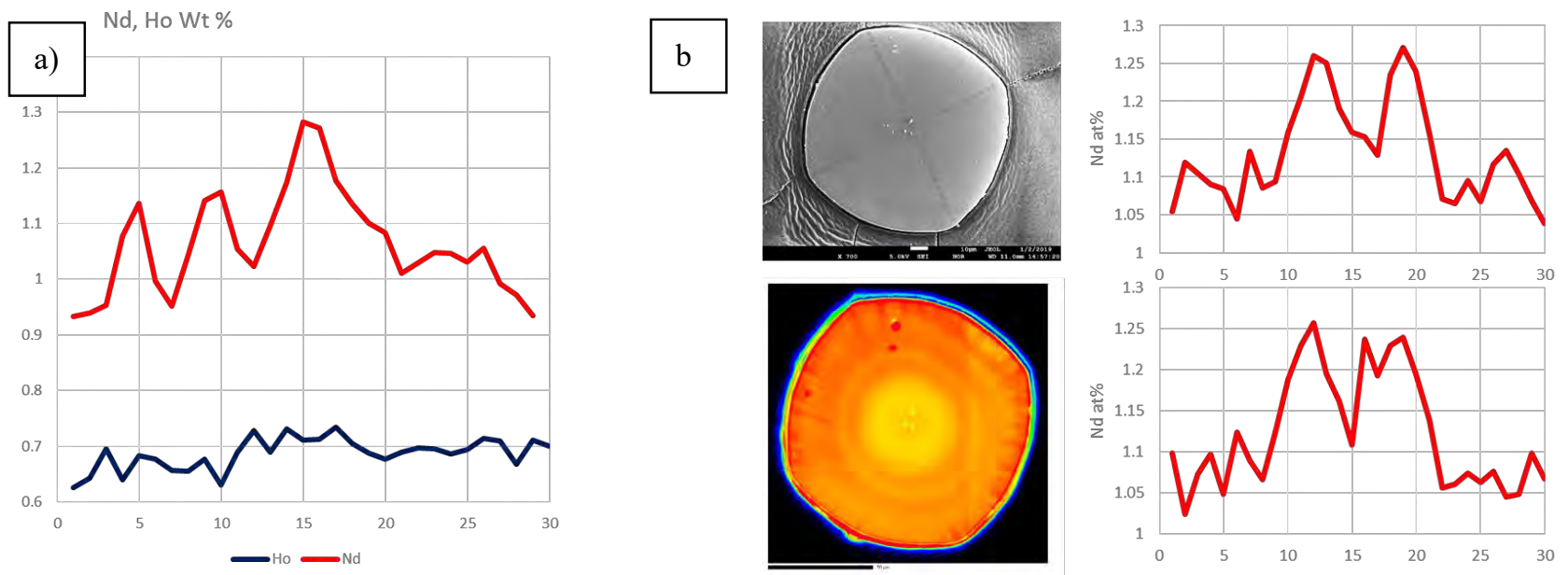

Figure 5a: Radial auto-segregation of ions in Nd, Ho:YAG; Figure 5b: Cross-section and radial auto-segregation in Nd:YAG

\section{Crystal Fibers with Post-Cladding}

For various sensing applications, in order to survive harsh environments such as boilers, turbines, gasifiers, etc., the cladding material should be thermally stable, chemically resistant and strongly adherent to the sapphire fiber over a wide range of temperature. Several strategies have been suggested for cladding single crystal fibers, of which wet chemical 
based post-cladding technique provides a valuable platform for experimentation[14]. Under this scheme, a cladding layer would be deposited on the crystal fiber via sol-gel dip-coating and subsequent thermal treatment. For a cladding, the material should be refractory material, with a refractive index lower than that of sapphire and have a CTE similar to sapphire. Magnesium aluminate spinel is one of the very few materials which meets these stringent requirements.

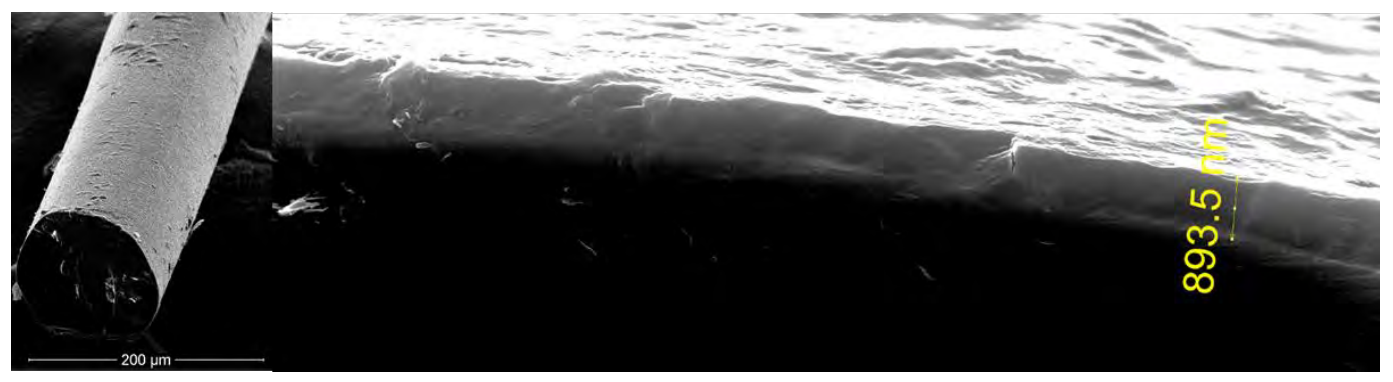

Figure 6: Spinel coated sapphire fiber. Magnified image showing the spinel film on fiber.

It has been demonstrated that despite the increase of solubility of alumina in spinel at high temperature, the deposited cladding layer still remains mostly intact for extended periods at temperatures up to around $1200^{\circ} \mathrm{C}$. The spinel cladding layer also offers improved resilience to the detrimental effects of coking on these fibers. This is evident from the fact that carbon monoxide gas at high temperatures do not have an irreversible effect on spinel clad fibers compared to bare sapphire fibers.

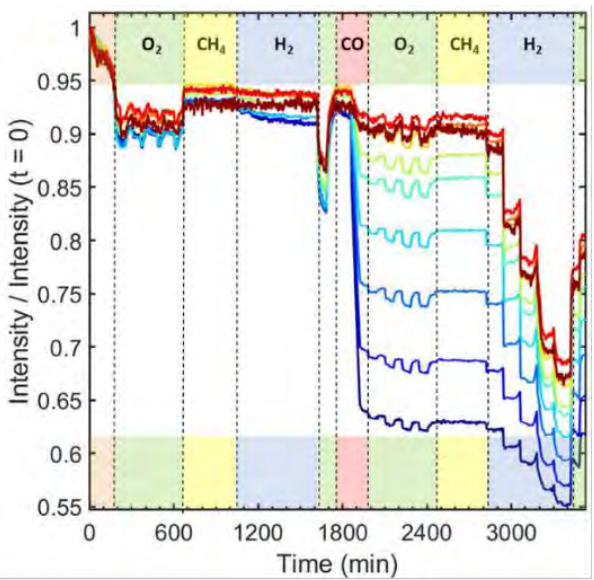

Uncoated Fiber

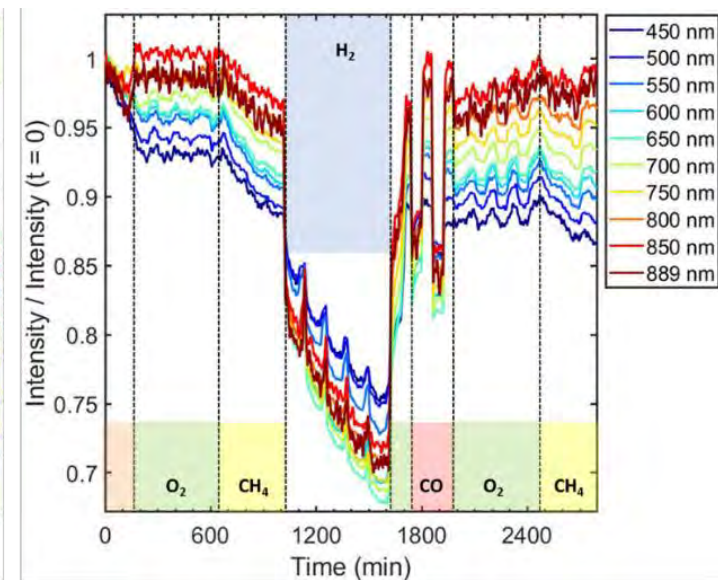

Coated Fiber

Figure 7: Normalized transmission spectrum for spinel-coated and uncoated fiber for gas exposure test[15].

\section{Conclusions}

Significant improvements in the LHPG technique have led to the growth of low-loss YAG SC fibers of consistent excellent single-crystalline quality, leading to the reporting of lowest attenuation values for SC fibers. Several methods for growing crystalline cladding for SC fibers were attempted with varying degrees of success. Magnesium aluminate spinel cladding was applied on to sapphire SC fibers, which led to improved resilience towards coking in harsh sensing environments. Using a simple low-cost sol-gel based approach, SC YAG fibers with varied dopant ion concentrations have been grown and lasing characteristics have been demonstrated.

\section{Acknowledgements}

The author would like to extend his sincerest gratitude towards his collaborators at NETL Albany, Rutgers University, Clemson University and University of Michigan. Part of the high-power laser work was supported by the HEL-JTO under 
contract number W911NF-12-1-0536. The harsh environment sensing work was performed in support of the US Department of Energy's Fossil Energy Crosscutting Technology Research Program. It was executed through the NETL Research and Innovation Center's Advanced Sensors and Controls FWP. Research performed by Leidos Research Support Team staff was conducted under the RSS contract 89243318CFE000003.

\section{Disclaimer}

This work was funded by the Department of Energy, National Energy Technology Laboratory, an agency of the United States Government, through a support contract with Leidos Research Support Team (LRST). Neither the United States Government nor any agency thereof, nor any of their employees, nor LRST, nor any of their employees, makes any warranty, expressed or implied, or assumes any legal liability or responsibility for the accuracy, completeness, or usefulness of any information, apparatus, product, or process disclosed, or represents that its use would not infringe privately owned

rights. Reference herein to any specific commercial product, process, or service by trade name, trademark, manufacturer, or otherwise, does not necessarily constitute or imply its endorsement, recommendation, or favoring by the United States Government or any agency thereof. The views and opinions of authors expressed herein do not necessarily state or reflect those of the United States Government or any agency thereof.

\section{References}

1. Zhang, Y., et al., Single-crystal sapphire-based optical high-temperature sensor for harsh environments. Optical Engineering, 2004. 43(1): p. 157-165.

2. Dawson, J.W., et al., Power scaling analysis of fiber lasers and amplifiers based on non-silica materials. 2010, Lawrence Livermore National Laboratory (LLNL), Livermore, CA.

3. $\quad$ Feigelson, R.S., Pulling optical fibers. Journal of Crystal Growth, 1986. 79(1): p. 669-680.

4. $\quad$ Nie, C.D., S. Bera, and J.A. Harrington, Growth of single-crystal YAG fiber optics. Optics Express, 2016. 24(14): p. $15522-15527$.

5. Bera, S., et al., Optimizing alignment and growth of low-loss YAG single crystal fibers using laser heated pedestal growth technique. Applied Optics, 2017. 56(35): p. 9649-9655.

6. Buric, M., et al., Fabrication and on-line evaluation of single crystal fiber via laser-heated pedestal growth. SPIE Defense + Commercial Sensing. Vol. 11000. 2019: SPIE.

7. Kim, W., et al., Hydrothermally cladded crystalline fibers for laser applications. Optical Materials Express, 2019. 9(6): p. 2716-2728.

8. Dubinskii, M., et al., Low-loss 'crystalline-core/crystalline-clad' (C4) fibers for highly power scalable high efficiency fiber lasers. Optics Express, 2018. 26(4): p. 5092-5101.

9. Li, Y., et al., Lasing characteristics of Ho: YAG single crystal fiber. Optics Express, 2016. 24(9): p. 9751-9756.

10. Bera, S., et al., Growth and lasing of single crystal YAG fibers with different $\mathrm{Ho}^{3+}$ concentrations. Optical Materials, 2018. 75: p. 44-48.

11. Cheng, L., et al., Single crystal Er ${ }^{3+}$ YAG fibers with tailored refractive index profiles. Applied Optics, 2018. 57(2): p. 362-370.

12. Bera, S., Rare-earth doped yttrium aluminum garnet single crystal fibers for high power laser applications. 2018.

13. Chang, C.-L., et al., Simulation and experiment on laser-heated pedestal growth of chromium-doped yttrium aluminum garnet single-crystal fiber. Journal of Crystal Growth, 2011. 318(1): p. 674-678.

14. Chen, H., et al., Review and perspective: Sapphire optical fiber cladding development for harsh environment sensing. Applied Physics Reviews, 2018. 5(1): p. 011102.

15. Bera, S., et al., Fabrication and evaluation of sapphire fiber cladding via magnesium aluminate spinel sol-gel based approaches. SPIE Defense + Commercial Sensing. Vol. 11000. 2019: SPIE. 


\title{
Titania Clad Fiber Fatigue Performance
}

\author{
Kevin W. Bennett*, Garth W. Scannell, G. Scott Glaesemann \\ Corning Research and Development Corporation, 1 Riverfront Drive, Corning NY 14831
}

\begin{abstract}
Improvement of the mechanical reliability of fibers deployed under tight bending can be achieved through addition of $\mathrm{TiO}_{2}$ to the outermost layer of the fiber cladding. Optimization of the layer thickness and composition can lead to performance enhancement relative to traditional silica clad fibers.
\end{abstract}

Keywords: titania, optical fiber, fatigue

*bennettkw@.corning.com; phone 1607-974-9000

\section{Introduction}

The use of an outer layer of $\mathrm{TiO}_{2}-\mathrm{SiO}_{2}$ doped glass has long been known to impact the fatigue performance of optical fiber. ${ }^{1,2}$ Recently interest in this material system has experienced a resurgence as data center applications have transitioned to ever greater speeds and higher physical densities. Packing ever greater numbers of fibers and associated terminations in smaller and smaller footprints leads to designers looking to implement tighter bending radii in their component designs. Additionally, as connections are reconfigured the patch cords can become tangled leading to unintentional bends that are beyond the recommended deployment conditions for traditional silica fibers. Fibers with optical profiles compatible with tight bends are readily available. As the mechanical space constraints become tighter a look into mechanical design guidelines is warranted.

\section{Body}

When an optical fiber is bent stress and strain are imparted upon the glass. The stress, $\sigma_{\mathrm{b}}$, is related to Young's Modulus of the material, E, bend radius and fiber radius and can be shown to be $\sigma_{b}=E \frac{r}{R}$ where $E=E_{0}\left(1+2.3 \frac{r}{R}\right), \mathrm{E}_{0}=10.2 \times 10^{6} \mathrm{psi}(70.3$ $\mathrm{GPa}$ ) for silica, $\mathrm{r}$ is the fiber radius and $\mathrm{R}$ is the bend radius, see Figure 1 for imparted stress versus bend radius for optical fiber. Fiber is proof tested to eliminate the weakest flaws at typical levels of 100 or $200 \mathrm{kpsi}$. Deploying fiber at stresses higher than $1 / 5^{\text {th }}$ the proof stress leads to the probability of finding a flaw that will fail due to fatigue during a 25 -year lifetime of the fiber. 


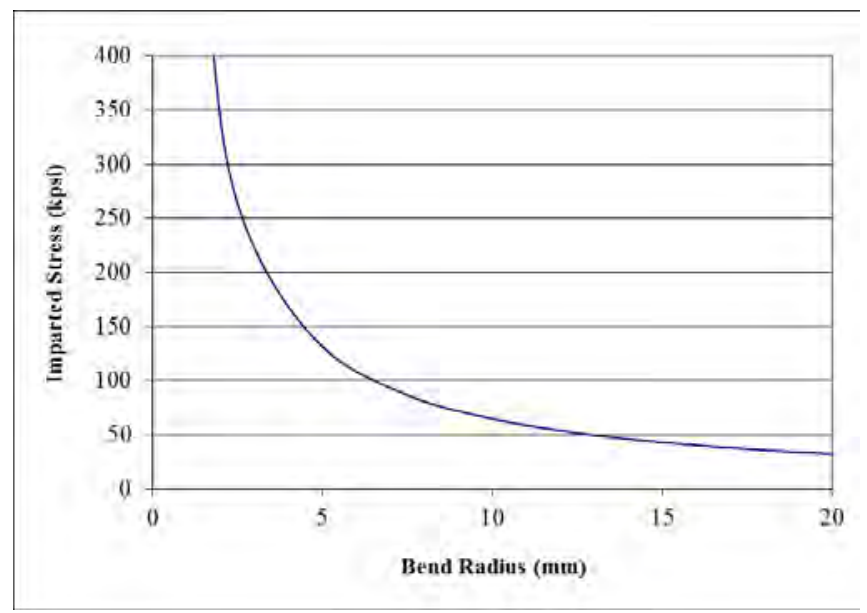

Figure 2. Imparted Stress as a function of bend radius for a $125 \mu \mathrm{m}$ diameter fiber.

Fatigue in glass follows an exponential crack velocity behavior. This means that it is initially a slow process where an existing crack will grow slowly for a long period of time and then rapidly fail. ${ }^{3,4}$ The main requirements for fatigue to occur are an applied stress, a flaw (crack), moisture and time. Remove any one of the required elements and fatigue is stopped. Dynamic fatigue is an experimental technique used to characterize the resistance of a material to fatigue. A material is tested to failure using a constant stressing rate and the stress at failure is recorded. Several stressing rates are tested, and the data is plotted on a log-log plot in stressing rate versus failure stress. At higher stressing rates there is less time for fatigue so the stress at failure will be higher. The slope of the resulting curve is equal to $1 /(n+1)$ where $n$ is the dynamic fatigue parameter. A higher value of $\mathrm{n}$ gives greater resistance to fatigue. Traditional silica clad fiber has a $\mathrm{n}$ value of about 20 .

\section{Results and Discussion}

Adding $\mathrm{TiO}_{2}$ to $\mathrm{SiO}_{2}$ as the outer cladding layer of optical fiber has three main effects that impact the mechanical performance of the fiber. First $\mathrm{TiO}_{2}$ lowers the Young's modulus relative to pure $\mathrm{SiO}_{2}$ resulting in less imparted stress at the same bend radius. The Young's modulus is about $10 \%$ lower than Silica at $8 \mathrm{Wt} \% \mathrm{TiO}_{2}$ and higher levels of $\mathrm{TiO}_{2}$ will further reduce the Young's modulus. Second, $\mathrm{TiO}_{2}$ reduces the coefficient of thermal expansion relative to Silica. A layer of $\mathrm{TiO}_{2}$ doped Silica on the outside of the fiber imparts a residual compressive stress. The combination of layer thickness and doping level will determine the actual compressive stress. Third, $\mathrm{TiO}_{2}$ increases the dynamic fatigue parameter, $\mathrm{n}$. Figure 3 shows the impact on fatigue as the concentration of $\mathrm{TiO}_{2}$ is increased. 


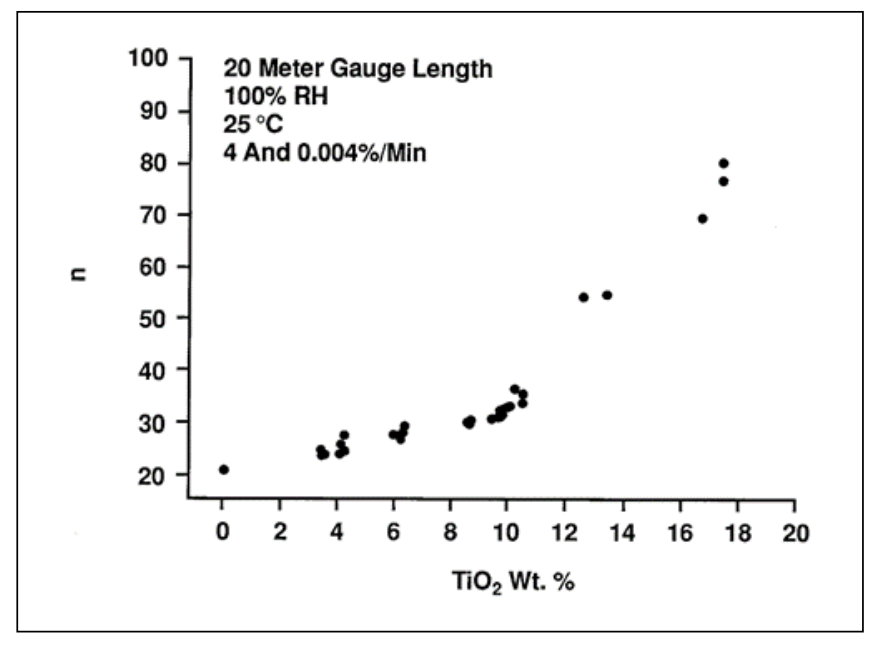

Figure 4. Fatigue resistance as a function of $\mathrm{TiO}_{2}$ in the cladding.

Given a set of proposed deployment conditions and a targeted lifetime, it is possible to develop an optimal design by using a combination of each of these three effects in concert with other design parameters such as glass diameter. Combination of both a bend resistant optical design with a robust mechanical design will lead to improved performance and reliability of the optical network.

\section{References}

[1] Gulati, S.T, Helfinstine, J.D. and Glaesemann, G.S., "Improvements in Optical Fiber Reliability via High Fatigue Resistant Composition," SPIE vol 842 Fiber Optics: Benign and Adverse Environments (1987).

[2] Backer et al., "Optical waveguide fiber with Titania-silica outer cladding and method of manufacturing", US5180411 (1993). [3] Michalske, T.A., and Freiman, S.W. "A Molecular Interpretation of Stress Corrosion in Silica," Nature, 295 (2), 511-12 (1982).

[4] Ritter, J.E., Glaesemann, G.S., Jakus, K. and Rampone, P. "Dynamic Fatigue of Soda-Lime Glass as a Function of Temperature," Phys. Chem. of Glass., 27 [2] 65-70 (1986). 


\title{
Radiation-Resistant Nanoparticle Erbium Doped Fibers for High Power Space Laser Communications*
}

\author{
E. Joseph Friebele*a, Colin C. Baker, ${ }^{\mathrm{b}}$ Ashley Burdett, ${ }^{\mathrm{c}}$ Jasbinder S. Sanghera ${ }^{\mathrm{b}}$, \\ Michael J. LuValle ${ }^{\mathrm{d}}$, and Stephanos Logothetis ${ }^{\mathrm{d}}$ \\ ${ }^{a}$ KeyW Corporation, 7740 Milestone Pkwy, Suite 400, Hanover, MD 21076, USA \\ baval Research Laboratory, 4555 Overlook Ave. SW, Washington, DC 20375, USA \\ ${ }^{c}$ University Research Foundation, 6411 Ivy Lane, Suite 110, Greenbelt MD 20770, USA \\ ${ }^{\mathrm{d}}$ Rutgers University, 110 Frelinghuysen Road, Piscataway, NJ 08854-8019, USA
}

\begin{abstract}
The in-situ radiation-induced degradation and recovery of the output optical power of actively pumped erbium-doped fiber amplifiers, during and after radiation exposure, has been studied to improve their radiation tolerance in the space environment. Significant progress has been made using nanoparticle doping and by incorporating specific dopants to reduce Er ion clustering and improve efficiency. Statistical kinetics modeling has been used to predict on-orbit performance.
\end{abstract}

Keywords: erbium doped fiber, radiation effects, space radiation, laser communications

*joe.friebele.ctr@nrl.navy.mil; phone 1-202-767-2270

\section{Introduction}

Space-based laser communication is based on erbium-doped fiber (EDF) because of the availability of reliable pump laser diodes from the telecommunications industry, as well as highly developed EDFs. However, two issues that are specific to the space environment need to be addressed: on orbit radiation exposure, and higher power. Radiation-induced degradation of EDFs occurs because of the generation of color centers associated with dopants that are incorporated to increase Er ion solubility in the silica glass matrix and reduce clustering. If the inter-ion separation is too small, excited energy transfer processes such as upconversion and quenching occur, which results in significant loss of efficiency. Progress has been made in reducing the radiation-induced attenuation in EDFs by co-doping with $\mathrm{Ce}[1,2]$, hydrogen loading[3], nanoparticle (NP) doping $[4,5]$ and other techniques, but only a few studies have addressed active fibers. Furthermore, clad pumping of doubleclad fibers is required for higher power to distribute pumping along the length of fiber and decrease thermal loading. The $\mathrm{Er}$ concentration of these fibers must be significantly increased because of the areal dilution factor (ratio of the areas of the core to the pump cladding).

In this study, we have measured the degradation of output optical power from Er-doped fiber amplifiers (EDFAs) configured as a master oscillator-power amplifier (MOPA). The measurements were made in-situ while the active EDF was exposed to $\gamma$ irradiation and for a significant recovery period afterwards. In addition to $\mathrm{Al}$, we have incorporated co-dopants such as $\mathrm{La}$ and $\mathrm{Yb}$, which further reduce Er ion clustering and improve efficiency[6]. Measurements have been made using both 980 and 1480 $\mathrm{nm}$ pump wavelengths. ( $\mathrm{Yb}$ is optically inactive in the latter case.)

Laboratory measurements can be used to characterize the radiation tolerance of EDFs, but typical laboratory dose rates are many orders of magnitude greater than those encountered during space deployment. Although simplistic models can be used to compare fibers and predict the end-of-life performance, they are incapable of incorporating variations in temperature or dose rate, such as might occur due to the spacecraft transiting through the van Allen radiation belts or periodic solar flares. We have used a sophisticated statistical kinetics model previously developed for establishing system reliability through accelerated

Sixth International Workshop on Specialty Optical Fibers and Their Applications (WSOF 2019),

edited by Liang Dong, John M. Ballato, Proc. of SPIE Vol. 11206, 1120614 · C 2019 SPIE

CCC code: $0277-786 X / 19 / \$ 21 \cdot$ doi: $10.1117 / 12.2552535$ 
lifetime testing to predict the degradation of EDFA output in various orbit scenarios, including geosynchronous orbits where the primary radiation experienced is due to periodic solar flares[7].

\section{Experimental}

NP dispersions were synthesized as described in reference 8, while solutions were prepared by dissolving salts of Er, Al, La or $\mathrm{Yb}$ in methanol. Preforms were fabricated using in-situ solution or NP doping on the MCVD lathe, and fibers were drawn on the conventional NRL silica fiber optic draw tower. The preform and fiber refractive index profiles were measured with a Photon Kinetics P104 preform analyzer and an Interfiber Analysis IFA-100 fiber measurement system, respectively. The Er content was determined from the absorption of the Er peak at $1.532 \mu \mathrm{m}$ using an Agilent broadband EELED source and Ando Optical Spectrum Analyzer and compared with electron microprobe analysis. Optical absorption spectra and cutoff wavelength were measured with a Photon Kinetics 2300 Fiber Measurement System to determine background loss and hydroxyl content from the $1.38 \mu \mathrm{m}$ second $\mathrm{OH}$ overtone peak.

Active measurements were made with a master oscillator power amplifier (MOPA) consisting of a $12 \mathrm{~mW}$ signal laser diode at $1.55 \mu \mathrm{m}$ combined with $0.5-1 \mathrm{~W}$ pump laser at either 0.98 or $1.48 \mu \mathrm{m}$. A sharp cutoff long pass filter was inserted between the fiber output and power meter to eliminate unabsorbed pump. Prior to radiation exposure, the optimum EDF length was determined by cutback measurements; typical lengths were $\sim 3 \mathrm{~m}$. For consistency and comparison between the fibers, a standard length of $3 \mathrm{~m}$ EDF was used for the active radiation experiments. The 3-m sample coil of each EDF was fusion spliced to $\sim 10 \mathrm{~m}$ leads connected to the MOPA and power meter inserted into a sealed sample can which was lowered into the ${ }^{60} \mathrm{Co} \gamma-$ ray pool source. The output power was measured prior to, during, and after radiation exposure using a LabView program, and the degradation is expressed as $\mathrm{dB}$ loss. Typical radiation exposures were $100 \mathrm{~Gy}\left(10^{4} \mathrm{rad}\right)$ and recovery times were $\sim 70 \mathrm{~h}(2.5$ $\left.\mathrm{x} 10^{5} \mathrm{~s}\right)$.

End-of-life extrapolation was first made using a simplistic $\mathrm{n}^{\text {th }}$-order kinetic model where $\sim 70 \mathrm{~h}$ recovery data following exposure was fit to the model. In the empirical $\mathrm{n}^{\text {th }}$-order reaction kinetics model, the annealing of the radiation-induced loss is based on standard chemical rate theory. During recovery, the induced attenuation $A(t)$ can be described by

$$
A(t)=\left(A_{i}-A_{p}\right)(1+c t)^{-[1 /(1-n)]}+A_{p},
$$

where

$$
c=(1 / \tau)\left[2^{n-1}-1\right],
$$

and $A_{i}$ is the initial induced attenuation at the end of the exposure, $A_{p}$ is the permanent induced attenuation, if any, $\tau$ is the halflife of the induced attenuation and $n$ is the order of the reaction, or kinetic order. Having fit the recovery data to equation (1), it is then possible to calculate the EOL loss $\mathrm{A}\left(\mathrm{t}_{\mathrm{f}}\right)$ for $\mathrm{t}_{\mathrm{f}}=10 \mathrm{y}$, for example. Although this model was developed for and has been demonstrated on passive fiber radiation-induced attenuation, it is equally applicable to the radiation-induced degradation in output power of an active device.

The more sophisticated statistical kinetics model[7] requires both active amplifier data and passive optical absorption spectra $(1.25-1.75 \mu \mathrm{m})$ data acquired using a design of experiments with different total doses, dose rates and temperatures. Because of the large input data requirement, the model was only used on one of the fibers. 


\section{Results}

Table 1 contains a summary of selected fibers that were fabricated for this study, together with the Er concentration, $\mathrm{Al}_{2} \mathrm{O}_{3}$ mol\%, and core effective areas. All fibers were doped with Er with the exception of Al1, which contained only $\mathrm{Al}_{2} \mathrm{O}_{3}$ and silica. The 2 fibers with an $\mathrm{S}$ in their label were prepared from solution-doped preforms, while all the others were doped with NPs. In the 3 cases where two MOPA pump wavelengths were used for a fiber, they are designated as either $0.98 \mu \mathrm{m}$ or $1.48 \mu \mathrm{m}$. If not specified, the experiments were done with $1.48 \mu \mathrm{m}$ pumping.

Table 1. Effective core area, Er concentration, radiation-induced loss and recovery for fibers.

For reference, $[\mathrm{Er}]=1 \times 10^{25} / \mathrm{m}^{3}$ corresponds to an Er peak height $=11.9 \mathrm{~dB} / \mathrm{m}$ at $1.532 \mu \mathrm{m}$.

\begin{tabular}{|c|c|c|c|c|c|c|c|}
\hline Fiber & $\begin{array}{l}A_{\text {eff }} \\
\mu m^{2}\end{array}$ & $\begin{array}{l}\text { Er conc. } \\
\mathrm{x} 10^{25} / \mathrm{m}^{3}\end{array}$ & $\begin{array}{l}\mathrm{Al}_{2} \mathrm{O}_{3} \\
\mathrm{~mol} \%\end{array}$ & $\begin{array}{c}\text { Loss dB } \\
100 \mathrm{~Gy}\end{array}$ & $\begin{array}{l}\text { Loss dB } \\
\text { @ } 8000 \mathrm{~s}\end{array}$ & $\begin{array}{c}\text { Loss } \\
\text { (norm) } \\
\text { @ } 8000 \mathrm{~s}\end{array}$ & $\begin{array}{c}\text { EOL Loss } \\
\mathrm{dB} \\
10 \mathrm{y}\end{array}$ \\
\hline ErAl1 & 94.3 & 1.58 & 2.08 & 5.83 & 5.19 & 0.89 & 3.77 \\
\hline ErA14 & 200.0 & 0.81 & 2.7 & 7.98 & 7.23 & 0.91 & 5.76 \\
\hline ErA16 & 104.8 & 1.90 & 3.08 & 4.84 & 4.29 & 0.89 & 3.19 \\
\hline ErA17 & 60.8 & 2.41 & 3.91 & 3.8 & 3.31 & 0.87 & 2.63 \\
\hline ErA18 & 71.0 & 2.91 & 2.78 & 5.27 & 4.60 & 0.87 & 3.00 \\
\hline LaErAl1-1.48 & 44.1 & 2.27 & 2.73 & 10.45 & 6.81 & 0.65 & 1.01 \\
\hline LaErAll-0.98 & & & & 5.47 & 3.10 & 0.57 & 0.61 \\
\hline LaErA12 & 63.2 & 1.97 & 3.82 & 11.44 & 7.80 & 0.68 & 1.66 \\
\hline LaErA13 & 51.8 & 1.59 & 1.96 & 13.21 & 8.88 & 0.67 & 2.54 \\
\hline LaErA1S1 & 92.8 & 0.97 & 1.69 & 25.39 & 18.59 & 0.73 & 4.37 \\
\hline GeLaErAl1-1.48 & 50.9 & 2.16 & 3.07 & 5.99 & 3.72 & 0.62 & 1.01 \\
\hline GeLaErAl1-0.98 & & & & 3.48 & 2.15 & 0.62 & 0.46 \\
\hline GeLaErA12-1.48 & 55.6 & 1.83 & 2.81 & 3.01 & 2.07 & 0.69 & 0.94 \\
\hline GeLaErA12-0.98 & & & & 2.3 & 1.32 & 0.57 & 0.18 \\
\hline YbErAlS1 & 67.5 & 1.60 & 1.95 & 2.36 & 2.29 & 0.97 & 2.12 \\
\hline Al1 & 40.3 & ---- & 6.76 & 0.82 & 0.70 & 0.86 & 0.49 \\
\hline ErAlS2 & 55.9 & 2.99 & 4.22 & 5.05 & 4.30 & 0.85 & 2.74 \\
\hline
\end{tabular}

Figure 1 shows an example of increase in loss of the output optical power during radiation exposure and the recovery following irradiation for a selection of EDFs. It is apparent from Figure 1(a) that: 1) the loss experienced during exposure can be significant; 2) the induced losses are higher in the EDFs co-doped with La; and 3) there are considerable variations in loss due to differences in fiber core composition. In contrast, the normalized recovery data shown in Figure 1(b) indicate little variation among EDFs with similar dopants, while the recovery of the loss is much greater in EDFs co-doped with La than in those with just Al.

Table 1 also contains the peak radiation-induced loss of optical output power and the amount of recovery that was measured 
after $8000 \mathrm{~s}$. The loss values $(\mathrm{dB})$ after $8000 \mathrm{~s}$ of recovery are the actual reduction in optical output power. Because of the differences in peak losses, the recovery has also been normalized to the peak value to elucidate the kinetics and facilitate comparison between the fibers. Note that because the loss has been normalized to the peak induced loss, larger normalized loss indicates less recovery i.e., for fiber ErAll the normalized loss $=0.89$ means that only $\sim 0.11$ of the radiation-induced loss has recovered in $8000 \mathrm{~s}$.
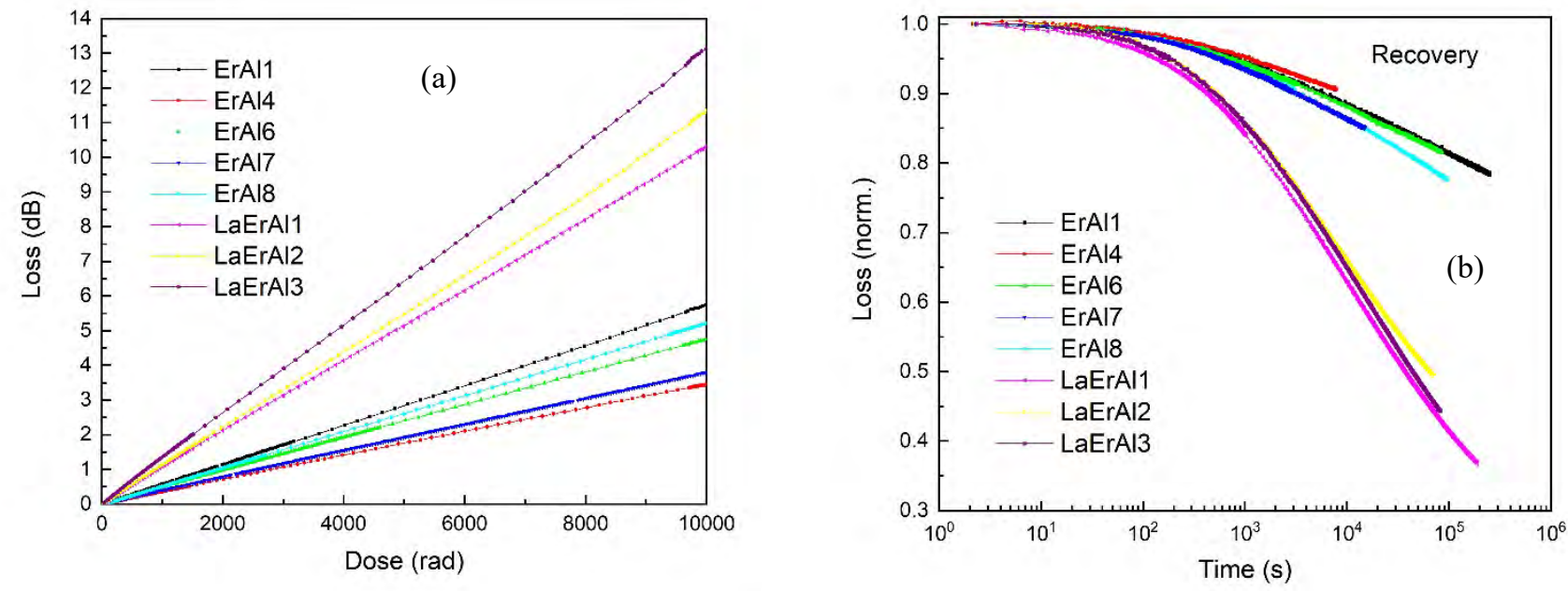

Figure 1. Example data of the (a) growth and (b) recovery of the loss of $1.48 \mu \mathrm{m}$ pumped MOPA output optical power for various EDFs. Because of the differences in peak losses, the recovery has been normalized to the peak value.

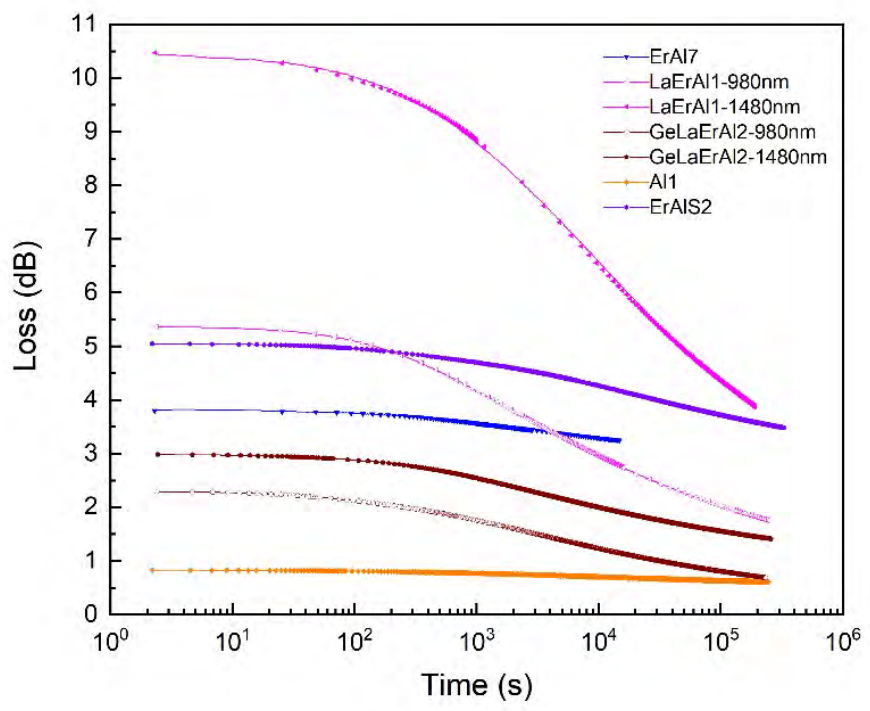

Figure 2. Recovery of the radiation-induced loss (points) and fits to $\mathrm{n}^{\text {th }}$-order kinetics (lines). 
The recovery data of all the fibers were fit to $\mathrm{n}^{\text {th }}$-order kinetics, e.g., Eq. (1), and the data and fits for some fibers are shown in Figure 2. It is apparent that there is excellent agreement between the data and fits, at least in the first $70 \mathrm{~h}$ or $2.5 \times 10^{5} \mathrm{~s}$. The parameters from the fits were used to perform end of life extrapolation for a nominal lifetime of $10 \mathrm{y}=3.2 \times 10^{8} \mathrm{~s}$, and these results are shown in Table 1. The EOL losses for the MOPAs resonantly pumped at $1.48 \mu \mathrm{m}$ vary from $\sim 1$ to $5.7 \mathrm{~dB}$, depending on the fiber composition. It is significant to note that the three fibers with the lowest EOL losses (LaErAl1, GeLaErAl1 and GeLaErAl2) have relatively large $\mathrm{Al}_{2} \mathrm{O}_{3}$ concentrations $\sim 3$ mole\%. However, their initial peak losses are low and their recovery is good. This result, together with the low initial loss of the Er-free All fiber containing much more $\mathrm{Al}_{2} \mathrm{O}_{3}$ indicates that the radiation damage mechanism in these EDFs is much more complicated than just color centers associated with $\mathrm{Al}_{2} \mathrm{O}_{3}$.

The results from the MOPA measurements of the same 3 fibers pumped at $0.98 \mu \mathrm{m}$ indicate much lower EOL losses. Indeed, the lowest value is only $0.18 \mathrm{~dB}$ for GeLaErAl2, which is substantially better than any other EOL losses of any of the other fibers or wavelengths. We attribute the lower induced losses and enhanced recovery of these fibers at 0.98 vs. $1.48 \mu \mathrm{m}$ to enhanced photo annealing due to the higher energy photons of the pump and the substantial amount of up-converted or ESA-generated green light in the fiber for $0.98 \mu \mathrm{m}$ pumping.

Three different types of experiments were used to acquire data for the statistical kinetics model: 1) Active radiation-induced loss of output optical power using the MOPA; 2) Passive radiation-induced attenuation spectra data over the $1.25-1.75 \mu \mathrm{m}$ range; and 3) Active/passive data where periodic spectral measurements were interleaved with active MOPA measurements. To elucidate the radiation damage kinetics, data at 1.3 and $1.65 \mu \mathrm{m}$ were extracted from the spectral measurements. Because of the intense $\mathrm{Er}^{3+}$ absorption at $1532 \mathrm{~nm}$, no radiation-induced spectral data could be acquired or extracted in this region. Rather, we have used the data at $1.65 \mu \mathrm{m}$ to indicate the loss at $1.532 \mu \mathrm{m}$.

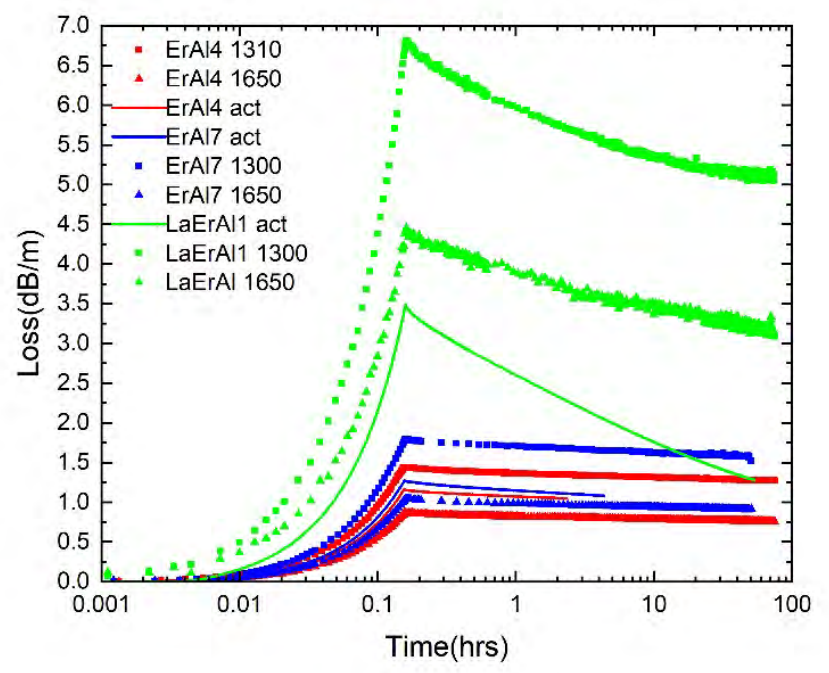

Figure 3. Growth and recovery of the loss of output optical power (active) and radiation-induced absorption measured spectrally at 1.31 and $1.65 \mu \mathrm{m}$.

A comparison of the passive and active loss measurements for 3 exemplar fibers is shown in Figure 3. It is apparent that the loss at $1.31 \mu \mathrm{m}$ is greater than that at $1.65 \mu \mathrm{m}$ due to the radiation-induced color center absorptions being at shorter wavelength. 
Likewise, the losses measured with the active MOPA are less and the recovery is much more rapid than the passive losses due to photo annealing. We also observed that the recovery inversely correlated with the core area since the power in the fibers cores was relatively constant while there was as much as a factor of 4 variation in the core areas (Table 1), resulting a significant difference in optical intensity and hence the photo annealing.

The statistical kinetics model takes into account both the growth and recovery kinetics and is therefore able to fit both the growth and recovery data. The consistency of this fitting over a range of dose rates, total doses and temperatures occurs due to the selection of the correct activation energies and pre-exponential factors for the different kinetic components.[7] Figure 4 shows the fit obtained for the LaErAl1 radiation data obtained at 25 and $80 \mathrm{C}$ and at 3 different dose rates and total doses. The fact that one set of kinetic parameters has successfully fit both the growth and recovery data over a wide range of variables is quite remarkable.

Having fit the accelerated lifetime laboratory data, it is possible to then extrapolate to a realistic spacecraft orbit, and these results are shown in Figure 5 for a geostationary orbit exposed to 5 solar flares of varying magnitude over 15 year lifetime. In this simulation, the fiber is assumed to be constantly optically pumped so that there is continuous photo annealing. Note that the large degradation from the solar flares is fully annealed after each event.

\section{Discussion and Summary}

The location of defects for silica and common dopants found in active and passive silica fiber lie in various areas of the electromagnetic spectrum. Defects associated with the silica lattice and the processing of fiber such as oxygen deficiency centers and non-bridging oxygen hole centers (NBOHC) lie in the region between $\sim 7.6 \mathrm{eV}$ to $1.2 \mathrm{eV}$ ( $\sim 163 \mathrm{~nm}$ to $1033 \mathrm{~nm})$. Many of the defects that cause trap states in silica fiber are introduced by the necessary co-dopants added in order to give proper waveguide (or active device) performance. These defect states have varying levels (energies) often being described as shallow or deep. For germanium, known defects lie in the region $\sim 6.8 \mathrm{eV}$ to $1.97 \mathrm{eV}(\sim 182 \mathrm{~nm}$ to $630 \mathrm{~nm})$. Conversely, we note that the addition of $\mathrm{GeO}_{2}$ has been shown to reduce RIA in RE doped aluminosilicate glass [9]. For phosphorus, defects are found at similar energies in the UV and visible region, while the P1 defect is centered near $0.8 \mathrm{eV}(1600 \mathrm{~nm})$, in the wavelength region of interest for EDF-based devices [10,11]. The fibers presented in this work have been free of phosphorous. And finally, for aluminum, defects such as aluminum oxygen hole centers (AlOHC) are known to exist in the region of $4.0 \mathrm{eV}$ to $2.2 \mathrm{eV}$. Thus, all of the defects anticipated in our fibers occur in the UV and VIS region of the spectrum, not in the near IR. Nevertheless, RIA is a well-observed phenomenon in $\mathrm{Al}_{2} \mathrm{O}_{3}$ doped fiber in the near IR. Studies for an $\mathrm{Al}_{2} \mathrm{O}_{3}$ doped fiber with no Er or other species have shown no difference in passive experiments for RIA in the infrared region than for Er doped fiber with other codopants [12]. However, the authors found essentially no thermal annealing for the $\mathrm{Al}_{2} \mathrm{O}_{3}$ doped fiber. Thus, with the exception of the phosphorous defect at $0.8 \mathrm{eV}$ these defects all lie in the region below $1.55 \mathrm{eV}(<800 \mathrm{~nm})$. These defects may have tail states that extend into the near IR but this does not explain the RIA and power degradation seen in active fibers [10].

Under active pumping these defects may be photo-annealed, reducing and even eliminating power degradation. We have seen that the dopants that are a concern for passive silica fibers are not necessarily a concern for active amplifiers, and even for passive amplifiers that are optically pumped (fiber Al1). Defects associated with $\mathrm{Al}_{2} \mathrm{O}_{3}$ that do extend in the near IR seem to be shallow and easily annealed. This may be the reason why the passive heavily doped $\mathrm{Al}_{2} \mathrm{O}_{3} \mathrm{NP}$ fiber All had very low degradation. This is a benefit, because it is highly desirable to retain aluminum in EDFA, as it is an important co-dopant, where, as previously mentioned, $\mathrm{Al}$ co-doping increases Er solubility, broadens the $\mathrm{Er}^{3+}$ gain spectrum and provides gain flatness. 


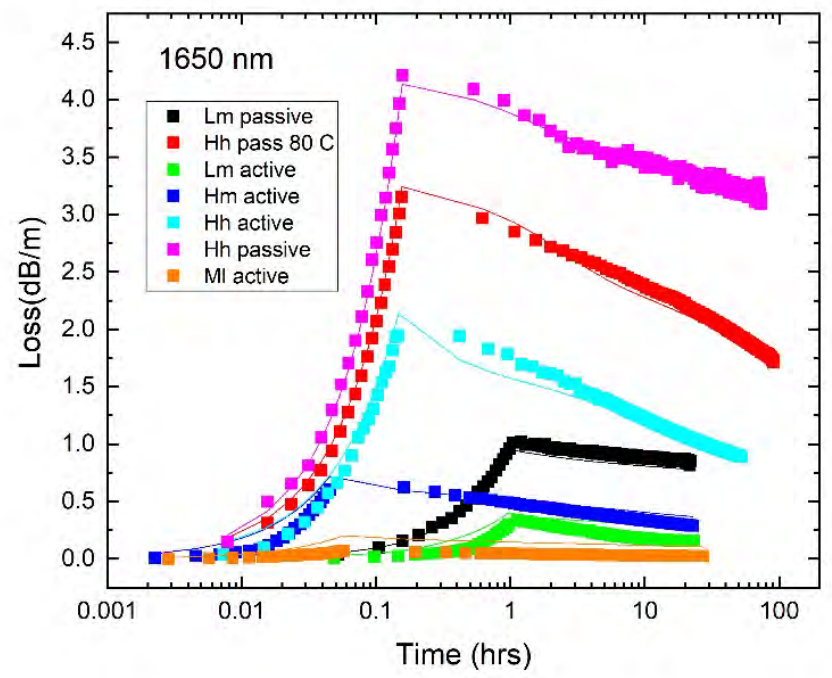

Figure 4. Results of 7 experiments with the LaErAl1 NP-doped fiber using different dose rates (L, M, H = 0.5, 2.5, 500 Gy/h) and total doses $(1, \mathrm{~m}, \mathrm{~h}=10,30,100 \mathrm{~Gy})$ together with the fits obtained via the statistical kinetics model. The lines are the data and the points are the fit.

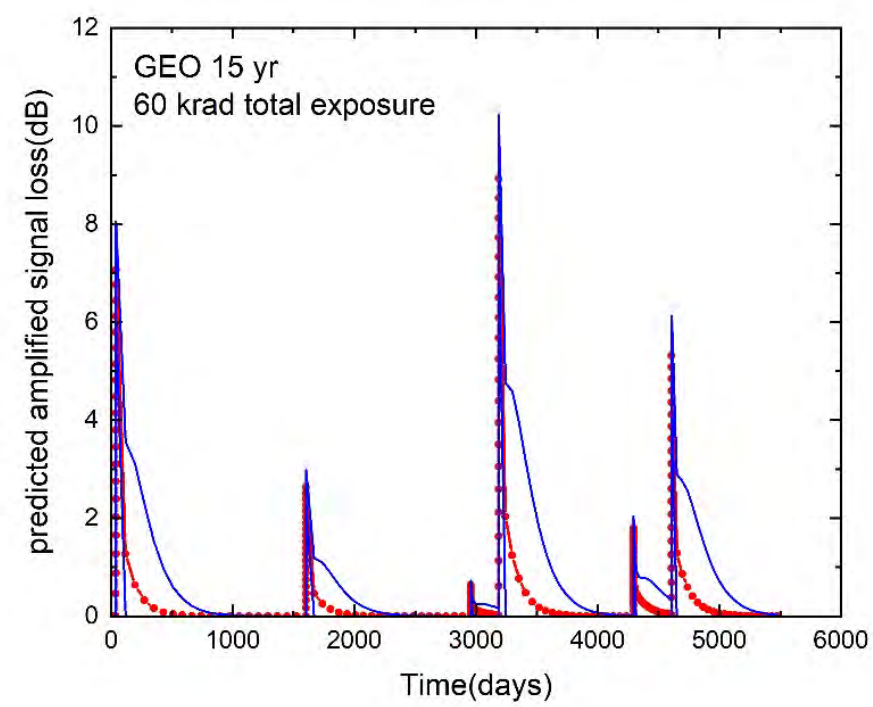

Figure 5. The predicted signal loss (red) in an EDFA made with LaErAll on-orbit in GEO exposed to a number of solar flares over a 15 -year lifetime together with $95 \%$ confidence bounds (blue). 
Indeed, active commercial fibers such as AstrogainTM[13] proprietary formulism claim to have been developed to exploit the photo-anneal process for EDFA and allow a fast recovery [11]. The proprietary formulism is composed of three trivalent materials, one of which is aluminum. It is most likely that the fiber also contains cerium. We note that the photo-anneal process is also important for maintaining a wide emission spectrum bandwidth, which has been shown to be narrowed due to radiation damage.

The mechanisms involved in an active amplifier degradation seem to be more complex than can be attributed to passive darkening and annealing alone. The erbium ions absorb pump light and provide gain of signal light. It is likely that there are defects associated with the combination of $\mathrm{Er}$ and $\mathrm{Al}_{2} \mathrm{O}_{3}$ doping. We have found that these defect states cause more signal degradation but photo-annealing appears to release many of the trap states. Other detrimental processes may exist for EDFA. Some studies have pointed out that there seems to be no influence of the $\mathrm{Er}^{3+}$ concentration in EDFA on darkening [14, 15]. Furthermore, it has been reported that the $\mathrm{Er}^{3+}$ absorption and emission cross section does not change during exposure to $\gamma-$ radiation [16]. However, there has also been a report of $\mathrm{Er}^{3+}$ reduction to $\mathrm{Er}^{2+}$ under $\mathrm{x}$-ray irradiation [17]. According to the authors, analysis of $\mathrm{Er}^{3+}$ absorption cross sections would not change pre- and post-irradiation, as is the case often reported in the literature. In this scenario, $\mathrm{Er} 3+$ ions are reduced to form $\mathrm{Er}^{2+}$ ions by trapping electrons, and a hole is consequently captured to form trapped-hole centers that contribute to RIA. A full recovery occurs after thermal bleaching to high temperatures [17]. Both europium and ytterbium are known to change valence states in glass, and so it is speculated that this may also occur for $\mathrm{Er}^{3+}$ in oxide glasses although it has never been previously observed.

The introduction of passive RE co-dopants such as La in this work seems to drastically affect the defect states. This RE codoping is effective in increasing MOPA slope efficiency, as discussed previously. Active losses are increased by the presence of La co-dopants, but the fiber strongly recovers with photo-annealing. The result is superior performance in a long term geostationary (GEO) orbit. Lanthanum in alumino-silicate glass is known to act as a charge compensator. Therefore, it preferentially locates itself closer to the tetrahedrally-coordinated $\mathrm{Al}$ species. This would then allow it to compete with the erbium in the same site and so reducing clustering [18]. Lanthanum co-doping has been shown to be effective in reducing pump light-induced photodarkening, but this is not necessarily the same behavior and mechanism as active power degradation and recovery in radiation rich environments. In all cases the photo-anneal behavior seems to be enhanced by the irradiance of the fiber, with greater power delivery for small core diameters.

\section{References}

1. M. Vivona, S. Girard, C. Marcandella, T. Robin, B. Cadier, M. Cannas, A. Boukenter, Y. Ouerdane, "Influence of Ce codoping and $\mathrm{H}_{2}$ pre-loading on $\mathrm{Er} / \mathrm{Yb}$-doped fiber: Radiation response characterized by Confocal Micro-Luminescence," J. Non-Crystal. Solids 357 (8) 1963-1965 (2011).

2. S. Girard, M. Vivona, L. Mescia, A. Laurent, Y. Ouerdane, C. Marcandella, F. Prudenzano, A. Boukenter, T. Robin, B. Cadier, E. Pinsard, M. Cannas and R. Boscaino, "Radiation hardening techniques for rare-earth based optical fibers and amplifiers," Proc. SPIE 8246, 82460Y-1-7 (2012).

3. S. Girard, A. Laurent, E. Pinsard, T. Robin, B. Cadier, M. Boutillier, C. Marcandella, A. Boukenter, and Y. Ouerdane, "Radiation-hardened erbium-doped optical fibers and amplifiers for future high-dose space missions," Proc. SPIE 8971, 89710E-1-6 (2014).

4. J. Thomas, M. Myara, L. Troussellier, E. Burov, A. Pastouret, D. Boivin, G. Mélin, O. Gilard, M. Sotom, and P. Signoret, "Radiation-resistant erbium-doped-nanoparticles optical fiber for space applications," Opt. Express 20 (3) 2435-2444 (2012). 
5. B. H. Babu, N. Ollier, I. Savelli, ; H. El Hamzaoui, A. Pastouret, B. Poumellec, M. Bouazaoui, L. Bigot, M. Lancry, "Study of radiation effects on $\mathrm{Er}^{3+}$-doped nanoparticles germano-silica fibers," J. Lightwave Technol. 34 (21) 4981-4987 (2016).

6. C.C. Baker, A. Burdett, E.J. Friebele, D.L. Rhonehouse, W. Kim, J. Sanghera, "Rare earth co-doping for increased efficiency of resonantly pumped Er-fiber lasers," Opt. Mat. Express 9 (3) 1041-1048 (2019).

7. M.J. LuValle, E. J. Friebele, F. Dimarcello, G. Miller, E. Monberg, L. Wasserman, P. Wisk, M. Yan and E. Birtch., "Radiation-Induced Loss Predictions for Pure Silica Core, Polarization-Maintaining Fibers," SPIE 6193, 6193OJ (2006).

8. C.C Baker, E.J. Friebele, A.A. Burdett, D.L. Rhonehouse, J. Fontana, W. Kim, S.R. Bowman, L.B. Shaw, J. Sanghera, J. Zhang, R. Pattnaik, M. Dubinskii, J. Ballato, C. Kucera, A. Vargas, A. Hemming, N. Simakov, J. Haub, "Nanoparticle doping for high power fiber lasers at eye-safer wavelengths," Opt. Express 25, 13903-13915 (2017).

9. M. Leo'n, M. Lancry, N. Ollier, B. H. Babu, L. Bigot, H. El Hamzaoui, I. Savelii, A. Pastouret, E. Burov, F. Trompier, B. Poumellec, and M. Bouazaoui, "Ge- and Al-related point defects generated by gamma irradiation in nanostructured erbiumdoped optical fiber preforms", J Mater Sci. 51:10245-10261 (2016).

10. S. Girard, A. Morana, A. Ladaci, T. Robin, L. Mescia, J-J. Bonnefois, M. Boutillier, J. Mekki, A. Paveau, B. Cadier, E. Marin, Y. Ouerdane and A. Boukenter "Recent advances in radiation-hardened fiber-based technologies for space applications," J. Opt. 20, 093001 (2018).

11. D.L. Griscom, E.J. Friebele, K.J. Long and J.W. Fleming, "Fundamental defect centers in glass: Electron spin resonance and optical absorption studies of irradiated phosphorus-doped silica glass and optical fibers," J. Appl. Phys. 54, 3743-3762 (1983).

12. B. Brichard, A. Fernandez Fernandez, H. Ooms, M. van Uffelen, F. Berghmans, "Study of the radiation-induced optical sensitivity in erbium and aluminium doped fibres," Proceedings of the 7th European Conference on Radiation and Its Effects on Components and Systems, Noordwijk, The Netherlands (2003)

13. M. Hill, R. Gray, J. Hankey and A. Gillooly, "Fibercore AstroGainTM fiber: multichannel Erbium doped fibers for optical space communications," Proc. of SPIE Vol. 8982, 898204-1 (2014).

14. B. P. Fox, K. Simmons-Potter, D. A. V. Kliner, and S. W. Moore, "Effect of low-earth orbit space on radiation-induced absorption in rare-earth-doped optical fibers", J. Non-Cryst. Solids 378, 79-88 (2013).

15. M. Lezius, K. Predehl, W. Stower, A. Turler, M. Greiter, Ch. Hoeschen, P. Thirolf, W. Assmann, D. Habs, A. Prokofiev, C. Ekstrom, T. W. Hansch and R. Holzwarth, "Radiation Induced Absorption in Rare Earth Doped Optical Fibers," IEEE Trans. Nucl. Sci. 59, (2) (2012).

16. B. Tortech, M. Van Uffelen, A. Gusarov, Y. Ouerdane, A. Boukenter, J. P. Meunier, F. Berghmans, and H. Thienpont, "Gamma radiation induced loss in erbium doped optical fibers," J. Non-Cryst. Solids 353, 477-480 (2007).

17. Y. Mebrouk, F. Mady, M. Benabdesselam, J-B. Duchez, and W. Blanc, "Experimental evidence of $\mathrm{Er}^{3+}$ ion reduction in the radiation-induced degradation of erbium-doped silica fibers" Opt. Lett. 39, 21 (2014).

18. J.-F. Lupi, M. Vermillac, W. Blanc, F. Mady, M. Benabdesselam, B. Dussardier, and D. R. Neuville, "Steady photodarkening of thulium alumino-silicate fibers pumped at $1.07 \mu \mathrm{m}$ : Quantitative effect of lanthanum, cerium and thulium," Optics Lett. 41 (12) 2771-2774 (2016). 


\title{
Single crystal semiconductor-core optical fiber*
}

\author{
Wei Wu ${ }^{\mathrm{a}}$, Seunghan Song ${ }^{\mathrm{a}, \mathrm{b}}$, Mustafa H. Balci ${ }^{\mathrm{a}}$, Fredrik Laurell ${ }^{\mathrm{c}}$ Patrick R. Cantwell ${ }^{\mathrm{d}}$, John Ballato ${ }^{\mathrm{e}}$ \\ and Ursula Gibson*a, b, c \\ a Department of Physics, Norwegian University of Science \& Technology, Høgskoleringen 5, \\ 7491Trondheim, Norway \\ bPoreLab, Department of Physics, Norwegian University of Science and Technology, Trondheim, \\ Norway \\ 'Department of Applied Physics, Royal Institute of Technology, Roslagstullsbacken 21, 10691 \\ Stockholm, Sweden \\ dDepartment of Mechanical Engineering, Rose-Hulman Institute of Technology, 5500 Wabash \\ Avenue Terre Haute, IN 47803 USA \\ ${ }^{e}$ Center for Optical Materials Science and Engineering Technologies (COMSET) School of Materials \\ Science and Engineering, Clemson University, Clemson, SC 29634 USA
}

\begin{abstract}
Semiconductor-core fibers are improved by removal of grain boundaries. We use a $\mathrm{CO}_{2}$ laser to process silicon-based cores; an alloying element reduces both the temperature and speed required for formation of a single crystal.
\end{abstract}

Keywords: semiconductor-core, laser-treated, single crystal, silicon, alloy

\section{Introduction}

Optical and potential THz applications of semiconductor-core fibers rely on low transmission losses, to which grain boundaries are significant contributors, as demonstrated by Healy, et $\mathrm{al}^{1}$ and Coucheron, et $\mathrm{al}^{2}$. Grain boundaries act as sinks for impurities (including deposits of the interface modifiers that reduce core oxidation), but can be eliminated by laser or other thermal treatment ${ }^{3}$ after fiber drawing. With elemental cores, a multiplicity of nucleation sites promote the formation of polycrystalline cores if there are local temperature variations and recrystallization of elemental core glass fibers is sensitive to a temperature gradient at the solidification front ${ }^{4}$. For smaller fiber cores a temperature gradient in the radial direction can be negligible, but because we heat the semiconductor core by conduction from the glass cladding, at larger diameters or faster scanning speed the temperature gradient in the radial direction can become significant. Especially for larger core materials such as those suitable for Thz applications, the thermal mass sets an upper limit on the translation rate at which a melt zone can be maintained. Making fibers small does not necessarily solve this problem, as it reduces the cross-section available for laser radiation absorption; extra glass is often added for studies of small cores. Fiber translation velocities of $\mathrm{mm} / \mathrm{s}$ were required to establish single crystals for $12 \mu \mathrm{m}$ Si core fiber, and that speed was insufficient to crystallize larger pure silicon cores. However, with an alloying element, small variations in the local temperature can be accommodated by changes in the local composition, which can be thermodynamically favorable to nucleating a new crystal. Thus alloys can be used to reduce the required melt zone velocity needed for single crystal growth. However, with an alloy system, the composition variation must be limited to avoid undue scattering, which can set an upper limit on the translation speed during recrystallization.

With a steep temperature gradient and a constant cooling rate (fixed power of the laser and constant velocity of the fiber), the effects of constitutional undercooling can be minimized in a solid solution such as $\mathrm{SiGe}^{2}$. The effects of capillarity, thermal gradient and concentration effects have been modelled by Tiller $^{5}$ and Mullins and Sekerka ${ }^{6}$, giving:

$$
v_{c}=\frac{D \nabla{ }_{L} k}{\nabla T_{L} x(k 1)}
$$

Sixth International Workshop on Specialty Optical Fibers and Their Applications (WSOF 2019), edited by Liang Dong, John M. Ballato, Proc. of SPIE Vol. 11206, 1120615 · C 2019 SPIE

CCC code: $0277-786 X / 19 / \$ 21 \cdot$ doi: $10.1117 / 12.2548428$ 
where $v_{c}$ is the critical velocity, $D$ is the diffusion coefficient in the liquid, $k$ is the segregation coefficient, $\nabla \Theta_{L}$ is the temperature gradient in the liquid, $\nabla T_{L}$ is the slope of the liquidus, and $x$ is the Ge composition in the liquid. With an alloy, speeds of hundreds of microns per second permit the formation of single crystal cores with diameters up to 100 microns, of interest for solar energy and $\mathrm{THz}$ transmission. In this paper, we discuss the use of alloying components for the crystallization of silicon cores using solid solutions of Si and Ge.

\section{Experimental}

We present results on fibers with cores that are pure Si (single crystal as drawn) and with Ge concentrations of 6 at $\%$ and 0.8 at $\%$ in silicon, used to promote crystallization of the core. Fibers were drawn at Clemson University, as described in Healy's work ${ }^{1}$. Fibers were translated at fixed velocities $(144 \mu \mathrm{m} / \mathrm{s}$ for $0.8 \%)$ using an LCS004 DTI piezoelectric motor with optical encoder, through the path of a $25 \mathrm{~W}$ Synrad $\mathrm{CO}_{2}$ laser operated at power levels chosen to create a melt zone of width $900 \mu \mathrm{m}$, and an intensity chosen to avoid damage to the cladding during the anneal. Additional experiments were performed using a scanning laser system suitable for treating longer fibers (BRM 4060, $80 \mathrm{~W} \mathrm{CO} 2,100 \mu \mathrm{m} / \mathrm{s}$ for $\mathrm{S}_{.44} \mathrm{Ge}_{.06}$ ). In the larger system, a cylindrical lens and substantial defocus was used. Optical measurements were made using butt coupling of a fiber coupled diode laser and a Watec 902H2 camera, and X-ray characterization was performed as described in a recent article. The TEM sample was prepared via in-situ lift out using a Zeiss 1540XB focused ion beam-scanning electron microscope (FIB-SEM), with final thinning done at $30 \mathrm{kV}$. Scanning transmission electron microscopy (STEM) and energy-dispersive X-ray spectroscopy (EDS) mapping were carried out on an FEI Tecnai F20ST TEM/STEM operating at $200 \mathrm{kV}$.

\section{Results and Discussion}

Figure 1 shows a) an STEM-EDS map which reveals a Ca-rich inclusion at a grain boundary in a SiGe fiber before annealing, b) the optical transmission of a section of pure silicon-core cane that was (111) single crystal over more than $1 \mathrm{~cm}$, and c) the optical transmission of a $6 \% \mathrm{Ge}$ in silicon material after laser annealing. The alloy core was opaque prior to annealing. While both samples are highly crystalline, the mode structure of the $\mathrm{SiGe}$ is clearly randomized by internal scattering. Oven annealing after the laser treatment shows some promise for reducing $\operatorname{loss}^{8}$ as it can promote Ge diffusion when slight variations exist. Reduction of inhomogeneity should both reduce loss and allow simpler mode structures.

a)
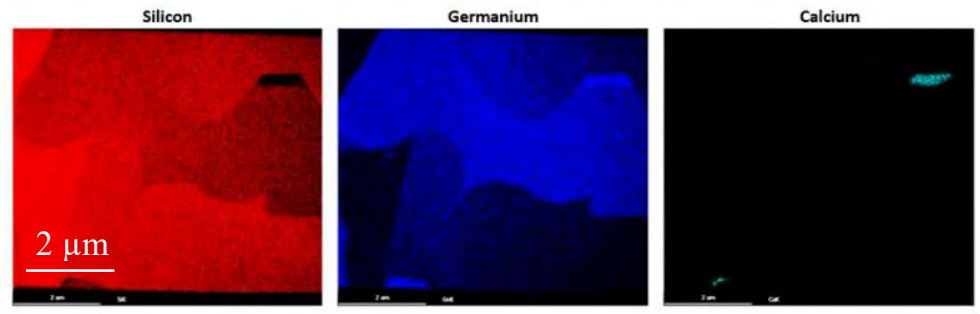

b)
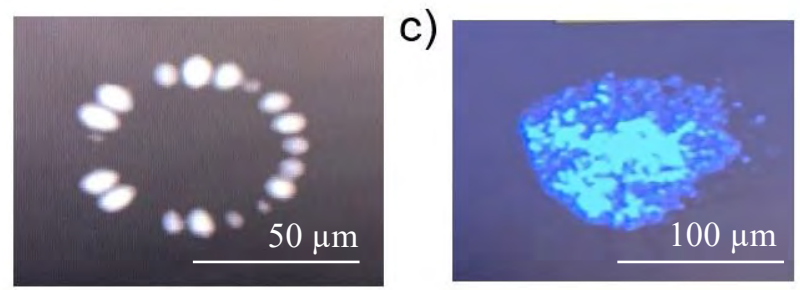

Figure 1. a) Calcium rich inclusion at an as-drawn fiber grain boundary, and optical transmission through b) single crystal silicon and c) bicrystal $\mathrm{Si}_{.94} \mathrm{Ge} .06$. 
The importance of establishing a single crystal core, to remove impurities and reduce scattering suggests using lower Ge concentration alloys. The liquidus slope approaches zero as the concentration of silicon increases, increasing the critical velocity so that the refractive index variations due to Ge concentration variation should be reduced. To test whether low concentrations would be adequate for single crystal growth, we drew fibers with 0.8 at $\% \mathrm{Ge}$, using commercial single crystal wafers (MTI) of this composition as the source material for the core. The cane, as drawn, was inhomogeneous, but after a single pass recrystallization, the core was seen to be single crystal and confirmed to be homogeneous using EDS mapping. XRD results for fibers are shown in Fig. 2; scans shown in a) and b) are for the fibers with optical results above. The intensity vs phi scan is an unusual presentation of the data, but gives an indication of whether a fiber has a single crystal core (and yields the axial orientation after analysis ${ }^{7}$ ). Figure $2 \mathrm{a}$ is a single crysta, 1 pure Si-core fiber, with [111] oriented along the axis (hence the 6 -fold symmetry in phi), $2 \mathrm{~b}$ is a laser annealed $0.6 \% \mathrm{Ge}$ fiber showing a small second grain, and $2 \mathrm{c}$ is a scan of $0.8 \% \mathrm{Ge}$ in $\mathrm{Si}$ after annealing, and is a single crystal. The brackets indicate which Bragg peak is presented for each fiber. The SiGe wafer used for these initial tests had a high conductivity, which limited IR transmission. Future work will include optimization of the preparation including higher resistivity starting materials, and/or an additional oven annealing process. In addition to solid solution forming alloys, eutectic systems may be used for recrystallizing the core, as demonstrated in reference [7].
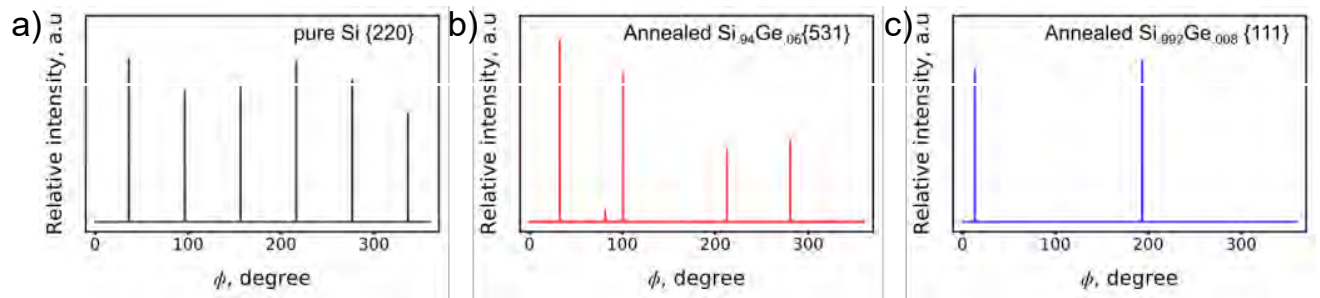

Fig. 2 XRD results on fibers a) pure silicon single crystal core fiber, b) annealed Si. $.4 \mathrm{Ge} .06$ with small secondary crystal and c) single crystal annealed $\mathrm{Si}_{.992} \mathrm{Ge} .008$. The $\{\mathrm{hkl}\}$ indicate the Bragg peak for each phi scan.

\section{Acknowledgements}

This work was supported by Norwegian Research Council grants 219686/O70 and 262232/O70, the Norwegian Microand Nano-Fabrication facility, NorFab, project number 245963/F50, Swedish Research Council (grant 2016-04488), the Swedish Foundation for Strategic Research (SSF) grant RMA15-0135, and the Knut and Alice Wallenberg Foundation (KAW) grant 2016.0104. The support of the J. E. Sirrine Foundation is gratefully acknowledged. Use of the Center for Nanoscale Materials, an Office of Science user facility, was supported by the U.S. Department of Energy, Office of Science, Office of Basic Energy Sciences, under Contract No. DE-AC02-06CH11357

\section{References}

[1] Healy, N., Fokine, M., Franz, Y., Hawkins, T., Jones, M., Ballato, J., Peacock, A. C. and Gibson, U. J., “CO 2 Laser-Induced Directional Recrystallization to Produce Single Crystal Silicon-Core Optical Fibers with Low Loss," Advanced Optical Materials 4(7), 1004-1008 (2016).

[2] Coucheron, D. A., Fokine, M., Patil, N., Breiby, D. W., Buset, O. T., Healy, N., Peacock, A. C., Hawkins, T., Jones, M., Ballato, J. and Gibson, U. J., "Laser recrystallization and inscription of compositional microstructures in crystalline SiGe-core fibres," Nature Communications 7, 13265 (2016).

[3] Franz, Y., Runge, A. F. J., Ren, H., Healy, N., Ignatyev, K., Jones, M., Hawkins, T., Ballato, J., Gibson, U. J. and Peacock, A. C., "Material properties of tapered crystalline silicon core fibers," Opt. Mater. Express, OME 7(6), 2055-2061 (2017). 
[4] Ji, X., Lei, S., Yu, S.-Y., Cheng, H. Y., Liu, W., Poilvert, N., Xiong, Y., Dabo, I., Mohney, S. E., Badding, J. V. and Gopalan, V., "Single-Crystal Silicon Optical Fiber by Direct Laser Crystallization," ACS Photonics 4(1), 8592 (2017).

[5] Tiller, W. A., Jackson, K. A., Rutter, J. W. and Chalmers, B., "The redistribution of solute atoms during the solidification of metals," Acta Metallurgica 1(4), 428-437 (1953).

[6] Mullins, W. and Sekerka, R., "Stability of Planar Interface During Solidification of Dilute Binary Alloy," J. Appl. Phys. 35(2), 444-451 (1964).

[7] Song, S., Lønsethagen, K., Laurell, F., Hawkins, T. W., Ballato, J., Fokine, M. and Gibson, U. J., "Laser restructuring and photoluminescence of glass-clad GaSb/Si-core optical fibres," Nature Communications 10(1), 1790 (2019).

[8] Sørgård, T., Mühlberger, K., Wu, W., Yang, X., Hawkins, T., Ballato, J., Laurell, F., Fokine, M., Gibson, U. J. and Gibson, U. J., "Reduced loss in SiGe-core optical fibers," Conference on Lasers and Electro-Optics (2018), paper SF3I.6, SF3I.6, Optical Society of America (2018). 


\title{
Chalcogenide fiber for long-wave infrared supercontinuum source
}

\author{
Francois Chenard ${ }^{*}$, Oseas Alvarez ${ }^{\mathrm{a}}$, Andrew Buff ${ }^{\mathrm{a}}$

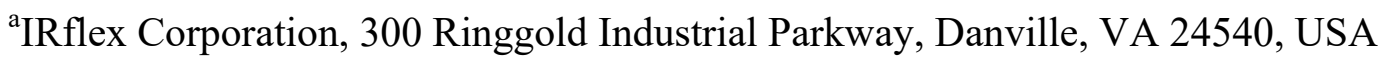

\begin{abstract}
We explain why chalcogenide glass fibers are well suited for long-wave infrared supercontinuum source. In particular we discuss the effects of the chemical composition and the fiber properties on the supercontinuum generation.

Keywords: chalcogenide, fiber, supercontinuum generation, mid-infrared, LWIR, laser, nonlinear optics

* francois.chenard@irflex.com; phone 1434-483-4304
\end{abstract}

\section{Introduction}

Broadband supercontinuum (SC) sources based on fiber nonlinearities are highly desired for sensing and spectroscopy applications in the infrared spectrum $\sim 2$ to $20 \mu \mathrm{m}$. SC generation in optical fibers was first demonstrated in 1976 [1]. SC sources offer high average power and good beam quality over a continuous wavelength range that is not possible with other alternatives like low-power blackbody source or tunable lasers. Nonlinear optical processes in the fibers pumped with short pulse lasers generate the SC. To date, SC has been generated in silica, tellurite, fluoride, and chalcogenide fibers. Long-wave infrared (LWIR) SC sources require the use of optical fibers that are transparent in the LWIR. Silica fiber has a strong absorption above $2.5 \mu \mathrm{m}$, tellurite fiber transmits up to $4.5 \mu \mathrm{m}$, and the fluoride fiber $\left(\mathrm{InF}_{3}\right)$ cuts off at $5.5 \mu \mathrm{m}$. Chalcogenide glass fibers are the best candidates to generate LWIR SC. Chalcogenide fibers have longer infrared transparency window and possess higher nonlinearity up to 1000 times greater than that of silica fiber. In this paper, we discuss the effects of the physical properties and chemical compositions of the chalcogenide fibers on the LWIR SC generation.

\section{Chalcogenide fiber for LWIR supercontinuum}

Chalcogenide glass contains one or more chalcogen element ( $\mathrm{S}, \mathrm{Se}, \mathrm{Te})$ with other additional elements (As, Ge, Ga, $\mathrm{Sb} . .$.$) . The heavier the chalcogen element, the further the glass transmits in the infrared and the higher the glass$ refractive index. Figure 1 shows the longer transmission of chalcogenide glasses and the relative higher refractive indices $\mathrm{n}_{\text {Telluride }}>\mathrm{n}_{\text {Selenide }}>\mathrm{n}_{\text {Sulfife }}[2]$.

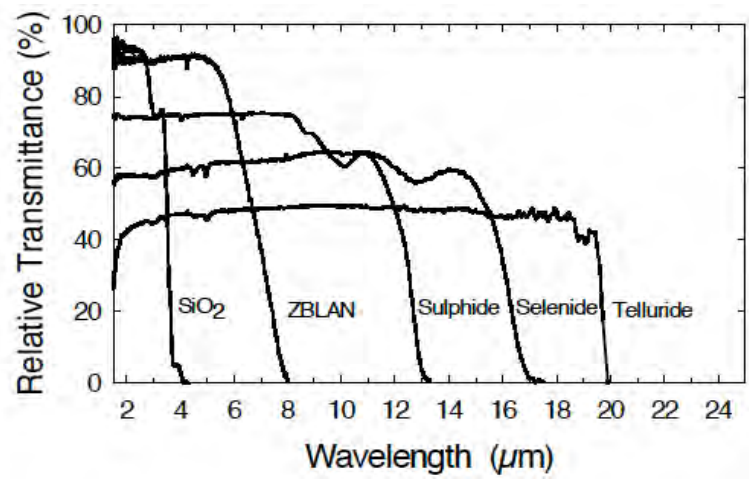

Figure 1. Infrared transmission for several bulk glass samples, thickness of about 2-3 mm [2] 
Glass-forming abilities and capabilities to fabricate optical fibers decrease with increasing molar weight of constituent elements; i.e., $\mathrm{S}>\mathrm{Se}>\mathrm{Te}$. Commercial $\mathrm{As}_{2} \mathrm{~S}_{3}$ and $\mathrm{As}_{2} \mathrm{Se}_{3}$ chalcogenide fibers are very stable, durable, insensitive to moisture, and transmit well up to $6.5 \mu \mathrm{m}$ and $10 \mu \mathrm{m}$ respectively [3,4]. Tellurium (Te) based chalcogenide glasses are not very stable and prone to crystallization, which makes Te-based fiber fabrication difficult. Te-As-Se unclad fiber has been developed to push the LWIR transmission but it has low glass transition temperature $\left(T_{g}\right)$ that may be a problem with high-power laser transmission [5]. Ge-As-Se-Te fibers are stable but have relatively high loss $(>2 \mathrm{~dB} / \mathrm{m})$ [6]. GeAs-Se fibers are stable (resistant to crystallization) and have higher $T_{g}$, which improves the damage threshold. Also GeAS-Se microstructured optical fiber with low optical loss and LWIR transmission window similar to $\mathrm{As}_{2} \mathrm{Se}_{3}$ fiber has been demonstrated [7].

The ideal fiber for LWIR SC source must have high nonlinearity, LWIR transmission, and low-loss. According to the above paragraph, $\mathrm{As}_{2} \mathrm{Se}_{3}$ is the current chalcogenide glass technology that satisfies all the required conditions. So we can safely argue that the best chalcogenide glass for the fiber core is $\mathrm{As}_{2} \mathrm{Se}_{3}$. Now we have several chalcogenide glass choices for the fiber cladding composition. The obvious one is to choose $\mathrm{As}_{2} \mathrm{Se}_{3}$ glass with higher $\mathrm{Se}$ content to lower the refractive index and increase the numerical aperture (NA). The cladding composition $\mathrm{AsSe}_{2}$ can be used with the $\mathrm{As}_{2} \mathrm{Se}_{3}$ core in order to achieve the high NA $(>0.5)$ required for efficient $\mathrm{SC}$ generation, but the large difference in thermal properties with the $\mathrm{As}_{2} \mathrm{Se}_{3}$ core leads to high fiber loss $(>5 \mathrm{~dB} / \mathrm{m})$ and lower SC efficiency [8]. Another approach is to use $\mathrm{As}_{2} \mathrm{~S}_{3}$ glass to make a cladding and produce extremely high NA of 1.4 with the $\mathrm{As}_{2} \mathrm{Se}_{3}$ core, but even the highly confined light in the $1.4 \mathrm{NA}$ core has enough evanescent light in the $\mathrm{As}_{2} \mathrm{~S}_{3}$ cladding (long wavelength edge $<6.5 \mu \mathrm{m}$ ) to prevent the full LWIR $\mathrm{As}_{2} \mathrm{Se}_{3}$ transmission [9]. Another cladding possibility is to add $\mathrm{S}$ to the $\mathrm{As}_{2} \mathrm{Se}_{3}$ glass (As-Se-S) to lower the refractive index, but the desired NA $>0.5$ to achieve efficient SC generation requires $\mathrm{S}>10 \%$, which is more than enough S content to reduce the LWIR transmission and induce LWIR fiber transmission loss with the evanescent field in the As-Se-S cladding [10].

The use of Ge-As-Se cladding with $\mathrm{As}_{2} \mathrm{Se}_{3}$ core is particularly appealing. Ge-As-Se has good stability, low-loss in the LWIR, and is thermally compatible with $\mathrm{As}_{2} \mathrm{Se}_{3}$. Ge-As-Se has a large glass forming range to produce stable chalcogenide glasses with a wide variety of physical and optical properties [11]. The Ge-As-Se composition can be selected to change the refractive index from 2.53 to 2.7 (compared to $\mathrm{As}_{2} \mathrm{Se}_{3} \mathrm{n}=2.79$ ). At the same time, the addition of $\mathrm{Ge}$ in the $\mathrm{As}_{2} \mathrm{Se}_{3}$ changes $\mathrm{T}_{\mathrm{g}}$ from $165^{\circ} \mathrm{C}$ to $350^{\circ} \mathrm{C}$ (compared to $\mathrm{As}_{2} \mathrm{Se}_{3} \mathrm{Tg}=190^{\circ} \mathrm{C}$ ). A fiber with $\mathrm{As}_{2} \mathrm{Se}_{3}$ core and $\mathrm{Ge}_{10} \mathrm{As}_{23} \mathrm{Se}_{67}$ cladding was made by a multi-stage process of extrusion and preform drawing [12]. The fiber NA was very high $\sim 1.0$ and the fiber loss was $>2 \mathrm{~dB} / \mathrm{m}$. A short $85 \mathrm{~mm}$ length of the fiber was used to generate LWIR SC covering 1.4-13.3 $\mu \mathrm{m}$.

Considering the previous published results, we developed a single mode chalcogenide fiber for LWIR SC generation [13]. The core composition was $\mathrm{As}_{2} \mathrm{Se}_{3}$ and the cladding was $\mathrm{GeAs}_{2} \mathrm{Se}_{5}$ to produce an $\mathrm{NA}=0.76$. The core diameter of $12 \mu \mathrm{m}$ was selected to bring the zero dispersion wavelength (ZDW) to $\sim 6 \mu \mathrm{m}$ and keep tight mode confinement over the LWIR. Figure 2 shows the loss spectra for three $\mathrm{As}_{2} \mathrm{Se}_{3}$ fibers with similar background loss $\sim 0.5 \mathrm{~dB} / \mathrm{m}$. The $\mathrm{As}_{2} \mathrm{Se}_{3} / \mathrm{GeAs}_{2} \mathrm{Se}_{5}$ (core/cladding) single mode fiber with $\mathrm{NA}=0.76$ has a Se-H absorption peak at $4.57 \mu \mathrm{m}$ of $\sim 3.5 \mathrm{~dB} / \mathrm{m}$. Another $\mathrm{As}_{2} \mathrm{Se}_{3} / \mathrm{As}_{2} \mathrm{Se}_{3}$ single mode fiber with lower $\mathrm{NA}=0.3$ has a similar Se-H absorption peak of $\sim 2.5 \mathrm{~dB} / \mathrm{m}$. The multimode fiber $\mathrm{As}_{2} \mathrm{Se}_{3} / \mathrm{As}_{2} \mathrm{Se}_{3}$ fiber with $\mathrm{NA}=0.3$ shows a lower Se-H peak of $\sim 1 \mathrm{~dB} / \mathrm{m}$. The fabrication process of the small core fibers seems to increase the $\mathrm{Se}-\mathrm{H}$ content. The $\mathrm{As}_{2} \mathrm{Se}_{3} / \mathrm{GeAs}_{2} \mathrm{Se}_{5}$ fiber shows the same long wavelength transmission edge as the one for typical $\mathrm{As}_{2} \mathrm{Se}_{3}$ fibers and demonstrated excellent $\mathrm{SC}$ generation $[9,13]$. 


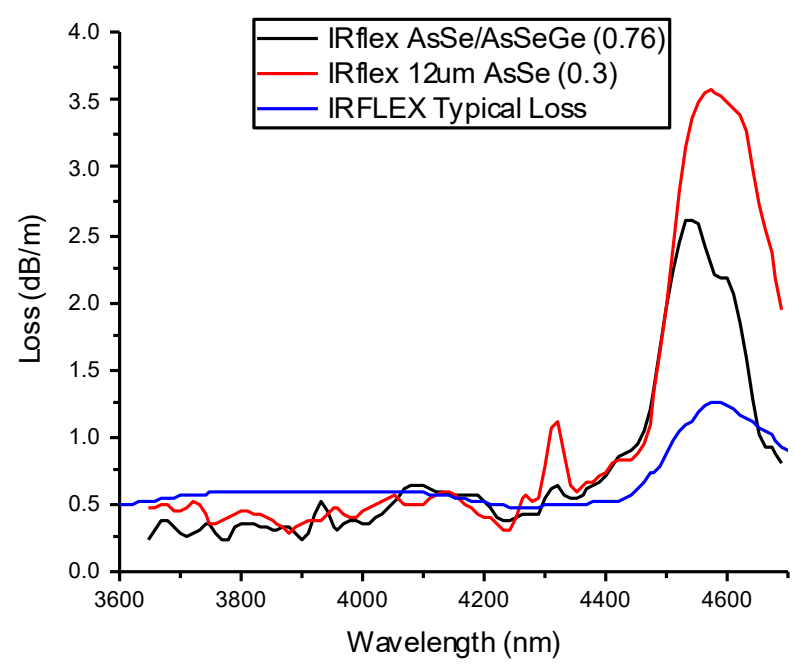

Figure 2. Loss spectra for three $\mathrm{As}_{2} \mathrm{~S}_{3}$ chalcogenide fibers.

The good SC generation performance of our $\mathrm{As}_{2} \mathrm{Se}_{3} / \mathrm{GeAs}_{2} \mathrm{Se}_{5}$ fiber comes from several factors. First, the selected glass compositions for the core and cladding provide low-loss fiber $\sim 0.5 \mathrm{~dB} / \mathrm{m}$ and a long wavelength edge up to $10 \mu \mathrm{m}$. The fiber NA (0.76) and its core diameter $(12 \mu \mathrm{m})$ provide good waveguiding properties that allow the light to be well confined to the core over its wide wavelength transmission window and also provide a suitable ZWD at $6 \mu \mathrm{m}$. Even though the theoretical single mode cutoff wavelength is at $12 \mu \mathrm{m}$, the SC output fiber exhibits single mode behavior over the entire LWIR. Higher NA fiber can produce shorter ZDW but at the expense of being more multimode and having more power coupling to higher-order modes, thus less SC generation. Also higher NA fiber with very small core can cause laser damage and higher coupling loss. On another side, lower NA fiber produces larger mode field diameter and leads to lower fiber nonlinearity, preventing SC expansion into LWIR. Therefore, it seems that the $\mathrm{As}_{2} \mathrm{Se}_{3} / \mathrm{GeAs}_{2} \mathrm{Se}_{5}$ fiber with $\mathrm{NA}=0.76$ and $12 \mu \mathrm{m}$ core diameter is more optimized to achieve good $\mathrm{SC}$ generation in the LWIR.

\section{Conclusion}

In conclusion, we have found that the chemical composition of the chalcogenide glass fiber is important for the LWIR $\mathrm{SC}$ generation. Core made of $\mathrm{As}_{2} \mathrm{Se}_{3}$ glass has the highest nonlinearity and also the longest transmission window in the LWIR. The best cladding materials is Ge-As-Se because of the good thermal compatibility with $\mathrm{As}_{2} \mathrm{Se}_{3}$ and comparable low-loss and LWIR transmission. We produced a $\mathrm{As}_{2} \mathrm{Se}_{3} / \mathrm{GeAs}_{2} \mathrm{Se}_{5}$ single mode fiber with NA=0.76 and $12 \mu \mathrm{m}$ core diameter. The chalcogenide fiber parameters seem to be optimum for the LWIR SC generation.

\section{References}

[1] Lin. C. and Stolen, R. H., "New nanosecond continuum for excited-state spectroscopy," Appl. Phys. Lett. 28,216 (1976)

[2] Aggarwal, I. D. and Sanghera, J. S., "Development and applications of chalcogenide optical fibers at NRL," J. Optoelectron. Adv. M. Vol. 4, No. 3, 665-678 (2002).

[3] IRF-S Series Chalcogenide Mid-Wave Infrared (MWIR) Fiber (1.5 to $6.5 \mu \mathrm{m}),<$ https://irflex.com/products/irf-sseries/> (July 2019).

[4] IRF-Se Series Chalcogenide Long-Wave Infrared (LWIR) Fiber (1.5 to $10 \mu \mathrm{m}),<$ https://irflex.com/products/irf-seseries/> (January 2019). 
[5] Shiryaev, V. S., Adam, J.-L., Zhang, X. H., Boussard-Pledel, C., Lucas, J., Churbanov, M. F., "Infrared fibers based on Te-As-Se glasses system with low optical losses," J. Non-Crystalline Solids 336, 113-119 (2004).

[6] Zhao, Z, Wang, X, Dai, S., Pan, Z., Liu, S., Sun, L., Zhang, P., Liu, X., Nie, Q., Shen, X., Wang, R., “1.5-14 $\mu \mathrm{m}$ midinfrared supercontinuum generation in a low-loss Te-based chalcogenide step-index fiber," Opt. Lett. Vol. 41, No. 22, 5222-5225 (2016).

[7] Petersen, C. R., Engelsholm, R. D., Markos, C., Brilland, L., Caillaud, C., Troles, J, Bang, O., "Increased midinfrared supercontinuum bandwidth and average power by tapering large-mode-area chalcogenide photonic crystal fibers," Optics Express Vol.25, No. 13, 15336-15347 (2017).

[8] Cheng, T., Nagaszka, K., Tuan, T. H., Xue, X, Matsumoto, M. Tezuka, H., Suzuki, T., Ohishi, Y, "Mid-infrared supercontinuum generation spanning 2.0 to $15.1 \mu \mathrm{m}$ in a chalcogenide step-index fiber," Opt. Lett. Vol. 41, No. 9, 2117 2120 (2016).

[9] Guo, K., Martinez, R. A., Plant, G, Maksymiuk, L., Janiszewski, B., Freeman, M. J., Maynard, R. L., Islam, M. N., Terry, F. L., Bedford, R., Gibson, R., Chenard, F., Chatigny, S., Ifarraguerri, A. I., "Generation of near-diffractionlimited, high-power supercontinuum from $1.57 \mu \mathrm{m}$ to $12 \mu \mathrm{m}$ with cascaded fluoride and chalcogenide fibers," Appl. Opt. Vol. 57, No. 10, 2519-2532 (2018).

[10] Guillevic, E., Zhang, X., Pain, T., Calvez, L., Adam, J.-L., Lucas, J., Guilloux-Viry, M., Ollivier, S., Gadret, G., "Optimization of chalcogenide glass in the As-Se-S system for automotive applications," Optical Materials 31, 1688-1692 (2009).

[11] Eggleton, B. J., Luther-Davies, B, Richardson, K., "Chalcogenide photonics," Nat. Photonics 5(3), 141-148 (2011)

[12] Petersen, C. R., Møller, U, Kubat, I., Zhou, B., Dupont, S., Ramsay, J., Benson, T., Sujecki, S., Abdel-Moneim, N., Tang, Z., Furniss, D., Seddon, A., Bang, O., "Mid-infrared supercontinuum covering the 1.4-13.3 $\mu \mathrm{m}$ molecular fingerprint region using ultra-high NA chalcogenide step-index fibre," Natur Photonics Vol. 8, 830-834 (2014).

[13] Martinez, R. A., Plant, G., Guo, K., Janiszewski, B., Freeman, M. J., Maynard, R. L., Islam, M. N., Terry, F. L., Alvarez, O., Chenard, F., Bedford, R., Gibson, R., Ifarraguerri, A. I., "Mid-infrared supercontinuum generation from 1.6 to $>11 \mu \mathrm{m}$ using concatenated step-index fluoride and chalcogenide fibers," Opt. Lett. · Vol. 43, No. 2, 296-299 (2018). 


\title{
Broadband mid-infrared supercontinuum generation in chalcogenide tapered fiber with all-normal dispersion
}

\author{
Shixun Dai*, Nan Zhang, Yingying Wang \\ aLaboratory of Infrared Material and Devices, The Research Institute of Advanced Technologies, \\ Ningbo University, Ningbo, 315211, China \\ ${ }^{b}$ Key Laboratory of Photoelectric Materials and Devices of Zhejiang Province, Ningbo, 315211 , \\ China
}

\begin{abstract}
We report broadband supercontinuum (SC) generation in chalcogenide $(\mathrm{ChG})$ tapered fibers pumped in the normal dispersion (ANDi) regime. The fibers were fabricated using the isolated stacked extrusion method. A homemade tapering platform allows us to accurately control tapering parameters. An SC generation spanning 1.4 - 7.2 $\mu \mathrm{m}$ was achieved by pumping an $\mathrm{As}_{2} \mathrm{~S}_{3}$ tapered fiber. To further extend the $\mathrm{SC}$, a Te-based ChG tapered fiber was pumped at $5.5 \mu \mathrm{m}$, and an $\mathrm{SC}$ generation covered $1.7-12.7 \mu \mathrm{m}$ with high coherence property was generated.

Keywords: nonlinear optics, chalcogenide glass fiber, tapered fiber, supercontinuum generation. *daishixun@nbu.edu.cn
\end{abstract}

\section{Introduction}

Mid-infrared (MIR) supercontinuum (SC) light sources with spatial coherence, broad bandwidth, and high brightness have received considerable attention due to their significant potential in various applications, such as spectroscopy [1], and hyper-spectral microscopy [2]. A variety of soft-glass fibers made of fluoride, tellurite or chalcogenide (ChG) glasses have been used to generate MIR SC. Particularly, ChG fibers have advantages of wider transparency window (over $20 \mu \mathrm{m}$ ) [3] and higher optical nonlinearity (up to thousand times greater than that of silica glasses)[4], making them good candidates for MIR SC generation. Recently, many broadband MIR SCs have been experimentally generated from $\mathrm{ChG}$ step-index fibers in the anomalous group velocity dispersion regime to obtain a maximum spectral bandwidth. However, the generated SC spectra usually suffer from uneven flatness and weak stability due to phase noise and unstable spectral amplitude. Tapered fibers allow us to modify the dispersion profile and increase the optical nonlinearity via dimensional control. Tapered fiber with ANDi characteristics can generate SC with high coherence property, but the width of $\mathrm{SC}$ is relatively narrow.

In this study, we report broadband SC generation in ChG tapered fibers pumped in the normal dispersion (ANDi) regime. The fibers were fabricated using the isolated stacked extrusion method. A homemade tapering platform allows us to accurately control tapering parameters. An SC generation spanning $1.4-7.2 \mu \mathrm{m}$ was achieved by pumping an $\mathrm{As}_{2} \mathrm{~S}_{3}$ tapered fiber. To further extend the $\mathrm{SC}$, a Te-based $\mathrm{ChG}$ tapered fiber was pumped at $5.5 \mu \mathrm{m}$, and an $\mathrm{SC}$ generation covered 1.7-12.7 $\mu \mathrm{m}$ was generated. In addition, the coherence property of SC spectrum was also discussed.

\section{Experiments}

\section{As-S tapered fiber fabrication}

The $\mathrm{ChG}$ fiber used in this work made of $\mathrm{As}_{2} \mathrm{~S}_{3}$ core and $\mathrm{As}_{38} \mathrm{~S}_{62}$ cladding were prepared using purified materials through the melt-quenching method. The fiber preform with a core-cladding diameter ratio of 1:5 was fabricated through the isolated stacked extrusion method [5]. The preform was drawn in a fiber drawing tower into a step-index fiber which

Sixth International Workshop on Specialty Optical Fibers and Their Applications (WSOF 2019), edited by Liang Dong, John M. Ballato, Proc. of SPIE Vol. 11206, 1120617 · C 2019 SPIE

CCC code: $0277-786 X / 19 / \$ 21 \cdot$ doi: $10.1117 / 12.2550293$ 
had a $\sim 60 \mu \mathrm{m}$ diameter core and a $\sim 300 \mu \mathrm{m}$ diameter cladding coated with a $\sim 30-\mu \mathrm{m}$-thick polyethersulfone (PES) jacket, which was used to improve the fiber mechanical robustness [6]. The fiber exhibits a minimum loss of $1.4 \mathrm{~dB} / \mathrm{m}$ at 3.25 $\mu \mathrm{m}$ and an average loss of $3.72 \mathrm{~dB} / \mathrm{m}$. The numerically calculated zero-dispersion-wavelength (ZDW) of fundamental mode (FM) for this fiber was around $5.3 \mu \mathrm{m}$.

A homemade tapering platform was adopted to taper the As-S ChG fiber. When the electric heating zone temperature was increased slowly to soften $\mathrm{ChG}$ fiber and a minor bending of the fiber was observed, the motorized stages pulling the two ends of the fiber were moved in opposite directions at a fixed traction speed. When the fiber diameter was much smaller than the size of the different heating zones, a tapered fiber with a decaying-exponential profile was produced (shown in Fig. 1(a)) [7]. Figure 1(b) is the image of a part of the transition region taken by SEM, and Fig. 1(c) shows the waist of a tapered fiber. The core diameter at taper waist and transition region length can be accurately controlled by changing the electric heating zone length and traction distance.
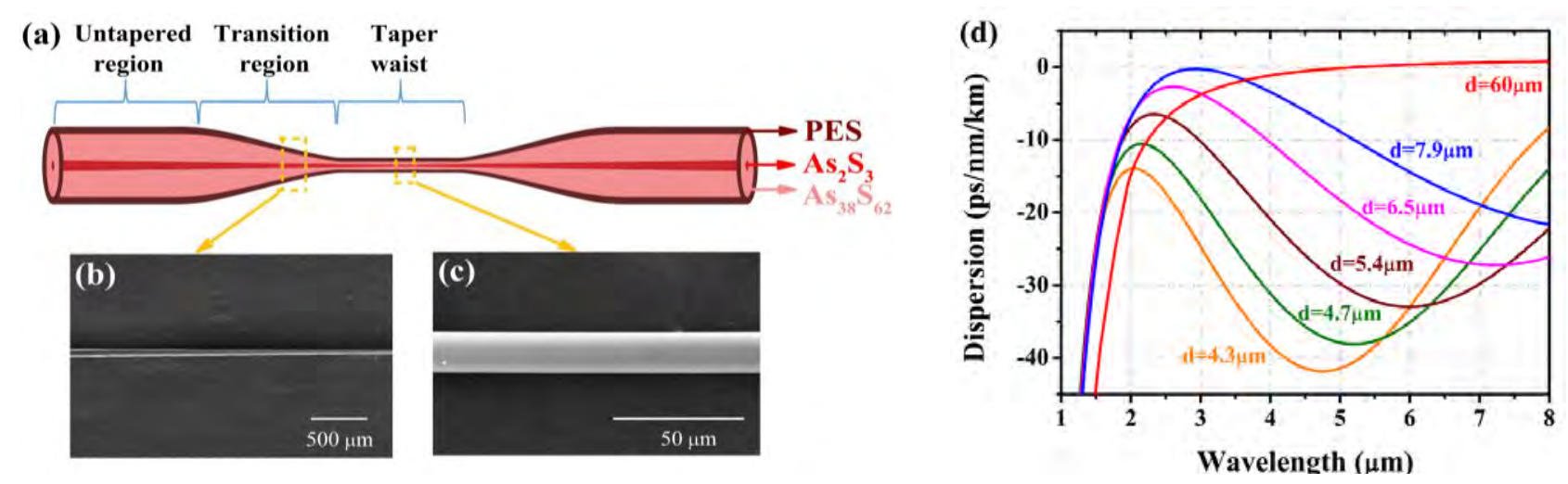

Figure 1. (a) Schematic of a ChG tapered fiber with a decaying-exponential profile. (b) SEM image of a part of transition region for a tapered fiber. (c) SEM image of the taper waist for a tapered fiber. (d) Calculated fundamental mode dispersion characteristic curves of $\mathrm{ChG}$ fibers with various core diameters.

The FM dispersion characteristic curves for various core diameters in the fiber tapered region were also simulated, as illustrated in Fig. 1(d). When the core diameter was below $8.0 \mu \mathrm{m}$, the fiber showed an all-normal dispersion in the range of 1-8 $\mu \mathrm{m}$, which may improve the generated SC spectral quality under the femtosecond pump laser [8].

\section{Te-based tapered fiber fabrication}

The Te-based tapered fiber consisting of $\mathrm{Ge}_{20} \mathrm{As}_{20} \mathrm{Se}_{2} \mathrm{Te}_{40}$ cladding and $\mathrm{Ge}_{20} \mathrm{As}_{20} \mathrm{Se}_{15} \mathrm{Te}_{45}$ core glasses, which have higher linear refractive indices than As-S glass, was fabricated in the way as mentioned above. The glass rods and tapered fiber are shown in Fig. 2. This fiber exhibits a minimum loss of $3.9 \mathrm{~dB} / \mathrm{m}$ at $6.8 \mu \mathrm{m}$. Due to the long wavelength of zero material dispersion of Te-based glass, this fiber has a ZDW of $10.4 \mu \mathrm{m}$ when core diameter was $60 \mu \mathrm{m}$.

The dispersion characteristic curves with various core diameters in the fiber-tapered region are illustrated in Fig. 3. With the decrease of the fiber core diameter at the taper waist from $60 \mu \mathrm{m}$ to $20 \mu \mathrm{m}$, the ZDW gradually blue shifted from $10.4 \mu \mathrm{m}$ to $8 \mu \mathrm{m}$. When the fiber core diameter was $\leq 14 \mu \mathrm{m}$, the dispersion of the fiber exhibited an ANDi characteristic in the wavelength range of $1.5-14 \mu \mathrm{m}$. 
(a) Glass rod
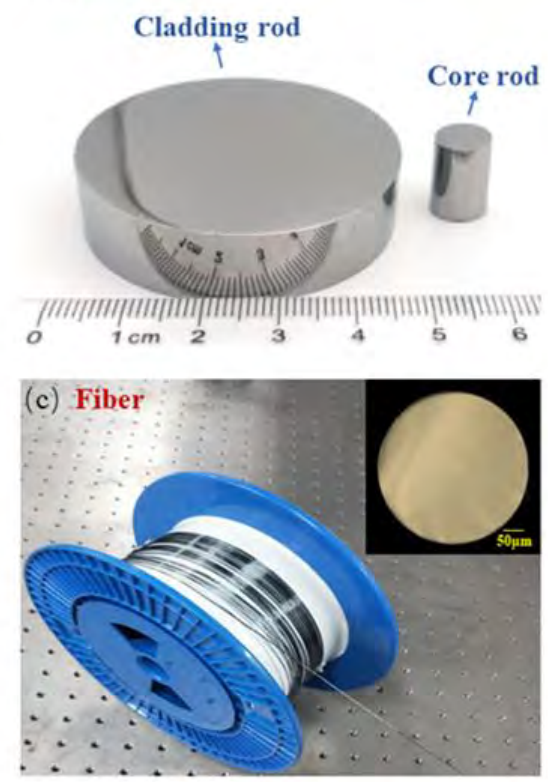

(b) Preform
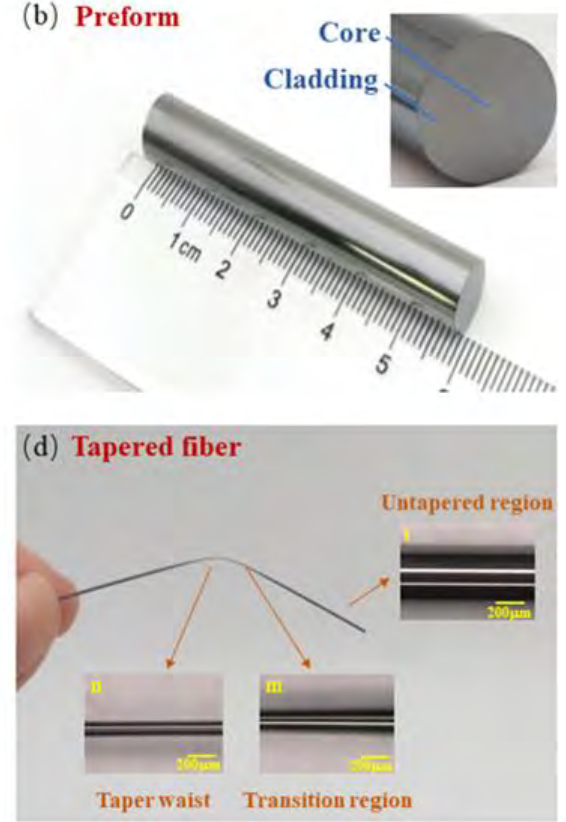

Figure 2. (a) Core and cladding glass rods. (b) Extruded preform. Inset: cross-section of the preform. (c) A roll of well-fabricated step-index fiber. Inset: cross-section image of the fiber. (d) Tapered fiber with the core waist diameter of $80 \mu \mathrm{m}$. Inset: I untapered region, II taper waist, III transition region.

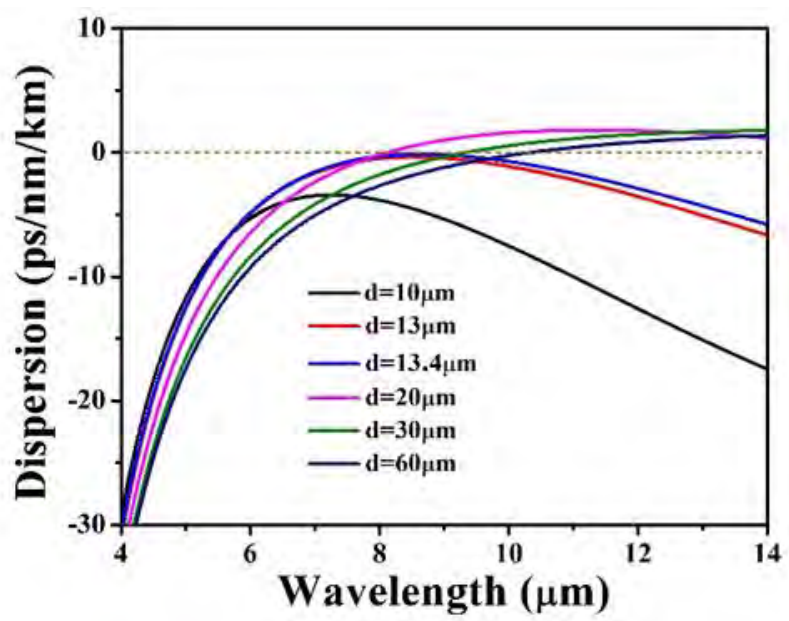

Figure 3. Calculated fundamental mode dispersion characteristic curves of Te-based ChG fibers with various core diameters.

\section{Results and Discussion}

The experimental set-up for SC generation and measurement from the ChG tapered fiber has been described in [9]. A tunable OPA system (Mirra $900+$ Legend Elite +OperA Solo) was used as the exciting source. The pump pulse has a duration of $150 \mathrm{fs}$ and a repetition rate of $1 \mathrm{kHz}$. The beam from the OPA was coupled via a calcium fluoride lens with a focal length of $75 \mathrm{~mm}$ into the fiber. The SC output from the fiber was collected by the input slit of a monochromator. A 
liquid nitrogen-cooled $\mathrm{HgCdTe}$ (MCT) detector with a wavelength range of 1-16 $\mu \mathrm{m}$ was used to measure the output of the monochromator.

\section{SC generation in As-S tapered fiber}

Figure 4(a) shows the dependence of the resulting SC spectra on transition region length of the tapered fiber pumped with the same average pump power of $25 \mathrm{~mW}$ at $3.25 \mu \mathrm{m}$. Several tapered fibers with almost same core diameters at waist of $5.4 \mu \mathrm{m}$ and same fiber length of $\sim 12 \mathrm{~cm}$ were used. The values of transition region length for these tapered fibers were $6.7,7.6,8.4,9.2$, and $10.5 \mathrm{~mm}$, respectively. Firstly, the pulses undergo strong self-phase modulation (SPM) behavior during the initial propagation since the pump laser experienced normal dispersion along the tapered fiber [10]. After that, self-steeping and third-order dispersion caused the optical wave breaking (OWB), leading to a significant blue shift of the spectrum [11]. As shown in Fig. 4(a), the generated spectrum on the long wavelength side was wider than that on the short wavelength side for all tapered fibers. This was because the group velocity dispersion slope on short wavelength side was much steeper than that on long wavelength side. Four-wave mixing (FWX) and stimulated Raman scattering also contributed to the spectral broadening [12]. Finally, a broad and relatively flat SC spectrum spanning from 1.4 to $7.2 \mu \mathrm{m}$ was obtained from the tapered fiber with transition region length of $10.5 \mathrm{~mm}$.
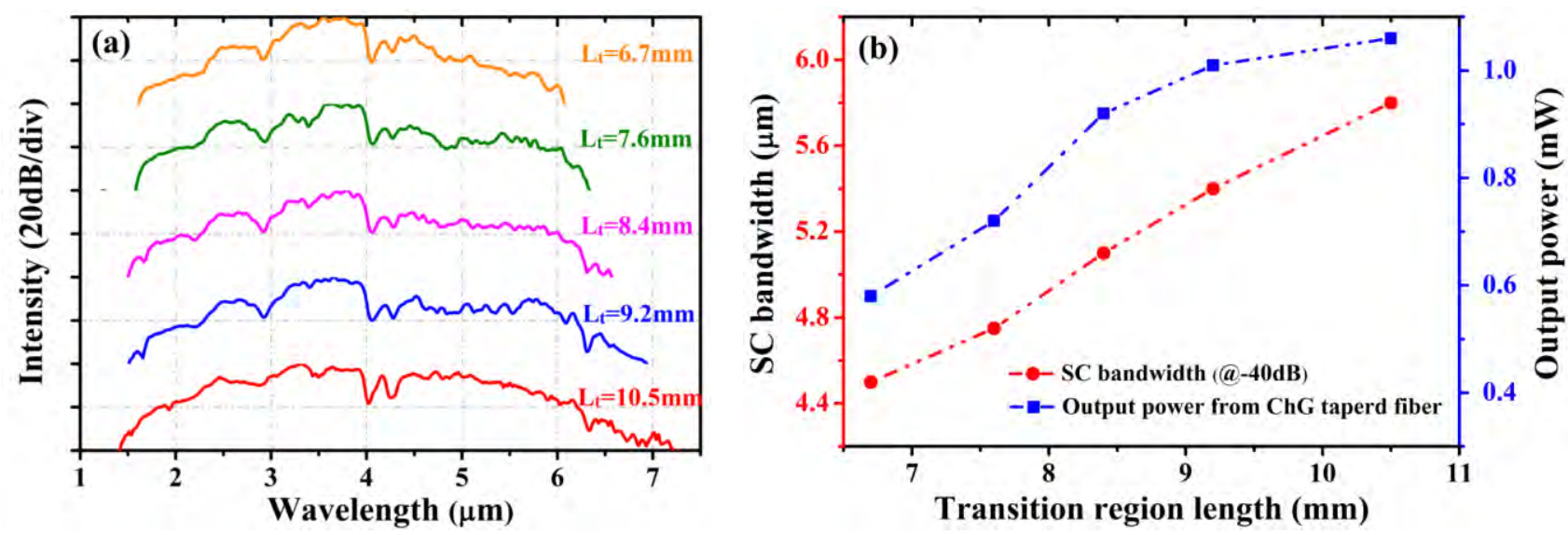

Figure 4. (a) Measured SC spectra generated from 12-cm-long As-S ChG tapered fibers with transition region length varying from $6.7 \mathrm{~mm}$ to $10.5 \mathrm{~mm}$ pumped at $3.25 \mu \mathrm{m}$. (b) Bandwidth of the generated SC spectra at $40 \mathrm{~dB}$ and the corresponding output power from tapered fibers as a function of transition region length.

The bandwidth of the generated SC spectrum and the corresponding output power from the above tapered fibers as a function of transition region length are depicted in Fig. 4(b). Both output power and SC bandwidth increased with increasing transition region length. The output power of $1.06 \mathrm{~mW}$ was achieved for the maximum spectral broadening of $5.8 \mu \mathrm{m}$ (from 1.4 to $7.2 \mu \mathrm{m}$ ) in the tapered fiber with the transition region length of $10.5 \mathrm{~mm}$. In tapered fibers with the same core diameter, the different transition region lengths led to various diameter changing rates along the fiber. When the diameter of the transition region changes slowly with a relatively larger transition region length, there is always accompanied by a less number of higher-order modes, and thus higher transmission efficiency of light, resulting in a higher output power and a larger spectral broadening from the tapered fiber [13].

\section{SC generation in Te-based tapered fiber}

A $7 \mathrm{~cm}$ long tapered fiber with $13.4 \mu \mathrm{m}$ waist core diameter was used to generate the SC. Figure 5 shows the dependence of the resulting SC spectrum at different pump wavelengths of 4.5, 5.5, and $6.5 \mu \mathrm{m}$ in the normal dispersion regime, respectively. When pumped at $4.5 \mu \mathrm{m}$, the SC bandwidth spanned only from $1.8 \mu \mathrm{m}$ to $10.5 \mu \mathrm{m}$, as shown in Fig. 5(a). When pumping wavelength was increased to $5.5 \mu \mathrm{m}$, the long wavelength edge of SC spectrum was extended, and the widest SC covering from $1.7 \mu \mathrm{m}$ to $12.7 \mu \mathrm{m}$ was generated, as shown in Fig. 5(b). On further increasing the pumping 
wavelength to $6.5 \mu \mathrm{m}$, the SC spectrum becomes slightly narrow from $2.2 \mu \mathrm{m}$ to $11.1 \mu \mathrm{m}$ under $19 \mathrm{~mW}$ laser power, as shown in Fig.5(c). The SC spectrum results pumped at different wavelength showed that a pump wavelength of $5.5 \mu \mathrm{m}$ was more efficient than $6.5 \mu \mathrm{m}$, which due to the higher pumping power at $5.5 \mu \mathrm{m}$. For comparison, the SC broadening in an untapered fiber with the same length of the taper fibers was also obtained under the same pumping conditions, as shown in Fig. 5(d). The result showed that the SC spectrum from untapered fiber spanning from $2.6 \mu \mathrm{m}$ to $10.4 \mu \mathrm{m}$ was relatively narrower than that of tapered fibers.
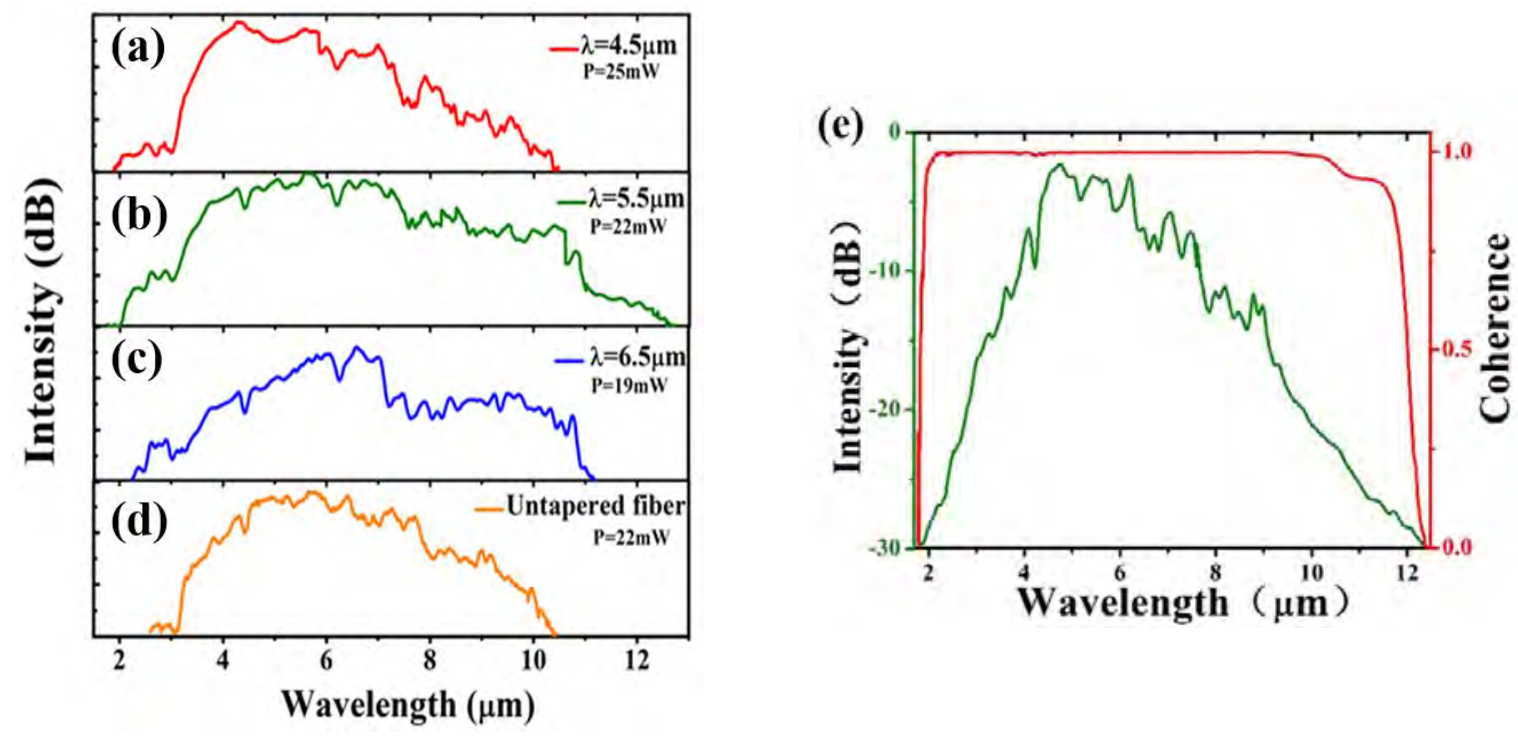

Figure 5. Measured SC spectral generated from Te-based $\mathrm{ChG}$ tapered fiber with different pumping wavelengths of (a) $4.5 \mu \mathrm{m}$, (b) $5.5 \mu \mathrm{m}$, and (c) $6.5 \mu \mathrm{m}$ and (d) an untapered fiber pumped at $5.5 \mu \mathrm{m}$.(e) Simulated the SC spectrum (green curve, left axis) and its coherence property (red curve, right axis).

In addition, the SC spectrum generated in Te-based fiber was simulated, which is shown in Fig. 5(e). As we can see, the SC over the wavelength range of 2-12.7 $\mu \mathrm{m}$ has a perfect coherence, which corresponded to perfect coherence. Both SPM and OWB play an important role for spectral broadening, which create new wavelength components with a phase related to the injected pulse, eliminating noise-sensitive soliton dynamics [14]. Therefore, the SC spectra can keep the coherence property of the pump laser.

\section{Conclusion}

An SC generation spanning $1.4-7.2 \mu \mathrm{m}$ was achieved in an As-S tapered fiber with a transition region length of 10.5 $\mathrm{mm}$, and SC generation spanning 1.7-12.7 $\mu \mathrm{m}$ was achieved in a Te-based tapered fiber when pumped in the normal dispersion regime. Our simulation result shows that the SC generated in ANDi region has a high coherence property.

\section{Acknowledgement}

This work was supported by National Natural Science Foundation of China (Grant nos. 61435009 and 61377099); K.C. Wong Magna Fund in Ningbo University; China Postdoctoral Science Foundation (Grant no. 2018M642386).

\section{References}

1. Ke, K., Xia, C., Islam, M. N., Welsh, M. J., and Freeman, M. J., "Mid-infrared absorption spectroscopy and differential damage in vitro between lipids and proteins by an all-fiber-integrated supercontinuum laser," Opt. Express 17, 12627-12640, (2009).

2. Dupont, S., Petersen, C., Thøgersen, J., Agger, C., Bang, O., and Keiding, S. R., "IR microscopy utilizing intense 
supercontinuum light source," Opt. Express 20, 4887-4892, (2012).

3. Shiryaev, V. S., and Churbanov, M. F., "Trends and prospects for development of chalcogenide fibers for midinfrared transmission," J. Non-Cryst. Solids 377, 225-230, (2013).

4. Slusher, R. E., Lenz, G., Hodelin, J., Sanghera, J., L. Shaw, B., and Aggarwal, I. D., "Large raman gain and nonlinear phase shifts in high-purity As2Se3 chalcogenide fibers," Journal of the Optical Society of America B: Optical Physics 21, 1146-1155, (2004).

5. Sun, Y., Dai, S., Zhang, P., Wang, X., Xu, Y., Liu, Z., Chen, F., Wu, Y., Zhang, Y., Wang, R., and Tao, G., "Fabrication and characterization of multimaterial chalcogenide glass fiber tapers with high numercial apertures," Opt. Express 23, 23472-23483, (2015)

6. Tao, G., Ebendorff-Heidepriem, H., Stolyarov, A. M., Danto, S., Badding, J. V., Fink, Y., Ballato, J., andAbouraddy, A. F., "Infrared fibers," Adv. Opt. Photonics 7, 379-458, (2015)

7. Harun, S. W., Lim, K. S., Tio, C. K., Dimyati, K., and Ahmad, H., "Theoretical analysis and fabrication of tapered fiber," Optik 124, 538-543, (2013)

8. Falk, P., Frosz, M. H., and Bang, O., "Supercontinuum generation in a photonic crystal fiber with two zerodispersion wavelengths tapered to normal dispersion at all wavelengths," Opt. Express 13, 7535-7540, (2005)

9. Luo, B., Wang, Y., Dai, S., Sun, Y., Zhang, P., Wang, X., and Chen, F., "Midinfrared supercontinuum generation in As2Se3-As2S3 chalcogenide glass fiber with high NA," J. Lightwave Technol. 35, 2464, (2017)

10. Price, J. H.V., Feng, X., Heidt, A. M., Brambilla, G., Horak, P., Poletti, F., Ponzo, G., Petropoulos, P., Petrovich, M., Shi, J., Ibsen, M., Loh, W. H., Rutt, H. N., and Richardson, D. J., "Supercontinuum generation in non-silica fiber," Opt. Fiber Technol. 18, 327-344, (2012).

11. Zhao, Z., Wang, X., Dai, S., Pan, Z., Liu, S., Sun, L., Zhang, P., Liu, Z., Nie, Q., Shen, X., and Wang, R., “1.5-14 mum midinfrared supercontinuum generation in a low-loss Te-based chalcogenide step-index fiber," Opt. Lett. 41, 5222-5225, (2016)

12. Wang, F., Wang, K., Yao, C., Jia, Z., Wang, S., Wu, C., Qin, G., Ohishi, Y., and Qin, W., "Tapered fluorotellurite microstructured fibers for broadband supercontinuum generation," Opt. Lett. 41, 634-7, (2016)

13. Ma, C., Ren, L., Xu, Y., Wang, Y., Liang, J., and Qu, E., "Design and fabrication of tapered microfiber waveguide with good optical and mechanical performance," J. Mod. Optic. 61, 683-687, (2014)

14. Hooper, L. E., Mosley, P. J., Muir, A. C., Wadsworth, W. J., and Knight, J. C., "Coherent supercontinuum generation in photonic crystal fiber with all-normal group velocity dispersion," Opt. Express 1, $4902-4907$ (2011). 


\title{
Mid-infrared supercontinuum generation in chalcogenide or heavy oxide fibers for sensing applications
}

\author{
Arnaud Lemière*, Frédéric Désévédavy, Pierre Mathey, Grégory Gadret, Jean-Charles Jules, \\ Christophe Aquilina, Bertrand Kibler, Pierre Béjot, Franck Billard, Olivier Faucher, Frédéric \\ Smektala
Laboratoire Interdisciplinaire Carnot de Bourgogne (ICB), UMR 6303 CNRS - Université Bourgogne Franche-Comté, 9 avenue Alain Savary, 21000 Dijon, France

\begin{abstract}
Significant improvements have been made in the processing of non-linear glasses and optical fibers since the early 2000s in order to carry out supercontinuum generation experiments. In this work, we present recent advances achieved in our laboratory on tellurite fibers as well as on arsenic and antimony free chalcogenide fibers. We report the resulting supercontinuum spanning between 2 and $14 \mu \mathrm{m}$ for chalcogenide fibers and the result of a supercontinuum absorption spectroscopy experiment above $3 \mu \mathrm{m}$ with tellurite fibers is reported.
\end{abstract}

Keywords: nonlinear, glass, optics, fiber, supercontinuum, laser, spectroscopy

*arnaud.lemiere@u-bourgogne.fr; phone+33 380395921

\section{Introduction}

During the last decade, many studies have been realized to develop new infrared nonlinear glasses for applications in molecular sensing [1], medicine [2] or free-space communications [3]. Many chemical species exhibit absorption bands in the near- and mid-infrared region $(1-20 \mu \mathrm{m})$, such as for example $\mathrm{CH}_{4}, \mathrm{CO}_{2}, \mathrm{~N}_{2} \mathrm{O}, \mathrm{CO}, \mathrm{SO}_{2}$ or $\mathrm{HCl}$ [4]. To work in this wavelength range which includes the two strategic atmospheric windows $(3-5 \mu \mathrm{m}$ and $8-14 \mu \mathrm{m})$, tellurite and chalcogenide glasses are ideal materials because of their transmission windows: $1-6 \mu \mathrm{m}$ for tellurite glasses and $1-30 \mu \mathrm{m}$ for chalcogenide glasses depending on composition as measured on bulk samples. Another advantage of these glasses is their high nonlinear refractive index $\mathrm{n}_{2}$ : around $4 \times 10^{-19} \mathrm{~m}^{2} / \mathrm{W}($ at $1.55 \mu \mathrm{m})$ for tellurite glasses and from $21 \times 10^{-19} \mathrm{~m}^{2} / \mathrm{W}$ (at $1.55 \mu \mathrm{m}$ ) to $130 \times 10^{-19} \mathrm{~m}^{2} / \mathrm{W}$ (at $1.06 \mu \mathrm{m}$ ) for chalcogenide glasses, upon their composition. These two types of glasses need specific synthesis and purification processes to reach efficient optical fibers after drawing operation [5]. Supercontinuum (SC) generation has been reached in tellurite optical fibers since 2006 [6] but the first SC covering almost the entire transmission window of a tellurite fiber has been obtained in 2013 [7]. A few years later, the broadest SC by pumping in the anomalous dispersion regime is achieved [8] and spans from 1.3 to $5.3 \mu \mathrm{m}$. From a similar SC, a supercontinuum absorption spectroscopy (SAS) experiment has been realized in 2016 [1] allowing for $\mathrm{CH}_{4}$ sensing between 2.2 and $2.5 \mu \mathrm{m}$. Concerning chalcogenide fibers, the first SC broader than $10 \mu \mathrm{m}$ is obtained in 2014 [9] when the largest SC generated in this kind of fibers has been reached in 2017 [10] and spans from 2 to $16 \mu \mathrm{m}$. In this work we present recent improvements in SC generation realized in our laboratory for tellurite and As-Sb-free chalcogenide fibers, as well as technological progress in SAS experiment by showing the detection of $\mathrm{CH}_{4}$ above $3 \mu \mathrm{m}$.

Sixth International Workshop on Specialty Optical Fibers and Their Applications (WSOF 2019), edited by Liang Dong, John M. Ballato, Proc. of SPIE Vol. 11206, 1120618 · (C) 2019 SPIE

CCC code: $0277-786 X / 19 / \$ 21 \cdot$ doi: $10.1117 / 12.2542200$ 


\section{Glasses synthesis and physical properties}

Our step-index tellurite fibers are drawn from a TZNF glass $\left(80 \% \mathrm{TeO}_{2}-5 \% \mathrm{ZnO}-10 \% \mathrm{Na}_{2} \mathrm{O}-5 \% \mathrm{ZnF}\right.$, at $\left.\%\right)$ for the core and $\mathrm{TNaGZ}$ glass $\left(60 \% \mathrm{TeO}_{2}-20 \% \mathrm{Na}_{2} \mathrm{O}-15 \% \mathrm{GeO}_{2}-5 \% \mathrm{ZnO}\right.$, at $\%$ ) for the clad. A first step-index preform is synthesized by the built-in-casting method [11] and is then drawn to capillaries with an outer diameter $\phi \approx 900 \mu \mathrm{m}$. A second clad rod is casted, mechanically drilled and filled with a previous capillary (rod-in-tube technique), before being drawn as a small-core fiber $\left(\phi_{\text {core }} \approx 3.5 \mu \mathrm{m}\right)$ [11]. The physical properties of the glasses are summarized in table 1 and the fiber attenuation is shown in figure 1(a).
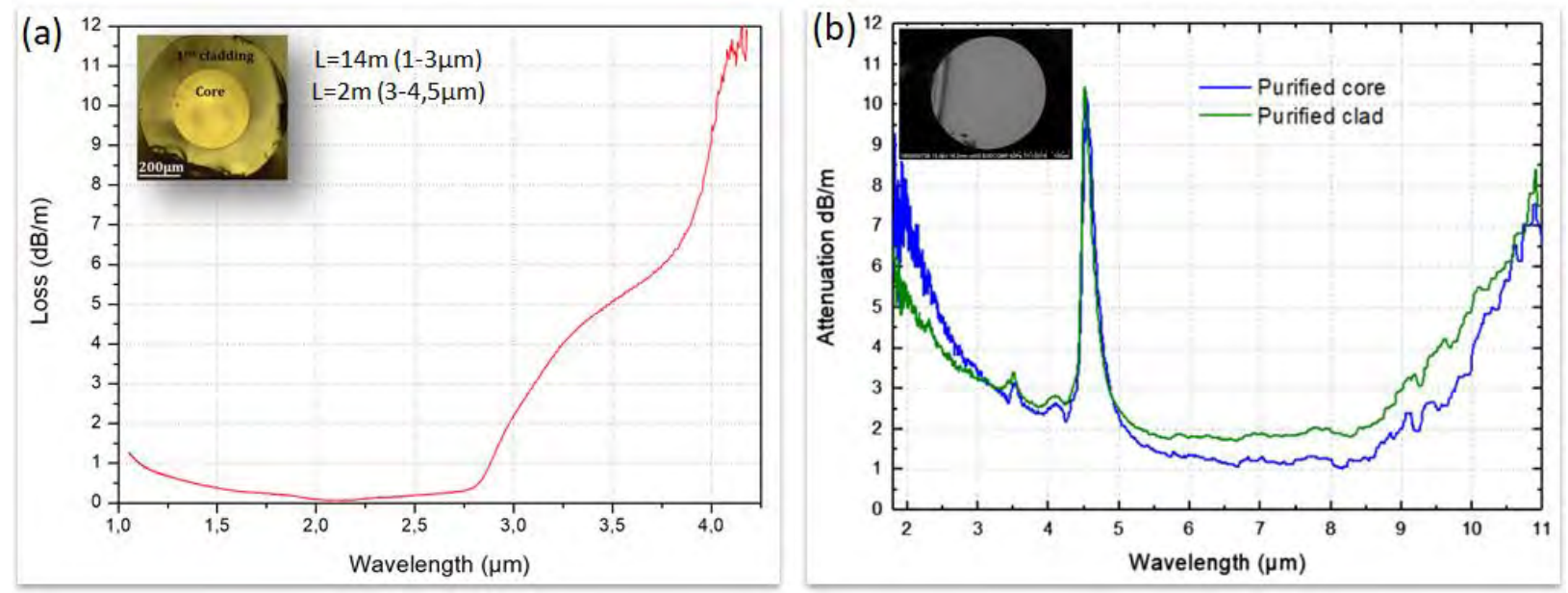

Figure 1. Attenuation curves of: (a) the large core (TZNF) step-index tellurite fiber; (b) both single index chalcogenide fibers from core GST20 and clad GST10 glasses.

Step-index chalcogenide fibers are drawn from a GST20 glass (20\% Ge $-60 \% \mathrm{Se}-20 \% \mathrm{Te}$, at $\%)$ for the core and a GST10 glass $(20 \% \mathrm{Ge}-70 \% \mathrm{Se}-10 \% \mathrm{Te}$, at $\%)$ for the clad. Core and clad glasses are synthesized after different purification processes (HF cleaning of the silica ampoule, dynamic and static distillations under secondary vacuum). The core glass is drawn as capillaries $(\phi=900 \mu \mathrm{m})$ which lead, after insertion in a drilled clad glass rod, to a small core fiber by the rod-in-tube technique $\left(\phi_{\text {core }} \approx 12 \mu \mathrm{m}\right)$. Physical properties of glasses are summarized in the table 1 and the attenuation of the single index chalcogenide fibers both drawn from core and clad glasses are presented in the figure $1(b)$.

For both types of fibers, table 1 shows that the glass transition temperatures $\left(T_{g}\right)$ of the core glasses are close to the ones of the clad glasses. This is necessary to ensure a good drawing process. Refractive indices are sufficiently different to ensure light confinement in the core: $\Delta \mathrm{n}_{\text {tellurite }}=0.128$ at $1.55 \mu \mathrm{m}$ and $\Delta \mathrm{n}_{\text {chalcogenide }}=0.09$ at $3 \mu \mathrm{m}$. Attenuation curve of the large-core step index tellurite fiber exhibits low losses, below $0.1 \mathrm{~dB} / \mathrm{m}$ at $2.1 \mu \mathrm{m}$ and $10 \mathrm{~dB} / \mathrm{m}$ at $4.0 \mu \mathrm{m}$. In the case of the chalcogenide single-index fibers the losses are also low, around $1 \mathrm{~dB} / \mathrm{m}$ at $7 \mu \mathrm{m}$, with a remaining $\mathrm{SeH}$ absorption band around $4.5 \mu \mathrm{m}$ whose amplitude is below $10 \mathrm{~dB} / \mathrm{m}$.

Table 1. Physical properties of tellurite and chalcogenide glasses

\begin{tabular}{|c|c|c|c|}
\hline Glass & $\mathbf{T}_{\mathrm{g}}\left({ }^{\circ} \mathrm{C}\right)$ & $\begin{array}{l}\text { Refractive index } \\
\quad n(\text { at } \lambda \mu \mathrm{m})\end{array}$ & $\left(\mathbf{x 1 0}^{-19} \mathrm{~m}^{2} / \mathrm{W}\right)$ \\
\hline TZNF & 282 & $1.995(1.55 \mu \mathrm{m})$ & \multirow{2}{*}{3.8 [12] } \\
\hline TNaGZ & 272 & $1.867(1.55 \mu \mathrm{m})$ & \\
\hline GST20 & 162 & $2.59(3 \mu \mathrm{m})$ & \multirow{2}{*}{80 [13] } \\
\hline GST10 & 159 & $2.50(3 \mu \mathrm{m})$ & \\
\hline
\end{tabular}




\section{Supercontinuum generation}

\section{Tellurite fiber}

A supercontinuum is generated in a $10 \mathrm{~cm}$-long step-index tellurite fiber by pumping it at $2.3 \mu \mathrm{m}$ by means of an optical parametric oscillator (OPO, $200 \mathrm{fs}, 80 \mathrm{MHz}$, up to $3 \mathrm{~kW}$ peak power) as a pump source, focused in the fiber with a $\mathrm{x} 20$ silica microscope objective. Tellurite fiber chromatic dispersion management will be discussed during the presentation. The output supercontinuum is coupled in an $\mathrm{InF}_{3}$ multimode fiber and analyzed with an Optical Spectrum Analyzer (OSA) and a FTIR (Fourier-Transform Infrared spectrometer). The resulting SC is shown in Figure 2 and spans from 1.2 to $4.2 \mu \mathrm{m}$ with $20 \mathrm{~dB}$ of dynamics by pumping the fiber at $2.35 \mu \mathrm{m}$.

\section{Chalcogenide fiber}

A supercontinuum is generated in a $4 \mathrm{~cm}$-long step-index chalcogenide fiber using non-collinear optical parametric amplifier (NOPA) pumped by a chirped pulse amplified Ti:sapphire system and followed by a difference frequency generation (DFG) module (65 fs, $1 \mathrm{kHz}$, up to $77 \mathrm{MW}$ peak power). The laser emitting at $7.7 \mu \mathrm{m}$ is focused in the fiber with a $6 \mathrm{~mm}$ focal length $\mathrm{ZnSe}$ objective. Chalcogenide fiber chromatic dispersion management will be discussed during the presentation. The output supercontinuum is collected with a $12 \mathrm{~mm}$ focal length ZnSe objective and then focused in a monochromator equipped with two diffraction gratings adapted for the wavelength range of interest Higher orders of diffraction are filtered using long pass filters. SC output light is imaged by means of gold-coated parabolic mirror $(\mathrm{f}=50 \mathrm{~mm})$ on a liquid nitrogen cooled $\mathrm{HgCdTe}$ detector. The resulting $\mathrm{SC}$ is shown in Figure 2 and spans from 2 to $13.5 \mu \mathrm{m}$.

\section{Supercontinuum Absorption Spectroscopy}

From the tellurite output supercontinuum, SAS experiment has been successfully realized by travelling through a $\mathrm{CH}_{4}$-filled multipass gas cell. The gas cell leads to 45 reflections corresponding to a path length of $3.50 \mathrm{~m}$. The output signal is then focused into an $\mathrm{InF}_{3}$ multimode fiber and then analyzed with an OSA and a FTIR. Resulting $\mathrm{CH}_{4}$ absorption is shown in figure 2. Comparing this SAS experiment with the HITRAN database shows a good agreement for $\mathrm{CH}_{4}$ absorption bands.
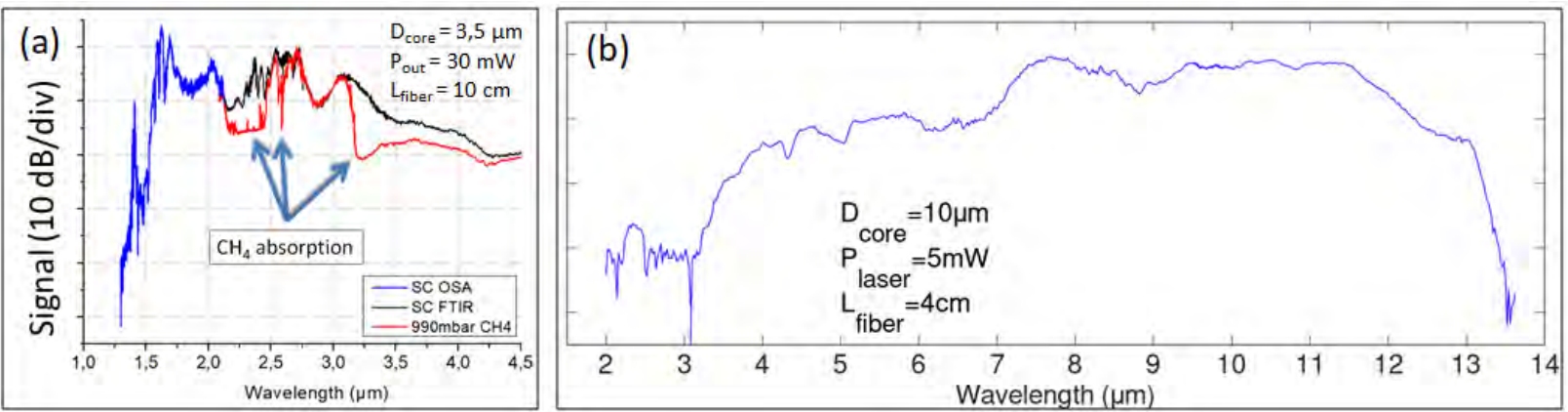

Figure 2. (a) SC generated in the step-index tellurite fiber measured with an OSA (blue line) and a FTIR (dark line). Red line shows the $\mathrm{SC}$ travelling into the $\mathrm{CH}_{4}$-filled gas cell. (b) $\mathrm{SC}$ generated in the chalcogenide step-index fiber.

\section{Conclusions and perspectives}

A first SAS experiment of $\mathrm{CH}_{4}$ above $3 \mu \mathrm{m}$ has been successfully realized with a tellurite fiber. This proof of principle result can be improved, for example, by pumping the tellurite fiber with new femtosecond laser sources commercially available emitting around $2 \mu \mathrm{m}$. Development of As-Sb-free chalcogenide fibers as well as improvements of synthesis and purification methods has been achieved. We succeed to generate efficient SC between 2 
and $13.5 \mu \mathrm{m}$ with a dispersion managed, arsenic- and antimony-free step-index fiber. The next step of this work is to realize SAS experiment with chalcogenide fibers to detect greenhouse pollutants such as $\mathrm{CH}_{4}$ and $\mathrm{CO}_{2}$ between 8 and $14 \mu \mathrm{m}$.

\section{Acknowledgements}

We acknowledge the financial support of the French Investissements d'Avenir program (ISITE-BFC ANR-15IDEX-0003, SCUVIRA project), the Council of the French Région Bourgogne Franche-Comté, and the European program FEDER, through the grant "Jeune Chercheur Entrepreneur" scheme.

\section{References}

[1] Strutynski, C., Picot-Clémente, J., Désévédavy, F., Jules, J.-C., Gadret, G., Kibler, B., Smektala, F. “Compact supercontinuum sources based on tellurite suspended core fibers for absorption spectroscopy beyond $2 \mu \mathrm{m}$," Laser Physics Letters, 13(7), 075402 (2016).

[2] Keirsse, J., Boussard-Plédel, C., Loreal, O., Sire, O., Bureau, B., Turlin, B., Leroyer, P., Lucas, J., "Chalcogenide glass fibers used as biosensors," Journal of non-crystalline solids 326, 430-433 (2003).

[3] Ebrahim-Zadeh, M., and Sorokina, I. T., [Mid-infrared coherent sources and applications], Springer (2007).

[4] Ferreira, M. F., Castro-Camus, E., Ottaway, D. J., López-Higuera, J. M., Feng, X., Jin, W., Jeong, Y., Picqué, N., Tong, L., Reinhard B. M., Pellegrino, P. M., Méndez, A., Diem, M., Vollmer, F., Quan, Q., "Roadmap on optical sensors," Journal of Optics 19( 8), 083001 (2017).

[5] Strutynski, C., "Fibres tellurites pour sources supercontinuum infrarouges : gestion des profils opto-géométriques et des absorptions extrinsèques," Ph.D. dissertation, Université de Bourgogne Franche-Comté (2015).

[6] Delmonte, T., Watson, M. A., O’Driscoll, E. J., Feng, X., Monro, T. M., Finazzi, V., Petropoulos, P., Price, J. H., Baggett, J. C., Loh W., Richardson, D. J., Hand, D. P., "Generation of mid-IR continuum using tellurite microstructured fiber," Conference on Lasers and Electro-Optics and 2006 Quantum Electronics and Laser Science Conference (pp. 1-2) IEEE (2006).

[7] Thapa, R., Rhonehouse, D., Nguyen, D., Wiersma, K., Smith, C., Zong, J., Chavez-Pirson, A., "Mid-IR supercontinuum generation in ultra-low loss, dispersion-zero shifted tellurite glass fiber with extended coverage beyond $4.5 \mu \mathrm{m}$," in Technologies for Optical Countermeasures X; and High-Power Lasers 2013: Technology and Systems, International Society for Optics and Photonics, 8898, 889808 (2013).

[8] Kedenburg, S., Strutynski, C., Kibler, B., Froidevaux, P., Désévédavy, F., Gadret, G., Jules, J.-C., Steinle, T., Mörz, F., Steinmann, A., Giessen, H., Smektala, F., "High repetition rate mid-infrared supercontinuum generation from 1.3 to $5.3 \mu \mathrm{m}$ in robust step-index tellurite fibers," Journal of Optical Society of America B, 34(3), 601-607 (2017).

[9] Petersen, C. R., Møller, U., Kubat, I., Zhou, B., Dupont, S., Ramsay, J., Benson, T., Sujecki, S., Abdel-Moneim, N., Tang, Z., Furniss, D., Seddon, A., Bang, O., "Mid-infrared supercontinuum covering the 1.4-13.3 $\mu$ m molecular fingerprint region using ultrahigh NA chalcogenide step-index fibre," Nature Photonics, 8(11), 830 (2014).

[10] Zhao, Z., Wu, B., Wang, X., Pan, Z., Liu, Z., Zhang, P., Shen, X., Nie, Q., Dai, S., Wang, R., "Mid-infrared supercontinuum covering 2.0-16 $\mu \mathrm{m}$ in a low-loss telluride single-mode fiber," Laser \& Photonics Reviews, 11(2), 1700005 (2017).

[11] Strutynski, C., Picot-Clémente, J., Lemière, A., Froidevaux, P., Désévédavy, F., Gadret, G., Jules, J. C., Kibler, B., Smektala, F., "Fabrication and characterization of step-index tellurite fibers with varying numerical aperture for near- and mid-infrared nonlinear optics," Journal of Optical Society of America B, 33(11), D12-D18 (2016).

[12] Froidevaux, P., Lemière, A., Kibler, B., Désévédavy, F., Mathey, P., Gadret, G., Jules, J.-C., Nagasaka, K., Suzuki, T., Ohishi, Y., Smektala, F., "Dispersion-engineered step-index tellurite fibers for mid-infrared coherent supercontinuum generation from 1.5 to $4.5 \mu \mathrm{m}$ with sub-nanojoule femtosecond pump pulses," Applied Sciences, 8(10), 1875 (2018).

[13] Lemière, A., Désévédavy, F., Kibler, B., Mathey, P., Gadret, G., Jules, J. C., Aquilina, C., Béjot, P., Billard, F., Faucher O., Smektala, F., "Mid-infrared two-octave spanning supercontinuum generation in a Ge-Se-Te glass suspended core fiber," Laser Physics Letters, 16(7), 075402 (2019). 


\title{
Specialty optical fibers for generation of light with extreme properties
}

\author{
Alexander M. Heidt*a , Dirk-Mathys Spangenberga ${ }^{\mathrm{a}}$, Sönke Pilz ${ }^{\mathrm{b}}$, Martin Hochstrasser ${ }^{\mathrm{b}}$, \\ Manuel Ryser ${ }^{\mathrm{a}}$, Valerio Romano ${ }^{\mathrm{a}, \mathrm{b}}$, and Thomas Feurer ${ }^{\mathrm{a}}$ \\ ${ }^{a}$ Institute of Applied Physics, University of Bern, 3012 Bern, Switzerland \\ ${ }^{b}$ Bern University of Applied Sciences, ALPS, Pestalozzistrasse 20, CH-3400 Burgdorf, Switzerland
}

\begin{abstract}
We review how specialty optical fiber technology can support the application-driven demand for light sources with increasingly extreme properties, such as extremely short pulse durations, exotic wavelength ranges, or ultra-low noise broadband coherent sources.
\end{abstract}

Keywords: ultrafast optics, photonic crystal fibers, hollow-core fibers, light-plasma interaction * alexander.heidt@iap.unibe.ch;

\section{Introduction}

The application of light with special properties is one of the most important cornerstones of today's scientific research and industrial development and has resulted in countless breakthroughs, e.g. in precision measurements, chemical analysis, microscopy, telecommunications, industrial manufacturing, or medical diagnostics and therapy. The rapid technological progress in these and other areas drives a demand for light sources with increasingly extreme properties. Attoscience and strong-field physics applications require the generation and control of extremely short and intense laser pulses on time scales of a single optical cycle and below [1]. Novel spectroscopy and imaging applications demand coherent light sources in exotic wavelength ranges from the soft x-ray to mid-IR spectral regions [2, 3]. Precision optical frequency measurements, biophotonic imaging such as optical coherence tomography and nonlinear micro-spectroscopy methods call for ultra-lownoise broad-bandwidth laser sources that will further push the sensitivity and precision of these techniques $[4,5]$.

In this contribution we explore some of the current developments and future perspectives of specialty optical fiber development and applications that can support the generation and control of light with such extreme properties.

\section{Results}

\subsection{Generation and amplification of ultra-low noise supercontinuum}

Driven by the invention of the photonic crystal fiber (PCF), optical fiber based supercontinuum (SC) generation has become a scientific and commercial success story in the past decades. From optical frequency metrology to bio-photonic imaging its unique spectral properties have revolutionized dozens of applications [6]. The more than octave-spanning spectral bandwidths of modern PCF-based SC are exploited, for example, for carrier-envelope phase measurement and stabilization of frequency combs (FC), the generation of ultrashort pulses in the single or sub-cycle regime, or for precision optical imaging in OCT. After the initial race to maximize spectral bandwidths, the noise properties of SC sources have now shifted into the center of attention as amplitude or phase noise has become the main performance-limiting factor in these applications [7]. Minimizing the SC noise becomes even more important when the SC is used as seed in broadband amplifiers in order 
to satisfy the increasing demand for high average power FC sources and few-cycle pulses with high pulse energy and peak power, as any noise generated in the $\mathrm{SC}$ generation process will be amplified in the subsequent amplifier chain [7].

Here we discuss experiments on ultra-low noise SC generation in an all-solid, all-normal dispersion soft glass PCF and subsequent broadband amplification in a Thulium-Holmium fiber amplifier. The fiber design is based on a hexagonal arrangement of rods made from two thermally compatible silicate glasses (Schott SF6 / F2), yielding an all-normal dispersion design that suppresses noise-amplifying nonlinear effects associated with the usual approach of pumping in the anomalous dispersion region $[8,9]$. The smooth and flat SC covers the range $1150-2150 \mathrm{~nm}(-20 \mathrm{~dB})$ and exhibits relative intensity noise (RIN) of only $0.05 \% \mathrm{rms}$ in the frequency range $1 \mathrm{~Hz}$ to $20 \mathrm{MHz}$, limited by the RIN of the pump laser. Due to the all-normal dispersion fiber design, a single ultrashort pulse is maintained in the time domain. The spectral bandwidth supports a high-quality single-cycle pulse (5 fs).

For testing the performance of this SC source as seed in an amplifier configuration, an all-fiber chirped pulse amplification system for the long-wavelength part of this SC is constructed based on a Thulium/Holmium doped fiber. This yields a $20 \mathrm{~dB}$ bandwidth of over $300 \mathrm{~nm}(1800-2110 \mathrm{~nm})$, a pulse duration of $66 \mathrm{fs}$ directly at the single-mode exit fiber at an average power of $500 \mathrm{~mW}$ ( $70 \mathrm{~kW}$ peak power). The measured RIN after the amplifier is as low as $0.15 \% \mathrm{rms}$. The remaining excess RIN was traced to noise-amplifying polarization modulation instability (PMI) [10], which will be suppressed in future implementations by realizing an all-PM system. The excellent noise properties and the overlap of the SC seed source with all major fiber-optic gain media paves the way for a new generation of low-noise, high power FC and single-cycle pulse sources based on (specialty) optical fiber technology.
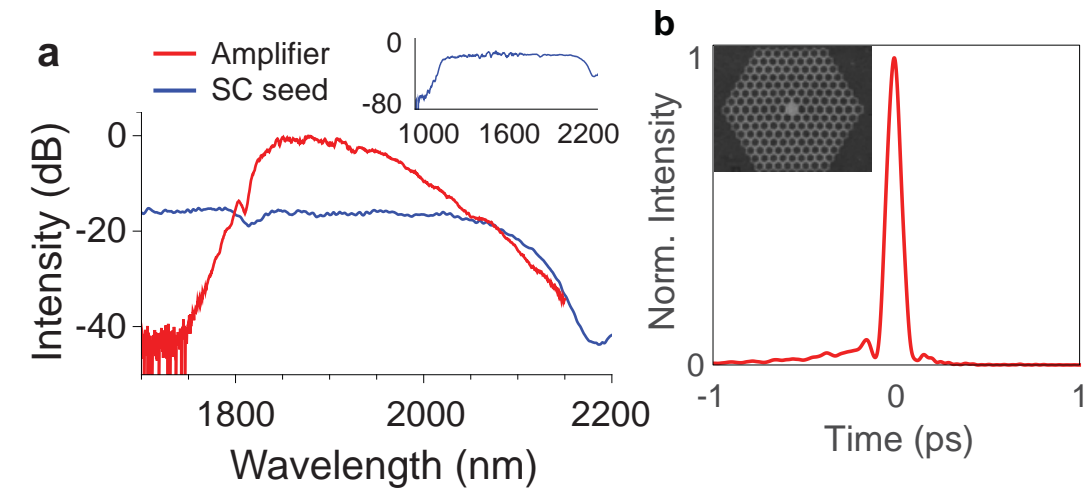

Figure 1. (a) Logarithmic spectrum of the long-wavelength part of the low noise SC seed pulse generated in all-solid, allnormal dispersion photonic crystal fiber and spectrum after subsequent amplification in an all-fiber chirped pulse amplifier. The complete SC spectrum is shown in the inset, supporting a single-cycle (5 fs) pulse. (b). Reconstructed pulse with $66 \mathrm{fs}$ duration at the exit of the amplifier. The inset shows a microscope image of the PCF used for SC generation.

\subsection{Sol-gel granulated silica fabrication technology for multi-dopant optical fibers}

With our in-house developed specialty optical fiber production technology based on granulated silica we merge the benefits of the granulate-based sol-gel method with the advantages of the powder-in-tube technique [11]. As a result, our method overcomes the fabrication constraints of conventional fiber fabrication techniques and offers complete freedom in the production of fibers with any geometry, a high degree of flexibility in dopant composition and concentrations, while it does not pose any inhomogeneity limits to the core diameter. Hence, it is ideally suited to complement the more 
conventional techniques like MCVD for the versatile and cost-effective "rapid prototyping" of specialty optical fiber preforms from in-stock sol-gel-based granulated silica.

Figure 3 shows an experimental multi-dopant fiber design highlighting the potential benefits that the freedom of dopant choice and composition may offer for the fabrication of fibers with unusual properties. Five different rare-earth ions have been chosen and combined into a single fiber core as dopants, i.e. $\mathrm{Nd}^{3+}, \mathrm{Ho}^{3+}, \mathrm{Tm}^{3+}, \mathrm{Er}^{3+}$, and $\mathrm{Yb}^{3+}$. When pumped around $800 \mathrm{~nm}$ wavelength, cross-excitation between the dopants leads to an ultra-broadband emission spectrum in the range $365-2300 \mathrm{~nm}$. The spectrum consists of narrow lines in the visible spectral region, and a continuous band spanning over more than one octave from $925 \mathrm{~nm}$ to about $2300 \mathrm{~nm}$, perfectly matched to the SC seed source described in Section 2.1. The displayed results were obtained with fibers fabricated directly from dry granulated oxides, and consequently the recorded background losses were in the order of several $\mathrm{dB} / \mathrm{m}$ [12]. However, using the recent progress in our sol-gel based granulated silica approach, where we achieved more than $130 \mathrm{~W}$ continuous-wave output power in a purely $\mathrm{Yb}$-doped fiber, we are currently working towards significantly improved broadband emitting fibers, which in combination with the ultra-low noise SC source discussed in Section 2.1 could be promising candidates for generation and subsequent direct amplification of single-cycle pulses in optical fibers.

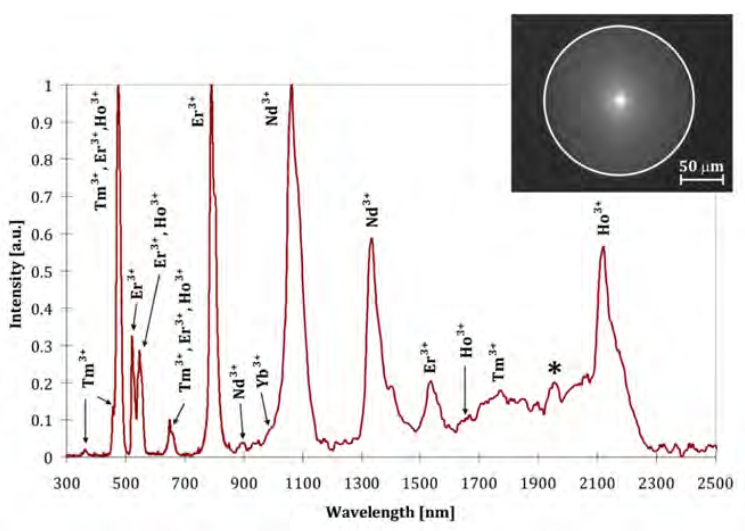

Figure 1. Typical fluorescence spectrum of a single-core, multi-dopant fiber containing $\mathrm{Nd}^{3+}, \mathrm{Ho}^{3+}, \mathrm{Tm}^{3+}, \mathrm{Er}^{3+}$, and $\mathrm{Yb}^{3+}$ pumped at $800 \mathrm{~nm}$.

\subsection{Plasma generation in hollow-core optical fibers}

Gas-filled hollow-core photonic crystal fibers (HC-PCF) have emerged as an ideal platform to investigate light-gas and light-plasma interactions in a well-controlled environment [13]. The tight confinement of both matter and light in the hollow core allows for much longer interaction lengths than would be possible in free space, and the minimal overlap of the guided mode with the surrounding cladding region enables low-loss guiding even in spectral regions where silica as the host material is highly absorptive, i.e. in the UV and mid-IR spectral regions [14].

Here we discuss experiments on the non-invasive generation of electric gas discharges in HC-PCF using capacitive coupled discharges driven by an external high voltage radio frequency (RF) source and the observation of stable gas discharges forming meter-scale plasma columns in the fiber core. These plasmas are an attractive active media for the realization of novel fiber-integrated gas lasers and broadband emission sources operating directly at (deep) UV, visible, or mid-IR wavelengths, which are otherwise difficult to directly access with fiber optic means. In future, the external excitation and control of such plasmas in HC-PCF and capillaries might also enable exciting new possibilities in nonlinear optics, 
where the interaction of plasmas with intense ultrashort light pulses facilitate efficient frequency conversion to the UV and mid-IR spectral range as well as the generation of ultra-broadband optical pulses with single-cycle durations [13].
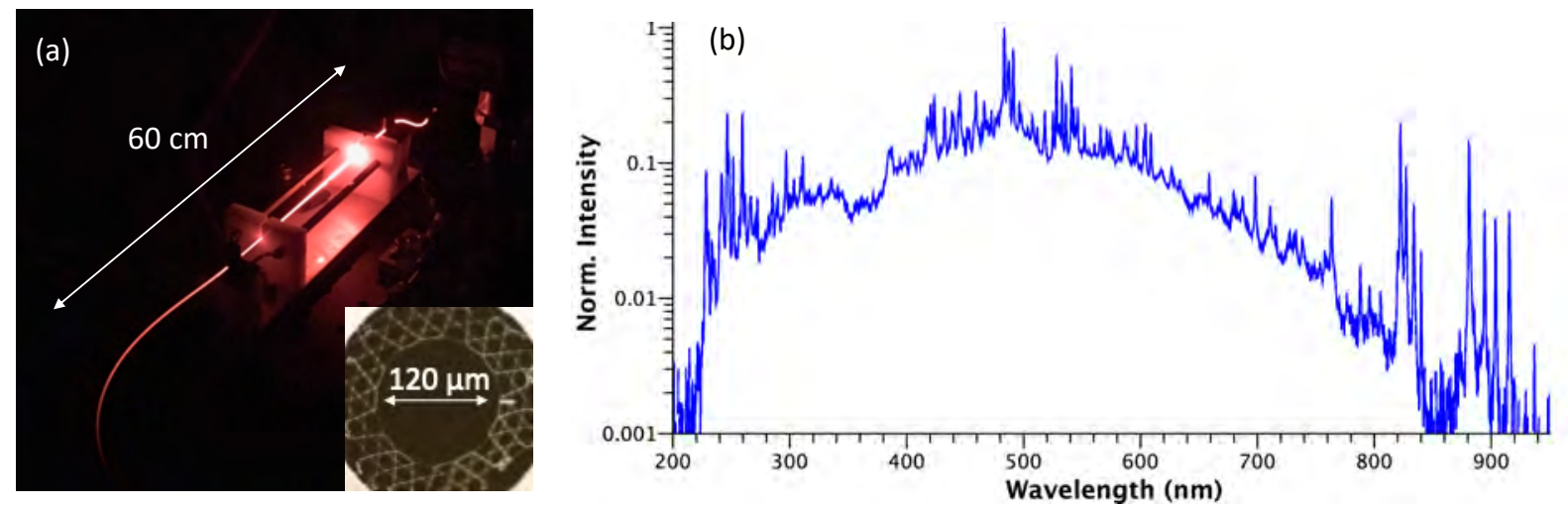

Figure 3: Excitation of a $60 \mathrm{~cm}$ long discharge in a helium/neon mixture at low pressure (10 mbar). The inset shows a microscope image of the HC-PCF cross section. (b) Spectrum of an atmospheric pressure discharge of a helium / xenon mixture, recorded at the fiber exit open to the atmosphere.

\section{Conclusion}

We have demonstrated how specialty optical fiber technology can support the application-driven demand for light sources with increasingly extreme properties, focusing on 3 different examples: generation of ultra-low noise broadband coherent ultrashort pulses; novel multi-dopant fibers for straightforward amplification of such broad bandwidths in a single fiber; and the non-invasive generation of meter-scale plasma columns in HC-PCF, which forms a novel platform for the exploration of emission sources in exotic wavelength ranges and might in future enable the manipulation of single-cycle pulses by controlled light-plasma interaction.

\section{References}

[1] Chang, Z. and Corkum, P., "Attosecond photon sources: the first decade and beyond,” J. Opt. Soc. Am. B 27, 9 (2010). [2] Muraviev, A. V., Smolski, V. O., Loparo, Z. E., and Vodopyanov, K. L. "Massively parallel sensing of trace molecules and their isotopologues with broadband subharmonic mid-infrared frequency combs," Nat. Photonics 12, 209 (2018).

[3] Gardner, D., Tanksalvala, M., Shanblatt, E., Zhang, X., Galloway, B., Porter, C., Karl, R., Bevis, C., Adams, D., Kapteyn, H., Murnane, M., and Mancini, G., "Sub-wavelength coherent imaging of periodic samples using a 13.5nm tabletop high harmonic light source," Nat. Photonics 11, 259 (2017).

[4] Jensen, M., Bravo Gonzalo, I., Engelsholm, R. D., Maria, M., Israelsen, M. N., Podoleanu, A. and Bang, O. "Noise of supercontinuum sources in spectral domain optical coherence tomography," J. Opt. Soc. Am. B 36, 154-160 (2019).

[5] Hänsch, T. W. "Nobel lecture: passion for precision," Rev. Mod. Phys. 78, 1297 (2006).

[6] Alfano, R., [The supercontinuum laser source], Springer, New York (2016). 
[7] Gaida, C., Heuermann, T., Gebhardt, M., Shestaev, E., Butler, T. P., Gerz, D., Lilienfein, N., Sulzer, P., Fischer, M., Holzwarth, R., Leitenstorfer, A., Pupeza, I., Limpert, J. "High-power frequency comb at $2 \mu \mathrm{m}$ wavelength emitted by a Tmdoped fiber laser system,” Opt. Lett. 43, 5178-5181 (2018).

[8] Klimczak, M., Siwicki, B., Skibiński, P., Pysz, D., Stępień, R., Heidt, A. M., Radzewicz, C., and Buczyński, R., "Coherent supercontinuum generation up to $2.3 \mu \mathrm{m}$ in all-solid soft-glass photonic crystal fibers with flat all-normal dispersion," Opt. Express 22, 18824-18832 (2014).

[9] Heidt, A. M., Feehan, J. S., Price, J. H. V., and Feurer, T., "Limits of coherent supercontinuum generation in normal dispersion fibers," J. Opt. Soc. Am. B 34, 764-775 (2017).

[10] Gonzalo, I. B., Engelsholm, R. D., Sørensen, M. P. and Bang, O. "Polarization noise places severe constraints on coherence of all-normal dispersion femtosecond supercontinuum generation," Sci. Rep.8, 6579(2018).

[11] Pilz, S., Najafi, H., El Sayed, A., Boas, J., Kummer, D., Scheuner, J., Etissa, D., Ryser, M., Raisin, J., Berger, S., and Romano, V. "Progress in the fabrication of optical fibers by the sol-gel-based granulated silica method," Proc. SPIE 9886, 988614 (2016).

[12] Di Labio, L., Lüthy, W., Romano, V., Sandoz, F., and Feurer, T. "Superbroadband fluorescence fiber fabricated with granulated oxides," Opt. Lett 33, 1050-1052 (2008).

[13] Markos, C., Travers, J. C., Abdolvand, A., Eggleton, B., and Bang, O. “Hybrid photonic-crystal fiber,” Rev. Mod. Phys. 89, 045003 (2017).

[14] Wheeler, N. V., Heidt, A. M., Baddela, N. K., Numkam Fokoua, E., Hayes, J. R., Sandoghchi, S. R., Poletti, F., Petrovich, M., and Richardson, D. J. "Low loss and low bend sensitivity mid-IR guidance in a hollow core photonic bandgap fiber," Opt. Lett. 39, 297 - 298 (2014). 


\title{
Random Lasing from Optical Fibers with Phase-Separated Glass Cores
}

\author{
Srinath Jagannathan ${ }^{\mathrm{a}}$, Liam Ackerman ${ }^{\mathrm{a}}$, Wynter Chen ${ }^{\mathrm{a}}$, Matthew Tuggle ${ }^{\mathrm{b}}$, Maxime Cavillon ${ }^{\mathrm{b}}$, \\ Thomas Hawkins $^{\mathrm{b}}$, John Ballato ${ }^{\mathrm{b}}$, Peter Dragic ${ }^{\mathrm{a}}$ \\ ${ }^{a}$ Department of Electrical and Computer Engineering, University of Illinois at Urbana- \\ Champaign, 306 N. Wright St., Urbana, Illinois 61801, USA \\ ${ }^{b}$ Center for Optical Materials Science and Engineering Technologies (COMSET) and the \\ Department of Materials Science and Engineering, Clemson University, 91 Technology Drive, \\ Anderson, South Carolina 29625, USA
}

\begin{abstract}
Random fiber lasers incorporating optical fiber with heterogeneous, phase-separated glass cores possessing high scattering coefficients are presented. In addition to having features characteristic of random lasers, these architectures offer the possibility of azimuthally uniform (sideways) illumination.

Keywords: random lasers, specialty optical fiber, optical fiber design, optical fiber fabrication, fiber lasers *p-dragic@illinois.edu; phone 1217 333-8399

\section{Introduction}

Random lasers are those that use a random scattering medium rather than a conventional 'mirror' for providing optical feedback [1]. Random fiber lasers are one form of such lasers, with some making use of intrinsic distributed Rayleigh scattering as the feedback mechanism [2-4]. Others utilize extrinsic scattering such as by filling a hollow core with a scattering medium [5], or modifying the glass to enhance scattering by adding, for example, random Bragg gratings [6] or random scattering or reflecting centers [7], or the use of specialty polymer fibers [8]. Those systems that make use of intrinsic Rayleigh scattering typically require long fiber lengths, often hundreds of meters to kilometers, to realize sufficient feedback for lasing $[9,10]$. Given the wide range of applications for random fiber lasers [11-14], there is merit in the development of optical fiber with large-scale scattering in order to significantly reduce the requisite length of scattering fiber. This may offer a path towards making Rayleigh-based random lasers somewhat more practical. Here, such fibers have been fabricated and their viability for use in random lasing is investigated. Enhancement to scattering is accomplished through the fabrication of fibers with heterogeneous cores resulting from phase separation. Lasing experiments are conducted with a commercial Yb-doped fiber as the gain medium.
\end{abstract}

\section{Optical Fiber}

Two phase-separated optical fibers (P-SFs) were fabricated using the molten core method (MCM). A powder $\left(100 \% \mathrm{Al}_{2} \mathrm{O}_{3}\right.$ for fiber 1 and $100 \% \mathrm{CaO}$ for 2$)$ was first inserted into a telecommunications-grade silica capillary preform ( $3 \mathrm{~mm}$ inner / $30 \mathrm{~mm}$ outer diameters) that will serve as the cladding material after drawing. The preform is thus placed inside a draw tower furnace at temperatures of $2000{ }^{\circ} \mathrm{C}$ (fiber 1) and $2100^{\circ} \mathrm{C}$ (fiber 2). At these temperatures, the core melts, and the silica cladding softens, enabling fiber drawing. Owing to the high cooling rates, the molten core is quenched upon drawing to a glassy state as the fiber cools. Fibers were drawn to a cladding diameter of $125 \mu \mathrm{m}$, and coated with a UV-curable convential acrylate, yielding a total fiber diameter of approximately $250 \mu \mathrm{m}$. By taking advantage of the draw conditions during fiber fabrication (essentially draw temperature here), phase separation in the fiber cores could be promoted [15]. In this case, spinodal phase separation dominates in the aluminosilicate fiber, while it is binodal phase separation for the calcium silicate one. Phase separation in the fiber cores are shown in Figure 1 (aluminosilicate) and Figure 2 (calcium silicate). 


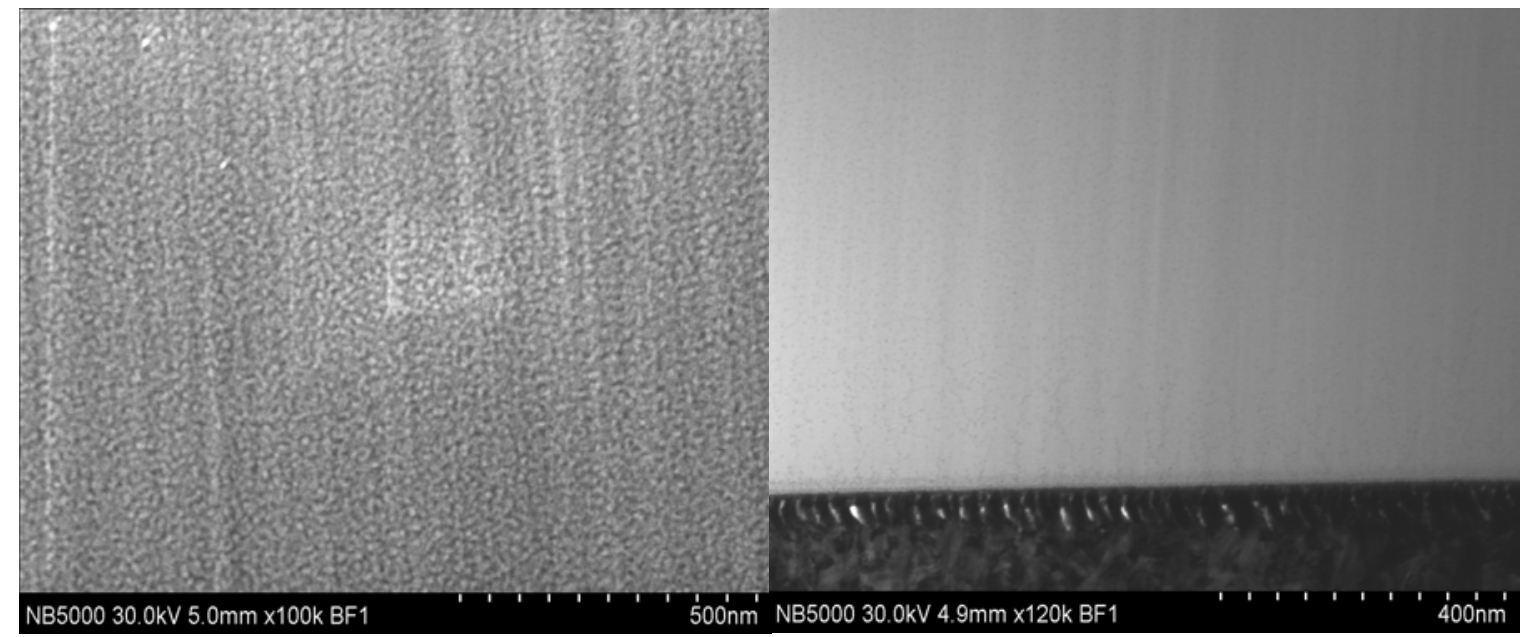

Figure 1. Tunneling electron microscope image of a typical spinodally phase-separated aluminosilicate fiber core (left) and pure silica cladding (right). The nano-scale phase separation in the core is evident from the image.

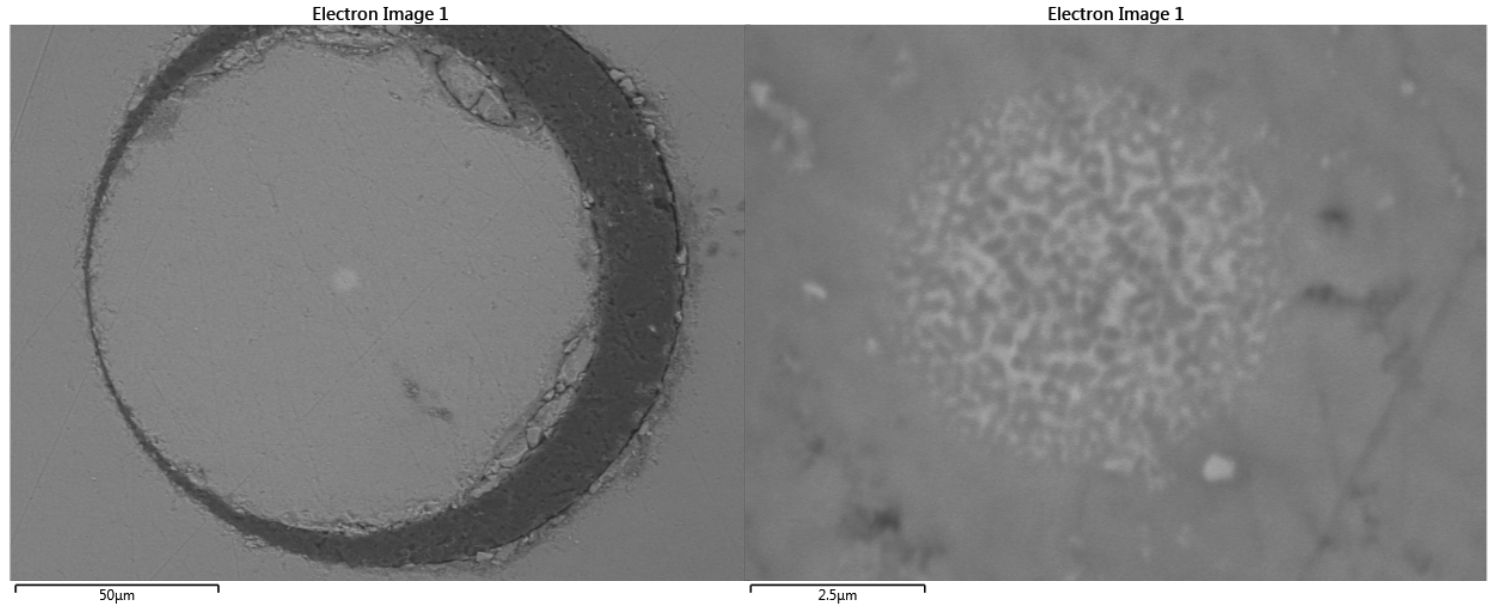

Figure 2. Scanning electron microscope image of the calcium silicate fiber core (roughly $5 \mu \mathrm{m}$ diameter). Note the larger scale relative to the aluminosilicate fiber. The sizes of the particles were on the order of $250 \mathrm{~nm}$. 


\section{Results and Discussion}

For brevity, the results presented in the abstract will be limited to the aluminosilicate fiber. More detailed results with the calcium silicate fiber will be presented at the conference.

\section{Scattering Characteristics}

The attenuation was determined to be $18 \mathrm{~dB} / \mathrm{m}$ at $1050 \mathrm{~nm}$ (lasing wavelength as described in the next section). In order to confirm that this loss is dominated by Rayleigh scattering (although absorptive impurity loss likely also is present), a scattering profile was measured for this fiber. To do this, a segment of fiber was passed axially perpendicular across a slit (with $1 \mathrm{~mm}$ width) and green light from a $532 \mathrm{~nm}$ doubled Nd:YAG laser was launched into one end of this fiber. The spatial intensity pattern emerging from the slit then was characterized by using a detector that was attached to a rotation stage. This secured equidistant rotation of the sensor about the slit. The result of this measurement (normalized) is shown in Figure 3. Also shown in the figure is the normalized Rayleigh

scattering pattern $\left(I \propto \frac{1}{2}\left(1+\operatorname{Cos}^{2} \theta\right)\right.$ ) for comparison. At small angles (less than $30^{\circ}$ or greater than $\left.150^{\circ}\right)$, the fiber, owing to total internal reflection at its surface with air, begins to capture scattered light, and so the scattering signal appears to approach zero. With that in mind, the region between $30^{\circ}$ and $150^{\circ}$ is strongly suggestive of a Rayleigh-dominated source of light scattering.

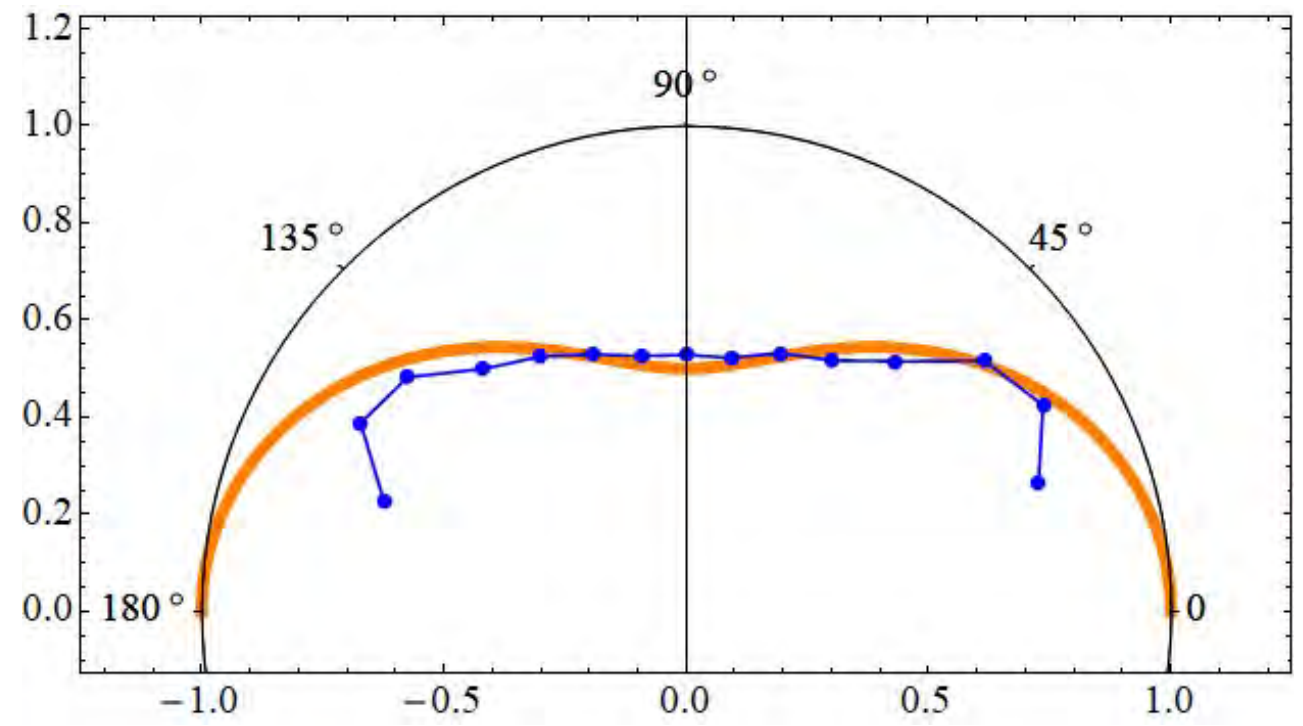

Figure 3. Normalized scattering pattern measured at $532 \mathrm{~nm}$ for the aluminosilicate optical fiber (blue) shown with the normalized Rayleigh pattern from theory. 


\section{Lasing Characteristics}

The laser configuration used in this analysis is shown in Figure 4. A $0.75 \mathrm{~m}$ length of commercial single mode $\mathrm{Yb}$-doped fiber served as the gain medium. This fiber was pumped through a wavelength division multiplexer (WDM), with feedback at the output end provided by a fiber Bragg grating (FBG, 50.68\% reflectivity, $1049.78 \mathrm{~nm}$ center wavelength, and $1.199 \mathrm{~nm}$ spectral width). The feedback from the other end came from either 1) flat-cleaving the WDM fiber or 2) the P-SF. The former was used to establish a benchmark non-random laser for comparison purposes. In the case of the latter, the fiber length was arbitrarily chosen such that no light emerged from the back fiber facet. Splicing was performed with a standard telecom splicer for all fibers comprising the system, including that between the WDM and scattering fiber. Output power versus pump current (LI) curves for the two cases are shown in Figure 5. Figure 6 gives the RF spectrum of the laser output when collected with a Si APD and analyzed with an electrical spectrum analyzer. The suppression of the cavity modes with the scattering fiber in place supports that this laser is operating in a random state. It should be pointed out that at the highest output powers, randomly timed and distributed peaks slowly appear/disappear in RF spectra, usually no more than two or three at a time, suggesting a significant level of dynamic activity within the laser. Work is underway to try to understand this phenomenon.

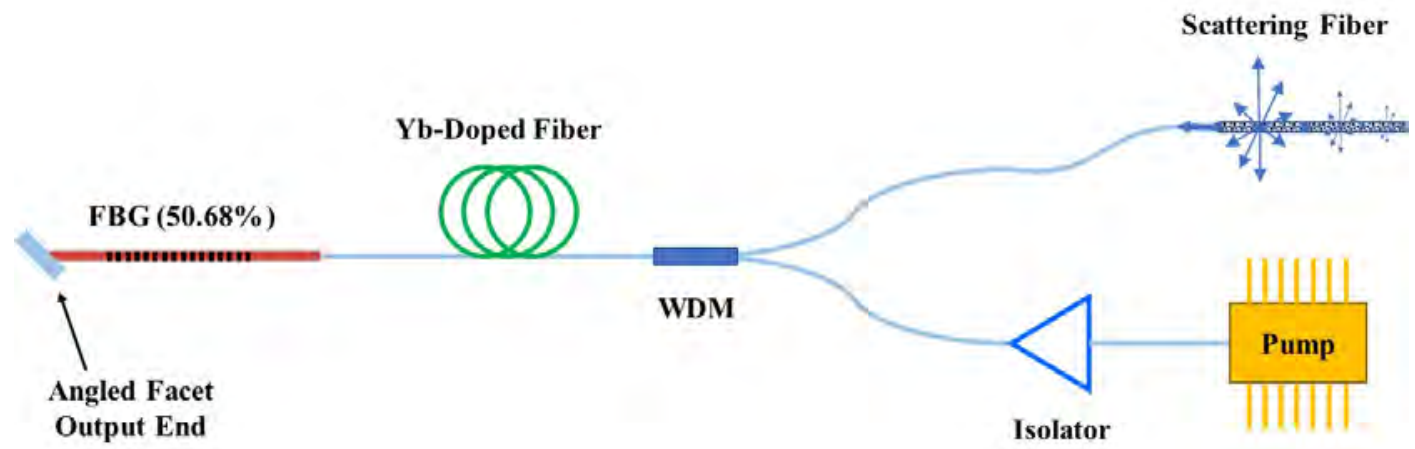

Figure 4. Random laser configuration. The commercial Yb-doped fiber is end pumped by a laser diode at $976 \mathrm{~nm}$. The broadband isolator was used to prevent lasing from the pump diode facet. The output was taken at the FBG end.

As is to be expected, the added cavity loss introduced by the highly scattering fiber leads to a higher lasing threshold and reduced lasing efficiency. The relatively low output power, however, potentially can be amplified, or the use of a double-clad active gain fiber can enable more pump power to be launched. Work is also underway to characterize the effect of length of P-SF on the lasing characteristics. As an interesting side note, Rayleigh scattering leads to significant laser power being scattered from the cavity. Given the short length of P-SF that is required for this application, the scattering fiber itself may be an interesting light delivery vehicle, in that it can offer an azimuthally-isotropic power distribution. Such an emission pattern can be useful for illumination in $360^{\circ}$ vehicular lidar system, or the broad transmission of optical signals. Measurements of the power distribution radiated from the side of the fiber will be shown at the conference. 


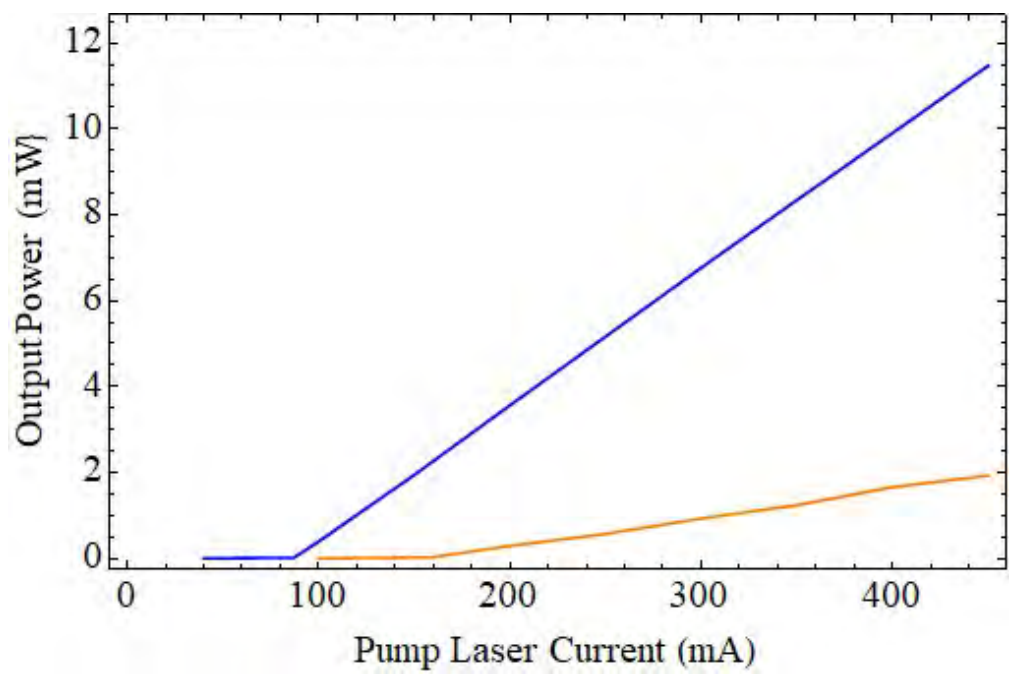

Figure 5. LI curves (current into the pump laser versus output laser power) for the laser without the P-SF (blue curve) and with the P-SF (orange curve). The output was taken to be the FBG end only. The random laser was found to have significant ASE and this power was integrated and subtracted from the measurement. Pump losses through the system have not yet been characterized, but will be done for the conference in order to convert the abscissa to launched pump power $(\mathrm{mW})$. The pump laser had a threshold of $28 \mathrm{~mA}$. From loss in the components, the maximum pump power launched into the active fiber is estimated to be roughly $200 \mathrm{~mW}$.

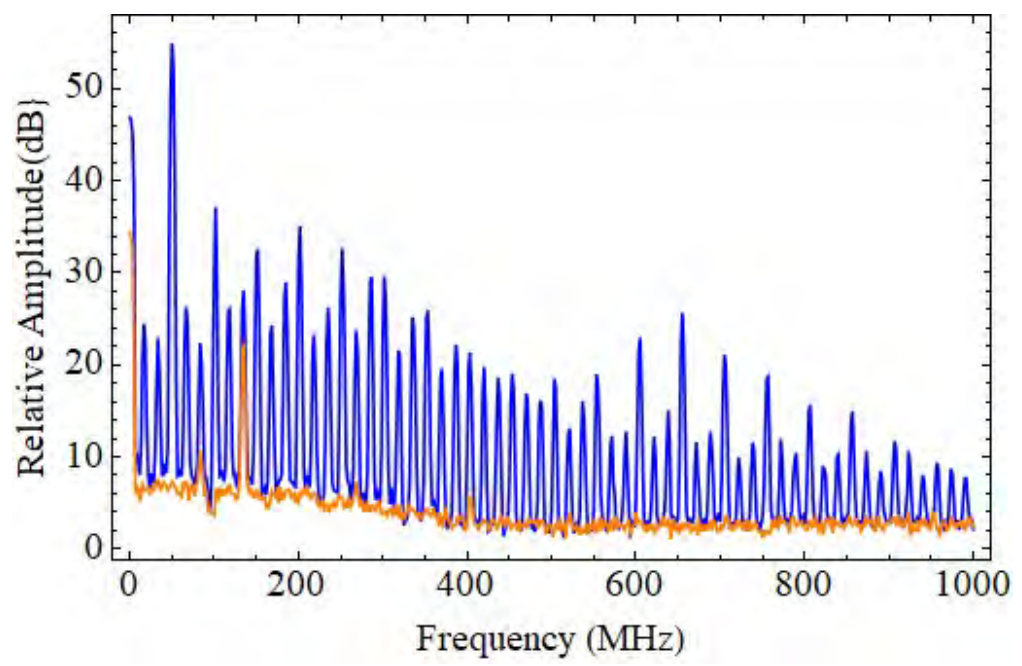

Figure 6. RF beat spectra taken at a laser output power of $2 \mathrm{~mW}$ for the laser without (blue) and with (orange) the P-SF. The beat signal between the large number of cavity modes, spaced at the laser free spectral range $(\sim 16 \mathrm{MHz})$ are clearly visible. These modes are suppressed in the case of the random laser. At the highest power the spectrum exhibits a random presence of one or two peaks, at random frequency. Such is an example that is shown here. 


\section{References}

[1] Ambartsumyan, R. V., Basov, N. G., Kryukov, P. G. \& Letokhov, V. S., "Non-resonant feedback in lasers, [Progress in Quantum Electronics], Pergamon, Vol. 1, Part 3, 107-185 (1970).

[2] Fotiadi, A. A., “An incoherent fibre laser,” Nat. Photon. 4, 204-205 (2010).

[3] Turitsyn, S. K., Babin, S. A., El-Taher, A. E., Harper, P., Churkin, D. V., Kablukov, S. I., Ania-Castañón J. D., Karalekas, V., and Podivilov, E. V., "Random distributed feedback fibre laser," Nat. Photon. 4, 231-235 (2010).

[4] Turitsyn, S. K., Babin, S. A., Churkin, D. V., Vatnik, I. D., Nikulin, M. Podivilov, E. V., "Random distributed feedback fibre lasers," Phys. Rep. 542(2), 133-193 (2014).

[5] de Matos, C. J. S., Menezes, L. d. S., Brito-Silva, A. M., Gámez, M. A. M., Gomes, A. S. L., and de Araújo, C. B., "Random Fiber Laser," Phys. Rev. Lett. 99, 153903 (2007).

[6] Gagné, J. and Kashyap, R., "Demonstration of a $3 \mathrm{~mW}$ threshold Er-doped random fiber laser based on a unique fiber Bragg grating," Opt. Express 17(21), 19067-19074 (2009).

[7] Wang, X., Chen, D., Li, H., She, L., and Wu, Q., "Random fiber laser based on artificially controlled backscattering fibers,” Appl. Opt. 57(2), 258-262 (2018).

[8] Sebastian, S., Linslal, C. L., Vallabhan, C. P. G., Nampoori, V. P. N., Radhakrishnan, P., and Kailasnath, M., "Random lasing with enhanced photostability of silver nanoparticle doped polymer optical fiber laser," Laser Phys. Lett. 11(5), 055108 (2014).

[9] Meng, Q., Wu, H. Han, B., Li, J., Wang, Z., "LD-Pumped Random Fiber Laser Based on Erbium-Ytterbium CoDoped Fiber," Photonic Sens. 1-5 (2019).

[10] Babin, S. A., "Random fiber laser based on Rayleigh scattering: Basic principles and experimental results," Proc. Photonics Global Conference, (2010), DOI/10.1109/PGC.2010.5706005.

[11] Churkin, D., Sugavanam, S., Vatnik, I. D., Wang, Z., Podivilov, E. V., Babin, S. A., Rao, Y., Turitsyn, S. K., "Recent advances in fundamentals and applications of random fiber lasers," Adv. Opt. Photon. 7(3), 516-569 (2015).

[12] López-Amo, M., Leandro, D., de Miguel, V., Bravo, M., Fernández-Vallejo, M., and Perez-Herrera, R. A., "Random Fiber Lasers: Application to Fiber Optic Sensors Networks," Presented at the International Conference on Transparent Optical Networks, Paper Tu. A1.5 (2017).

[13] Redding, B., Choma, M. A., Cao, H.,'Speckle-free laser imaging using random laser illumination," Nat. Photon. 6, 355-359 (2012).

[14] Wiersma, D. S., “The physics and applications of random lasers,” Nat. Phys. 4, 359-367 (2008).

[15] Cavillon, M., Dragic, P., Greenberg, B., Garofalini, S. H., and Ballato, J., "Observation and practical implications of nano-scale phase separation in aluminosilicate glass optical fibers," J. Am. Ceram. Soc. 102, 879883 (2019). 


\title{
Challenges in Preform Material Preparation for Laser-Active Fibers
}

\author{
Katrin Wondraczek*a, Robert Müller ${ }^{\mathrm{a}}$, Volker Reichel ${ }^{\mathrm{a}}$, Tom Trautvetter ${ }^{\mathrm{a}}$, Lothar Wondraczek ${ }^{\mathrm{b}}$ \\ ${ }^{a}$ Leibniz-IPHT Jena e.V., Albert-Einstein-Str. 9, 07745 Jena, Germany; ${ }^{b}$ Laboratory of Glass \\ Science, Friedrich-Schiller-University, Fraunhofer Str. 8, 07743 Jena, Germany
}

\begin{abstract}
We present a novel approach in creating extremely uniform, actively doped core material for laser-active fibers produced by reactive powder-sintering (REPUSIL) or plasma enhanced deposition with dimensions around $\varnothing 15 \mathrm{~mm} \times 100 \mathrm{~mm}$ or even larger.
\end{abstract}

Keywords: optical fiber, REPUSIL, plasma enhanced deposition, rare earth elements, co-doping, laser-active fibers

\section{INTRODUCTION}

Fiber based laser systems are key components for applications, e.g. in medicine for medical imaging and minimalinvasive treatments, industrial production and material processing. The current trend aims at improving performance by realizing higher optical power stability and encountering less non-linear effects. Large mode area fibers support high mode field diameters at reduced intensity and high beam quality. One key aspect for high quality material is the uniform distribution of dopants within the silica matrix, especially the absence of clustering. This uniformity directly effects the refractive index profile variations within the core. Low fiber attenuation can only be achieved for high uniformity in dopant distribution.

The REPUSIL technology is a suitable method to fabricate large quantities of rare-earth doped silica core material for laser-active fibers [1]. Up to now, even though the optical performance of REPUSIL derived fibers is of outstanding quality, dopant distribution homogeneity can further be enhanced and thus variations in refractive index values still can be reduced. Attenuation with a present threshold value of about $20 \mathrm{~dB} / \mathrm{km}$ appears to be a limit that cannot be overcome on a reproducible scale. In achieving the required $\mathrm{Al}$ doping level certain problems have to be considered, resulting in additional efforts in the fabrication chain.

However, new trends in fiber preform material preparation appear to set promising accents especially when adapted or combined to the REPUSIL technology. In detail, preparation of fiber preform using plasma-assisted processes, modification of single steps within the REPUSIL process as well as combination with sol-gel-technology appears to be promising. While the standard REPUSIL process starts with doping silica particles or siloxanes, which are being doped, the improved process steps profit from starting with silane monomers in direct reaction with the doping compounds. Such an in-situ generated network of Si-O and doping elements offers new possibilities in creating high performance material.

We exemplarily present our efforts on both, the reproducibility of the absolute values of the doping concentration and the influence of different production technologies on the homogeneity of the distribution for individual dopants in the glass matrix of the prepared preforms and fibers. These are important parameters for a stable fabrication chain and essential for reliable absolute values and distributions of the refractive index in the preform material.

\section{REPRODUCIBILITY OF THE DOPING CONCENTRATION}

Fabrication of large batch material requires very reproducible process steps. The initial step of preparing doped silica precursor material defines the maximum achievable dopant concentration in the final preform material.

Considering the final dopant content, the early process steps until the fabrication of a compressible powder for green body preparation are essential. These are (i) the wet chemical precipitation of dopant oxides/hydroxides from salt solutions, e.g., $\mathrm{AlCl}_{3} \times 6 \mathrm{H}_{2} \mathrm{O}$ and rare earths $(\mathrm{RE})$ chlorides in presence of high-purity gas phase formed silica

Sixth International Workshop on Specialty Optical Fibers and Their Applications (WSOF 2019),

edited by Liang Dong, John M. Ballato, Proc. of SPIE Vol. 11206, 112061B · C 2019 SPIE

CCC code: $0277-786 X / 19 / \$ 21 \cdot$ doi: $10.1117 / 12.2547445$ 
nanoparticles, (ii) removal of unwanted side products (salts) by washing, and (iii) drying of the material to an oxide powder. After drying the doped suspension, a moldable granulate is produced with the help of isostatic pressure mostly in cylindrical shapes.

In a standard procedure, defined amounts of the doping solution are mixed into a silica suspension under controlled adjustment of the $\mathrm{pH}$ value. As a result, the doping material is precipitated on the surface of the silica. The mixing is carried out under reproducible conditions (dosing, stirring power and duration). Despite of the mentioned efforts, a lack of $\mathrm{Al}_{2} \mathrm{O}_{3}$ in the final preform with a considerable variation could be observed (Figure 1). After analyzing the source of errors, two main parameters could be identified and were subsequently improved. That implies changes in the preparation of the initial salt solutions to correct variations in the Al-concentration and the washing procedure of the material. The impact of such adjustment is shown in Fig. 1. The standard deviation of more than tens of experiments was improved from initially $14 \%$ down to low $2 \%$. At the same time, exemplary EPMA measurements indicated an increase of the $\mathrm{Al}$-content from about $90 \%$ to about $97 \%$ of samples with a desired $\mathrm{Al}$-content of 1.5 to $3.0 \mathrm{~mol} \% \mathrm{Al}_{2} \mathrm{O}_{3}$ in the glass composition, connected with a respective increase of the refractive index. A further improvement of the Al-content seems to be possible, however intensified "correction steps" lead to challenges in the handling of the material in subsequent processing steps.

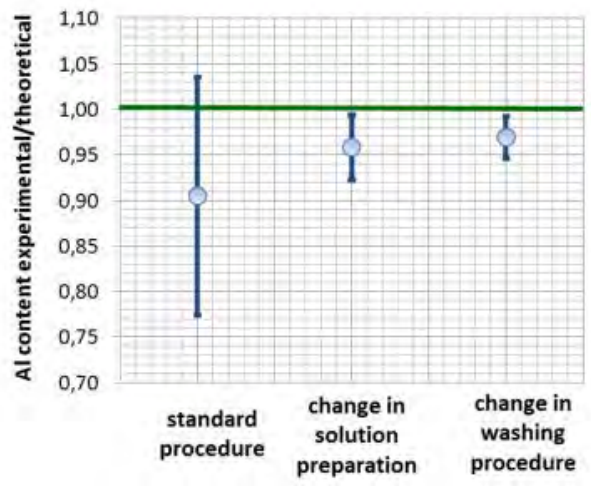

Figure 1: Impact of changing preparation of solution and subsequent washing procedure of doped particles on reproducibility of $\mathrm{Al}_{2} \mathrm{O}_{3}$-doped $\mathrm{SiO}_{2}$ powder. The given Al-content is the average EPMA value measured in a line scan over the preform core diameter.

\section{IMPACT ON SPATIAL HOMOGENEITY}

Beside the absolute amount of dopants in the glass their spatial homogeneity is important to achieve good attenuation properties. The REPUSIL process allows fabricating preforms with active core diameters up to $30 \mathrm{~mm}$ but shows limitations regarding the dopant homogeneity. In terms of dopant homogeneity, the sol-gel-technology is offering high potential since it is a bottom up technology, causing single-molecule components to react on a molecular scale to homogeneous complex systems. To overcome the limitations in terms of spectral attenuation which is especially linked to the process related resulting content of $\mathrm{OH}$ groups as well as residual organics in the final material, [2, 3], a new fabrication technology has been elaborated by combining the sol-gel-technology with a plasma based approach [4].

Direct comparison of EPMA-derived radial dopant profiles of preform samples obtained from plasma approach and from the well-established REPUSIL-technology demonstrates advanced homogeneity for $\mathrm{Al}_{2} \mathrm{O}_{3}$ as depicted in Figure 2.

To verify the structural homogeneity, Raman spectra have been recorded. A line scan with a step size of $15 \mu \mathrm{m}$ across 2 $\mathrm{mm}$ has been performed with a Raman Microscope (Renishaw Invia) using a 50x objective with a $514 \mathrm{~nm}$ Argon laser. The band obtained at $490 \mathrm{~cm}^{-1}\left(\mathrm{D}_{1}\right.$ peak) is corresponding to symmetric bridging oxygen bending vibrations in 4membered rings [5]. The relative intensity decreases with increasing alumina content, indicating that a change of $\mathrm{Al}_{2} \mathrm{O}_{3}$ concentration affects the ring-size distribution in the silica glass system [6]. The variation of intensities between undoped and doped silica with $3.2 \mathrm{~mol} \% \mathrm{Al}_{2} \mathrm{O}_{3}$ is in the range of about $5 \%$ for the sharp $\mathrm{D}_{1}$ peak at $490 \mathrm{~cm}^{-1}$ [6]. Our acquired spectra exhibit almost no deviation in intensity (less than $0.2 \%$ ) between different measuring spots obtained during line 
scan, suggesting almost no variation in $\mathrm{Al}_{2} \mathrm{O}_{3}$ content. This corresponds well to the shown EPMA measurements in Figure 2 and supports the idea of very homogenous material on a structural level.

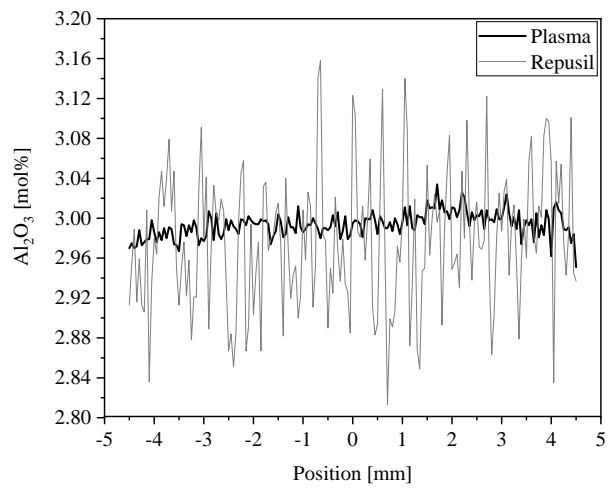

Figure 2: Dopant profiles of $\mathrm{Al}_{2} \mathrm{O}_{3}$ by EPMA for the REPUSIL process and the novel plasma based approach.

\section{CONCLUSION}

The plasma based approach offers the possibility of green body preparation as a pre-step in the REPUSIL process, to obtain samples with extremely uniform dopant distribution. Therefore, the REPUSIL process and the plasma enhanced deposition complement each other on various aspects.

\section{REFERENCES}

[1] Schuster, K. et al., "Material and technology trends in fiber optics", Adv. Opt. Techn. 3, no.4, 447-468, (2014).

[2] Sarkar A., et al., "Sol-Gel Optical Fiber Preforms", Key Engineering Materials, vol. 150, 153-160 (1998).

[3] Brinker C. J. et al., "Sol-Gel Science", Elsevier Science, Burlington (1990).

[4] Trautvetter T. et al., "Novel approach for high-performance optical fibers: multiple-doped silica powders with plasma-enhanced processes", Proc. SPIE (2019).

[5] Galeener, F. L., "Planar rings in vitreous silica", J. Non-Cryst. Solids 49, 53-62 (1982).

[6] Ando M. F. et al., "Boson peak, heterogeneity and intermediate-range order in binary $\mathrm{SiO}_{2}-\mathrm{Al}_{2} \mathrm{O}_{3}$ glasses", Scientific reports, vol. 8(1), 5394 (2018). 


\title{
Multicore fiber amplifier based on the Talbot effect
}

\author{
Cesar Jauregui*a ${ }^{*}$ Albrecht Steinkopff ${ }^{a}$, Jens Limpert ${ }^{\mathrm{a}, \mathrm{b}, \mathrm{c}}$ \\ anstitute of Applied Physics, Abbe Center of Photonics, Friedrich-Schiller-Universität Jena, \\ Albert-Einstein-Str. 15, 07745 Jena, Germany \\ ${ }^{\mathrm{b}}$ Helmholtz-Institute Jena, Fröbelstieg 3, 07743 Jena, Germany \\ ${ }^{c}$ Fraunhofer Institute for Applied Optics and Precision Engineering, Albert-Einstein-Str. 7, 07745 \\ Jena, Germany
}

\begin{abstract}
We present a multicore fiber design for coherent beam combination. This fiber allows reducing the complexity of these systems since, by exploiting the Talbot effect, the beam splitting and recombination happen inside of the fiber.

Keywords: multicore fiber, coherent beam combination, high-power fiber laser systems, Talbot effect.

*cesar.jauregui-misas@uni-jena.de; phone +49 3641947816
\end{abstract}

\section{Introduction}

Fiber laser systems have become extremely popular in industrial, medical and scientific environments due to their high efficiency and beam quality, their power scalability, their robustness and their maintenance-free operation ${ }^{1}$. However, novel applications are always demanding more performance from these systems, which brings them to their limits. These limitations come in the form of non-linear effects ${ }^{2}$, which either degrade pulsed operation or set a power limit in CWoperation, or in the form of thermally-induced beam fluctuations (the so-called mode instabilities-TMI) ${ }^{1}$.

One way to get around the limitations and compromises imposed by any particular fiber design is the coherent combination of the emission of several fiber emitters into a single beam ${ }^{3,4}$. Hereby several emitters are operating below the bounds imposed by their respective limitations, and their beams are combined into a single one. This way, ideally, the performance of a laser system can be increased by a factor equal to the number of emitters. This is a very powerful approach in which the further scalability of the performance is not limited by fundamental physical restrictions but by engineering and economic considerations. There are two main drawbacks to this approach: first is that the system becomes typically bulky; the second drawback is that the system complexity also grows significantly. This is because the coherent combination of beams requires interferometric accuracies in the spatial and temporal overlap of the different beams. This, usually, requires the use of active stabilization systems to ensure long-term operation. The first of these drawbacks can be mitigated by the use of multicore fibers, in which all the different fiber emitters are densely packaged and integrated into a single fiber ${ }^{5}$. This approach allows partially restoring the compactness of coherently combined fiber laser systems. However, the growth of system complexity is not palliated by the use of multicore fibers. This is because before coupling into the multicore fiber an incoming beam has to be divided into $\mathrm{N}$-beams ( $\mathrm{N}$ being the number of cores) and, after amplification, the beams emitted by the different cores have to be externally recombined into a single beam with interferometric accuracy (which still requires active stabilization).

In this work we present a novel multicore fiber for the high-power amplification of optical signals which is designed so that the splitting and recombination of the beams happen automatically inside of the fiber. Since most of the complexity in coherently combined systems stems from the splitting and recombination of the beams (and the active stabilization thereof), this desing allows using a multicore fiber without increasing the system complexity. In other words, the handling and use of the fiber is similar to that of single-core designs.

Sixth International Workshop on Specialty Optical Fibers and Their Applications (WSOF 2019),

edited by Liang Dong, John M. Ballato, Proc. of SPIE Vol. 11206, 112061C · C 2019 SPIE

CCC code: $0277-786 X / 19 / \$ 21 \cdot$ doi: $10.1117 / 12.2541974$

Proc. of SPIE Vol. 11206 112061C-1 


\section{Talbot Fiber}

The multicore fiber described in this work represents a clear departure from the conventional architecture of multicore fibers for high-power applications since its cores are designed to interact/couple with each other. It is this intrinsic coupling between the cores of the fiber which allows that the light, initially coupled in only one of them, spreads to the other cores upon propagation along the fiber (this represents the beam splitting) and then comes back to the original core (which represent the beam recombination), as schematically illustrated in Fig.1a. This way the intensity pattern generated by the light as it propagates along the fiber changes in a periodic way encompassing first one core, then multiple cores and then one core again. This cycle can be repeated several times along the fiber but, by choosing the right fiber length, it can be ensured that most of the energy comes out of one core.

a)

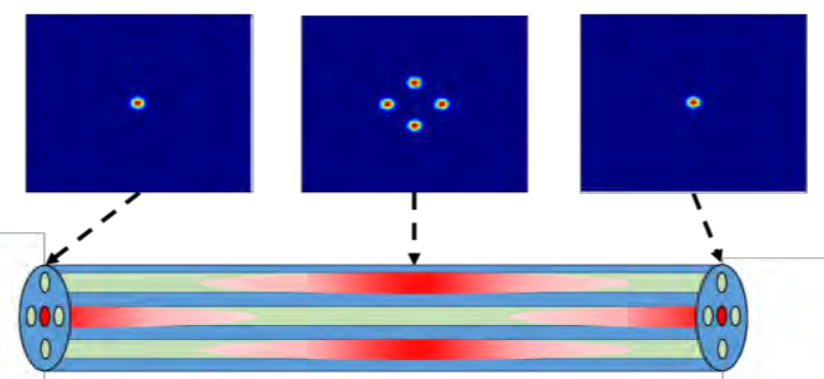

b)

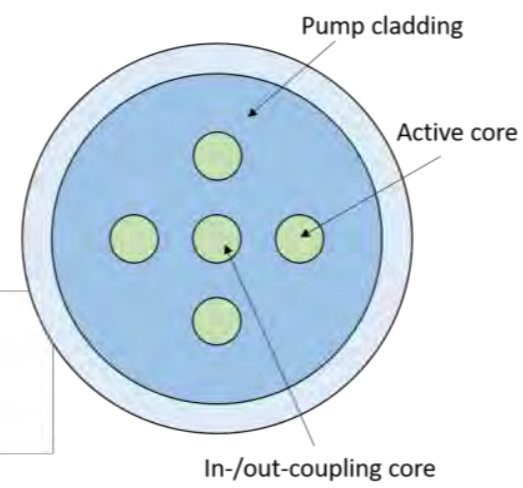

Figure 1. a) Schematic illustration of light propagation along the Talbot fiber. b) Cross-section of the proposed fiber design.

The reason why the beams, after a certain propagation length recombine in the original core is the Talbot effect ${ }^{6}$, which leads to a periodic re-imaging of a periodic arrangement of objects (e.g. cores, slits, etc) in planes that are separated a certain distance from one another. The reason why the Talbot effect occurs in multicore fibers with coupled cores is because of the periodic interference of supermodes in the fiber.

The proposed operating principle of the Talbot fiber brings a twofold advantage for high-power operation: first since the power is distributed across several cores over sections of the fiber, the non-linear effects will be mitigated. Simulations have shown that the B-integral can be decreased by a factor of 2 to 3 using a 5 core fiber such as the one shown in Fig.1b), as will be discussed in detail at the conference. Second, the spread of the amplification over several cores reduces the heat-load in each one of the individual core, which will also increase the TMI-threshold. These benefits, unlike in traditional multicore fiber laser systems, are not accompanied by an increase of the system complexity, since the splitting and recombination of the beams take place inside of the fiber and, therefore, do not need to be actively stabilized (under normal circumstances).

Fig. 2 shows a simulation of light amplification in a fiber with the cross-section shown in Fig. $1 \mathrm{~b}$. In this simulation, done solving the rate equations for the amplification process and using the supermode theory ${ }^{7}$ to analyze the coupling between cores, the fiber has a length of $1.27 \mathrm{~m}$, and the $30 \mu \mathrm{m}$ diameter cores have a V-parameter of 3. The core-to-core distance is $50 \mu \mathrm{m}$ and the pump cladding has a diameter of $375 \mu \mathrm{m}$. Each core is doped with $7.5^{*} 10^{25} \mathrm{ions} / \mathrm{m}^{3}$. The central core is seeded with $5 \mathrm{~W}$ at $1030 \mathrm{~nm}$ and the fiber is pumped by $50 \mathrm{~W}$ at $976 \mathrm{~nm}$ in the counter-propagating direction. As can be seen, as expected, the light couples periodically from the center core into the satellite cores and back, and by choosing the 
right length, ideally, all the light returns to the central core at the end of the fiber. In the simulated configuration, the Bintegral accumulated by the light as it propagates along the Talbot fiber is less than half of the one that would have been accumulated if the fiber had just a single core. Finally, the amplification efficiency is better than $80 \%$.
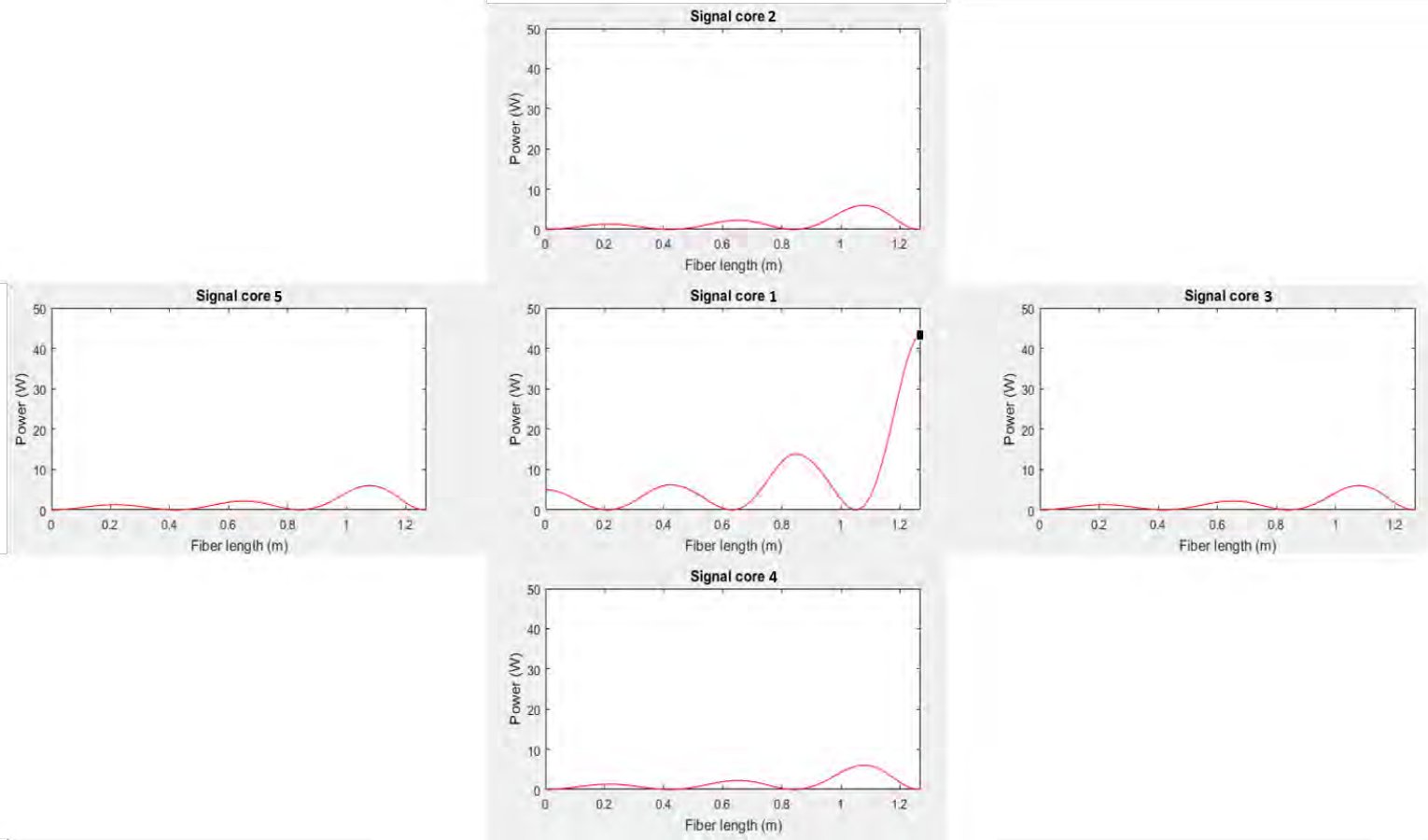

Fig.2. Simulation of the power evolution along the different cores of the proposed Talbot fiber.

A detailed analysis of the performance of these fibers under high-power operation will be presented at the conference. This includes the impact of temperature, the impact of the pump direction, the spectral properties of the design as well as a discussion on why these fibers should be able to also mitigate TMI.

The authors want to acknowledge funding by the German Research Foundation (DFG) (IRTG 2101, JA 2625/2-1, JA 2625/3-1), the European Research Council (ERC) (MIMAS, 670557).

\section{References}

[1] Jauregui, C., J. Limpert and A. Tünnermann 2013. High-power fibre lasers. Nat. Photonics 7(11): 861-867.

[2] Agrawal, G. P. 2006. Nonlinear Fiber Optics, 3rd Ed.. Academic Press, Orlando, Fla.

[3] Augst, S. J., J. K. Ranka, T. Y. Fan and A. Sanchez 2007. Beam combining of ytterbium fiber amplifiers (Invited). J. Opt. Soc. Am. B 24(8): 1707.

[4] Kienel, M., M. Müller, A. Klenke, J. Limpert and A. Tünnermann 2016. $12 \mathrm{~mJ} \mathrm{kW-class} \mathrm{ultrafast} \mathrm{fiber} \mathrm{laser}$ system using multidimensional coherent pulse addition. Opt. Lett. 41(14): 3343.

[5] Klenke, A., M. Müller, H. Stark, F. Stutzki, C. Hupel, T. Schreiber, A. Tünnermann, and J. Limpert 2018. Coherently combined 16-channel multicore fiber laser system. Opt. Lett. 43(7): 1519.

[6] Latimer, P. and R. F. Crouse 1992. Talbot effect reinterpreted. Appl. Opt. 31(1): 80-89.

[7] Szostkiewicz, L., M. Napierala, A. Ziolowicz, A. Pytel, T. Tenderenda and T. Nasilowski 2016. Cross talk analysis in multicore optical fibers by supermode theory. Opt. Lett. 41(16): 3759. 


\title{
Cleaning protocols for fabrication of fibers for kilowatt-class lasers
}

\author{
Michael Runkel*, Diana VanBlarcom, Derrek Drachenberg, Marcus Monticelli, \\ Gabriel Davalos, Steve Davis, Nick Schenkel, Matt Cook, Parker Crist, \\ Michael Messerly, Jay Dawson, and Tayyab Suratwala \\ Lawrence Livermore National Laboratory, 7000 East Avenue, Livermore, CA, USA 94550
}

\begin{abstract}
Stack-and-draw methods allow the fabrication of complex waveguide designs, including those that require control of refractive indices to the $1 \times 10^{-4}$ level. Unfortunately, stacking steps can introduce power-limiting contaminants into the glass matrix. We present protocols, originally developed for cleaning and handling large optics at the National Ignition Facility, that are appropriate for fabrication of fibers for kilowatt-class lasers.
\end{abstract}

Keywords: optical fibers, optical fiber-based lasers, laser damage

* runkel1@LLNL.gov phone (925) 424-2210

\section{Introduction}

Stack-and-draw methods allow for the fabrication of complex waveguide designs, including those that support modes having relatively large cross-sectional areas [1]. In addition, by mixing two or several different glasses in appropriate proportions, the methods also allow for refractive index control to better than $1 \times 10^{-4}$, which often essential for very large area modes.

Unfortunately, contaminants can severely limit the efficiency, laser damage threshold, and strength of stack-and-draw fibers. The contamination can be due to environmental exposure during the pulling or stacking of canes and capillaries, or to improper handling and storage of the pieces. Though most contaminating particulates evaporate long before they reach the hottest portion of the glass-melting furnace, their residue (usually charred carbon) can become embedded in a fiber, limiting its power and reliability. For example, Figure 1 shows the cross-section of an early fiber fabricated by our group, that fractured when its power was raised to just several hundred watts. The picture suggests that the laser heated an embedded bit of carbon, which expanded and fractured the fiber.

Clearly, a key to eliminating glass failures is to eliminate contaminants. Toward that end, we have adopted cleaning and handling protocols originally developed for large optics at the National Ignition Facility [2 - 5], and now use them routinely when fabricating stack-and-draw fibers.

Sixth International Workshop on Specialty Optical Fibers and Their Applications (WSOF 2019),

edited by Liang Dong, John M. Ballato, Proc. of SPIE Vol. 11206, 112061D · C 2019 SPIE

CCC code: $0277-786 X / 19 / \$ 21 \cdot$ doi: $10.1117 / 12.2548659$

Proc. of SPIE Vol. 11206 112061D-1 


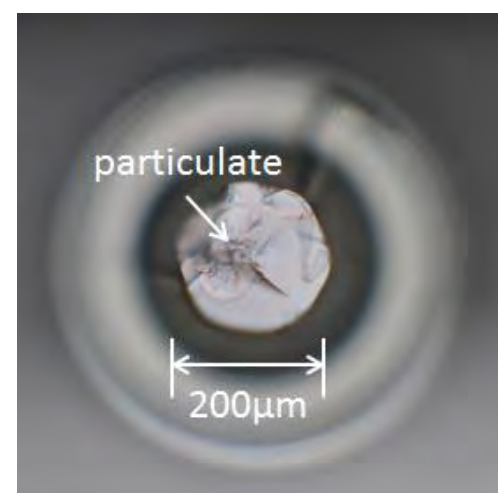

Figure 1 Cross section of fiber that failed at roughly $300 \mathrm{~W}$, showing an embedded particulate that likely have generated the failure.

\section{Experiments}

To test the efficacy of the AMP cleaning processes, we fabricated two stack-and-draw preforms. Both consisted of roughly 200 canes drawn from a telecom-grade fused silica rod. However, one was cleaned with methanol and lint-free wipes (a common protocol), and the other was cleaned via the AMP (Advanced Mitigation Process) protocols developed for processing large fused silica optics - optics that must withstand extreme fluences of $351 \mathrm{~nm}$ light - for the National Ignition Facility; see Figure 2. In addition, all cleaning, fixturing, and assembly processes used to prep the second stack were performed in a Class 100 cleanroom.

For the adapted AMP process, glass rods (1-3mm in diameter and 10" long) are assembled into a Teflon fixture that only contacts the rods at each end. The loaded fixture receives $120 \mathrm{kHz}$ ultrasonic cleaning in $10 \%$ sodium hydroxide at $45 \mathrm{C}$ and $3 \%$ Brulin 1696 detergent at 55C. Parts are thoroughly rinsed using ultrasonicated $18 \mathrm{Mohm}$ ultrapure water and spray rinses. A $200 \mathrm{~nm}$ etch in buffered hydrofluoric acid (6:1 BOE diluted 2:1 in DI water) is followed by additional ultasonicated $(120 \mathrm{kHz}-270 \mathrm{kHz})$ ultrapure water and spray rinse. Finally, the components are allowed to fully dry inside the Teflon frame. The rods are stacked and assembled into a fused silica tube. The preform stack is then returned to a non-cleanroom area to be pulled into fiber using standard telecom fiber-based draw tower equipment and without clean air filters around the draw area.

Figure 3 compares the losses of fibers drawn from wipe-cleaned and AMP-cleaned preforms. The loss of the fiber pulled from the wipe-cleaned preform were roughly $20 \mathrm{~dB} / \mathrm{km}$ at $1 \mu \mathrm{m}$, while the loss of fiber pulled from the AMPcleaned preform was much lower: less than $3 \mathrm{~dB} / \mathrm{km}$ at $1 \mu \mathrm{m}$. For comparison, note that the Rayleigh-scattering limit for optical fibers is approximately $1.2 \mathrm{~dB} / \mathrm{km}$ at $1 \mu \mathrm{m}$.

Encouraged by the early results, we applied the AMP process to a mixture of Yb-doped fused silica and F-doped fused silica (Figure 4a). The mixture was created to fine-tune the refractive index of the Yb-doped material. First, an appropriate ratio of the materials was stacked inside a tube, drawn to canes, and then the starting tube was acidetched away with a weak solution of HF acid. Then the etched canes were combined into a new tube, drawn to 
canes, and then starting tube from the second draw was also acid-etched away. The AMP process was applied between all mixing steps, and after all acid-etching steps, and all stacking was conducted in a Class 100 cleanroom.

The Figure 4a mixture was then built into a cladding-pumped optical fiber. Figure $4 \mathrm{~b}$ shows that the losses of this fiber were very low, and in subsequent testing, the material achieved $1 \mathrm{~kW}$ of power at a quantum efficiency within $2 \%$ of the quantum limit. These results suggest that the AMP procedure can achieve a level of cleanliness that is appropriate for kilowatt-class fiber lasers.
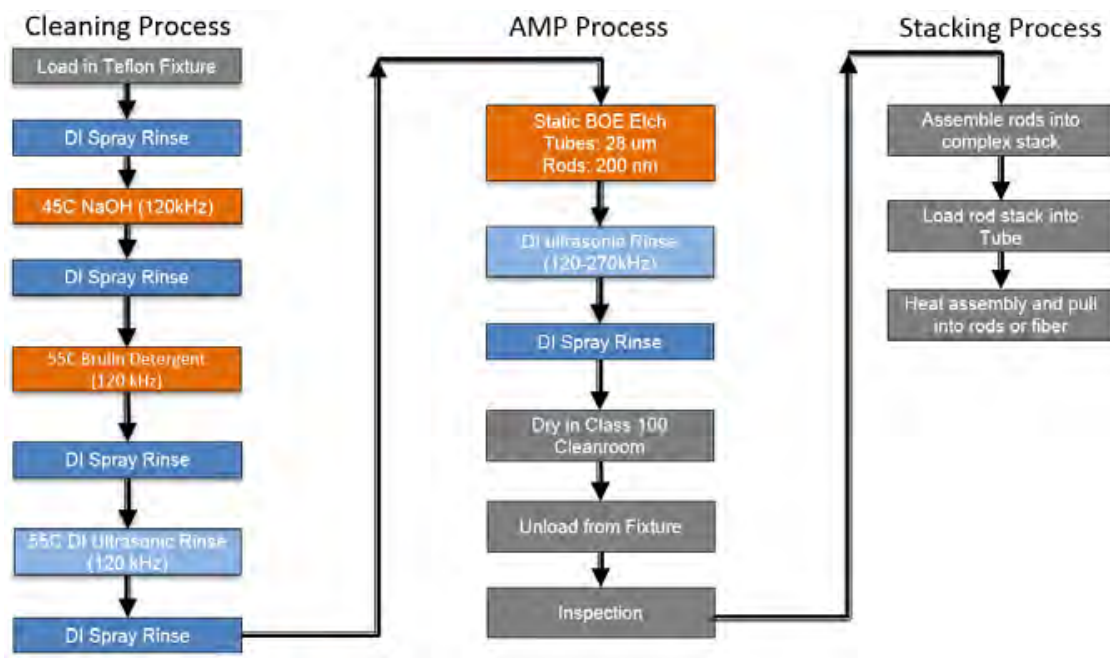

Figure 2 Flow diagram for AMP process. From [5].

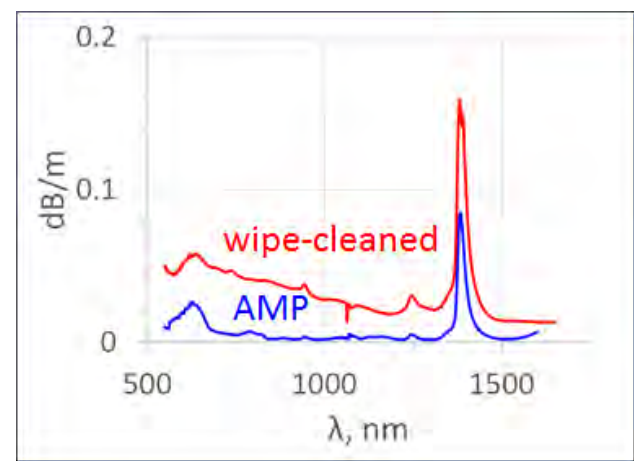

Figure 3 Losses of fibers drawn from wipe-cleaned and AMP-cleaned preforms. From [5]. 

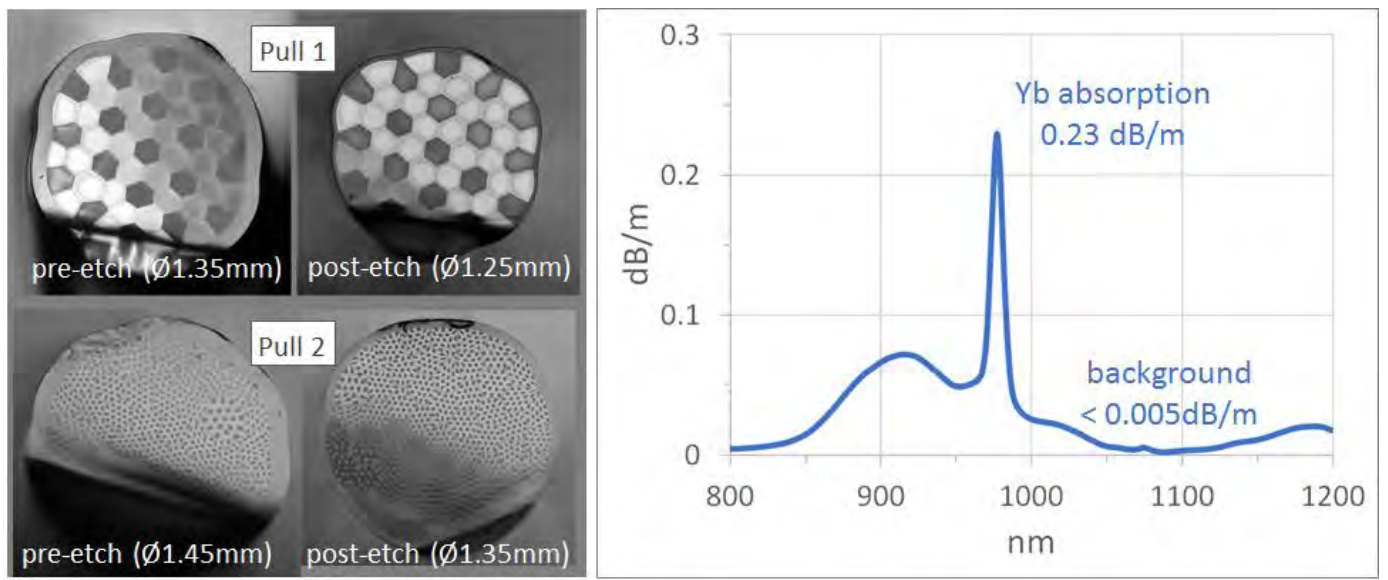

Figure 4a Mixtures of $\mathrm{Yb}$ doped glass (brighter regions) and F-doped glass (darker region). After mixing twice (Pull 2), a cane was built into a cladding-pumped optical fiber. Figure $4 \mathrm{~b}$ suggests that the losses of the cladding-pumped fiber are suitably low for high power applications.

\section{Summary}

In summary, we have shown that a modified version of the AMP process, which was originally developed to reduce damage in large optics at the National Ignition Facility, can be a valuable tool for the fabrication of stackand-draw optical fibers, especially for very high power applications.

Lawrence Livermore National Laboratory (LLNL) (DE-AC52-07NA27344)

\section{References}

[1] J. Limpert, T. Schreiber, S. Nolte, H. Zellmer, A. Tünnermann, R. Iliew, F. Lederer, J. Broeng, G. Vienne, A. Petersson, C. Jakobsen, "High-power air-clad large-mode-area photonic crystal fiber laser," Optics Express $11(2003)$.

[2] Suratwala, Tayyab I., Miller, Phil E., Bude, Jeffery D. et al., "HF-Based Etching Processes for Improving Laser Damage Resistance of Fused Silica Optical Surfaces," Journal of the American Ceramic Society 94(2), 416-428 (2011).

https://onlinelibrary.wiley.com/doi/full/10.1111/j.1551-2916.2010.04112.x

[3] Pryatel, James A., Gourdin, William H., Frieders, Susan C., Ruble, Gerald S., Monticelli, Marcus V., "Cleaning practices and facilities for the National Ignition Facility (NIF)," Proc. SPIE 9237, Laser-Induced Damage in Optical Materials: 2014, 92372H (31 October 2014).

https://www.spiedigitallibrary.org/conference-proceedings-of-spie/9237/1/Cleaning-practices-and-facilities-forthe-National-Ignition-Facility-NIF/10.1117/12.2075927.full 
[4] Bude, J.D., Miller, P.E., Shen, N., Suratwala, T.I., Laurence, T.A., Steele, W.A., Baxamusa, S.H., Wong, L.L. Carr, C.W., Cross, D.A., Monticelli, M.V., Feit, M.D., Guss, G., "Silica laser damage mechanisms, precursors and their mitigation," Proc. SPIE 9237, Laser-Induced Damage in Optical Materials: 2014, 92370S (31 October 2014)

https://www.spiedigitallibrary.org/conference-proceedings-of-spie/9237/1/Silica-laser-damage-mechanismsprecursors-and-their-mitigation/10.1117/12.2070017.full?SSO=1

[5] D. VanBlarcom, D. Drachenberg, M. Monticelli, G. Davalos, S. Davis, N. Schenkel, M. Cook, R. Crist, M. Messerly, and J. Dawson, "Novel AMP surface treatment for improving optical fiber strength and laser gain," Proceedings SPIE Laser Damage, Volume 10805 (2018). 


\title{
Improved wavelength selective filtering design in a $\mathrm{Nd}$ doped silica fiber for E-band amplification
}

\author{
Leily Kiani, Paul Pax, Derrek Reggie Drachenberg, Jay Dawson, Charles Boley, Cody Mart, Victor \\ Khitrov, Charles Yu, Robert Crist, Matthew Cook, Nick Schenkel, Michael Runkel, Michael \\ Messerly
}

Lawrence Livermore National Laboratory, Livermore, California 94550, USA

\begin{abstract}
We present the design of a Nd doped silica fiber for $1400 \mathrm{~nm}$ amplification. The waveguide design includes elements that induce core loss at selective wavelengths enabling efficient amplification of $1400 \mathrm{~nm}$. The effect of these elements is modeled in COMSOL in order to produce a fiber design that is applicable to telecom amplifiers.
\end{abstract}

Keywords: microstructure fibers, E-band, telecommunications

*kiani2@1lnl.gov

\section{Introduction}

A focus of our recent work has been to promote lasing on rare-earth transitions that have previously been inaccessible to laser action because of competition from stronger lasing transitions in the same rare-earth element ${ }^{1}$. Neodymium has two useful transitions other than the well-known transition that produces light a $1 \mu \mathrm{m}$, the ${ }^{4} \mathrm{~F}_{3 / 2}$ to ${ }^{4} \mathrm{I}_{9 / 2}$ that produces light at 0.9 $\mu \mathrm{m}$ and the ${ }^{4} \mathrm{~F}_{3 / 2}$ to ${ }^{4} \mathrm{I}_{132}$ that produces light at $1.4 \mu \mathrm{m}$. By suppressing gain via filtering away the light at the $1 \mu \mathrm{m}$ line, amplification in the other bands can be promoted. Light in the $0.9 \mu \mathrm{m}$ band is useful as a route to blue-green light and amplifiers in the $1.4 \mu \mathrm{m}$ region are useful primarily for telecommunications applications.

There are a few fiber design approaches for suppressing competitive gain by spectrally filtering the light in a fiber core. Schemes using rare-earth absorption such as that in samarium have been used to deplete spectrum for gain tailoring ${ }^{2}$. Fibers having W-profiles, sometimes referred to as depressed-well or trench profiles, have been developed for inducing loss of the fundamental guided mode at long wavelengths. Here the confinement losses grow as the mode field expands for long wavelengths generating an extended short-pass filter. This technique applied to Nd-doped silica fibers can be used to promote $0.9 \mu \mathrm{m}$ amplification ${ }^{3}$. In contrast to the depressed-well fibers, we have demonstrated a wavelength selective fiber filter where core losses are influenced by coupling to high index elements near the core, creating a spectral notch filter ${ }^{4}$. We have applied the wavelength selective filter to suppress the $1 \mu \mathrm{m}$ transition to allow amplification at $0.925 \mu \mathrm{m}^{4}$, and we have suppressed both $1 \mu \mathrm{m}$ and $0.9 \mu \mathrm{m}$ light to allow amplification at $1.4 \mu \mathrm{m}^{5}$. Here, we present designs for an improved fiber that allows amplification at $1.4 \mu \mathrm{m}$, thus serving as an amplifier in the telecom E-band window (1360 to $1460 \mathrm{~nm}$ ). The primary improvement is reduction of the mode-field diameter allowing for favorable gain dynamics in a $10 \mathrm{~m}$ length of fiber, representing a performance comparable to conventional telecom amplifiers.

In a first demonstration of a $\mathrm{Nd}$ doped fiber for telecom applications, the fiber design was such that it achieved a mode field diameter of $10.5 \mu \mathrm{m}$ and an outer diameter of $125 \mu \mathrm{m}$, closely resembling a standard telecom fiber ${ }^{5}$. Importantly, the design of the first fiber had a core index matched to silica and the core confinement was generated by a photonic crystal

Sixth International Workshop on Specialty Optical Fibers and Their Applications (WSOF 2019),

edited by Liang Dong, John M. Ballato, Proc. of SPIE Vol. 11206, 112061E · C 2019 SPIE

CCC code: $0277-786 X / 19 / \$ 21 \cdot$ doi: $10.1117 / 12.2548664$ 
lattice of low index elements. The raised core index was reduced by mixing with low index fluorine doped glass which also diluted to concentration to $40 \%$ of its original value. Details can be found in Dawson et al. 2017. The fiber produced $>13 \mathrm{~dB}$ gain in $14 \mathrm{~m}$ of fiber. Improvements identified for a next fiber include development of a design where the doped core is not mixed to reduce it to the index of fused silica to avoid dilution of the $\mathrm{Nd}$ dopant and avoid the introduction of contaminants that likely contributed to the relatively high background loss $(>0.1 \mathrm{~dB} / \mathrm{m})$ in the first attempt.

\section{Wavelength selective filter designs}

\section{Methods}

The basis of the filtering fiber is spectrally dependent reduction of core guidance, in this case brought on by auxiliary waveguide elements that form a bridge spanning the confining "wall" surrounding the core ${ }^{4}$. The auxiliary elements are chosen to be resonant with the core at the wavelength to be filtered, so that light in the core couples to and spreads into them. They are also chosen to have higher dispersion than the core so that the resonance is constricted to a limited spectral band.

In our refined design, the core confinement is formed by a step index region in contrast to the photonic crystal fiber lattices used in the previous fiber ${ }^{1}$. The core index is raised to $0.135 \mathrm{NA}$ with respect to the inner cladding region - that is, the region that immediately neighbors the core. Auxiliary elements surrounding the core are strings of graded-index (GRIN) waveguides with relatively high waveguide dispersion due to their high NA (0.20 to 0.30) and small size. These GRINs are arranged in six groups or "spokes" of five inclusions. The GRINs support modes of their own, which are designed to be resonant with the fundamental core mode at the wavelength we wish to filter; at resonance, the core mode couples to these inclusions. An outer region, which immediately neighbors the inner cladding, is raised to be higher than that of the core to ensure coupling from the resonant elements toward an outer reservoir rather than back into the core of the fiber. The overall effect is that the core guidance is lost when the resonance condition is satisfied or nearly satisfied, thus creating a wavelength-selective filter.

\section{Modeling}

We model the structure and waveguide losses via a Finite Element Method based mode solver (COMSOL). Figure 1 shows a schematic of the design (left) and an example of the mesh required to model the core (right), GRIN elements and outer reservoir. The reservoir is outside the boundary shown as a hexagon. Beyond the reservoir is an artificial structure, a ring of absorbing material, used to characterize the resonant loss from the core mode. In the actual fiber, there may or may not be dissipation in the reservoir: light that makes its way from the core to the reservoir will not return on the length scales of interest, due to the mode coupling in the large mode volume of the reservoir. In the model we introduce dissipation to avoid having to explicitly track the modal population of the reservoir.

The core is not a simple step index but rather has a gradation of the index at the outer edge. The core diameter is $5.02 \mu \mathrm{m}$ but considering this gradation the effective core diameter is $4.09 \mu \mathrm{m}$. The NA between the core and cladding is 0.135 and the mode field diameter is $8.18 \mu \mathrm{m}$ at $1450 \mathrm{~nm}$. The fundamental mode in the core couples to the GRIN LP11 modes. The GRIN elements are $5.13 \mu \mathrm{m}$ in diameter and on a center-to-center pitch of $6 \mu \mathrm{m}$. There are five elements in each GRIN 'spoke' with a spatial offset from the core of one additional unit of the pitch. The modeled inner cladding is the hexagon enclosing the core and GRIN elements. The hexagon is $70.1 \mu \mathrm{m}$ face-to-face and the outer cladding diameter is $125 \mu \mathrm{m}$. Lastly, to model the dissipation of outcoupled light, an absorbing boundary is added to the model with a ramped profile that extends $15 \mu \mathrm{m}$ from the outer cladding diameter. 

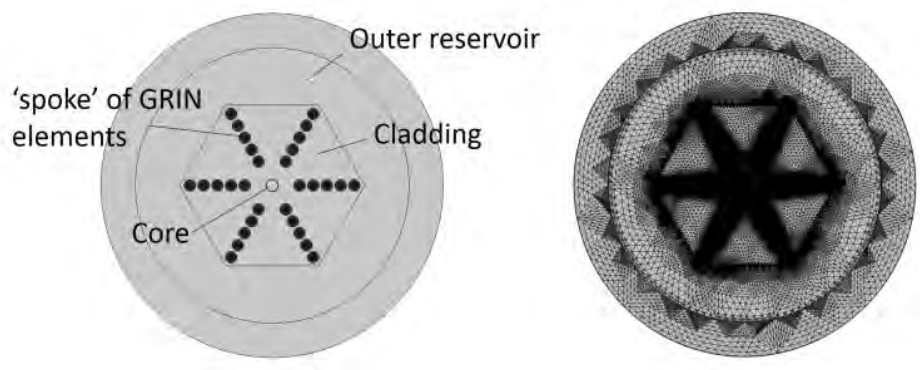

Figure 1. Example of the fiber design geometry (left) and the corresponding mesh (right) in COMSOL. The high index GRIN elements extend out from the core in the center of the design and are surrounded by the background region. The ends of the GRIN element 'spokes' are located close to a raised index region that serves as a reservoir for the outcoupled light.

\section{Results and Discussion}

The reduced core size and increased NA in this fiber reduces the mode field diameter by $20 \%$ compared to the previous fiber. An amplifier based on this fiber could be pumped at 808 or $880 \mathrm{~nm}$. Figure 2 shows the calculated losses over the range between the pump at $808 \mathrm{~nm}$ and the signal at $1400 \mathrm{~nm}$. Modeling results indicate that gain can be suppressed for both competitive transitions at $0.9 \mu \mathrm{m}$ and $1.1 \mu \mathrm{m}$ while maintaining low losses at the pump and signal wavelengths.

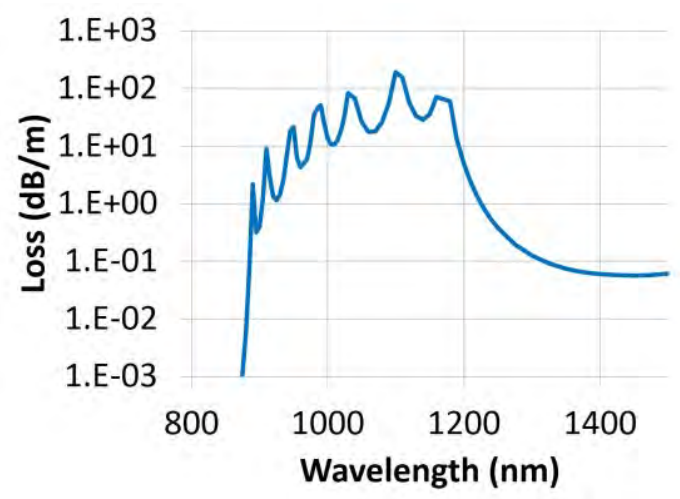

Figure 2. Calculated losses from COMSOL modeling showing loss exceeding $1 \mathrm{~dB} / \mathrm{m}$ between $0.9 \mu \mathrm{m}$ and $1.2 \mu \mathrm{m}$.

In summary, we have designed a new Nd doped fiber with reduced mode field diameter to achieve high inversion over short lengths and thus reduce the pump power needed to reach $30 \mathrm{~dB}$ of gain, a specific technical goal for telecommunications applications. We intend to fabricate this fiber design via the stack and draw method. This stack will require roughly 300 canes to be stacked in a hexagonal array which will be enclosed in a silica tube and finally drawn to fiber with dimensions comparable to standard telecom fiber.

Lawrence Livermore National Laboratory (LLNL) (DE-AC52-07NA27344)

LLNL-ABS-784901 


\section{References}

[1] Kiani, L.S., Beach, R.J., Dawson, J.W., Pax, P.H., Allen, G.S., Drachenberg, D.R., Khitrov, V.V., Schenkel, N., Cook, M.J., Crist, R.P. and Messerly, M.J., 2018. E-band fiber laser performance and power analysis with longitudinally averaged population modeling. JOSA B, 35(11), pp.2833-2841.

[2] Liaw, S.K. and Chen, Y.K., 1996. Passive gain-equalized wide-band erbium-doped fiber amplifier using samarium-doped fiber. IEEE Photonics Technology Letters, 8(7), pp.879-881.

[3] Soh, D.B., Yoo, S., Nilsson, J., Sahu, J.K., Oh, K., Baek, S., Jeong, Y., Codemard, C., Dupriez, P., Kim, J. and Philippov, $\mathrm{V}$., 2004. Neodymium-doped cladding-pumped aluminosilicate fiber laser tunable in the $0.9-/ \mathrm{spl} \mathrm{mu} / \mathrm{m}$ wavelength range. IEEE Journal of Quantum Electronics, 40(9), pp.1275-1282.

[4] Pax, P.H., Khitrov, V.V., Drachenberg, D.R., Allen, G.S., Ward, B., Dubinskii, M., Messerly, M.J. and Dawson, J.W., 2016. Scalable waveguide design for three-level operation in Neodymium doped fiber laser. Optics express, 24(25), pp.28633-28647.

[5] Dawson, J.W., Kiani, L.S., Pax, P.H., Allen, G.S., Drachenberg, D.R., Khitrov, V.V., Chen, D., Schenkel, N., Cook, M.J., Crist, R.P. and Messerly, M.J., 2017. E-band Nd 3+ amplifier based on wavelength selection in an all-solid microstructured fiber. Optics express, 25(6), pp.6524-6538. 


\title{
Optical fibers for downhole oil and gas applications* \\ Dr. Paul F. Wysocki*, Dr Zhao Li \\ Baker Hughes, a GE company, 2851 Commerce St, Blacksburg, VA 24060
}

\begin{abstract}
Optical fiber optimization for downhole applications in the oil and gas industry is discussed. Fiber coatings, packaging and glass chemistry offer unique challenges in oil wells with harsh chemicals, high pressure and temperatures up to $300 \mathrm{C}$. The use of FBGs to enhance distributed sensing in these environments is discussed.
\end{abstract}

Keywords: distributed sensing, oil and gas industry, optical fibers, hydrogen resistance, enhanced scattering

*Paul.wysocki@bakerhughes.com; phone 540-961-9523

\section{Introduction}

In the oil and gas industry, optical fibers are used almost exclusively as sensors of environmental quantities such as temperature, pressure, strain, vibration and acoustic signals. In some cases, the fiber is used to provide a distributed measurement while other times, a discrete single location measurement is needed. The applications can also be divided into surface sensing applications and downhole sensing applications. Generally, surface sensing applications in oil and gas are not very different from such applications in other fields. Applications such as pipeline monitoring on or near the surface using distributed acoustic sensing (DAS) require the use of techniques and fiber hardware similar to those used in telecommunications or perimeter sensing. Downhole applications are more unique in that the environment downhole often includes harsh chemicals, hydrogen exposure, high and variable pressures up to 15000 psi and temperatures up to 300C. This presentation focuses on the harsh downhole environments and how they affect the deployment and application of optical fiber sensing. Often optical fibers are used rather than electronic sensors for the very reason that they can stand up to the rigors of such environments.

\section{Downhole Environments}

\section{Downhole Optical Sensing}

The downhole environmental requirements for optical fibers are evolving over time towards both higher pressures and temperatures. Typical deployments in conventional production might require temperatures up to $150 \mathrm{C}$ and pressures below 5000 psi. However, unconventional wells with steam injection can reach temperatures of 300C and pressures up to 15000 psi. Optical fibers fortunately are able to survive such conditions but other materials used in cables and as coatings pose the greater challenge.

In addition to such pressure and temperature, optical fiber must either be kept isolated from harsh chemicals and high pressure steam, or they must be designed to survive these as well. Even if protected, in high T hydrocarbon production, large quantities of hydrogen gas is liberated. Hydrogen diffuses through most materials over time at high temperatures and so any fiber degradation caused by hydrogen must be anticipated and minimized. Typically it is assumed that the environment downhole may reach a steady-state hydrogen level of 1 Atmosphere partial hydrogen pressure.

\section{Limiting Deployment Challenges}

The deployment of equipment into downhole environments precludes the use of standard optical fiber cables. Downhole cables are pulled, dragged and pushed into a well and exposed to many chemicals. Consequently, optical fibers deployed in such cables only survive if the cables are made of chemical resistant metals such as stainless steel or Inconel. Often 
such cables include a fiber in metal tube (FIMT) inside the armored cable, providing 2 layers or sealed metal protection. The connection/splicing of such cables to each other or to other metal clad equipment downhole requires special metal splice housings and seals and techniques for breaking out the fibers to make a fusion splice. Fibers passing out of a well often must pass through a well head outlet (WHO) that provides a barrier between the surface and harsh downhole environments. The custom cables, hardware and processes to interconnect cables are expensive. Consequently, earliest fiber deployments avoided complex interconnections downhole as much as possible.

There are also substantial limitations on the types of optical fiber components that can be deployed downhole. The collection of components developed for telecommunications applications are useful for building surface interrogators to launch light into downhole fibers, but none of them are typically packaged or designed to survive temperatures of 100C or higher. Consequently, discrete sensors for downhole use usually require custom development, qualification and packaging, paying particular attention to how they may be integrated and connected to downhole cables.

A further challenge is posed by the need to get an optical fiber based sensor into contact with the measured quantity at the point of interest. To measure local temperature, either the thermal conductivity must be high so the downhole packaged sensor responds quickly to the environment in the vicinity, or substantial delay must be built in and the system designed to respond only slowly. To measure local pressure, a transducer is required to convert pressures into optical changes. To perform distributed temperature sensing (DTS), DAS or distributed strain sensing (DSS) the temperature, acoustic waves or strain respectively must be transmitted through all the packaging to the optical fiber. This is further complicated by the myriad of ways a cable can be deployed including strapped outside the casing of the well, inside the casing of the well, cemented in place around a casing, or in an open hole.

\section{Application Specifics}

Some downhole sensing applications utilize an optical fiber to transmit light from an interrogator to a discrete sensor location and back to an interrogator. One of the earliest uses of optical sensing downhole was to provide discrete pressure/temperature (PT) readings at particular locations. In this case, the PT gauge is an optical device and the fiber only provides a way to interrogate it without placing the interrogator downhole. Similarly, fiber might be used downhole to transmit the light to a specific fiber that serves as a distributed sensor. In all these cases, the main requirement of the fiber is that it provide low loss at all wavelengths of interest when exposed to the harsh environments downhole.

Other downhole sensing applications such as DAS, DTS or DSS utilize the fiber as a distributed sensor. In some cases, fiber Bragg grating (FBGs) arrays may be added to provide discrete distributed sensing locations or to generally enhance the scattering of the fiber. Such distributed sensing optical fibers must certainly maintain low loss, but they also might meet other requirements such as specific FBG strengths and wavelengths, loss that does not change over time, or uniformity of scatter level.

For any specific application, the optical wavelength might be selected based on several criteria including fiber loss profile, fiber loss change wavelength dependence, and available laser sources, components and detectors for building an interrogator. Fibers might also be multi-moded or single-moded at the wavelength of choice. Often the environments and current fiber technology dictate the choices made to a very small number of best options.

\section{Temperature Rating}

\section{Optical Fibers to Meet the Challenge}

Optical fiber coatings for downhole are first selected to meet temperature requirements of the particular application. Figure 1 shows thermogravitational analysis (TGA) performed on a mid-temperature acrylate (MTA) and polyimide coating used on CoreBright single mode fiber broduced by Baker Hughes, a GE company (BHGE). ${ }^{1}$ The MTA is rated at $150 \mathrm{C}$ and the plots show that $20 \%$ weight loss occurs (typical criteria for failure) at $150 \mathrm{C}$ in about $400000 \mathrm{hrs}$, or about 45 years. On the other hand, by the time this coating reaches $200 \mathrm{C}$, that same case is reached in 400 hrs, or about 17 days. 
Polyimide coating is selected for temperatures up to 300C where the lifetime according to TGA is hundreds of years. Other coatings are sometimes used for intermediate temperatures such as PFA or PEEK because they have other beneficial properties, such as greater mechanical robustness than typical thin polyimide coatings.
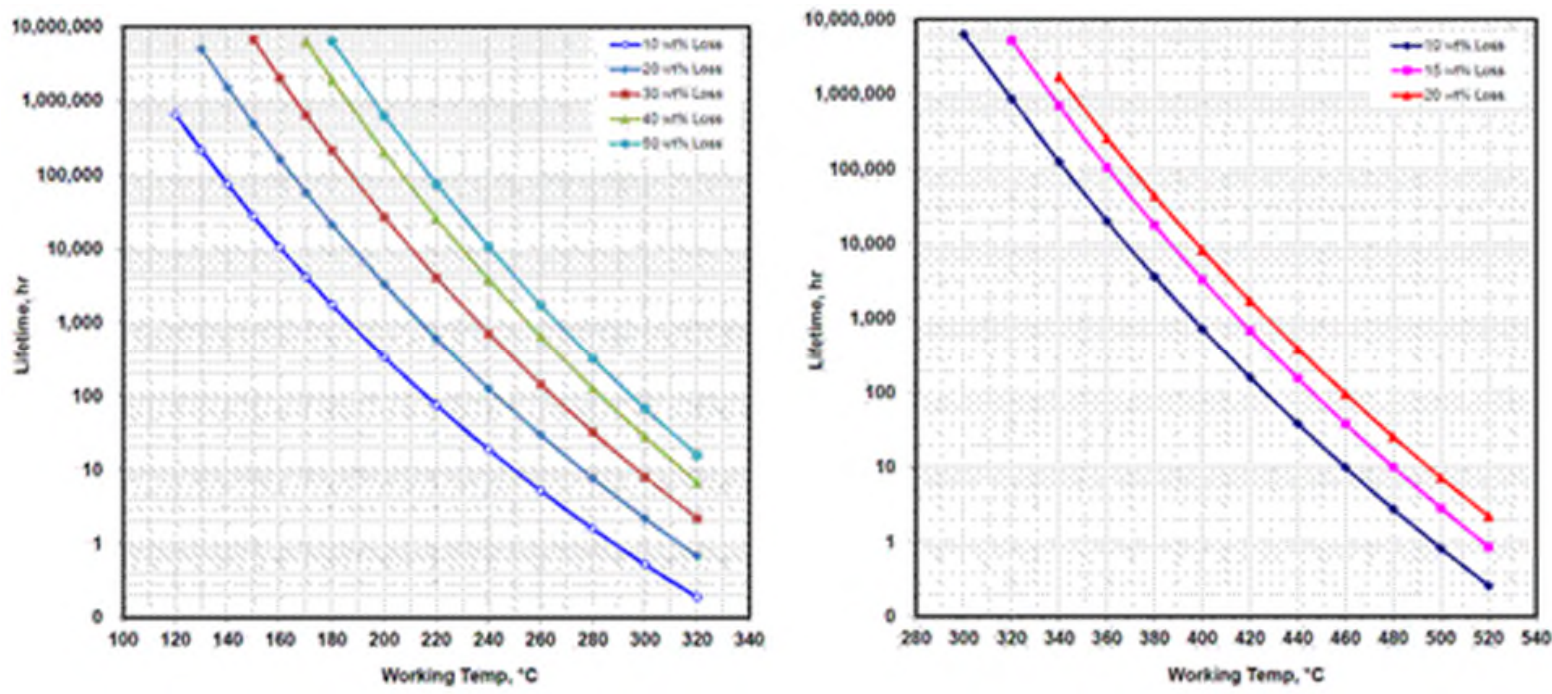

Figure 1. TGA for MTA (LEFT) and polyimide coating (RIGHT) used on BHGE CoreBright fibers.

\section{Hydrogen induced losses}

Performance in hydrogen rich atmospheres has often been the driving force for downhole fiber selection. ${ }^{2}$ Hydrogen diffuses so rapidly through most materials that, even within a sealed metal cable, hydrogen arrives at the core of a fiber within hours, days, weeks or months depending upon the temperature and exact cable design. Figure 2 shows the hydrogen induced loss after short duration exposure of a Ge-doped Allwave single-mode fiber and a pure-silica core Corebright single-mode fiber to 5 Atm hydrogen. The spectrum between 1080 and $1260 \mathrm{~nm}$ is the second overtone of molecular hydrogen that appears in all glasses. The tail of loss above $1500 \mathrm{~nm}$ is the edge of the primary hydrogen absorption peaked above $2000 \mathrm{~nm}$. These molecular hydrogen losses disappear when hydrogen is removed.

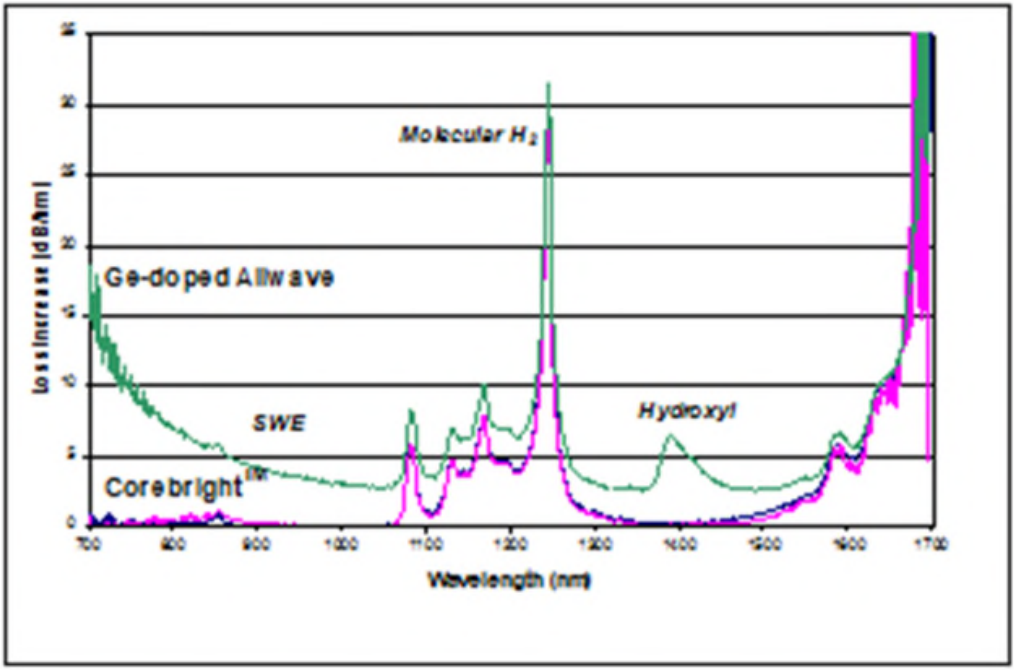

Figure 2: Hydrogen induced loss in Germanium containing SMF and in pure core Corebright SMF. 
Permanent loss changes also occur as $\mathrm{OH}$ is formed due to $\mathrm{H}$ reaction with oxygen defects in the fiber core. This is shown in the Ge containing fiber which has 2 overlapping peaks, one at $1390 \mathrm{~nm}$ and the other near $1410 \mathrm{~nm}$, due to Si and $\mathrm{Ge}$ associated $\mathrm{O}$ defects respectively. The number of such defects depends on the details of the glass manufacturing processes. In Ge-doped fibers, there is also a short-wavelength edge (SWE) loss that tails across the entire spectrum. In pure core fibers, this loss does not occur, but the Si associated $\mathrm{OH}$ peak at $1390 \mathrm{~nm}$ occurs, in proportion to the number of defects present. The CoreBright pure-core fiber in Figure 2 has so few defects that no $\mathrm{OH}$ peak is visible.

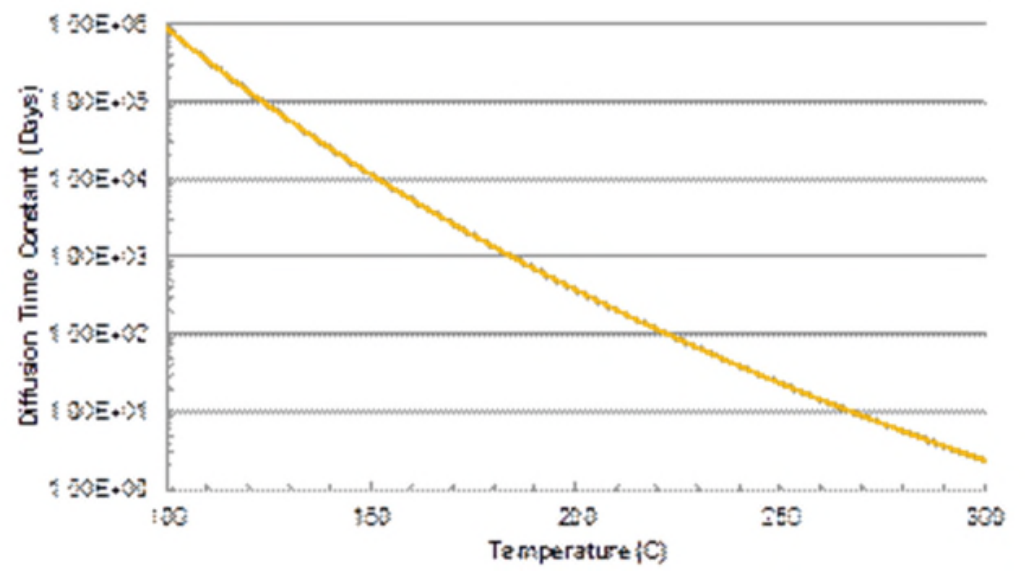

Figure 3: Typical diffusion time through high quality carbon coating vs temperature.

All hydrogen induced losses can be stopped or slowed down by the addition of carbon coating to stop hydrogen ingress. This is typically only effective for a length of time, as depicted in Figure 3, as a function of temperature for a particular high quality carbon coating. Above $150 \mathrm{C}$ even the best carbon coatings are considered ineffective for a long enough time to be relied on for resisting hydrogen ingress to the fiber core downhole.

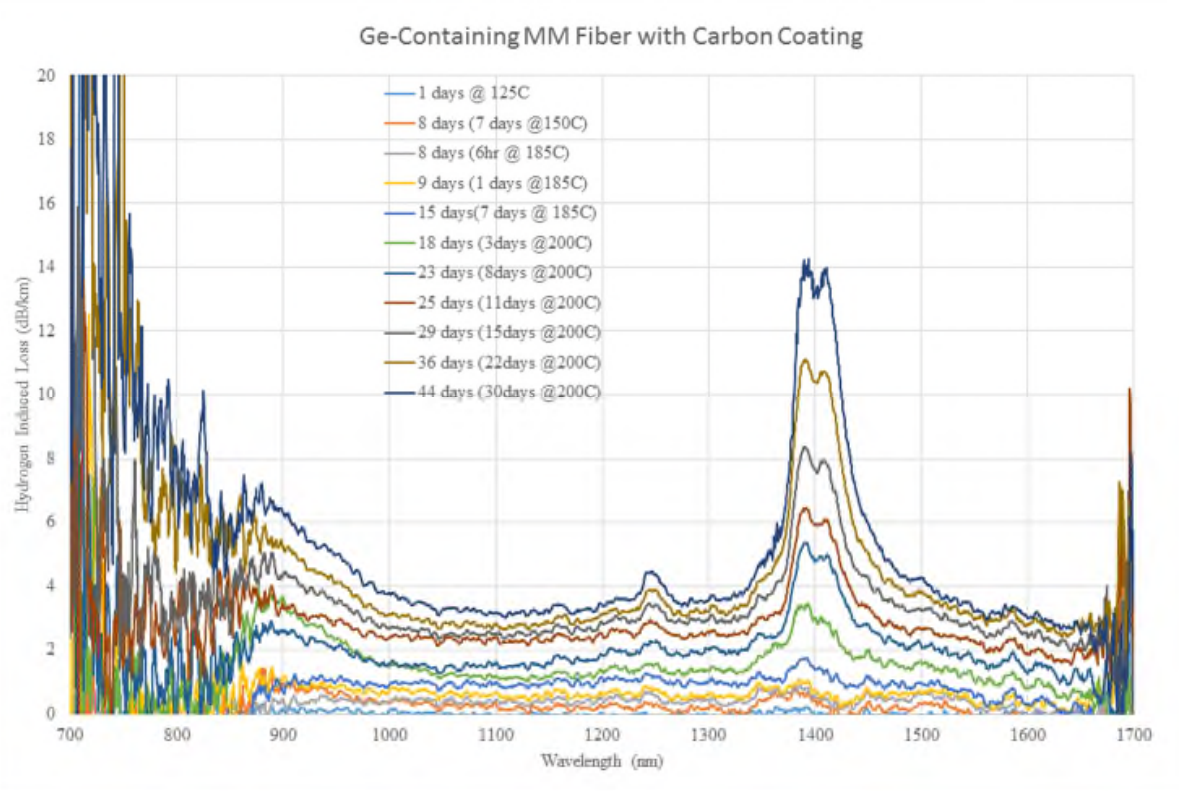

Figure 4: Hydrogen induced loss in a typical GE containing MM graded index fiber 
Single-mode pure silica core fiber like CoreBright are readily available, but some applications are better suited for multimode fiber system use. Both Ge-containing and non Ge-containing ("pure-core") MM fibers have been used. Figure 4 shows the loss seen in a MM Ge-containing fiber tested with $2 \mathrm{Atm}$ of hydrogen over increasing $\mathrm{T}$ in several time steps. Below $150 \mathrm{C}$ (first several data sets at bottom), this carbon-coated fiber does not exhibit losses, but above this temperature, losses appear and, at 200C they are continuing to grow after 44 days on test. Typical "pure-core" MM fibers contain a graded profile of fluorine to down-dope and build the core index profile. While it is possible to make a low defect MM fiber, all available "pure-core" MM fibers we have sampled contain substantial defects, we believe because they are made by plasma CVD. The behavior of such a fiber is shown in Figure 5 over the same series of tests with 2 Atm hydrogen. Note the large number of defects apparently in this fiber and their rapid reaction rate as compared with the Ge containing fiber. There is no way to utilize this fiber at $1550 \mathrm{~nm}$ without a carbon-coating barrier, which is only effective up to $150 \mathrm{C}$. However, it has a useful window of operation from 1000-1060 nm.

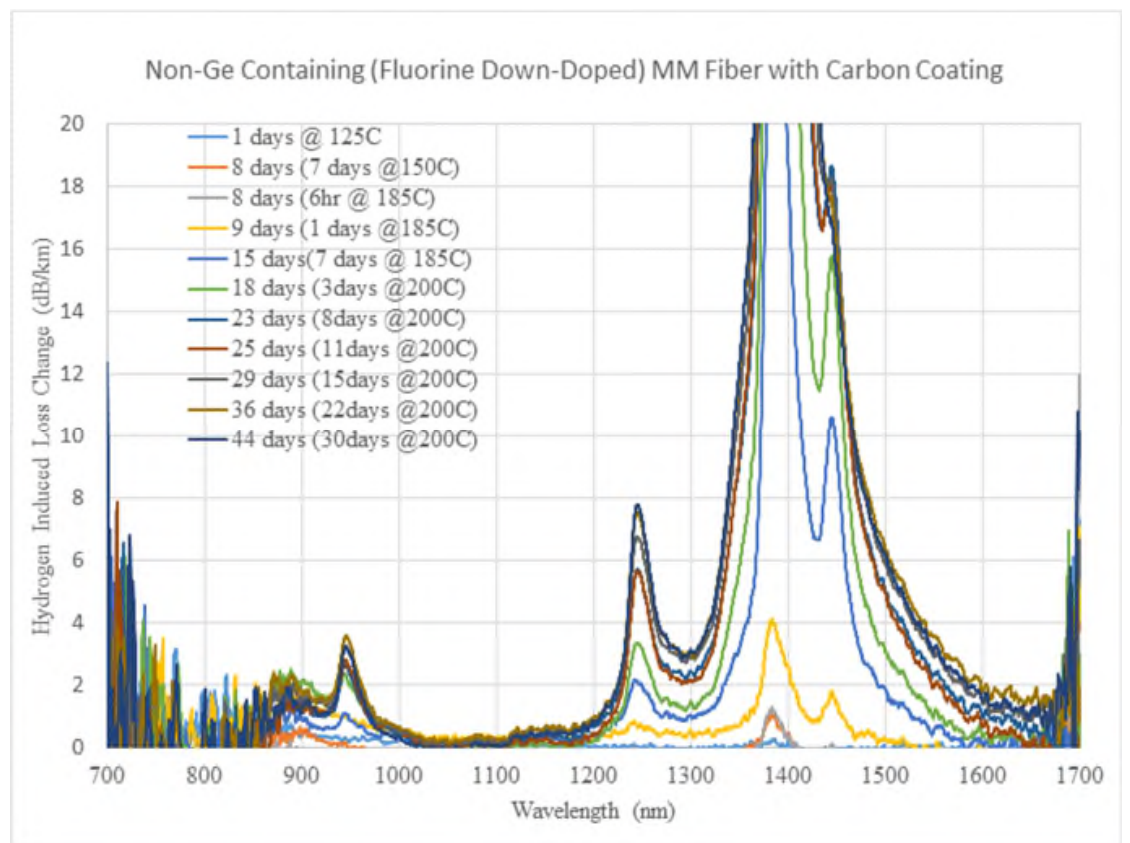

Figure 5: Hydrogen induced loss in a non-GE containing "Pure-core" MM graded index fiber

There are operators in the Oil and Gas industry that pump fibers into the well and replace them occasionally. That is to say, rather than deploy permanent cables, they deploy an empty tube and then pump a sensing fiber into the tube downhole using fluid pressure. Such a deployment allows for the removal of a fiber that has darkened and replacement with a pristine fiber. The fiber choice then becomes an economic decision of whether to deploy a more expensive hydrogen resistant fiber or deploy a standard fiber and replace it often.

\section{Microbend losses}

Another significant effort for downhole fibers has been to reduce microbend induced losses, which are a complex function of the index profile and stresses acting on the fiber in harsh environments in metal cables. Polyimide-coated fibers are more prone to microbending than acrylate-coated fibers because the thin hard polyimide coatings allow contact stresses to be transmitted to the glass. When multiple fibers are deployed in a cable, they can induce losses on each other, especially if pulled taught downhole. The pure-core fiber index of refraction is limited by the amount of fluorine that can be 
incorporated but has been pushed up over time to reduce microbending. MM and SM fibers both have undergone significant design efforts to reduce microbending losses.

\section{FBG based sensors}

While DTS and DAS are often performed in standard downhole fibers, DSS systems typically have relied on FBGs to provide discrete distributed sensors. One such system, SureView WIRE measures strain up to $2 \%$ at $2 \mathrm{~cm}$ spacing, but does so using optical frequency domain reflectometry (OFDR) and is limited to about $12500 \mathrm{FBG}$ sensors over $250 \mathrm{~m}$. The easiest ways to make such FBG arrays is to write them using a phase mask either during fiber draw or afterwards through a write-through coating. This requires a Ge doped fiber, which is prone to hydrogen induced losses and limits the length of the system as well. One might write FBGs in pure-core fibers using femtosecond laser induced damage based FBGs, but this is a far less developed technology, though an area of active development.

\section{Enhanced scatter fiber}

For some scatter based sensing systems, particularly for DAS, it has become an established fiber improvement to enhance fiber scattering to increase the detected signal level. While it is possible to add scattering particles or random index variations to a fiber by many methods, such scattering is omnidirectional and so increases loss at least in proportion to the scatter increase, which is unacceptable for long systems. It limits the effectiveness since the signal for distant parts of the fiber must travel through a higher loss path. Chirped FBG based solutions, with more directional enhancement of scattering in a particular wavelength band are advantageous in this regard. Enhanced scatter fiber are offered by fiber vendors and/or used in the industry, but the details of how they are made is not revealed. In principle, the interrogator and enhanced scatter fiber should be optimized together. Depending on the approach, the bandwidth, FBG spacing/overlap and FBG strength might be chosen to provide the optimal signal consistent with the interrogation approach. Designs of such optimized tailored fibers is an ongoing area of development. Additionally, different schemes are proposed for enhancing the transmission of acoustic waves to the fiber, and the optimization of this is an area of active development.

\section{Conclusion}

This presentation provides a survey of fiber usage issues in downhole oil and gas sensing applications. Due to unique environmental and deployment requirements, and application specific requirements, oil and gas fiber sensing utilizes:

- MM and SM sensing fibers

- $1060 \mathrm{~nm}$ and $1550 \mathrm{~nm}$ wavelength interrogators

- scattering based and FBG based sensing

- standard fibers and scatter enhanced fibers

- several deployment approaches, including prepacked cables and pumped fibers

- OFDR, OTDR and wavelength multiplexed systems

- multiple different coating types for different temperatures and properties

Interestingly, after years of development and deployment, no single choice has emerged as the preferred method or fiber for even the majority of such applications.

\section{References}

[1] Stolov, Andre A., Simoff, Debra and Li, Jie, "Thermal Stability of Specialty Optical Fibers," IEEE Journal of Lightwave Technologies, 26, 3443-3451 (2008)

[2] Baldwin, C., "Optical fiber sensing in the oil and gas industry:overcoming challenges", Proc SPIE, 9157, 23 ${ }^{\text {rd }}$ International Confernece on Optical Fiber Sensors, 9157C4 (2014). 
Invited Paper

\title{
Making optical fibers sensitive and selective to the environment via functionalization and nanostructuring
}

\author{
Heike Ebendorff-Heidepriem*a, Erik P. Schartner ${ }^{\mathrm{a}}$, Roman Kosteckia , Akash Bachhuka ${ }^{\mathrm{a}}$, Stephen C. \\ Warren-Smith ${ }^{\mathrm{a}}$

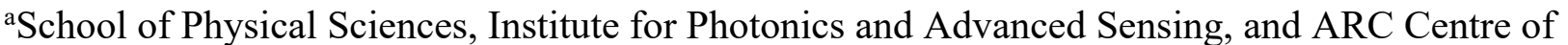 \\ Excellence for Nanoscale BioPhotonics, University of Adelaide, Adelaide, SA 5005, Australia
}

\begin{abstract}
This paper presents optical fiber based approaches of sensing various physical, chemical and biological parameters. The sensitivity and selectivity for the fibers are achieved via new types of active glass, micro/nano-structures and surface functionalization.

Keywords: optical fiber sensing, Fiber Bragg gratings, microstructured optical fibers, surface functionalization, fluorescence based sensing, plasmonic based sensing

*heike.ebendorff@adelaide.edu.au

\section{Introduction}

Optical fibers provide an attractive platform for sensing physical, chemical and biological parameters [1]. Microstructured fibers present an opportunity for high-sensitivity fluorescence measurements through their long interaction length [2]. The exposed core fiber (ECF) is particularly suited for nanostructuring and surface functionalization due to easy access to the fiber core [3-7]. Fiber tip sensors have lower sensitivity due to less efficient fluorescence capture, but they allow rapid, localized and repeated measurements $[8,9]$.

Traditional fiber materials and structures are not sensitive to the environment. Therefore, the sensing capability of an optical fiber relies on the functionalization of the fiber [10]. Here we report different strategies for fiber functionalization, such as micro/nanostructuring of optical fibers for physical or refractive index, or for fluorescence-based sensing where surface functionalization of fibers utilizing sensitive and selective fluorophores are widely used. An alternative approach is the direct doping of the sensitive fluorescence species into the fiber material, where the fiber itself becomes the sensing element such as for detection of magnetic fields.
\end{abstract}

\section{Micro/nanostructuring of optical fibers}

Micro/nanostructuring of optical fibers has been used to add sensitivity and functionality to optical fibers. Modern techniques in micro/nano-structuring include the use of femtosecond laser processing and focused ion beam milling. Femtosecond lasers provide the capability to ablate material with micron-scale resolution through non-thermal multi-photon ionization [11]. This produces stable and well controlled features that are particularly suited to producing fiber Bragg gratings (FBGs) in microstructured optical fibers as no photosensitivity is required. These FBGs can be written into ECFs (Fig. 1(a) and 1(b)) for refractive index based sensing [3,12] or into suspended-core fibers for temperature sensing in excess 
of $1000^{\circ} \mathrm{C}[13,14]$. Alternatively, focused ion beam milling uses a highly controlled ion beam to structure with resolution at the nanoscale [15]. The rate of milling is typically very slow, therefore post-processing such as tapering the optical fiber is usually performed first to reduce the amount of material. Fabry-Perot cavities as small as $2.8 \mu \mathrm{m}$ can then be written into the fiber, which produce an interferometric signal that is sensitive to the optical path length inside the cavity (Fig. 1(c)) [16-18]. These devices are sensitive to refractive index and can be used to detect biochemicals that bind to the surface. The cavities can also be made in series to perform multiplexed sensing [19] or simultaneous measurements with temperature [20].
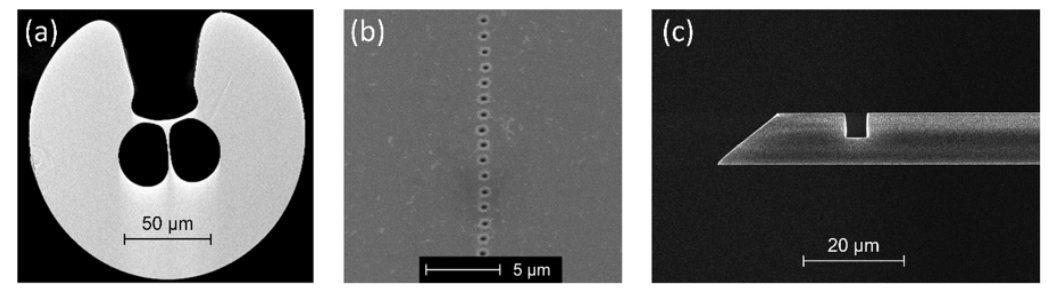

Figure 1. (a) Scanning electron microscope image of an exposed-core microstructured optical fiber (ECF). (a) Femtosecond laser ablation fiber Bragg grating written onto the core of the ECF shown in (a). (c) Tapered optical fiber with a $5 \mu \mathrm{m}$ cavity fabricated using focused ion beam milling. (a) and (b) reprinted from [12].

\section{Surface functionalization at optical fiber tips}

Surface functionalization of the tip of a multimode fiber allows localized sensing, whereby placing the tip in direct contact with the sample the signal can be recorded over time. Larger areas can be monitored via scanning the fiber tip across the sample. Fluorescence is captured less efficiently by the tip compared to a microstructured fiber, so typically a bulk coating is applied to the surface of either a polymer or glass containing the fluorescent indicators [2].

Several examples of this have been demonstrated for biological sensing applications, covering both chemical and physical sensing of the environment. One sensor employed a $\mathrm{pH}$ indicator dye, encapsulated in an acrylamide layer on the tip of the fiber (Fig. 2(a) and (b)) [8]. This sensor was used for measurements of cancerous tissue, where the $\mathrm{pH}$ of the surface could be used to give an indication as to whether the location was healthy or cancerous. The fiber tip can be sensitized to virtually any molecule simply by changing the fluorescent indicator in the polymer to one that reacts with the target species. Alternatively, the tip of the fiber can be sensitized with a rare-earth doped glass layer, where the signal from the tip changes depending on the environmental temperature (Fig. 2(c) and (d)). This sensor has been used for in-vivo measurements of temperature, examining the impacts of MDMA on brain temperature by implanting the probe within the brain of an ambulatory rat [9]. This type of sensor also has the advantage that it can be multiplexed with other sensing methods, either through different indicators located on the tip, or by imaging through the coating to simultaneously perform sensing measurements and imaging through a single fiber [21].
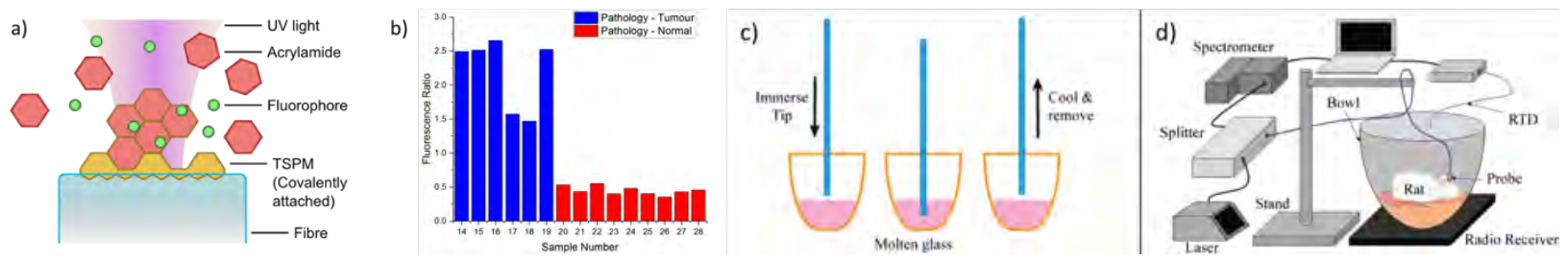

Figure 2. (a) Attachment method when embedding fluorescent dyes into a polymer coating (b) Measurement of $\mathrm{pH}$ on human tissue samples, where the $\mathrm{pH}$ of the tissue is correlated to whether the tissue is healthy or cancerous [8]. (c) Coating method for application of sensing region to the tip of an optical fiber by dipping the tip into molten glass. (d) Experimental setup for in-vivo measurements using the tip temperature sensor [9]. 


\section{Surface functionalization along the fiber core}

Fluorescence-based sensing along the core of a microstructured fiber offers enhanced sensitivity [2]. The ECF is particularly suited for immobilization sensitive and selective fluorophores onto the fiber core. The local molecular nanoenvironment of the fluorophore can be used to influence binding interactions between fluorophore and analyte, without changing the chemical structure of the fluorophore. This was demonstrated for photoswitchable spiropyran-ionophore (SI1) fluorophore, which can form both a single-ligand complex with $\mathrm{Ca}^{2+}$ and a dual-ligand complex with $\mathrm{Al}^{3+}$, (Fig. 3(Left)). The selectivity of SI1 to these ions was controlled by the nano-environment around SI1 [5]; being in solution (Fig. 3(Right,A)), chemisorbed onto the core surface of an ECF (Fig. 3(Right,B)), or physisorbed into a thin-film PMMA on the core surface of an ECF (Fig. 3(Right,C)) using methods shown in Refs. [6,22]. Solution-based measurement showed strong fluorescence for the single-ligand complex with $\mathrm{Ca}^{2+}$, but only modest fluorescence for the dual-ligand complex with $\mathrm{Al}^{3+}$ (Fig. 3(Right,A)). The response from the SI1 physisorbed within the thin-film PMMA on the ECF core (Fig. 3(Right,B)) showed that the fluorescence from the dual-ligand complex with $\mathrm{Al}^{3+}$ was twice that of the single-ligand complex with $\mathrm{Ca}^{2+}$. This is in contrast with the solution-based measurements that showed the opposite. The response from SP1 chemisorbed on the ECF core surface (Fig 3(Right,C)) showed an increase in the fluorescence intensity when exposed to $\mathrm{Ca}^{2+}$, however in the presence of $\mathrm{Al}^{3+}$ did not result in an increase in fluorescence intensity. This result was significantly different from the solution-based experiments. The control of the selectivity of a fluorophore by the nano-environment of the specific surface functionalization approach is an attractive proposition towards optical fiber-based sensing elements with highly selective surfaces for detection of biologically and/or environmentally relevant ions.
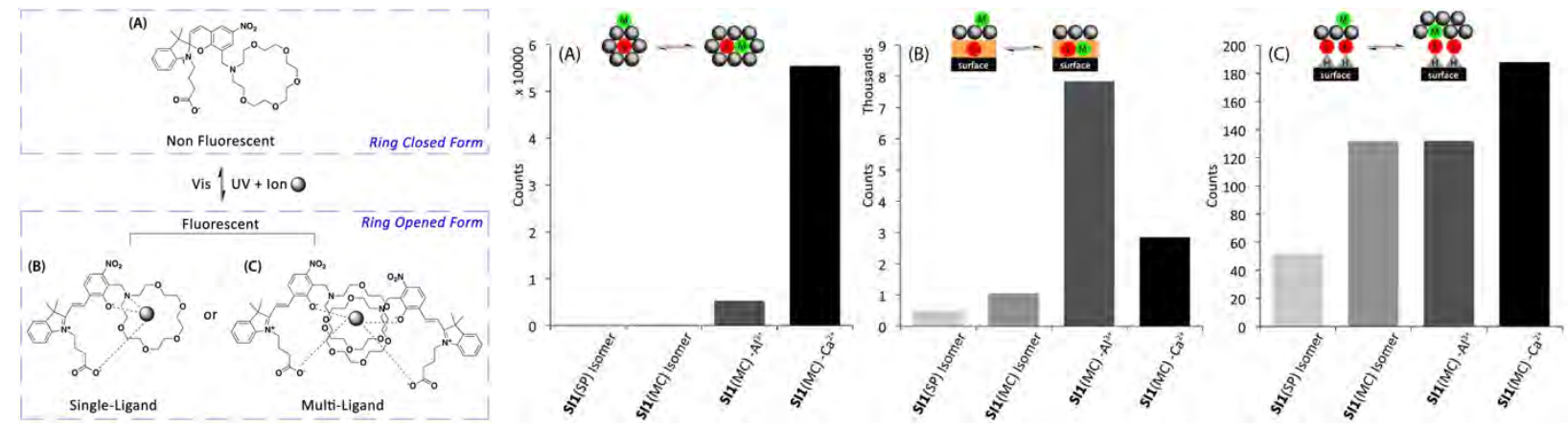

Figure 3. (Left) Cation interaction with Spiropyran-ionophore molecule (SI1) (A) represents the spiropyran (SP) ring-closed, non fluorescent form. (B) and (C) represent the ring-opened, fluorescent forms of merocyanine (MC) isomer single- and multiliganded complexes with cations. (Right) Comparison of peak fluorescence intensity from SP isomer without ions, MC isomer without ions, (SI1)MC-Ca ${ }^{2+}$ complex, and 2(SI1)MC-Al ${ }^{3+}$ complex. (A) in solution, (B) physisorbed, and (C) chemisorbed.

Plasma polymerization technique is an attractive alternative for surface functionalization of ECF [7]. A key benefit of this technique is that it is substrate independent and can generate coatings of desired physicochemical properties without requirement of any surface pre-modification $[23,24]$. In addition, it is a solvent free process, which deposits uniform and homogeneous thin films in a single step coating procedure [25]. The high potential of the plasma polymerization technique for optical fiber functionalization was recently demonstrated for $\mathrm{Al}^{3+}$ sensing with ECFs [7]. Plasma parameters such as RF power were found to control both the immobilization and the orientation of the fluorophore based sensing molecule on the surface of the ECF core. High homogeneity, reproducibility and sensitivity of plasma coated ECFs was demonstrated for equal-length sections $(15 \mathrm{~cm})$ of the same fiber. (Fig. 4) An order of magnitude improvement in sensitivity of the same 
fluorophore attached to a microstructured fiber core was achieved with the plasma polymerization technique [7] compared to conventional surface functionalization technique [26]. Using plasma polymerization technique, a vast variety of precursors can be polymerized on fibers to generate coatings of desired physical and chemical properties. This opens new avenues for immobilizing sensing molecules ranging from fluorophores to antibodies to expand optical fiber sensing capabilities.
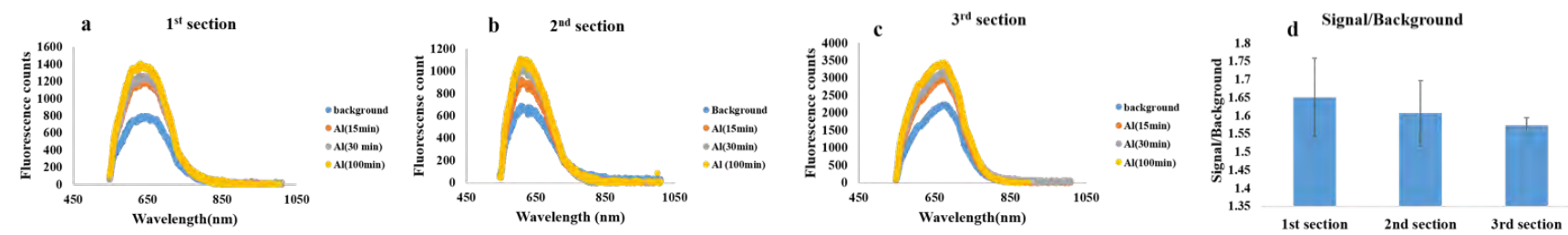

Figure 4. Fluorescence counts for a plasma polymer surface functionalized ECF (at plasma power of 25W) based aluminium sensor. a-c) Each plot corresponds to a different section of the same fiber. Blue curves correspond to background fluorescence from the fluorophore coated fiber. Orange, grey and yellow curves were recorded at 15, 30 and 100 min after exposing the fiber to $1 \mathrm{mM}$ aluminium ion solution. d) Bar plot corresponds to the average of signal-to-background ratio recorded at $100 \mathrm{~min}$ for sections (1st, 2nd and 3rd) of 3 fibers each. Figure after [7].

\section{Hybrid glass optical fibers}

Optical fibers can attain sensitivity by directly embedding the active species into the fiber material itself. This approach was successfully demonstrated for doping nanodiamond (ND) containing nitrogen vacancy (NV) centers into tellurite glass $[27,28]$. NV centers in diamond are widely explored for magnetic field sensing at room temperature. By embedding NVdiamond in tellurite glass and then fabricating an optical fiber from this hybrid glass, the first fiber with intrinsic magnetic sensitivity was demonstrated [29]. Optically detected magnetic resonance (ODMR) spectra measured with $532 \mathrm{~nm}$ CW excitation and using microwave radiation and external magnetic field (Fig. 5) demonstrate a room-temperature magnetic field sensitivity of $11 \mu \mathrm{T} / \sqrt{ } \mathrm{Hz}$. Such sensitivity is comparable to that achieved in fiber taper platforms with NDs coated on the taper surface [E3 29,30].
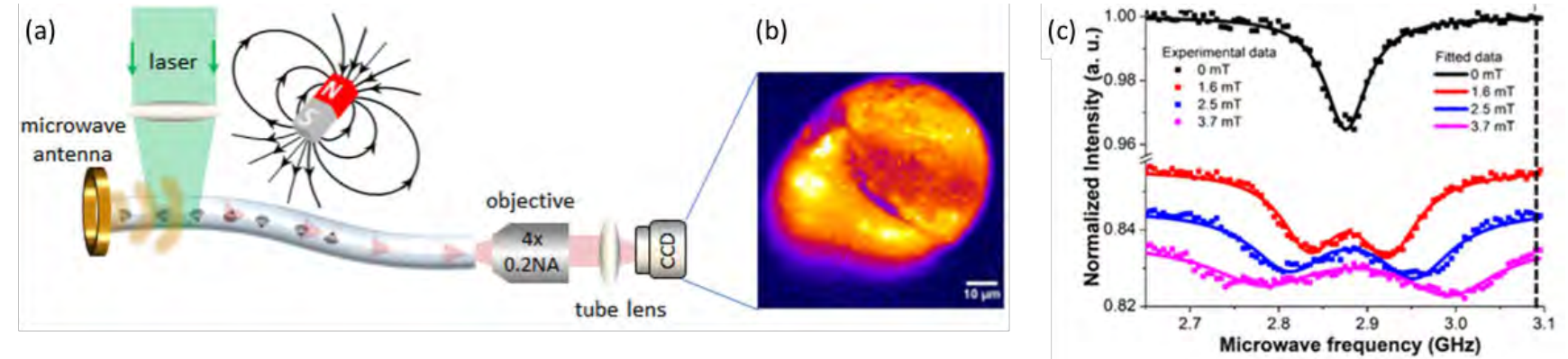

Figure 5. Optically detected magnetic resonance (ODMR) of ND doped tellurite fiber. (a) Schematic of experimental set-up for ODMR measurement using laser light at $532 \mathrm{~nm}$ for side excitation and (b) imaging the NV emission from the endface onto an camera. (c) ODMR spectra from a $\sim 200 \mu \mathrm{m}^{2}$ region of the fiber for varying external magnetic fields. The square points are measured data, and the solid lines are fitted curves. Figure after [29]. 


\section{Conclusions}

Functionalization of optical fibers is a powerful approach to add sensitivity and selectivity to optical fibers for detection of a large range of physical, chemical and biological parameters. Combination of functionalization approaches such as surface coatings and nanostructuring paves the way towards enhanced sensitivity and label-free sensing. Future work in the area of fluorescence-based sensing focusses on the development of robust coatings and customized fluorophores.

\section{Acknowledgment}

The work was supported by the following funding: Ramsay Fellowship from the University of Adelaide; Optofab node of the Australian National Fabrication Facility utilizing Commonwealth and South Australian State Government funding; Australian Research Council grants (CE14010003, FT110100225, FT160100357, FL130100119, DP120100901, DP130102494, LP150100657, LP110200736); Defence Science and Technology Group.

\section{References}

[1] E.P. Schartner, G. Tsiminis, A. François, R. Kostecki, S.C. Warren-Smith, L. Viet Nguyen, S. Heng, T. Reynolds, E. Klantsataya, K.J. Rowland, A.D. Abell, H. Ebendorff-Heidepriem, T.M. Monro, "Taming the light in microstructured optical fibers for sensing," International Journal of Applied Glass Science 6, 229-239 (2015).

[2] E. P. Schartner, G. Tsiminis, M. R. Henderson, S. C. Warren-Smith, T. M. Monro, "Quantification of the fluorescence sensing performance of microstructured optical fibers compared to multi-mode fiber tips”, Opt. Express 24, 1854118550 (2016).

[3] S. C. Warren-Smith and T. M. Monro, "Exposed core microstructured optical fiber Bragg gratings: refractive index sensing", Opt. Express 22, 1480-1489 (2014).

[4] R. Kostecki, H. Ebendorff-Heidepriem, C. Davis, G. McAdam, S. Warren-Smith, T. M. Monro, "Silica exposed-core microstructured optical fibers", Opt. Mater. Express 2, 1538-1547 (2012).

[5] R. Kostecki, S. Heng, A. M. Mak, H. Ebendorff-Heidepriem, T. M. Monro, A. D. Abell, "Control of Molecular Recognition via Modulation of the Nanoenvironment”, ACS Appl. Mater. Interfaces 10, 41866-41870 (2018).

[6] R. Kostecki, H. Ebendorff-Heidepriem, S. Afshar V., G. McAdam, C. Davis, T. M. Monro, "Novel polymer functionalization method for exposed-core optical fiber”, Opt. Mater. Express 4, 1515-1525 (2014).

[7] A. Bachhuka, S. Heng, K. Vasilev, R. Kostecki, A. Abell, H. Ebendorff-Heidepriem, "Surface functionalization of exposed core glass optical fiber for metal ion sensing", Sensors 19, 1829 (2019).

[8] E. P. Schartner, M. R. Henderson, M. Purdey, D. Dhatrak, T. M. Monro, P.G. Gill, D. F. Callen, "Cancer detection in human tissue samples using a fiber-tip pH probe”, Cancer Research 76, 6795-6801 (2016).

[9] S. T. Musolino, E. P. Schartner, M. R. Hutchinson, A. Salem, "Improved method for optical fiber temperature probe implantation in brains of free-moving rats", Journal of Neuroscience Methods 313, 24-28 (2019).

[10] J. Li, H Ebendorff-Heidepriem, B C. Gibson, A D. Greentree, M. R. Hutchinson, P. Jia, R. Kostecki, G. Liu, A. Orth, M. Ploschner, E. P. Schartner, S. C. Warren-Smith, K. Zhang, G. Tsiminis, E. M. Goldys, "Perspective: Biomedical sensing and imaging with optical fibers - innovation through convergence of science disciplines", APL Photonics 3, article $100902,1-20$ (2018)

[11] R. R. Gattass and E. Mazur, "Femtosecond laser micromachining in transparent materials", Nat. Photonics 2, 219-225 (2008). 
[12] S. C. Warren-Smith, R. Kostecki, L. V. Nguyen, T. M. Monro, "Fabrication, splicing, Bragg grating writing, and polyelectrolyte functionalization of exposed-core microstructured optical fibers", Opt. Express 22, 29493-29504 (2014).

[13] S. C. Warren-Smith, L. V. Nguyen, C. Lang, H. Ebendorff-Heidepriem, T. M. Monro, “Temperature sensing up to $1300^{\circ} \mathrm{C}$ using suspended-core microstructured optical fibers", Opt. Express 24, 3714-3719 (2016).

[14] S. C. Warren-Smith, E. P. Schartner, L. V. Nguyen, D. E. Otten, Z. Yu, D. G. Lancaster, H. Ebendorff-Heidepriem, "Stability of grating-based optical fiber sensors at high temperature", IEEE Sens. J. 19, 2978-2983 (2019).

[15] C. A. Volkert and A. M. Minor, "Focused ion beam microscopy and micromachining", MRS Bulletin 32, 389-399 (2007).

[16] T. Wieduwilt, J. Dellith, F. Talkenberg, H. Bartelt, M. A. Schmidt, "Reflectivity enhanced refractive index sensor based on a fiber-integrated Fabry-Perot microresonator", Opt. Express 22, 25333-25346 (2014).

[17] S. C. Warren-Smith, R. M. André, J. Dellith, T. Eschrich, M. Becker, H. Bartelt, "Sensing with ultra-short Fabry-Perot cavities written into optical micro-fibers", Sensor. Actuat. B-Chem. 244, 1016-1021 (2017).

[18] S. C. Warren-Smith, R. M. André, C. Perrella, J. Dellith, H. Bartelt, "Direct core structuring of microstructured optical fibers using focused ion beam milling”, Opt. Express 24, 378-387 (2016).

[19] S. C. Warren-Smith, R. M. André, J. Dellith, H. Bartelt, "Multiplexed refractive index-based sensing using optical fiber microcavities", Proc. SPIE $98991 \mathrm{G}$ (April 29, 2016).

[20] R. M. André, S. C. Warren-Smith, M. Becker, J. Dellith, M. Rothhardt, M. I. Zibaii, H. Latifi, M. B. Marques, H. Bartelt, O. Frazão, "Simultaneous measurement of temperature and refractive index using focused ion beam milled Fabry-Perot cavities in optical fiber micro-tips", Opt. Express 24, 14053-14065 (2016).

[21] J. Li, E. P. Schartner, S. Musolino, B. C. Quirk, R. W. Kirk, H. Ebendorff-Heidepriem, R. A. McLaughlin, "Miniaturized single-fiber-based needle probe for combined imaging and sensing in deep tissue" Opt. Letters 43, 16821685 (2018).

[22] S. Heng, M. Nguyen, R. Kostecki, T. M. Monro, A. D. Abell, "Nanoliter-scale, regenerable ion sensor: sensing with a surface functionalized microstructured optical fiber", RSC Adv. 3, 8308-8317 (2013).

[23] A. Michelmore, P. Martinek, V. Sah, R.D. Short, K. Vasilev, "Surface Morphology in the Early Stages of Plasma Polymer Film Growth from Amine-Containing Monomers", Plasma Processes and Polymers 8, 367-372 (2011).

[24] K. Vasilev, A. Michelmore, H.J. Griesser, R.D. Short, "Substrate influence on the initial growth phase of plasmadeposited polymer films", Chem. Commun. 28, 3600-3602 (2009).

[25] A. Michelmore, D.A. Steele, J.D. Whittle, J.W. Bradley, R.D. Short, "Nanoscale deposition of chemically functionalised films via plasma polymerisation", RSC Advances 3, 13540-13557 (2013).

[26] S.C. Warren-Smith, S. Heng, H. Ebendorff-Heidepriem, A.D. Abell, T.M. Monro, "Fluorescence-based aluminum ion sensing using a surface-functionalized microstructured optical fiber", Langmuir 27, 5680-5685 (2011).

[27] M. R. Henderson, B. C. Gibson, H. Ebendorff-Heidepriem, K. Kuan, S. Afshar V., J. O. Orwa, I. Aharonovich, S. Tomljenovic-Hanic, A. D. Greentree, S. Prawer, T. M. Monro, "Diamond in tellurite glass: A new medium for quantum information," Advanced Materials 23, 2806-2810 (2011).

[28] Y. Ruan, H. Ji, B. C. Johnson, T. Ohshima, A. D. Greentree, B. C. Gibson, T. M. Monro, H. Ebendorff-Heidepriem, "Nanodiamond in tellurite glass Part II: practical nanodiamond-doped fibers", Opt. Mater. Express 5, 73-87 (2015).

[29] Y. Ruan, D. A. Simpson, J. Jeske, H. Ebendorff-Heidepriem, D. W. M. Lau, H. Ji, B. C. Johnson, T. Ohshima, S. Afshar V., L. Hollenberg, A. D. Greentree, T. M. Monro, B. C. Gibson, "Magnetically sensitive nanodiamond-doped tellurite glass fibers", Scientific Reports 8, article 1268 (2018).

[30] I. Fedotov, et al. "Fiber-optic magnetometry with randomly oriented spins", Opt. Lett. 39, 6755-6758 (2014). 


\title{
Femtosecond laser inscribed bridging cladding waveguides combining single- and multi-core optical fibres for shape sensing
}

\author{
K. Kalli ${ }^{\mathrm{a}^{*}}$, A. Theodosiou ${ }^{\mathrm{a}}$, S. S. Stojanovic ${ }^{\mathrm{a}}$, A. Ioannou ${ }^{\mathrm{a}, \mathrm{b}}$ \\ ${ }^{a}$ Photonics and Optical Sensors Research Laboratory, Cyprus University of Technology, Limassol, \\ Cyprus; ${ }^{b}$ University of Mons, 7000, Belgium
}

\begin{abstract}
We show that femtosecond laser inscribed "bridging waveguides" can couple the displaced cores of different types of optical fibres. We explore coupling between single- and four-core fibre, incorporating multiple Bragg gratings. The sensor is calibrated for bend and shape sensing.

Keywords: Femtosecond laser inscription, fibre Bragg gratings, optical sensing, shape sensing *kyriacos.kalli@cut.ac.cy; phone 35725002609
\end{abstract}

\section{Introduction}

The interest in multi-core optical fibres results principally from improved data density on telecommunication networks or for the development of new types of vectorial optical fibre sensors. This fibre type would benefit from efficient methods for coupling multi-core fibre to existing networks or for coupling to single-core optical fibres. In particular, multiple core fibres have important applications in sensing, since the simultaneous interrogation of more than one core could be solve cross sensitivity issues, or could be used for shape sensing applications. In this work, we consider coupling between the displaced cores of different types of optical fibres. We demonstrate a novel, direct, and efficient coupling technique between single-core and four-core fibres, via femtosecond laser inscribed bridging waveguides, once the two fibre types are fusion spliced. We also incorporate fibre Bragg grating (FBG) arrays that are inscribed into three of the four cores. The bridging waveguides direct light from the single core fibre to the four-core fibre and recover the reflection spectra, so that the single core fibre can be connected to a conventional spectrometer, allowing for standard FBG characterization and demodulation. We can selectively inscribe the bridging waveguides and monitor the FBG growth or first inscribe the FBGs and assess the waveguide quality in recovering the FBG spectra. The FBGs' response were measured and calibrated for bend and shape sensing.

\section{Results}

All inscriptions were performed using a fs-laser system (HighQ laser femtoREGEN) operating at $517 \mathrm{~nm}$, with a 220fs pulse duration. The repetition rate was controlled using a pulse picker, allowing a variable repetition rate from 1-100 $\mathrm{kHz}$, with pulse energies $\sim 100 \mathrm{~nJ}$. The fibres were mounted on an air bearing translation system (Aerotech) for accurate two-axis motion and the laser beam was focused from above using a long working distance x50 objective (Mitutoyo). Accurate synchronization of the laser pulse repetition rate and stage motion allowed for suitable laser processing, and the direct-write, plane-by-plane (Pl-by-Pl), "inscribe and step" method was used to build all components (bridging waveguides and FBGs) in a piece-wise process [1-3]. A standard single mode silica optical fibre (SMF28), with core diameter of $8.2 \mu \mathrm{m}$ and a cladding diameter of $125 \mu \mathrm{m}$, was used with a 4-core fibre (core diameter $8 \mu \mathrm{m}$, cladding diameter $125 \mu \mathrm{m}$ ); the cores were distributed uniformly around to the fibre's central axis by $50 \mu \mathrm{m}$, Fig. $1 \mathrm{a}$.

Sixth International Workshop on Specialty Optical Fibers and Their Applications (WSOF 2019), edited by Liang Dong, John M. Ballato, Proc. of SPIE Vol. 11206, 112061H · C 2019 SPIE CCC code: $0277-786 X / 19 / \$ 21 \cdot$ doi: $10.1117 / 12.2547755$ 
The bridging waveguides were formed as follows. The SMF28 fibre was fusion spliced to the multi-core fibre (Fibercore Ltd), Fig. 1b; of course light launched through the SMF28 was not injected into any of the spatially-separated four cores. By inscribing waveguides near to the single fibre core (Fig. 1c), we evanescently coupled light from that core to one or more cores in the multi-core fibre, thereby acting as bridging waveguides; now the cores could transfer light signals between them. To demonstrate the efficiency of the method and the design of new kinds of optical fibre sensors, we first selectively inscribing three FBGs in three of the four cores of the multi-core fibre, using the $P l$-by- $P l$ inscription method (prior to waveguide inscription). The gratings were $4^{\text {th }}$ order, having a period of $\sim 2.15 \mu \mathrm{m}$ that were physically separated by $3 \mathrm{~mm}$, Fig. 2a. The FBGs were monitored (but not yet observed) via the SMF28 fibre; as the bridging waveguides were inscribed the FBG-array appeared with the increasing waveguide size (Fig. 2b). The final spectrum for three of the four cores connected with the SMF28 is shown in Fig. 2c.

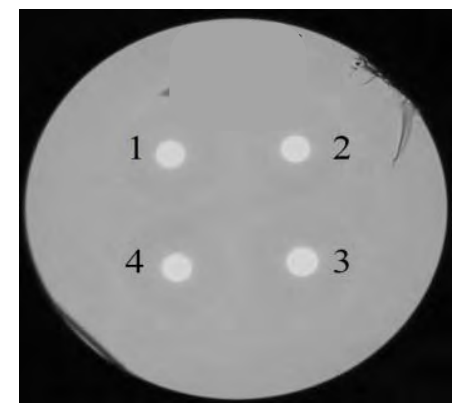

Fig. 1a. 4-core fibre endface

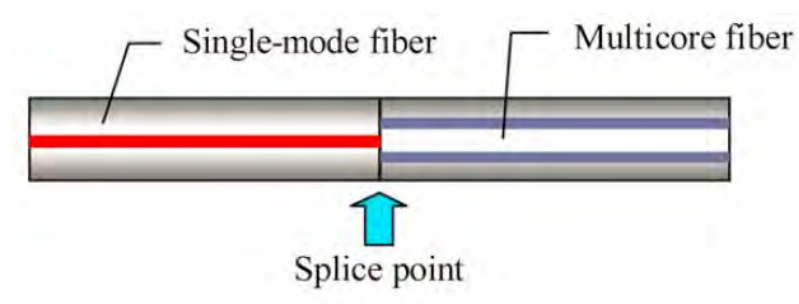

Fig. 1b. Dissimilar fibres are spliced prior to bridging waveguide inscription

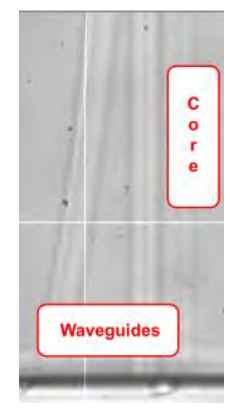

Fig. 1c. Bridging waveguides in single-core fibre cladding

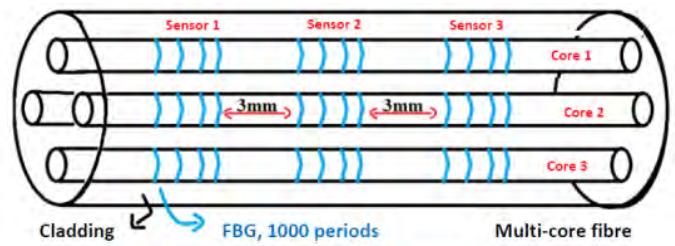

Fig. 2a. The FBGs arranged in the multi-core fibre

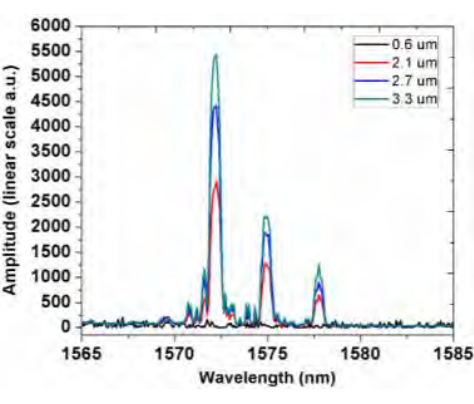

Fig. 2b. Appearance of the FBG array as the bridging waveguide width increases, recovered with SMF28

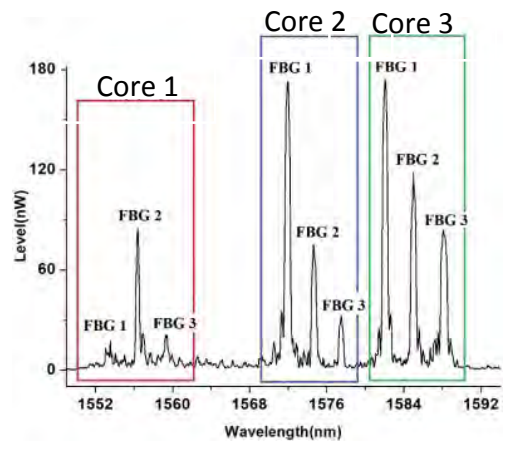

Fig. 2c. Final 9-sensor array spectrum, as recovered with the SMF28 fibre

The multi-core, FBG sensor array was calibrated for bend and used as a shape sensing element. Fig. 3a shows an example of the dependence of the Bragg wavelength shifts on the inverse of the radius of curvature for a particular rotation angle. We note that the angle is an arbitrary value, where $0^{\circ}$ represents the sensor as first mounted. However, we garner information on the sensor position from each FBG-core sensor's wavelength shift. Fig. 3b shows the applied fibre compression, the Bragg sensor separation in series (3-mm apart in each core), whereas, the y-axis displacement indicates the potential fibre travel perpendicular to the bend, for which there is zero displacement, for a fibre curvature of $\mathrm{C}=$ $4.7855 \mathrm{~m}^{-1}$.

We have shown the potential for a new type of single- to multi-core optical fibre coupler that uses femtosecond laser inscribed "bridging" waveguides to couple the cores. The approach offers a flexible approach to couple selectively to any 
one of the many cores. Moreover, we have demonstrated that this also allows for the development of a novel, multi-core, multi-sensor element that has applications to shape sensing.

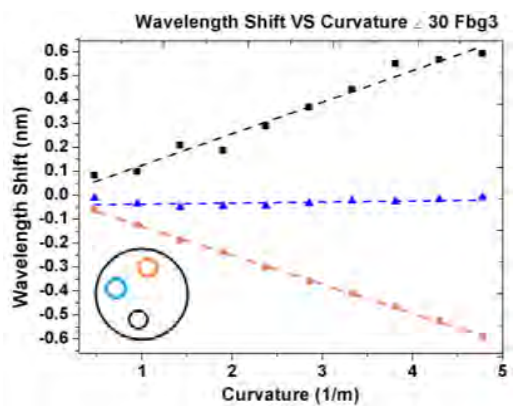

Fig. 3a. Core 3 lies on the neutral axis, core 1 experiences strain, and core 2 compression

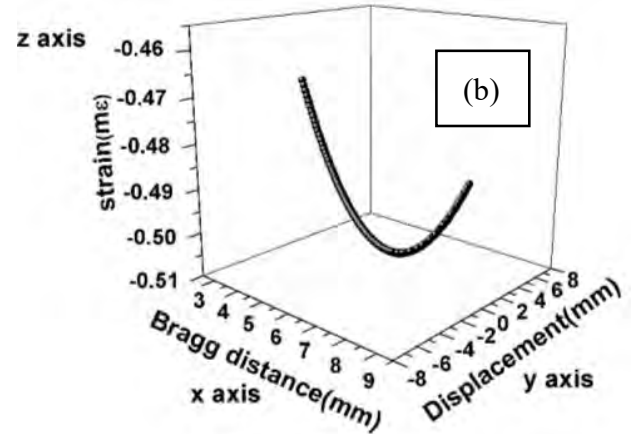

Fig. 3b. The shape of the fibre curvature

\section{References}

[1] Theodosiou, A., Lacraz, A., Polis, M, Kalli, K., Tsangari, M., Stassis, A., and Komodromos, M., "Modified fs-laser inscribed FBG array for rapid mode shape capture of free-free vibrating beams," IEEE Photonics Technology Letters 28(14), 1509-1512 (2016). [2] Theodosiou, A., Lacraz, A., Stassis, A., Koutsides, C., Komodromos, M., and Kalli, K., "Plane-by-Plane femtosecond laser inscription method for single-peak Bragg gratings in multimode CYTOP polymer optical fiber," Journal of Lightwave Technology 35(24), 5404-5410 (2017).

[3] Ioannou, A., Theodosiou, A., Caucheteur, C., and Kalli, K., "Direct writing of plane-by-plane tilted fiber Bragg gratings using a femtosecond laser," Optics Letters 42(24), 5198 - 5200 (2017). 


\title{
Polymer end-capped fiber sensors for biomedical applications
}

\author{
Ricardo Defas-Brucil ${ }^{\mathrm{a}}$, Mildred Cano-Velázquez ${ }^{\mathrm{a}}$, Juan Hernández-Cordero*a \\ ${ }^{a}$ Instituto de Investigaciones en Materiales, Universidad Nacional Autónoma de México, Circuito \\ Exterior, Cd. Universitaria, México City, 04510, México
}

\begin{abstract}
We demonstrate a simple fabrication procedure for polymer end-capped Fabry-Pérot sensors. We demonstrate the potential of these devices for biosensing, as well as for developing fiber probes for pressure and temperature measurements.
\end{abstract}

Keywords: Fabry-Pérot sensors, fiber biosensors, biomedical fiber devices.

*jhcordero@iim.unam.mx; phone+52 55 5622-4588

\section{Introduction}

Fiber optic sensors based on Fabry-Perot interferometers have been used extensively for measuring chemical and physical parameters. Strain, molar concentration, temperature and refractive index, to name a few, have been successfully monitored using fiber-based devices. Amid the different techniques for fabricating extrinsic Fabry-Perot (F-P) cavities, the use of polymer overlays on the tip of standard single-mode optical fibers has gained a lot of attenntion [1-5]. This is mostly due to the ease of processing of polymeric materials, which are typically easy to deposit and readily curable on the tip of the fibers. Several UV-curable polymers have been shown to provide a suitable platform for developing the so-called polymer end-caps forming the F-P cavity in these structures. Refractive index, temperature, pressure and humidity measurements have been carried out with such polymers [1-4], yielding robust structures and in some cases with multiparameter sensing (pressure and temperature) capabilities [5]. For biosensing applications, adequate biocompatible polymeric platforms capable to support bioactive molecules for biorecognition are always required [6]. Polydimethylsiloxane (PDMS) for instance, has become one of the main choices for biomedical related applications owing to its biocompatibility, chemical inertness, haemocompatibility, and optical transparency. It is further easy to process, flexible and has low surface energy allowing for creating complex arrays for microfluidic biosensors and for miniaturizable detection systems.

PDMS end-caps have been used in extrinsic F-P sensors thereby offering new opportunities for biomedical applications [4]. In this work, we report on advances on the fabrication of PDMS-based extrinsic F-P sensors. A key step in the fabrication process is the deposition of the PDMS end-cap and, as shown below, we are able to obtain highly reproducible results through a controlled dip-coating procedure. The potential for developing biosensors using this F-P end-capped devices is demonstrated upon funciontalizing the PDMS end-cap with a bioactive lipid antigen cocktail from Mycobacterium fortuitum, used as a surrogate source of antigens for tuberculosis diagnosis [7]. In addition, we also demonstrate a novel 
approach for fabricating a temperature and pressure sensor using these end-capped devices. Our goal is to develop a fiber probe based on a single PDMS end-cap with enhanced sensitivity and capable to register both parameters for intracranial applications [8]. To achieve this, we have explored the use of a double cavity F-P sensor fabricated upon "inflating" a microbubble inside the PDMS end-cap. The microbubble surfaces serve as the reflectors of the F-P cavities whose features are affected differently by pressure and temperature. We describe the fabrication process as well as the performance of these PDMS-microbubbles sensors. Our results show that these PDMS end-capped sensors offer attractive features and great potential for developing novel lipidomic analytical tools (e.g. for biomarker screening or serodiagnostics), as well as for fabricating fiber probes for biomedical applications.

\section{Sensor fabrication}

\section{PDMS end-cap deposition}

The F-P fiber sensor tip comprises a PDMS (Sylgard 184, Dow Corning) cap on the cleaved end face of a standard singlemode optical fiber (SMF-28e). A mixture of PDMS precursor and cross-linker (10:1 by mass) was prepared, mixed and degassed. The polymer end-cap is deposited by dip coating; to avoid Marangoni effects that usually displace the polymer upwards along the fiber [9], the viscosity of the PDMS mixture was increased following a pre-curing process $\left(70^{\circ} \mathrm{C}\right.$ during $15 \mathrm{~min}$ ). After pre-curing, the flat end face of the fiber was dipped into a drop of PDMS using a computer-controlled system for fiber positioning and pulling. This allows for setting the appropriate velocities for driving the fiber in and out of the precured PDMS. Based on previous experiments for liquids analysis on optical fiber tips [9], the fiber displacement velocity was set to $1.7 \mathrm{~mm} / \mathrm{s}$ yielding a semi-spherical cap on the end face of the optical fiber. The end-capped sensor was subsequently placed in an oven $\left(180^{\circ} \mathrm{C}\right.$ during $50 \mathrm{~min}$.) to fully solidify the PDMS. During the dip-coating process, the back-reflected signal from the fiber sensor was continuously monitored using a fiber Bragg grating (FBG) interrogator (Micron Optics, sm125).

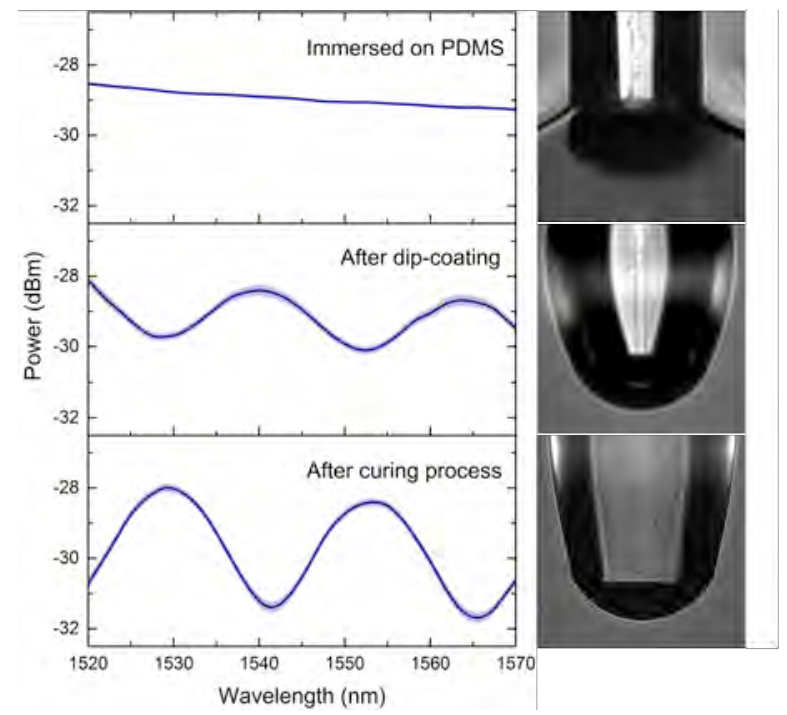

Figure 1. Back-reflected spectra obtained during the end-cap deposition on the tips of single-mode fibers. Note that the spectra include results obtained from three different probes fabricated using the same immersion parameters. The images show the fiber tip during immersion, after extraction from the PDMS and after curing. 
Reflections from the fiber end-face and the PDMS produce an interference pattern that can be monitored continuously during the fabrication of the sensors (see Fig. 1). As shown in the figure, the controlled dipping process allows for obtaining similar spectra for three different fiber tips (notice that all spectra are plotted on the same graph), even at different stages of the fabrication process (i.e., after PDMS deposition and after curing). Since all the relevant parameters for the dipping process are kept under controlled (i.e., PDMS volume and pre-curing, fiber pulling velocities), the fabrication process consistently yields sensors with similar features. For all three cases, the spectral features and calculated free spectral range FSR is similar $(\approx 24.876 \mathrm{~nm}$ ) corresponding to an effective cavity length (cap size) of approximately $33.423 \mu \mathrm{m}$.

\section{End-cap functionalization: biosensor fabrication}

Biosensors generally rely on hydrophilic molecules (e.g., enzymes and other proteins, nucleic acids or peptides) as biorecognition. Since PDMS is highly hydrophobic, surface modification methods must be done in order to promote the attachment of these molecules. In general, the hydrophobicity of PDMS has been considered as a disadvantageous feature for biosensing, since most measurements are typically performed on aqueous-based systems. To overcome this limitation, we have explored the use of this polymer for the attachment of lipidic molecules showing affinity to hydrophobic surfaces. Specifically, Mycobacterium fortuitum, a rapidly growing mycobacterial species, was used as a source of antigens [7]. After fabrication of the PDMS end-caps, mycobacterial lipid antigens were immobilized on the PDMS simply by immersing the device in $200 \mu \mathrm{L}$ of ethanol-hexane $(1: 1, \mathrm{v} / \mathrm{v})$ with an antigen concentration of $8(\mu \mathrm{g} / 100 \mu \mathrm{L})$ for 30 minutes, followed by a 15 minutes drying process at room temperature. Free binding sites were blocked by immersing the sensor tip in $200 \mu \mathrm{L}$ of $3 \% \mathrm{BSA}$ for $30 \mathrm{~min}$ at $37^{\circ} \mathrm{C}$, followed by five consecutive immersion washes in PBS (300 $\left.\mu \mathrm{L}\right)$. A total of three sensors were functionalized and treated following this procedure.

\section{Microbubble generation within the PDMS endcap: double cavity F-P devices}

Several approaches have been demonstrated for simultaneously measuring pressure and temperature with polymer endcapped F-P sensors. However, they usually involve elaborated fabrication processes and most devices are typically intended for temperature and pressure ranges way beyond those required for intracranial applications. Our approach for increasing the sensitivity of the end-capped sensors is based on the fabrication of a microbubble inside the PDMS end-cap [8]. The microbubble introduces an additional cavity within the sensor thus yielding a modulated interference pattern. Because the bubble and the PDMS surfaces will show different sensitivities to pressure and temperature, information about these parameters may be obtained through spectral analysis of the modulated signal.

The micro-bubble end-capped sensors are fabricated using optical fiber microheaters [8]. These are obtained upon depositing a thin layer of carbon nanoparticles (CNPs) on the cleaved end-face of a single-mode optical fiber (SMF-28e) by means of a simple optically assisted deposition process []. After deposition of the CNPs, the fiber tips are immersed in precured PDMS following the same dip-coating procedure described previously. Subsequently, a micro-bubble is formed within the polymer drop by pumping the micro-heater with a fiber-coupled laser diode (Thorlabs, $980 \mathrm{~nm}, 250 \mathrm{~mW}$ maximum output power) (see Fig. 2). Micro-bubble formation arises from photo-thermal effects observed in the carbon nanotubes layer creating highly localized heat. The polymer with the micro-bubble is then cured for 1 hour at $170{ }^{\circ} \mathrm{C}$. As depicted in Fig.2, a double cavity F-P is created with this configuration: one cavity defined by the micro-bubble surfaces and a second cavity formed by the remaining polymer layer. The setup for fabricating the sensors (also shown in Fig. 2) includes a WDM $(980 / 1550 \mathrm{~nm})$ to feed the pump signal required for generating the micro-bubble, and the FBG interrogator (Micron Optics, SM-125) for monitoring the interference pattern of the sensors. 

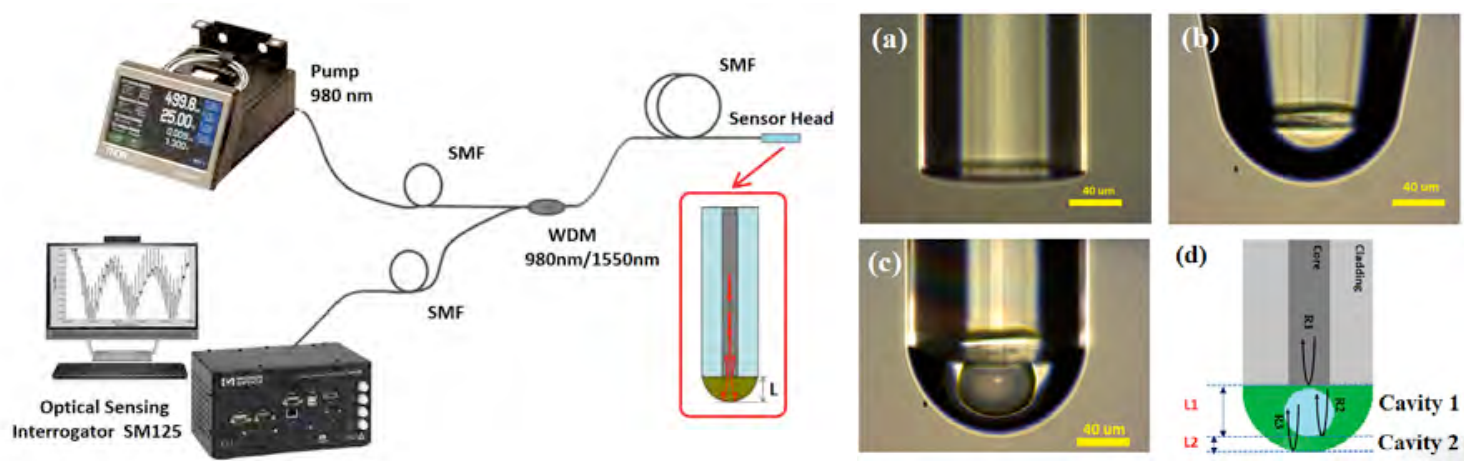

Figure 2. Experimental setup used for fabricating the microbubble F-P end-capped sensors (left); the interference pattern is monitored with an FBG sensor interrogator. The images show the different steps for sensor fabrication: an optical fiber with a thin layer of CNPs on the end-face prior immersion on PDMS (a); the fiber tip after deposition of the PDMS end-cap (b); a F-P sensor with a microbubble after curing (see text for details); (d) illustrates the two cavities obtained with this fabrication process.

\section{Results and Discussion}

\section{Biosensor performance}

The performance of the PDMS end-capped biosensors was evaluated by immersing the devices in pre-immune and hyperimmune serums. These were obtained from two white New-Zealand rabbits as previously described in [7]. Each sensor was first immersed in $200 \mu \mathrm{L}$ of pre-immune serum dilution $(1: 400 \mu \mathrm{L} / \mu \mathrm{L})$ during $30 \mathrm{~min}$ at $37^{\circ} \mathrm{C}$. Subsequently, the same sensors were used for testing hyper-immune serum after removing the bound lipid ligands attached to the PDMS cap. This was done by immersion ( 5 times) in a denaturalizing buffer solution with $\mathrm{pH} 3$. The free binding sites on the fiber tips were immersed again in BSA for blocking and finally washed five consecutive times in PBS $(300 \mu \mathrm{L})$. After this process was finished, the tips were immersed in $200 \mu \mathrm{L}$ of $1: 400(\mu \mathrm{L} / \mu \mathrm{L})$ hyper-immune serum dilution during 30 min at $37^{\circ} \mathrm{C}$. While immersed in the serums, the back-reflected interferometric signal from the sensors was continuously monitored and the shift in wavelength over time $(\Delta \lambda)$ was obtained by cross-correlation of the acquired spectra. For control purposes, we also tested non-functionalized sensors (i.e., without antibodies) during the experiments.

The resulting wavelength shifts for all the tested solutions and for the three functionalized sensors are shown in Fig. 3. Notice first that thermal expansion effects are clearly noticeable from the sensor readout: after the sensor tip is immersed in PBS at $37{ }^{\circ} \mathrm{C}, \Delta \lambda$ increases reaching a maximum after 30 minutes (curve A). At this time, the PDMS cap seems to reach its maximum length due to thermal expansion. Thermal expansion effects are also noticeable in the untreated FPI sensors (curves B and C); notice further that upon immersing them in the serums (either pre-immune or hyper-immune), the registered $\Delta \lambda$ is different than that obtained for the PBS solution. This is attributed to the difference in refractive indices of both solutions (PBS and serums). The time evolution of $\Delta \lambda$ follows a similar trend in both cases and, as before, the maximum wavelength shift was achieved after 30 minutes. These results confirm that thermal expansion effects lead to an increase in the length of the PDMS cavity. We can also conclude that there is no attachment of antibodies on the sensors without the antigen; thus, it is not possible to discern between the pre-immune and the hyper-immune serums. In contrast, the response of the functionalized sensors during immersion in the serums is noticeably different (curves D and $\mathrm{E}$ in Fig. 3). While $\Delta \lambda$ still 
changes over time showing the thermal expansion effects of PDMS, the maximum wavelength shifts obtained with both serums (pre-immune and hyper-immune) are larger than those obtained with the untreated FPI devices. This is attributed to antibody binding on the functionalized PDMS cap, which may in turn affect the spectral response of the sensors. Moreover, the $\Delta \lambda$ registered during immersion in the hyper-immune serum (curve E) is larger than that obtained with the pre-immune serum (curve D). This is a reasonable outcome as the former serum contains a larger number of specific antibodies, thus increasing the binding events and thereby leading to a larger $\Delta \lambda$. Notice further that the readouts for the hyper-immune serum were obtained after washing the probes. Hence, the antigen coating is preserved on the PDMS cap and the sensors can be readily reused.

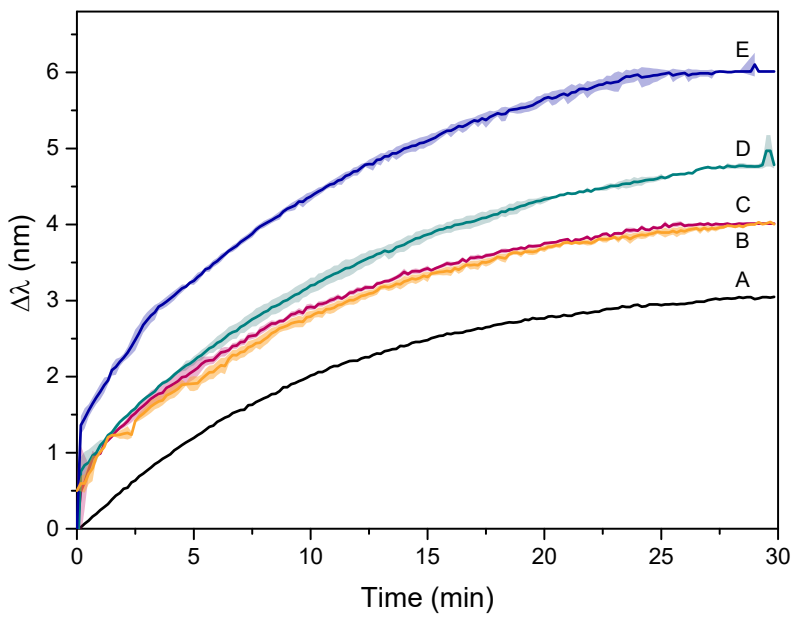

Figure 3. Wavelength shift $(\Delta \lambda)$ during detection of antigen-antibody binding on PDMS. Curves A, B and $\mathrm{C}$ show the performance of untreated (i.e., without the lipid antigen) sensors during incubation in $\mathrm{PBS}\left(\mathrm{T}=37^{\circ} \mathrm{C}\right)(\mathrm{A})$; (B) and (C) were obtained during immersion in serums from lipid-immunized rabbits (pre-immune and hyper-immune, respectively). Curves D and E were obtained with functionalized sensors immersed in pre-immune and hyper-immune serums, respectively. The shadows on the curves represent the standard deviation from triplicate measurements.

\section{Microbubble sensor performance}

A typical reflection spectrum of the double cavity F-P sensor is shown in Fig. 4; this was obtained under room temperature and standard atmospheric pressure conditions. The back-reflected signal registered by the FBG interrogator clearly resembles a multi-mirror F-P interferometer, showing a modulated interference pattern. Theoretically, the back-reflected intensity from a three-mirror F-P model may be used to represent a double cavity device; this can be expressed as:

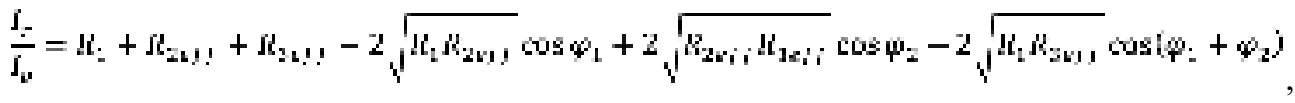

where $R_{1}, R_{2 \text { eff }}$ and $R_{3 e f f}$ denote the effective reflectivity on the three mirror surfaces, and $\varphi_{1}$ and $\varphi_{2}$ represent the phase difference experienced by the beam when traversing the polymer and the air cavity, respectively. As shown in Fig. 4, the interference pattern obtained with this model agrees with the experimental spectrum. Using this model we have observed a linear shift towards longer wavelengths with an increase in temperature. Similarly, when simulating pressure changes the 
spectrum varies owing to a reduction in the cavity formed by the microbubble, leading to a spectral shift in the opposite direction to that observed for changes in temperature.
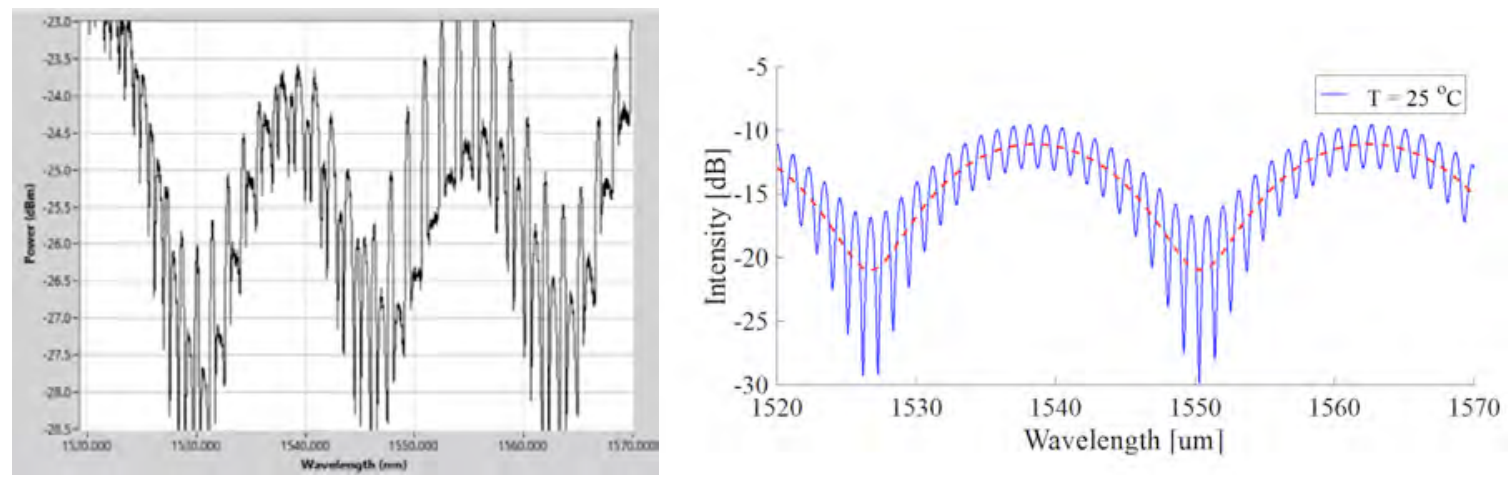

Figure 4. Measured reflected spectra from the microbubble PDMS end-capped fiber sensor (left). The spectrum obtained using a three-mirror F-P device (right) shows similar features to those obtained with the fabricated sensor.

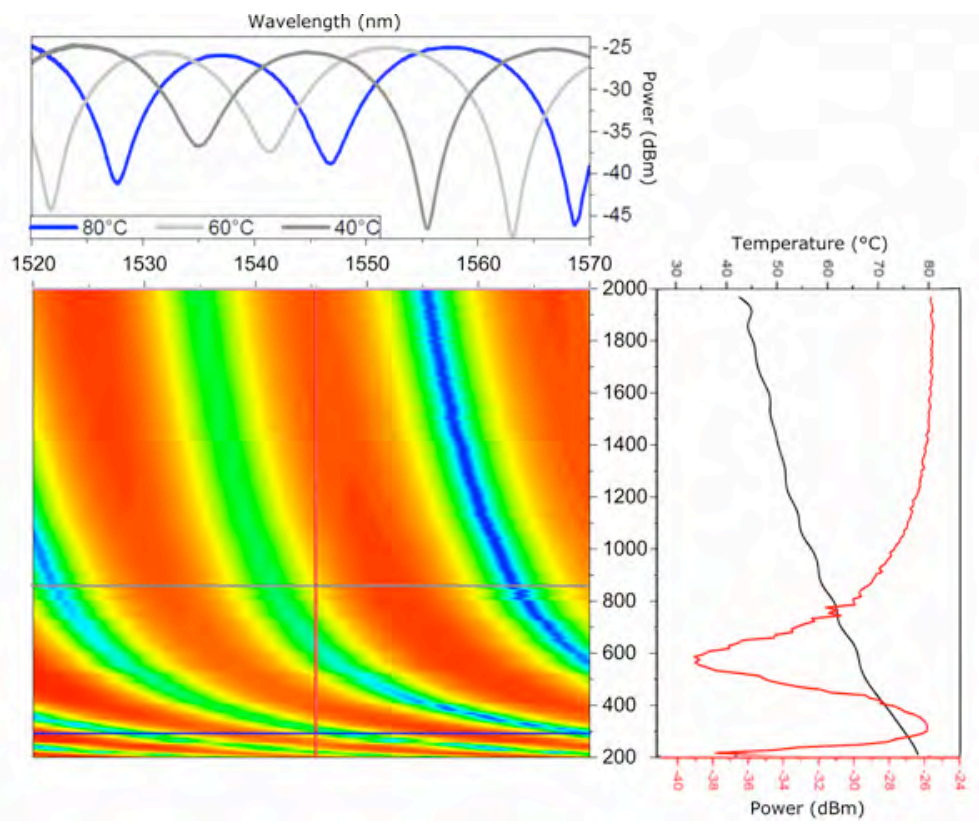

Figure 5. Reflected spectra from the microbubble F-P sensor.

As an example of the temperature tracking capabilities of the microbubble device, we monitored the spectral features of the sensor while subjected to temperature changes in an oven running a temperature cycle from $40{ }^{\circ} \mathrm{C}$ to $80{ }^{\circ} \mathrm{C}$ and subsequently cooled back to $40^{\circ} \mathrm{C}$. Fig. 5 shows a contour plot obtained upon stacking the resulting spectra while the temperature was decreasing for the cooling part of the cycle. During heating, the PDMS and the microbubble experience thermal expansion 
according to their thermal properties. During cooling, the spectra show a blue shift since the PDMS returns to its original dimensions. The microbubble also experiences a reduction in size during cooling and thus contributes to the registered wavelength shift as well.

\section{Conclusions}

PDMS end-capped fiber sensors offer attractive features for developing sensors intended for biomedical applications. Aside from their ease of fabrication, the polymer offers adequate qualities for fabricating biocompatible devices. The fabrication procedure followed to obtain the proposed devices is simple and yields reproducible results. For biosensing applications, the end-caps based on PDMS provide a suitable support for lipidomic entities that have not been fully explored. This therefore opens new possibilities for developing novel biosensors showing attractive features such as ease of fabrication and lipid functionalization, as well as label-free, real-time, direct detection, and reusability. As for the microbubble sensor, the fabrication process is also simple, although more experiments are required in order to fully evaluate its performance for monitoring temperature and pressure in biomedical applications.

\section{Acknowledgments}

This work was partially funded by Conacyt (FONCICYT, grant 246648) and by DGAPA UNAM (PAPIIT IG100519).

\section{References}

1. Tan, X. L., Geng, Y. F., Li, X. J., Deng, Y. L., Yin, Z., \& Gao, R. (2014). UV-curable polymer microhemisphere-based fiber-optic Fabry-Perot interferometer for simultaneous measurement of refractive index and temperature. IEEE Photonics Journal, 6(4), 1-8.

2. Zhang, X. Y., Yu, Y. S., Zhu, C. C., Chen, C., Yang, R., Xue, Y., \& Sun, H. B. (2013). Miniature end-capped fiber sensor for refractive index and temperature measurement. IEEE photonics technology letters, 26(1), 7-10.

3. Hernández-Romano, I., Cruz-Garcia, M. A., Moreno-Hernández, C., Monzón-Hernández, D., López-Figueroa, E. O., Paredes-Gallardo, O. E., \& Villatoro, J. (2016). Optical fiber temperature sensor based on a microcavity with polymer overlay. Optics express, 24(5), 5654-5661.

4. Arrizabalaga, O., Velasco, J., Zubia, J., de Ocáriz, I. S., \& Villatoro, J. (2019). Miniature interferometric humidity sensor based on an off-center polymer cap onto optical fiber facet. Sensors and Actuators B: Chemical, 126700.

5. Guermat, A., Guessoum, A., Demagh, N. E., Zaboub, M., \& Bouhafs, Z. (2018). Fibre-optic temperature and pressure sensor based on a deformable concave micro-mirror. Sensors and Actuators A: Physical, 270, 205-213.

6. Tseng, Y. T., Chuang, Y. J., Wu, Y. C., Yang, C. S., Wang, M. C., \& Tseng, F. G. (2008). A gold-nanoparticle-enhanced immune sensor based on fiber optic interferometry. Nanotechnology, 19(34), 345501.

7. López-Marín, L. M., Segura, E., Hermida-Escobedo, C., Lemassu, A., \& Salinas-Carmona, M. C. (2003). 6, 6'Dimycoloyl trehalose from a rapidly growing Mycobacterium: an alternative antigen for tuberculosis serodiagnosis. FEMS Immunology \& Medical Microbiology, 36(1-2), 47-54.

8. Pimentel-Dominguez, R., \& Hernandez-Cordero, J. (2016, August). Fabrication and Applications of Optical Fiber Microheaters. In Latin America Optics and Photonics Conference (pp. LW2C-1). Optical Society of America.

9. Márquez-Cruz, V. A., \& Hernández-Cordero, J. A. (2014). Fiber optic Fabry-Perot sensor for surface tension analysis. Optics express, 22(3), 3028-3038. 


\title{
Data acquisition from a bundle of 1,000 optical fibers
}

\author{
David Ward, SemQuest Inc. United States
}

In existing fiber technology areas there are multi-core fibers or fiber bundles which can be used for applications such as endoscopic imaging. Those systems enable transmission of imagery from one end of the cable to the other by utilizing one optical channel for each fiber core "pixel". In those systems, a number of imaging technologies can be utilized such as CCD or CMOS imagers in order to receive the optical information [1-4]. However, when each fiber contains uncorrelated data at high bandwidth these techniques can limit the system. The bandwidth of any fiber in the system is limited to the frame rate of the imager and in addition, all pixels in the imager are sampled regardless of a fiber being present in that portion of the imaging array.

An integrated circuit containing a mixture of CMOS devices and photo-detectors was created to enable a low SWaP solution to achieving high bandwidth and low cost coupling to a fiber bundle of 1,000 randomly positioned fibers. The fiber bundle is manufactured to achieve a polished surface and mated to an integrated circuit with a small gap. An array of detector pixels is positioned in a rectangular form factor similar to a traditional imaging array. Each pixel contains a photo-detector in addition to a collection of analog switches and programmable memory registers. The $180 \mathrm{~nm}$ process geometry utilized in our prototype enables a $70 \%$ photo-detector fill factor for a $20 u \mathrm{~m}$ pixel. The conductivity of the switches is controlled by the state of the memory bits. The switches enable an electrical connection between neighboring pixels so that groups of pixels can be ganged together to form one large photo-detector. The pixels being one sixth the size of a fiber, a group of about 25 pixels can be formed anywhere within the pixel array to convert optical power projected by one fiber into electrical charge. In addition to forming connections between pixels, the switches also connect the photodetector and associated group of pixels to peripheral readout circuits which are aligned to the rows and columns of the array and fill the edges thereby enabling readout from any pixel. These readout circuits enable high dynamic range signal sampling range since they are not confined to the small area available in the pixels.

With the ability to gang pixels together anywhere within the array and route them electrically to a readout channel, a variety of functions could exist in the readout circuits. We have demonstrated an integrating amplifier which converts photo-charge to voltage. Channel outputs are multiplexed together into a single serial analog readout where they are converted to digital values using an off the shelf analog to digital converter. In this way, fiber optical power can be sampled at over 1,000 times per second utilizing a single multiplexed analog line. Using this technology we have demonstrated a data acquisition system in a 1" diameter circuit board using less than 1 Watt of power consumption. This system is integrated into the payload section of an $81 \mathrm{~mm}$ diameter mortar or rocket and collect laser irradiance data in flight.

Future systems will utilize the same detector array architecture coupled to level sensitive channels and digital processing circuits. In this way, fiber optical bandwidths over 100 $\mathrm{MHz}$ are possible. In addition the combination of larger arrays, smaller pixels, and smaller fiber diameter all lead to single chip systems that can handle multi-1,000 fiber bundles.

Sixth International Workshop on Specialty Optical Fibers and Their Applications (WSOF 2019), edited by Liang Dong, John M. Ballato, Proc. of SPIE Vol. 11206, 112061J · (C) 2019 SPIE

CCC code: $0277-786 X / 19 / \$ 21 \cdot$ doi: $10.1117 / 12.2542237$ 


\title{
Fabrication of a tellurite hollow core optical fiber for mid- infrared transmission
}

\author{
Tong Hoang Tuan*, Nobuhiko Nishiharaguchi, Takenobu Suzuki and Yasutake Ohishi \\ Research Center for Advanced Photon Technology, Toyota Technological Institute, 2-12-1 \\ Hisakata, Tempaku, Nagoya, 468-8511, Japan.
}

\begin{abstract}
We experimentally demonstrated a successful fabrication of a tellurite hollow core optical fiber with 6 nontouching air holes in the cladding to obtain low loss transmission up to $6 \mu \mathrm{m}$ in mid-infrared region.

Keywords: Hollow core fiber, Microstructured optical fiber, Fiber fabrication and characterization.

*tonghoangtuan@gmail.com; phone +81-52-809-1862
\end{abstract}

\section{Introduction}

Hollow core optical fibers (HCOFs) which enable light confinement in a low-refractive-index air core by antiresonant reflection effect [1] have been widely studied [2-5] because it does not require a complicated air-hole structure in the cladding and the first ring of air holes mainly determines the guiding properties [6]. It is mentioned that the antiresonant reflection mechanism allows a much broader spectral transmission than that achieved by photonic bandgap fibers $[7,8]$. In addition, low loss transmission bands, even in the mid-IR wavelength region $(\lambda>$ $4 \mu \mathrm{m}$ ), can be obtained by using one ring of air hole despite of very high material losses of silica [6]. Due to their superior properties, HCOFs have many potential applications including data communications, optical data transmission, terahertz propagation, power beam delivery for industrial applications, such as cutting, welding, engraving, chemical sensing and medical applications.

Recently, non-silica glasses have been used for HCOFs but the development of non-silica HCOFs has encountered several fabrication difficulties [9-11]. With wide transmission regions up to IR region (from 0.35 to $6 \mu \mathrm{m}$ ), high thermal stability and good corrosion resistance [12], tellurite glasses can also be potential candidates for non-silica HCOFs. However, the performance of tellurite HCOFs has not been demonstrated yet. In this work, the effect of fiber structure on the confinement loss is numerically investigated. The results show that tellurite HCOFs with 6 nontouching air holes in the cladding can be a good candidate to obtain low loss transmission up to the mid-infrared region. For the first time, a tellurite HCOF with six non-touching air holes in the cladding is experimentally demonstrated.

\section{Results and discussions}

\section{Fiber development}

Fiber geometry and confinement loss were investigated numerically by using the commercial software Comsol Multiphysics, the finite element method and the perfectly-matched boundary condition. The mesh resolution was automatically optimized to maintain the high calculation accuracy and reduce the calculation time. However, the largest mesh size was equal to $0.25 \mu \mathrm{m}$ which is equal to $1 / 8$ [13] of the shortest wavelength in the range from 2 to 6 $\mu \mathrm{m}$. Figure 1 shows the effect of the air-hole number $(\mathrm{N})$ on the confinement loss calculated for the fundamental mode in the air-core of the fiber by the solid line $(\mathrm{N}=5)$, the dashed line $(\mathrm{N}=6)$ and the dotted line $(\mathrm{N}=7)$. In the inset, 
schematic cross-sectional images of the fibers corresponding to $N=5,6$ and 7 were plotted. As can be seen, the confinement loss is less than $10 \mathrm{~dB} / \mathrm{m}$ in four wavelength bands in the range from 2 to $6 \mu \mathrm{m}$. The confinement loss becomes much lower when $\mathrm{N}$ is larger than 5 , but it slightly increases when $\mathrm{N}$ is larger than 6 .

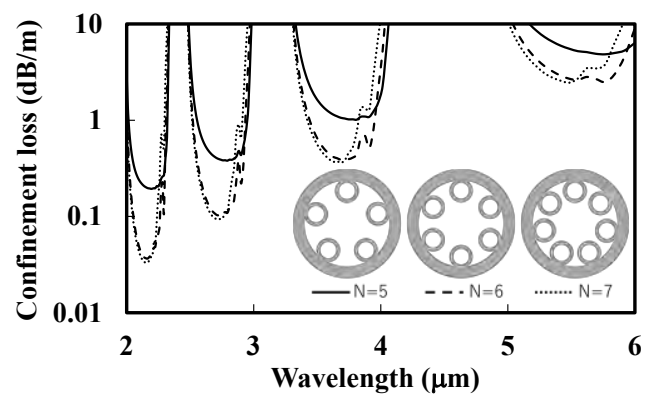

Figure 1. Calculated confinement loss spectra of the fundamental mode with different value of N (5, 6 and 7).

The schematic image of a tellurite HCOF with 6 non-touching cladding air-hole structure was plotted in Fig. 2. The geometrical parameter D1 is the air-core diameter, D2 is the cladding air-hole diameter, D is the outer diameter of the full air-hole structure, $t$ is the wall thickness of cladding air-hole and $\mathrm{R}$ is the ratio between D2 and D1. In Fig. 2 , images of intensity distribution profiles calculated for the fundamental mode are also plotted when $\mathrm{R}$ are $0.1,0.5$ and 0.86 , respectively. As can be seen, when $\mathrm{R}$ is as small as 0.1 , the air-core diameter is very large and the gap between two adjacent cladding air holes is also large. When $\mathrm{R}$ increases, the gap becomes smaller and it disappears when $\mathrm{R}$ is 0.86 . At this value of $\mathrm{R}$, the walls of two adjacent cladding air holes touch each others.

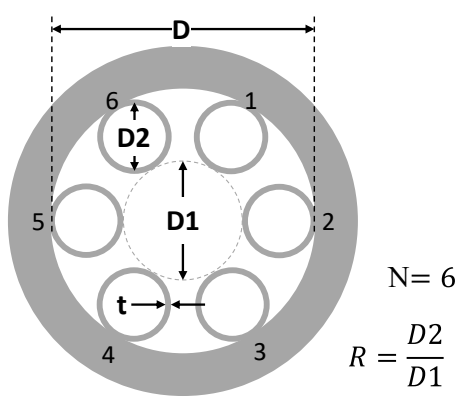

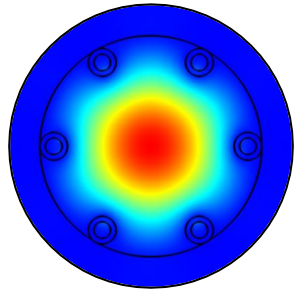

$\mathbf{R}=\mathbf{0 . 1}$

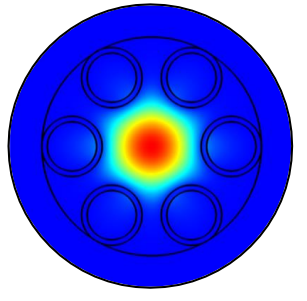

$\mathbf{R}=\mathbf{0 . 5}$

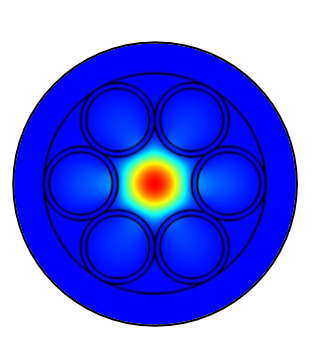

$\mathbf{R}=\mathbf{0 . 8 6}$
$\operatorname{Max}$

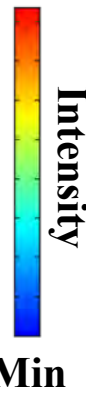

Figure 2. Calculated intensity distribution of the fundamental mode in the air-core when $\mathrm{N}=6$ and $\mathrm{R}=0.1,0.5$ and 0.86 , respectively.

The confinement loss of the fundamental mode and the $1^{\text {st }}$ order mode when $\mathrm{R}$ changed from 0.1 to 0.86 were plotted in Fig. 3. Firstly, it can be noticed that the confinement loss of the $1^{\text {st }}$ order mode is larger than that of the fundamental mode regardless of $\mathrm{R}$ value. Secondly, the confinement loss reduces when $\mathrm{R}$ increases from 0.1 to 0.5 , but when $\mathrm{R}$ becomes larger than 0.5 , the confinement loss increases rapidly, especially for the $1^{\text {st }}$ order mode as shown in Fig. 3. Similar results were obtained in Ref [14]. When the gap between two cladding air-hole is too large $(\mathrm{R}<0.5)$, the confinement loss is high due to the leakage of the electric field through the air gap [14, 15]. On the other hand, when the gap is smaller ( $\mathrm{R}>0.5)$, the diameter of cladding air-holes becomes larger than the diameter of the air-core and a weak coupling between the fundamental mode and the cladding air-hole mode occurs. Because of this weak coupling, the confinement loss in the air core increases. When the gap disappears, the walls of two 
cladding air-holes connect to each other forming nodes where the mode field can reside [15-17] and it causes a rapid increase in confinement loss.
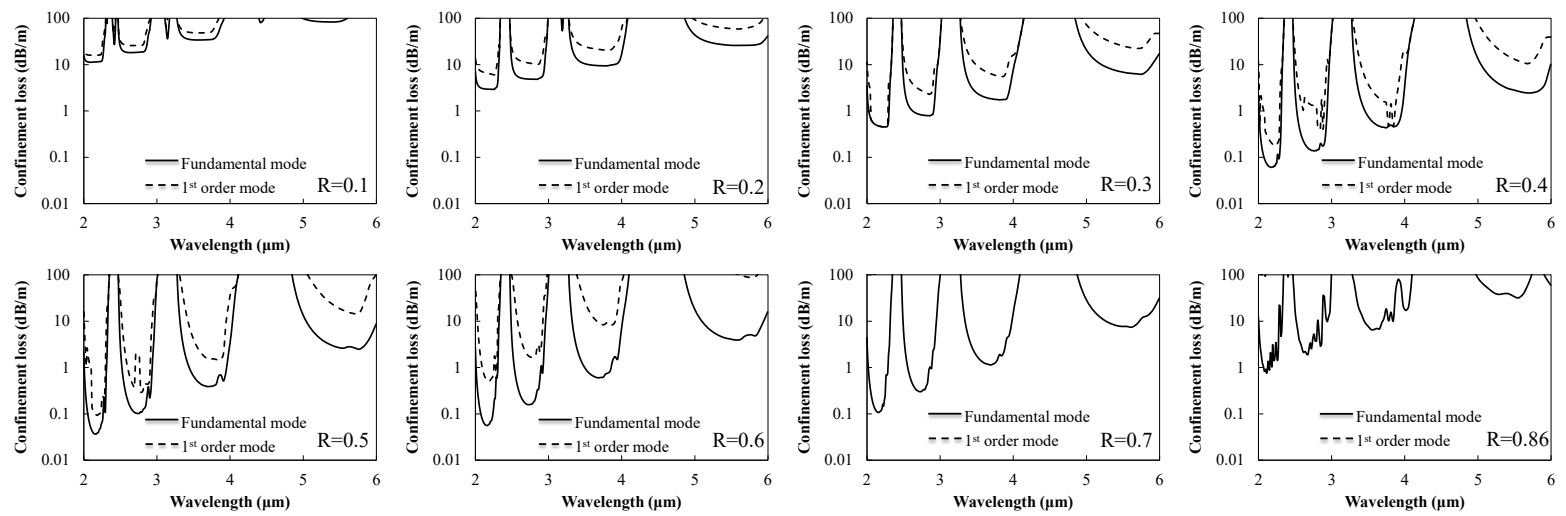

Figure 3. Calculated confinement loss of the fundamental mode and $1^{\text {st }}$ order mode with different value of $\mathrm{R}$ (from 0.1 to 0.86$)$.

\section{Fiber fabrication}

Following the finding of the above numerical analysis, we demonstrated the fabrication of a tellurite HCOF with 6 non-touching air holes in the cladding by using the stack-and-draw technique. A schematic diagram which illustrates the fiber fabrication process was shown in Fig. 4.
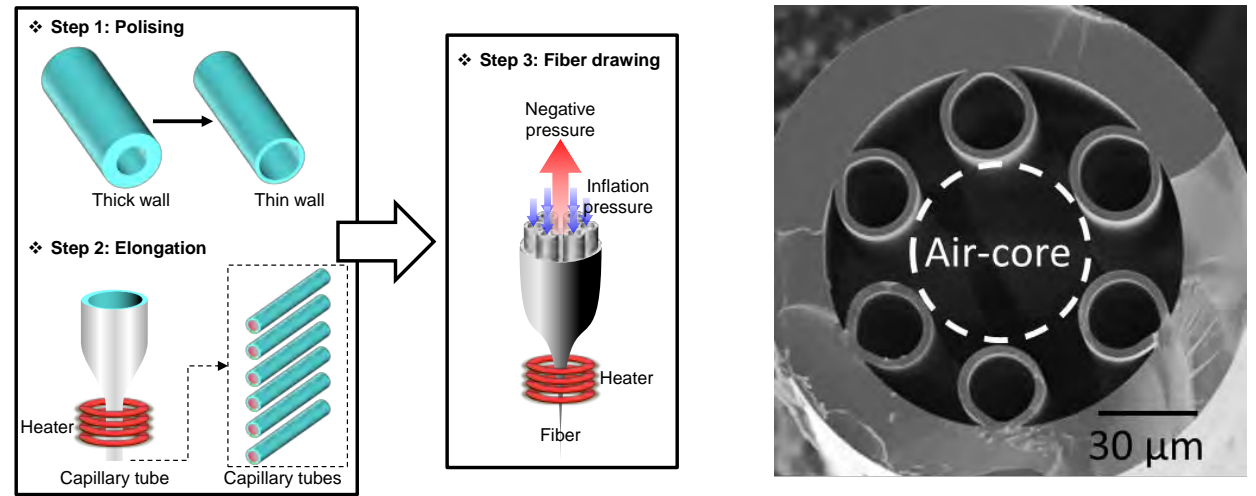

Figure 4. Schematic diagram for the fiber fabrication of tellurite HCOF with a 6 non-touching cladding air-hole structure and the cross-sectional image of the fabricated tellurite HCOF taken by a scanning electron microscope.

A cylindrical TZLB tube was first prepared by using the rotational casting method [12]. Its outer and inner diameters were 15 and $10 \mathrm{~mm}$, respectively. The outer of the tube was polished so that its wall thickness was controlled to be as thin as $1 \mathrm{~mm}$. An elongation process was carried out to obtain TZLB capillary tubes which have $0.25-\mathrm{mm}$ wall thickness. Each tube was about $15-\mathrm{cm}$ long. After that, a set of 6 capillary tubes was used to form a preform which has a hexagonal cladding air-hole structure by stacking and soldering them inside a cylindrical TZLB jacket tube. Finally, the preform was drawn into fiber whose diameter was about $150 \mu \mathrm{m}$. During this fiber drawing process, an inflation pressure was kept inside of 6 capillary tubes to prevent their shape-deformation. At the same time, a negative pressure was applied in the center of the TZLB jacket tube. The cross-sectional image of the fiber 
was taken by a scanning electron microscope and shown in Fig. 4. The air-hole structure is depicted by the black area. The wall thickness of each cladding air-hole was about $2.8 \mu \mathrm{m}$. The outer diameter of the full air-hole structure D was $110 \mu \mathrm{m}$ and the parameter $\mathrm{R}$ was about 0.4 .

\section{Conclusions}

Numerical calculation in this work shows that when the air gap between two cladding air holes of a tellurite HCOF is large or when their walls are connected, the confinement loss of the fundamental mode in the air core will be high because of the leakage energy. Therefore, tellurite HCOFs with 6 non-touching air holes in the cladding can be used to obtain low loss transmission spectra up to $6 \mu \mathrm{m}$ in the mid-infrared region. In addition, we experimentally demonstrated a successfully fiber fabrication for the first time.

This work was supported by the Japan Society for the Promotion of Science (JSPS) KAKENHI (Grant Number $15 \mathrm{H} 02250,17 \mathrm{~K} 18891$ and 18H01504) and the JSPS-CNRS joint research program.

\section{References}

[1] M. Duguay, Y. Kokubun, T. L. Koch, and L. Pfeiffer, "Antiresonant reflecting optical waveguides in SiO2 - Si multilayer structures," Appl. Phys. Lett. 49(1), 13-15 (1986).

[2] W. Belardi, and J. C. Knight, "Effect of core boundary curvature on the confinement losses of hollow antiresonant fibers," Opt. Express 21(19), 21912-21917 (2013).

[3] W. Belardi, and J. C. Knight, "Hollow antiresonant fibers with reduced attenuation," Opt. Lett. 39(7), 1853-1856 (2014).

[4] W. Belardi, and J. C. Knight, "Hollow antiresonant fibers with low bending loss," Opt. Express 22(8), 1009110096 (2014).

[5] M. S. Habib, J. E. Antonio-Lopez, C. Markos, A. Schulzgen, and R. Amezcua-Correa, "Single-mode, low loss hollow-core anti-resonant fiber designs," Opt. Express 27(4), 3824-3836 (2019).

[6] A. D. Pryamikov, A. S. Biriukov, A. F. Kosolapov, V. G. Plotnichenko, S. L. Semjonov, and E. M. Dianov, "Demonstration of a waveguide regime for a silica hollow - core microstructured optical fiber with a negative curvature of the core boundary in the spectral region $>3.5 \mu \mathrm{m}$," Opt. Express 19(2), 1441-1448 (2011).

[7] J. C. Knight, J. Broeng, T. A. Birks, and P. S. J. Russell, "Photonic band gap guidance in optical fibers," Science 282(5393), 1476-1478 (1998).

[8] P. Russell, "Photonic crystal fibers," Science 299(5605), 358-362 (2003).

[9] A. F. Kosolapov, A. D. Pryamikov, A. S. Biriukov, V. S. Shiryaev, M. S. Astapovich, G. E. Snopatin, V. G. Plotnichenko, M. F. Churbanov, and E. M. Dianov, "Demonstration of CO2-laser power delivery through chalcogenide-glass fiber with negative-curvature hollow core," Opt. Express 19(25), 25723-25728 (2011).

[10]V. S. Shiryaev, "Chalcogenide glass hollow-core microstructured optical fibers," Front. Mater. 2(24), (2015).

[11]V. S. Shiryaev, A. F. Kosolapov, A. D. Pryamikov, G. E. Snopatin, M. F. Churbanov, A. S. Biriukov, T. V. Kotereva, S. V. Mishinov, G. K. Alagashev, and A. N. Kolyadin, "Development of technique for preparation of As2S3 glass preforms for hollow core microstructured optical fibers," J. Optoelectron. Adv. M. 16(9-10), 10201025 (2014).

[12]A. Mori, "Tellurite-based fibers and their applications to optical communication networks," J. Ceram. Soc. Jpn. 116(1358), 1040-1051 (2008).

[13]M. I. Hasan, N. Akhmediev, and W. Chang, "Empirical Formulae for Dispersion and Effective Mode Area in Hollow-Core Antiresonant Fibers," J. Lightwave Technol. 36(18), 4060-4065 (2018). 
[14]C. L. Wei, R. J. Weiblen, C. R. Menyuk, and J. Hu, "Negative curvature fibers," Adv. Opt. Photon. 9(3), 504561 (2017).

[15]C. L. Wei, C. R. Menyuk, and J. Hu, "Impact of cladding tubes in chalcogenide negative curvature fibers," IEEE Photonics J. 8(3), (2016).

[16]F. Poletti, "Nested antiresonant nodeless hollow core fiber," Opt. Express 22(20), 23807-23828 (2014).

[17]A. N. Kolyadin, A. F. Kosolapov, A. D. Pryamikov, A. S. Biriukov, V. G. Plotnichenko, and E. M. Dianov, "Light transmission in negative curvature hollow core fiber in extremely high material loss region," Opt. Express 21(8), 9514-9519 (2013). 


\title{
3D printed optical fibre preforms from silica contained resin
}

\author{
Yushi $\mathrm{Chu}^{\mathrm{a}, \mathrm{b}, \mathrm{d}}$, John Canning*a, Xinghu Fu ${ }^{\mathrm{b}, \mathrm{c}}$, Yanhua Luo ${ }^{\mathrm{b}}$, Kevin $\mathrm{Cook}^{\mathrm{a}}$, Jianzhong Zhang ${ }^{\mathrm{d}}$, Gang- \\ Ding Peng, \\ ${ }^{a}$ interdisciplinary Photonic Laboratories $(i \mathrm{PL})$, Tech Lab, School of Electrical and Data Engineering, \\ University of Technology Sydney, Sydney, NSW 2007 \& 2019, Australia \\ ${ }^{\mathrm{b}}$ Photonics and Optical Communications, School of Electrical Engineering and Telecommunications, \\ University of New South Wales, Sydney, NSW 2052, Australia \\ ${ }^{c}$ The Key Laboratory for Special Fiber and Fiber Sensor of Hebei Province, School of Information \\ Science and Engineering, Yanshan University, Qinhuangdao, 066004, China \\ ${ }^{\mathrm{d}} \mathrm{Key}$ Lab of In-Fiber Integrated Optics of Ministry of Education, School of Science, Harbin \\ Engineering University, Harbin, 150001, China \\ *john.canning@uts.edu.au; phone+61295145025
}

\begin{abstract}
3D printed glass optical fiber preforms have been fabricated from silica containing resin. The fabrication process includes resin preparation, preform printing, debinding and sintering. Results demonstrate the silica concentration greatly influenced on the transmittance of resin and shape of preforms.
\end{abstract}

Keywords: Silica based resin, 3D printing fibers, optical fiber fabrication, debinding, sintering

\section{Introduction}

Silica optical fibres form the backbone of today's telecommunications networks, enabling the bandwidth necessary for internet-of-things (IoT) transmission of data. The fibre itself has gone beyond a transmission medium to an integral IoT instrument component in its own [1]. Fabrication of these fibres exploits a range of methods but only a few dominate, including modified chemical vapor deposition and stack-and-draw [2-4]. Despite their maturity, these existing methods have limited capability in both material and structure flexibility for diverse and custom designed functionalities, primarily based around a labour intensive, centre spin model on a lathe deposition system. The emergence of additive manufacturing (3D printing) technologies offers the possibility of overcoming these limits [5]. The first report in this field was focused on $3 \mathrm{D}$ printed plastic optical fibers [6]. Until now, there is no report about silica-based fibre, mainly because of the very high temperature processing required along with the occupational health and safety (OHS) challenges associated with traditional fibre manufacture. Although Massachusetts Institute of Technology (MIT) demonstrated large scale ultra high temperature 3D printing of glass using high temperature zirconate nozzles and specialty ovens, the viscosity of glass prevented high resolution printing with a nozzle size $\sim 4 \mathrm{~mm}$ [7]. In 2017, Karlsruhe Technology Institute (KIT) achieved low temperature 3D printing of silica glasses by combining a bottom up nanoparticle approach with polymers and subsequently sintering [8]. They were limited by sintering challenges to demonstrating small sized optical components. Variations of this approach were also demonstrated by others and establishes a low temperature route to demonstrating the first silica based preforms if the issue of cracking can be resolved so that reasonable sized preforms could be manufactured.

The reported method consists four steps: (1) preparing UV sensitive resin contained silica; (2) printing designed structure utilizing a commercial direct light projection (DLP) 3D printer; (3) annealing driven debinding process to remove polymer support; and (4) higher temperature sintering to further remove impurities, fuse nanoparticles and leave only silica glass.

The UV sensitive resin plays an important role among all these steps. Whilst there are range of available monomer materials that will do the job, each needs to be optimized to consider properties such as viscosity and flow. This is particularly true if one wishes to extend the previous work

Sixth International Workshop on Specialty Optical Fibers and Their Applications (WSOF 2019), edited by Liang Dong, John M. Ballato, Proc. of SPIE Vol. 11206, 112061L · (C) 2019 SPIE

CCC code: $0277-786 X / 19 / \$ 21 \cdot$ doi: $10.1117 / 12.2547436$ 
from small optical elements from a few $\mathrm{mm}$ to larger preform pieces at least many cms in length and width. In this work we describe this optimization process focusing on structured optical fibres [9].

\section{Experiment}

Concentrations equivalent to $\left[\mathrm{SiO}_{2}\right] \sim 33.3,42.8$ and 50 $\mathrm{wt} \%$ of silica nanoparticles were dispersed in the mixture which contains 60 vol\% 2-hydroxyethyl methacrylate, 30 vol\% 2-phenoxyethanol and 10 vol\% tetra(ethylenglycol) diacrylate monomers, respectively. Afterwards, $0.2 \mathrm{wt} \%$ diphenyl(2,4,6-trimethylbenzoyl) phosphine oxide and 0.1 wt $\%$ hydroquinone were added to the mixture. The silica nanoparticles were bought from Evonik, Australia (Aerosil OX50 $\phi \sim 40 \mathrm{~nm}$ ), and other chemicals were purchased from Sigma-Aldrich, Australia.

The transmission spectra of resin were measured with UV-VIS spectrophotometer (UV 2401 PC, Shimadzu, Japan). The resin was printed with designed structures using a commercial DLP 3D printer (PRO2, Asiga, Australia). The printed preforms were undertaken the debinding and sintering processes in a high temperature furnace (1700M, Furnace Technologies, Australia).

\section{Results and Discussion}

The addition of silica resulted in large Rayleigh scattering, changing the preform colouration to blue-white (Fig. 1 (e), Fig. 2 (a)). There is also Rayleigh scattering in the silica contained resin. Figure 1(a) shows the attenuation arising from this as a function of wavelength - for all concentrations there is an approximate $\mathrm{A} / \lambda^{4}+B \times \lambda+C$ fit, which $\lambda$ is the wavelength, $\mathrm{A}, \mathrm{B}, \mathrm{C}$ are constants, indicating the attenuation was effect by absorption edge and Rayleigh scattering.

Taking advantage of 3D printing any arbitrary structure, we have fabricated both step-index and structured preforms, the former done using germanosilicate in the core after printing a structured inner hole. Here, structured preforms with one or two rings of holes are considered to study the impact on holes for eventual structured and photonic crystal fibre fabrication. Fig. 1(c) illustrates an example of two ring photonic crystal fiber preforms, where $D=25 \mathrm{~mm}$, $d=3 \mathrm{~mm}$ and $\Lambda=0.7 \mathrm{~mm}$, respectively. The length was $L$ $=20 \mathrm{~mm}$. The DLP printing is shown in Fig. 1(d), where near-UV light cured the resin layer by layer. The printing parameters were $I=5.8 \mathrm{~mW} / \mathrm{cm}^{2}$, curing time $t=3.5 \mathrm{~s}$ and
$75 \mu \mathrm{m}$ of every curing layer. Preform samples after printing and cleaning are shown in Fig. 1 (e) and (f) respectively. As shown in Fig. 1(g), the masses of preforms increased with the increment of silica concentration as expected.


Fig. 1. (a) Silica induced attenuation of resins using plastic cuvettes; (b) Rayleigh and linear fitting of attenuation, insert: resins photo; (c) Designed preform structure; (d) DLP 3D printing; (e) preforms after 3D printing. (f) Preforms after cleaning; and (g) the preform mass vs silica concentration.

The resulting preforms were annealed to remove the polymer binder and sintered to fuse the silica nanoparticles and reduce scattering. The thermal treatment setting was showed in Fig. 2(a). The debinding and sintering process ranged from $T=25$ to $600^{\circ} \mathrm{C}$ (green background) and $T=$ 600 to $1200{ }^{\circ} \mathrm{C}$ (yellow background). Shrinkage and weight loss occurred as polymer material is removed initially and 
the glass left behind, held together by van der Waals forces (Fig. 2(b) $\sim$ (e)). Interestingly by having the structure present, the effect of silica concentration on outer diameter and height was small but hole diameter reduced substantially when $\left[\mathrm{SiO}_{2}\right] \sim 50 \mathrm{wt} \%$. After sintering, the preforms can be drawn into optical fibre using a commercial fibre-drawing tower, to be reported later.
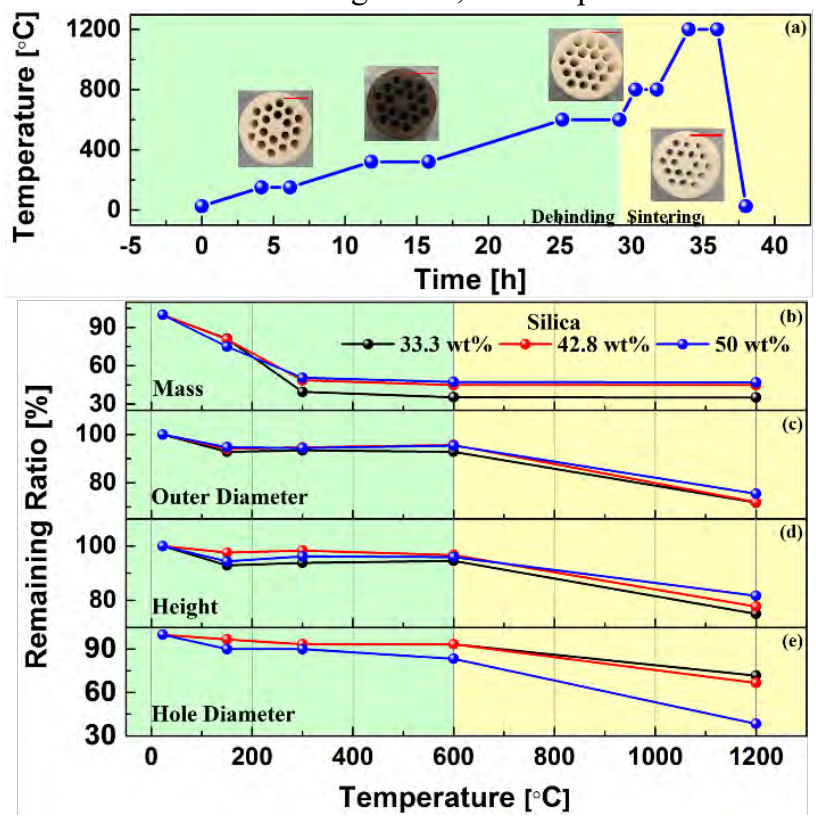

Fig. 2. (a) Thermal process of debinding and sintering, inset photos: preforms of $33.3 \mathrm{wt} \%$ silica after thermal treatment at 150 , 300,600 and $1200{ }^{\circ} \mathrm{C}$. (b) (e) Remaining ratio of mass, outer diameter, height and hole diameter after thermal treatment in (a).

\section{Conclusion}

In this paper, the effect of silica concentration on 3D printing silica fibre preforms was studied. Rayleigh scattering arises from non-fused particles both in the composite polymer silica stage and after sintering when the polymer materials and organic radicals are removed. The material shrinks and loses weight during polymer removal in the initial annealing, or debinding, stage. The largest shrinkage occurred during sintering as the silica is dried thoroughly and residual material removed, allowing van der Waals forces to hold the preform together.

\section{Acknowledgements}

Y. Chu and X. Fu also thank for the support of China Scholarship Council (CSC No. 201706680054 and 201708130199).

\section{References}

[1] Peng, G. D., [Handbook of Optical Fibers], Springer, Singapore, (2019).

[2] Luo, Y., Wen, J., Zhang, J., Canning, J. and Peng, G. D., "Bismuth and erbium codoped optical fiber with ultrabroadband luminescence across O-, E-, S-, C-, and L-bands," Opt. Lett. 37(16), 3447-3449 (2012).

[3] Chu, Y., Tian, Y., Fan, D., Xiao, G., Wei, S., Zhang, B., Fu, X., Ma, Z., Ren, J., Chai, Q., Luo, Y., Zhang, J. and Peng, G. D., "Fabrication and Characterization of Birefringent Bismuth and Erbium Co-Doped Photonic Crystal Fiber for Broadband Polarized Near Infrared Emission," OSA, CLEO: QELS_Fundamental Science, JW2A-107 (2019).

[4] Luo, L., Chu, Y., Cook, K., Tafti, G., Wang, S., Wang, W., Tian, Y., Canning J. and Peng, G. D., "Spun High Birefringence Bismuth/Erbium Co-Doped Photonic Crystal Fibre with Broadband Polarized Emission," IEEE, Asia Communications and Phononics Conference, 1-3 (2018).

[5] Bikas, H., Stavropoulos, P. and Chryssolouris, G., "Additive manufacturing methods and modelling approaches: a critical review," The International Journal of Advanced Manufacturing Technology 83(14), 389-405 (2016).

[6] Cook, K., Canning, J., Leon-Saval, S., Reid, Z., Hossain, M. A., Comatti, J.-E., Luo, Y. and Peng, G.D., "Air-structured optical fiber drawn from a 3D-printed preform,” Opt. Lett. 40(17), 3966-3969 (2015).

[7] Klein, J., Stern, M., Franchin, G., Kayser, M., Inamura, C., Dave, S., Weaver, J. C., Houk, P., Colombo, P., Yang, M. and Oxman, N., "Additive Manufacturing of Optically Transparent Glass," 3D Printing and Additive Manufacturing 2(3), 92-105 (2015).

[8] Kotz, F., Arnold, K., Bauer, W., Schild, D., Keller, N., Sachsenheimer, K., Nargang, T. M., Richter, C., Helmer, D. and Rapp, B.E., "Three-dimensional printing of transparent fused silica glass," Nature 544(7650), 337 (2017).

[9] Canning, J., Optical Fiber: Structured, Vol 4. Encyclopedia of Optical and Photonic Engineering, 2nd Ed, Taylor \& Francis, (2015). 


\title{
Wavelength dependence of transverse Anderson localization in disordered glass-air fiber
}

\author{
Paul Roth*a, Gordon K. L. Wong ${ }^{\mathrm{a}}$, Jian Zhao ${ }^{\mathrm{b}}$, Jose Enrique Antonio-Lopez ${ }^{\mathrm{b}}$, \\ Rodrigo Amezcua-Correa ${ }^{b}$, Michael H. Frosz ${ }^{a}$, Philip St.J. Russell ${ }^{\mathrm{a}, \mathrm{c}}$, and Axel Schülzgen ${ }^{\mathrm{b}}$ \\ ${ }^{a}$ Max Planck Institute for the Science of Light, Staudtstr. 2, 91058 Erlangen, Germany \\ ${ }^{b}$ CREOL, College of Optics and Photonics, University of Central Florida, Orlando, FL 32816, USA \\ 'Department of Physics, University of Erlangen-Nuremberg, Staudtstr. 2, 91058 Erlangen, Germany
}

\begin{abstract}
Wavelength-independent transverse localization of light propagating through disordered glass-air fibers is observed from $540 \mathrm{~nm}$ to $1600 \mathrm{~nm}$. The effect opens the door to the transmission of color images through fibers in fluorescence imaging and endoscopy with white light illumination.
\end{abstract}

Keywords: fiber optics, imaging, microstructured fibers, localization

*paul.roth@mpl.mpg.de

\section{Introduction}

Image transmission through intentionally disordered optical fibers was proposed decades ago [1,2] and was recently experimentally demonstrated [3-5]. It has been shown that the novel waveguiding mechanism based on transverse Anderson localization (TAL) of light can result in high-fidelity image transmission in disordered polymer and glass-air fibers with image quality comparable to commercial multicore imaging fiber bundles [4,5]. Low-loss transmission at visible wavelengths in glass-air localizing optical fibers (GALOFs) allows image transmission through meter-long disordered fiber segments [5] and the combination with machine learning algorithms and deep convolutional neural networks (DCNNs) has brought further significant improvements in the performance of imaging systems based on GALOFs [6,7].

While powerful imaging systems using disordered fibers have already been demonstrated, some fundamental properties of light transport through such disordered fibers are still the subject of scientific debate. One particular aspect, the wavelength dependence of the TAL effect, is not only of fundamental interest [8], but also of great practical relevance for future fiber optic imaging systems since a strong wavelength dependence would limit the ability of multi-color and white light imaging. Considering biomedical applications, the wavelength dependence of light transmission through imaging fibers is particularly relevant when contrast enhancing staining methods using one or several different dyes are applied, or in optogenetics, where light sensitive proteins are introduced into brain cells to monitor and control their activity using light signals.

Recent theoretical and experimental studies have produced different and sometimes even contradictory results. The first study on polymer fibers predicted a strong variation of the TAL effect with wavelength, calculating different localization radii for $633 \mathrm{~nm}$ and $405 \mathrm{~nm}$ of $\sim 30 \mu \mathrm{m}$ and $\sim 8 \mu \mathrm{m}$, respectively [9]. The first experimental study on glassair disordered fibers found a slight decrease in image resolution, from $\sim 18 \mu \mathrm{m}$ to $\sim 22 \mu \mathrm{m}$ as the wavelength increased from $405 \mathrm{~nm}$ to $635 \mathrm{~nm}$ [5]. Meanwhile, a recent theoretical study showed contradictory results for different implementations of randomness in the wave equation, while additional experiments in polymer fibers showed minimal

Sixth International Workshop on Specialty Optical Fibers and Their Applications (WSOF 2019),

edited by Liang Dong, John M. Ballato, Proc. of SPIE Vol. 11206, 112061M · C 2019 SPIE

CCC code: $0277-786 \mathrm{X} / 19 / \$ 21 \cdot$ doi: $10.1117 / 12.2547473$ 
variations of the localization radii for wavelengths between $550 \mathrm{~nm}$ and $1050 \mathrm{~nm}$ [8]. Because of this ongoing discussion, we have investigated the wavelength dependence of the localization radii over a large wavelength range for two GALOFs with different average air hole sizes.
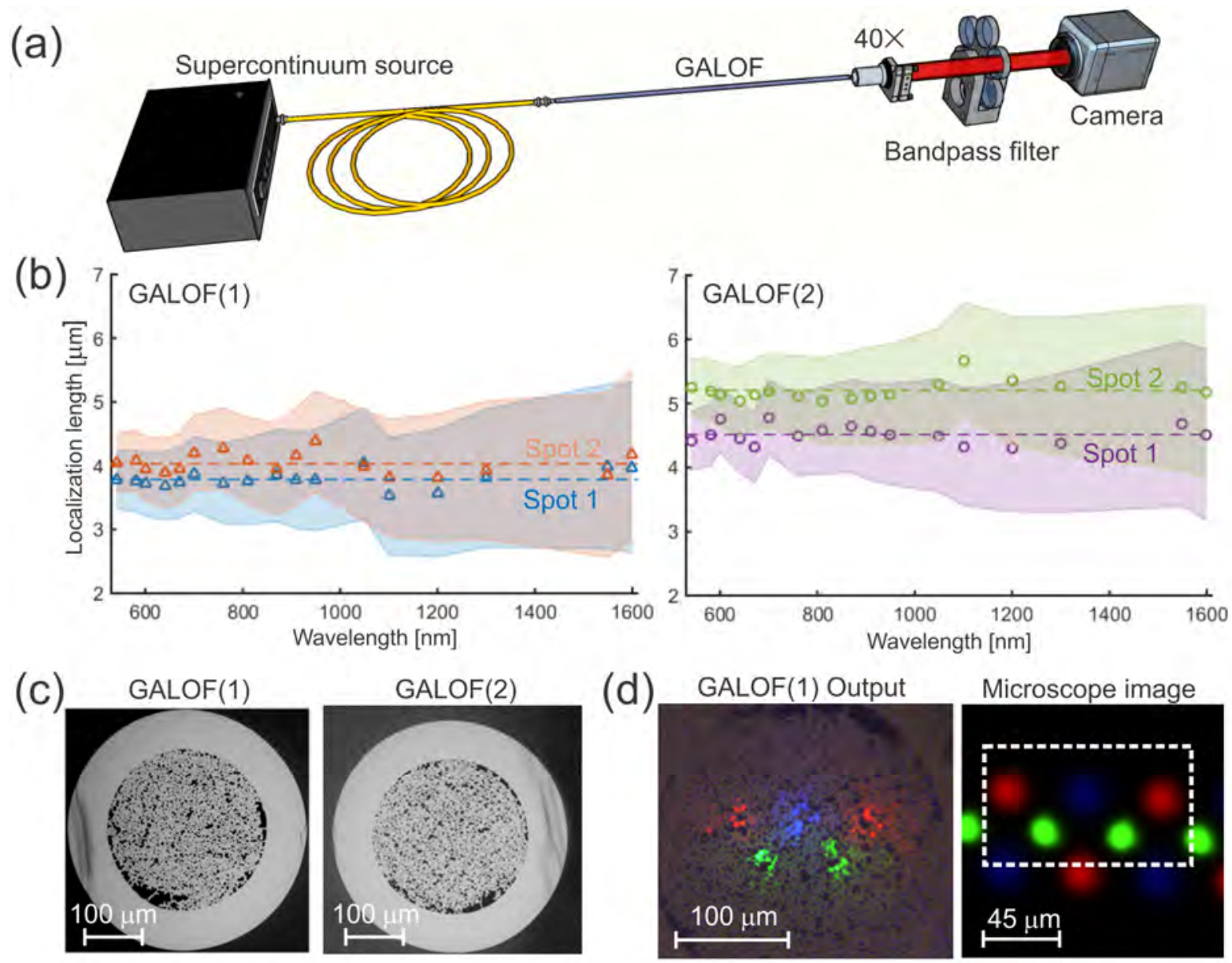

Figure 1. a) Experimental setup for measuring the wavelength-dependent spread of the output beam size. (b) Radius of the transmitted beams at the GALOF output face vs. wavelength. The shaded regions indicate the uncertainty given by the aberration limit calculated for an objective with an NA of 0.6. The dashed lines correspond to the mean value of the measured localization lengths for each output spot, acting as a guide for the eye. (c) Scanning electron micrographs of the GALOF cross-sections. (d) Nearfield image of the output facet of GALOF(1) after imaging smartphone screen pixels onto the input facet of the fiber. The microscope image shows the pixel arrangement which is imaged onto the input facet of GALOF(1) (dashed box).

\section{Experiments and results}

A schematic of the experimental setup to investigate the wavelength dependence of the beam spreading in the GALOF is shown in Fig. 1 (a). The single-mode fiber output of a supercontinuum source is butt-coupled into the GALOF, resulting in a localized spot at the fiber output.

The output face of the GALOF is imaged onto a CCD camera using a microscope objective. A set of different optical bandpass filters ranging from $540 \mathrm{~nm}$ to $1600 \mathrm{~nm}$ with bandwidths of $10 \mathrm{~nm}$ are used to observe the output spot-size for 
different wavelengths. Two GALOF samples, GALOF(1) and GALOF(2) with lengths of $27 \mathrm{~cm}$ and $25 \mathrm{~cm}$, respectively, were tested. Cross-sectional images of the two fiber samples are shown in Fig. 1 (c). It is found that the two GALOFs have significantly different average air-hole diameters of $\sim 3 \mu \mathrm{m}$ and $\sim 5 \mu \mathrm{m}$, respectively, but a similar air-to-glass volume fraction of $\sim 30 \%$. For the measured spot-sizes the localization length is estimated using [5]:

$$
\frac{1}{L^{2}}=\frac{\int I(x, y)^{2} d x d y}{\left[\int I(x, y) d x d y\right]^{2}},
$$

where $L$ is the localization length and $I(\mathrm{x}, \mathrm{y})$ is the intensity of the output beam at position $I(\mathrm{x}, \mathrm{y})$. The localization length of the output beam size vs. wavelength for two different input spots in each GALOF sample is illustrated in Fig. 1 (b). The measured output spots show an average localization length of 4 to $5 \mu \mathrm{m}$ even in fiber structures with feature sizes on the order of $5 \mu \mathrm{m}$. This small transverse spreading of light can be attributed to a small wavevector in the plane of disorder. The localized spots show almost no change in size over the wavelength range from $540 \mathrm{~nm}$ to $1600 \mathrm{~nm}$. To further demonstrate the capability of transmitting color images of a light-emitting sample through GALOFs, an array of smartphone screen pixels was imaged on to the input face of GALOF(1) with a 20x microscope objective (Fig. 1 (d)). Due to the wavelength independence of the output spot-size the individual smartphone pixels are clearly separable after transmission through a $27 \mathrm{~cm}$ length of fiber.

\section{Conclusion}

In summary, we have demonstrated that two different GALOFs show almost wavelength-independent spreading of launched single mode beams between $\sim 540 \mathrm{~nm}$ and $1600 \mathrm{~nm}$, in agreement with previous results using disordered polymer fibers. This feature opens the door to fiber-based imaging systems that transmit color images-certainly a great benefit for future GALOF-based endoscopy devices. In addition, we demonstrate the transmission of colored pixel emission from a light emitting object without any external illumination. Both demonstrations illustrate the great potential of GALOF-ased imaging systems for practical applications in neuroscience and clinical diagnosis.

\section{References}

[1] S. S. Abdullaev and F. K. Abdullaev, "On propagation of light in fiber bundles with random parameters," Radiofizika 23(6), $766-767$ (1980).

[2] H. De Raedt, A. Lagendijk, and P. de Vries, "Transverse localization of light," Phys. Rev. Lett. 62(1), 47-50 (1989).

[3] S. Karbasi, K. W. Koch, and A. Mafi, "Image transport quality can be improved in disordered waveguides," Opt. Comm. 311, 72-76 (2013).

[4] S. Karbasi, R. J. Frazier, K. W. Koch, T. Hawkins, J. Ballato, and A. Mafi, "Image transport through a disordered optical fibre mediated by transverse Anderson localization," Nat Commun 5, 3362 (2014).

[5] J. Zhao, J. E. A. Lopez, Z. Zhu, D. Zheng, S. Pang, R. A. Correa, and A. Schülzgen, "Image transport through meter-long randomly disordered silica-air optical fiber," Sci. Rep. 8, 3065 (2018).

[6] J. Zhao, Y. Sun, Z. Zhu, J. E. Antonio-Lopez, R. A. Correa, S. Pang, and A. Schülzgen, "Deep learning imaging through fully-flexible glass-air disordered fiber," ACS Photonics 5(10), 3930-3935 (2018).

[7] J. Zhao, M. Peysokhan, J. E. Antonio-Lopez, Y. Sun, B. Abaie, A. Mafi, R. Amezcua-Correa, S. Pang, and A. Schülzgen, "A path to high-quality imaging through disordered optical fibers: a review," Appl. Opt. 58(13), D50-D60 (2019).

[8] W. Schirmacher, B. Abaie, A. Mafi, G. Ruocco, and M. Leonetti, "What is the right theory for Anderson localization of light? An experimental test," Phys. Rev. Lett. 120(6), 067401 (2018).

[9] S. Karbasi, C. R. Mirr, R. J. Frazier, P. G. Yarandi, K. W. Koch, and A. Mafi, "Detailed investigation of the impact of the fiber design parameters on the transverse Anderson localization of light in disordered optical fibers," Opt. Express 20(17), 18692-18706 (2012). 


\title{
Noise-like pulse pumped all-fiber supercontinuum laser source
}

\author{
Xing Luo, Tong Hoang Tuan, Than Singh Saini, Hoa Phuoc Trung Nguyen, Takenobu Suzuki, \\ and Yasutake Ohishi \\ Research Center for Advanced Photon Technology, Toyota Technological Institute, \\ 2-12-1 Hisakata, Tempaku, Nagoya 468-8511, Japan
}

\begin{abstract}
Flat supercontinuum spanning from $1370 \mathrm{~nm}$ to $2200 \mathrm{~nm}$ with the direct pumping of a noise-like pulse mode locked fiber laser was demonstrated. The all-fiber supercontinuum source has very flat spectrum and weak residual-pump.

Keywords: Noise-like Pulse, supercontinuum generation, all-fiber laser

*luoxing@toyota-ti.ac.jp; phone 0528091862
\end{abstract}

\section{Introduction}

The supercontinuum (SC) generation in fibers has been widely studied in the last decades. Because of its special properties, fiber-based SC laser sources have found the wide range of applications in optical communication, fiber sensing and spectral metrology [1]. Generally, two main parts are included in the SC source, i.e, the high peak power pulse laser and the highly nonlinear fiber. Both silica single-mode fibers and Microstructured fibers have been used for SC generation [2, 3]. In some cases, all-solid single mode fibers are very suitable for SC generation due to its low loss splicing with the pigtail of the pump laser, which makes the system highly efficient and compact. On the other hand, the pump laser is also a critical part for the SC generation. Most SC generation experiments were performed with picosecond or femtosecond mode locked lasers. Nanosecond pulse and continuous wave laser were also demonstrated to pump nonlinear fibers for SC generation [4]. The noise-like pulse (NLP) is a pulse packet composed by numerous ultra-short pulses with random pulse width, intensity and phase, which has received extensive attention in recent years.

In this paper, we report broad and flat SC generation in a highly nonlinear fiber pumped with a NLP mode locked fiber laser directly. The fiber laser achieves NLP mode locking based on nonlinear amplifying loop mirror (NALM). Pumping a piece of 102-m highly nonlinear fiber with the NLP mode locked fiber laser directly, the SC spectrum spanning from less than $1200 \mathrm{~nm}$ to $\sim 2200 \mathrm{~nm}$ was achieved. Moreover, the spectrum energy within $1865 \mathrm{~nm}$ to 2200 $\mathrm{nm}$ was enhanced and contained more than $45 \%$ of the whole energy of the SC. This SC laser source showed compact all-fiber structure, weak residual-pump and mid-infrared enhanced spectrum

\section{Experimental Setup}

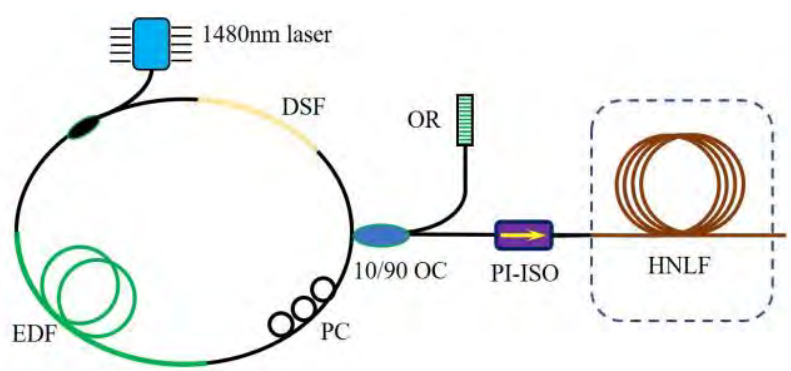

Fig. 1. Configuration of the all-fiber NLP mode locked fiber laser based on nonlinear amplifying loop mirror. EDF: Erbiumdoped fiber; OC: optical coupler; PC: polarization controller; OR: optical reflector; ISO: isolator; WDM: wavelength division multiplexer; HNLF: highly nonlinear fiber.

Sixth International Workshop on Specialty Optical Fibers and Their Applications (WSOF 2019), edited by Liang Dong, John M. Ballato, Proc. of SPIE Vol. 11206, 112061N · C 2019 SPIE

CCC code: $0277-786 \mathrm{X} / 19 / \$ 21 \cdot$ doi: $10.1117 / 12.2547634$ 
The configuration of the proposed mode locked fiber laser is shown in Fig.1. The laser adopted a very simple all-fiber structure and realizes NLP mode locking based on a NALM. The NALM consisted of a 10/90 optical coupler (OC), a 1480/1550 wavelength division multiplexer (WDM), a piece of $8 \mathrm{~m}$ Erbium-doped fiber (EDF, OFS, LP980) and a polarization controller (PC). A piece of $10 \mathrm{~m}$ dispersion shift fiber was inserted between the WDM and the OC. A 1480 $\mathrm{nm}$ Raman laser with maximum power of $5 \mathrm{~W}$ was used to pump the EDF through the WDM. One pigtail of the OC was connected to an optical reflector (OR) with a broad bandwidth. The other pigtail was connected to a polarization independent isolator (ISO) as the output. The total length of the laser cavity of was $\sim 26.7 \mathrm{~m}$ and the round-trip time was estimated to be $\sim 129 \mathrm{~ns}$, which corresponded to the fundamental repetition rate of $\sim 7.75 \mathrm{MHz}$. The dispersions of the EDF, SMF and DSF were $-48 \mathrm{ps} / \mathrm{nm} / \mathrm{km}, 20 \mathrm{ps} / \mathrm{nm} / \mathrm{km}$ and $2 \mathrm{ps} / \mathrm{nm} / \mathrm{km}$ at $1580 \mathrm{~nm}$, respectively. The net dispersion of the cavity was estimated to be $\sim-0.204 \mathrm{ps}^{2}$.

\section{Results and Discussion}

By increasing the pump power gradually and adjusting the PC carefully, the NLP mode locking can be achieved easily with a pump power of $\sim 830 \mathrm{~mW}$. The output spectrum of the laser is shown in Fig. 2(a). The spectrum has the central wavelength of $\sim 1581 \mathrm{~nm}$ and the $3-\mathrm{dB}$ bandwidth of $\sim 35.8 \mathrm{~nm}$. Besides, there is a secondary spectral peak at $\sim 1471 \mathrm{~nm}$. The broad and smooth spectrum is the typical characteristic of NLP mode locking. In order to confirm the NLP mode locking further, a high-speed sampling oscilloscope combined with a photodetector was used to measure the pulse train. Figure 2(b) presents a typical measured pulse, which has the pulse width of $\sim 720 \mathrm{ps}$. Besides, an autocorrelator was used to measure the laser pulse and the corresponding autocorrelation trace is shown in Fig. 2(c). The autocorrelation trace shows a strong narrow spike riding on a broad but weak pedestal. The inset of Fig. 2(c) is the local view of the autocorrelation trace in the range of 2 ps. The full width at half-maximum (FWHM) of the autocorrelation trace was $\sim 80$ fs and the width of the spike was $\sim 52 \mathrm{fs}$ assuming $\operatorname{sech}^{2}$ pulse shape. The pulse traces recorded by the oscilloscope and the autocorrelation trace indicate that each pulse is a pulse packet which consists of numerous femtosecond sub-pulses with random separation. All the results indicate that the laser operates in the NLP mode locking regime.
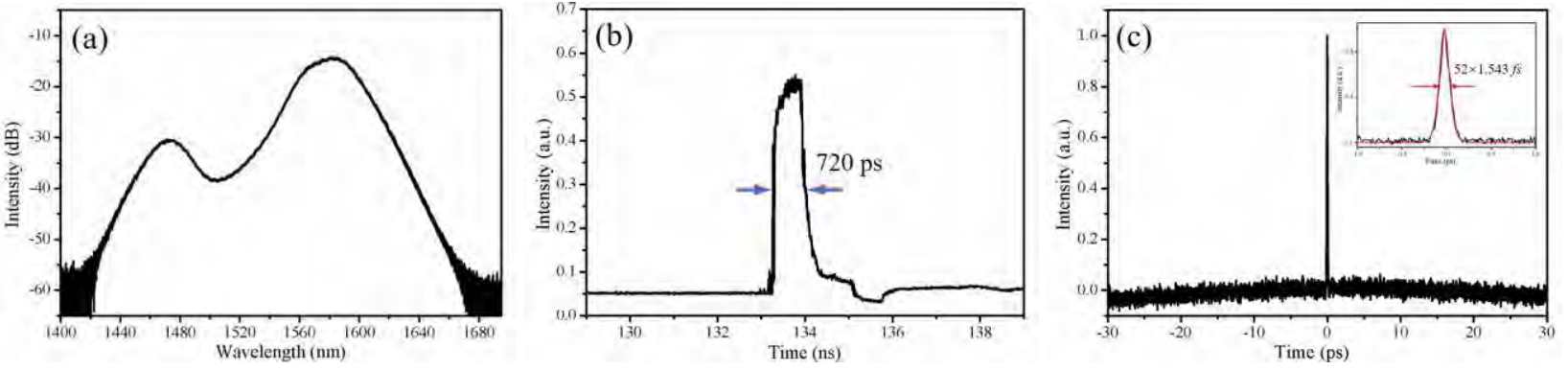

Fig. 2. (a) Output spectrum of the NLP mode locking. (b) A pulse measured by the wide-band oscilloscope. (c) Autocorrelation

trace measured in the range of $60 \mathrm{ps}$ and (inset) the local view of autocorrelation trace in range of $2 \mathrm{ps}$.

To obtain broad SC, the pigtail of the mode locked fiber laser was connected to a $102 \mathrm{~m}$ highly nonlinear fiber (HNLF) directly. The HNLF had an effective area of $11.5 \mu \mathrm{m}^{2}$, the estimated nonlinear coefficient of $11.6 / \mathrm{W} / \mathrm{km}$ at $1550 \mathrm{~nm}$ and the cut-off wavelength of $1170 \mathrm{~nm}$. The zero dispersion wavelength (ZDW) of the HNLF was $\sim 1584 \mathrm{~nm}$. The dispersion and dispersion slope of the HNLF at $1550 \mathrm{~nm}$ were $-0.45 \mathrm{ps} / \mathrm{nm} / \mathrm{km}$ and $0.016 \mathrm{ps} / \mathrm{nm}^{2} / \mathrm{km}$, respectively. It should be pointed out that no amplifier stages are adopted in the SC laser source, which makes it very compact and cost-effective. The spectra of the SC from the HNLF with different pump power are shown in Fig. 3(a). The SC has very broad spectra spanning from less than $1200 \mathrm{~nm}$ to $\sim 2200 \mathrm{~nm}$. The spectrum of the SC doesn't change obviously with increasing in the pump power, though the spectral intensity increases as a whole. It's worth noting that the 3-dB bandwidth of the SC spans from $\sim 1370 \mathrm{~nm}$ to $\sim 1935 \mathrm{~nm}$ including the weak residual pump. In our case, the spectrum of the NLP covers both the slight normal and anomalous dispersion regime around the ZDW. In the initial stage, the physical mechanisms which contribute to spectral broadening are four wave mixing (FWM) and high order solitons formation. The spectrum 
extended to the anomalous dispersion regime and the pre-existing spectrum of the NLP in the anomalous dispersion regime form high order soliton due to the self-phase modulation combined with the anomalous dispersion. The high order solitons split into a series of fundamental solitons due to the perturbation like stimulated Raman scattering and high order dispersion. These solitons in the anomalous dispersion regime have very short pulse width and broad spectrum, which is very beneficial to soliton self-frequency shift (SSFS) leading to the spectrum extending to the longer wavelength regime dramatically. The output power of the laser and the SC with different pump power are shown in the inset of Fig. 3(b).


Fig.3 (a) Spectra of the SC from the HNLF with different pump power of the $1480 \mathrm{~nm}$ Raman Laser. (b) Output power of the NLP mode locked fiber laser and power of the SC from the highly nonlinear fiber.

In conclusion, we demonstrated a simple and compact all-fiber SC laser source with broad and ultra-flat spectra. The fiber laser achieved stable NLP mode locking based on the NALM. Using this NLP mode locked fiber laser to pump a $102 \mathrm{~m}$ HNLF directly, the SC spectrum spanning from less than $1200 \mathrm{~nm}$ to $2200 \mathrm{~nm}$ with the power of $196 \mathrm{~mW}$ was generated with $1480 \mathrm{~nm}$ pump power of $1630 \mathrm{~mW}$.

\section{References}

[1] J. M. Dudley, G. Genty, and S. Coen, "Supercontinuum generation in photonic crystal fiber," REV MOD PHYS 78, 1135 (2006).

[2] Z. Zheng, D. Ouyang and J. Zhao, et. al., "Dual-Operation Regime Thulium-Doped Fiber Laser and Its Applications in Cascaded Raman Light and Supercontinuum Generation," IEEE Photonics J 10, 1-9 (2018).

[3] S. Coen, A. H. L. Chau and R. Leonhardt, et. al., "White-light supercontinuum generation with 60-ps pump pulses in a photonic crystal fiber," Opt. Lett. 26, 1356-1358 (2001).

[4] J. M. Dudley, L. Provino and N. Grossard, et. al., "Supercontinuum generation in air- silica microstructured fibers with nanosecond and femtosecond pulse pumping," JOSA B 19, 765-771 (2002). 


\title{
Designing silicon fiber tapers for efficient mid-IR supercontinuum
}

\author{
J. Campling*a ${ }^{*}$ P. Horak ${ }^{\mathrm{a}}$ and A. C. Peacock ${ }^{\mathrm{a}}$ \\ ${ }^{a}$ Optoelectronics Research Centre, University of Southampton, UK SO17 1BJ
}

\begin{abstract}
We simulate supercontinuum generation for mid-infrared spectroscopy in three silicon fiber taper designs, demonstrating that a $2.1 \mu \mathrm{m}$ fiber laser input can be efficiently converted to the $3-4.5 \mu \mathrm{m}$ spectral range.

Keywords: supercontinuum, spectroscopy, silicon, optics, mid-infrared, taper, fiber laser, optical fibers *j.campling@soton.ac.uk
\end{abstract}

\section{Introduction}

In recent years there has been a great deal of interest in supercontinuum (SC) generation that spans the mid-infrared (mid-IR) spectrum, particularly between 2.7 and $4.3 \mu \mathrm{m}$ as this can be used for spectroscopy of greenhouse gases ${ }^{1-3}$. Although various systems have produced SC across this region and further, the output power levels involved are generally very small. With the advance of highpowered fiber lasers around $2 \mu \mathrm{m}$, the opportunity has arisen to produce a higher-powered SC across the $3-4 \mu \mathrm{m}$ range $(\sim 0.3 \mathrm{~mW})$, but the conversion efficiencies (CE) tend to be very low, at around $0.5 \%$, thus requiring extremely high peak input powers (tens of $\mathrm{kW})^{2}$. Recently, a higher CE has been achieved, but the mid-IR output is more narrow-band $(\sim 500 \mathrm{~nm})$ as it relies on dispersive wave (DW) generation in planar silicon nitride waveguides ${ }^{1}$. Changing the DW wavelength for different applications requires altering the waveguide width. Although the authors estimate that they can generate $1 \mathrm{~mW}$ across the entire $3-4 \mu \mathrm{m}$ range, this still requires tens of $\mathrm{kW}$ peak power, and very short pulse durations $(<100 \mathrm{fs})$ to achieve a $\mathrm{CE}$ of $\sim 2 \%$.

Silicon fibers offer a potential solution for efficient SC generation in the mid-IR that can tolerate more moderate input powers due to its high intrinsic nonlinearity ${ }^{4}$. Furthermore, SC can be produced with more readily achievable pulse widths from a fiber laser (100 $200 \mathrm{fs}$ ), so that additional compression stages are not needed ${ }^{5}$. Our designs can achieve a $2 \%$ CE across the $3-4 \mu \mathrm{m}$ range with $130 \mathrm{fs}$ pulses and input peak powers of only $\sim 5 \mathrm{~kW}$. By tapering the fiber, this power can be spread more evenly, producing a flatter SC, and also can be used to extend the SC to $4.5 \mu \mathrm{m}$ and beyond, thus covering the $4.3 \mu \mathrm{m}$ carbon dioxide absorption line and providing a solution for spectroscopy of all the major greenhouse gases.

In this paper, we show the results of SCG simulations using three taper designs to maximise the power conversion from a $2.1 \mu \mathrm{m}$ fiber laser to the entire $3-4.5 \mu \mathrm{m}$ range. The designs have the same length, waist diameter and input and output facets, but vary in their precise shape to change the distribution of power in the three $500 \mathrm{~nm}$ ranges covering this region.

\section{Designing the taper}

Silicon fibers are most commonly produced by the molten core drawing technique ${ }^{4}$, which is an adaption of the traditional fiber drawing method. The fiber preform is made by sleeving a silicon rod inside a silica capillary, which has been coated with an interface layer of calcium oxide to prevent diffusion of oxygen from the silica to the silicon. The interface layer also reduces stresses on the materials during the drawing process. The preform is then drawn into a fiber using a standard tower, under conditions where the molten core is contained in the softened glass cladding. A schematic of a silicon core fiber is shown in Figure 1(a).

As the silicon core fibers are clad in silica, they are robust and can be subsequently heated and stretched to further reduce their crosssection, producing a tapered structure. These tapers can be used for improving the input and output coupling efficiencies, and also for improving the splicing of silicon core fibers to single-mode fibers (SMFs) to produce all-fiber systems. In this paper we look at three taper designs, shown in Figure 1(b). All are 3mm long and have input and output core diameters of 2600nm and a waist diameter of $1700 \mathrm{~nm}$. The designs differ in the rate of diameter change and position of waist. Design 1 tapers down over $0.5 \mathrm{~mm}$, has a waist region of $1 \mathrm{~mm}$ and then tapers up over $0.5 \mathrm{~mm}$. The final $1 \mathrm{~mm}$ has constant diameter. The second and third designs taper down and then up again continuously. The difference is that Design 2 has a steeper down-taper $(1 \mathrm{~mm})$ and longer up-taper, whereas Design 3 has

Sixth International Workshop on Specialty Optical Fibers and Their Applications (WSOF 2019),

edited by Liang Dong, John M. Ballato, Proc. of SPIE Vol. 11206, 112061O · C 2019 SPIE

CCC code: $0277-786 \times / 19 / \$ 21 \cdot$ doi: $10.1117 / 12.2548323$

Proc. of SPIE Vol. 11206 1120610-1 
symmetrical tapered regions. These designs result in different distributions of the output spectrum, such that each one maximizes the power in one of the three $500 \mathrm{~nm}$ ranges studied here $(3-3.5 \mu \mathrm{m}, 3.5-4 \mu \mathrm{m}$ and $4-4.5 \mu \mathrm{m})$.

We chose the waist diameter, $1700 \mathrm{~nm}$, because the pump lies in the anomalous dispersion regime close to the zero-dispersion wavelength (ZDW), so that soliton fission can occur in addition to self-phase modulation for maximum initial spectral broadening. The up-taper region then redistributes the power to longer wavelengths, because the dispersion parameters continuously change, allowing new phase-matching conditions to transfer power via non-degenerate four-wave-mixing. The output diameter (2600nm) produces optimal phase-matching conditions for transfer of power to the $4-4.5 \mu \mathrm{m}$ range.

(a)

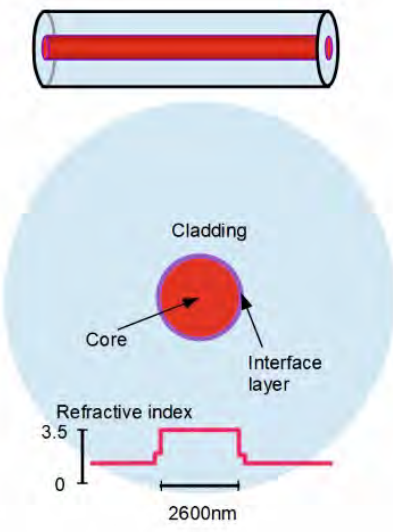

(b)

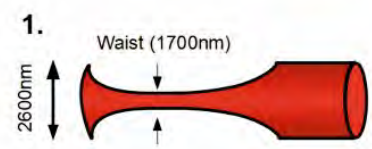

2. Waist $(1700 \mathrm{~nm})$
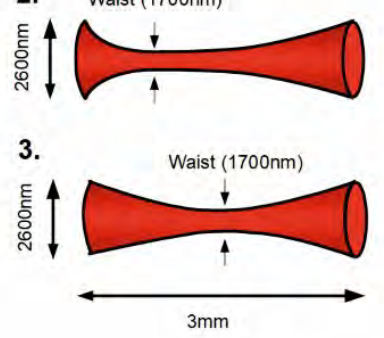

(c)

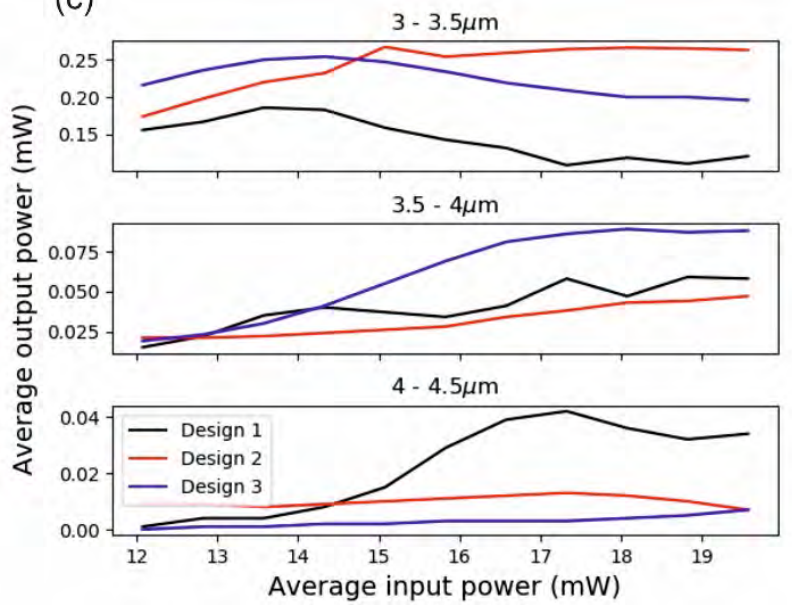

Figure 1. (a) Cross section of silicon fiber showing core, cladding and interface layers. (b) $1-3$ show the three taper designs (core dimensions only). (c) Power outputs of simulations in three wavelength ranges according to input power. Black lines show Design 1, red lines Design 2 and blue lines Design 3.

\section{Results and Discussion}

We tested the designs with propagation simulations using a pulse width of $130 \mathrm{fs}$, which is a typical pulse width achievable with thulium-doped fiber lasers ${ }^{5}$. The simulations used the nonlinear Schrödinger equation ${ }^{6}$, which includes two-photon absorption, free carrier absorption, and also wavelength-dependent linear loss to account for higher cladding losses beyond $4 \mu \mathrm{m}$.

(a)

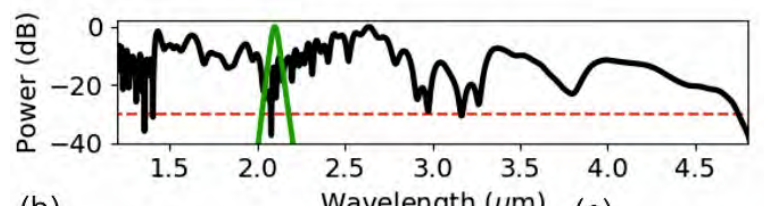

(b)

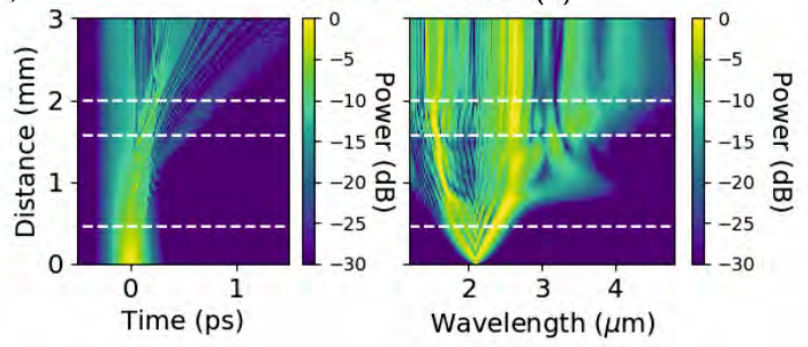

(d)

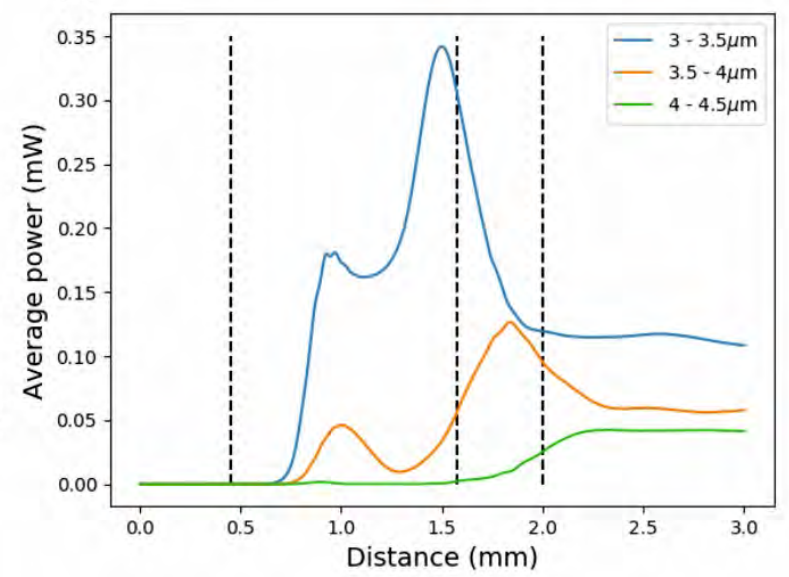

Figure 2. (a) Output SC (green line is input spectrum, red dashed line shows -30dB level), (b) temporal evolution and (c) spectral evolution with $17.3 \mathrm{~mW}$ (5.75kW peak) 130fs pulses in Design 1. (d) Power evolution of the mid-IR ranges of interest. Dashed lines show the ends of the down-taper, waist and up-taper. 
Figure 2(a) shows the SC produced with $5.75 \mathrm{~kW}$ peak pulses $(17.3 \mathrm{~mW}$ average) in Design 1. Figure 2(b) shows the evolution of pulses in the time domain. Soliton fission occurs just before the up-taper region. 2(c) shows the evolution of the spectrum. It can be seen how the up-taper and final section both extend the long-wavelength side of the SC. 2(d) Shows the average power levels in the three wavelength ranges of interest. The waist region transfers the initial power, but this is concentrated below 3.5 $\mu \mathrm{m}$. The up-taper redistributes some of this power into the $3.5-4 \mu \mathrm{m}$ range, and finally, the end-section further redistributes power into the $4-4.5 \mu \mathrm{m}$ range. This design produces the maximum output in this longest wavelength region because the phase-matching conditions in the end section remain constant over $1 \mathrm{~mm}$.

The other two designs produce less power beyond $4 \mu \mathrm{m}$, but the SC (measured at $-30 \mathrm{~dB}$ level) can still extend beyond $4.5 \mu \mathrm{m}$, and the power transfer to the whole $3-4 \mu \mathrm{m}$ range is more efficient. Figure 1(b) shows the output power in each of the three ranges according to input power for all three designs.

Design 2 consistently produces the most power in the $3-3.5 \mu \mathrm{m}$ range, while Design 3 redistributes this power more effectively into the $3.5-4 \mu \mathrm{m}$ range, producing more power overall across $3-4 \mu \mathrm{m}(\sim 0.3 \mathrm{~mW})$. With an input of $15 \mathrm{~mW}$ this is a $\mathrm{CE}$ of $2 \%$.

\section{Conclusion}

In this paper we have shown that tapered silicon fibers pumped with a fiber laser are an efficient platform for generating a SC that covers the entire greenhouse gas absorption window $(2.7-4.3 \mu \mathrm{m})$. These fibers can be spliced to SMF fibers, allowing for a portable, all-fiber solution to mid-IR spectroscopy.

\section{References}

[1] Grassani, D., Tagkoudi, E., Guo, H. , Herkommer, C., Yang, F., Kippenberg, T. J. and Brès, C.-S., "Mid infrared gas spectroscopy using efficient fiber laser driven photonic chip-based supercontinuum," Nat. Commun. 10(1), 1553 (2019).

[2] Hickstein, D. D., Jung, H., Carlson, D. R. , Lind, A., Coddington, I., Srinivasan, K., Ycas, G. G., Cole, D. C., Kowligy, A., Fredrick, C. et al., "Ultrabroadband supercontinuum generation and frequency-comb stabilization using on-chip waveguides with both cubic and quadratic nonlinearities,” Phys. Rev. Appl. 8(1), 014025 (2017).

[3] Cossel, K. C., Waxman, E. M., Finneran, I. A., Blake, G. A., Ye, J. and Newbury, N. R., "Gas-phase broadband spectroscopy using active sources: progress, status, and applications [Invited],” J. Opt. Soc. Am. B 34, 104-129 (2017).

[4] Ren, H., Aktas, O., Franz, Y., Runge, A. F. J., Hawkins, T., Ballato, J., Gibson, U. J. and Peacock, A. C., “Tapered silicon core fibers with nano-spikes for optical coupling via spliced silica fibers," Opt. Express 25, 24157-24163 (2017).

[5] Rudy, C. W., Digonnet, M. J. F. and Byer, R. L., “Advances in $2 \mu \mathrm{m}$ Tm-doped mode-locked fiber lasers,” Opt. Fiber Technol. 20(6), 642-649 (2014).

[6] Lin, Q., Painter, O. J. and Agrawal, G. P., "Nonlinear optical phenomena in silicon waveguides: Modeling and applications," Opt. Express 15(25), 16604-16644 (2007). 


\title{
Heat mitigation via anti-Stokes fluorescence cooling in Core/Cladding Yb-doped fiber amplifiers
}

\author{
Esmaeil Mobini ${ }^{a, b}$, Mostafa Peysokhan ${ }^{a, b}$, and Arash Mafi ${ }^{a, b}$ \\ ${ }^{a}$ Department of Physics \& Astronomy, University of New Mexico, Albuquerque, NM 87131, \\ USA \\ ${ }^{b}$ Center for High Technology Materials, University of New Mexico, Albuquerque, NM 87106, \\ USA
}

\begin{abstract}
Radiation-balancing is proposed to offset the heating in a laser via anti-Stokes fluorescence cooling. Here, we report a Core/Cladding ion-doped fiber amplifier where the radiation-balancing can efficiently offset the generated heat in high-power operation.
\end{abstract}

Keywords: Radiation-Balanced Laser, Solid-state laser cooling, Fiber laser, Fiber amplifier

\section{INTRODUCTION}

Heat management has been an important issue in high-power fiber amplifiers over the past few decades. In high-power operation of the fiber amplifiers, the temperature variation inside the core of the fiber amplifiers becomes large enough to initiate modal instabilities. ${ }^{1}$ Radiation Balanced Laser (RBL) has been introduced first by S. Bowman in 1999 .2,3 This method relies on anti-Stokes fluorescence cooling where the generated heat from quantum defect is simultaneously canceled out by the heat extraction from anti-Stokes fluorescence cooling. ${ }^{3}$

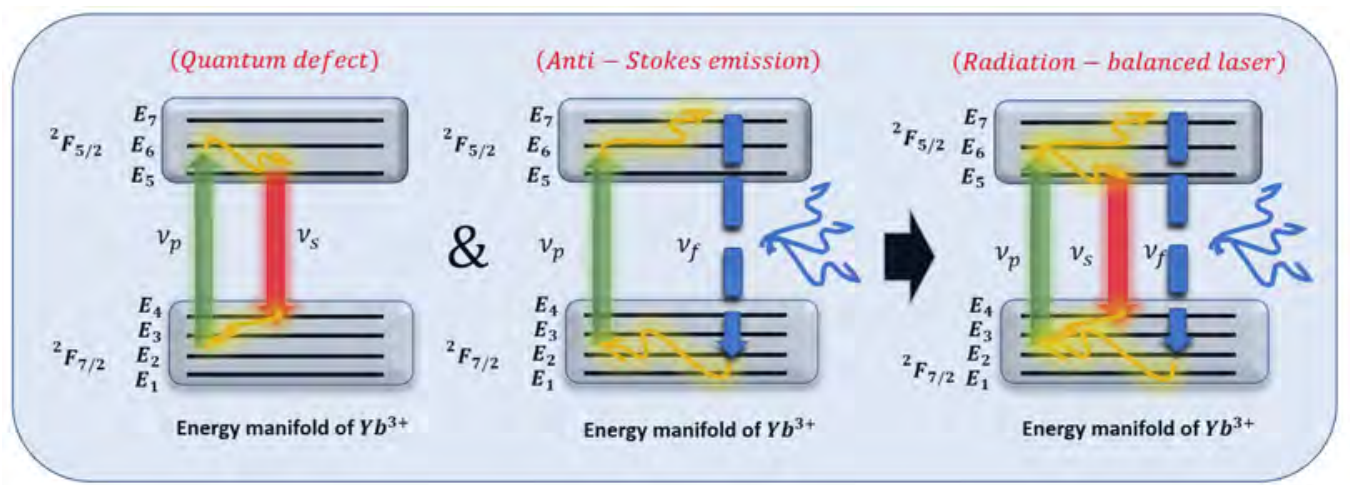

Figure 1. Schematic of radiation-balancing process in a laser with a Yb-doped gain. As it is obvious, quantum defect which is an intrinsic process in a laser can be canceled out simultaneously by anti-Stokes fluorescence cooling.

As it is obvious from Fig. 1, as a laser functions, it turns part of its input energy into heat through quantum defect which is proportional to $q_{1} \propto\left(1-\nu_{s} / \nu_{p}\right) P_{p}$.If the gain medium of the laser is capable of anti-Stokes fluorescence cooling, then part of the input energy proportional to $q_{2} \propto\left(1-\nu_{f} / \nu_{p}\right) N_{2}$ will be offset simultaneously by anti-Stokes fluorescence emission. If the pump and signal wavelengths satisfy $\nu_{s}<\nu_{p}<\nu_{f}$ which is the precondition of anti-Stoke fluorescence cooling, under a very delicate balanced condition between the pump and signal intensities, the heat generation from quantum defect can be canceled out by the heat extraction from anti-Stokes fluorescence cooling, $q_{1}+q_{2}=0$. Here, $\nu_{s}$ is the signal wavelength, $\nu_{p}$ is the pump wavelength, $\nu_{f}$ is

Further author information: (Send correspondence to Arash Mafi)

Arash Mafi: E-mail: mafi@unm.edu

Sixth International Workshop on Specialty Optical Fibers and Their Applications (WSOF 2019),

edited by Liang Dong, John M. Ballato, Proc. of SPIE Vol. 11206, 112061P · (C) 2019 SPIE

CCC code: $0277-786 X / 19 / \$ 21 \cdot$ doi: $10.1117 / 12.2548341$

Proc. of SPIE Vol. 11206 112061P-1 
the mean fluorescence wavelength, $P_{p}$ is the pump power and $N_{2}$ is the ion density in the upper level of the $\mathrm{Yb}$ ions.

In practice there are a few issues that make it very difficult to implement radiation-balancing in a laser or amplifier especially in a fiber amplifier that has a small cross-sectional area. On the one hand, we know that the heat generation from the background absorption linearly scales up with the input power, but on the other hand, the heat extraction from the anti-Stokes fluorescence cooling is proportional to the ion density in the upper level $\left(N_{2}\right)$ that gets saturated with the increase of the power. ${ }^{4}$ Meaning that as the power goes up in a laser or amplifier, the heat extraction from anti-Stokes fluorescence cooling cannot catch up with the heat generation from background absorption and consequently the required balanced condition for RBL fails to hold. To solve the problem in high-power amplifier, we come up with a new configuration that will be explained in the next session.

\section{CORE/CLADDING YB-DOPED FIBER AMPLIFIER}

In Ref., ${ }^{4}$ we explored the heat mitigation by anti-Stokes fluorescence cooling in a typical Yb-doped silica DC fiber amplifier. The calculations show that the anti-Stokes fluorescence cooling can offset the generated heat in a DC fiber amplifier for powers up to a few tens of Watts. But at higher powers, e.g., hundreds of Watts and more, the background absorption of the inner cladding dominates over the other factors contributing to the heat generation and extraction mechanisms. The heat generated due to the background absorption in the inner cladding dominates significantly the maximum heat extraction that can be delivered by the anti-Stokes fluorescence cooling inside the core; therefore, for the high power operation of a typical DC fiber amplifier, the anti-Stokes fluorescence cooling fails to offset the generated heat effectively. Hence, as mentioned earlier, a new configuration needs to be introduced in which the heat extraction by the anti-Stokes fluorescence cooling is large enough to counterbalance the significant heat load from the background absorption generated in the inner cladding in high-power operation.

In the new configuration, we dope the inner cladding with the same ions as in the core. Because the inner cladding area is much larger than the core area, a properly doped inner cladding can increase the cooling power sufficiently to counterbalance the heat generation especially in the high-power operation. Because in the configuration both the core and inner cladding are doped with the same $\mathrm{Yb}$ ion, we coin the term Core/Cladding $(\mathrm{C} / \mathrm{C}) \mathrm{Yb}$-doped fiber amplifier to refer to the new configuration. The cross-sectional schematic of the proposed $\mathrm{C} / \mathrm{C}$ ion-doped fiber is shown in Fig. 2, where $a$ and $b$ represent the core and inner cladding radii, respectively. The total dopant distribution $\left(N_{0}\right)$ in the core and the inner cladding is defined by

$$
N_{0}(\rho)=\left\{\begin{array}{ll}
N_{01}, & \rho \leq a \\
N_{02}, & a<\rho \leq b
\end{array},\right.
$$

where $N_{01}$ and $N_{02}$ are the dopant densities in the core and inner cladding, respectively, and $\rho$ represents the radial coordinate.

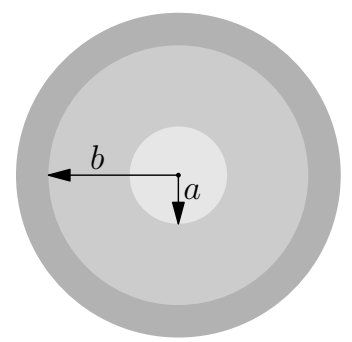

Figure 2. Schematic of a C/C ion-doped fiber amplifier.

In order to calculate the pump and signal intensities along the fiber, we follow the formalism introduced in. ${ }^{4}$ Unlike a typical DC fiber amplifier in which the pump power is only attenuated by the background absorption in 
the inner cladding, here, in the suggested $\mathrm{C} / \mathrm{C}$ ion-doped fiber amplifier, the pump power in the inner cladding is attenuated by the background absorption and resonant absorption that comes from the dopant in the inner cladding. Therefore, the impact of the dopant in the inner cladding should be considered in the modeling. The detailed modeling of the $\mathrm{C} / \mathrm{C}$ ion-doped configuration can be found in. ${ }^{5}$

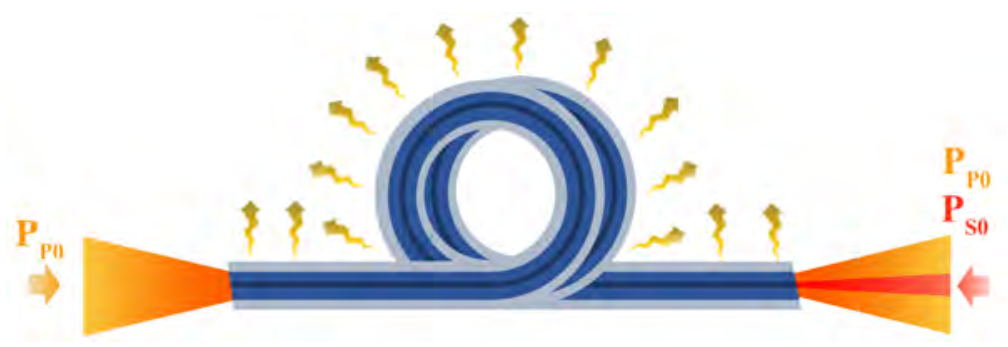

Figure 3. Schematic of a C/C ion-doped fiber amplifier.

Figure 3 describes the schematic of the $\mathrm{C} / \mathrm{C}$ Yb-doped fiber amplifier. As it is obvious from Fig. 2, the suggested configuration consists of a core and inner cladding with radii of $a$ and $b$, respectively. As it is clear in Fig. 3, the pump power can be coupled into the fiber amplifier from either port 1 or port 2 at $\mathrm{z}=0$ and $\mathrm{z}=\mathrm{L}$, respectively. The signal power is introduced into the fiber amplifier from port 2 . We also take the fractional signal power in the core to be $\eta_{a}=0.9$.

In the modeling, for the host material we choose ZBLAN glass which is amenable to anti-Stokes florescence cooling. We take the core and inner cladding to be $a=13 \mu \mathrm{m}$ and $b=182 \mu \mathrm{m}$. The fiber amplifier is pumped from port 1 at $\lambda_{p}=1020 \mathrm{~nm}$ with an input power of $P_{p 0}^{+}=0.5 \mathrm{KW}$ and seeded at $\lambda_{s}=1050 \mathrm{~nm}$ with the seed power of $P_{s 0}^{-}=1 \mathrm{~W}$. The radiative lifetimes of both core and inner cladding are assumed to be $\tau_{r}=1.8 \mathrm{~ms}$. The mean fluorescence wavelength and the fiber length are also taken to be $\lambda_{f}=995 \mathrm{~nm}$ and $\mathrm{L}=9 \mathrm{~m}$, respectively. We also take the $\mathrm{Yb}$ ion density in the core and inner cladding to be $N_{01}=3 \times 10^{26} \mathrm{~m}^{-3}$ and $N_{02}=5 \times 10^{25} \mathrm{~m}^{-3}$, respectively. Here, we also assume that the absorptive and scattering parts of the background absorption $\left(\alpha_{b}=\alpha_{b a}+\alpha_{b s}\right)$ are $\alpha_{b a}=10 \mathrm{~dB} / \mathrm{km}$ and $\alpha_{b s}=10 \mathrm{~dB} / \mathrm{km}$, respectively. Needless to say, the absorptive part of the background absorption only contributes to the heat generation. We also assume that the internal quantum efficiency of both core and inner cladding in the configuration is near unity $\eta_{q}=1$.

Figure 4 (a) describes the pump and signal powers along the fiber amplifier. The pump power is totally absorbed along the amplifier, and signal power is amplified up to $P_{s}^{-}(0)=0.06 \mathrm{KW}$, which is equivalent to a signal efficiency of $\eta_{s}=12 \%$. Figure 4 (b) also describes the temperature distribution along the fiber amplifier. It is clear that, in less than $2 \mathrm{~m}$ after port 1, the amplifier temperature goes below the ambient temperature $\left(T_{0}\right)$. The calculations show that the longitudinal average of the temperature is $\overline{\Delta T}=-5.1 \mathrm{~K}$ where $\overline{\Delta T}=(1 / L) \int_{0}^{L} T(\rho=0, z) d z-T_{0}$. The detailed calculations also show that the temperature variation across the fiber core at port 1 is $\delta T=T(\rho, 0)-T(\rho=a, 0)=0.08 \mathrm{~K}$ which is in an acceptable range.
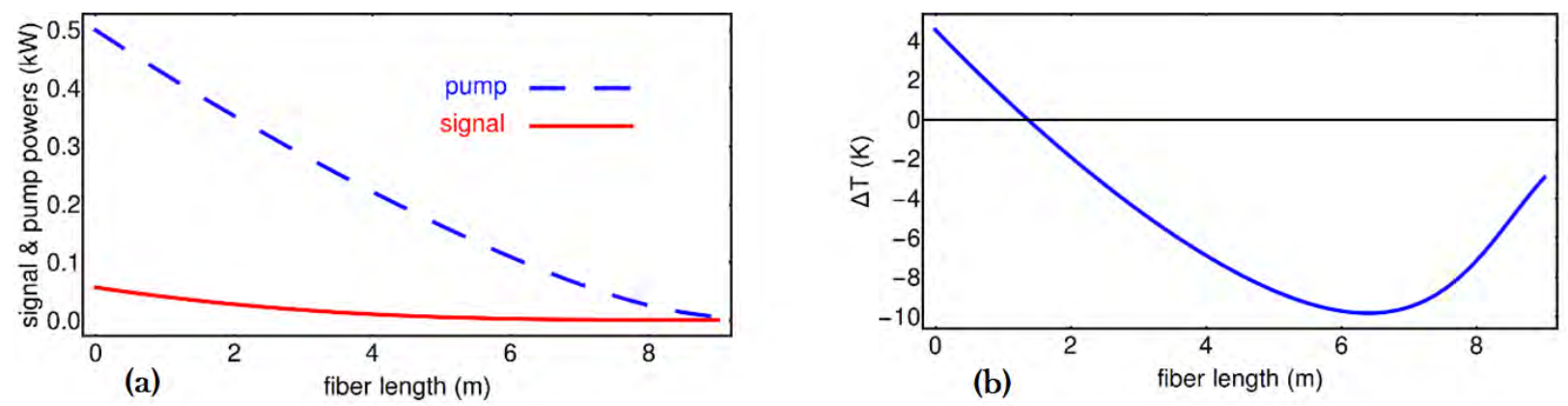

Figure 4. (a) Propagation of right and left moving pump and signal powers along the single-pass pump C/C Yb-doped ZBLAN fiber amplifier at $\lambda_{p}=1020 \mathrm{~nm}$ and $\lambda_{s}=1050 \mathrm{~nm}$ with $P_{p 0}^{+}=0.5 \mathrm{~kW}$, (b) Longitudinal temperature distribution $(\Delta T)$ of the single-pass pump $\mathrm{C} / \mathrm{C}$ Yb-doped ZBLAN fiber amplifier. 
In conclusion, we introduced a new design of the DC fiber amplifier where the anti-Stokes fluorescence cooling can effectively offset the generated heat from the quantum defect and background absorption in high-power operation. Unlike a conventional DC fiber amplifier, the C/C Yb-doped fiber amplifier benefits from a Yb-doped cladding that bestows heat extraction mechanism from anti-Stokes fluorescence cooling on the configuration so that the anti-Stokes fluorescence cooling can cancel out the generated heat efficiently. We have shown that the temperature of the configuration can go below the ambient temperature.

\section{ACKNOWLEDGMENT}

This material is based upon work supported by the Air Force Office of Scientific Research under award number FA9550-16-1-0362 titled Multidisciplinary Approaches to Radiation Balanced Lasers (MARBLE).

\section{REFERENCES}

[1] Dawson, J. W., Messerly, M. J., Beach, R. J., Shverdin, M. Y., Stappaerts, E. A., Sridharan, A. K., Pax, P. H., Heebner, J. E., Siders, C. W., and Barty, C., "Analysis of the scalability of diffraction-limited fiber lasers and amplifiers to high average power," Opt. Express 16, 13240-13266 (2008).

[2] Bowman, S. R., "Lasers without internal heat generation," IEEE Journal of Quantum Electron 35, 115-122 (1999).

[3] Yang, Z., Meng, J., Albrecht, A. R., and Sheik-Bahae, M., "Radiation-balanced Yb: Yag disk laser," Optics Express 27, 1392-1400 (2019).

[4] Mobini, E., Peysokhan, M., Abaie, B., and Mafi, A., "Thermal modeling, heat mitigation, and radiative cooling for double-clad fiber amplifiers," J. Opt. Soc. Am. B 35, 2484-2493 (2018).

[5] Mobini, E., Peysokhan, M., and Mafi, A., "Heat mitigation of a core/cladding Yb-doped fiber amplifier using anti-Stokes fluorescence cooling," J. Opt. Soc. Am. B 36, 2167-2177 (2019). 


\title{
Measuring the anti-Stokes cooling parameters of a Yb-doped ZBLAN fiber for radiation balancing
}

\author{
Mostafa Peysokhan, Esmaeil Mobini, and Arash Mafi \\ Department of Physics \& Astronomy, University of New Mexico, Albuquerque, NM 87131, \\ USA \\ Center for High Technology Materials, University of New Mexico, Albuquerque, NM 87106, \\ USA
}

\begin{abstract}
Anti-Stokes fluorescence cooling can mitigate the generated heat in fiber lasers. Performing various tests on a ytterbium-doped ZBLAN fiber, we show that the ZBLAN glass is a viable host material for implementation in radiation-balanced laser.
\end{abstract}

Keywords: anti-Stokes fluorescence, laser cooling, radiation balanced laser, Yb-doped, ZBLAN, fiber laser

\section{INTRODUCTION}

Rare-earth-doped fiber lasers have proven to be efficient and reliable sources of high-power radiation. They have many unique properties like mode-hop-free, narrower linewidths, less noise, compact all-fiber designs, and diffraction-limited beam qualities. ${ }^{1,2}$ To tackle the demand for higher-power lasers, effective heat management in fiber lasers is crucial. Current approaches to power scaling are restricted by the thermally-induced mode instabilities, which degrade the output beam quality. ${ }^{3-7}$ The conventional approach for heat dissipation in fiber lasers benefits from external agents such as water- and air-forced cooling. Whereas Anti-Stokes fluorescence (ASF) cooling has been proposed as a self-cooling mechanism to address such thermal issues without assistance of any external agents. ${ }^{8,9}$ Radiation-balancing can mitigate the heat-load from quantum defect and background absorption which are inherent to any laser or amplifier via ASF cooling. ${ }^{10-13}$

To implement the radiation-balancing in a fiber laser, first one needs to characterize the material and optical properties of the gain medium such as resonant and background absorption coefficients to investigate its amenability to ASF cooling. It has been shown that the parasitic absorption of the host material is the dominant factor in the heat generation in radiation-balanced lasers and amplifiers in high-power operation. ${ }^{14}$ Therefore, it is critical to characterize the parasitic absorption of the Yb-doped ZBLAN glass fibers for implementation in radiation-balanced lasers. In this paper, we combine two techniques: the "Laser-Induced Temperature Modulation Spectrum" (LITMoS) test ${ }^{15}$ and the recently developed "Measuring the Absorption Coefficient via Side-Light Analysis" (MACSLA) method ${ }^{16,17}$ to first of all, investigate the amenability of the Yb-doped ZBLAN glass to the ASF cooling and secondly, determine its resonant and parasitic absorption coefficients. It is shown that by selecting the right pump and signal wavelengths and optimizing fiber laser geometry combined with implementing new designs of optical fibers like Core/Cladding ion doped configuration, efficient heat mitigation using radiation-balancing in a Yb-doped ZBLAN glass fiber laser is achievable. ${ }^{18,19}$

\section{COOLING EFFICIENCY}

The implemented experimental setup of the LITMoS test for measuring the cooling efficiency, $\eta_{\mathrm{abs}}$, is shown in Fig. 1. The implemented fiber in the experiment is a $\left(1 \% Y b F_{3}\right)$ ZBLAN glass with a core diameter of $300 \mu \mathrm{m}$ and a cladding diameter of $430 \mathrm{\mu m}$. In order to avoid any heat-load on both facets of the fiber, we performed a cooling-grade polishing on the both facets of the fiber. For the polishing we used a cooling grade polishing technique that was developed in our lab. As it is illustrated in Fig. 1a., the ZBLAN fiber is pumped by a

Further author information: (Send correspondence to Arash Mafi)

Arash Mafi: E-mail: mafi@unm.edu

Sixth International Workshop on Specialty Optical Fibers and Their Applications (WSOF 2019),

edited by Liang Dong, John M. Ballato, Proc. of SPIE Vol. 11206, 112061Q · C 2019 SPIE

CCC code: $0277-786 \mathrm{X} / 19 / \$ 21 \cdot$ doi: $10.1117 / 12.2548362$

Proc. of SPIE Vol. 11206112061 Q-1 
tunable Ti:Sapphire laser. Light is coupled to the ZBLAN fiber through a 20x microscope objective. The pump is reflected-back into the fiber by an objective-mirror component in a double-pass configuration. For measuring the fiber temperature, a thermal camera on top of the fiber is used. Two thin fiber as it is shown in Fig. 1b. is also used to support and minimize the thermal contact with the ZBLAN fiber.

The fluorescence spectral power density of the ZBLAN fiber, $S(\lambda)$, is measured from the fiber side by a multimode optical fiber connected to a spectrometer. We note that the multimode optical fiber does relocate during the LITMoS test. Therefore, the absorbed power density, $P_{\text {abs }}$ at each pump wavelength, $\lambda_{p}$, is proportional to the total collected fluorescence spectral power. In other words, $P_{\text {abs }}\left(\lambda_{p}\right) \propto \int d \lambda S_{p}(\lambda)$, where the integral is performed over the entire fluorescence spectrum, and the subscript $p$ in $S_{p}(\lambda)$ signifies that the fluorescence spectral power density relates to pumping the ZBLAN fiber at $\lambda_{p}$. We emphasize that by changing $\lambda_{p}$, only the overall intensity of $S_{p}(\lambda)$ is rescaled and its spectral form does not change. $P_{\text {net }}$ is proportional to the change in the temperature, $\Delta T$, of the fiber, which is proportional to $\Delta$ (pixel) of the image captured by the thermal camera at each $\lambda_{p}$. Therefore, for at pump wavelength, $\lambda_{p}$, the cooling efficiency is approximated by $\eta_{c} \propto \Delta$ (pixel) $/ \int d \lambda S_{p}(\lambda)$. To quantify ASF cooling, it is customary to use the cooling efficiency, $\eta_{c}$.
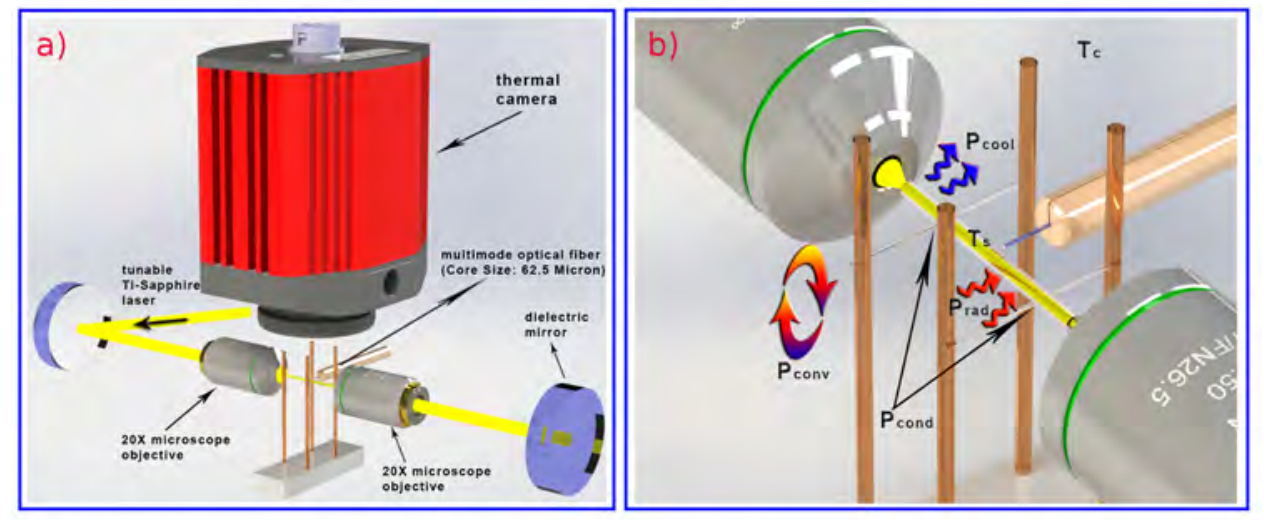

Figure 1. a) Experimental setup for LITMoS test of the Ytterbium doped ZBLAN fiber. b) Magnified fiber holder part and illustration of three source of heat load, convective, conductive and radiative

Here, $\eta_{c}$ is the net power density (per unit volume) deposited in the material $\left(P_{\text {net }}\right)$ per unit power density absorbed or scattered $\left(P_{\mathrm{abs}}\right): \eta_{c}=P_{\text {net }} / P_{\text {abs }}$. Therefore, we have $P_{\text {net }}=P_{\text {abs }}-P_{\text {asf }}$ where $P_{\text {asf }}$ is the ASF power density that escapes the cooling material. The absorbed power density is given by $P_{\text {abs }}=\left(\alpha_{r}+\alpha_{b}\right) I_{P}$, where $I_{P}$ is the pump intensity, and $\alpha_{r}$ and $\alpha_{b}$ are the resonant and parasitic absorption coefficients, respectively. The ASF power density is given by $\eta_{e} N_{2} W_{r}\left(h \nu_{f}\right)$ where $\nu_{f}$ is the mean florescence frequency, $N_{2}$ is the number density of the excited upper level in the quasi two-level $\mathrm{Yb}$ ions, and $W_{r}\left(W_{n r}\right)$ is the radiative (non-radiative) decay rate of the excited state of the doped ions. $\eta_{e}$ is the extraction (escape) efficiency and $1-\eta_{e}$ is the fraction of photons which are radiated but are trapped inside the host. Finally, the rate equation of the ASE cooling can be expressed by

$$
d N_{2} / d t=\alpha_{r} I_{P} /\left(h \nu_{p}\right)-\left(W_{r}+W_{n r}\right) N_{2}+\left(1-\eta_{e}\right) W_{r} N_{2},
$$

where we have assumed that the trapped florescence is reabsorbed by the $\mathrm{Yb}$ ions. In a steady-state situation, $d N_{2} / d t=0$, we can solve for $N_{2}$ and obtain $P_{\text {asf }}=-\alpha_{r} I_{P} \eta_{q}\left(\lambda_{p} / \lambda_{f}\right)$, where the external quantum efficiency is given by $\eta_{q}=\eta_{e} W_{r} /\left(\eta_{e} W_{r}+W_{n r}\right)$, and $\lambda_{p}\left(\lambda_{f}\right)$ is the pump (mean florescence) wavelength. Hence, we have

$$
P_{\text {net }}=\left(\alpha_{r}+\alpha_{b}\right) I_{P}-\alpha_{r} I_{P} \eta_{q}\left(\lambda_{p} / \lambda_{f}\right) .
$$

We can use these results to present the cooling efficiency as

$$
\eta_{c}\left(\lambda_{p}\right)=1-\frac{\lambda_{p}}{\lambda_{f}} \eta_{q} \eta_{\mathrm{abs}}\left(\lambda_{p}\right)
$$


where the absorption efficiency is given by

$$
\eta_{\mathrm{abs}}\left(\lambda_{p}\right)=\frac{\alpha_{r}\left(\lambda_{p}\right)}{\alpha_{r}\left(\lambda_{p}\right)+\alpha_{b}} .
$$

The ASF cooling requires $P_{\text {net }}$ to be negative which means that the cooling efficiency should be negative, $\eta_{c}<0$. From Eq. 3, one immediately realized that the pump wavelength should be larger than the mean-fluorescence wavelength, $\lambda_{p}>\lambda_{f}$, for satisfying the necessary condition for ASF cooling given that $0 \leq \eta_{q}, \eta_{\mathrm{abs}} \leq 1$. In practice, both $\eta_{q}$ and $\eta_{a b s}$ must be very close to unity to satisfy the ASF cooling condition due to the fact that $\lambda_{p}$ cannot be much longer than $\lambda_{f}$, otherwise the pump absorption coefficient, $\alpha_{r}\left(\lambda_{p}\right)$, becomes too small that the absorption efficiency nearly vanishes $\eta_{\mathrm{abs}}\left(\lambda_{p}\right)$. Therefore, in an ASF cooling experiment, $\lambda_{p} / \lambda_{f}\left(\eta_{\mathrm{abs}}\left(\lambda_{p}\right)\right)$ is a monotonically increasing (decreasing) function of the pump wavelength. Therefore, the cooling condition requires the product of $\lambda_{p} / \lambda_{f}$ and $\eta_{\text {abs }}\left(\lambda_{p}\right)$ to lie in an acceptable range to guarantee a negative cooling efficiency considering $\eta_{q} \approx 1$. As mentioned earlier, using the LITMoS test, we can measure the $\eta_{\mathrm{c}}$ from which finally $\eta_{q}$ and $\eta_{\mathrm{abs}}$ can be extracted for the cooling-grade material. Once $\eta_{\mathrm{abs}}$ is determined, we will then apply the MACSLA method to find the values of $\alpha_{b}$, as well as $\alpha_{r}\left(\lambda_{p}\right)$.

In Fig. 2a, the blue circles correspond to $\Delta$ (pixel) and the red asterisks represent $\int d \lambda S_{p}(\lambda)$. The ratio is plotted in Fig. 2b and is fitted to Eq. 3, where the $\Delta$ (pixel) $/ \int d \lambda S_{p}(\lambda)$ ratio is renormalized by a single overall scaling factor to conform to Eq. 3. We note that $\alpha_{r}\left(\lambda_{p}\right)$ follows a strict spectral function of the form: ${ }^{20-22}$

$$
\alpha_{r}(\lambda) \propto \lambda^{5} S(\lambda) \exp \left(\frac{h c}{\lambda k_{B} T}\right)
$$

which is used in Eq. 4 and Eq. 3 to perform the fit. Here, $h$ is the Planck constant, $k_{B}$ is the Boltzmann constant, $c$ is the speed of light in vacuum, and $T$ is assumed to be the room temperature as long as the temperature variation due to ASF cooling is not large. Equation 5 allows us to replace $\alpha_{r}(\lambda)$ in Eq. 4 with $\alpha_{r}^{p} \times \widetilde{\alpha}_{r}(\lambda)$, where $\widetilde{\alpha}_{r}(\lambda)$ is the absorption coefficient normalized to its peak value, $\alpha_{r}^{p}=\alpha_{r}\left(\lambda_{\text {peak }}\right)$. Therefore, the fitting procedure in Fig. $2 \mathrm{~b}$ becomes a two-parameter fit (besides the overall scaling) in which we ca determine the $\alpha_{b} / \alpha_{r}^{p}$ and quantum external efficiency $\left(\eta_{q}\right)$. Which are found to be $2.3 \times 10^{-4}$ and $99.6 \%$ respectively.
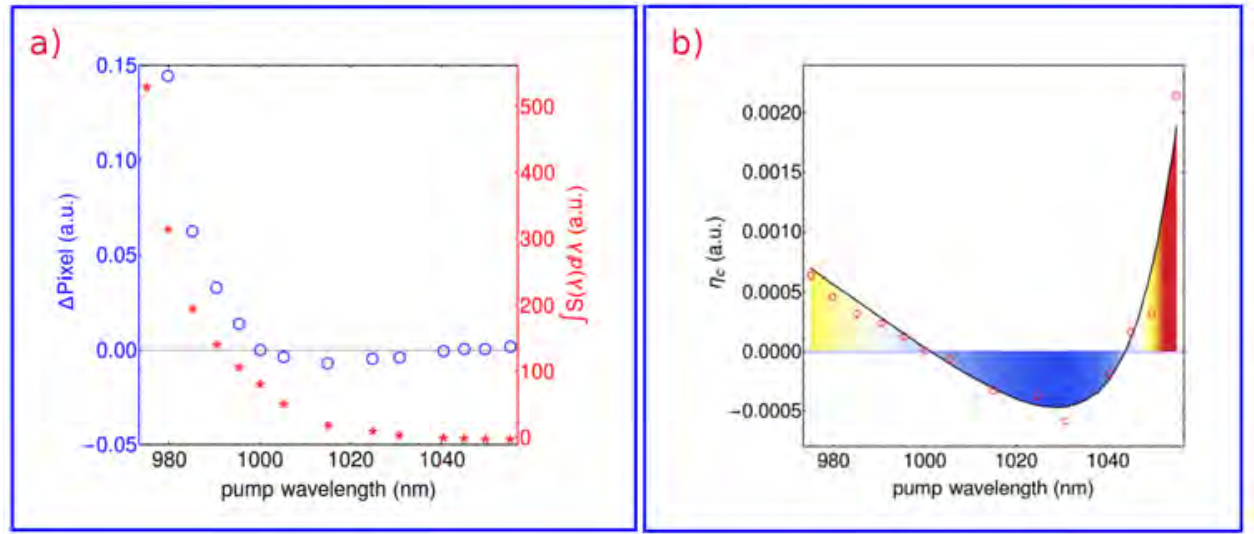

Figure 2. a) Blue circles are the $\Delta$ Pixel of the thermal camera at each wavelengths, and red asterisks are the integrate under the $S(\lambda)$. b) Measured and fitting of the cooling efficiency $\left(\eta_{c}\right)$ for the Yb:ZBLAN fiber. The solid curve shows a fit of $\eta_{c}$ and the red circles shows data points at different wavelength.

\section{MEASURING THE RESONANCE ABSORPTION}

In the previous section, we managed to determine the external quantum efficiency, $\eta_{q}$, along with the ratio of the parasitic background absorption to the peak resonant absorption, $\alpha_{b} / \alpha_{r}^{p}$. In order to find the actual values of $\alpha_{b}$ and $\alpha_{r}^{p}$, we can now use the method we recently proposed, dubbed as "measuring the absorption coefficient via side-light analysis" (MACSLA). ${ }^{16,23}$ The MACSLA method is based on comparing the amount of collected spontaneous emission power at two arbitrary points along the fiber for different pump wavelengths. 

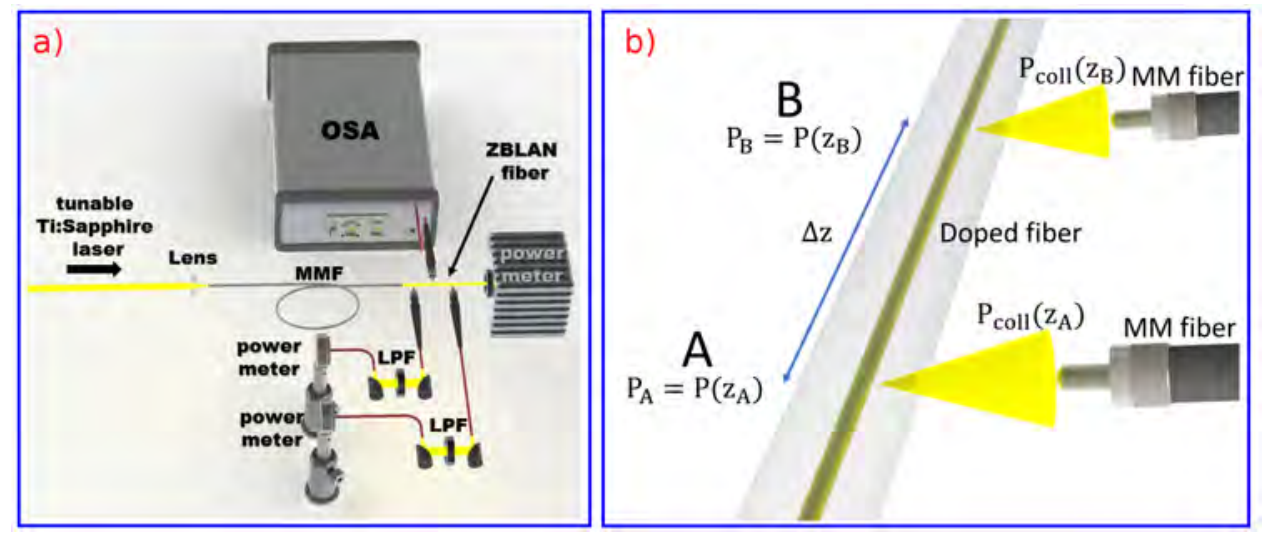

Figure 3. a) Schematic of the experimental setup which is used for the MACSLA method. OSA stands for optical spectrum analyzer, LPF for long-pass filter, and MMF for multimode fiber. b) Schematic of the propagation of the pump power in the core of the optical fiber. And collecting the spontaneous emission from the side of the Yb-doped ZBLAN fiber by two multimode passive optical fibers.

For multimode optical fibers, due to their large core size, unlike a single-mode fiber they emit enough emission from their side to capture; therefore, a lock-in amplifier is not required for capturing emission. ${ }^{16}$ Then the spontaneous emission power from the side of the fiber could be measured directly by a power meter. And also instead of a passive single-mode fiber, a multimode fiber is utilized to lunch the pump power to the core of the Yb-ZBLAN fiber.

The implemented experimental setup shown in Fig. 3(a) consists of a tunable Ti:Sapphire laser beam that is coupled to a multimode fiber (infinicore 300, Corning) with the length of $3 \mathrm{~m}$ which is butt-coupled to the ZBLAN fiber. Two multimode fibers (M124L02, Thorlabs) are employed to collect the spontaneous emission from the side of the doped fiber at two different locations, points $\mathbf{A}$ and $\mathbf{B}$ marked by positions $z_{A}$ and $z_{B}$, respectively, alongside the ZBLAN fiber. The collected side light is filtered with a $1.0 \mathrm{\mu m}$ long-pass filter to remove the pump power scattering and the filtered collected fluorescence is measured with a sensitive power meter (S120C, Thorlabs). Figure 3(b) shows a schematic of the MACSLA method.
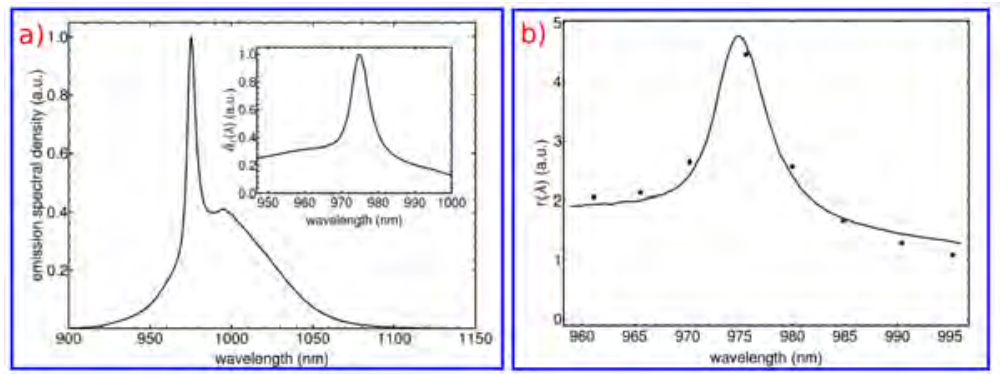

Figure 4. a) Emission power spectral density $S(\lambda)$ which is measured by the optical spectrum analyzer, is plotted in arbitrary units. The inset shows the resonant absorption coefficient, which is normalized to its peak value and is calculated by using the McCumber theory. b) The points with error-bars indicate the values of $r(\lambda)$ measured different wavelengths near the peak of the resonant absorption coefficient.

The power spectral density $S(\lambda)$ of the Yb-ZBLAN fiber is shown in Fig. 4(a) where the inset shows the resonant absorption coefficient which is normalized to its peak value and calculated by using the McCumber theory. ${ }^{24}$ The fitted line over the experimental measurements related to $r(\lambda)=\ln \left(P_{\text {coll }}\left(z_{B}\right) / P_{\text {coll }}\left(z_{A}\right)\right)$ are shown in Fig. 4(b). The points (with error-bars) indicate the values of $r(\lambda)$ measured at eight different wavelengths. The fitting curve comes directly from the resonant absorption spectrum shown as the inset in Fig. 4(a). The final outcome of the fitting process is the peak value of the absorption coefficient $\alpha_{r}^{p}=1.86 \pm 0.12 \mathrm{~cm}^{-1}$. 


\section{DISCUSSION AND CONCLUSION}

We have shown the ASF cooling of a Yb-doped ZBLAN fiber in atmospheric pressure through a set of experiments. The cooling efficiency of the Yb-doped ZBLAN fiber for different wavelengths was measured and finally the optimum value of the cooling efficiency was extracted. Using the ASF cooling model and MACSLA method we developed in our lab, we could extract two of the most important cooling parameters such as external quantum efficiency and the background absorption. The novel, non-contact, non-destructive MACSLA method was used to characterize the ZBLAN fiber. The results showed that the optimum pump wavelength for the ASF cooling is $1030 \mathrm{~nm}$; Therefore, all the optimization processes of the signal wavelength and other factors in radiationbalancing of the ZBLAN fiber should evolve around this optimum cooling wavelength.

\section{ACKNOWLEDGMENTS}

The authors would like to acknowledge M. Sheik-Bahae, R. I. Epstein, M. P. Hehlen and A. R. Albrecht for informative discussions. This material is based upon work supported by the Air Force Office of Scientific Research under award number FA9550-16-1-0362 titled Multidisciplinary Approaches to Radiation Balanced Lasers (MARBLE).

\section{REFERENCES}

[1] Richardson, D., Nilsson, J., and Clarkson, W., "High power fiber lasers: current status and future perspectives," JOSA B 27(11), B63-B92 (2010).

[2] Zervas, M. N. and Codemard, C. A., "High power fiber lasers: a review," IEEE Journal of selected topics in Quantum Electronics 20(5), 219-241 (2014).

[3] Brown, D. C. and Hoffman, H. J., "Thermal, stress, and thermo-optic effects in high average power doubleclad silica fiber lasers," IEEE Journal of quantum electronics 37(2), 207-217 (2001).

[4] Zenteno, L., "High-power double-clad fiber lasers," Journal of Lightwave Technology 11(9), 1435-1446 (1993).

[5] Ward, B., Robin, C., and Dajani, I., "Origin of thermal modal instabilities in large mode area fiber amplifiers," Optics Express 20(10), 11407-11422 (2012).

[6] Dawson, J. W., Messerly, M. J., Beach, R. J., Shverdin, M. Y., Stappaerts, E. A., Sridharan, A. K., Pax, P. H., Heebner, J. E., Siders, C. W., and Barty, C., "Analysis of the scalability of diffraction-limited fiber lasers and amplifiers to high average power," Optics express 16(17), 13240-13266 (2008).

[7] Jauregui, C., Eidam, T., Otto, H.-J., Stutzki, F., Jansen, F., Limpert, J., and Tünnermann, A., "Physical origin of mode instabilities in high-power fiber laser systems," Optics express 20(12), 12912-12925 (2012).

[8] Epstein, R. I., Buchwald, M. I., Edwards, B. C., Gosnell, T. R., and Mungan, C. E., "Observation of laser-induced fluorescent cooling of a solid," Nature 377(6549), 500 (1995).

[9] Seletskiy, D. V., Melgaard, S. D., Bigotta, S., Di Lieto, A., Tonelli, M., and Sheik-Bahae, M., "Laser cooling of solids to cryogenic temperatures," Nature Photonics 4(3), 161 (2010).

[10] Bowman, S. R., "Lasers without internal heat generation," IEEE journal of quantum electronics 35(1), 115-122 (1999).

[11] Bowman, S. R., O'Connor, S. P., Biswal, S., Condon, N. J., and Rosenberg, A., "Minimizing heat generation in solid-state lasers," IEEE Journal of Quantum Electronics 46(7), 1076-1085 (2010).

[12] Bowman, S. R., "Low quantum defect laser performance," Optical Engineering 56(1), 011104 (2016).

[13] Yang, Z., Meng, J., Albrecht, A. R., and Sheik-Bahae, M., "Radiation-balanced yb: Yag disk laser," Optics express 27(2), 1392-1400 (2019).

[14] Mobini, E., Peysokhan, M., Abaie, B., and Mafi, A., "Thermal modeling, heat mitigation, and radiative cooling for double-clad fiber amplifiers," JOSA B 35(10), 2484-2493 (2018).

[15] Peysokhan, M., Abaie, B., Mobini, E., Rostami, S., and Mafi, A., "Measuring quantum efficiency and background absorption of an ytterbium-doped zblan fiber," in [CLEO: Applications and Technology], JW2A118, Optical Society of America (2018).

[16] Peysokhan, M., Mobini, E., Abaie, B., and Mafi, A., "Method for measuring the resonant absorption coefficient of rare-earth-doped optical fibers," Applied Optics 58(7), 1841-1846 (2019). 
[17] Peysokhan, M., Mobini, E., Abaie, B., and Mafi, A., "A non-destructive method for measuring the absorption coefficient of a yb-doped fiber," in [Frontiers in Optics / Laser Science], Frontiers in Optics / Laser Science, JW3A.138, Optical Society of America (2018).

[18] Mobini, E., Peysokhan, M., and Mafi, A., "Heat mitigation of a core/cladding yb-doped fiber amplifier using anti-stokes fluorescence cooling," arXiv preprint arXiv:1903.05738 (2019).

[19] Peysokhan, M. and Mafi, A., "Minimizing heat generation by radiative cooling in a ytterbium-doped silica fiber laser," in [Frontiers in Optics], JTu3A-106, Optical Society of America (2017).

[20] Mobini, E., Peysokhan, M., Abaie, B., Hehlen, M. P., and Mafi, A., "Spectroscopic investigation of Yb-doped silica glass for solid-state optical refrigeration," Phys. Rev. Applied 11, 014066 (Jan 2019).

[21] Newell, T., Peterson, P., Gavrielides, A., and Sharma, M., "Temperature effects on the emission properties of yb-doped optical fibers," Optics communications 273(1), 256-259 (2007).

[22] Aull, B. and Jenssen, H., "Vibronic interactions in nd: Yag resulting in nonreciprocity of absorption and stimulated emission cross sections," IEEE Journal of Quantum Electronics 18(5), 925-930 (1982).

[23] Peysokhan, M., Souchelmaei, E. M., Abaie, B., and Mafi, A., "A non-destructive method for measuring the absorption coefficient of a doped optical fiber," in [Photonic Heat Engines: Science and Applications], 10936, 109360K, International Society for Optics and Photonics (2019).

[24] McCumber, D., "Einstein relations connecting broadband emission and absorption spectra," Physical Review 136(4A), A954 (1964). 


\title{
Investigation of Low-Bending-Loss Single-Mode Anti-resonant Hollow- core THz Fiber
}

\author{
Shuai Sun ${ }^{\mathrm{a}, \mathrm{b}}$, Wei Shi*a,b, Quan Sheng ${ }^{\mathrm{a}, \mathrm{b}}$, Guo Zhang ${ }^{\mathrm{a}, \mathrm{b}}$, Yao Zhang ${ }^{\mathrm{a}, \mathrm{b}}$, Jianquan Yaa ${ }^{\mathrm{a}, \mathrm{b}}$ \\ ${ }^{a}$ College of Precision Instrument and Optoelectronics Engineering, Tianjin University, Tianjin \\ 300072, China \\ ${ }^{\mathrm{b}}$ Tianjin Institute of Modern Laser \& Optics Technology, Tianjin 300384, China
}

\begin{abstract}
An anti-resonant hollow-core THz fiber with six half-ellipse cladding tubes is demonstrated. The high order mode suppression in the hollow core fiber with half-ellipse cladding tubes is investigated. By optimizing the cladding tube, single mode and low bending loss characteristics are achieved.

Keywords: single-mode, $\mathrm{THz}$ fiber, anti-resonant, hollow-core fiber, high order mode suppression, Cyclic Olefin Copolymer

* shiwei@tju.edu.cn;

\section{Introduction}

Terahertz (THz) wave with excellent optical properties and electrical characteristics has a variety of applications and THz fiber will play an important role in Sensors, Communication, Bioimaging and so on[1]. But almost all the optical materials have considerable absorption loss of $\mathrm{THz}$ wave, fortunately the anti-resonant hollow-core fiber (ARF) transferring light in the center air hole could help to ignore the absorption loss of fiber materials and achieve low-loss $\mathrm{THz}$ wave transmission[2]. Single-mode ARF with compact structure could be realized by high-ordermode (HOM) suppression or twisting the fiber[3, 4]. In this paper, the HOM suppression and bending loss in THz ARF is investigated and a low-bending-loss single-mode ARF based on half-ellipse cladding tubes is described for all fiber laser system.
\end{abstract}

\section{Study of anti-resonant effect}

\section{Simulation and Result}

A Cyclic Olefin Copolymers (COC) ARF with six half-ellipse cladding tubes is presented in this paper and the cross section of the fiber is shown in Figure 1(a). The shell thickness of cladding tubes is $T$ and elliptical tubes has the radius of $R_{\mathrm{a}} / R_{\mathrm{b}}=1000 \mu \mathrm{m} / 850 \mu \mathrm{m}$ which are covered by external cladding by $D_{\mathrm{c}}=1000 \mu \mathrm{m}$ making the fiber compact. This ARF is designed for transferring $2.5 \mathrm{THz}$ laser which is the major output frequency of optically pumped methanol gas THz laser, and COC material has low absorption loss of this laser. For $2.5 \mathrm{THz}$ wave, COC material has complex refractive index of $1.53+0.0037$ i. The ARF is simulated based on the Finite-Element-Method software-Comsol Multiphysic and a scattering boundary condition (SBC) is applied out of the external cladding to ensure the simulation corresponds to reality. The confinement loss (CL) is calculated by the formula: $\mathrm{CL}=\frac{20}{\ln 10} * \frac{2 \pi f}{\mathrm{c}} *$ Neff_imag, where $f$ is the incident frequency and Neff_imag is the imaginary part of effective refractive index. To investigate the anti-resonant effect in the fiber composed of lossy materials, the imaginary part of material refractive index is simplified to zero as control group. CL and the real part of effective refractive index (Neff_real) of fundamental mode changing with the $T$ are shown in Figure 1(b) and (c). The ARFs calculated based on actual material parameter and simplified material parameter, both show clear anti-resonant

Sixth International Workshop on Specialty Optical Fibers and Their Applications (WSOF 2019), edited by Liang Dong, John M. Ballato, Proc. of SPIE Vol. 11206, 112061R · C 2019 SPIE CCC code: $0277-786 \mathrm{X} / 19 / \$ 21 \cdot$ doi: $10.1117 / 12.2548421$ 
effect and the resonant period is both $52 \mu \mathrm{m}$. But the simplified fiber shows a right-shift and has lower minimal CL than the actual one. Neff_real of both fibers are almost coincident and in each best anti-resonant point, Neff_real is maintained at 0.9991 which could be another reference for judging anti-resonant effect.
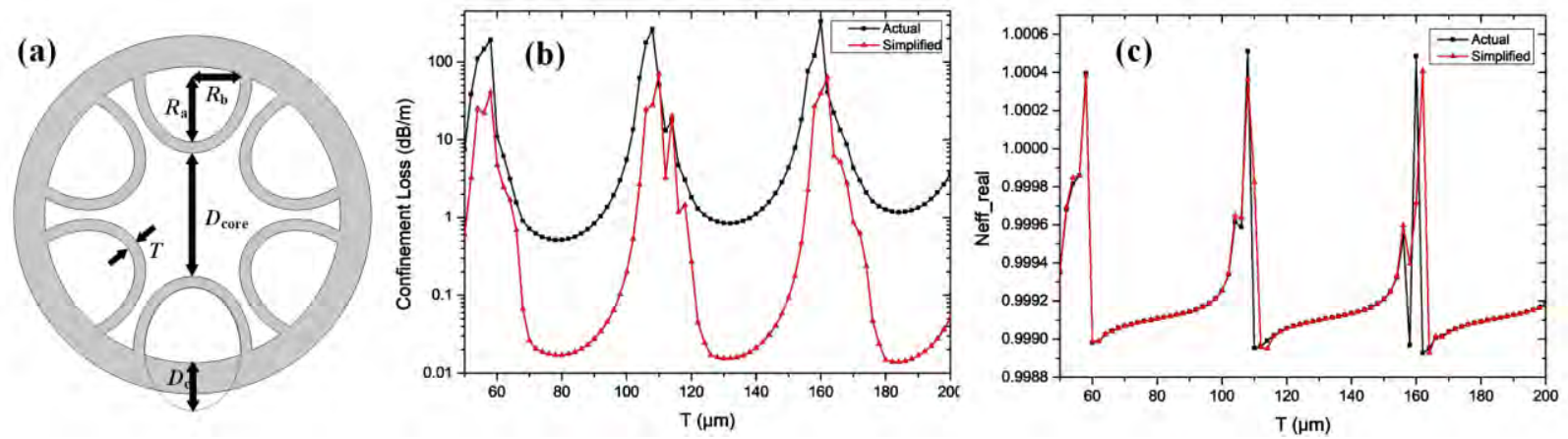

Figure 1. (a)Schematic diagram of ARF with six half-ellipse cladding tubes. (b, c) CL and Neff_real changing with the shell thickness of cladding tubes.

\section{Study of high order mode suppression}

In single-layer-tubes ARF, the single-mode characteristic could be realized by the HOM suppression effect which realized by the coupling between center air mode to cladding tubes. In the ARF with cladding tube radius $R_{\mathrm{b}}=850$ $\mu \mathrm{m}$ and shell thickness $T=78 \mu \mathrm{m}$, the loss of fundamental mode (LP01) and high-order-mode (LP11 mode) changing with $R_{\mathrm{a}}$ and $D_{\mathrm{c}}$ are show in Figure 2. With the changing of cladding tubes, the loss of LP01 mode fluctuates within a narrow range and mainly effected by $R_{\mathrm{a}}$. At the same time, the loss of LP11 mode appears a linear relationship with the changing of cladding tubes. When $R_{\mathrm{a}}$ is $900 \mu \mathrm{m}, D_{\mathrm{c}}$ is $600 \mu \mathrm{m}$, the loss of LP11 mode is more than $22 \mathrm{~dB} / \mathrm{m}$ at the same time the loss of LP01 mode is just $0.55 \mathrm{~dB} / \mathrm{m}$. According to reference, HOM suppression is major determined by the radius rate between cladding tube and center air hole. In this paper, according to the distribution of the maximal LP11 mode loss in Figure 2 (b), it could be concluded that the HOM suppression in ARF with halfellipse tubes is determined by the cross-sectional area rate between cladding tubes and center air hole.
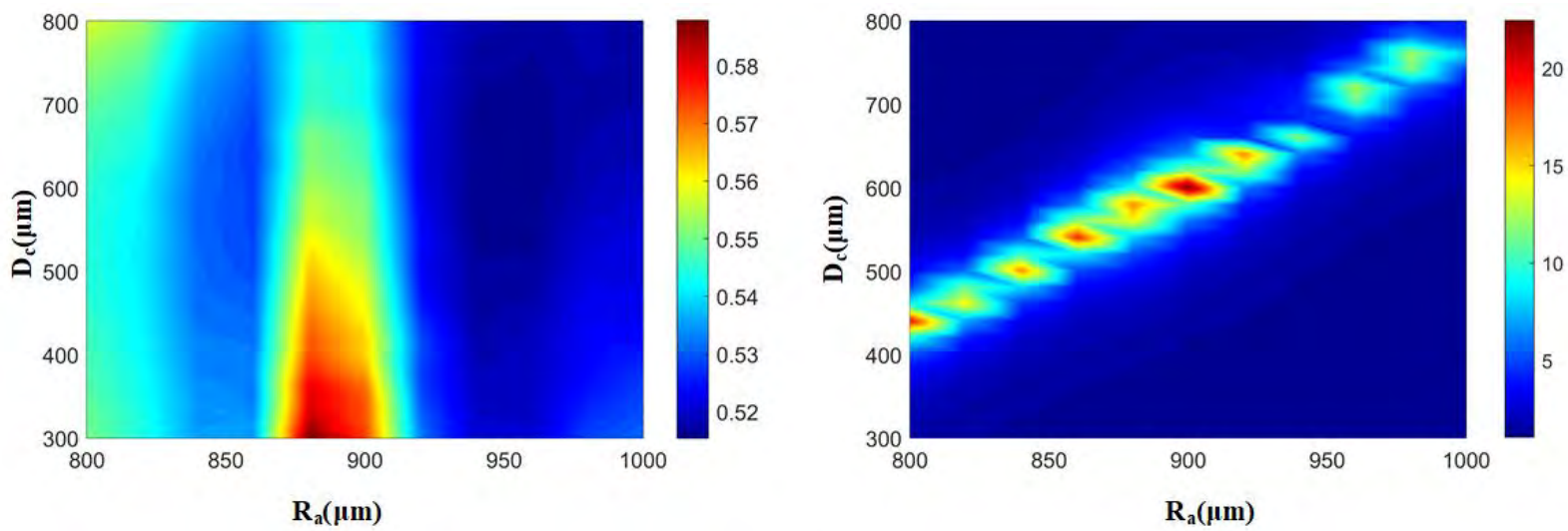

Figure 2 Loss of fundamental mode (a) and HOM (b) changing with Ra and Dc

\section{Study of bending loss}

Bending loss is always a shortage of ARF, and by the way of inserting additional tubes into the cladding tubes could prevent the light coupling into the cladding tubes which will make the fiber fabrication more difficult. In this paper, bending loss characteristic of ARF with half-ellipse cladding tubes is studied. Keeping $T$ at $78 \mu \mathrm{m}$ maintaining ARF in best anti-resonant state, bending ARF at bending radius of $50 \mathrm{~cm}$ and the bending loss changing with $D_{\mathrm{c}}$ is shown in Figure 3(a). It's obvious with the increase of $D_{\mathrm{c}}$, there is a peak value of bending loss and for different $R$ a each peak value is corresponding to different $D_{\mathrm{c}}$. According to the electric field distribution of fiber 
cross section as shown in Figure 3(b, c), when bending the fiber at particular bending radius, strong coupling will occur between center-air mode with cladding tubes. When $D_{\mathrm{c}}$ is $600 \mu \mathrm{m}$ and $R_{\mathrm{b}}$ is $900 \mu \mathrm{m}$, the bending loss is less than $0.8 \mathrm{~dB} / \mathrm{m}$.

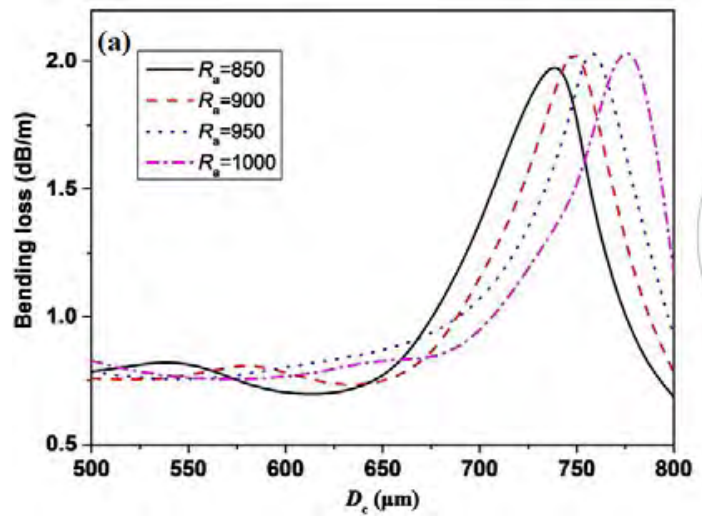

(b)

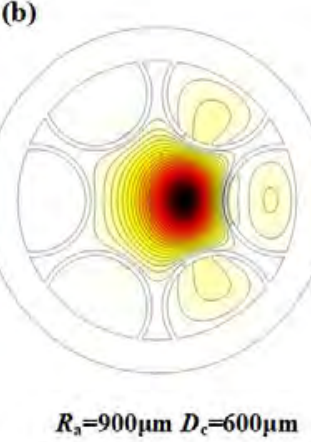

(c)

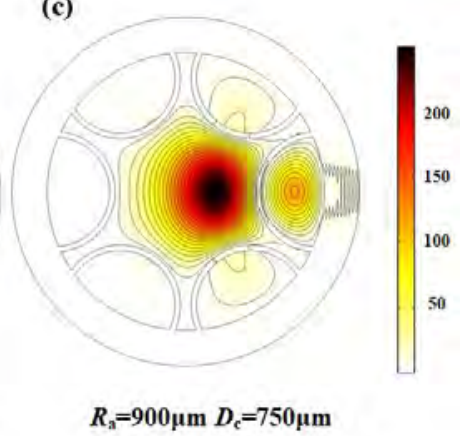

Figure 3 (a) Bending loss of ARF changing with cladding tubes when the bending radius is $50 \mathrm{~cm}$. The electric field distribution of fiber cross section when $R_{a}$ is $900 \mu \mathrm{m}$, Dc is $600 \mu \mathrm{m}(\mathrm{b})$ and when $\mathrm{R}_{\mathrm{a}}$ is $900 \mu \mathrm{m}$, Dc is $750 \mu \mathrm{m}(\mathrm{c})$.

\section{Conclusion}

The high-order-mode suppression in $\mathrm{THz}$ hollow-core fiber is investigated. The major factor effecting the single-mode characteristics of ARF with half-ellipse cladding tubes is the cross-sectional area rate of cladding tubes inner to center air core. Based on the investigation, a low bending-loss single-mode ARF based on non-round cladding tubes is designed for $2.5 \mathrm{THz}$ laser in this paper and by optimizing the structure, the confinement loss of high-order-mode could to be more than $22 \mathrm{~dB} / \mathrm{m}$ at the same time fundamental mode is less than $0.6 \mathrm{~dB} / \mathrm{m}$. The bending loss of ARF with bending radius of $50 \mathrm{~cm}$ is less than $0.8 \mathrm{~dB} / \mathrm{m}$.

\section{References}

[1] Zhong, K., Shi, W., Xu, D. G., Liu, P. X., Wang, Y. Y., Mei, J. L., Yan, C., Fu, S. J. and Yao, J. Q., “Optically pumped terahertz sources," Science China Technological Sciences, (2017).

[2] Yu, F. and Knight, J. C., "Negative Curvature Hollow-Core Optical Fiber," IEEE Journal of Selected Topics in Quantum Electronics, 22(2), 146-155 (2016).

[3] Uebel, P., Günendi, M. C., Frosz, M. H., Ahmed, G., Edavalath, N. N., Ménard, J.-M. and Russell, P. St.J., "Broadband robustly single-mode hollow-core PCF by resonant filtering of higher-order modes," 41(9), 19611964 (2016).

[4] Edavalath, N. N., Günendi, M. C., Beravat, R., Wong, G. K. L., Frosz, M. H., Ménard, J.-M. and Russell, P. St.J., "Higher-order mode suppression in twisted single-ring hollow-core photonic crystal fibers," Opt Lett, 42(11), 2074-2077 (2017). 


\title{
Low-loss terahertz pulse transmission through commercially available porous tubes with PTFE*
}

\author{
Yong Soo Lee ${ }^{\mathrm{a}}$, Soeun $\mathrm{Kim}^{\mathrm{b}}$, Inhee Maeng ${ }^{\mathrm{b}}$, Chul Kang ${ }^{\mathrm{b}}$, and Kyunghwan $\mathrm{Oh}^{* \mathrm{a}}$ \\ ${ }^{a}$ Department of Physics, Yonsei University, Seoul 03722, South Korea \\ ${ }^{b}$ Intergrated Optics Laboratory, Advanced Photonics Research Institute, GIST, Gwangju 61005, \\ South Korea
}

\begin{abstract}
Terahertz waveguide with porous is investigated in the $0.1-1.0 \mathrm{THz}$ frequency range. The waveguide has 17 air holes and are arranged air holes throughout the PTFE tubes in a hexagonal lattice structure without defect. We have experimentally confirmed that a terahertz signal can also be guided in a core-free waveguide and investigate the propagation characteristics of porous PTFE tubes of different lengths experimentally and numerically analyzed their optical properties using finite element method with perfectly matched layer. This porous tube with PTFE exhibit very low effective material loss than bulk material and experimentally measure high transmittance in the low frequency region.
\end{abstract}

Keywords: terahertz, waveguide, porous fiber, low-loss

*koh@yonsei.ac.kr; phone 822 2123-7657

\section{Introduction}

Over the decades, technological advances in the generation and detection of terahertz (THz) waves have continued steadily. With the advancement of technology and growing interest in $\mathrm{THz}$, research on $\mathrm{THz}$ in several fields including spectroscopy, imaging, sensing, and communication is actively in progress [1-3]. However, most THz systems still rely on free-space propagation and processing of $\mathrm{THz}$ waves. There is a very important issue here. The use of optics to guide and manipulate terahertz radiations in free space requires advanced experience and skill in optical technology. In addition, $\mathrm{THz}$ propagation in free space using optics is subject to many constraints due to the atmosphere attenuation, which is highly absorbed by atmospheric water vapor and oxygen molecules. These difficulties lead to the development of waveguides that can guide $\mathrm{THz}$ radiations and are independent of the external environment. Waveguides for $\mathrm{THz}$ applications include circular and square metallic pipe waveguides, parallel plate waveguide, single-crystal sapphire fibers, metallic wire waveguides and photonic crystal fiber (PCF) [4-6], but have difficulties due to their higher bending loss, lower coupling efficiency, and high material absorption loss. In recent times, there has been interest in polymer fibers such as polymer Bragg fiber, polystyrene foam, plastic fiber, and porous core PCF [7-9]. Using porous core PCF has important advantages such as lower material absorption loss and lower dispersion. However, PCF with porous core has a disadvantage that the manufacturing difficulty is very high.

In this work, we conducted experiments to transmit terahertz signals using porous tubes with PTFE and numerically analyzed the optical properties. This tube is commercially available from ZEUS and is characterized by the absence of a core as a waveguide [10]. We have experimentally confirmed that a terahertz signal can also be guided in a core-free waveguide. This porous structure minimizes the material area with air holes, resulting in low transmission losses. This work analyzes confinement loss and effective material loss (EML) of the porous tube with PTFE using finite element method (FEM) with perfectly matched layer (PML).

Sixth International Workshop on Specialty Optical Fibers and Their Applications (WSOF 2019), edited by Liang Dong, John M. Ballato, Proc. of SPIE Vol. 11206, 112061S · (C) 2019 SPIE

CCC code: $0277-786 X / 19 / \$ 21 \cdot$ doi: $10.1117 / 12.2548460$ 


\section{Experiment setup}

The experimental set-up used for the THz transmission experiment is shown in Figure 1(a). THz-TDS setup as in figure was used to characterize the fibers, where both the $\mathrm{THz}$ generator and detector consisted of photoconductive detector. The $\mathrm{THz}$ beam was collimated and focused using parabolic mirror and lenses for THz. A photograph of the PTFE tube which has 17 air holes with a hexagonal structure with a diameter of $\Lambda=560 \mu \mathrm{m}$ and $D=430 \mu \mathrm{m}$ is shown Figure 1(b). Photograph of the figure is shown in the porous tube with PTF. Upper right-hand corner inset shows the cross-section of the tube where 17 air holes are arranged. Lower right-hand is the enlarged cross-section. And the noncore cross-sectional structure can be confirmed. The tube lengths used in the experiments were $1 \mathrm{~cm}$ and $2 \mathrm{~cm}$, respectively.

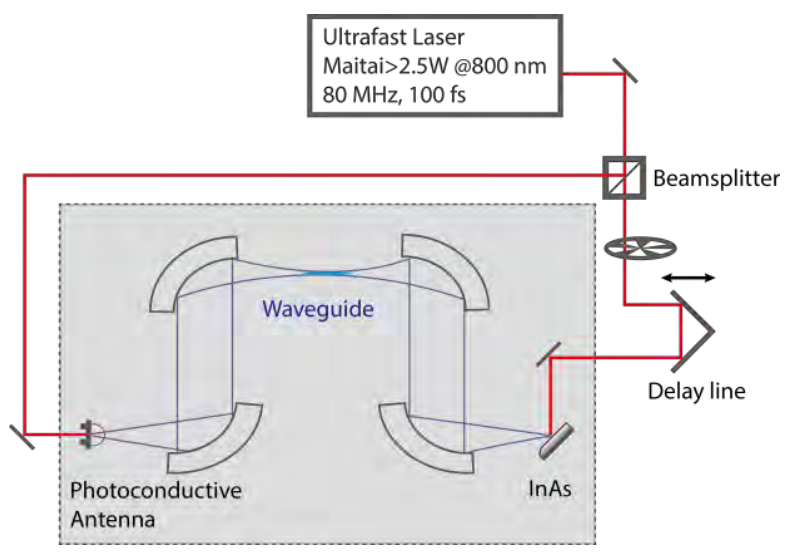

(a)

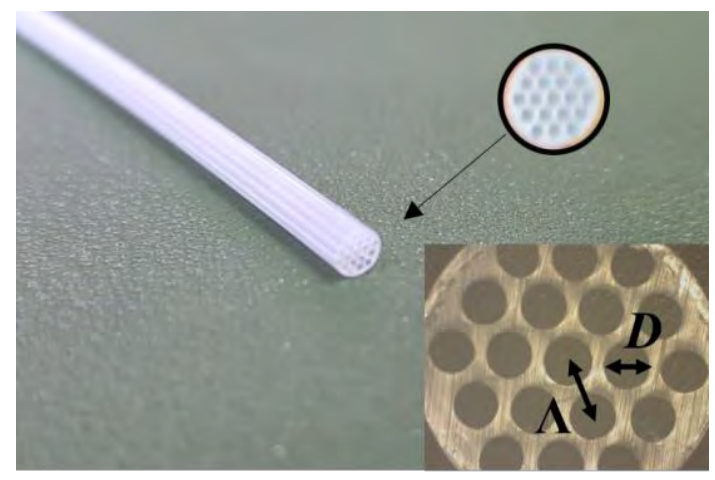

(b)

Figure 1. (a) A diagram of the optical set-up for waveguide measurements. (b) Photograph showing the porous PTFE tube constructed. Upper right-hand corner inset shows the cross-section of the tube where 17 air holes are arranged. Lower right-hand is the enlarged cross-section.
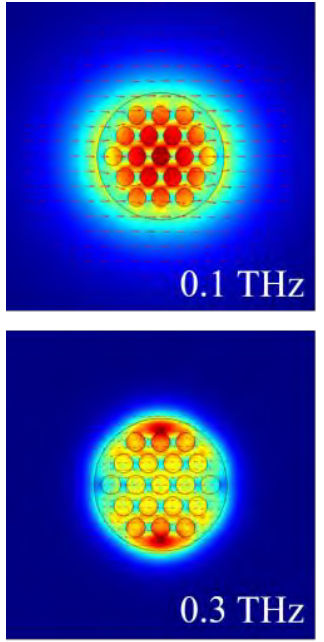

$0.3 \mathrm{THz}$
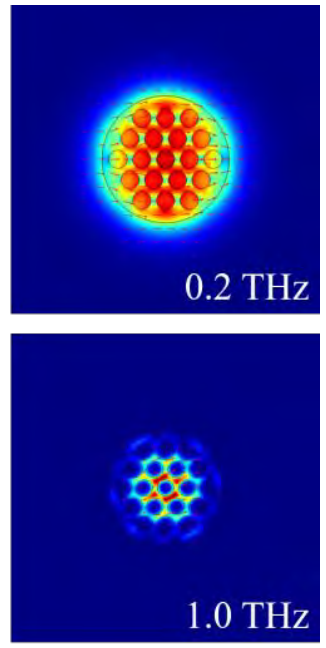

(a)

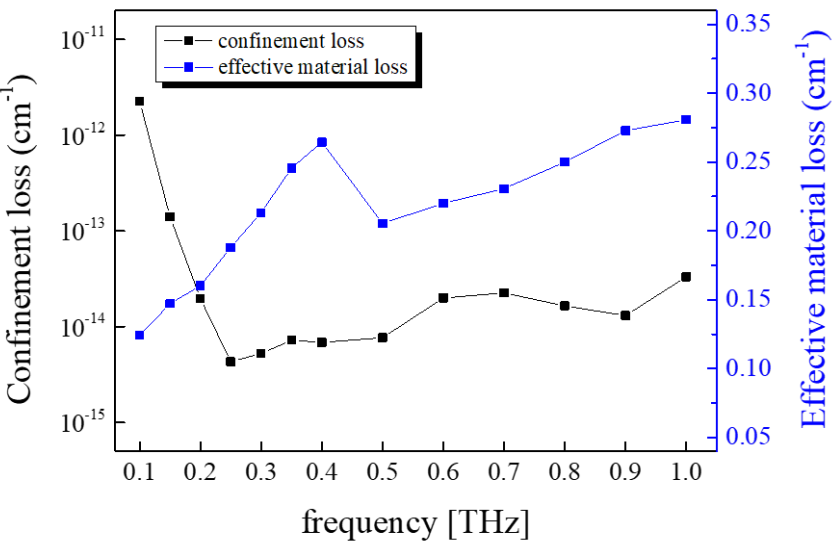

(b)

Figure 2. Mode distribution of the porous tube with PTFE depending on the frequency. In the low frequency band, the mode is distributed evenly in the air holes of the tube. 


\section{Results and Discussion}

Figure 2 shows the numerical results. Figure 2(a) shows the mode distribution depending on frequency in the porous tube with PTFE. One can clearly see that the mode distribution of the porous tube is different according to the frequency. The mode distribution of the porous tube in the low frequency band is shown throughout the tube, but in the high frequency band, the mode distribution is confined to the material region around the central air hole. Figure 2(b) shows the confinement loss and EML. The black and blue line indicate confinement loss and EML, respectively. The units were unified in $\mathrm{cm}^{-1}$ for easy comparison. Confinement loss and EML of the porous tube are calculated using the following expression [11]:

$$
L_{\mathrm{c}}=\frac{4 \pi f}{c} \operatorname{Im}\left[n_{\text {eff }}\right]
$$

and

$$
\alpha_{\text {eff }}=\sqrt{\frac{\varepsilon_{0}}{\mu_{0}}}\left(\frac{\int_{\text {mat }} n_{\text {mat }}|E|^{2} \alpha_{\text {mat }} d A}{\left|\int_{\text {all }} S_{z} d A\right|}\right)
$$

In general, confinement loss is negligible in calculated frequency region. Then, the loss of the waveguide of porous tube is more affected by the EML than the confinement loss. What is remarkable here is that it represents a very large EML near $0.4 \mathrm{THz}$. At this point, the distribution of the mode formed in the porous tube is greatly changed. It is the frequency at which the mode formed throughout the tube starts to distribute from the material around the central air hole.

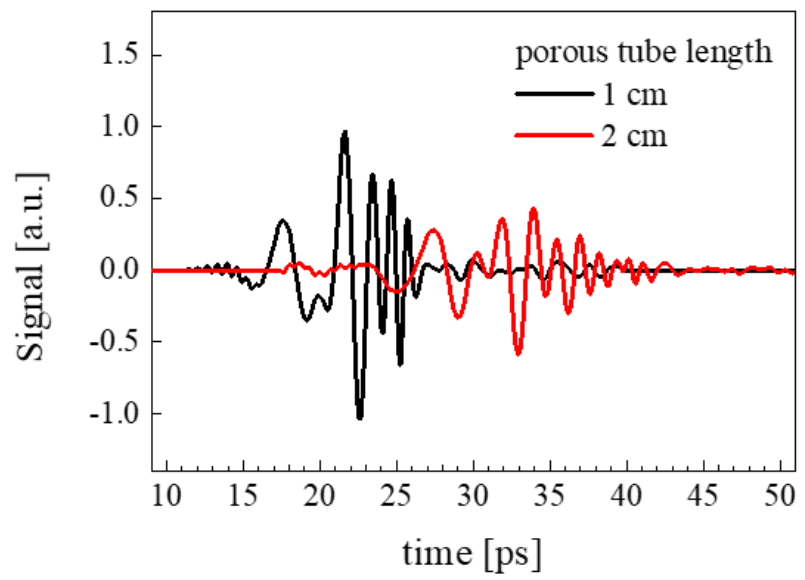

(a)

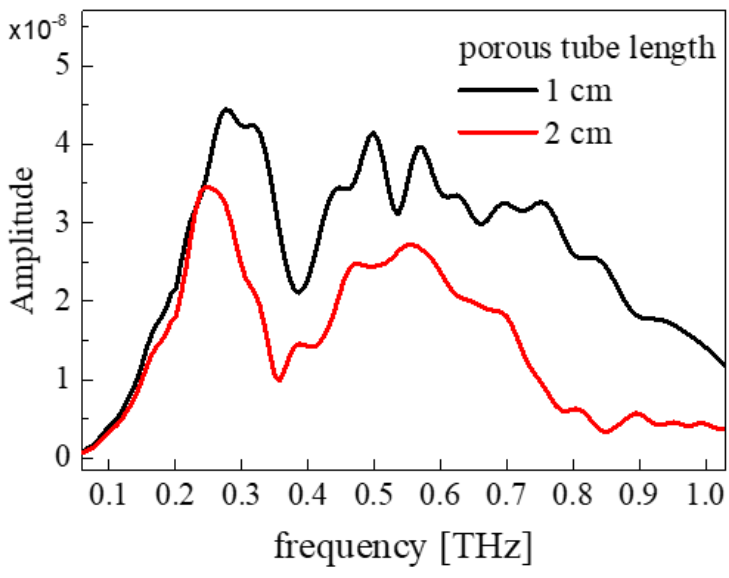

(b)

Figure 3. (a) Measured time-domain waveform of $\mathrm{THz}$ pulse passing through the porous tube. (b) Corresponding amplitude spectrum.

Figure 3 shows the experimental results. We initially measured the properties of the THz pulse incident in the porous with the photoconductive detector. Figure 3(a) shows the measured time-domain waveform in this experimental configuration, demonstrating that $\mathrm{THz}$ pulse passed through the porous tube. Figure 3(b) shows the corresponding amplitude spectrum. The black and red line indicate $1 \mathrm{~cm}$ and $2 \mathrm{~cm}$ of the porous tube, respectively. Near of $0.4 \mathrm{THz}$, where the largest EML was observed, the amplitude dropped significantly. 


\section{Acknowledgements}

This work is supported by the Korea government (MSIT) (No. 2019R1A2C2011293) and the Basic Science Research Program through the National Research Foundation of Korea (NRF) funded by the Ministry of Education (No.

2018R1D1A1B07049349).

\section{References}

[1] Lothar, M., John, F., Alexander, S., Chongjin, X., Hee, C. L. and Randy, C. G., "Data encoding on terahertz signals for communication and sensing," Opt. Lett. 33(4), 393-395 (2008).

[2] Inhee, M., Young, M. L., Jinwoo, P., Sonia, R. R., Chul, K., Chul-Sik, K., Byung, D. Y., Suklyun, H., Luis, K. O., Yabing, Q., Min-Cherl, J. and Masakazu, N., "Significant THz absorption in CH 3 NH 2 molecular defectincorporated organic-inorganic hybrid perovskite thin film," Scientific reports 9(1), 5811 (2019).

[3] Gyuseok, L., Inhee, M., Chul, K., Myoung-Kyu, O. and Chul-Sik, K., "High-efficiency optical terahertz modulation of aligned Ag nanowires on a Si substrate," Appl. Phys. Lett. 112(11), 111101 (2018).

[4] Mendis, R. and Grischkowsky, D., " Undistorted guided-wave propagation of subpicosecond terahertz pulses," Opt. Lett. 26(11), 846-848 (2001).

[5] Andrey, M., Hichem, G. and Maksim, S., "Hybrid metal wire-dielectric terahertz waveguides: challenges and opportunities," J. Opt. Soc. Am. B 31(11), 2587-2600 (2014).

[6] Soeun, K., Yong, S. L., Chul-Sik, K. and Chung, G. L., "Dispersion flattened terahertz photonic crystal fiber with high birefringence and low confinement loss," Proc. of SPIE 8985, 89851L (2014).

[7] Alexandre, D., Karen, S., Bora, U., Charles, D. and Maksim, S., " Transmission measurements of hollow-core THz Bragg fibers," J. Opt. Soc. Am. B 28(4), 896-907 (2011).

[8] Bora, U., Anna, M., Alexandre, D., Mathieu, R. and Maksim, S., "Polymer microstructured optical fibers for terahertz wave guiding," Opt. Express 19(26), B848-B861 (2011).

[9] Jianfeng, L., Fengjun, T., Hongkun, Q., Li, L., Jianzhong, Z., Xinhua, Y. and Libo, Y., " Design and numerical analysis of a THz square porous-core photonic crystal fiber for low flattened dispersion, ultrahigh birefringence," Appl. Opt. 56(24), 6993-7001 (2017).

[10] ZEUS, https://www.zeusinc.com/

[11] Mohammad, F. and Md. Shariful, I., " Extremely high birefringent terahertz fiber using a suspended elliptic core with slotted airholes," Appl. Opt. 57(13), 3340-3347 (2018). 


\title{
Low quantum defect fiber lasers via Yb-doped fluorosilicate optical fiber
}

\author{
Nanjie Yu*a ${ }^{* a}$ Maxime Cavillon ${ }^{\mathrm{b}}$, Courtney Kucera ${ }^{\mathrm{b}}$, Thomas Hawkins ${ }^{\mathrm{b}}$, John Ballato ${ }^{\mathrm{b}}$, Peter Dragic ${ }^{\mathrm{a}}$ \\ ${ }^{a}$ Department of Electrical and Computer Engineering, University of Illinois at Urbana-Champaign, \\ 306 N. Wright St., Urbana, Illinois 61801, USA \\ ${ }^{\mathrm{b}}$ Center for Optical Materials Science and Engineering Technologies (COMSET) and the \\ Department of Materials Science and Engineering, Clemson University, 91 Technology Drive, \\ Anderson, South Carolina 29625, USA
}

\begin{abstract}
Core pumped fiber lasers exhibiting a quantum defect of $0.92 \%, \lambda=985.7 \mathrm{~nm}$, and best-case slope efficiency of $68.6 \%$ based on $\mathrm{Yb}$-doped multicomponent fluorosilicate optical fiber are demonstrated. Power scaling through the use of a double-clad fiber in a MOPA configuration is discussed.
\end{abstract}

Keywords: fiber lasers, quantum defect, thermal effects, fiber optics amplifiers, fiber Bragg gratings *nanjiey2@illinois.edu; phone 1217 333-8399

\section{Introduction}

High-power fiber lasers are valuable light sources in numerous industrial and defense related applications ${ }^{1}$ because of their high beam quality, high brightness, low transmission loss, extraordinary capacity of heat dissipation, and low sensitivity to outside perturbation. However, as the requirement for output power continues to climb, thermal management of high-power fiber lasers has become an increasingly important issue to consider. Although processes such as impurity absorption and imperfect splicing between various fibers can lead to thermal energy generation in fiber laser systems, these processes can always be managed through optimization of the fiber fabrication process and splice quality. Ultimately, it is the quantum defect (QD) heating that sets the minimum generated thermal energy.

The QD is defined to be

$\mathrm{QD}=1-\frac{\lambda_{p}}{\lambda_{s}}$

in which $\lambda_{p}$ and $\lambda_{s}$ refer to pump and signal wavelengths of the system, respectively. Since the pump wavelength should be shorter than the signal wavelength to obtain optical gain in a laser system, the QD will have a value between 0 and 1 and represents the percentage of pump power transferred to thermal energy in the quantum limit. In other words, for every photon that is used to pump the system, there will always be a photon emitted out at the signal wavelength, and the only thermal energy generated through this process results from the energy difference between these two photons.

Problems from the parasitic ${ }^{2,3}$ to the catastrophic ${ }^{4,5}$ can result from such QD-related thermal energy generation. The latter, permanent damage to the fiber, obviously sets the upper limit to the heat power that could be introduced in the active fiber core for a certain length, while the former, such as transverse mode instability (TMI), can have a major impact on beam quality. Therefore, if the QD can significantly be reduced from $5 \%$ (typical for commercial Yb-doped aluminosilicate fiber operating at wavelengths longer than $1 \mu \mathrm{m}^{6}$ ), this may offer significant mitigation of the problems mentioned above. For instance, reducing the QD from $5 \%$ to $1 \%$ in a $10 \mathrm{~kW}$ fiber laser system removes $400 \mathrm{~W}$ of thermal energy.

As defined in (1), the QD could be reduced through two approaches, namely increasing the pump wavelength or reducing the signal wavelength. The former approach has found success through tandem pumping, such as with the $10 \mathrm{~kW}$ fiber laser manufactured by

Sixth International Workshop on Specialty Optical Fibers and Their Applications (WSOF 2019),

edited by Liang Dong, John M. Ballato, Proc. of SPIE Vol. 11206, 112061T · C 2019 SPIE

CCC code: $0277-786 X / 19 / \$ 21 \cdot$ doi: $10.1117 / 12.2548491$ 
IPG photonics ${ }^{7}$ (pumping at $1018 \mathrm{~nm}$ ). However, since laser diode technology is not yet mature in this wavelength range $(1018 \mathrm{~nm})$, tens of fiber laser-based pumps at $1018 \mathrm{~nm}$ are required to be manufactured in order to obtain a $10 \mathrm{~kW}$ signal output power at the last amplifier stage, clearly adding great complexity to the system. The latter approach (reduced signal wavelength) is usually challenging because in most of the conventional $\mathrm{Yb}$-doped fibers, amplified spontaneous emission (ASE) near a local peak of the gain curve $(\sim 1030 \mathrm{~nm})$ will introduce detrimental influences on laser performance, especially on the slope efficiency. Instead, lowerwavelength laser operation can be achieved by a judicious selection of host glass, such as phosphosilicates or fluorosilicates, while maintaining the $976 \mathrm{~nm}$ diode pumping. Recent works ${ }^{8,9}$ have shown that fluorine (F) co-doping into a multicomponent silica glass renders the emission spectrum of $\mathrm{Yb}^{3+}$ similar to that of the fluoride glasses, which has a favorable characteristic of a blue-shift of the local peak originally at $1030 \mathrm{~nm}$. An example of measured normalized absorption and emission cross section spectra are shown in Fig. 1 with a fluorosilicate core fiber shown in comparison to a commercial aluminosilicate. Because of this blue-shift, efficient lasing at short wavelengths $(<1000 \mathrm{~nm})$ should be achievable. Meanwhile, it has also been demonstrated ${ }^{8}$ that this glass has a reduction of thermo-optic coefficient so that the TMI threshold could be further raised. Therefore, Yb-doped fluorosilicate glass fiber becomes an attractive choice for high-power laser applications.

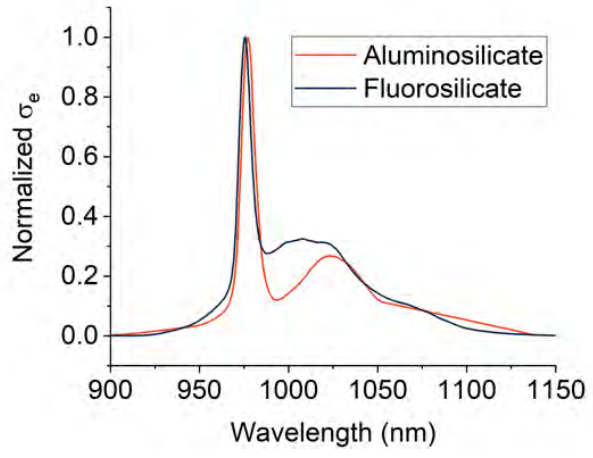

(a)

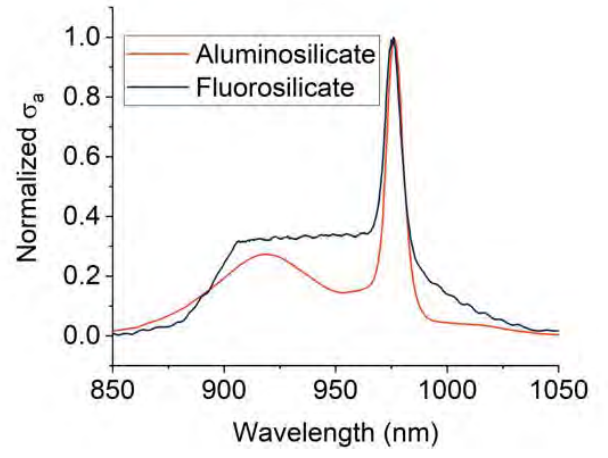

(b)

Fig. 1. Normalized (a) emission cross section $\left(\sigma_{e}\right)$ and (b) absorption cross section $\left(\sigma_{a}\right)$ spectra for Yb-doped commercial aluminosilicate fiber and the fluorosilicate fiber (studied in this work)

\section{Fiber Fabrication}

The fibers used herein were fabricated using the molten core method ${ }^{10}$. A precursor material in the form of a powder mixture of $\mathrm{YbF}_{3}, \mathrm{SrF}_{2}$, and $\mathrm{Al}_{2} \mathrm{O}_{3}$ was inserted into a pure silica capillary tube ( $3 \mathrm{~mm}$ inner diameter and $30 \mathrm{~mm}$ outer diameter). Then this preform was heated to $\sim 2000{ }^{\circ} \mathrm{C}$, whereby the core precursor mixture becomes molten and the pure silica cladding draws into fiber. As the fiber cools down, the core leads to a kinetically hindered glassy state in the resulting fiber. The drawn optical fiber possesses a cladding diameter of $125 \mu \mathrm{m}$ and a standard acrylate coating diameter of $250 \mu \mathrm{m}$. Compositional and refractive index profiles of the fiber are provided in Fig. 2 (a). A scanning electron microscope (SEM) micrograph is also shown in Fig. 2 (b). In addition to $\mathrm{Si}, \mathrm{O}, \mathrm{F}$ and $\mathrm{Yb}$, the fiber contains Strontium ( $\mathrm{Sr}$ ) and Aluminum (Al) in the core. The main role for $\mathrm{SrF}_{2}$ is to deliver $\mathrm{F}$ to the core, while $\mathrm{Al}_{2} \mathrm{O}_{3}$ is used to assist in the mixing of materials when the core is molten, ultimately promoting glass formation and avoidance of phase separation. The strontium is believed to exist in both oxide and fluoride form in the glass ${ }^{11}$, with the former (in addition to the $\mathrm{Al}_{2} \mathrm{O}_{3}$ ) offering reduction in strength of relevant nonlinearities, including Brillouin scattering ${ }^{8}$. The fiber possesses a high numerical aperture (NA), calculated to be $\sim 0.35$, which is moderated somewhat by the presence of the index-reducing fluorine. This may compromise single- or few-moded operation in the fiber, especially if it is fabricated with a larger core diameter for later amplifier stages (for example, $20 \mu \mathrm{m}$ core diameter). However, high-index cladding materials (such as $\mathrm{SiO}_{2}-\mathrm{Al}_{2} \mathrm{O}_{3}-\mathrm{La}_{2} \mathrm{O}_{3}$ glass) could be used to reduce $\mathrm{NA}^{12}$. 


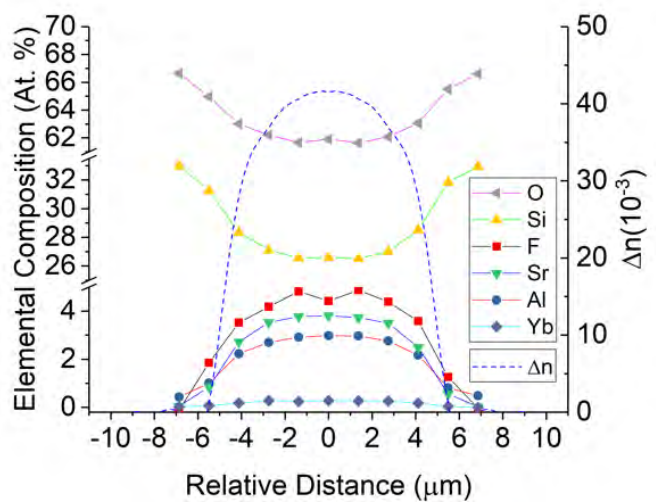

(a)

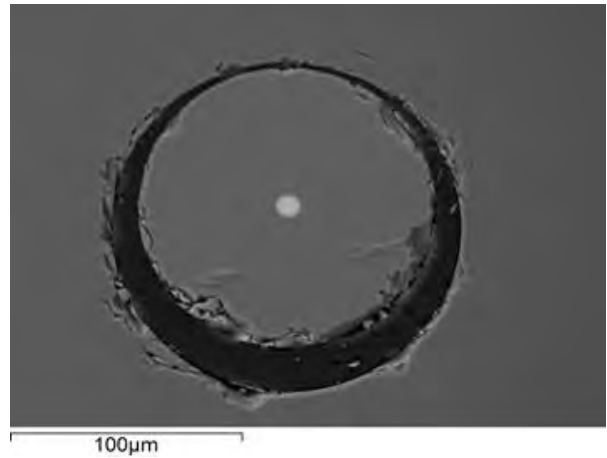

(b)

Fig. 2. (a) Compositional and refractive index profiles and (b) SEM micrograph of the Yb-doped fluorosilicate fiber.

\section{Experimental Setup, Results and Discussion}

The experimental setup is provided in Fig. 3. A commercial 976.6nm single-mode fiber-coupled diode laser was used as pump. A matched pair of fiber Bragg gratings (FBGs) were used to construct the cavity. Specifically, FBG 1 and FBG 2 have $99.02 \%$ and $38.34 \%$ reflectivity at $985.7 \mathrm{~nm}$, respectively. An isolator was placed between the pump and the cavity to avoid reflections from the cavity FBGs destabilizing the pumping wavelength. According to (1), the QD is calculated to be $0.92 \%$.

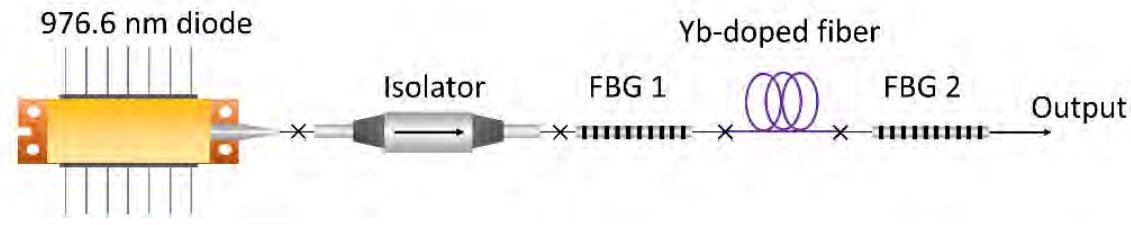

Fig. 3. Experimental Setup (an ' $X$ ' indicates a fusion splice).

Different lengths of Yb-doped fiber were spliced between the two FBGs to reach the optimized lasing condition. Fig.4 (a) is a representative example of the output spectra using five different active fiber lengths, Fig.4 (b) shows the slope efficiencies measured for the different active fiber lengths, and Fig 4 (c) shows the output power vs pump power at the maximum slope efficiency with a comparison to a theoretical mode $1^{13}$. From Fig.4 (a), it is obvious that with longer active fiber, pump power is more completely absorbed. At the same time, any non-inverted part of the fiber imparts reabsorption of the signal wavelength at $985.7 \mathrm{~nm}$, resulting in a higher level of amplified spontaneous emission (ASE) or even selfoscillation at around $1022 \mathrm{~nm}$. Clearly, greater pump absorption will lead to a higher slope efficiency, while larger ASE will lead to a lower slope efficiency, rendering length optimization a critical step in the development of this laser. As such, the slope efficiency is expected to have a maximum value at a particular optimal fiber length, consistent with data shown in Fig.4 (b). The power data for the near-optimized length $(12.7 \mathrm{~cm})$ is shown in Fig.4 (c) and the slope efficiency is measured to be $68.6 \%$. 


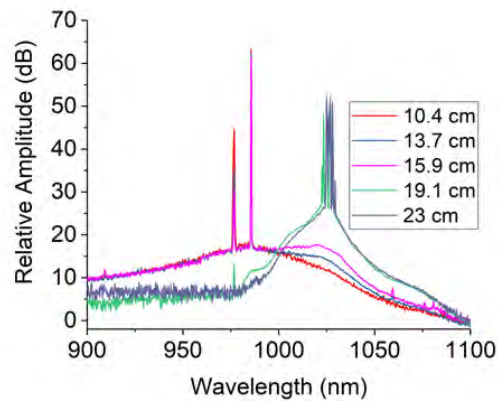

(a)

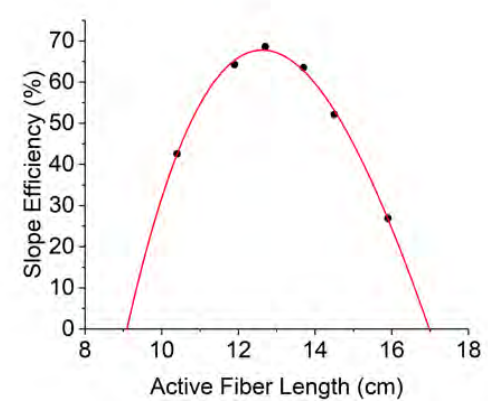

(b)

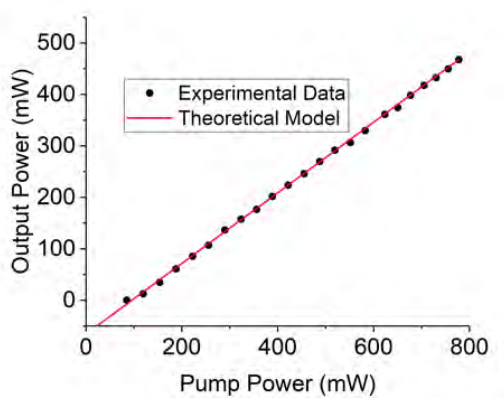

(c)

Fig. 4. (a) Output spectra with different active fiber lengths. (b) slope efficiency with different fiber lengths (the curve is a polynomial fit and only for visual aid). (c) Experimental and theoretical output power versus launched pump power at the near-optimized active fiber length.

The slope efficiency does not reach the theoretical quantum limit (in this case should be $100 \%-0.92 \%=99.08 \%$ ) mainly because of the splice loss between active fiber and the cavity FBGs as well as background loss in the active fiber. Background loss is measured to be $1.36 \mathrm{~dB} / \mathrm{m}$ at $985.7 \mathrm{~nm}$ and is mainly due to scattering and impurity absorption. Splice loss $(0.09 \mathrm{~dB} /$ splice $)$ is mainly influenced by the mode mismatch between the two very dissimilar fibers, with the observation that the output power will change considerably when twisting the fiber. It should be noted that splicing was achieved with a standard Fujikura telecom splicer set to 'Auto' mode. A more careful taper splice could be applied to decrease the splice loss and increase the slope efficiency, however, low-QD operation is much more important in the subsequent power amplifier stages.

As such, a double-clad version of this fiber is currently being fabricated and is intended for a master oscillator power amplifier (MOPA) configuration that will be seeded by the fiber laser discussed here. A cross section of the target doubleclad fiber is shown in Fig.5 (a), which gives a core and cladding diameters of $25 \mu \mathrm{m}$ and $125 \mu \mathrm{m}$, respectively. The precursor for the core will be the same as the one discussed in Part II, the inner cladding will be pure silica, and the outer cladding will be a low index coating material. The D-shape design is for suppressing the helically-propagating higher order modes in the inner cladding, which have an unfavorably small overlap with the core and is bad for pump absorption. Theoretical calculations ${ }^{13}$ were performed assuming a $\mathrm{Yb}^{3+}$ concentration of $1.65 \times 10^{26} \mathrm{~m}^{-3}$, a seed signal power of 500 $\mathrm{mW}$, an effective ASE input power of $1 \mathrm{~mW}$, a signal background loss of $0.5 \mathrm{~dB} / \mathrm{m}$ in the core, and a pump background loss of $15 \mathrm{~dB} / \mathrm{km}$ in the inner cladding. First, as discussed above, active fiber length optimization is a critical issue for maximum slope efficiency. Therefore, simulation of the output power versus active fiber length in the presence of a $15 \mathrm{~W}$ pump power was performed and the result is shown in Fig. 5 (b). Similar to Fig.4 (b), an optimized length was observed. Second, output power versus pump power at the optimized fiber length (calculated in the last step) was calculated and provided in Fig.5 (c), which indicates a slope efficiency of $78.3 \%$. If the background loss can be reduced to $5 \mathrm{~dB} / \mathrm{km}$, same calculations give a slope efficiency of $81.4 \%$, although this will not be achieved in this first iteration. Fabrication of this fiber is currently underway and primary results of amplifier experiments with this fiber will be discussed during the conference. 


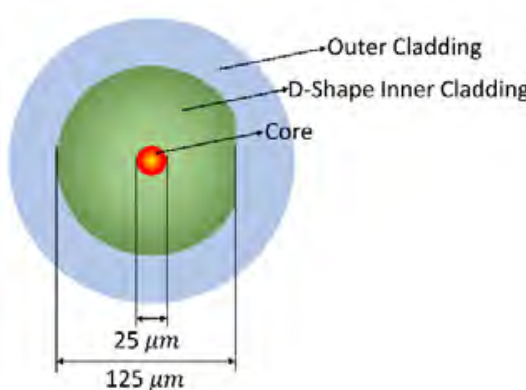

(a)

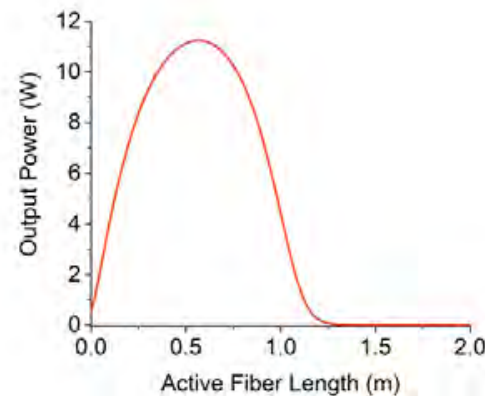

(b)

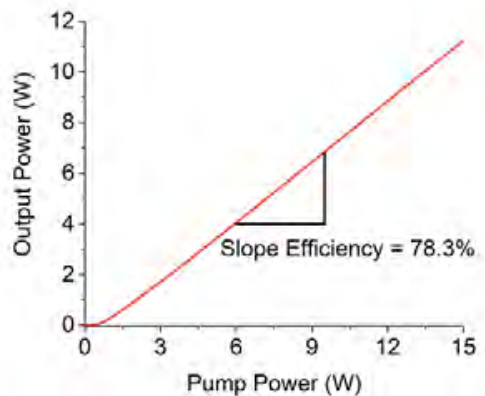

(c)

Fig. 5. (a) Cross section of the fluorosilicate double-clad fiber (b) output power versus active fiber length with $15 \mathrm{~W}$ of pump power (c) output power versus pump power with $0.78 \mathrm{~m}$ fluorosilicate double-clad fiber.

\section{References}

[1] Zervas, M. N. and Codemard, C. A., "High power fiber lasers: A review," IEEE J. Sel. Top. Quantum Electron. 20(5), 219-241 (2014).

[2] Jauregui, C., Limpert, J. and Tünnermann, A., "High-power fibre lasers,” Nat. Photonics 7(11), 861-867 (2013).

[3] Dong, L., "Stimulated thermal Rayleigh scattering in optical fibers," Opt. Express 21(3), 2642 (2013).

[4] Dawson, J. W., Messerly, M. J., Beach, R. J., Shverdin, M. Y., Stappaerts, E. A., Sridharan, A. K., Pax, P. H., Heebner, J. E., Siders, C. W. and Barty, C. P. J., "Analysis of the scalability of diffraction-limited fiber lasers and amplifiers to high average power," Opt. Express 16(17), 13240 (2008).

[5] Lapointe, M.-A., Chatigny, S., Piché, M., Cain-Skaff, M. and Maran, J.-N., "Thermal effects in high-power CW fiber lasers," Fiber Lasers VI Technol. Syst. Appl. 7195(February 2009), 71951U (2009).

[6] Chu, Q., Zhao, P., Lin, H., Liu, Y., Li, C., Wang, B., Guo, C., Tang, X., Tang, C. and Jing, F., "kW-level 1030 $\mathrm{nm}$ polarization-maintained fiber laser with narrow linewidth and near-diffraction- limited beam quality," Appl. Opt. 57(12), 2992-2996 (2018).

[7] "IPG Photonics.", <https://www.ipgphotonics.com/en>.

[8] Cavillon, M., Kucera, C., Hawkins, T. W., Yu, N., Dragic, P. and Ballato, J., "Ytterbium-doped multicomponent fluorosilicate optical fibers with intrinsically low optical nonlinearities," Opt. Mater. Express 8(4), 744-760 (2018).

[9] Yu, N., Cavillon, M., Kucera, C., Hawkins, T. W., Ballato, J. and Dragic, P., "Less than 1\% quantum defect fiber lasers via ytterbium-doped multicomponent fluorosilicate optical fiber,” Opt. Lett. 43(13), 3096 (2018).

[10] Ballato, J. and Snitzer, E., "Fabrication of fibers with high rare-earth concentrations for Faraday isolator applications,” Appl. Opt. 34(30), 6848-6854 (1995).

[11] Cavillon, M., Faugas, B., Zhao, J., Kucera, C., Kukuoz, B., Dragic, P., Qiao, X., Du, J. and Ballato, J., "Investigation of the structural environment and chemical bonding of fluorine in $\mathrm{Yb}$-doped fluorosilicate glass optical fibres," J. Chem. Thermodyn. (2019).

[12] Cavillon, M., Kucera, C. J., Hawkins, T. W., Runge, A. F. J., Peacock, A. C., Dragic, P. D. and Ballato, J., "Oxyfluoride Core Silica-Based Optical Fiber with Intrinsically Low Nonlinearities for High Energy Laser Applications," J. Light. Technol. 36(2), 284-291 (2018).

[13] Barnard, G., Myslinski, P., Chrostowski, J. and Kavehrad, M., "Analytical Model for Rare-Earth-Doped Fiber Amplifiers and Lasers,” IEEE J. Quantum Electron. 30(8), 1817-1830 (1994). 


\title{
Long-term behaviour of water vapour absorption in hollow core fibres
}

\author{
Shuichiro Rikimi ${ }^{*}$, Yong Chen ${ }^{1,2}$, Thomas D. Bradley ${ }^{1}$, Marcelo A. Gouveia ${ }^{2}$, Raymond J. Horley ${ }^{2}$, \\ Andrew T. Harker ${ }^{2}$, Simon Bawn ${ }^{2}$, Francesco Poletti ${ }^{1}$, Marco N. Petrovich ${ }^{1,2}$, David J. Richardson ${ }^{1}$ \\ and Natalie V. Wheeler ${ }^{1}$ \\ ${ }^{1}$ Optoelectronics Research Centre, University of Southampton, Southampton, United Kingdom \\ ${ }^{2}$ Lumenisity Ltd, Unit 7, The Quadrangle, Premier Way, Romsey, United Kingdom
}

\begin{abstract}
We report on the long-term behaviour of water vapour absorption in 19 cell HC-PBGFs in open and spliced conditions. Two main trends were observed as a function of time: increase in absorption of water vapour after opening sealed ends and reduction of the absorption after splicing ends. Furthermore, attenuation at $1550 \mathrm{~nm}$ was not significantly influenced by water vapour dynamics at $22^{\circ} \mathrm{C}$ and $85^{\circ} \mathrm{C}$.

Keywords: hollow core fibre, optical fibre testing, gas sensing

* srly15@soton.ac.uk
\end{abstract}

\section{INTRODUCTION}

Recent advances in hollow core fibre (HCF) fabrication ${ }^{1}$ are driving increasing interest in these fibres for future applications in telecommunications, ultra-high power delivery and sensing. While guidance in a hollow core leads to the unique properties of HCFs, such as ultimate low latency, low nonlinearity and the potential for ultra-low loss, the gas content within the core and the surrounding microstructure has the potential to impact both the optical and mechanical properties of the fibre ${ }^{2,3}$.

In several applications (usually requiring reasonably short fibre lengths), such as gas sensing and nonlinear optics ${ }^{4,5}$, the gas content in the hollow core region is carefully controlled. However, in many other experiments, little attention is paid to the composition of the gas inside a HCF. This gas composition is likely to be largely ambient air and accordingly measurements have recorded the presence of nitrogen, oxygen, water vapour and carbon dioxide in $\mathrm{HCFs}^{6,7}$. Yet the gas composition is also influenced by the fibre fabrication process (the exact grade of silica glass used as the raw material, the fibre draw parameters and the gas used to control the hole size during fabrication), which can lead to the presence of additional gas species such as inert gasses and hydrogen chloride ${ }^{2}$. Finally, it will also be influenced by whether the HCF is used in a sealed condition (for example, spliced at both ends to conventional fibre for use in telecommunications) or with both ends open to the surrounding environment (as in several sensing configurations). Optically, absorption resonances from different gas species can increase the fibre attenuation in specific spectral regions. Furthermore, it is feasible that chemical reactions between gas species within the fibre or operation at very low temperatures could lead to increased scattering loss.

In this work, we focus on the effects of water vapour in HCFs. Water presents an especially complex behaviour as it can exist in the vapour phase and therefore be observed in transmission measurements but it also interacts with the silica glass membranes of the fibre. Previous work ${ }^{8}$, reported in 2012, studied water ingress into a $50 \mathrm{~m}$ length of HCF over a period of 28 weeks and showed growth of a broadband spectral feature around $1398 \mathrm{~nm}$ which was attributed to the interaction with the silica surfaces within the fibre. Although water vapour absorption spectra were also shown, the development of these with time was not discussed.

Here, we describe optical measurements of water vapour absorption in HCFs over a range of time scales in both open and sealed conditions and at elevated temperatures. The absorption at $1369.85 \mathrm{~nm}$ varied with time in both of those

Sixth International Workshop on Specialty Optical Fibers and Their Applications (WSOF 2019),

edited by Liang Dong, John M. Ballato, Proc. of SPIE Vol. 11206, 112061U · C 2019 SPIE

CCC code: $0277-786 X / 19 / \$ 21 \cdot$ doi: $10.1117 / 12.2548508$

Proc. of SPIE Vol. $11206112061 \mathrm{U}-1$ 
conditions and the temperature behaviour was observed. Furthermore, the attenuation was also recorded and it was found that loss at $1550 \mathrm{~nm}$ was not significantly influenced by water vapour dynamics at $22^{\circ} \mathrm{C}$ and $85^{\circ} \mathrm{C}$.

\section{EXPERIMENTAL SETUP AND RESULTS}

For this work, all the fibres used were 19-cell HC-PBGFs with a similar design to the fibre reported ${ }^{9}$. A typical crosssectional image is shown in Fig. 1(c). The fibres were designed for operation around $1550 \mathrm{~nm}$ but also have low attenuation between $1350 \mathrm{~nm}$ and $1550 \mathrm{~nm}$ which overlaps with the $v_{1}+v_{3}$ and $2 v_{1}$ absorption bands of water vapour (HITRAN data Fig. 1(a), PBGF transmission Fig. 1(b)).
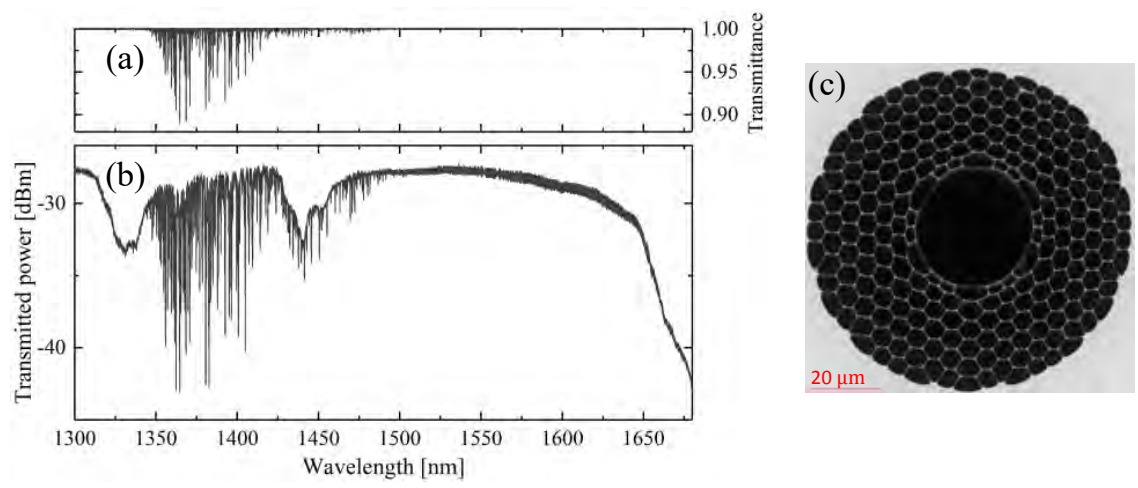

Fig. 1: (a) Transmittance of water vapour from HITRAN database (1 ppm, $1 \mathrm{~km}, 0.05 \mathrm{~nm}$ filter) (b) transmitted spectra recorded at $0.05 \mathrm{~nm}$ resolution and (c) scanning electron micrograph of PBGF-A (55 m).

\subsection{Water vapour ingress into open HCFs}

(a)

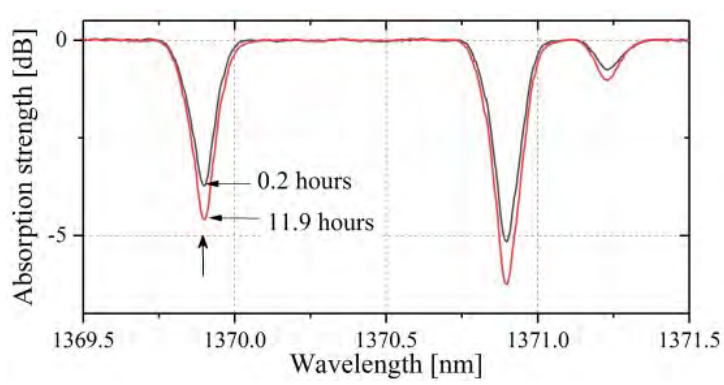

(b)

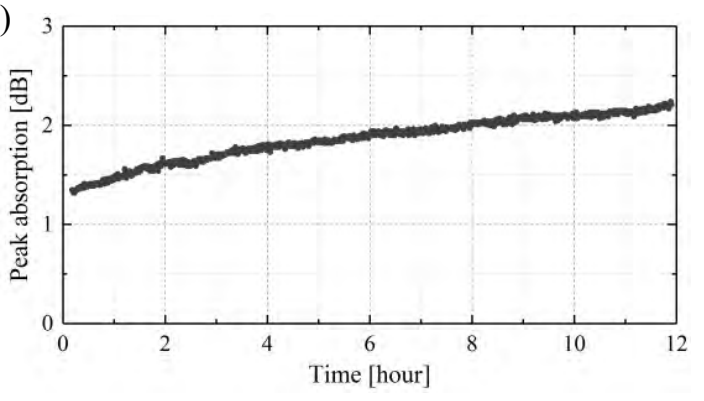

Fig. 2: (a) Evolution of spectral absorption of water vapour in $35 \mathrm{~m}$ of PBGF-A with time. The arrowed line is at 1369.85 $\mathrm{nm}$. (b) Ingress of water vapour in PBGF-A; the sample was open to the atmosphere for 12 hours. The measurement time was started when the first fibre end was cleaved.

In this section the ingress of water vapour from the atmosphere is investigated. A $35 \mathrm{~m}$ length of HC-PBGF (PBGFA) was kept in a laboratory with sealed ends prior to this experiment. The aim of the experiment was to measure the variation of water vapour absorption in the HCF immediately after opening the fibres to atmospheric conditions by cleaving both ends. For this measurement, a supercontinuum (SC) laser was used as a light source because high power spectral density was required to obtain high resolution measurements of the gas absorption lines. The transmitted power as a function of wavelength was recorded using an optical spectrum analyser (OSA) at $0.05 \mathrm{~nm}$ resolution. The SC light was launched into the HC-PBGF via a single mode fibre (SMF) and the output was collected by a SMF. A $\sim 0.3 \mathrm{~mm}$ gap was set between the SMFs and the sample to allow atmospheric air access to the hollow core region. The laboratory relative humidity and temperature were $35.5 \%$ and $24{ }^{\circ} \mathrm{C}$ for the duration of the test. In order to eliminate any contribution other than from the HC-PBGF assembly (e.g. water vapour in the free-space path within the SC laser and 
the OSA), reference data was separately recorded and subtracted (Fig. 2(b)). Note, reference data was also collected for measurements presented later in Fig. 3(a) and 4.

The time variation of water vapour absorption between $1369.5 \mathrm{~nm}$ and $1371.5 \mathrm{~nm}$ in PBGF-A is shown in Fig. 2(a). In this paper we selected the absorption line at $1369.85 \mathrm{~nm}$ for detailed analysis of water vapour dynamics because it is a relatively isolated peak and has moderate absorption strength (excluding PBGF-C measurements where absorption at $1399.65 \mathrm{~nm}$ was monitored due to the limited transmission at $1369.85 \mathrm{~nm}$ ).

Fig. 2(b) shows that the water vapour absorption strength at $1369.85 \mathrm{~nm}$ gradually increased for 12 hours after opening the ends in PBGF-A. It indicates that water vapour present in air diffused into the hollow core when the fibre was exposed to the atmosphere. The initial absorption at the start of the measurement is attributed to water vapour preexisting in the HCF as well as water vapur that has previously ingressed. Further data (not shown) showed that the rate of increasing absorption and the initial level of water absorption varied between different HCF samples.

\subsection{Water vapour absorption in sealed HCFs}

In this section, the behaviour of water vapour in sealed HCFs is discussed. Two different HCFs (PBGF-B, length 625 $\mathrm{m}$ and PBGF-C, $498 \mathrm{~m}$ ) were prepared by splicing them at both ends to SMFs via solid buffer fibres (BFs). The BFs were used to adjust the mode field diameter between the HC-PBGF and the SMF. During the splicing process, the fibres were open to the atmosphere for $\sim 1$ hour. After splices at both ends are applied, the HC-PBGFs become effectively hermetically sealed; in this condition, further atmospheric ingression is prevented and cannot impact the fibre's transmission properties.
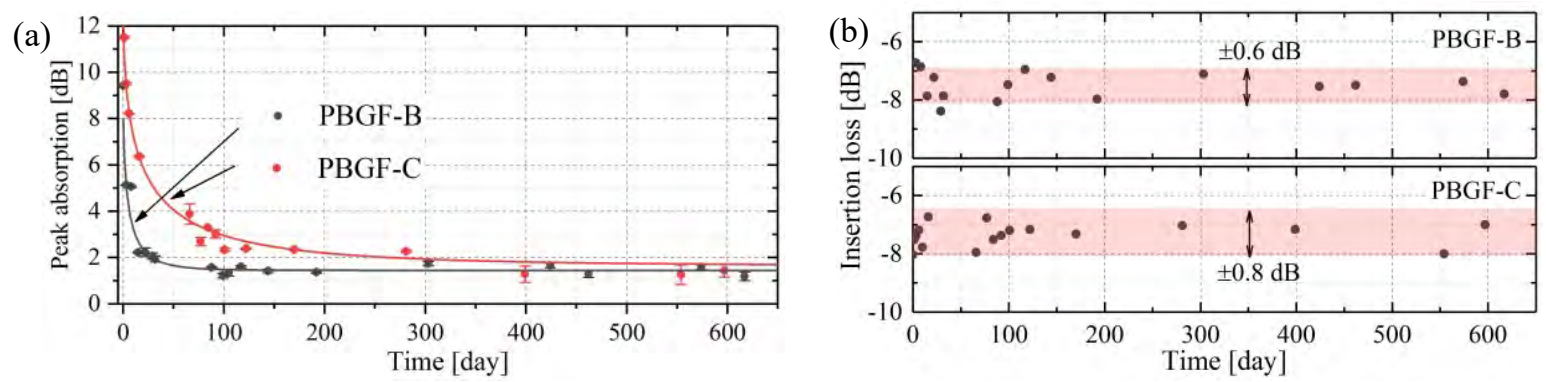

Fig 3: (a) Evolution of water vapour absorption strength with respect to time; black circle: PBGF-B at $1369.85 \mathrm{~nm}$, red circle: PBGF-C at $1399.65 \mathrm{~nm}$. (b) Insertion loss at $1550 \mathrm{~nm}$ at $10 \mathrm{~nm}$ resolution.

The two fibres showed similar trends over time and the results are shown in Fig. 3(a). The absorption by water vapour significantly decreases over a time period of $\sim 14$ days post-splicing. Following this, the observed absorption level continues to decrease, slowly approaching a stable state, but does not disappear even after 600 days. The decreasing trend is not a simple exponential decay; following previous work ${ }^{10}$, the absorption strength, $P(t)$, can be described by

$$
P(t)=A \times \exp \left(-\left(\frac{t}{\tau}\right)^{a}\right)+B
$$

Here, $\tau$ is defined as the absorption decay time and $a$ is a constant depending on the adsorption mechanics. $A$ and $B$ are the absorption strengths for the initial and equilibrium states, respectively. Fitting (1) to the experimental data in Fig. 3(a) gives 0.57 and 0.47 for $a$ and 5.17 day and 18.6 day for $\tau$ in PBGF-B and PBGF-C, respectively.

In parallel, the transmitted power at $1550 \mathrm{~nm}$ through the samples was recorded. Fig. 3(b) shows the measured insertion loss of the HC-PBGFs (including the splice losses) as a function of time. The shaded areas represent the measurement uncertainty due to variable connection loss at the input which was separately measured by removing and reattaching the connectors. The standard deviation of the transmitted power due to the variable connection loss was \pm 
$0.6 \mathrm{~dB}$ and $\pm 0.8 \mathrm{~dB}$ at $1550 \mathrm{~nm}$ for PBGF-B and PBGF-C respectively. Overall, no significant power change was observed with time within the measurement uncertainty.

Section 2.1 suggests that water vapour in the atmosphere is one of the sources of the water absorption post-splicing (day 0). This vapour can, through diffusion, move along the fibre length. The data in Fig. 3(a) shows that in a sealed condition, water vapour absorption decreases, indicating less water is in the light path. Possible scenarios which explain our result are that water molecules are sticking to silica surfaces within the HCF or chemically reacting with another gas species, such as hydrogen chloride, in the core. It is well known that water is a very reactive molecule with glass, both chemically and physically ${ }^{8,11}$. Water molecules that chemically react with silica can exist on the glass surface as a silanol (Si-OH) and can also physically be adsorbed. An $\mathrm{OH}$-covered glass surface can capture further water vapour through hydrogen bonding $\left(\mathrm{SiOH}-\mathrm{OH}_{2}\right)$ and thus there are possible reactions describing the interaction of the water molecules with the glass involving mono- and multiple layers.

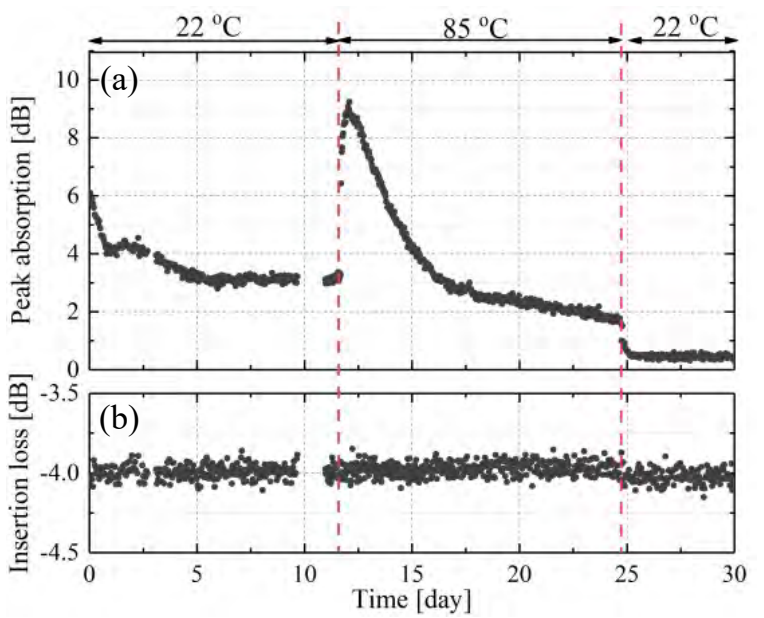

Fig 4: Variation of (a) water vapour absorption strength and (b) insertion loss at $1550 \mathrm{~nm}$ recorded at $2 \mathrm{~nm}$ resolution in PBGF-D with high time resolution.

In order to finely capture the trend of the water vapour absorption, the same measurement was performed using PBGF-D (55 m) but with much higher time resolution. Furthermore, the temperature behaviour of the water vapour was also recorded. The $55 \mathrm{~m}$ fibre sample was exposed to atmospheric conditions for 3 hours before both ends were sealed using the splicing process described above. Subsequently, water absorption spectra were recorded for 12 days at $22^{\circ} \mathrm{C}$ and a further 12 days at $85{ }^{\circ} \mathrm{C}$ before the temperature was reduced again to $22{ }^{\circ} \mathrm{C}$. Fig. 4 shows the evolution of absorption strength at $1369.85 \mathrm{~nm}$ during this time period. Day 0 corresponds to just after splicing. The water absorption strength decreased and approached the equilibrium state at $22^{\circ} \mathrm{C}$ as observed in Fig. 3. When the sample was baked at $85^{\circ} \mathrm{C}$, the absorption quickly increased and then gradually decreased again. When the temperature returned to $22^{\circ} \mathrm{C}$, the absorption quickly reduced and did not show any further change. In addition, it was confirmed from the transmission at $1550 \mathrm{~nm}$ during these measurements (Fig. 4) that the insertion loss did not significantly vary due to water vapour dynamics or temperature changes (a very small change in insertion loss $\sim 0.07 \mathrm{~dB}$ occured when the temperature was returned to $22^{\circ} \mathrm{C}$ ). Here, there was no variable connection loss as all connections were fixed during the measurement. This result suggests that transmitted power at wavelengths away from water vapour absorption bands is not affected by the dynamics of water vapour.

The high-time resolution measurement indicates that the reduction of the water vapour absorption strength does not follow a simple exponential decay; in fact, initially there is an approximately linear trend. There are two possible explanations to the discontinuous point at day 1. Firstly, different reaction processes with the glass surface; chemi- and physi-sorption were observed in the adsorption kinetics of water vapour onto borosilicate glass surfaces ${ }^{12}$. Secondly, the 
gas flow dynamics within the fibre; water molecules could be removed from the optical interaction path due to the combination of convection and diffusion of water vapour ${ }^{13}$ along the fibre length in the hollow core. Through these processes, water vapour driven along the fibre length could move to regions with lower overlap with the optical path (e.g. near the inner surface) or with surfaces with a higher probability of reaction with the molecules.

The large jump of the absorption strength at the change to $85^{\circ} \mathrm{C}$ suggests that the water vapour adsorbed on the inner glass surfaces prior to day 12 returned to the core region by desorption of physically adsorbed water molecules ${ }^{11}$. Prior to day 12 , these water molecules existing on the surface would not be optically detectable due to the small overlap of the mode field with the glass. The desorbed water molecules would then be captured by other available sites on the silica surface during the high temperature period, which results in the subsequent reduction of the absorption strength. In addition the high absorption strength $(\sim 9 \mathrm{~dB})$ observed at the change to $85^{\circ} \mathrm{C}$ indicates that there had already been some water molecules adsorbed onto the inner glass surfaces prior to day 0 , possibly originating from previous exposure to the atmosphere, because it surpassed that observed at day 0 by $\sim 3 \mathrm{~dB}$.

The reduction of the absorption strength at day 24 could be due to the adsorption-desorption process of water molecules. The physical and chemical reactions of water vapour with a silica surface ${ }^{11}$ are given by

$$
\begin{aligned}
& \mathrm{Si}-\mathrm{OH}+\mathrm{H}_{2} \mathrm{O} \underset{k_{p d}(T)}{\stackrel{k_{p a}(T)}{\rightleftarrows}} \text { Si-OH } \ldots \mathrm{OH}_{2} \quad T \leq 400^{\circ} \mathrm{C} \\
& \mathrm{Si}-\mathrm{O}-\mathrm{Si}+\mathrm{H}_{2} \mathrm{O} \underset{k_{c c}(T)}{\stackrel{k_{c d^{(T)}}}{\leftrightarrows}} 2 \cdot(\mathrm{Si}-\mathrm{OH}) \quad T>400^{\circ} \mathrm{C}
\end{aligned}
$$

where $k_{p a}(T)$ and $k_{p d}(T)$ are the physical adsorption and desorption rate coefficients, and $k_{c a}(T)$ and $k_{c d}(T)$ are the chemical adsorption and desorption rate coefficients, respectively. When the temperature returned to $22{ }^{\circ} \mathrm{C}$ in our sample, the physical coefficients adjusted to satisfy the new equilibrium state, which resulted in the quick reduction of the absorption strength.

\section{CONCLUSIONS}

Water vapour has the potential to impact the optical properties in HCFs. In this study, water vapour absorption in HC-PBGFs was monitored to obtain insight into the behaviour of water molecule in HCFs. HC-PBGFs in an open condition showed an increase in water vapour absorption. This indicates that water vapour in the atmosphere diffuses into the hollow core. The diffusion rate varies between different fibre samples and due to changes in the surrounding environment. A reduction of water vapour absorption was observed in spliced HCFs and the hermetic condition suggests that some chemical and physical reactions occurred inside the HCFs between the confined water vapour and the silica glass surfaces. Although trends were consistent across a range of fibre samples, different rates of change of the water absorption were observed which could be linked to the condition of the silica surfaces and possibly the fibre length. No negative effects of these reactions (e.g. increased insertion loss from surface scattering) were observed as the transmitted power at $1550 \mathrm{~nm}$ did not significantly change at $22^{\circ} \mathrm{C}$ or $85^{\circ} \mathrm{C}$.

\section{REFERENCES}

[1] Bradley, T. D., Hayes, J. R., Chen, Y., et al., "Record low-loss $1.3 \mathrm{~dB} / \mathrm{km}$ data transmitting antiresonant hollow core fibre," Proc. Eur. Conf. on Optical Communication, Th3F: Post-deadline papers (2018).

[2] Wheeler, N. V., Petrovich, M. N., Baddela, N. K., et al., "Gas Absorption between 1.8 and $2.1 \mu \mathrm{m}$ in Low Loss (5.2 dB/km) HC-PBGF," Proc. Conference on Lasers and Electro-Optics (CLEO), paper CM3N.5 (2012).

[3] Glaesemann, G. S., "Optical Fiber Mechanical Reliability Review of Research at Corning's Optical Fiber Strength laboratory," CORNING, 1-62 (2017). 
[4] Hodgkinson, J. and Tatam, R. P., "Optical gas sensing: a review," Measurement Science and Technology 24(1), 012004 (2013).

[5] Mousavi, S. A., Mulvad, H. C. H., Wheeler, N. V., et al., "Nonlinear dynamic of picosecond pulse propagation in atmospheric air-filled hollow core fibers," Optics Express 26(7), 8866-8882 (2018).

[6] Buric, M. P., Chen, K. P., Falk, J., et al, "Enhanced spontaneous Raman scattering and gas composition analysis using a photonic crystal fiber," Applied Optics 47(23), 4255-4261 (2008).

[7] Chen, Y., Wheeler, N. V., Baddela, N. K., et al., "Understanding Wavelength Scaling in 19-Cell Core Hollow-Core Photonic Bandgap Fibers," Proc. Optical Fiber Communication Conference, OSA Technical Digest (online) (Optical Society of America, 2014), paper M2F.4 (2014).

[8] Gris-Sánchez, I. and Knight, J.C., "Time-Dependent Degradation of Photonic Crystal Fiber Attenuation Around OH Absorption Wavelengths," Journal of Lightwave Technology 30(23), 3597-3602 (2012).

[9] Chen, Y., Liu, Z., Sandoghchi, S. R., et al., "Multi-kilometer Long, Longitudinally Uniform Hollow Core Photonic Bandgap Fibers for Broadband Low Latency Data Transmission," Journal of Lightwave Technology 34(1), 104-113 (2016).

[10] Zeng, Q., Xu, S., "A two-parameter stretched exponential function for dynamic water vapor sorption of cementbased porous materials," Material and Structure 50(2), 1-13 (2017).

[11] Feng, A., Mccoy, B. J., Munir, Z. A., et al., "Water Adsorption and Desorption Kinetics on Silica Insulation," Colloid and Interface Science 180(1), 276-284 (1996).

[12] Tuzi, Y., "Sorption of Water Vapour on Glass Surface in Vacuum Apparatus," Journal of the Physical Society of Japan 17(1), 218-227 (1962).

[13] Masum, B. M., Aminossadati, S. M., Kizil, M. S., et al., "Numerical and experimental investigations of pressuredriven gas flow in hollow core photonic crystal fibers," Applied Optics 58(4), 963-972 (2019). 


\title{
All-optically-driven and all-optical-fiber modulator via luminescence- quenched Yb-doped fiber
}

\author{
Nanjie $\mathrm{Yu}^{* \mathrm{a}}$, Matthew Tuggle ${ }^{\mathrm{b}}$, Courtney Kucera ${ }^{\mathrm{b}}$, Thomas Wade Hawkins ${ }^{\mathrm{b}}$, John Ballato ${ }^{\mathrm{b}}$, Peter \\ Dragic $^{\text {a }}$

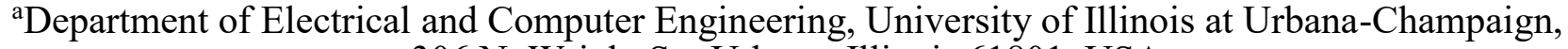 \\ 306 N. Wright St., Urbana, Illinois 61801, USA \\ ${ }^{\mathrm{b}}$ Center for Optical Materials Science and Engineering Technologies (COMSET) and the \\ Department of Materials Science and Engineering, Clemson University, 91 Technology Drive, \\ Anderson, South Carolina 29625, USA
}

\begin{abstract}
An all-optically-driven and all-optical-fiber modulator based on the phase delay introduced by an optically pumped, luminescence-quenched, $\mathrm{Yb}$-doped fiber microheater is presented. Results indicate that the system has the potential to achieve $\sim \mathrm{MHz}$ modulation speeds.
\end{abstract}

Keywords: Modulation, luminescence quenching, thermal effects, fiber materials, fiber design and fabrication *nanjiey2@illinois.edu; phone 1217 333-8399

\section{Introduction}

External modulation is useful in fiber research such as Q-switched fiber lasers ${ }^{1}$, narrow linewidth lasers ${ }^{2}$, femtosecond pulse shaping ${ }^{3}$, and the writing of Bragg gratings ${ }^{4}$. Conventionally, external modulation is achieved using bulk-devices via the acousto-optic ${ }^{1,3,4}$ or electro-optic ${ }^{2}$ effects. However, these devices often suffer from low damage threshold, large insertion losses, and high cost, making them unsuitable in many applications, such as high-power lasers. An alternative approach to overcome these disadvantages is by using fiber-based Mach-Zehnder interferometers (MZI), which rely on phase control of either one or both arms of the MZI. Specifically, it is first assumed that the optical fields in the two arms are initially in phase, having the same polarization, and initial intensities of $I_{1}$ and $I_{2}$. After propagating in both arms, if a phase difference of $\Delta \phi^{\prime}$ is introduced, the output intensity yields

$$
I_{\text {out }} \propto\left[I_{1}+I_{2}+2 \sqrt{I_{1} I_{2}} \cos \Delta \phi^{\prime}\right]
$$

Therefore, external modulation can be achieved by controlling $\Delta \phi^{\prime}$ to switch between two values such as 0 and $\frac{\pi}{2}$. This phase difference can be introduced by heating a segment (length $L^{\prime}$ ) of fiber. The phase delay in this heated segment of fiber (the "heat fiber," or HF) is

$$
\phi^{\prime}=\frac{2 \pi n_{e f f}^{\prime}}{\lambda} L^{\prime}
$$

where $\lambda$ is the signal wavelength and $n_{e f f}^{\prime}$ is the modal index. Taking the derivative with respect to temperature yields

$$
\frac{d \phi^{\prime}}{d T}=\frac{2 \pi}{\lambda} \frac{d n_{e f f}^{\prime}}{d T} L^{\prime}+\frac{2 \pi}{\lambda} \frac{d L^{\prime}}{d T} n_{e f f}^{\prime}=\frac{2 \pi L^{\prime}}{\lambda}\left(T O C+T E C \cdot n_{e f f}^{\prime}\right)
$$


in which $T O C$ is the thermo-optic coefficient and TEC is thermal expansion coefficient. Since typical values in optical fiber for the TOC $\left(\sim 10^{-5} \mathrm{~K}^{-1}\right)$ are usually much bigger than the TEC $\left(\sim 5 \times 10^{-7} \mathrm{~K}^{-1}\right)$, Eqn. (3) can be simplified to

$$
\Delta \phi^{\prime} \approx \frac{2 \pi L^{\prime}}{\lambda} \cdot T O C \cdot \Delta T
$$

Several approaches have been applied to introduce a change in temperature, $\Delta T$. One way is through the controlled pumping of an absorbing dopant which exhibits non-radiative relaxation from the upper excited state. For instance, this can include pumping rare earth ions to a non-radiating energy level ${ }^{5}$ or pumping ions in a clustered state ${ }^{6}$. However, this rare earth-based approach suffers should the dopant absorb and optically re-radiate any of the pump power, which leads to inefficient fiber heating. The use of absorbing transition metals is also non-ideal since it is difficult to obtain a high concentration of them in glass and they have broad absorption bands that can render large losses to the signal wavelength. Here, efficient fiber heating is accomplished through the novel fabrication of a very highly Yb-doped fiber (23.4 wt\% $\mathrm{Yb}_{2} \mathrm{O}_{3}$ ). When pumped with a $978 \mathrm{~nm}$ diode, no evidence of luminescence is observed, indicating efficient conversion from optical energy to thermal energy. The signal wavelength can be chosen to be far from the absorption band (signal wavelength in this case is $1550 \mathrm{~nm}$ ) and the system loss then is mostly limited by splicing the HF to the commercial passive fibers comprising the components. The high $\mathrm{Yb}^{3+}$ concentration enables the use of very short active fibers $(\sim \mathrm{mm})$ giving rise to the possibility of a high-temperature (in a very low volume), optically controlled thermal element. Here, this microheater is demonstrated in an all-optically-controlled phase and intensity modulator.

\section{Fiber Fabrication}

The optical fiber investigated herein was fabricated using a reactive molten core (rMC) approach ${ }^{7}$. Specifically, a $1 \mathrm{~mm}$ diameter ytterbium wire (99.9\% purity, Sigma-Aldrich) was placed inside a pure sapphire $\left(\mathrm{Al}_{2} \mathrm{O}_{3}\right)$ sleeve (Saint-Gobain), which measured $1.1 \mathrm{~mm}$ inner diameter and $1.5 \mathrm{~mm}$ outer diameter. This $\mathrm{Yb}$ wire- $\mathrm{Al}_{2} \mathrm{O}_{3}$ assembly was inserted into a pure silica capillary tube (Heraeus Tenevo Inc., Buford, GA) with $3 \mathrm{~mm}$ inner diameter and $30 \mathrm{~mm}$ outer diameter, which served as the fiber cladding. The fiber was drawn at a temperature of about $2050^{\circ} \mathrm{C}$ and to a cladding diameter of $110 \mu \mathrm{m}$, during which the $\mathrm{Yb}$ metal oxidizes to $\mathrm{Yb}_{2} \mathrm{O}_{3}$. A conventional single UV-curable acrylate coating was applied during the drawing process yielding a fiber diameter of $250 \mu \mathrm{m}$. The use of pure $\mathrm{Yb}$ wire significantly increased the $\mathrm{Yb}^{3+}$ concentration in comparison to other $\mathrm{Yb}$-doped fibers used for lasers ${ }^{8}$, leading to one that, while bad for laser applications, forms the basis for a very efficient optically-pumped high- $\mathrm{T}$ thermal element. A measurement of temperature change vs pump power for a $2.4 \mathrm{~mm}$ segment of this fiber is shown in Fig.1. Note that the HF temperature was measured using a bulk metal probe, in the open air, with much larger mass/volume than the HF. Therefore, Fig. 1 represents a minimum temperature, with the true temperatures likely being at least somewhat higher. Fig. 1 also suggests a thermal power density on the order of $\sim 10 \mathrm{~W} / \mathrm{nL}$ generated in the core of the fiber. Considering a phase shift of $\Delta \phi^{\prime}=\pi$ (at $\left.\lambda=1550 \mathrm{~nm}\right)$, the required $\Delta \mathrm{T}$ is $32.3 \mathrm{~K}$, corresponding to only $\sim 31 \mathrm{~mW}$ of pump power. In addition, the fact that all the optical power was absorbed within $2.4 \mathrm{~mm}$ of HF gives a small thermal volume (that of the fiber core), rendering a short response time and therefore high modulation speed. 


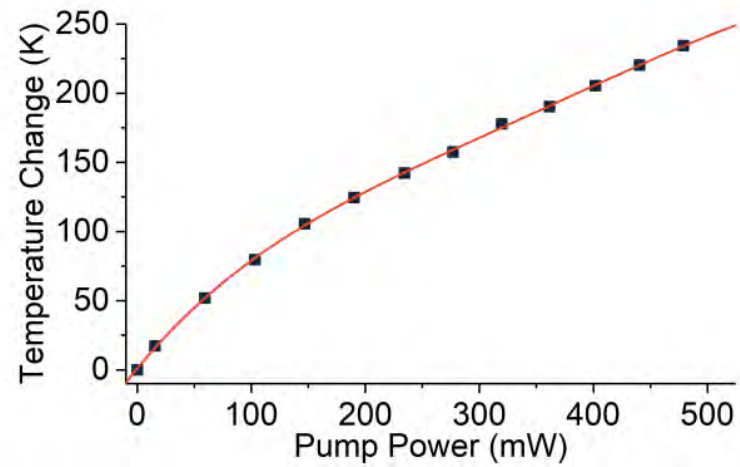

Fig. 1. Temperature change of $2.4 \mathrm{~mm}$ HF with different pump power, the dots are measured data and the line is a polynomial fit to the data meant as a visual aid to the reader.

\section{Experimental Setup, Results and Discussion}

The experimental set-up is shown in Fig. 2. A Mach-Zehnder-based modulator was constructed using a 75:25 splitter and a 50:50 coupler. Signal at $1550 \mathrm{~nm}$ was generated by a narrow-linewidth tunable laser source followed by an isolator to prevent feedback from destabilizing the source laser. In Arm 1, a WDM was introduced to combine the 1550nm signal and the $976 \mathrm{~nm}$ diode laser (LD) pump. A $2.4 \mathrm{~mm}$ segment of uncoated HF was spliced after the WDM, which absorbs the $976 \mathrm{~nm}$ pump while passing the $1550 \mathrm{~nm}$ signal with only $0.4 \mathrm{~dB}$ loss per unoptimized splice. To roughly balance the MZI, $1.65 \mathrm{~m}$ of passive fiber was used to extend the length of Arm 2. Finally, after the 50:50 coupler, the output signal was collected for the different measurements discussed below.

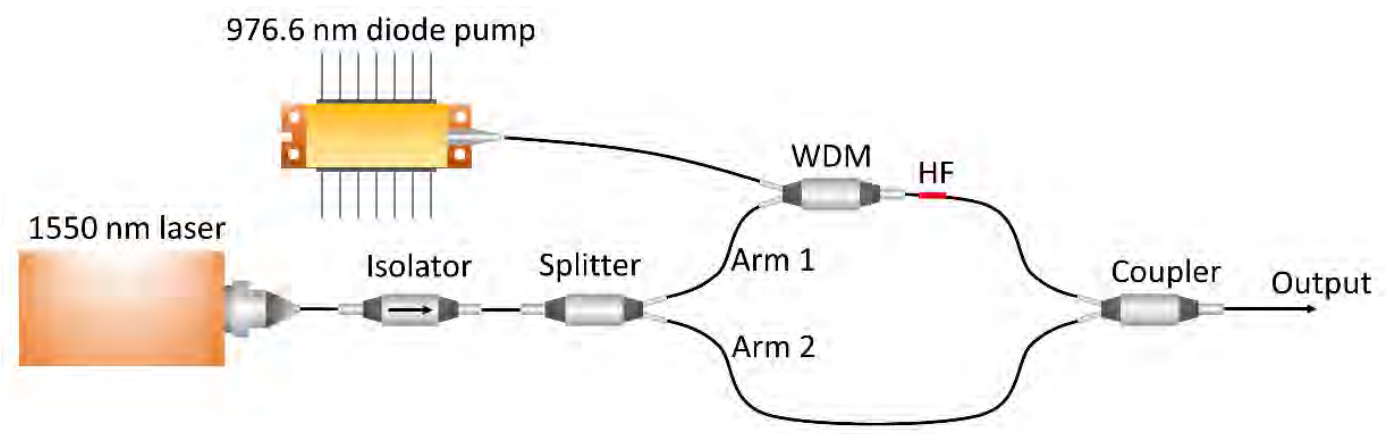

Fig. 2. Experimental Setup

First, a DC current was supplied to the pump and a measurement of modulator output power versus $976 \mathrm{~nm}$ LD power was performed using an optical power meter; see Fig.3 (a). Using Fig.1 to convert pump power into temperature change yielded the result shown in Fig.3 (b). The results directly show the intensity modulation introduced by the phase change in the pumped HF. The measured extinction ratio, $\sim 60 \%$, was limited by the unbalanced power between the two combined arms. The average temperature difference between neighboring peaks in Fig. 1 (d) (corresponding to $\Delta \phi^{\prime}=2 \pi$ ) is 11.7 $\mathrm{K}$. This, according to the equation for $\Delta \phi^{\prime}$ above, should theoretically be $64.6 \mathrm{~K}$. This result indicates that the neighboring passive fiber near the HF is also heating via thermal conduction, corresponding to a longer $L^{\prime}$ and therefore a smaller required $\Delta T$. 


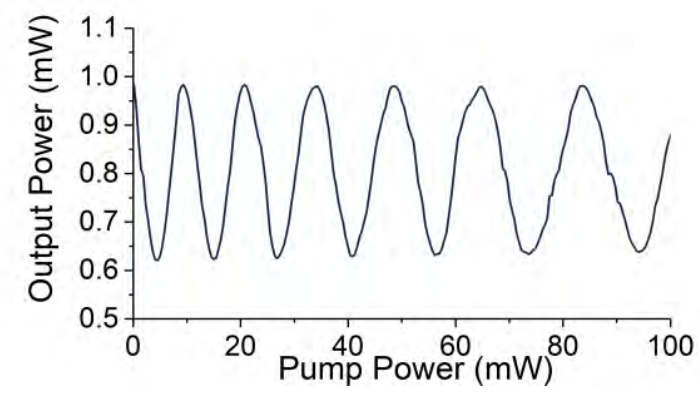

(a)

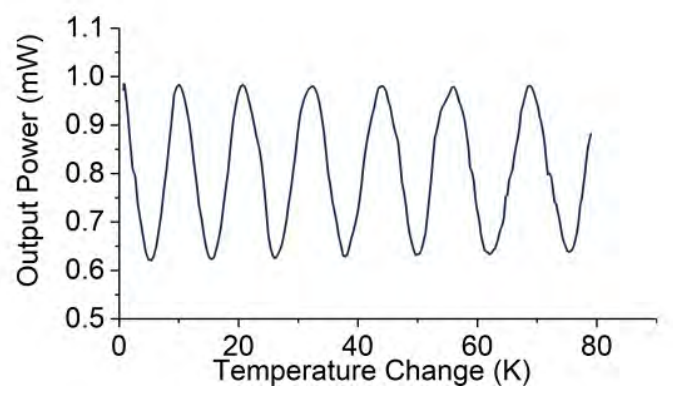

(b)

Fig. 3. (a) Output power versus launched pump power to HF. (b) Output power versus temperature change of HF

Second, to determine the modulation speed, a pulsed current (pulse width $5 \mathrm{~ms}$, pulse repetition interval $6.5 \mathrm{~ms}$ ) was supplied to the pump, as shown in Fig.4 (a). The long pulses were used in order to observe both slow and fast responses of the MZI. The results for the output signal with three different pump powers are shown in Fig.4 (b)-(d), which correspond to $\cos \left(\Delta \phi^{\prime}\right)=-1,0$, and 1 , respectively. When fitting the signal within one pulse width to a double exponential function, as shown in Fig.5 (e), both fast and slow temporal components are observed with the former and latter having characteristic times $\left(\mathrm{e}^{-1}\right)$ of 95 and $800 \mu \mathrm{s}$, respectively. The former time constant is limited by the rate at which energy is deposited into the core, or simply by the peak pump power, while the latter is limited by the slow thermal diffusion from the core through the much more voluminous $(\sim 121 \times)$ cladding. Using the Flash Method outlined in reference ${ }^{9}$, the thermal diffusivity, heat capacity, and thermal conductivity of $\mathrm{SiO}_{2}$ are used to determine the time $(-3$ $\mathrm{dB}$ as opposed to the $\mathrm{e}^{-1}$ time) it takes for thermal diffusion from core to cladding to reach equilibrium, resulting in $\sim 540 \mu \mathrm{s}$, which is in reasonable agreement with the measurement. Therefore, increasing the pump power (therefore faster thermal energy deposition) and etching some of the passive HF cladding (therefore faster thermal diffusion) should help to ultimately increase the modulation speed to $\sim \mathrm{MHz}$ range. These and other issues are currently under investigation and will be discussed at the conference.
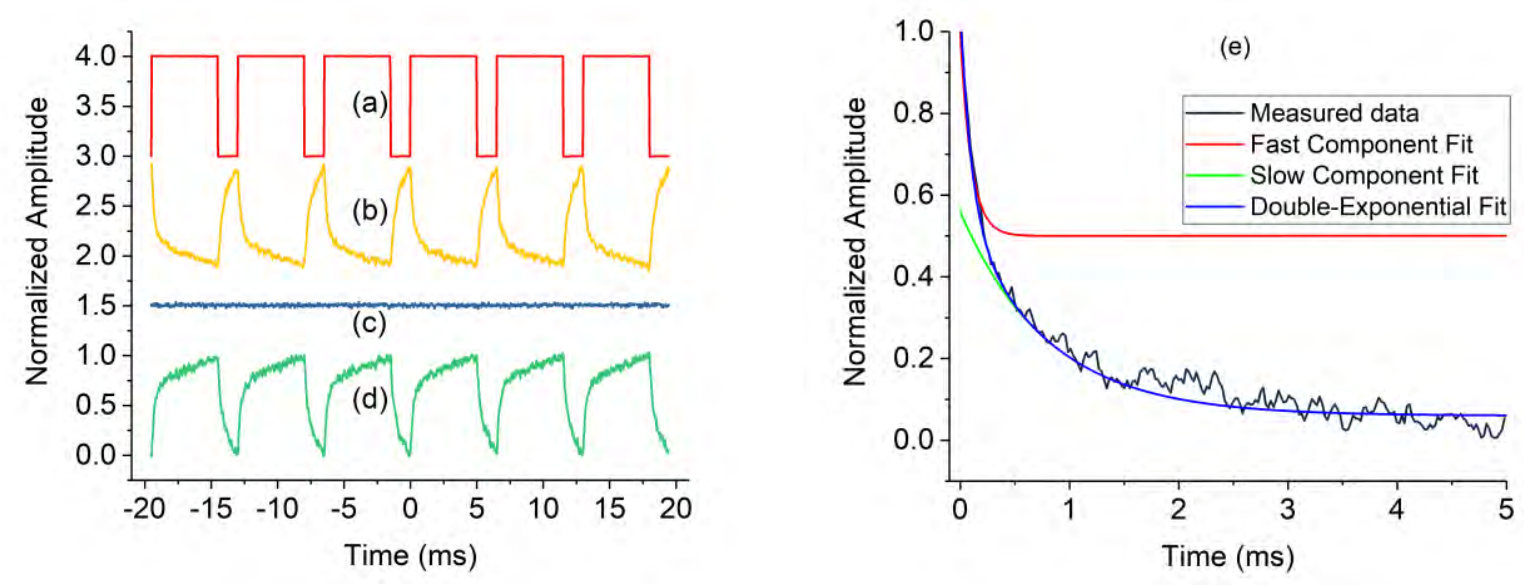

Fig. 4. (a) Input current into the laser diode. (b) Output signal with $\cos \left(\Delta \phi^{\prime}\right)=-1$ (c) $\cos \left(\Delta \phi^{\prime}\right)=0$ (d) $\cos \left(\Delta \phi^{\prime}\right)=1$.

\section{Reference}

[1] Alvarez-Chavez, J. A., Offerhaus, H. L., Nilsson, J., Turner, P. W., Clarkson, W. A. and Richardson, D. J., "High-energy, high-power ytterbium-doped Q-switched fiber laser," Opt. Lett. 25(1), 37 (2000). 
[2] Kee, H. H., Lees, G. P. and Newson, T. P., "Narrow linewidth CW and Q-switched erbium-doped fibre loop laser," Electron. Lett. 34(13), 1318 (2002).

[3] Shim, S.-H., Strasfeld, D. B., Fulmer, E. C. and Zanni, M. T., "Femtosecond pulse shaping directly in the mid-IR using acousto-optic modulation," Opt. Lett. 31(6), 838 (2006).

[4] Zhang, H., Eaton, S. M. and Herman, P. R., "Single-step writing of Bragg grating waveguides in fused silica with an externally modulated femtosecond fiber laser," Opt. Lett. 32(17), 2559 (2007).

[5] Wu, B. and Chu, P. L., "Fast optical switching in Sm3+-doped fibers," IEEE Photonics Technol. Lett. 8(2), 230232 (1996).

[6] Sadowski, R. W., Digonnet, M. J. F., Pantell, R. H. and Shaw, H. J., "Microsecond optical-optical switching in a neodymium-doped two-mode fiber," Opt. Lett. 18(11), 927 (1993).

[7] Tuggle, M., Kucera, C., Hawkins, T., Cavillon, M., Pan, G., Yu, N., Dragic, P. and Ballato, J., "Novel Reactive Molten Core Fabrication Employing in-situ Metal Oxidation: Erbium-Doped Intrinsically Low Brillouin Scattering Optical Fiber,” Opt. Mater. X 1(March), 100009 (2019).

[8] Yu, N., Cavillon, M., Kucera, C., Hawkins, T. W., Ballato, J. and Dragic, P., "Less than 1\% quantum defect fiber lasers via ytterbium-doped multicomponent fluorosilicate optical fiber," Opt. Lett. 43(13), 3096 (2018).

[9] Parker, W. J., Jenkins, R. J., Butler, C. P. and Abbott, G. L., "Flash Method of Determining Thermal Diffusivity, Heat Capacity, and Thermal Conductivity,” J. Appl. Phys. 32(9), 1679-1684 (1961). 


\title{
Tunable wavelength Q-switched All-Fiber Laser Based on
}

\section{Two-Dimensional Perovskite Solution}

\author{
Byungjoo Kim, Seongjin Hong, and Kyunghwan Oh* \\ Institute of Physics and Applied Physics, Yonsei University, Seoul 03722, South Korea
}

\begin{abstract}
We experimentally demonstrate an all-fiber Q-switched laser in L-band using two-dimensional(2D) hybrid organicinorganic $\left(\left(\mathrm{C}_{6} \mathrm{H}_{5} \mathrm{C}_{2} \mathrm{H}_{4} \mathrm{NH}_{3}\right)_{2} \mathrm{PbI}_{4}\right)$ perovskite solution. by adjusting an optical fiber end face micro air-gap distance, we also achieve a tunable peak wavelength blue shift of $7 \mathrm{~nm}$ at the center wavelength of $1600.6 \mathrm{~nm}$ using Fabry-Perot structure.
\end{abstract}

Keywords: Two-dimensional material, perovskite, Q-switching fiber laser, Liquid saturable absorber, L-band

*koh@yonsei.ac.kr; Office +82-2-2123-5608

\section{Introduction}

Saturable absorbers(SAs) have been well demonstrated using a different kind of materials, such as a two-dimensional material, carbon-based material, even biomaterial[1-3]. Since the discovery of the two-dimensional structure of perovskite, perovskite has been at the center of solar cell and quantum dot research.[4] Along with these applications, perovskite has recently attracted been attention as a new photonics material for optoelectronics applications. In the case of perovskite, nonlinear optical properties of 2D perovskite thin film were reported.[5] We demonstrate for a Qswitching all-fiber laser based on 2D perovskite solution in N, N-Dimethylformamide(DMF) using nonlinear optical phenomena of the tunable L-band region.

\section{Method}

(a)

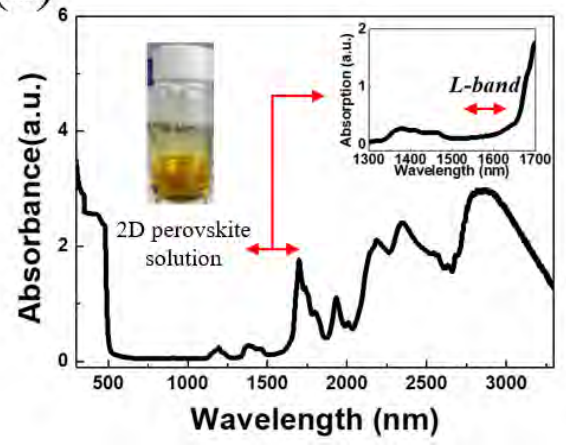

(b)

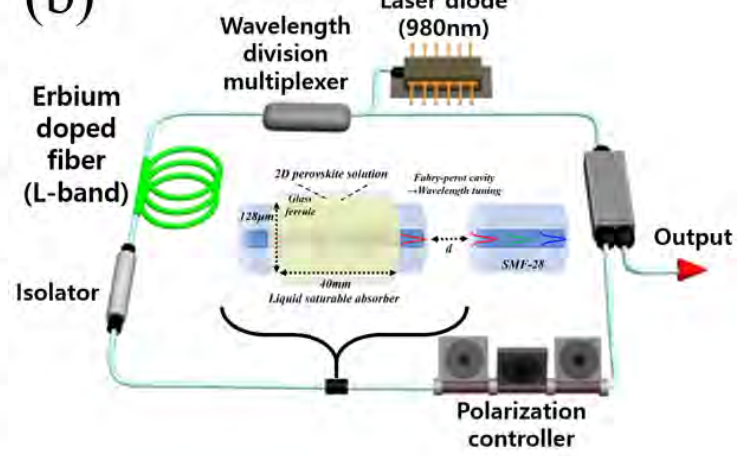

Figure 1. (a) 2D perovskite $40 \mathrm{wt} \%$ solution VIS-NIR spectrum (b) the fabrication process of 2D perovskite liquid saturable absorber and configuration of a laser ring cavity

As shown in Figure 1(a), the near-infrared(NIR) spectrum of a $40 \mathrm{wt} \%$ perovskite solution. In the NIR region, depending on the wavelength, the absorbance decreased at the C- and L-band. The end face of optical fiber was immersed into glass ferrule filled with the prepared $40 \mathrm{wt} \%$ perovskite solution. The configuration of the fiber laser cavity is schematically shown in Figure. 1(b). The optical gain medium is a $1.2 \mathrm{~m}$ Erbium-doped fiber(EDF) length

\footnotetext{
Sixth International Workshop on Specialty Optical Fibers and Their Applications (WSOF 2019), edited by Liang Dong, John M. Ballato, Proc. of SPIE Vol. 11206, 112061W · C 2019 SPIE

CCC code: $0277-786 X / 19 / \$ 21 \cdot$ doi: $10.1117 / 12.2548534$
} 
forward pumped by a 980nm laser diode (LD) via a 980/1550 wavelength division multiplexer(WDM) coupler. Following the EDF, an independent polarization isolator(ISO) and a polarization controller(PC) are used to make the adjusting the polarization state, respectively.
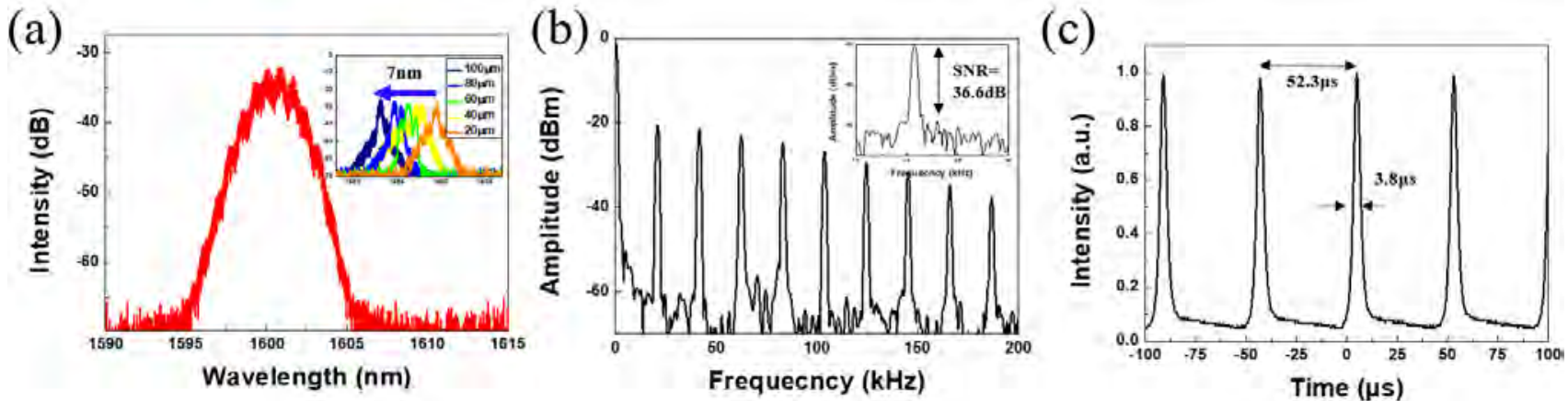

Figure 2. Q-switching fiber laser output at $458.5 \mathrm{~mW}$ power pump. (a) Optical spectrum analyzer data (b) RF spectrum data (c) oscilloscope trace data

By turning on the pump laser, the stable Q-switching pulse will be generated in the L-band regime, Figure 2.(a) shows the optical spectrum output of the Q-switching operation at the pump power of $458.5 \mathrm{~mW}$ with the central wavelength of 1600.6nm. Application of Fabry-Perot structure using fusion splicer program, we achieved a $7 \mathrm{~nm}$ of tuning wavelength data in the L-band by controlling the optical fiber end face air gap distance from $20 \mu \mathrm{m}$ to $100 \mu \mathrm{m}$, each $\mathrm{z}$-axis moving distance is $20 \mu \mathrm{m}$. Figure 2.(b) shows radio frequency(RF) spectrum indicates the repetition rate of $19.1 \mathrm{kHz}$ and signal to noise(SNR) is $36.6 \mathrm{~dB}$. The stable Q-switched pulse train is shown in Figure 2.(c). The repetition rate increased from $9.7 \mathrm{kHz}$ to $19.1 \mathrm{kHz}$ while the pump power increasing from $127.21 \mathrm{~mW}$ to $458.5 \mathrm{~mW}$, and the pulse width decreased from $12.0 \mu \mathrm{s}$ to $3.8 \mu \mathrm{s}$, and the output power increased from $0.53 \mathrm{~mW}$ to $4.23 \mathrm{~mW}$, respectively.

\section{Results and Discussion}

In conclusion, we have fabricated liquid saturable absorber using 2D perovskite solution and glass ferrule. The proposed liquid SA has several advantages: all-fiber configuration, efficient fabrication process, recovery, and high optical damage threshold. Stable passive Q-switched pulse was achieved with the repetition rate from $9.7 \mathrm{kHz}$ to $19.1 \mathrm{kHz}$. This result confirms the characteristics of liquid saturable absorber by using the Fabry-Perot structure as a novel method for tunable wavelength ultrafast pulse generation.

\section{References}

[1] Woodward, R., \& Kelleher, E. 2D saturable absorbers for fibre lasers. Applied Sciences, 5(4), 1440-1456. (2015). [2] Martinez, A., Zhou, K., Bennion, I \& Yamashita, S. In-fiber microchannel device filled with a carbon nanotube dispersion for passive mode-lock lasing. Optics express, 16(20), 15425-15430. (2008).

[3]Khazaeinezhad, R., Kassani, S. H., Paulson, B., Jeong, H., Gwak, J., Rotermund, F \& Oh, K. Ultrafast nonlinear optical properties of thin-solid DNA film and their application as a saturable absorber in femtosecond mode-locked fiber laser. Scientific reports, 7, 41480. (2017).

[4] Zhang, S., Audebert, P., Wei, Y., Al Choueiry, A., Lanty, G., Bréhier, A \& Deleporte, E. Preparations and characterizations of luminescent two dimensional organic-inorganic perovskite semiconductors. Materials, 3(5), 33853406. (2010).

[5] Hong, S., Lédée, F., Park, J., Song, S., Lee, H., Lee, Y. S \& Oh, K. Mode-Locking of All-Fiber Lasers Operating at Both Anomalous and Normal Dispersion Regimes in the C-and L-Bands Using Thin Film of 2D Perovskite Crystallites. Laser \& Photonics Reviews, 12(11), 1800118. (2018) 


\title{
O-band bismuth-doped fiber amplifier and its temperature dependent
}

\author{
performance
}

\author{
Y. Wang*, N. K. Thipparapu, S. Wang, P. Barua, D. J. Richardson, and J. K. Sahu \\ Optoelectronics Research Centre, University of Southampton, University Road, Southampton, SO17 1BJ, \\ United Kingdom \\ *Corresponding author: yw9n17@soton.ac.uk \\ Abstract
}

We report temperature-dependent gain and NF of single-pass and double-pass O-band BDFA from -60 to $+80^{\circ} \mathrm{C}$. A maximum gain of $41 \mathrm{~dB}$, NF $3.8 \mathrm{~dB}$ was achieved at $-60^{\circ} \mathrm{C}$ for $-23 \mathrm{dBm}$ input-signal. The temperature-dependent-gain coefficient was $-0.04 \mathrm{~dB} /{ }^{\circ} \mathrm{C}$.

Keywords: optical fibers, doped fiber amplifiers, bismuth, temperature dependence, optical fiber communication

\section{Introduction}

The demand for optical fiber communication is growing dramatically in the last three decades owing to the increased internet based applications such as cloud services, live video streaming, virtual and augmented reality technologies etc. The transmission capacity of standard single mode fiber (SSMF), in contrast, is now approaching the fundamental limits of 100-200Tbit/s [1]. Potential technologies such as space division multiplexing (SDM) using multi-core fibers (MCFs) and few-mode fibers (FMFs) are under investigation. However, the immediate solution is to explore the low-loss window of silica fibers from 1250 to $1700 \mathrm{~nm}$ by developing efficient fiber amplifiers [2]. Bismuth (Bi)-doped fibers (BDFs) have attracted significant attention because of its broadband near-infrared (NIR) luminescence properties. It has been reported that emission around 1.2, 1.3, 1.45 and $1.7 \mu \mathrm{m}$ can be realized in BDFs with different hosts [3, 4]. Recently, various types of BDFs have been developed and used to construct fiber amplifiers covering the O, E and U bands [5-8]. However, there are very few reports focused on the thermal behavior of gain and noise figure (NF) characteristics of Bi-doped fibre amplifiers (BDFAs). Such studies are of significant importance in understanding the system stability and robustness when incorporated in the communication network where the ambient temperatures fluctuate. Here, we present our work on temperature dependent performance of a BDFA operating from 1300 to 1360 $\mathrm{nm}$ in a temperature range of -60 to $+80{ }^{\circ} \mathrm{C}$, in both single-pass and double-pass amplifier configurations. The temperature-dependent-gain (TDG) coefficient was investigated at different signal wavelengths. The gain and NF characteristics with pump power and signal power was studied at $-60,+20$ and $+80{ }^{\circ} \mathrm{C}$. Also, we observed that similar gain and NF performance as in the case of single-pass amplifier can be achieved with double-pass configuration with a reduced length and pump power.

\section{O-band Bi-doped phosphosilicate fiber amplifier}

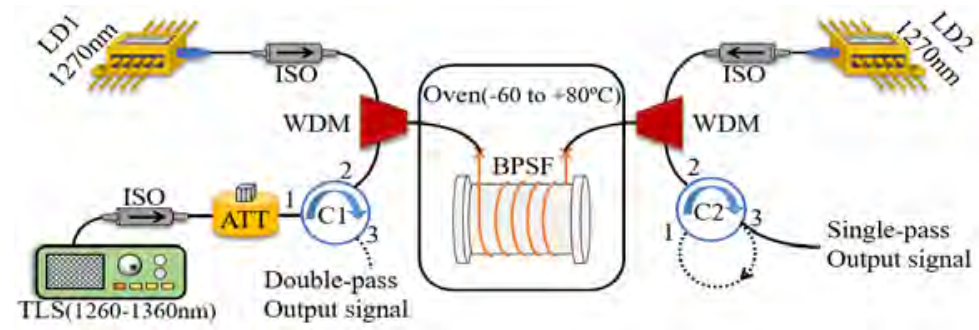

Figure 1. Schematic of experimental setup used to characterize the temperature dependent performance of the BDFA. Solid line: single-pass BDFA; and dashed line: double-pass BDFA.

A Bi-doped phosphosilicate preform was fabricated using an in-house MCVD-solution doping technique. The Bidoped phosphosilicate fiber (BPSF) was drawn with a core and cladding diameter of $13 \mu \mathrm{m}$ and $125 \mu \mathrm{m}$ respectively.

Sixth International Workshop on Specialty Optical Fibers and Their Applications (WSOF 2019), edited by Liang Dong, John M. Ballato, Proc. of SPIE Vol. 11206, 112061X · C 2019 SPIE

CCC code: $0277-786 X / 19 / \$ 21 \cdot$ doi: $10.1117 / 12.2548565$ 
The index difference $(\Delta \mathrm{n})$ between the core and cladding was $\sim 0.005$. The small signal absorption at $1270 \mathrm{~nm}$ and unsaturable loss (UL) at $1240 \mathrm{~nm}$ were measured to be $\sim 0.5 \mathrm{~dB} / \mathrm{m}$ and $13 \%$, respectively. The background loss at 1550 $\mathrm{nm}$ was $0.02 \mathrm{~dB} / \mathrm{m}$. The BDFA experimental setup used in this work is shown in Fig. 1. Two laser diodes (LDs) operating at $1270 \mathrm{~nm}$ were used to pump bi-directionally. A tunable laser source (TLS) operating in the wavelength region of 1260-1360 nm provided the input signals. The BPSFs were placed inside a temperature-controlled oven with an operating temperature range covering from -60 to $+80{ }^{\circ} \mathrm{C}$. The input and output signal spectra were recorded using an optical spectrum analyzer (OSA, YOKOGAWA AQ6370). Two wavelength division multiplexer (WDMs) were used to combine or separate pump and signal wavelengths, while three isolators (ISOs) were utilized to prevent back reflections from damaging the pump and signal sources. Two circulators $(\mathrm{C} 1$ and $\mathrm{C} 2)$ were used to construct either a single-pass or a double-pass amplifier configuration. The fiber length of the BPSF was optimized to $212 \mathrm{~m}$ at room temperature (RT) of $20^{\circ} \mathrm{C}$ with respect to gain performance. The input signal power was fixed to $-23 \mathrm{dBm}$. The maximum available pump powers launched into the BPSF was $386 \mathrm{~mW}$ and $359 \mathrm{~mW}$ from the forward and backward pumps, respectively.

\section{Temperature dependent performance of the BDFA}

Initially, the gain and NF characteristics of the BDFA were measured at RT. For a signal power of $-23 \mathrm{dBm}$, the maximum gain was $22.3 \mathrm{~dB}$ and $39 \mathrm{~dB}$ with a $\mathrm{NF}$ of $4 \mathrm{~dB}$ and $4.2 \mathrm{~dB}$ in the single-pass and double-pass BDFA, respectively. Then, the temperature dependent gain and NF performance of the BDFA was measured from -60 to $+80{ }^{\circ} \mathrm{C}$ at intervals of $20^{\circ} \mathrm{C}$, as shown in Fig. 2(a) and Fig. 2(b). Increment in gain and reduction in NF were observed as the temperature decreases at signal wavelengths from 1300 to $1360 \mathrm{~nm}$. The maximum gain increased to $25.4 \mathrm{~dB}$ and $41 \mathrm{~dB}$ at $-60{ }^{\circ} \mathrm{C}$ for the single-pass and double-pass BDFA. The gain and NF variation with temperature was found to be linear. In order to quantify the gain variation with temperature, the TDG coefficient was calculated, which is defined as the amount of signal gain change per unit of temperature change (in $\mathrm{dB} /{ }^{\circ} \mathrm{C}$ ). Fig. 2 (c) shows the TDG coefficient in the spectral region of 1300-1360 $\mathrm{nm}$. As can be seen, the TDG coefficient of the single-pass and double-pass BDFA is similar at longer wavelengths $(1340-1360 \mathrm{~nm})$, however it is different towards the shorter wavelength side (1300-1340 $\mathrm{nm})$. The TDG coefficient at maximum gain wavelength $(1350 \mathrm{~nm})$ was around $-0.04 \mathrm{~dB} /{ }^{\circ} \mathrm{C}$.
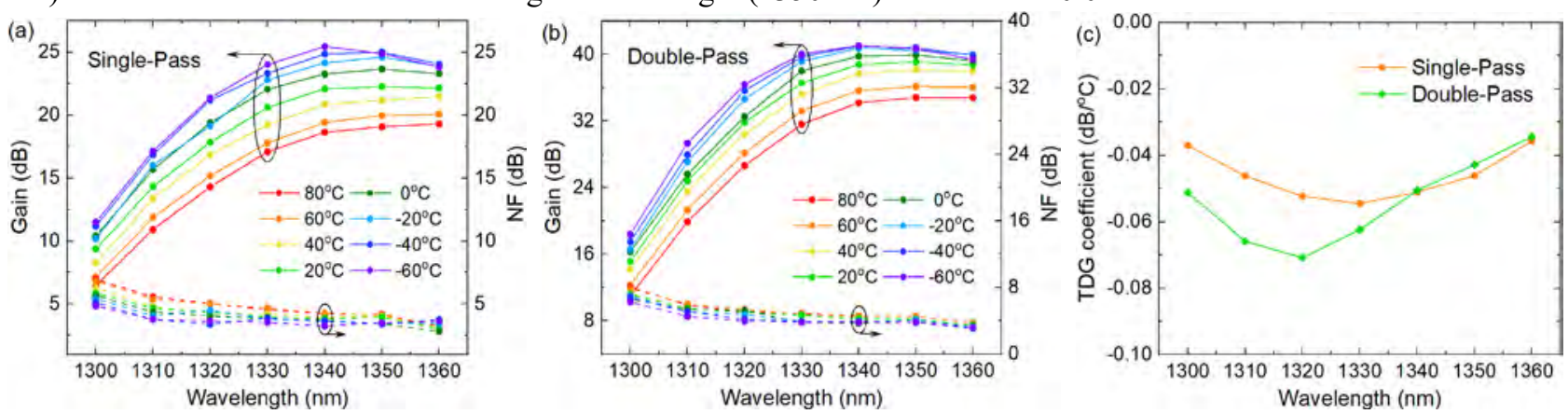

Figure 2. Gain and NF spectra at different temperatures from -60 to $+80{ }^{\circ} \mathrm{C}$ of (a) a single-pass and (b) a double-pass BDFA (c) TDG coefficient from 1300 to $1360 \mathrm{~nm}$ of the single-pass and double-pass BDFA (fiber length $=212 \mathrm{~m}$, signal power $=$ $23 \mathrm{dBm}$, total launched pump power $=745 \mathrm{~mW}$ )

Later, the gain and NF variation with pump power and signal power was measured at $-60,+20$ and $+80{ }^{\circ} \mathrm{C}$ in the double-pass BDFA, as shown in Fig. 3(a) and Fig. 3(b). The gain was increased whereas the NF was slightly decreased as the pump power increased or the signal power decreased. The gain coefficient was $0.073 \mathrm{~dB} / \mathrm{mW}$ at RT, and increased to $0.083 \mathrm{~dB} / \mathrm{mW}$ at $-60{ }^{\circ} \mathrm{C}$. At $\mathrm{RT}$, a small signal gain of $40 \mathrm{~dB}$ was obtained for a signal power of $-30 \mathrm{dBm}$, and it increased to $42.8 \mathrm{~dB}$ at $-60{ }^{\circ} \mathrm{C}$. In addition, the optical signal to noise ratio (OSNR) for a signal power of $-23 \mathrm{dBm}$ was observed to be $\sim 20 \mathrm{~dB}$ over the spectral region of $1300-1360 \mathrm{~nm}$.

Furthermore, the gain and NF were measured in the double-pass configuration again with the fiber length shortened to $159 \mathrm{~m}$ and the total pump power reduced to $370 \mathrm{~mW}$. As shown in Fig. 3(c), the gain and NF was found to achieve a similar performance as that of the single-pass BDFA with a fiber length of $212 \mathrm{~m}$ and a total pump power of $745 \mathrm{~mW}$. 
This shows the advantage of double-pass configuration over single-pass configuration with a reduced device length and pump power.
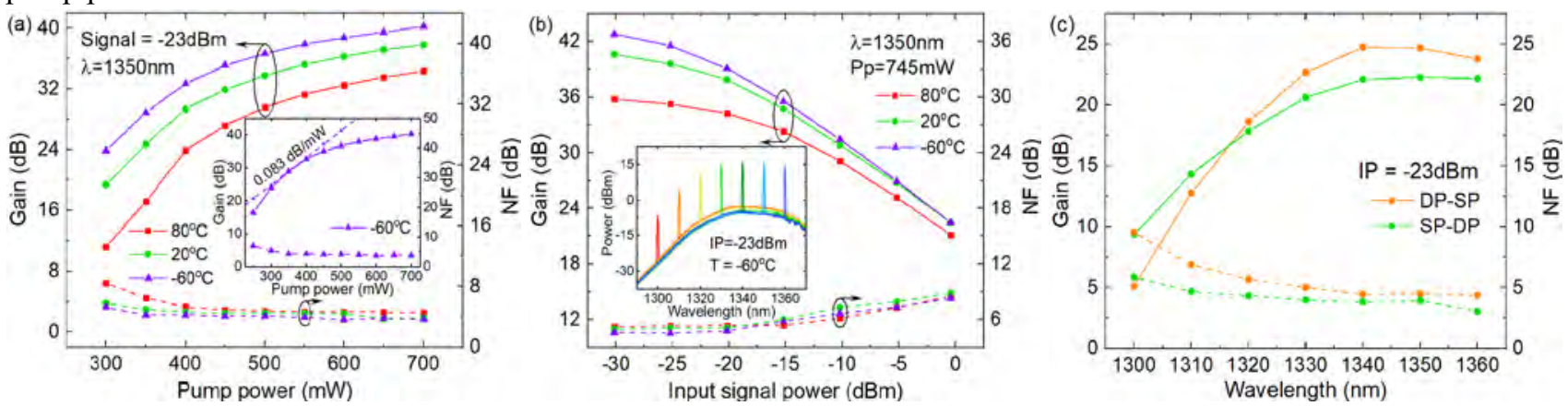

Figure 3. Gain and NF variation with (a) pump power and (b) signal power of the double-pass BDFA at $-60,+20$ and +80 ${ }^{\circ} \mathrm{C}$, for a fiber length of $212 \mathrm{~m}$ (inset of Fig. 3(a) and Fig. 3(b) presents the gain coefficient, and the OSNR spectrum for -23 $\mathrm{dBm}$ signal at $-60^{\circ} \mathrm{C}$ ) (c) Gain and NF of $212 \mathrm{~m}$ long BPSF in the single-pass double-pump (SP-DP, total pump power $=$ $745 \mathrm{~mW}$ ) and $159 \mathrm{~m}$ long BPSF in the double-pass single-pump (DP-SP, total pump power $=370 \mathrm{~mW}$ ) BDFAs

\section{Conclusions}

In conclusion, an O-band BDFA was demonstrated having a maximum gain of $39 \mathrm{~dB}$ with $4.2 \mathrm{~dB} \mathrm{NF}$ at $\mathrm{RT}$ and $41 \mathrm{~dB}$ with a NF of $3.8 \mathrm{~dB}$ at $-60{ }^{\circ} \mathrm{C}$ respectively, in the double-pass configuration for a signal power of $-23 \mathrm{dBm}$. The TDG coefficient was $-0.05 \pm 0.01 \mathrm{~dB} /{ }^{\circ} \mathrm{C}$ on the wavelength range of $1300-1360 \mathrm{~nm}$. The gain and $\mathrm{NF}$ variations with pump power and signal power were also reported at different temperatures. Furthermore, a similar amplifier performance was observed in the double-pass configuration to that of the single-pass configuration with a $25 \%$ reduction in fiber length and a $50 \%$ decrease in pump power.

\section{Acknowledgments}

This work was supported by the UK Engineering and Physical Sciences Research Council (EPSRC) under the "Airguide Photonics" Programme Grant (EP/P030181/1) and II-VI Foundation studentship. Data for this work can be accessed at doi: 10.5258/SOTON/D1029.

\section{References}

[1] Richardson, D. J., Fini, J. M., and Nelson, L. E., "Space-division multiplexing in optical fibers," Nat. Photonics. 7, 354-362 (2013).

[2] Taengnoi, N., Bottrill, K., Thipparapu, N. K., Umnikov, A.A., Sahu, J.K., Petropoulos, P., Richardson, D.J., "Amplified O-band WDM Transmission using a Bi-doped Fiber Amplifier," ECOC, Rome, Italy, 1-3 (2018).

[3] Melkumov, M. A., Alyshev, S. V., Firstov, S. V., Dianov, E. M., "Bismuth-doped Fiber Lasers and Amplifiers: Review and Prospects," ICLO, St. Petersburg, Russia, S1-19 (2016).

[4] Firstov, S. V., Alyshev, S. V., Riumkin, K. E., Khegai, A. M., Kharakhordin, A. V., Melkumov, M. A., and Dianov, E. M., "Laser-Active Fibers Doped with Bismuth for a Wavelength Region of 1.6-1.8 $\mu \mathrm{m}, "$ IEEE J. Sel. Top. Quantum Electron. 24, (5), 1-15 (2018).

[5] Thipparapu, N. K., Jain, S., Umnikov, A. A., Barua, P., and Sahu, J. K., "1120 nm diode-pumped Bi-doped fiber amplifier," Opt. Lett. 40, (10), 2441-1444 (2015).

[6] Thipparapu, N. K., Wang, Y., Umnikov, A. A., Barua, P., Richardson, D. J., and Sahu, J. K., "High gain Bi-doped all fiber amplifier for O-band DWDM optical fiber communication," OFC, San Diego, USA, paper M1J.5 (2019).

[7] Melkumov, M. A., Bufetov, I. A., Shubin, A. V., Firstov, S. V., Khopin, V. F., Guryanov, A. N., Dianov, E. M., "Bismuth-Doped Optical Fiber Amplifier for $1430 \mathrm{~nm}$ Band Pumped by $1310 \mathrm{~nm}$ Laser Diode," OFC, Los Angeles, USA, paper OMH1 (2011).

[8] Firstov, S. V., Alyshev, S. V., Riumkin, K. E., Khopin, V. F., Guryanov, A. N., Melkumov, M. A., and Dianov, E. M., "A 23-dB bismuth-doped optical fiber amplifier for a 1700-nm band," Sci. Rep. 40, (10), 1-6 (2015). 


\title{
Direct extrusion of hollow-core $\mathrm{THz}$ fiber using a 3D printer
}

\author{
Wanvisa Talataisong*a, Lieke D. Van Putten ${ }^{\mathrm{a}}$, Rand Ismaeel ${ }^{\mathrm{a}, \mathrm{b}}$, Martynas Beresna ${ }^{\mathrm{a}}$, \\ Gilberto Brambilla ${ }^{a}$ \\ ${ }^{a}$ Optoelectronics Research Centre, University of Southampton, Southampton, SO17 1BJ, UK

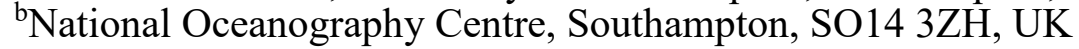

\begin{abstract}
We report a novel fabrication technique to achieve a hollow-core antiresonant polymer optical fiber for THz guidance. The fiber can be directly extruded from a 3D printer using a modified structured nozzle in a single step procedure. The possibility to use fibers made from acrylonitrile butadiene styrene (ABS) for guiding in the THz region is assessed through the profile of the confinement loss.
\end{abstract}

Keywords: hollow-core fiber, direct extrusion, THz fiber, 3D printer, antiresonant fiber

*w.talataisong@,soton.ac.uk

\section{Introduction}

Terahertz (THz) or T-waves, electromagnetic waves with frequency ranging from $0.1 \mathrm{THz}$ to $10 \mathrm{THz}$ (wavelength from $30 \mu \mathrm{m}$ to $3 \mathrm{~mm}$ ), have been widely studied because of their unique characteristic to be able to penetrate most of dielectric materials. Security scanning, non-destructive testing, and imaging are amongst the most promising applications of THz waves, which exploit wavelengths relatively shorter than microwave and millimeter waves providing better resolution in imaging [1, 2]. Because of the non-ionizing nature, $\mathrm{THz}$ waves can pass through the organic tissue without causing any damage, thus it can be safely applied for medical and biomedical sensing $[3,4]$. In chemical sensing, THz waves are also of great interest for detecting wide variety of chemicals and biological compounds because vibrational modes of many macromolecules are associated to strong absorption peaks in the THz regime $[5,6]$. Moreover, $\mathrm{THz}$ waves can be also applied for the applications in wireless communication and have been proposed to increase data transmission rates due to the large bandwidth of the terahertz band [7].

Although THz waves have been shown to be beneficial for imaging and sensing, most of the THz systems are based on free-space optics that require delicate alignment and is associated with high loss due to the absorption of water vapor which cannot be avoided when working in free-space. Because of this, a large amount of $\mathrm{THz}$ research has concentrated on achieving low-loss and lowdispersion $\mathrm{THz}$ wave guiding. Metal wires are the very first material that have been used in $\mathrm{THz}$ waveguides due to low material absorption, but their applications are limited owing to their finite conductivity [8,9]. An alternative is represented by dielectric waveguides. Several polymer optical fibers were exploited as $\mathrm{THz}$ waveguides because of the low cost, accessibility of materials, and relatively low loss when compared to other dielectrics in the THz spectral range [10, 11]. However, most of polymers have high material absorption in the THz range leading to the high loss. To overcome this issue, hollow-core waveguides or fibers have been proposed because the mode is mostly confined in the hollow air-core, and the effect of material loss can be minimized. The hollowcore can be easily filled with dry air eliminating water vapors and their absorption.

In this paper, we report the design and fabrication of a hollow-core antiresonant polymer optical fiber (HC-MOPF) for guiding $\mathrm{THz}$ waves. The traditional extrusion technique and 3D printing were combined to fabricate HC-MOPFs for THz guidance in a single fabrication step. Simulations confirmed the possibility to guide $\mathrm{THz}$ using the fabricated fiber.

\section{Hollow-core fiber design and fabrication}

A hexagonal fiber was chosen for extruding HC-MPOFs because of its relatively simple geometry. 3D models of the nozzle with the fiber cross section inverse structure profile were designed with the Fusion 360 (Autodesk) software [Fig. 1(a)]. A structured nozzle was fabricated from stainless steel using a metal 3D printer [Fig. 1(b)].

Sixth International Workshop on Specialty Optical Fibers and Their Applications (WSOF 2019), edited by Liang Dong, John M. Ballato, Proc. of SPIE Vol. 11206, 112061Y · (C) 2019 SPIE

CCC code: $0277-786 \mathrm{X} / 19 / \$ 21 \cdot$ doi: $10.1117 / 12.2548583$

Proc. of SPIE Vol. 11206 112061Y-1 
The metal 3D printed nozzle was mounted into the micro-heater of the printing head allowing to heat the nozzle up to $250^{\circ} \mathrm{C}$. The polymer fiber was extruded by feeding a commercially available 3D printer filament (acrylonitrile butadiene styrene (ABS)) through the heated nozzle. The 3D printer interface was used to control the temperature of the nozzle and the extrusion speed. In the first experiments, bubbles were forming on the surface of the extruded fiber as the result of the high fictive temperature experienced by the extruded polymer, which was caused by the combination of a high nozzle temperature and the low polymer feeding speed. The quality of extruded fiber was improved by optimizing extrusion parameters. The optimization process provided good surface roughness of extruded fiber that has an average surface roughness ( $\mathrm{Ra}$ ) less than $100 \mathrm{~nm}$ and the processing parameters: the polymer fiber was extruded at temperature $\mathrm{T} \sim 240{ }^{\circ} \mathrm{C}$ through the structured nozzle at the feeding speed $\mathrm{s} \sim 250 \mathrm{~mm} / \mathrm{min}$ by using a built-in feeding motor [Figs. 1(c)-1(d)].
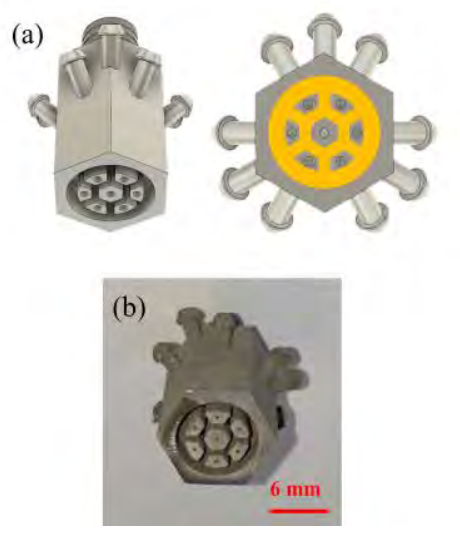
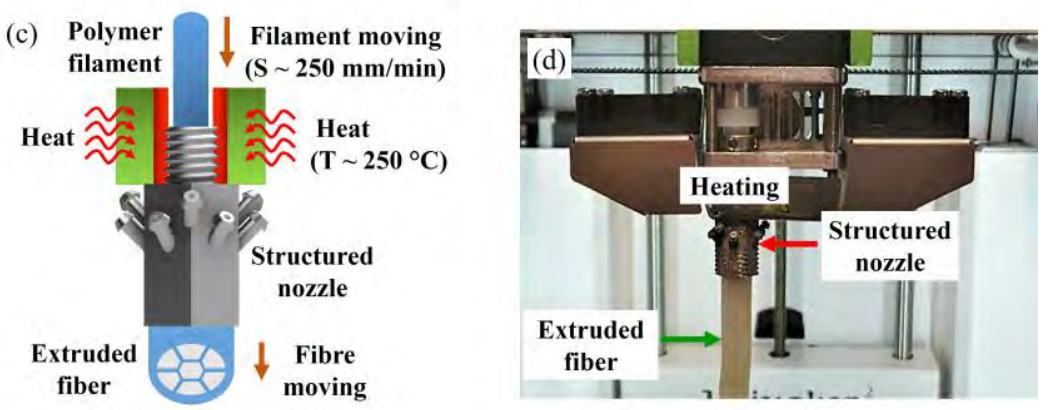

Figure 1. (a) Structured nozzle design including a 3D model and a bottom-view, from left to right respectively. Yellow colors represent the cross-section of the extruded fiber from structured nozzle. (b) Metal 3D printed structured nozzle. (c) Schematic and (d) photograph of the experimental setup used to extrude HC-MPOF.

\section{Results and Discussion}

The HC-MPOF used in this experiment consists of a hexagonal air-core with the diameter of $2165 \mu \mathrm{m}$. The thin layer surrounding the fiber core has a thickness of $650 \mu \mathrm{m}$ and the outer diameter of the extruded fiber is $8 \mathrm{~mm}$. Light in the hollow-core antiresonant fiber can be confined to the central air-core owing to the resonant reflection of the guided wave in the air-core with membrane surrounding the core which behaves effectively as a Fabry-Perot cavity. To observe the cross-section of the extruded HC-MPOF, the fiber was briefly heated at a temperature higher than the glass transition temperature of the material $\left(100{ }^{\circ} \mathrm{C}\right)$, then cleaved by using a heated razor blade. The cross-section image of the extruded HC-MPOF showed that the microstructure inside fiber was maintained after the extrusion process [Figs. 2(a)-2(b)].

In order to evaluate the HC-MPOF optical guidance properties in the THz regime, simulations of the mode propagating through the HC-MPOF structure were performed by importing the cross-section image of the fiber into a commercial finite element method software (COMSOLC). Figure 2(c) shows the supported fundamental mode confined in the air-core at frequency $f=0.7 \mathrm{THz}$. The loss spectrum was calculated from the created HC-MPOF profile with the same structure and dimension as the extruded HC-MPOF. The loss profile in Fig. 2(d) confirms that THz waves in the range $f=0.5-2.0 \mathrm{THz}$ experience the antiresonance effect. This means that the fabricated HC-MPOF effectively guides THz radiation in the antiresonance regions and also that the resonance peaks (where losses are high) are clearly defined. It can also be seen that the loss decreases with increasing frequency because the core size is small compared to the wavelength at lower frequencies pushing light into the surrounding material. To achieve guidance at different frequencies, a different thickness of the cladding elements is needed to shift the resonance peaks. This can be experimentally fabricated by applying pressure through the air holes while extruding the fiber. 

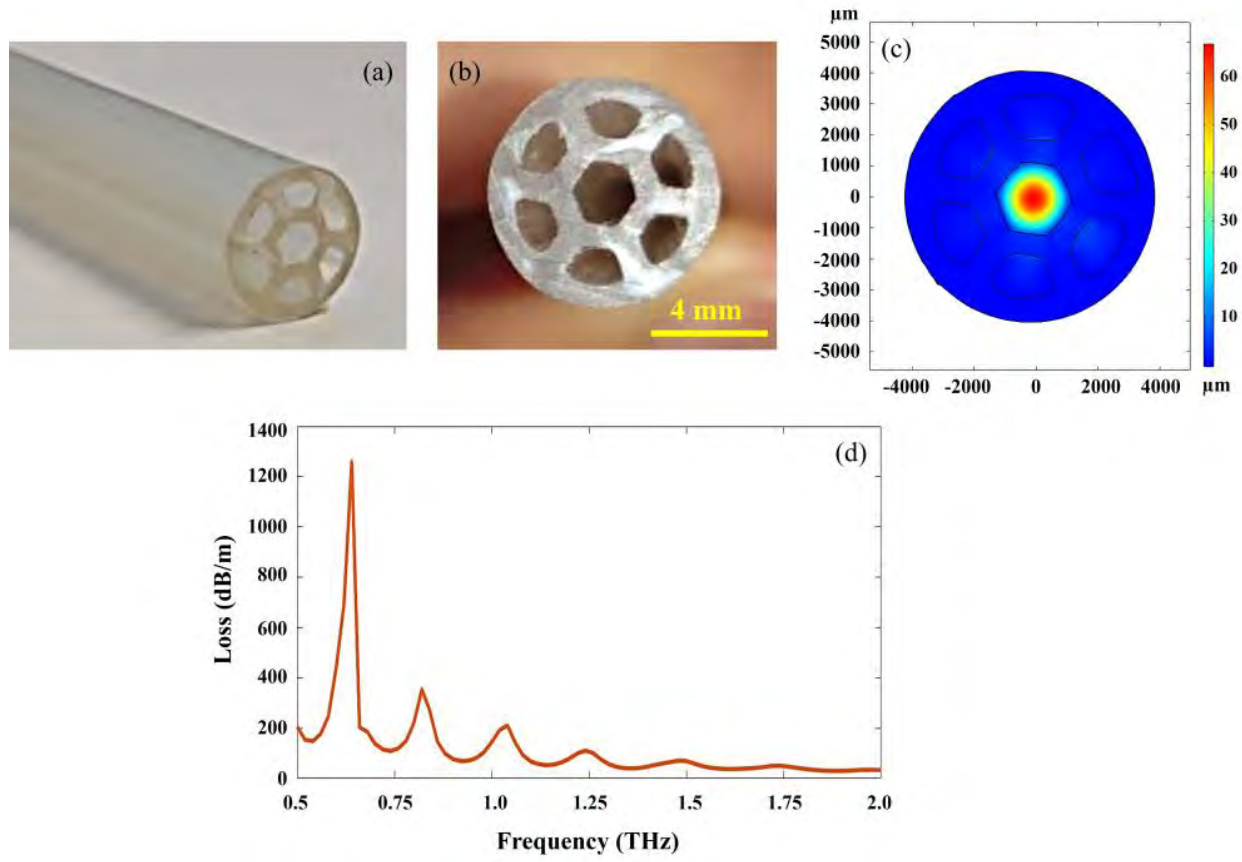

Figure 2. (a) Photograph and (b) cross-section of the hollow-core antiresonant fiber extruded from the metal 3D printed structured nozzle. (c) Simulated intensity profile of the fundamental mode at $f=0.7 \mathrm{THz}$. (d) Simulated transmission loss.

\section{Conclusion}

In conclusion, a polymer hollow-core optical fiber fabricated from a direct extrusion technique using a 3D printer has been demonstrated. By using this technique, 1 meter of fiber can be fabricated within 30 mins. The structure profile of the extruded fiber is clearly observed and maintained after extrusion. The modal profile simulations show the ability of the fiber to confine THz waves in the air-core. The calculated transmission loss, reveals a potential to use the extruded fiber as $\mathrm{THz}$ waveguide in the spectral range $0.5-$ $2.0 \mathrm{THz}$.

\section{Reference}

[1] D. M. Mittleman, "Twenty years of terahertz imaging [Invited]," Opt. Express 26, 9417-9431 (2018).

[2] E. V. Yakovlev, K. I. Zaytsev, I. N. Dolganova, and S. O. Yurchenko, "Non-Destructive Evaluation of Polymer Composite Materials at the Manufacturing Stage Using Terahertz Pulsed Spectroscopy," IEEE Transactions on Terahertz Science and Technology 5, 810-816 (2015).

[3] M. Borovkova, M. Khodzitsky, P. Demchenko, O. Cherkasova, A. Popov, and I. Meglinski, "Terahertz time-domain spectroscopy for non-invasive assessment of water content in biological samples," Biomed. Opt. Express 9, 2266-2276 (2018).

[4] M. Zhang and J. T. W. Yeow, "Nanotechnology-Based Terahertz Biological Sensing: A review of its current state and things to come," IEEE Nanotechnology Magazine 10, 30-38 (2016).

[5] M. S. Islam, J. Sultana, K. Ahmed, M. R. Islam, A. Dinovitser, B. W. Ng, and D. Abbott, "A Novel Approach for Spectroscopic Chemical Identification Using Photonic Crystal Fiber in the Terahertz Regime," IEEE Sensors Journal 18, 575-582 (2018).

[6] D. F. Swearer, S. Gottheim, J. G. Simmons, D. J. Phillips, M. J. Kale, M. J. McClain, P. Christopher, N. J. Halas, and H. O. Everitt, "Monitoring Chemical Reactions with Terahertz Rotational Spectroscopy," ACS Photonics 5, 3097-3106 (2018).

[7] I. F. Akyildiz, J. M. Jornet, and C. Han, "Terahertz band: Next frontier for wireless communications," Physical Communication 12, 16-32 (2014).

[8] K. Wang and D. M. Mittleman, "Metal wires for terahertz wave guiding," Nature 432, 376-379 (2004).

[9] K. Wang and D. M. Mittleman, "Guided propagation of terahertz pulses on metal wires," J. Opt. Soc. Am. B 22, 2001-2008 (2005).

[10]B. Ung, A. Mazhorova, A. Dupuis, M. Rozé, and M. Skorobogatiy, "Polymer microstructured optical fibers for terahertz wave guiding," Opt. Express 19, B848-B861 (2011).

[11]A. Argyros, "Microstructures in Polymer Fibres for Optical Fibres, THz Waveguides, and Fibre-Based Metamaterials," ISRN Optics 2013, 22 (2013). 


\title{
Non-destructive tomography for the characterization of extruded optical
}

\section{fiber triplet 3D shape sensors}

\author{
Pierre Lorre*a $^{* a}$ Arthur Poiffaut ${ }^{\mathrm{a}}$, Antoine Drouin ${ }^{\mathrm{a}}$, Samuel Kadoury ${ }^{\mathrm{b}}$ and Raman Kashyap ${ }^{\mathrm{a}, \mathrm{c}}$ \\ aDepartment of Engineering Physics, École Polytechnique Montréal, 2900 Édouard-Montpetit, \\ Montreal, QC, H3T 1J4, Canada \\ ${ }^{b}$ Department of Computer and Software Engineering, École Polytechnique Montréal, 2900 Édouard- \\ Montpetit, Montreal, QC, H3T 1J4, Canada \\ ${ }^{\mathrm{c}}$ Department of Electrical Engineering, PolyGrames, École Polytechnique Montréal, 2900 Édouard- \\ Montpetit, Montreal, QC, H3T 1J4, Canada
}

\begin{abstract}
This article proposes a non-destructive imaging system providing a sliced view of an extruded coated fiber triplet. It gives the relative positions of the fibers in the polymer coating with a $\pm 1.5 \mu \mathrm{m}$ precision. The characterization of a $3 \mathrm{D}$ shape sensor optical fiber triplet used for minimally invasive surgery is made using this system.

Keywords: imaging system, inverse Radon transform, optical fiber, shape sensing, extrusion

*Pierre.1orre@polymtl.ca; phone 1514 340-4711 \#4083

\section{Introduction}

Performing minimally invasive surgery requires the knowledge of the catheter or needle's position at all times. 3D shape sensing allows a real time guidance for the surgeon [1]. 3D shape sensing can be performed through the analysis of the Rayleigh backscatter signal of three optical fibers [2-4]. The fibers are positioned in a triangular cross-section called the optical fiber triplet. When compared with a reference, the backscatter signal gives the information of the strain along each fiber. Knowing the relative fibers positions, the triplet curvature values and directions can be calculated at each point, making possible the 3D shape reconstruction. For high precision, the fibers positions within the sensor must be well known. To ensure that the fibers are positioned at the same relative distance to one another while experiencing different strains, it is possible to coat them together through a polymer extrusion process, as we have recently demonstrated $[5,6]$. The triplet position within the coating is necessary for the $3 \mathrm{D}$ reconstruction. A non-destructive imaging method is thus required to locate the fibers and characterize the sensor. A transverse transmission image of the sensor is the equivalent of taking a projection slice. By using multiple rotation angles, a tomogram is created that can reconstruct the sliced view of the sensor through an inverse Radon transform [7].
\end{abstract}

\section{Physical system}

\section{Methodology}

Figure 1 shows the set up used to perform the imaging. The triplet sensor holder is filled with a refractive index matching liquid in order to minimize light refraction at the coating surface. A stepper motor rotates the stage holding the fibers over 180 degrees. A white and spatially uniform light source under the holder illuminates the sample so the transmission image of the sensor can be seen at the microscope CCD camera. An image is taken every 0.9-degree rotation. The polymer used for the triplet coating is a polypropylene copolymer. This polymer is transparent, has a good flexibility and its melt strength is high enough so when the coating undergoes strain, it is fully transferred to all the fibers.

Sixth International Workshop on Specialty Optical Fibers and Their Applications (WSOF 2019),

edited by Liang Dong, John M. Ballato, Proc. of SPIE Vol. 11206, 112061Z · C 2019 SPIE

CCC code: $0277-786 X / 19 / \$ 21 \cdot$ doi: $10.1117 / 12.2548640$ 


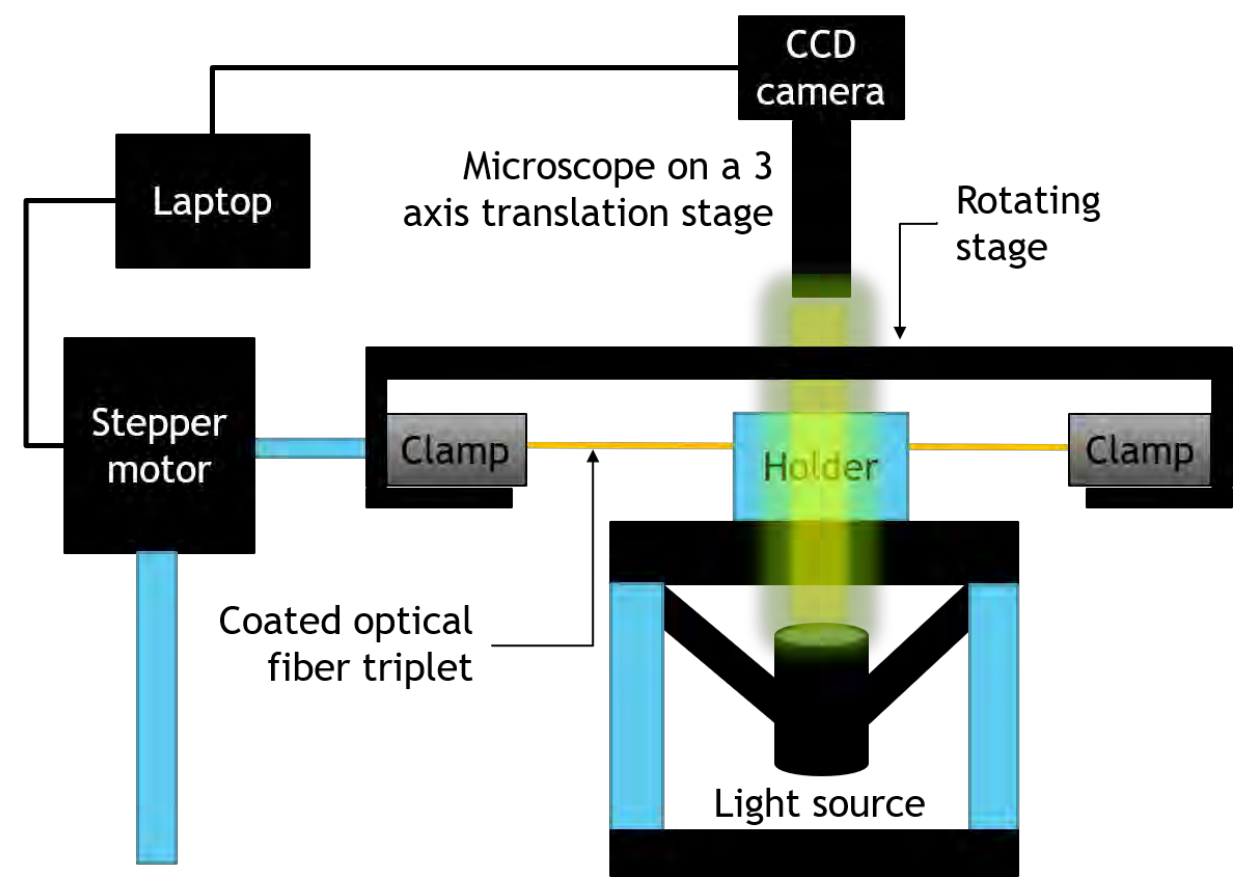

Figure 1: Scheme of the imaging system used for the sensor characterization

\section{Inverse Radon transform reconstruction}

The tomogram shown in Figure 2 is made by averaging the transmission image on a $1 \mathrm{~mm}$ length section on the longitudinal axis, over 180 degrees. That tomogram can be described as

$$
f(t, \theta)=I
$$

where $I$ is the light intensity.

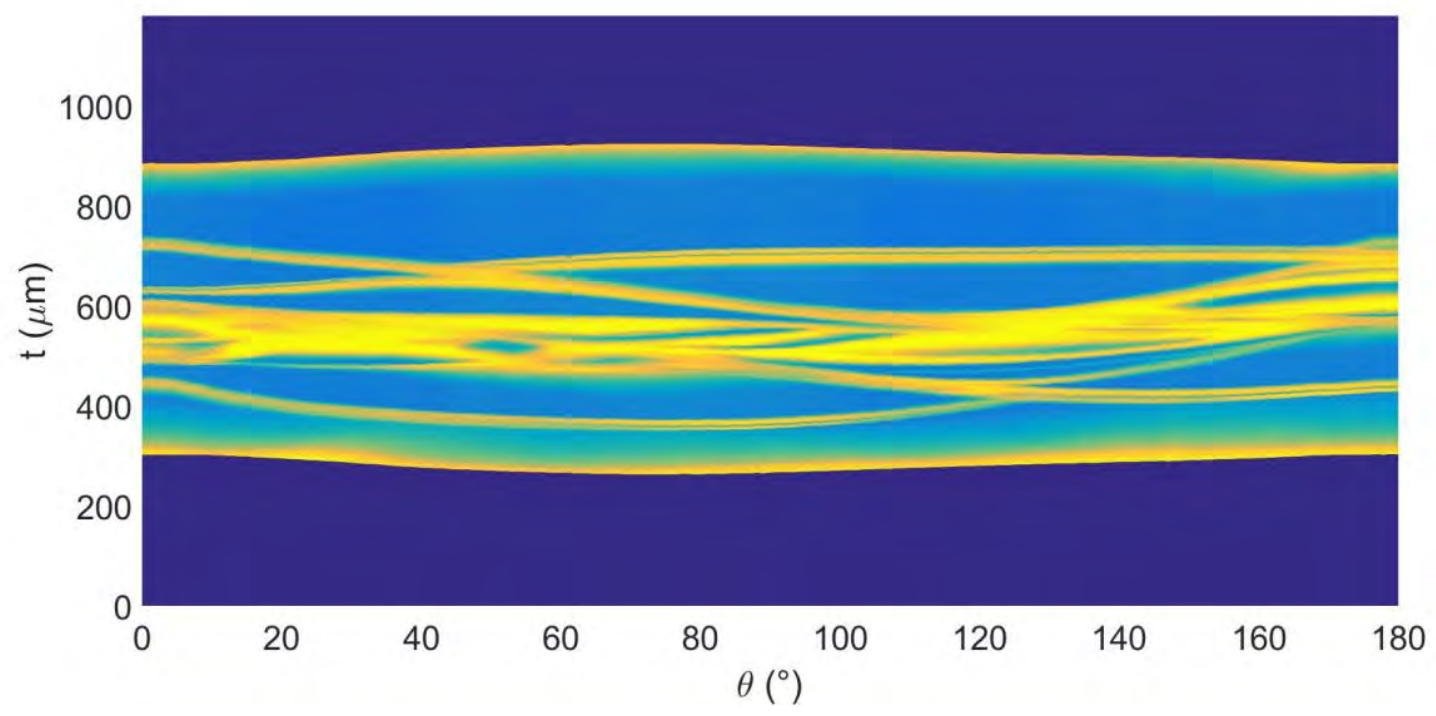

Figure 2: Tomogram of the coated triplet transmitted intensity 
Applying an inverse Radon transform on this function allows the reconstruction of the sliced view of the triplet:

$$
R^{-1} f(t, \theta)=g(\omega \cos (\theta), \omega \sin (\theta))
$$

Here, we can identify $\omega \cos (\theta)$ and $\omega \sin (\theta)$ respectively to the $x$ and $y$ parameters of a $2 \mathrm{D}$ cartesian coordinate system.

\section{Results and Discussion}

Figure $3 \mathrm{~A}$ ) shows the 2D reconstruction made through the inverse Radon transform of the tomogram shown in Fig. 2. The three optical fibers are discernable in the polymer coating and it allows the position of the fibers to be determined without cutting the sensor. As a comparison, Figure 3 B) shows an image taken with a destructive method consisting of cutting the sensor, polishing the tip and directly observing the sliced view with a microscope. The triangular shape of the triplet can be seen clearly. The darker ring around the fiber is a $15 \mu \mathrm{m}$ polyimide coating, which is thinner than the usual $60 \mu \mathrm{m}$ acrylate coating and protects the fiber to be handled during the extrusion process. The polyimide coating absorbs more light than the optical fibers or the coating itself, which is why it can be distinguished on the reconstructed image. It must be noted that the image on Figure 3 B) has been taken a few centimeters of fiber away from the measurement used for the Figure $3 \mathrm{~A}$ ) reconstruction, which is why there is are differences between them.

Our preliminary results show that the non-destructive method has two main advantages. First, cutting the sensor deforms it, so the tip view is not exactly the true form the triplet had in the coating. The non-destructive method does not have this issue. The second one is that it makes the calibration of the 3D shape sensor possible without destroying it. Even if the extrusion process usually provides stable results, the fibers positions within the sensor still need to be confirmed in order to properly calculate the curvature and then reconstruct the $3 \mathrm{D}$ shape. The destructive method would make the sensor unusable.

The curvature and direction values for 3D shape reconstruction are calculated using the strain difference between the fibers. Doing so, what really matters is the position of a fibers relative to each other. An image recognition algorithm is used to find the circles corresponding to the fibers. With the Radon reconstructed image, the relative positions of their centers can be determined with a $\pm 1.5 \mu \mathrm{m}$ precision.
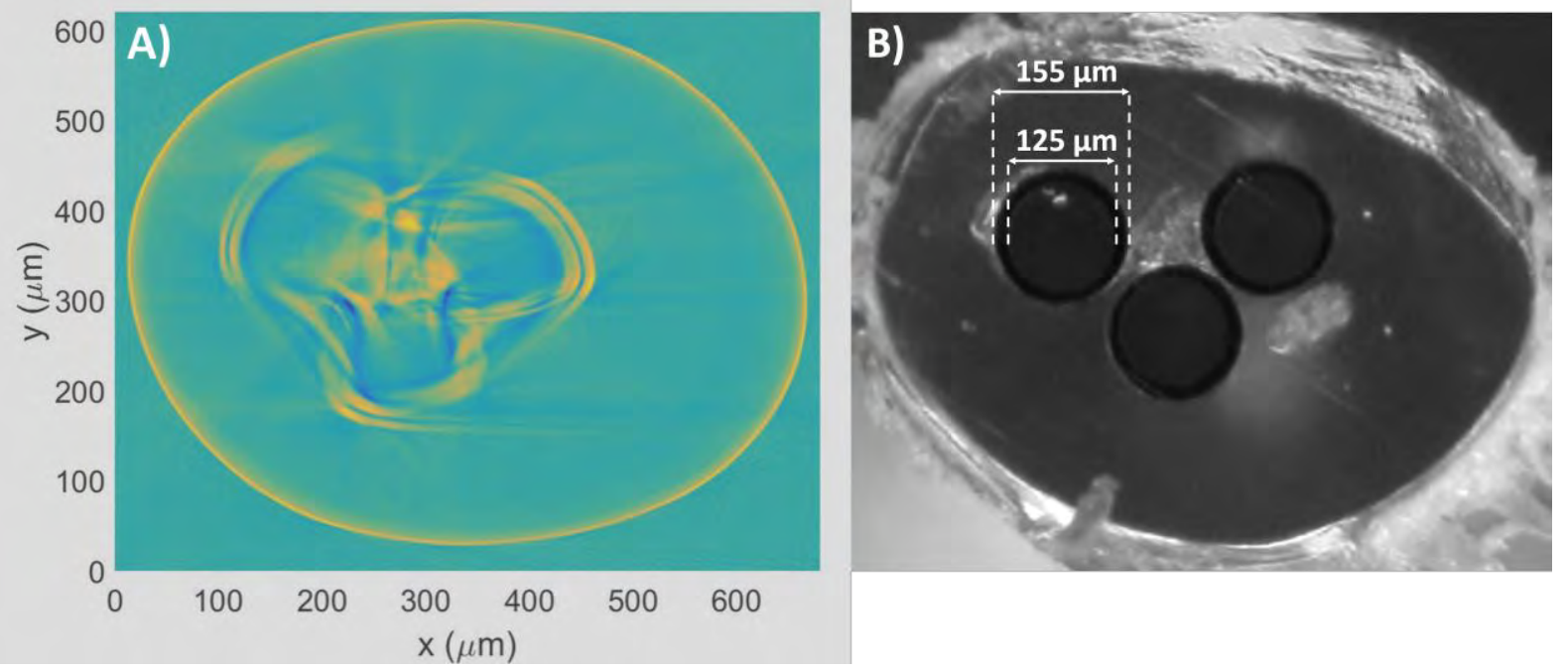

Figure 3: A) Sliced view of the coated triplet obtained applying an inverse Radon transform on the tomogram shown in Fig.2. B) Direct image of the coated triplet polished face. 


\section{Conclusion}

We have shown that using tomography, it is possible to image a relatively complex structure in a transparent polymer coating with a very good precision. This method is not only useful for characterizing the sensor itself and thus improve the 3D shape reconstruction, but also in order to validate that the extrusion process is stable. For mass production, one can imagine a similar system with the microscope and the light source rotating instead of the sensor. This system could be directly set up on the production line in order to check, in real time that the extrusion process is functioning properly.

\section{References}

[1] F. Parent, S. Loranger, K. K. Mandal et al., "Enhancement of accuracy in shape sensing of surgical needles using optical frequency domain reflectometry in optical fibers," 8(4), 2210-2221 (2017).

[2] R. G. Duncan, M. E. Froggatt, S. T. Kreger et al., "High-accuracy fiber-optic shape sensing." 6530, 65301S.

[3] M. Froggatt, and J. J. A. O. Moore, "High-spatial-resolution distributed strain measurement in optical fiber with Rayleigh scatter," 37(10), 1735-1740 (1998).

[4] J. Song, W. Li, P. Lu et al., "Long-range high spatial resolution distributed temperature and strain sensing based on optical frequency-domain reflectometry," IEEE Photonics Journal, 6(3), 1-8 (2014).

[5] H. F. Giles Jr, E. M. Mount III, and J. R. Wagner Jr, [Extrusion: the definitive processing guide and handbook] William Andrew, 48: Wire and Cable Coating (2004).

[6] P. Lorre, F. Monet, M. Gauthier et al., "Extruded optical fiber triplets for 3D shape sensing for minimally invasive surgery." Accepted Seventh European Workshop on Optical Fibre Sensors, Cyprus, EOF100-131 (2019).

[7] M.-Y. Chiu, H. H. Barrett, and R. G. Simpson, "Three-dimensional reconstruction from planar projections," JOSA, 70(7), 755-762 (1980). 


\title{
Spectroscopic properties of highly erbium doped fluorosilicate fiber
}

\author{
G. Pan*a, M. Cavillon ${ }^{\mathrm{b}}$, T. Hawkins ${ }^{\mathrm{b}}$, J. Ballato ${ }^{\mathrm{b}}$, P. Dragic ${ }^{\mathrm{a}}$ \\ ${ }^{a}$ Department of Electrical and Computer Engineering, University of Illinois at Urbana- \\ Champaign, Urbana, IL, 61822, USA \\ ${ }^{\mathrm{b}}$ Center for Optical Materials Science and Engineering Technologies (COMSET) and the \\ Department of Materials Science and Engineering, Clemson University, Clemson, SC, \\ 29625, USA
}

\begin{abstract}
A highly erbium doped (up to $5 \mathrm{wt} \% \mathrm{Er}_{2} \mathrm{O}_{3}$ ) optical fiber was fabricated using the molten core method (MCM), with core elemental constituents that include $\mathrm{Sr}, \mathrm{Al}, \mathrm{Er}, \mathrm{Si}, \mathrm{O}$ and $\mathrm{F}$. Its spectroscopic and lasing properties are investigated.
\end{abstract}

Keywords: Erbium doped fiber (EDF), high concentration, fluorescent lifetime, slope efficiency, fiber

lasers, fiber design, fiber fabrication

*gpan2@illinois.edu; phone 1217 333-8399

\section{Introduction}

The development of erbium doped fiber (EDF) has driven the progress of active fiber technology in the $1550 \mathrm{~nm}$ region, with sites currently set on passing the $1 \mathrm{~kW}$ benchmark in power scaling [1]. In order to generate $1550 \mathrm{~nm}$ $\left({ }^{4} I_{13 / 2} \rightarrow{ }^{4} I_{15 / 2}\right)$ emission from EDF, the fiber may be pumped at either $1480 \mathrm{~nm}$ or $980 \mathrm{~nm}$. The former is often referred to as in-band 'resonant' pumping $\left({ }^{4} \mathrm{I}_{15 / 2} \rightarrow{ }^{4} \mathrm{I}_{13 / 2}\right)$, while the latter pumps the system to the ${ }^{4} \mathrm{I}_{11 / 2}$ energy level from the ground state. In glasses of high phonon energy, the relaxation from ${ }^{4} \mathrm{I}_{11 / 2}$ to the ${ }^{4} \mathrm{I}_{13 / 2}$ level is non-radiative, whereas this level may become metastable in the case of low phonon energies [2]. Compounded with the relatively large quantum defect, the latter case results in low-efficiency laser operation.

The efficiency of the EDFA system can further be influenced by up-conversion and cross relaxation processes. Due to multitude of energy levels associated with $\mathrm{Er}^{3+}$, up-conversion processes become quite probable. Excitation to higher lying energy levels from a metastable or pumping level can occur through the absorption of a signal or pump photon [3,4]. Furthermore, with high concentrations of $\mathrm{Er}^{3+}$, energy transfer between neighboring excited ions in the metastable state becomes more likely and can adversely impact system efficiency [5]. The concentration of different dopants and the host glass will also impact the properties of the EDF [3]. For instance, fluoride glasses can incorporate relatively higher rare-earth ion concentrations prior to the onset of significant concentration quenching [6]. To enhance the absorption near $980 \mathrm{~nm}$, EDF routinely is co-doped with $\mathrm{Yb}^{3+}$ [7], where energy transfer from ytterbium (possessing a much higher absorption cross section) to erbium can be very efficient. However, at high power these systems ultimately are limited by amplified spontaneous emission or self-lasing by the ytterbium ion [8].

Here, a mixed anionic (O and F) fluorosilicate fiber, fabricated with cationic elemental constituents including $\mathrm{Sr}$, $\mathrm{Al}, \mathrm{Er}$, and $\mathrm{Si}$, is presented and discussed. Much like was shown with the case of similar Yb-doped glasses [9], a relatively high rare earth concentration can be doped into the glass while still maintaining good amplifier efficiency.

Sixth International Workshop on Specialty Optical Fibers and Their Applications (WSOF 2019), edited by Liang Dong, John M. Ballato, Proc. of SPIE Vol. 11206, 1120620 · () 2019 SPIE

CCC code: $0277-786 X / 19 / \$ 21 \cdot$ doi: $10.1117 / 12.2548648$ 
Spectroscopic and lasing experiments are presented, suggesting a path for a highly Er-doped silicate fiber for high power lasers.

\section{Experiment}

The optical fiber investigated here was fabricated using the molten core method (MCM) [10]. In brief, a precursor powder mixture composed of $5 \% \mathrm{ErF}_{3}, 23.75 \% \mathrm{Al}_{2} \mathrm{O}_{3}$, and $71.25 \% \mathrm{SrF}_{2}$ (in molar percentage), was inserted inside a fused silica capillary preform ( 3 and $30 \mathrm{~mm}$ inner and outer diameters). The preform was then heated to $\sim 2000{ }^{\circ} \mathrm{C}$ while inside a graphite draw tower furnace. At this temperature, the silica softens and the precursor mixture melts, enabling fiber drawing. As the fiber is being drawn, silica from the cladding is progressively incorporated into the molten precursor due to diffusion/dissolution processes, yielding to a graded-index profile silicate core - silica cladding - optical fiber. Approximately $800 \mathrm{~m}$ of fiber length was drawn, and few meters were collected and investigated for this work.

Composition analysis was performed using Wavelength Dispersive X-ray (WDX) analysis. Fiber refractive index profiles (RIPs) were measured using a spatially resolved Fourier transform interferometer with a sub-micron spatial resolution [11]. The fluorescence lifetime $(\tau)$ was measured by pulse-pumping $(977 \mathrm{~nm})$ a short fiber segment and analyzing end-emission with an avalanche photodiode. The background attenuation was obtained at $1310 \mathrm{~nm}$ using the cutback technique. By setting up a standard EDFA system (Figure 1) with a saturating $1550 \mathrm{~nm}$ signal and 1480 $\mathrm{nm}$ pumping, the slope efficiency was characterized. Low efficiency was observed when pumping at $980 \mathrm{~nm}$ and this is believed to be a result of the phonon environment in the vicinity of the Er ion. More details will be presented at the conference. As shown in Figure 1, the $1550 \mathrm{~nm}$ signal was generated by an active fiber laser system. The isolator was applied to guarantee the wave was propagating in one direction, and to prevent feedback from destabilizing the seed source. There was an angle at the end of the output passive fiber which prevented the reflected wave from being coupled back into core of fiber.

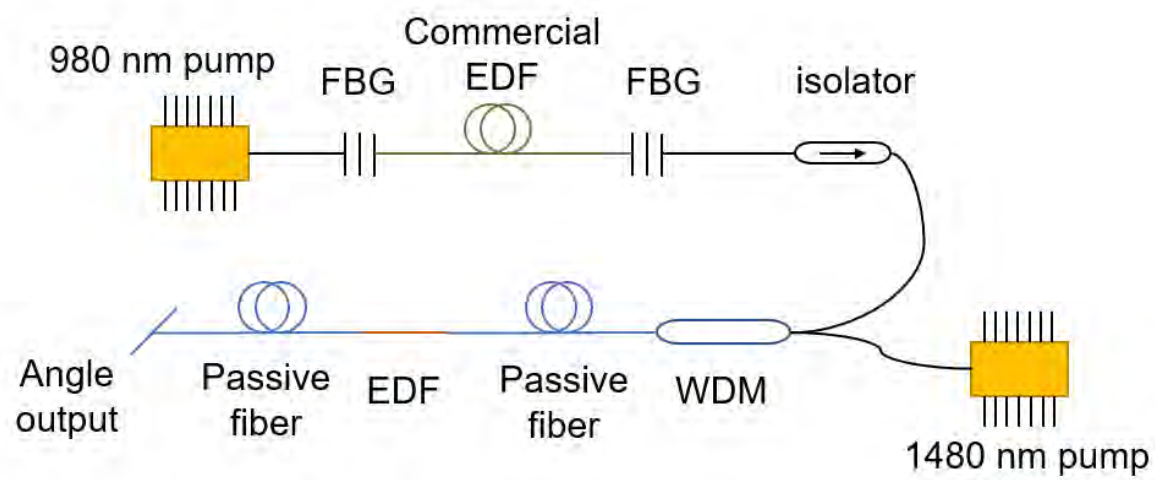

Figure 1. EDFA system setup for measuring the slope efficiency of EDF.

\section{Results and Discussions}

Five different fibers with different Er concentrations were fabricated and are summarized in Table 1. Relative to maximum absorption coefficients that can typically be found in commercial fibers [12], these fibers represent a significant increase in Er concentration. For brevity, only selected results from Fiber 1 will be shown in this abstract. This fiber core center consists of $2.2 \mathrm{wt} \% \mathrm{Er}, 15 \mathrm{wt} \% \mathrm{Sr}, 3.3 \mathrm{wt} \% \mathrm{Al}$ and $2.2 \mathrm{wt} \% \mathrm{~F}$, as indicated by the WDX data in Figure 2, in addition to $\mathrm{Si}$ and $\mathrm{O}$ (not shown). Recent work [13] has suggested that the F preferentially coordinates to the heavier cations (i.e. Sr and Er). Figure 2 also provides the measured refractive index profile (RIP). 
The maximum refractive index between the core and cladding $\Delta \mathrm{n}$ is about 0.04 . The background loss $(1310 \mathrm{~nm})$ is about $1 \mathrm{~dB} / \mathrm{m}$.

The measured fluorescence lifetime $(\tau)$ is shown in Figure 3, which is found to be roughly $8.38 \mathrm{~ms}$, which remains roughly constant across all erbium concentrations. By using the lifetime and the Füchtbauer-Ladenburg equation, the maximum emission cross section can be calculated as $9.61 \times 10^{-25} \mathrm{~m}^{2}$. The emission cross section spectrum is consequently shown in Figure 4. Figure 5 shows the absorption spectra in the near infrared region (NIR), in both the $980 \mathrm{~nm}$ and $1530 \mathrm{~nm}$ wavelength ranges. By setting up the saturated EDFA system described above, and employing in-band $1480 \mathrm{~nm}$ pumping, the slope efficiency was measured as shown in Figure 6. Since the tested fiber has a length of approximately $5 \mathrm{~cm}$, the background loss can be considered negligible. From Figure 6 , the slope efficiency takes the values of $39.6 \%$.

Results for the other fibers, with different $\mathrm{Er}^{3+}$ doping concentrations, will be shown at the conference. Power scaling for more reliable measurements (up to $10 \mathrm{X}$ the pumping power at $1480 \mathrm{~nm}$ ) is currently underway, and these results will also be shown at the conference. Results for $980 \mathrm{~nm}$ pumping will also be discussed in terms of the glass comprising the erbium host. Other practical issues, including splicing, handling, etc. will also be discussed.

\begin{tabular}{|c|c|c|c|}
\hline $\begin{array}{c}\text { Fiber } \\
\text { Number }\end{array}$ & $\begin{array}{c}\text { Lifetime } \\
(\mathbf{m s})\end{array}$ & $\begin{array}{c}\text { Absorption } \\
\text { Coefficient } \\
\text { at } \mathbf{1 5 3 0} \mathbf{~ n m} \\
\mathbf{( d B / m )}\end{array}$ & $\begin{array}{c}\text { Emission } \\
\text { Cross Section } \\
\text { at 1530 } \mathbf{~ n m} \\
\mathbf{( m}^{\mathbf{2}} \mathbf{)}\end{array}$ \\
\hline 1 & 8.38 & 250 & $9.61 \times 10^{-25}$ \\
\hline 2 & 8.40 & 263 & $8.78 \times 10^{-25}$ \\
\hline 3 & 8.58 & 373 & $9.03 \times 10^{-25}$ \\
\hline 4 & 8.91 & 491 & $8.55 \times 10^{-25}$ \\
\hline 5 & 9.12 & 391 & $8.67 \times 10^{-25}$ \\
\hline
\end{tabular}

Table 1. Properties of five different fibers.

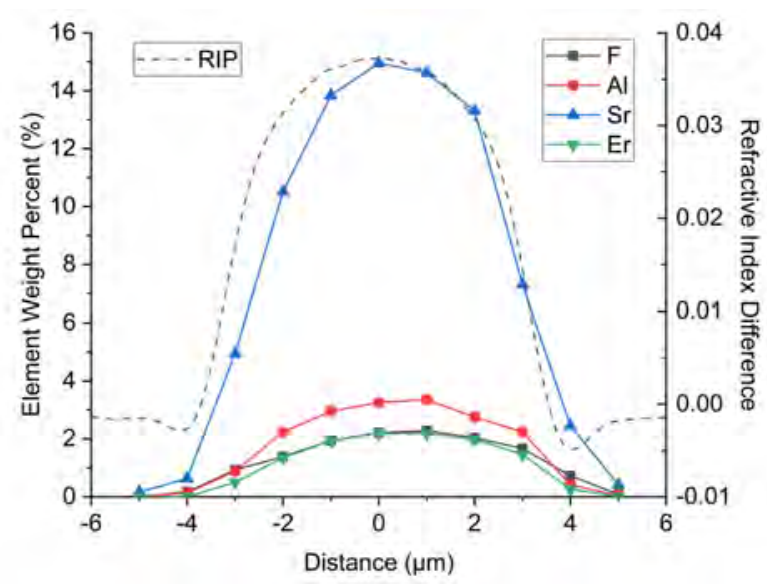

Figure 2. Composition profiles measured by WDX and fiber RIP with $0 \mu \mathrm{m}$ corresponding to the fiber core center. 


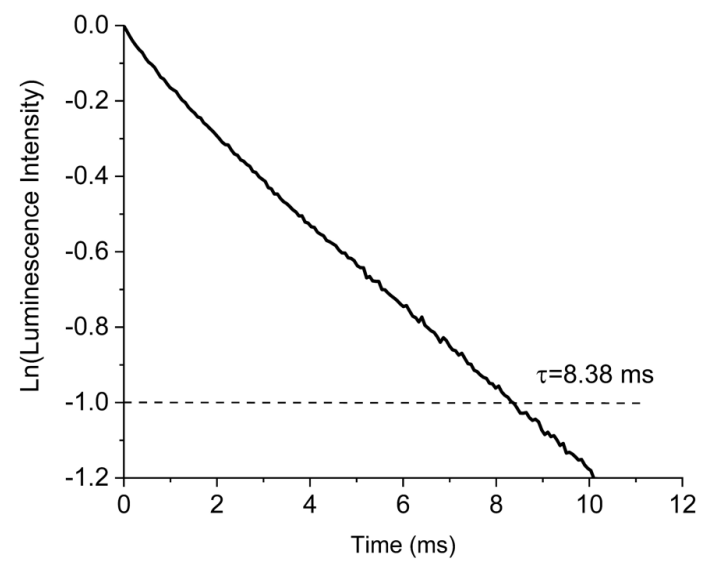

Figure 3. Fluorescent lifetime in the erbium-doped fluorosilicate fiber.

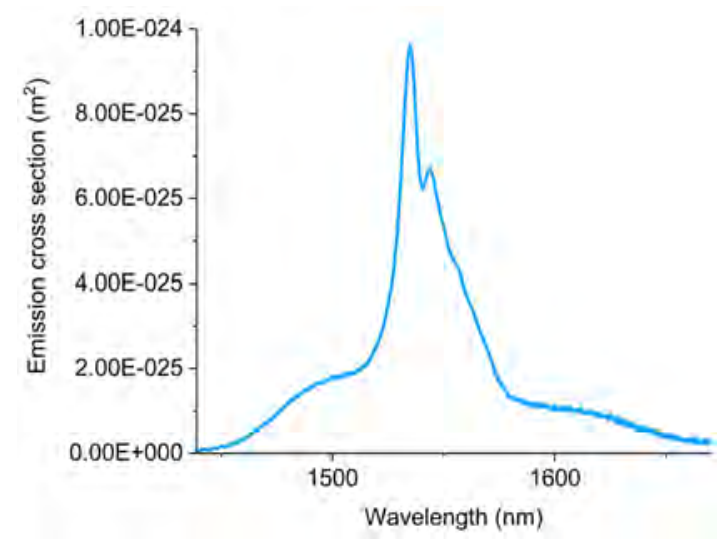

Figure 4. Emission cross section spectrum measured for Fiber 1. The emission spectrum is relatively narrow compared with aluminosilicate glasses [14].
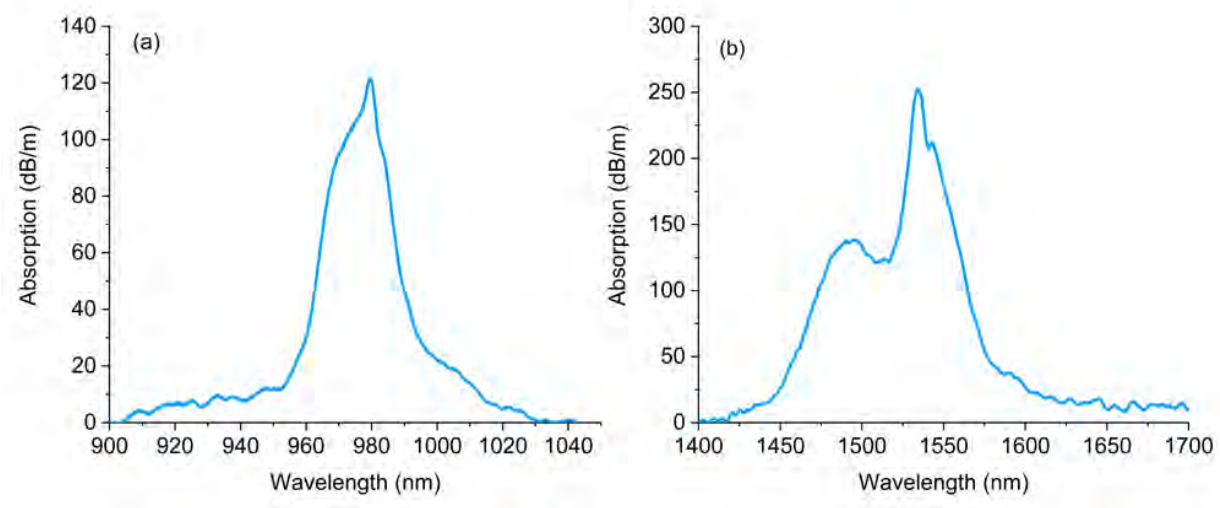

Figure 5. Fiber 1 absorption spectrum in infrared region (a) 900-1050 nm and (b) 1400-1700 nm. 


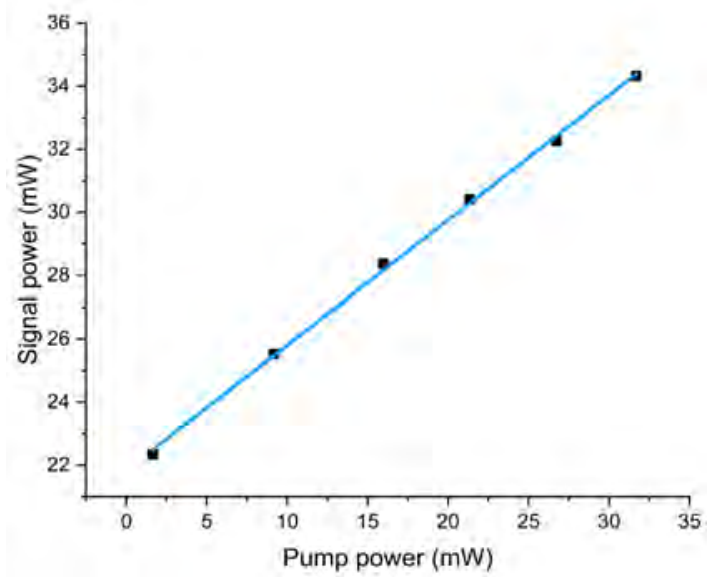

Figure 6. EDFA output power versus pump power with saturated signal input power. The slope efficiency is about $39.6 \%$. A major source of loss is believed to originate from poor mode matching between the active EDF and the EDFA system fiber.

\section{References}

[1] Kotov L.V., Likhachev M.E. (2019) High Power Continuous-Wave Er-doped Fiber Lasers. In: Ribeiro P., Andrews D., Raposo M. (eds) Optics, Photonics and Laser Technology 2017. Springer Series in Optical Sciences, vol 222. Springer, Cham

[2] Meng, Z., Kamebayashi, J., Higashihata, M. and Nakata, Y., "1.55- $\mu \mathrm{m}$ Ce,Er:ZBLAN fiber laser operation under 980-nm pumping: experiment and simulation," IEEE Photonics Technology Letters, vol. 14, no. 5, pp. 609-611, May 2002.

[3] Dragic, P. D., Cavillon, M. and Ballato, J., "Materials for optical fiber lasers: A review," Applied Physics Reviews, vol. 4, no. 4, pp. 041301, 2018.

[4] Nikolai Nikonorov, Alexander Przhevuskii, Michael Prassas, and David Jacob, "Experimental determination of the upconversion rate in erbium-doped silicate glasses," Appl. Opt. 38, 6284-6291 (1999).

[5] Blixt, P., Nilsson, J., Carlnas, T. and Jaskorzynska, B., "Concentration-dependent upconversion in Er/sup 3+/doped fiber amplifiers: Experiments and modeling," in IEEE Photonics Technology Letters, vol. 3, no. 11, pp. 996-998, Nov. 1991.

[6] Mohammed Saad, "High purity fluoride glass synthesis: a review," Proc. SPIE 7228, Laser Refrigeration of Solids II, 72280 G (9 February 2009).

[7] Colin C. Baker, E. Joseph Friebele, Ashley A. Burdett and Daniel L. Rhonehouse, "Yb-Er co-doping for resonantly pumped fiber lasers at eye-safer wavelengths (Conference Presentation)," Proc. SPIE 10528, Optical Components and Materials XV, 1052807 (14 March 2018).

[8] Grzegorz Sobon, Pawel Kaczmarek, Arkadiusz Antonczak, Jaroslaw Sotor, and Krzysztof M. Abramski, "Controlling the $1 \mu \mathrm{m}$ spontaneous emission in Er/Yb co-doped fiber amplifiers," Opt. Express 19, 19104-19113 (2011).

[9] Cavillon, M., Kucera, C., Hawkins, T. W., Yu, N., Dragic, P. and Ballato, J., "Ytterbium-doped multicomponent fluorosilicate optical fibers with intrinsically low optical nonlinearities," Optical Materials Express. 8. 744. 10.1364/OME.8.000744. 
[10] Morris, S., Ballato, J., "Molten Core Fabrication of Novel Optical Fibers," American Ceramic Society Bulletin. 92. 24-29.

[11] Andrew D. Yablon, "Multi-Wavelength Optical Fiber Refractive Index Profiling by Spatially Resolved Fourier Transform Spectroscopy," J. Lightwave Technol. 28, 360-364 (2010).

[12] https://www.thorlabs.com/newgrouppage9.cfm?objectgroup id=1504

[13] M. Cavillon, B. Faugas, J. Zhao, C. Kucera, B. Kokuoz, P. Dragic, X. Qiao, J. Du, and J. Ballato, "Investigation of the structural environment and chemical bonding of fluorine in Yb-doped fluorosilicate glass optical fibres," J. Chem. Therm. 128, 119-126 (2019).

[14] Miniscalco, W. J., "Erbium-doped glasses for fiber amplifiers at $1500 \mathrm{~nm}$," in J. Lightwave Technol. 9, 234-250 (1991). 


\title{
Applications of novel metal-derived optical fibers
}

\author{
Matthew Tuggle ${ }^{\mathrm{a}}$, Thomas Hawkins ${ }^{\mathrm{a}}$, Nanjie Yu ${ }^{\mathrm{b}}$, Guanyi Pan ${ }^{\mathrm{b}}$, Jane Gragg $^{\mathrm{a}}$, Courtney Kucera $^{\mathrm{a}}$, \\ Peter Dragic ${ }^{\mathrm{b}}$, John Ballato*a \\ ${ }^{a}$ Center for Optical Materials Science and Engineering Technologies (COMSET) and the \\ Department of Material Science and Engineering, Clemson University, Clemson SC 29625, USA \\ bepartment of Electrical and Computer Engineering, University of Illinois at Urbana-Champaign, \\ 306 N. Wright St., Urbana, Illinois 61801, USA
}

\begin{abstract}
Reported herein are two amorphous, all-oxide optical fibers derived from metal precursors, each possessing unique attributes. The first is a novel erbium-doped fiber (EDF) utilized within a traditional erbium-doped fiber amplifier (EDFA). The second is a highly doped aluminosilicate core possessing a very high ytterbium concentration sufficient for luminescence quenching. While inefficient for lasing, the luminescence quenching promotes heat generation for use as a novel all-optically driven fiber microheater.
\end{abstract}

Keywords: metal precursor, aluminum, specialty optical fiber, optical fiber fabrication, amplification, luminescence quenching

*iballat@,clemson.edu; phone 1864 656-1853

\section{Introduction}

As the demand for specialty optical fiber increases, so increases the drive for new dopants, fiber profiles, and methods for developing these new fibers. Some recently developed specialty fibers include bend-insensitive fibers for FTTH [1], multimaterial fibers [2], low Brillouin scattering optical fibers [3], low quantum defect fibers for high energy lasers [4], and semiconductor core fibers $[5,6]$. Presented within this abstract are two novel amorphous, all-oxide optical fibers derived from metals developed using the reactive molten core method ( $\mathrm{rMC}$ ). The precursor constituents of the first fiber is aluminum metal wrapped around an erbium-doped $\mathrm{YAG}\left(\mathrm{Y}_{3} \mathrm{Al}_{5} \mathrm{O}_{12}\right)$ crystal, and the second a ytterbium metal wire set inside a sapphire $\left(\mathrm{Al}_{2} \mathrm{O}_{3}\right)$ tube. The former yielded an erbium doped yttrium aluminosilicate core fiber for use within a traditional erbium-doped fiber amplifier system [7] with the added aluminum concentration in the core leading to a reduction in Brillouin gain coefficient. The latter yielded an aluminosilicate core fiber with exceptionally an high concentration of ytterbium, sufficient for luminescence quenching. While bad from a lasing standpoint, the pump power is converted to heat, considered the fiber an all-optically driven fiber microheater, generating sufficient temperatures for damaging biological cells and ablating tissue. This fiber has also found use within a Pirani gas gauge as an improved source for the thermal energy within the system [8]. Since conventional electrical heating elements tend to be impacted by electromagnetic interference, which limits their capabilities within certain applications, an all-optic fiber reduces the amount of electrical components that can be affected, potentially leading to higher accuracy gas pressure sensors.

\section{Fabrication of metal precursor derived optical fibers}

Both fibers described through this abstract were developed using the reactive molten core method (rMC) [9]. The first of the optical fibers is an erbium-doped fiber (EDF) for use within an erbium doped fiber amplifier (EDFA) system, and

Sixth International Workshop on Specialty Optical Fibers and Their Applications (WSOF 2019),

edited by Liang Dong, John M. Ballato, Proc. of SPIE Vol. 11206, 1120621 · C 2019 SPIE

CCC code: $0277-786 \mathrm{X} / 19 / \$ 21 \cdot$ doi: $10.1117 / 12.2548746$ 
the second is a highly $\mathrm{Yb}$-doped fiber utilized within an all-optic fiber microheater. For the EDF, a $1.5 \mathrm{~mm}$ diameter 2 mol\% Er:YAG $\left(\mathrm{Y}_{3} \mathrm{Al}_{5} \mathrm{O}_{12}\right)$ crystal rod (Northrop Grumman - Synoptics, Charlotte, NC) was wrapped in a $0.25 \mathrm{~mm}$ thick, high purity aluminum foil (99.999\% purity, Sigma-Aldrich). This metal foil/YAG rod assembly was inserted in a telecommunications grade, $3 \mathrm{~mm}$ inner by $30 \mathrm{~mm}$ outer diameter silica capillary preform that served as the cladding for the fiber. For the $\mathrm{Yb}$-doped microheater, a $1 \mathrm{~mm}$ diameter $\mathrm{Yb}$ metal wire (99.9\% purity, Sigma-Aldrich) was sleeved inside a sapphire $\left(\mathrm{A}_{2} \mathrm{O}_{3}\right)$ sleeve (Saint-Gobain) measuring $1.1 \mathrm{~mm}$ inner by $1.5 \mathrm{~mm}$ outer diameter. The $\mathrm{Yb}$-wire/sapphire sleeve assembly was inserted into an identical silica preform. Both fibers were drawn at a temperature of $2050^{\circ} \mathrm{C}$ to a target cladding diameter of $125 \mu \mathrm{m}$. A UV-curable acrylate coating was applied yielding a total fiber diameter of about $250 \mu \mathrm{m}$. At the draw temperature, the core constituents are molten, and the silica cladding sufficiently past its glass transition to yield a tension sufficient for drawing fiber. Further studies are necessary to understand the exact dynamics of the oxidation process of converting the $\mathrm{Al}$ metal foil to $\mathrm{Al}_{2} \mathrm{O}_{3}$ and $\mathrm{Yb}$ to $\mathrm{Yb}_{2} \mathrm{O}_{3}$ during the draw. The use of the metal constituents is intended to incorporate higher concentrations of the respective oxides in the final core for reduced Brillouin scattering for the $\mathrm{Al}$, and luminescence-quenching for the $\mathrm{Yb}$.

\section{Results and Discussion}

For brevity, the results presented herein are limited to select characteristics and performance features of the EDF and resulting EDFA. More detailed results of the Yb-doped all-optic fiber microheater will be presented at the conference.

\section{Composition}

The fiber composition was determined by energy dispersive $\mathrm{x}$-ray spectroscopy (EDX) using a variable pressure Hitachi SU-6600 scanning electron microscope operating with a beam acceleration voltage of $15 \mathrm{kV}$, an environmental pressure of $30 \mathrm{~Pa}$, and a working distance of $10 \mathrm{~mm}$. The refractive index profile (RIP) was measured transversely through the fiber using a spatially resolved Fourier transform interferometer with sub-micron resolution and is presented in Figure 1. The $\mathrm{Al}$-metal/YAG-derived fiber core possessed a composition of $1.05 \mathrm{~mol} \% \mathrm{Y}_{2} \mathrm{O}_{3}, 4.75 \mathrm{~mol} \% \mathrm{Al}_{2} \mathrm{O}_{3}$, and $94.20 \mathrm{~mol} \% \mathrm{SiO}_{2}$ (from the cladding) at the core center. The parabolic shape of the RIP is indicative of a graded index fiber, therefore the dopant concentration is expected to decrease from the core center. The erbium ion concentration was below the detectable limit, due to the low initial concentration in the precursor crystal, and during the draw, the core becomes further diluted with silica. Considering the fiber possessed a rather large refractive index difference of 0.031 between the core and cladding $(\Delta \mathrm{n})$, the measured composition is somewhat lower than expected. For example, assuming the same ratio of $\mathrm{Y}_{2} \mathrm{O}_{3}$ to $\mathrm{Al}_{2} \mathrm{O}_{3}$ (from EDX), modeling suggests the composition should be $2.0 \mathrm{~mol} \%$ and $8.9 \mathrm{~mol} \%$ respectively to achieve the same $\Delta \mathrm{n}$.

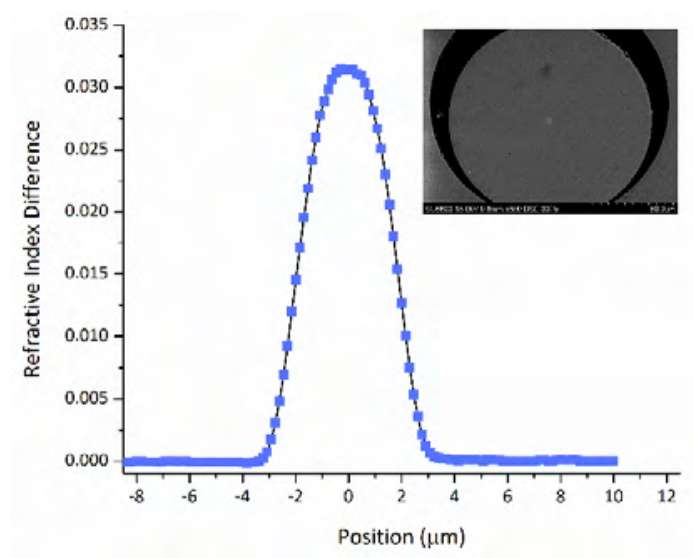

Figure 1: Refractive index profile with the 0 position being the center of the core with an image of the fiber inset. 


\section{Spectroscopy}

Attenuation was measured in the fiber using the standard cutback method and determined to be $0.25 \mathrm{~dB} / \mathrm{m}$, which is comparable to other fibers developed using the molten core method. Figure 2 provides the attenuation spectrum from 900 - $1700 \mathrm{~nm}$ showing the main $\mathrm{Er}^{3+}$ peaks at 980 and $1530 \mathrm{~nm}$ with a magnified plot inset showing the region of lowest loss between 1000 and $1500 \mathrm{~nm}$. The fluorescence lifetime was measured by pulse pumping a short segment of the fiber at 977 $\mathrm{nm}$ and analyzing the end emission with an avalanche photodiode. The pump driver used for these measurements only had a pulse duration of $\sim 6.5 \mathrm{~ms}$, therefore the measurement was carried out to $6.5 \mathrm{~ms}$ and then extrapolated to the 1/e point, found to be approximately $9.40 \mathrm{~ms}$. This value is somewhat shorter than those reported in literature, however since the lifetime is impacted by the $\mathrm{Er}^{3+}$ concentration and the local environment about the ions in the glass, it becomes notoriously difficult to directly compare glass samples. The combination of a comparable attenuation, and only a relatively shorter lifetime, further supports the hypothesis of complete oxidation of the metal precursor during fiber fabrication.

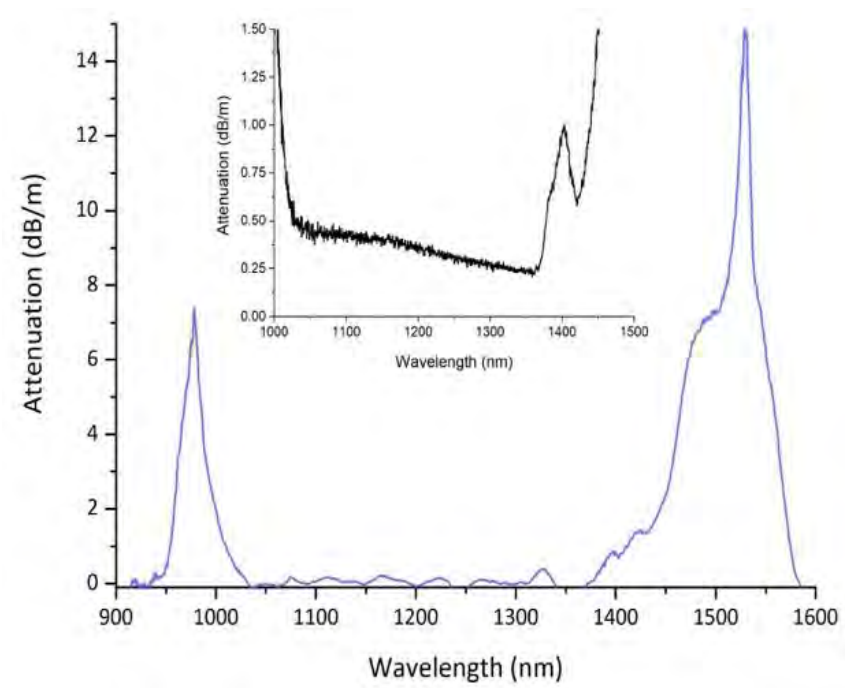

Figure 2: Attenuation plot of the metal-derived EDF over the region of $900-1700 \mathrm{~nm}$. Inset is a magnification of the region of lowest loss between $1000-1500 \mathrm{~nm}$.

\section{EDFA performance}

To test the performance of the novel EDF as an active gain medium, a traditional co-propagating EDFA system was developed. It consisted of roughly 4 meters of the EDF, a $976 \mathrm{~nm}$ pump source, $23.6 \mathrm{~mW}$ of input signal at $1550 \mathrm{~nm}$, an isolator and a wavelength division multiplexer. To minimize feedback at the facet, the fiber was cleaved at $12^{\circ}$. Figure 3 provides the output power as a function of the input pump power. The fiber exhibited a slope efficiency of $38.2 \%$ in this deeply saturated state. The relatively low slope efficiency (considering theoretical limit of $976 \mathrm{~nm} / 1550 \mathrm{~nm}=63 \%$ ) is believed to be a result of background losses at the pump and signal wavelengths and not the metal precursor. The slope efficiency was modeled using an equation found in Ref 10 . When setting the background losses to zero, the model suggests a new slope efficiency of $58.5 \%$ along a fiber of the same length. Further improvements can be made to increase the measured slope efficiency by reducing the impurity driven attenuation by incorporating higher purity precursor materials.

\section{References}

[1] Li, M.-J., Tandon, P., Bookbinder, D. C., Beckham, S. R., McDermott, M. A., Descorcie, R. B., Nolan, D. A., Johnson, J. J., Lewis, K. A., and Englebert, J. J., "Ultra-low bending loss single-mode fiber for FTTH," Journal of Lightwave Technology 27(3), 376-382 (2009) 
[2] Abourrady, A. F., Bayindir, M., Benoit, G., Hart, S. D., Kuriki, K., Orf, N., Shapiro, O., Soren, F., Temelkuran, B., and Fink, Y., "Towards multimaterial multifunctional fibres that see, hear, sense, and communicate," Nature Materials 6(5) 336-347 (2007)

[3] Cavillon, M., Kucera, C., Hawkins, T., Runge, A., Peacock, A. C., Dragic, P. D., and Ballato, J., Oxyfluoride core silica optical fiber with intrinsically low nonlinearities for high energy applications," IEEE Journal of Lightwave Technology 36(2), 284-291 (2018)

[4] Yu, N., Cavillon, M., Kucera, C., Hawkins, T. W., Ballato, J., and Dragic, P. D., "Less than 1\% quantum defect fiber lasers via ytterbium-doped multi component fluorosilicate optical fiber," Optics Letters 43(13), 3096-3099 (2018)

[5] Peacock, A. C., Gibson, U., and Ballato, J., "Silicon optical fibres - past, present, and future," Advanced Physics X 1(1) 114-127 (2016)

[6] Suhailin, F. H., Shen, L., Healy, N., Xiao, L., Jones, M., Hawkins, T., Ballato, J., Gibson, U., and Peacock, A. C., "Tapered polysilicon core fibers for nonlinear photonics," Optics Letters 41(7), 1360-1363 (2016)

[7] Tuggle, M., Kucera, C., Hawkins, T., Cavillon, M., Pan, G., Yu, G., Dragic, P., and Ballato, J., "Novel Reactive Molten Core Fabrication employing in-situ metal oxidation: erbium-doped intrinsically low Brillouin scattering optical fiber," Optical Materials X 1, 10009 (2019)

[8] Mironov, A. E., Yu, N., Park, S., Tuggle, M., Gragg, J., Kucera, C., Hawkins, T., Ballato, J., Eden, J. G., and Dragic, P., "All optical fiber thermal vacuum gauge," Journal of Physics: Photonics Special issue (Submitted 2019)

[9] Morris, S., Hawkins, T., Foy, P., McMillen, C., Fan, J., Zhu, L., Stolen, R., Rice, R., and Ballato, J., "Reactive molten core fabrication of silicon core optical fiber," Optical Materials Express 1(6), 1141-1149 (2011)

[10] Barnard, G., Myslinski, P., Chrostowski, J., and Kavehard, M., "Analytical model for rare-earth doped fiber amplifiers and lasers,” IEEE Journal of Quantum Electronics 30(8), 1817-1830 (1994) 